Slavistische Beiträge · Band 384

(eBook - Digi20-Retro)

\title{
Martin Bergmann
}

\section{Eine diskursanalytische}

\section{Betrachtung des rok-samizdat}

in der Sowjetunion und ihren

Nachfolgestaaten in der Periode zwischen 1967 und 1994

Verlag Otto Sagner München · Berlin · Washington D.C.

Digitalisiert im Rahmen der Kooperation mit dem DFG-Projekt „Digi20“

der Bayerischen Staatsbibliothek, München. OCR-Bearbeitung und Erstellung des eBooks durch den Verlag Otto Sagner:

http://verlag.kubon-sagner.de

( $)$ bei Verlag Otto Sagner. Eine Verwertung oder Weitergabe der Texte und Abbildungen, insbesondere durch Vervielfältigung, ist ohne vorherige schriftliche Genehmigung des Verlages unzulässig. 


\title{
SLAVISTISCHE BEITRÄGE
}

\author{
Begründet von \\ Alois Schmaus \\ Herausgegeben von \\ Peter Rehder \\ Beirat: \\ Tilman Berger - Walter Breu - Johanna Renate Döring-Smirnov \\ Walter Koschmal - Ulrich Schweier - Milos Sedmidubský - Klaus Steinke
}

BAND 384

\section{VERLAG OTTO SAGNER \\ MÜNCHEN 1999}




\section{Martin Bergmann}

\section{Eine diskursanalytische Betrachtung des rok-samizdat in der Sowjetunion und ihren Nachfolgestaaten in der Periode zwischen 1967 und 1994}

\section{VERLAG OTTO SAGNER MÜNCHEN 1999}


Bayeriscree Startsbibllothak München

ISBN 3-87690-743-8

(C) Verlag Otto Sagner, München 1999

Abteilung der Firma Kubon \& Sagner

D-80328 München

Gedruckt auf alterungsbeständigem Papier 


\section{Danksagung}

Die vorliegende Arbeit wurde im Dezember 1998 als Dissertation an der Ruhr-Universität Bochum angenommen. Mein Dank hierfür gebührt Herm Privat-Dozent Dr. Michael Fleischer und Herrn Professor Dr. Christian Sappok, dem Zweitgutachter der Abeit. Darüber hinaus möchte ich mich bei Frau Petra Wittrock für die tatkraftige Unterstützung in allen Lebensbereichen und bei Frau Ute Ackers für die Korrektur des Manuskripts bedanken. In erhöhtem Maße bin ich gleichfalls meinen Eltern zu Dank verpflichtet, ohne die ich dieses Projekt sicherlich nicht hätte realisieren können. 

Inhalt

0. Einleitung

11

\section{Teil I}

1. Der Konstruktive Funktionalismus von Finke ................................................... 15

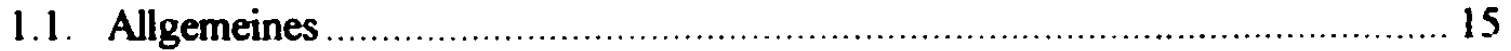

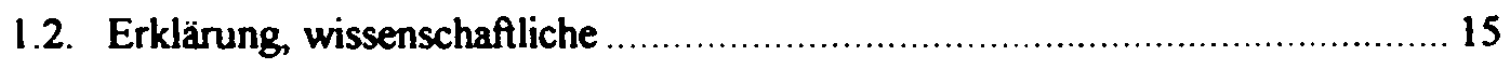

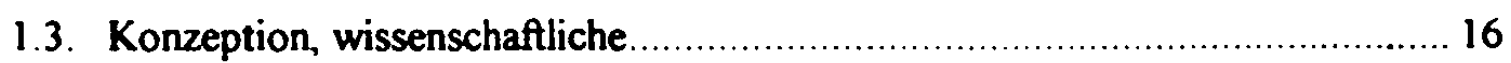

1.4. Sprachen, natürliche und wissenschaftliche ..................................... 17

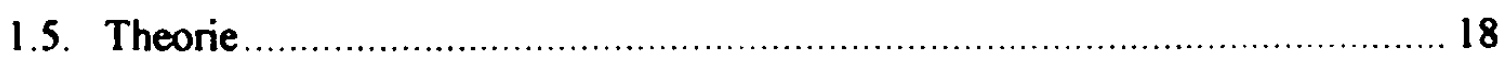

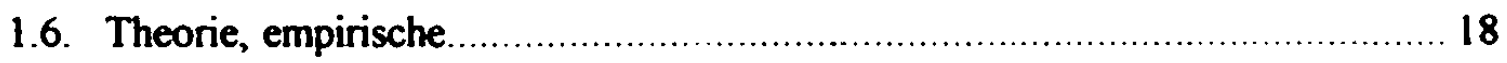

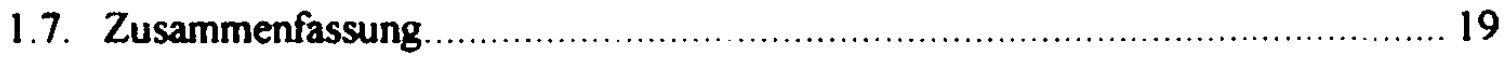

2. Die Theorie der Zweiten Wirklichkeit ......................................................... 21

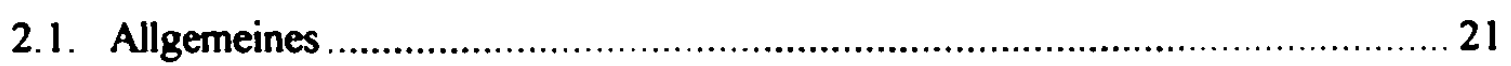

2.2. Der Objektbereich Soziales System vs. kulturelles System............................. 21

2.3. Die Begriffe Kultur und Zweite Wirklichkeit .................................... 22

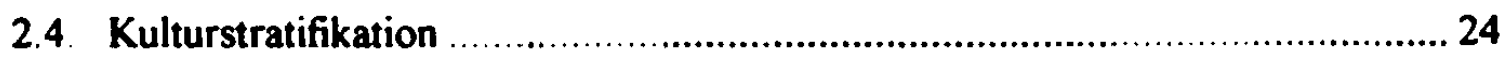

2.5. Die Begriffe Diskurs und Interdiskurs ............................................ 26

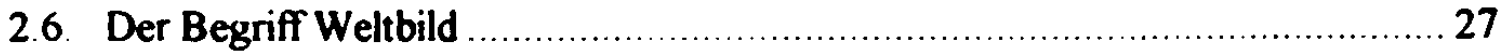

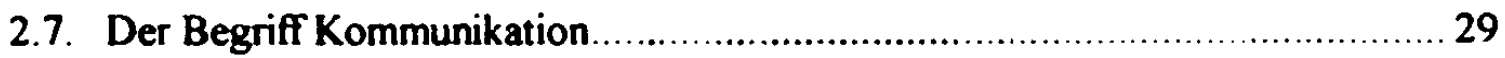

2.8. Kultursymbole: Diskurssymbole und Kollektivsymbole ............................... 31

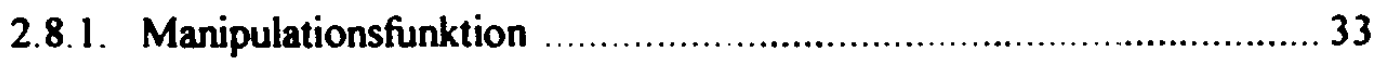

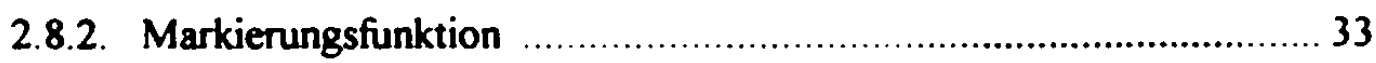

2.8.3. Zur Operationalisierbarkeit ..................................................... 33

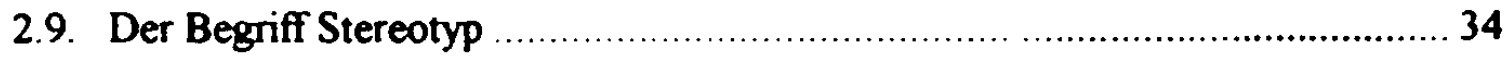

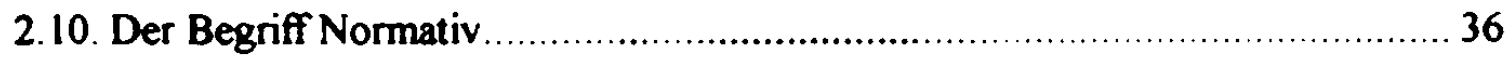

2.11. Semiotische Diskursanalyse als Grundlage empirischer Systemforschung ......... 37

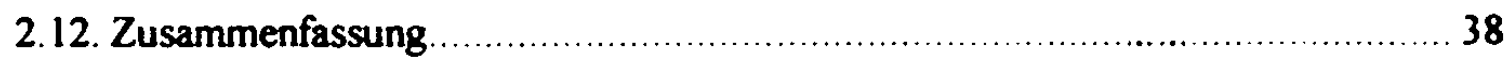




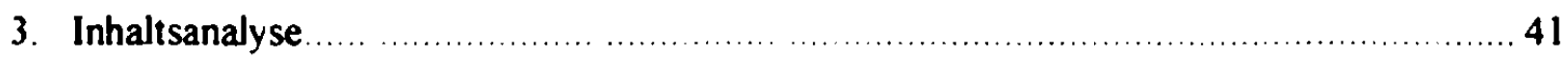

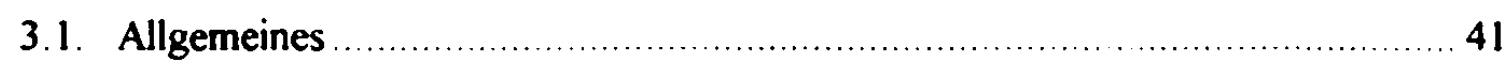

3.2. Theoretischer Ausgangspunkt .................................................... 41

3.3. Die Stellung der Inhaltsanalyse zwischen Hermeneutik und Empirie ............... 43

3.4. Die klassische Inhaltsanalyse: Auswahl-, Analyse- und Kontexteinheit............. 43

3.5. Ausgewähite inhaltsanalytische Verfahren ........................................ 45

3.5.1. Syntaktische Analyse .......................................................... 45

3.5.1.1. Die Wort-Satz-Relation: WSR ................................ 45

3.5.1.2. Das Type-Token Verhältnis (Type-Token-Ratio: TTR) ...... 47

3.5.2. Semantische Analyse ...................................................... 47

3.5.2.1. Themenanalyse ................................................ 47

3.5.2.2. Kontingenzanalyse (auch: Assoziationsanalyse) .............. 48

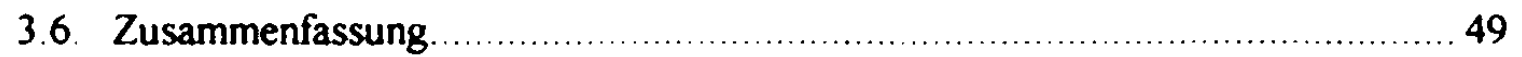

4. Sंepanskaja: Simvolika molodernoj subkul'tury. Opyt ètnograficeskogo issledovanija

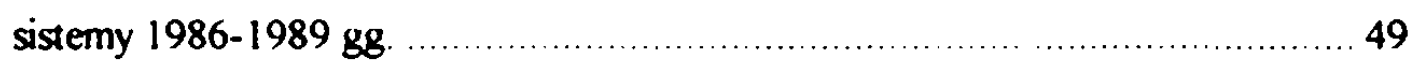

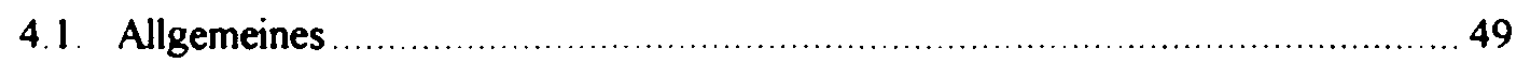

4.2. Sistema: der Versuch einer allgemeinen Bestimmung ............................... 50

4.3. Sistema und rok-samizdat ................................................................. 51

4.4. Sprachliche Aspekte der Jugendsubkultur Sistema ................................ 53

4.5. Aspekte des äußeren Erscheinungsbilds der Sistema-Mitglieder..................... 55

4.6. Aspekte der (Freizeit-) Beschäftigung der Sistema-Mitglieder ...................... 56

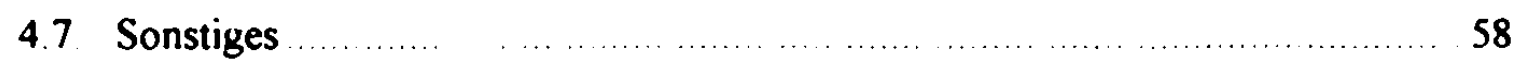

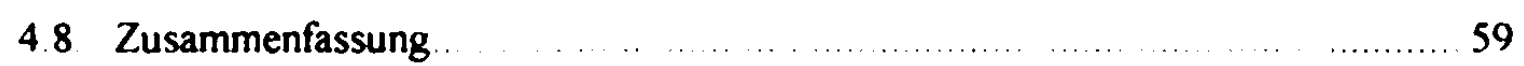

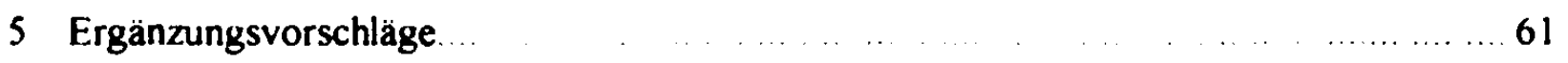

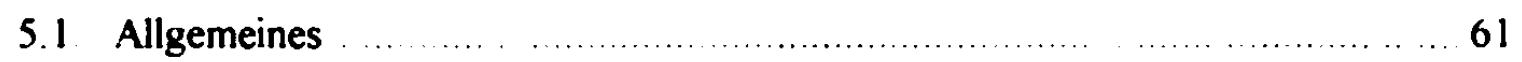

5.2. Das Bewertungskriterium charakteristisch ...................................61 61

5.3 Das Bewertungskriterium homogen ................................... 62

5.4. Nicht-interdiskursive Sprachelemente: Diskursiva . ... .........................63 63

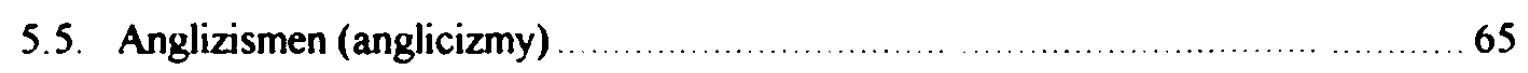

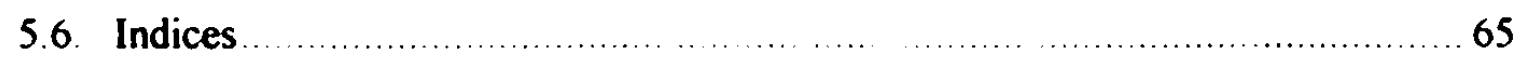

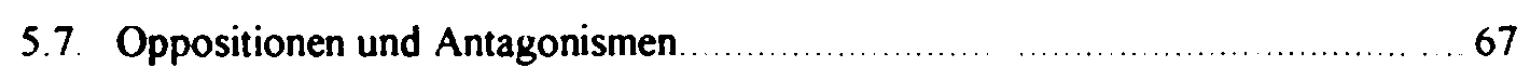

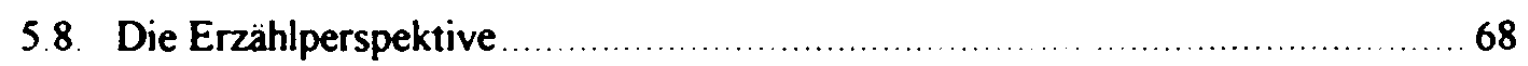

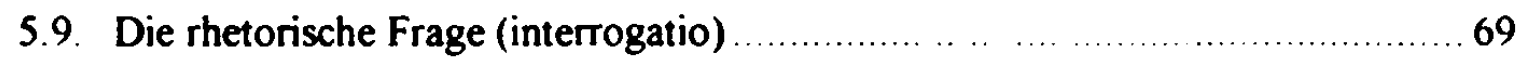

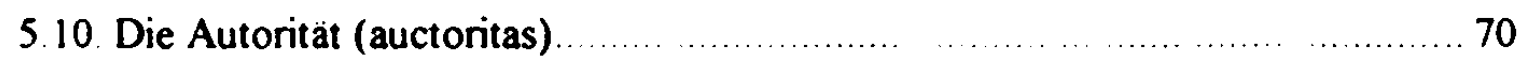

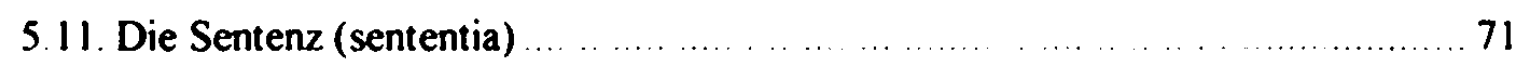

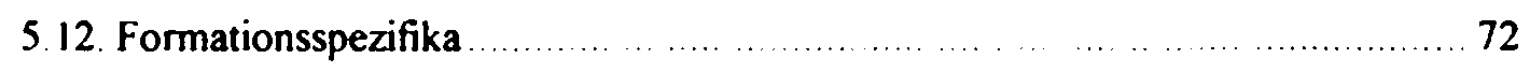

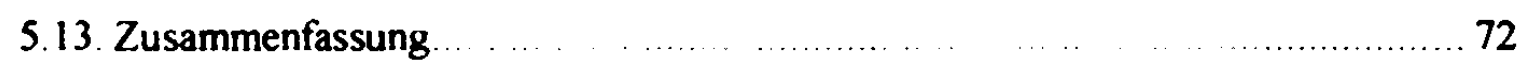




\section{Teil II}

6. Das Sample

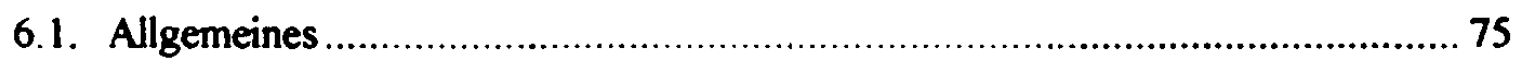

6.2. Umfang, Auflage, Herstellungs- und Vervielfältigungsart der Erstausgaben...... 76

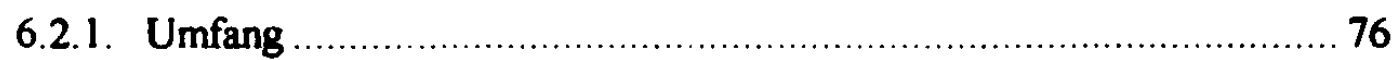

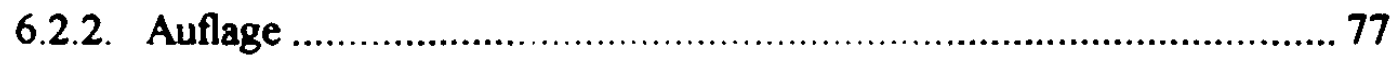

6.2.3. Herstellungs- und Vervielfaltigungsart ............................................... 78

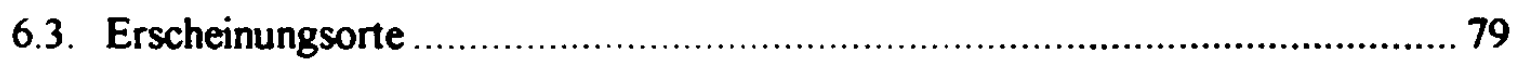

6.3.1. Die Anzahl verschiedener Publikationen pro Erscheinungsort............. 79

6.3.2. Die Anzahl verschiedener Ausgaben pro Erscheinungsort ...................8 80

6.4. Die Entwicklung des rok-samizdat: Erstveröffentlichungsjahre und

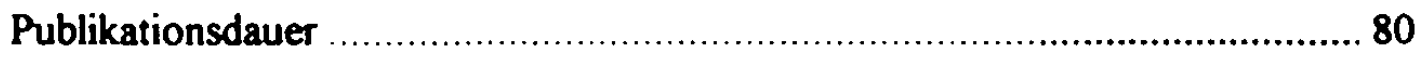

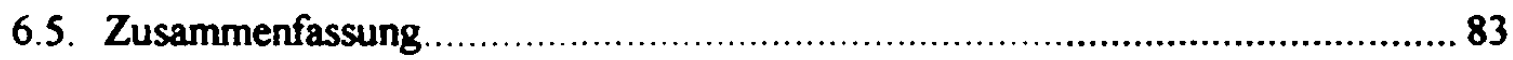

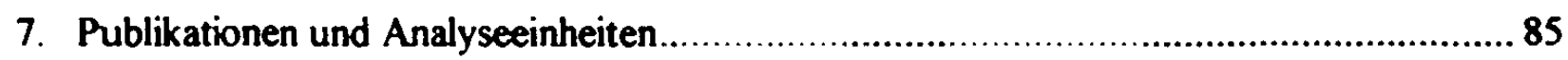

7.1. Algemeines

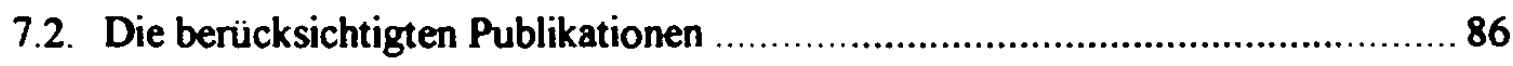

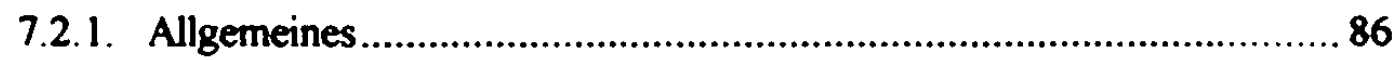

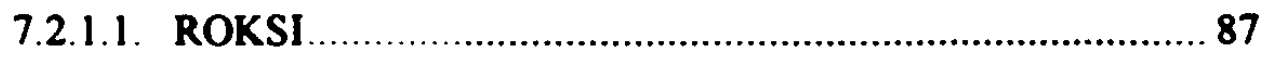

7.2.1.2. ZERKALO

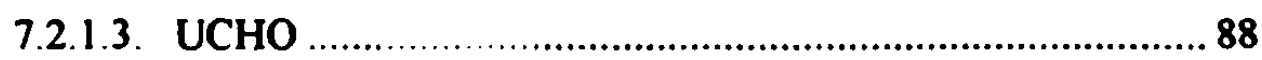

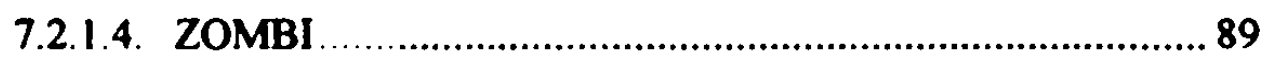

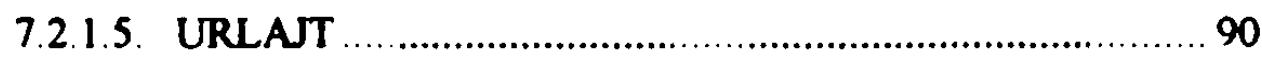

7.2.1.6. TUSOVKA (ROK-VESTNIK) .................................... 92

7.2.1.7. DVR (DALNEVOSTOCNYJ RADIO) ………................... 92

7.2.1.8. SUB"EKTIKON ………................................................ 93

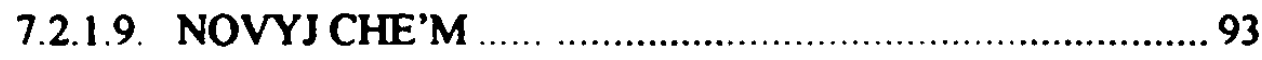

7.2.1.10. TIF (TEKUSCAAA INFORMACIJA FILOFONISTA) ........ 94

7.2.1.11. KONTR KUL'T UR'A …........................................... 94

7.2.1.12. CHERR (S MOSLOM) bZW. CHERR (BEZ MOSLA)........ 95

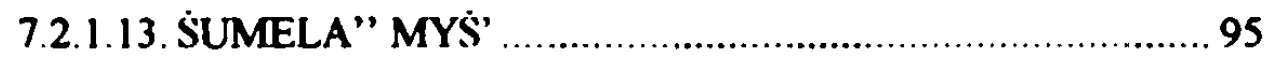

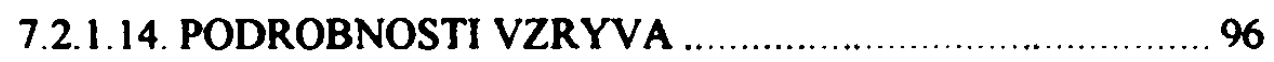

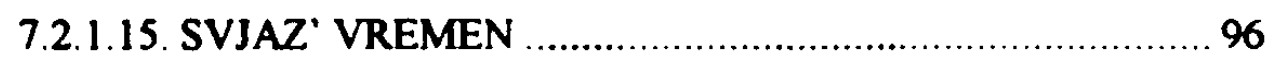

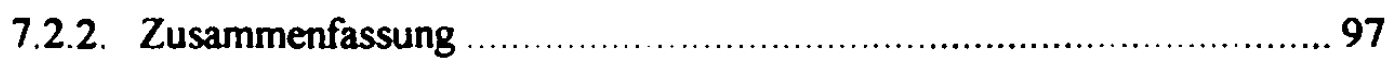

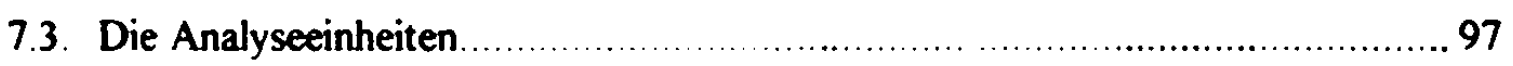


8. Eine diskursanalytische Betrachtung von: anon. [Il'ja Smimov], 1990,

Kolonka redaktora. In: URLAJT, 7 100

9. Ergebnisse der diskursanalytischen Betrachtung des Korpus ........................ 109

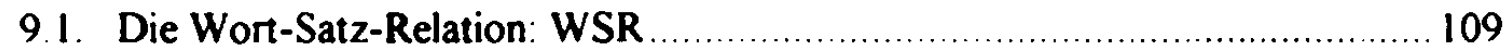

9.2. Das Type-Token-Verhältnis (Type-Token-Ratio: TTR) $\ldots \ldots \ldots \ldots \ldots \ldots \ldots \ldots \ldots \ldots$

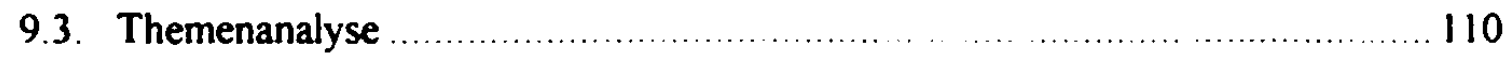

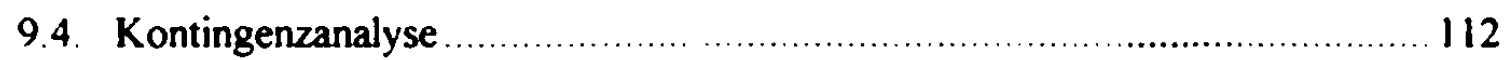

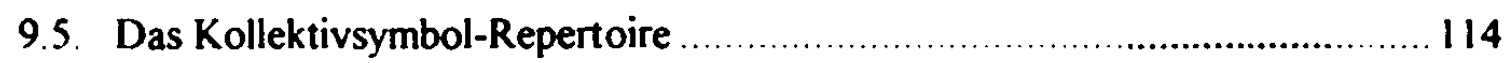

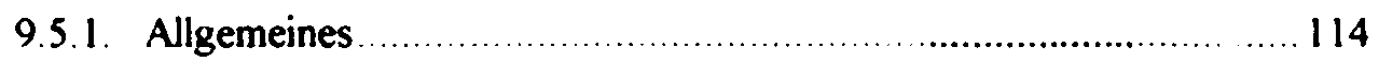

9.5.2. Zur Erhebung des Materials ............................................ 114

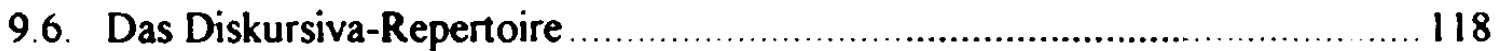

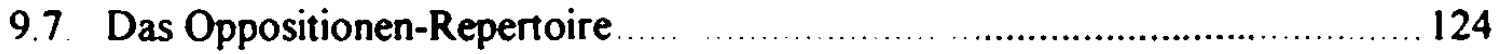

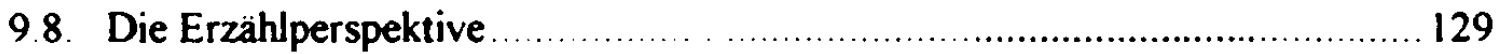

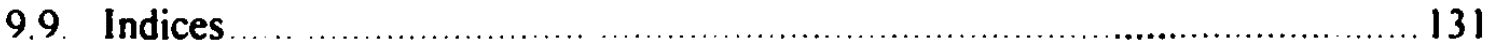

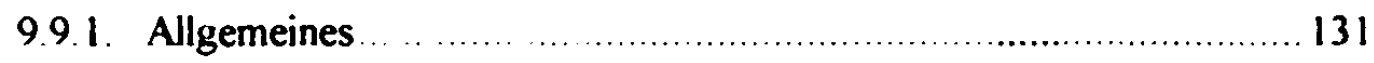

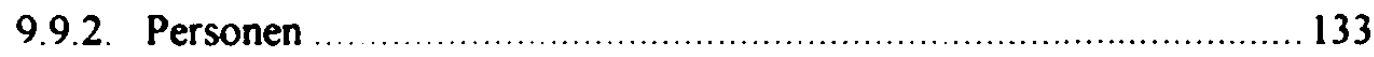

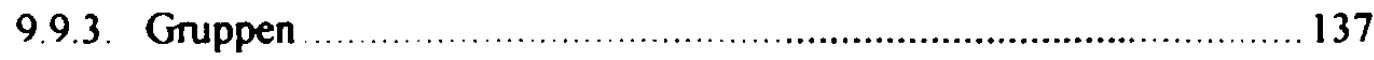

9.9.4. Geographica und Institutionen ............................................... 142

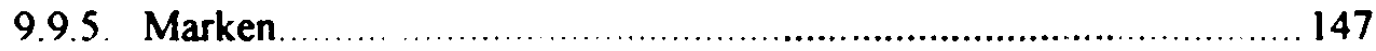

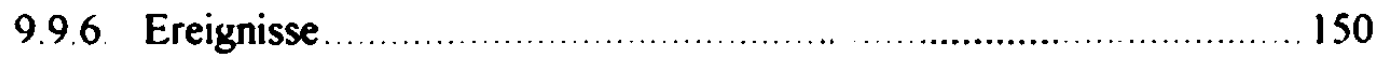

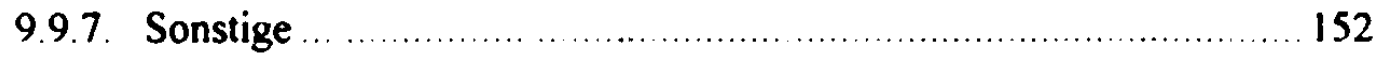

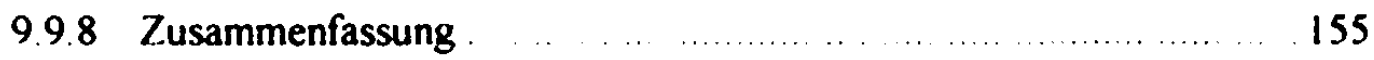

9. 10. Autoritaten $\ldots \ldots \ldots \ldots \ldots \ldots$

911 Sentenzen ... . . . . . . . . . . . . 162

912 Abgrenzungen und interne Differenzienungen 166

9.12.1 Antagonismen................................................ 166

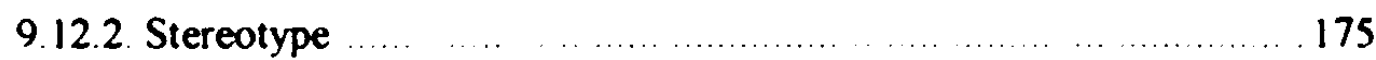

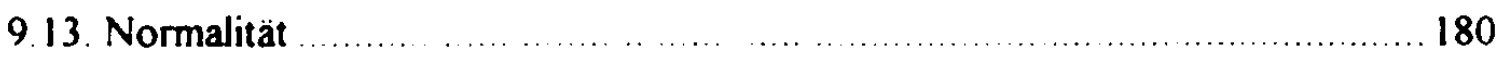

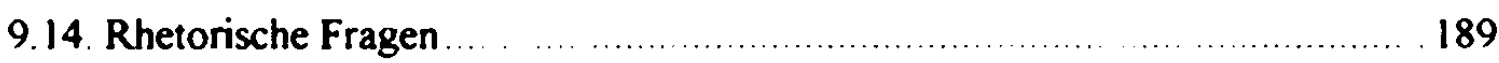

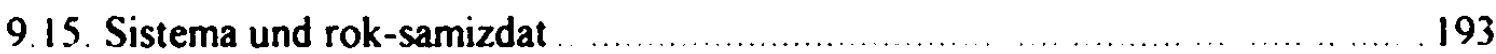

9. 16. Die Homogenität und Heterogenität des Korpus, des Diskurses und der

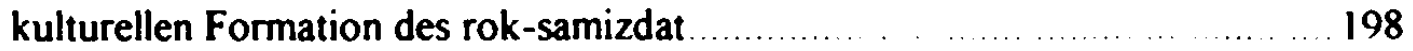

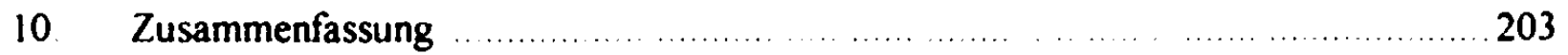

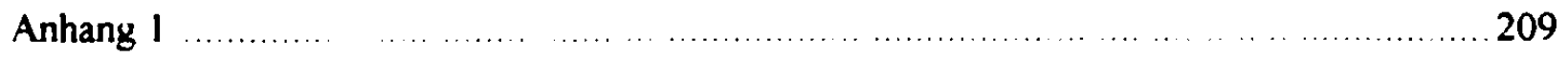

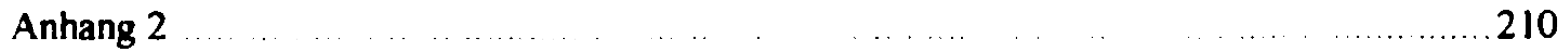

Literatur $\quad \ldots \ldots \ldots \ldots \ldots \ldots .231$ 


\section{Einleitung}

In der vorliegenden Arbeit wird das Phänomen des rok-samizdat in der Sowjetunion und deren Nachfolgestaaten in der Periode zwischen 1967 und 1994 diskursanalytisch betrachtet. Im ersten Teil der Arbeit wird das verwendete diskursanalytische Instrumentarium dargestellt, im zweiten Teil wird selbiges auf ausgewählte Texte angewendet.

Der rok-samizdat stellt eine Form des samizdat dar, der, wie im Laufe der vorliegenden Arbeit gezeigt werden soll, thematisch schwerpunktmäßig auf den Bereich der (Rock-) Musik beschränkt ist. Auf das weite Feld des samizdat, unter dem in bezug auf die Sowjetunion allgemein die illegale Tätigkeit des Selbstverlegens von Schriften verstanden wird, die sich hauptsächlich mit politischen, sozialen und religiösen Fragestellungen beschäftigten sowie seine "kulturelle Bedeutung»', wird nicht eingegangen. Bezüglich dieses Themenkomplexes sei auf die folgenden Quellen verwiesen: Meerson-Aksenov, 1977 und Skilling, 1989.

Ausgangspunkt der vorliegenden Arbeit ist zum einen die Tatsache, daß die Texte des roksamizdat bisher nicht diskursanalytisch betrachtet worden sind ${ }^{2}$. Zum anderen mangelt es an textanalytisch orientierten diskursanalytischen Verfahren, die als Eckpfeiler der forschungsleitenden Rahmenbedingungen die metatheoretischen Werte Theoretizität und Empirizität aufweisen. Der Untersuchung liegen letztendlich zwei Ziele zugrunde: ein theoretisches und ein praktisches. In theoretischer Hinsicht - dies ist das primäre Ziel - geht es um die Operationalisierbarkeit der gewählten Theorie bzw. des entsprechenden Diskursbegriffs, in praktischer Hinsicht um die Schaffung einer Alternative zu empirischen Erhebungen. Das praktische Ziel stellt ein sekundäres Ziel dar, welches sich aus dem primären Ziel ergibt. Ob das im Laufe dieser Arbeit zu entwickelnde, diskursanalytische Instrumentarium eine Alternative zu empirischen Erhebungen darstellt, soll daher nicht weiter diskutiert werden.

Grundlegend für die durchzufuhrende Analyse, die auf der Kultur- und Diskurstheorie von Fleischer (vgl : Fleischer 1994, 1995, 1996, 1997a 1997b) basiert, ist das Axiom, daß sich hinter dem Phänomen des rok-samizdat eine kulturelle Formation (vgl: 2.5.) verbirgt, die mittels eines spezifischen Diskurses kommuniziert. In diesem Diskurs werden visuell-wahmehmbare, sprachliche Kommunikationsbasen ${ }^{3}$, die im weiteren Verlauf dieser Arbeit als Texte bezeichnet werden sollen, unter Anerkennung bestimmter Regeln generient. In der vorliegenden Arbeit gett es in diesem Sinne um spezifische „Redegewohnheitsnotwendigkeiten“" (Röttgers 1988, 123), d.h.

\footnotetext{
I Der Begriff kulturelle Bedeutung soll an dieser Stelle umgangssprachlich verstanden werden. Auf die Bedeutung des Begriffs im Rahmen der zugnundeliegenden Theorie wird in Abschnitt 2.8. eingegangen.

2 Unter dem Begriff Diskursanalyse wird eine Analysemethode verstanden. die letztendlich auf Michel Foucault (vgl.: Foucault 1971, 1973. 1974 ) murickgeht und die aufgrund von (Re-) Interpretationen in verschiedenen Varianten vorliegt. Als Beispiele in dieser Hinsicht konnen die Diskurstheorien von Link (vgl: Drews: Gerhard: Link 1985: Link: Paar 1989: Link: Link-Heer 1989: Link 1975. 1979. 1982. 1983. 1986. 1991. 1992) und Fleischer (Fleischer 1994. 1995. 1996. 1997a. 1997b) genannt werden.

3 Schmidt fühn den Begriff der sprachlichen Kommunikationsbasis gemeinsam mit dem Begriff des Kommunikats ein. Unter cinen Kommunikat versteht er „... die Gesamtheit der kognitiven und emotionalen Operationen. die ein Kommunikationsteilnchmer $\mathbf{K}$ in einer Kommunikationssituation KSit einer von ihm wahrgenommenen sprachlichen Kommunikationsbasis SKB zoordnet" (Schmidt 1980. \%6. zit. nach Fleischer 1994. 393-394).
} 
Textgenerierungsregeln dieses Diskurses. Es geht darum, anhand einer Korpusanalyse festzustellen, ob charakteristische Textgenerierungsregeln und -merkmale des betrachten Diskurses nachgewiesen werden können, um auf dieser Grundlage Schlüsse über die entsprechende kulturelle Formation zu ziehen. In einem weiteren Sinne geht es darum, ein diskursanalytisches Instrumentarium zu entwickeln, welches erlaubt. Diskurse und somit kulturelle Formationen auf der Grundlage von Textgenerierungsregeln zu bestimmen.

In Abschnitt $1 \mathrm{ff}$ werden ausgewählte Aspekte der metatheoretischen Grundlage der vorliegenden Arbeit und der gewählten Objekttheorie, Finkes Konstruktiver Funktionalismus, dargestellt Danuber hinaus werden die metatheoretischen Werte eingeführ, die für die vorliegende Arbeit Gültigkeit besitzen.

Anschließend erfolgt in Abschnitt 2.ff. die Darstellung der objekttheoretischen Basis des diskursanalytischen Instrumentariums, die Kultur- und Diskurstheorie nach Fleischer. Neben dem Objektbereich werden die zentralen Begriffe und Aspekte dieser Theorie samt ihrer Operationalisienungsmöglichkeit im vorliegenden Rahmen erläutert. $\mathrm{Da}$ Fleischers Erkenntnisinteresse primär qualitativer Art ist, wird im darauf folgenden Abschnitt $3 \mathrm{ffr}$. zunachst allgemein auf die sozialwissenschaftliche Methode der Inhaltsanalyse eingegangen. Anschließend erfolgt eine Konzentration auf ausgewählte quantitative inhaltsanalytische bzw. semantische Verfahren, die sich u.a. fur vielfaltige Strukturbeschreibungen zeichenhaften Materials eignen.

In Abschnitt $4 \mathrm{ff}$ wird eine Arbeit dargestellt, die sich mit den Symbolsystemen einer als Sistema bezeichneten Jugendsubkultur beschäftigt. Die Darstellung dieser Arbeit im vorliegenden Rahmen erfolgt, da einerseits Assoziationen zwischen der genannten Jugendsubkultur und der betrachteten kulturellen Formation vermutet werden und andererseits, da sie ein heuristisches Potential bietet, das diskursanalytische Instrumentarium durch Einführung verschiedener diskursiver Objekte zu erweitern

Abschnitt $\mathbf{5} \boldsymbol{f f}$ ist der Erlauterung des crganzenden Instrumentariums gewidmet.

Das der Betrachtung zugrundeliegende Sample wird in Abschnitt $6 \mathrm{ff}$ analysiert Es geht in diesem Abschnitt im weitesten Sinne um Rahmenbedingungen der Textgenerierung.

In Abschnitt 7.ff werden ausgewählte Publikationen, aus denen die Texte des Korpus entnommen werden, charakterisiert Es soll versucht werden, personale Verbindungen aufzuzeigen, die belegen. daß das genannte Axiom sinnvoll ist.

Anschließend erfolgt in Abschnitt 8 die Anwendung des eingeführten diskursanalytischen Instrumentariums auf einen ungekurzten Text, um zu zeigen, wie auf dieser Grundlage Schlusse uber den kulturellen Kontext dieses Textes gezogen werden können.

Abschnitt $9 \mathrm{ff}$ ist der Analyse des Korpus gewidmet, welches aus 35 nahezu umfanggleichen Textfragmenten besteht

Eine Zusammenfassung der Ergebnisse. in der es einerseits um die untersuchte Formation. andererseits um die Produktivität des gewahlten Instrumentariums geht. erfolgt abschließend in Abschnitt 10 
10

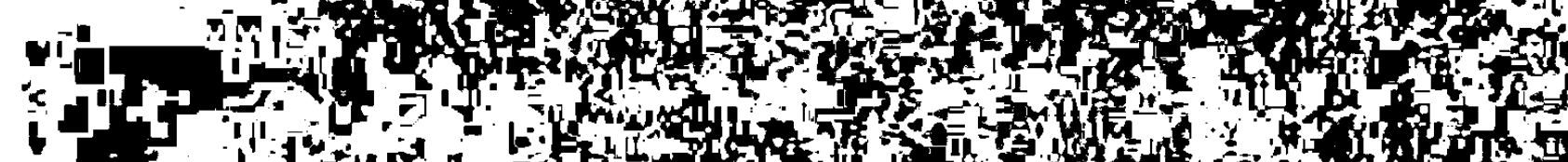

T1 5 -

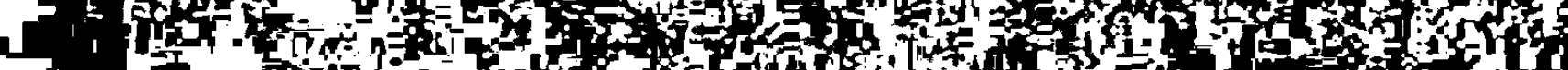
3 1.

If

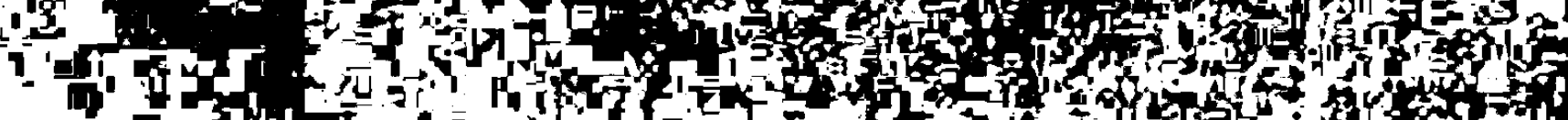
II If IThin

Ring 2110 10 of 1 1

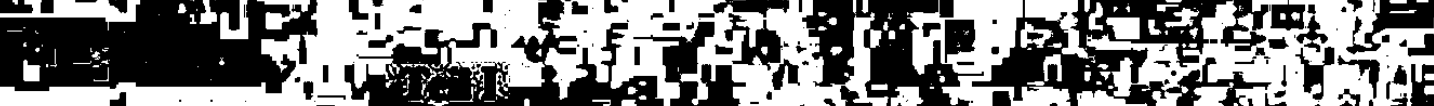

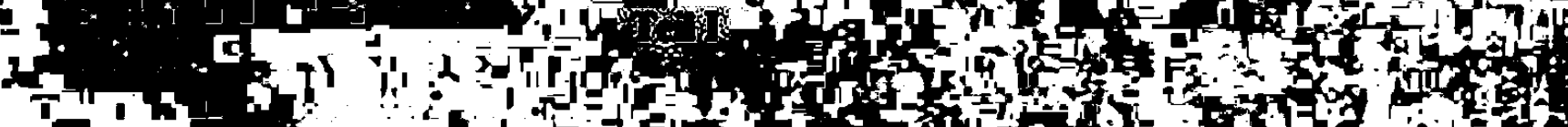
T. 슬.

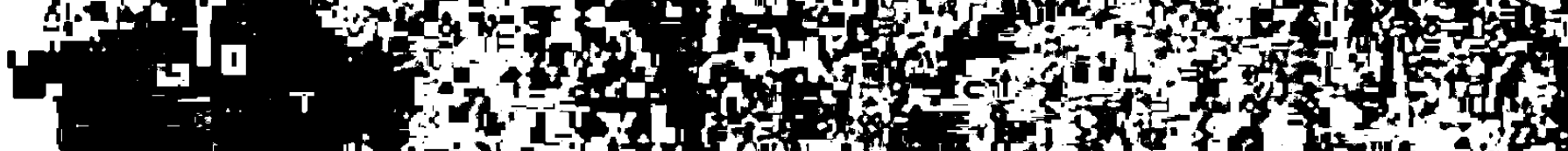

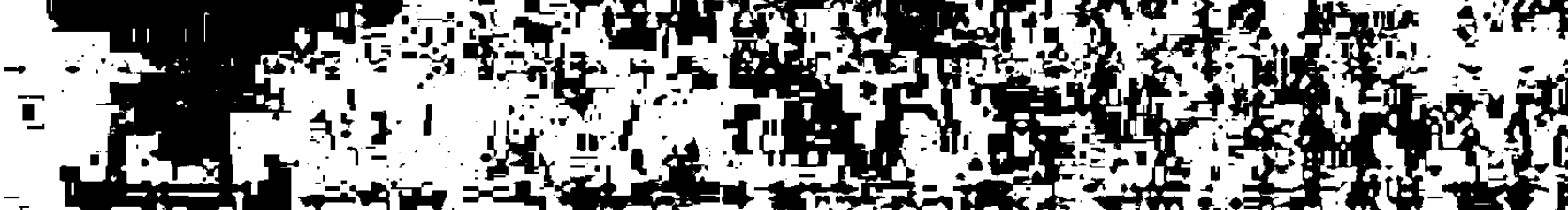

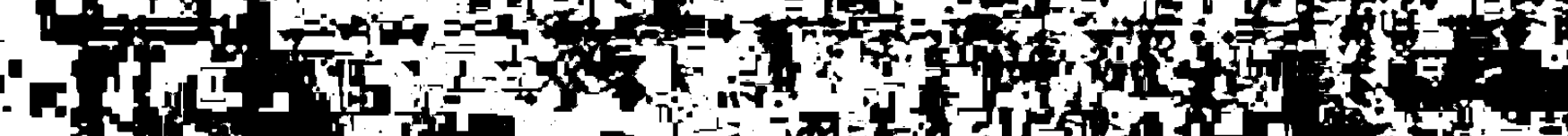

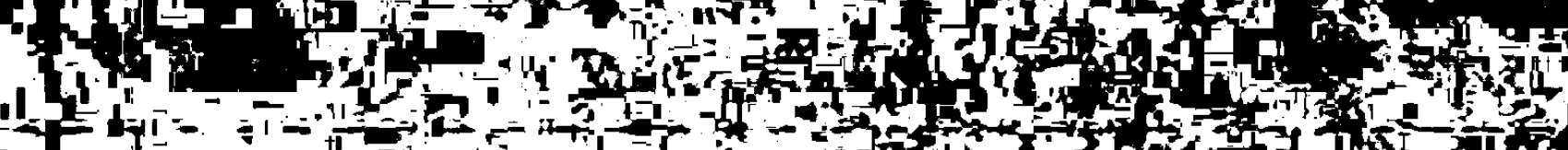

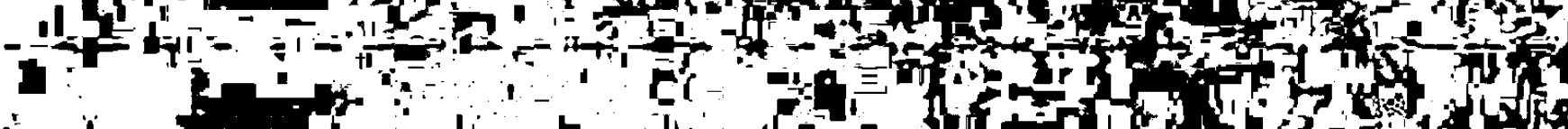

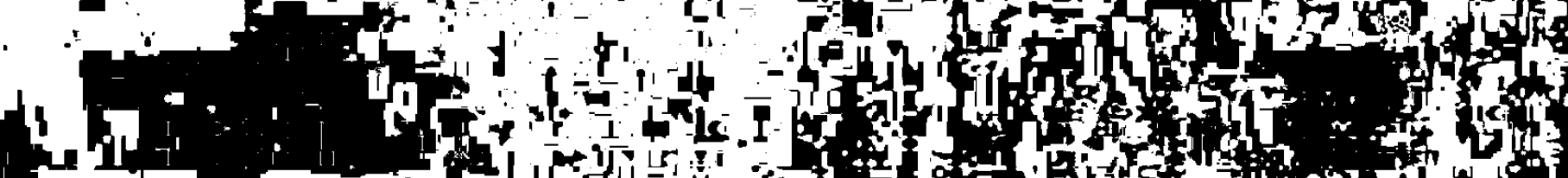
11. 1 i

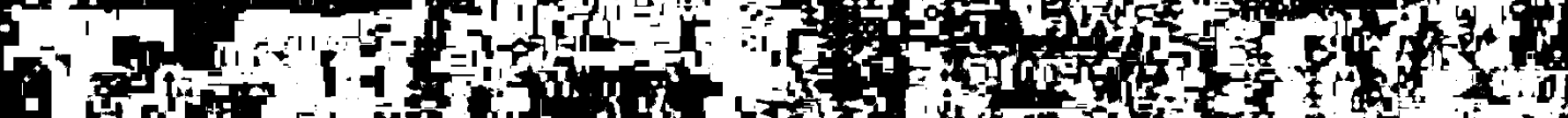

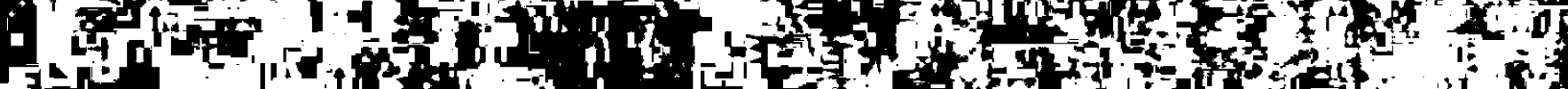

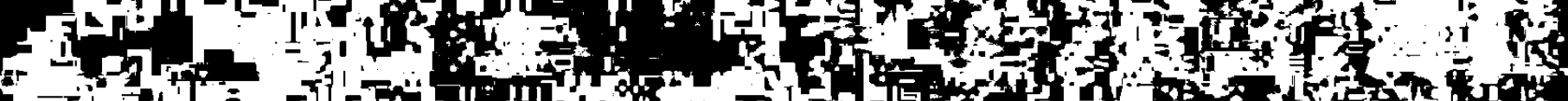

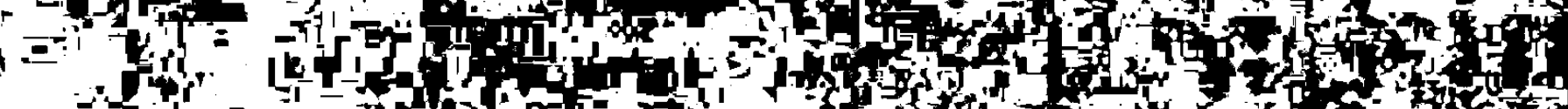
2. -1 a

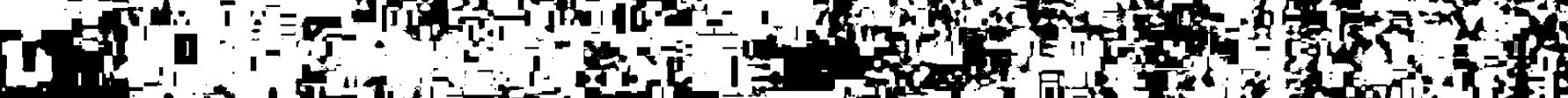

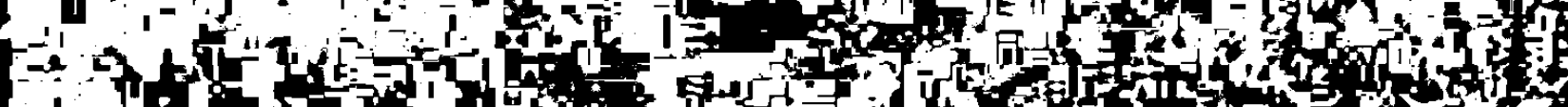

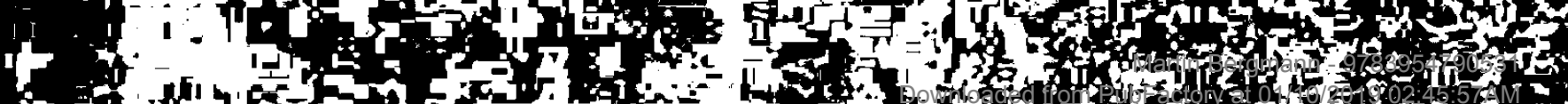


Martin Bergmann - 9783954790531

Downloaded from PubFactory at 01/10/2019 02:45:57AM

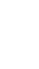




\section{Der Konstruktive Funktionalismus von Finke}

\subsection{Allgemeines}

Als Erweiterung der sogenannten Analytischen Wissenschaftstheorie ${ }^{4}$, deren Wesenszug ein rekonstruktiver Strukturalismus sei und das Phänomen Wissenschaft zu stark idealisiere, entwickelt Finke die Konzeption des Konstruktiven Funktionalismus, die als intradisziplinäre wissenschaftstheoretische Basis einer anwendungsbezogenen, empirischen Literaturwissenschaft gedacht ist. Die Empirische Literaturwissenschaft, die eine Theoretisierung des Konstruktiven Funktionalismus darstellt, interessiert an dieser Stelle nicht: es geht darum, eine wissenschaftstheoretische Konzeption darzustellen und zu analysieren, die laut Finke als eine Art Erzeugersystem fur wissenschaftliche Theorien verstanden werden kann (vgl.: Finke 1982, 229). Obwohl in der vorliegenden Arbeit keine neue wissenschafliche Theorie formuliert wird, können bestimmte Elemente dieses Systems als grundlegend für die durchzuführende, objektbezogene Untersuchung angesehen werden. Nicht alle Aspekte und Begriffe, die Finke anspricht bzw. einfuhrt, sind in diesem Zusammenhang relevant. Da die einzelnen Aspekte und Begriffe voneinander unabhängig besprochen werden, erfolgt die Darstellung in alphabetischer Reihenfolge

\subsection{Erklärung, wissenschaftliche}

Der Begriff wissenschaftliche Erklärung bezeichnet das primäre theoretische Ziel jeglicher Wissenschaft (vgl.: Finke 1982, 202). In funktionaler Hinsicht sind ,Erklärungen [...] hefriedigende Antworten auf warum-tragen" (Finke 1982, 203). Dies impliziert, daß Erklärungen Begrundungen enthalten müssen und keine reinen Beschreibungen sein können. Hinsichtlich der Struktur von Erkläungen konnen als allgemeine Begrundungstypen Strukturund Funktionserklärungen unterschieden werden: „Strukturerklärungen sind solche Erklärungen, die Fragen [... der] Form [Warum ist $X$ der Fall?] mit Antworten der Form: Weil $Y$ der Fall ist beantworten. Funktionserklänngen tun dies mit Antworten der Form: Damit $Y$ der Fall isr" (Finke 1982, 204). Die Funktion von Struktur- und Funktionserklärungen ist „die Befriedigung von bestimmten Erkenntnisinteressen, nämlich Erklärungsbedürfnissen" (Finke, 1982, 205). Hinsichtlich der Struktur von Funktions- und Strukturerklärungen gilt folgendes: „Eine hinreichende, formale Explikation dieser Struktur dürte einstweilen [...] kaum möglich sein, weil die allgemeine Erklärungstheorie noch eine Reihe von philosophischen Problemen kennt" (Finke 1982. 206). Das sogenannte D-N-Modell (Deduktiv-Nomologische Erklärung) von Hempel und Oppenheim grenzt jedoch die Anzahl der möglichen Strukturen von Erklärungen ein. „Jede deduktive wissenschaftliche Erklärung [ ...] hat danach den folgenden

4 Es soll an dieser Stelle darauf hingewiesen werden. daB der Begriff Analytische Wissenschaftstheoric eng mit dem Namen Popper (vgl.: Popper. 1973. 1973a) und dem Paradigma des Kritischen Rationalismus verbunden ist. Da Finkes Arteit nicht primatr als Kritik an Poppers Werk godacht ist. sondem allgemein anahytische Ansătze im Mittelpunkı des Interesses stehen. Wăhlt Finke die allgemeine Bereichnung. 
Strukturrahmen Das Emplanandum $H$ wird erklärt, indem man ein Explanans $E$ angibt, aus $\operatorname{dem} \mathbf{H}$ logisch folgt. Dazu muß ein Explanans zwei Klassen von Aussagen $\mathrm{A}$ und $\mathbf{G}$ enthalten, wobei die Aussagen der ersten Klasse sogenannte Antecedensbedingungen formulieren, die Aussagen der zweiten Klasse empirische Gesetzeshypothesen. Eine Erklarung hat also die Struktur: Wern $A_{1} \ldots, A_{n}$ gilt und $G_{l}, \ldots G_{m}\left[\right.$ sic!: $G_{n}$ ] gilt. damn gilt $H$. Die strukturale Interpretation dieses formalen Rahmens ist nun keinesfalls schwierig: $H$ ist deshalb der Fall. weil $A_{1} \ldots, A_{n}$ und $G_{1} \ldots$ ( $i_{n}$ " (Finke 1982, 206). Finke érgänzt das D-N-Modell um eine weitere Komponente, die er als Postcedensbedingungen $P$ bezeichnet, um den Erklärungsprozeß auch in funktionaler Hinsicht verständlich zu machen. Die Komponente $P$ steht für die Zwecke. die vermittels eines Erklärungsprozeßes erfült werden sollen. Finkes Modell hat demnach die Form: "H ist deshalh der Fall, weil $A_{1} \ldots, A_{n}$ und $G_{1}, \ldots G_{m}$ und damit

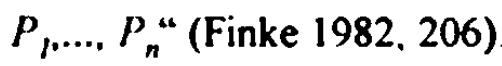

\subsection{Konzeption, wissenschaftliche}

Der Konstruktive Funktionalismus ist eine wissenschaftstheoretische Konzeption. „Wissenschaftiche Konzeptionen [...] sind Theorien, genauer: eine bestimmte Teilklasse von Theorien, nämlich Metatheorien“" (Finke 1982. 223). Der Begriff beabsichtigt eine Präzisierung des Kuhn'schen Paradigmabegriffs (vgl. Kuhn 1973): „Konzeptionen sind [...] eine Teilklasse der moglichen Metatheorien einer Theorie, nämlich genan diejenige Teilklasse, die die möglichen und faktischen Systeme unn Rahmenbedingungen. unter denen die ohjektheoretische Forschming in einer Disziplin möglich wird, definieren" (Finke 1982, 26). Bereits Kuhn hat. nachdem ihm unterschiedliche Verwendungen des Begriffs Paradigma nachgewiesen wurden. versucht. selbigen durch Erläuterung der sogenannten forschungsleitenden Rahmenbedingungen zu präzisieren Er ,hat hierfür die [. ] Idee entwickelt, Zustände von wissenschaftlichen Disziplinen durch ein System von Analyseebenen zu charakterisieren“ (Finke 1982. 214) Diese Analyseebenen. die als offenes System aufgefaßt werden. bezeichnet Kuhn zusammenfassend als Disziplinare Matrix. Diese weist die folgenden Koordinaten auf symbolische Verallgemeinerungen. Modelle, Werte und Musterbeispiele (vgl. Finke 1982, 214). .Symbolische Verallgemeinerungen sind Elemente der Sprache, die in einer Disziplin gesprochen wird; genauer diejenigen Elemente einer Fachsprache, die gesetzesartige Aspekte des disziplinären Wissens zu repräsentieren gestatten, wie "f = ma”, „(x)Fx" oder ,S $\rightarrow$ NP + VP”“" (Finke 1982, 215). Werte sind beispielsweise Einfachheit, Übereinstimmung. Widerspruchsfreiheit. Vollständigkeit. Anwendbarkeit u.a.m. (vgl.: Finke 1982. 215). Modelle sind, in der Gruppe [d.h., in einer wissenschaftiche Gemeinschaft] bevorzugte oder zulassige Analogien und Metaphem" (Weingart 1972, 295, zit nach Finke 1982, 215). Musterbeispiele umfassen „exemplarische Problemlösungen, die dazu geeignet sind. die weitere Forschung innerhalb der Gemeinschaft durch ihr Vorbild anzuleiten und auf dem rechten Wege zu halten“ (Finke 1982, 215). Zusammenfassend kann festgestellt werden, daß „der Begriff der Disziplinaren Matrix [...] eine Präzisierung dessen [ist], was man sich 
unter einem Paradigma, also einem System von forschungsleitenden Rahmenbedingungen wissenschaftlichen Handelns, vorzustellen hat. Wir können sagen: eine wissenschafiliche Konzeption $K$ ist dasjenige System forschungsleitender Rahmenbedingungen, das eine wissenschafiliche Gemeinschafi zur verbindlichen Orientierung ihres wissenschaftlichen Handelns akzeptiert und das von der Disziplinären Matrix DM ${ }_{K}$ anfgespanmt wird" (Finke 1982, 217). Aber: Der Kuhn'sche Begriff der Disziplinaren Matrix präzisiere, so Finke, zwar den Begriff eines Paradigmas, er sei jedoch selbst präzisierungsbedürttig, denn a) sind die Koordinaten kaum durch Relationen aufeinander bezogen; b) wird die interne Hierarchie der einzelnen Koordinaten nicht berücksichtigt; c) sind die Koordinaten unvollständig (vgl.: Finke 1982, 217). Da die Disziplinäre Matrix als offenes System konzipiert ist, kann der letzte Kritikpunkt vernachlässigt werden. Finke versucht Kuhns Ansatz zu präzisieren, indem er ein vernetztes Ebenensystem, in dem einzelne Ebenen aufeinander reduzierbar sind, entwickelt. Unter Hinweis auf die Reduktionsbehauptung, daß „die Erkenntnisleistungen, die vermittels von Strukturerklärungen erbracht werden können. [...] in vollem Umfange in denen enthalten [sind], die mit Funktionserklärungen erbracht werden können" (Finke 1982, 225-226), soll die Erläuterung des angesprochenen Begriffs beendet werden.

\subsection{Sprach en, natürtiche und wissenschaftliche}

Jegliche wissenschafliche Theorie wird u.a. durch sprachliche Mittel formuliert. Um die Funktion der wissenschaftlichen Sprachen, in der auch der Konstruktive Funktionalismus formuliert wird, zu verdeutlichen, soll zunächst die Funktion der sogenannten natürlichen Sprachen erläutert werden. Natürliche Sprachen sind dynamische Systeme, die stets struktur- und zweckadäquat sind und die in einen Zustand funktionaler Adäquatheit uberfuhrt werden können (vgl.: Finke 1982. 127). „Jedes kommunikative Handeln bedarf einer minimalen intersubjektiven Stabilität der in ihm verwendeten Sprachsysteme vor allem auf deren semantischer Ebene“ (Finke 1982, 126). Diese Stabilität ist das approximativ erreichte Resultat von Prozessen, die auf die Ausbildung und gemeinschaftliche Benutzung konventionell geltender Regelsysteme abzielen (vgl.: Finke 1982, 126). Diesen Prozessen liegt ein spezifischer Bedarf zugrunde, der als Sprachbedarf bezeichnet werden kann. ..Individuelle Kommunikationsbedürfnisse [...] konstituieren [...] einen überindividuellen Kommunikationsbedarf, der ein soziales Phänomen ist [...]. Dieser Bedarf wird [...] gedeckt durch die Ausbildung speziell strukturierten [sic!] Strategien, namlich Kommunikationssystemen, insbesondere Sprachen. [...] ein Kommunikationsbedarf erzeugt [...] im Bereich der menschlichen Sozialsysteme einen spezifischen Sprachbedarf und Sprachen sind diejenigen zweckadäquaten. dynamischen Systeme, die einen erheblichen Teil unserer Kommunikationsbedürfnisse zu befriedigen gestatten. Dies aber gestatten sie grundsätzlich vollständig und funktional adäquat, auch wenn keinesfalls jeder ihrer Zustände alle Lagen des Sprachbedarfs vollständig abzudecken geeignet ist“" (Finke 1982, 127-128). Der wissenschaftliche Sprachbedarf hingegen ist nur theoretisch, d.h. durch die Konstruktion von 
geeigneten Theorien adäquat abdeckbar ( $\mathrm{gl}$ : Finke 1982, 128). Er unterliegt speziellen Restriktionen, die von den jeweils forschungsleitenden Rahmenbedingungen gesetzt werden. Um den wissenschaftichen Sprachbedarf abzudecken, ist es notwendig. strukturell und funktional adaquate Sprachen, sogenannte Theoriesprachen. zu entwickeln. „Theoriesprachen sind hochspezialisierte. durch die Restriktion auf die Erklarungsfunktion als einzige Sprachfunktion spezialisierte, funktionale Erweiterungen natürlicher Sprachen. Genau genommen sind sie also nicht selber Sprachen. sondern nur Sprachausschnitte" (Finke 1982, 128)

\subsection{Theorie}

Der Konstruktive Funktionalismus ist eine wissenschaftliche Theorie. genauer: Metatheorie Metatheorien sind Theorien, deren Modelle theoretische Systeme, also andere Theorien sind. Objekttheorien hingegen sind Theorien, deren Modelle keine Theorien, sondern nichttheoretische Systeme sind. Es wird deutlich, daß der Begriff Theorie kein natürliches Objekt, sondern ein Konstrukt bezeichnet. Dieses Konstrukt besitzt für denjenigen, der es konstruiert, d.h. einen oder mehrere Wissenschaftler, die Funktion eines "Werkzeugs", welches bemuht wird, um ein Problem zu lösen. Voraussetzung für die Konstruktion einer Theorie ist ein Problem, d.h. ein Erklärungsdefizit. ..Die angezielten Lösungen sind Erklärungen, die Probleme Artikulationen von Teilen eines Erklärungsbedarfs. [...] Theorien sind [...] keine Lösungen von Problemen, sondern funktionale. zweckbestimmte Mittel zur Gewinnung solcher Lösungen. Die Lösungen sind Erklarungen, die funktionalen Mittel spezifische Strategien, die im Wissenschaftsprozeß zweckadaquat konstruiert werden müssen" (Finke 1982, 129). Zwischen Problemen und Theorien muß folglich eine funktionale Adaquatheitsrelation bestehen, wenn ein Erklarungsziel erreicht werden soll

\subsection{Theorie, empirische}

Der Begriff empirische Theorie ist ein zentraler Begriff der Konzeption des Konstruktiven Funktionalismus $\mathrm{Er}$ enthalt als wesentliche Merkmale die Werte Theoretizitat und Empirizitat, die auch als komplexes Merkmal einer empirischen Theoretizitat zusammengefaßt werden konnen. Die Aufnahme des Wertes der Empirizität in das System der theoretischen Werte wird nicht durch den Wert der Theoretizität notwendigerweise bedingt Vielmehr ist diese Aufnahme ..eine Entscheidung für einen komplexen theoretischen Wert den der empirischen Theoretizität" (Finke 1982, 104). Es kann ein struktureller und ein funktionaler Aspekt der theoretischen Empirizitat unterschieden werden „Die generelle theoretische Funktion empirischer wissenschafflicher Theorien im allgemeinen ist die ralıonaler Sirategıen sum tirwerh und zur (Iptimienung inı empirischem Wissen" (Finke 1982. 109). Es gilt in diesem Zusammenhang explizit darauf hinzuweisen, .daß es keimen scharfen oder Irivialen ('nterschied zwixchen Wissen und Meimen gibr" (Finke 1982, 110). Als empirisches Wissen konnen alle Meinungen gelten, für deren Wirklichkeitsbezug es eine Rechtfertigungsmöglichkeit über direkte oder indirekte Begründungsstrategien gibt .J Direkse Be- 
griundungsstrategien sind direkte Beobachtungen, indirekte Begriuntungsstrategien die widerspruchsfreie Anschließbarkeit an einen bereits bestehenden Erfahrungszusammenhang" (Finke 1982, 110). Alle Beobachtungen und jegliche Erfahrung, die als kohärent organisiertes System von Beobachtungen verstanden wird, sind theoriegeladen. Dieses gilt ebenfalls für direkte Beobachtungen. „Die Begründungsstrategien, die für eine Rechtfertigung der Empirizität unseres Wissens in Frage kommt, sind daher allemal theoretisch. Oder, anders ausgedrückt: Empirisches Wissen als von bloßen Meinungen unterschieden rechtfertigen heißt, es theoretisch rechtfertigen. [...] Daraus folgt, daß je expliziter und präziser unsere Theorien eines Bereiches sind, desto größer unsere Gewißheit sein darf, daß das über und durch sie formulierte Wissen ein Tatsachenwissen ist" (Finke 1982, 114). Da empirisches Wissen immer approximativ ist, geht Finke der Frage nach, ob der Typ dieser grundsätzlichen, partiellen Inadäquatheit genauer bestimmt werden kann. Er stellt fest, daß Idealisienungen eine prinzipielle Rolle in empirischen Wissenschaftsprozessen spielen. „Idealisierungen sind [...] keinesfalls mur Kennzeichen inadäquater Anfänge von Theoriebildungsprozessen. sondern grundsätzlich unvermeidbare. ja positiv zu bewertende Eigenschafien jeglicher empirischer Wissenschafr" (Finke 1982, 133).

Hinsichtlich der Struktur empirischer wissenschaftlicher Theorien weist Finke auf Folgendes hin: „Es gibt gegenwärtig zwei miteinander konkurrierende Konzeptionen des Begriffs einer empirischen wissenschaftichen Theorie" (Finke 1982, 140): die sogenannte Aussagenkonzeption (engl: statement view) und die sogenannte Begriffskonzeption (engl: non-statement-view). „Nach der Aussagenkonzeption ist eine Theorie ein in einer bestimmten Weise strukturiertes System von Aussagen, nach der Begriffskonzeption die Bedeutung eines Prädikats" (Finke 1982, 140). Die Begriffskonzeption, die auf E. W. Adams zurückgeht und v.a. von J. D. Sneed sowie Stegmüller, Moulines, Balzer und Diederich weiterentwickelt wurde. ist, so Finke, die empirisch adäquatere Konzeption, da sie eine einfache Beschreibung der Theorienidentitat und -verschiedenheit ermogglicht. .Auf dem Hintergrund dieser Konzeption können wir eine wissenschaftliche Theorie als die Bedeutung eines Prädikats auffassen. Die Bedeutung eines Prädikats ist ein Begriff. und so könmen wir auch sagen, daß Theorien Begriffe sind" (Finke 1982, 144).

\subsection{Zusammenfassung}

Es sollen die wesentlichen Aspekte des Konstruktiven Funktionalismus, die auf die gewählte Diskurstheorie bezogen werden, in zusammengefaßter Form dargestellt werden. Zunachst muß festgehalten werden. daß die Gültigkeit bestimmter metatheoretischer Werte für einen Forschungszusammenhang eine Wahl darstellt. Wenn die genannten Werte Einfachheit, Widerspruchsfreiheit, Anwendbarkeit, Empirizität, Theoretizitä und Intersubjektivität vertreten werden, so handelt es sich, abgesehen von den Werten, die für jegliche wissenschaftliche Arbeit gelten, um eine Entscheidung, die auch anders hätte ausfallen können. Ist die Entscheidung zugunsten bestimmter Werte gefallen. dann muß darauf geachtet 
werden, daß diese Werte eingehalten werden. Werden die gewählten Werte mißachtet, so kann dies als Hinweis auf die Unzulänglichkeit und letztendlich Unbrauchbarkeit einer wissenschaftlichen Theorie aufgefaßt werden

In dem hier vertretenen Sinne stellt eine Theorie ein Konstrukt dar, welches mittels einer Erklärung eine Lösung für ein Problem liefern soll. In bezug auf Erklärungen kann zwischen Struktur- und Funktionserklärungen unterschieden werden, wobei Funktionserklärungen Strukturerklärungen beinhalten bzw. voraussetzen. Beide Erklärungstypen sollen im Rahmen der durchzuführenden Diskursanalyse berücksichtigt werden. Es geht in diesem Sinne einerseits um die Struktur bestimmter diskursiver Phänomene, andererseits um deren Funktion.

Da eine empirische Theorie der Begriffskonzeption empirischer Theorien folgend, als Begriff aufgefaßt wird, markiert die Begriffsbestimmung den Aufbau der Theorie. Aus diesem Grund wird begrifflichen Bestimmungen, d.h. der Theoriesprache, im Rahmen dieser Arbeit ein besonderer Stellenwert beigemessen. Einen Punkt, auf den im Rahmen dieser Arbeit geachtet werden soll, stellt das Problem der Operationalisierbarkeit, d.h. der empirischen Prüfbarkeit des begrifflichen Instrumentariums und damit der Theorie dar. Es geht in diesem Zusammenhang u.a. um den Wert der Einfachheit, denn das Instrumentarium einer unangemessen komplizierten Theorie ist weitaus schwieriger operationalisierbar, als das einer vom Schwierigkeitsgrad angemessenen. Die maximale Einfachheit der Diskurstheorie stellt im vorliegenden Rahmen dementsprechend, von einem metatheoretischen Standpunkt aus betrachtet, eine Notwendigkeit dar.

Wie bereits angemerkt, ist das theoretische Ziel dieser Arbeit das der Problemlösung. Wie gezeigt werden kann, liegen fur den vorliegenden Zusammenhang. die diskursanalytische Betrachtung textuellen Materials, Probleme im theoretischen Bereich vor, die damit zusammenhangen, daß das primare Erkenntnisinteresse der zugrundeliegenden Diskurstheorie nicht auf textuelles Material ausgerichtet ist Zwischen begrifflichem Instrumentarium und dem zu analysierenden Objekt liegen in diesem Sinne Adaquatheitsprobleme vor, die gelöst werden können, indem die Adäquatheitsrelation «hergestellt» wird Es geht m.a.W. darum, aufzuzeigen, wie das vorhandene begriffliche Instrumentarium und somit die Theorie in bezug auf textuelles Material operationalisient werden kann Da das textuelle Material einem Diskurs ${ }^{5}$ entstammt. kann betrachtet werden, wie bestimmte Diskurselemente, beispielsweise Kollektivsymbole (vgl: 2.8.), in Texten realisient werden Daruber hinaus kann die Hypothese geaußert werden, daß die zu definierenden diskursiven Phanomene in allen Diskursen funktionieren, so daß sich Diskurse lediglich hinsichtlich ihrer faktischen Ausprägungen unterscheiden. Die Verschiedenheit der Diskurse kann als Problem aufgefaßt werden, welches einen Erklärungsbedarf evoziert. Ob dieses Problem gelöst wird. beruht auf politischen, nicht wissenschaftlichen Entscheidungen

\footnotetext{
${ }^{5}$ Der Begniff Diskurs wird an dieser Stelle in schr allgemeiner Weise veruendet. Er bercichnet an dieser Siclle sowohl dic "Sprache" cines kulturellen Systems als auch dieses selbst. Eine Differenzierung a wischen beiden Ebenen. wie sic in Abschnitt 2.5. cingefuihn wird. ist im vorliegenden Zusammenhang nicht notwendig.
} 


\section{Die Theorie der $Z$ weiten Wirklichkeit}

\subsection{Allgemeines}

In den folgenden Abschnitten soll die objekttheoretische Grundlage der vorliegenden Analyse, die von Fleischer konzipierte Kultur- und Diskurstheorie, die Theorie der Zweiten Wirklichkeit, dargestellt werden. Ein besonderes Augenmerk wird bei der Darstellung, wie bereits angekündigt, auf die Operationalisierbarkeit der zu diskutierenden Theorieelemente gelegt.

Die Theorie der Zweiten Wirklichkeit ist eine konstruktivistisch fundierte, empirische und systemtheoretische Kulturtheorie, die auf a) der funktional-semiotischen Peirce'schen Zeichentheorie (vgl: Bense; Walther 1973; Bense 1967, 1971; Fleischer 1989; Nöth, 1985; Peirce 1993), b) den Prämissen des Radikalen Konstruktivismus, wie er von Glasersfeld (Glasersfeld 1987) konzipiert wurde, c) der thermodynamisch-biologischen Systemtheorie nach Riedl (Riedl 1975), d) der Diskurstheorie nach Link (Drews; Gerhard; Link 1985; Link; Paar 1989; Link; Link-Heer 1989; Link 1975, 1979, 1982, 1983, 1986, 1991, 1992) und Fleischer (Fleischer 1994, 1995, 1996, 1997a, 1997b) und e) dem im vorangehenden Kapitel dargesteliten Konstruktiven Funktionalismus von Finke (Finke 1982) basiert.

Nicht alle Aspekte. Begriffe und Methoden der Theorie der Zweiten Wirklichkeit können und sollen im vorliegenden Zusammenhang dargestellt und analysiert werden, so daß eine Beschrănkung auf die für die vorliegende objektbezogene Untersuchung als relevant erachteten Theoricelemente erfolgt

\subsection{Der Objektbereich: Soziales System vs. kulturelles System}

Die Unterscheidung zwischen einem sozialen System und einem kulturellen System stellt eine theoretische Unterscheidung dar, die vorgenommen wird, um einen Objektbereich abzugrenzen. In bezug auf eine Wirklichkeit im Sinne des Radikalen Konstruktivismus (vgl.: Stadler; Kruse, 1990) gilt die Unterscheidung in allgemeiner Hinsicht nicht: „Beide Bereiche gehen ineinander über, beeinflussen und bedingen sich gegenseitig, sind isoliert undenkbar und brauchen auch nicht isolient gedacht zu werden" (Fleischer 1997a, 226). Das soziale System wird von Fleischer als erste Wirklichkeit bezeichnet. Hierunter versteht er .das vernetzte. hierarchische, normative, interdependente und tradierte Interaktionsgeflecht menschlicher (physikalisch meßbarer) Aktivitäten ( $\approx$ Handlungen)" (Fleischer 1997a, 226). Dieser Auffassung folgend, wird die erste Wirklichkeit als das Untersuchungsobjekt der Soziologie angesehen. Das kulturelle System, die sogenannte zweite Wirklichkeit, hingegen, die als das vernetzte, hierarchische, normative, interdependente und tradierte Interaktionsgeflecht 
zeichenhafter Prozesse und Phänomene verstanden wird (vgl: Fleischer 1997a, 226), stellt in diesem Sinne das Untersuchungsobjekt der Kulturwissenschaft dar.

\subsection{Die Begriffe Kultur und $Z$ weite Wirklichkeit}

Unter dem Begriff Kultur wird „ein zeichenhaftes Phänomen, das systemischen Charakter besitzt und als offenes dynamisches tatsächlich existierendes System zu begreifen ist" (Fleischer 1996, 166), verstanden. Der Begriff Kultur bezeichnet kein theoretisches oder hypothetisches Konstrukt (vgl: Benninghaus 1996, 15), sondern ein tatsächlich exjstierendes Objekt. Aufgrund der theoretischen Auffassung dieses Objekts als System im Sinne der thermodynamisch-biologischen Systemtheorie können verschiedene Merkmale und Eigenschaften des Systems Kultur hypothetisch angefürt werden: das System Kultur evoluiert. es ist nicht gezielt steuerbar, die Evolution nicht voraussagbar (vgl: : Fleischer 1996. 166). Kultur ist ein Phänomen, welches die Eigenschaft der Kontingenz aufweist. Des weiteren besitzt das System „Eigenschaften, die auf das System als solches zurückzuführen sind und den Systemelementen nicht zukommen (Systemeigenschafien), und Eigenschaften, die auf dessen (einzelne oder komplexe) Elemente zunükgehen" (Fleischer 1996, 166). Das System weist darüber hinaus die Eigenschaft der Selbstorganisation auf (vgl.: Fleischer 1996, 27). Kultur in diesem Sinne ist in der Lage. Schwankungen auszugleichen und einen stabilen Zustand zu erhalten (vgl: Fleischer 1996, 27). Weiterhin weist das angesprochene System eine interne Differenzienung auf, es gliedert sich in Subsysteme Jedes dieser Subsysteme besitzt konkrete Ausprägungen Es gilt das Prinzip der vernetzten und funktionalen Kausalität (vgl : Fleischer 1996, 166) Das System Kultur ist ein relationales und funktionales Gebilde (vgl. Fleischer 1996, 166), dessen Grundlage Zeichen und Zeichenprozesse sind Sowohl Zeichen als auch Zeichenprozesse erfullen bestimmte Funktionen im System Kultur bzw in den verschiedenen Subsystemen desselben und werden dort nach bestimmten Regeln generiert. Diese Funktionen und Generienungsregeln benuhen auf vier Ordnungsmustern. „Funktionen und Generierungsregeln unterliegen auf der Basis von vier Ordnungsmustern (nach Riedl 1975) - Norm, Hierarchie. Interdependenz. Tradition - Fixierungen und Determinationsprozessen, die sich jedoch gleichzeitig durch den Mechanismus der grundsatzlich gegebenen Variabilität einer endgültigen Fixierung entziehen können, sich weiterentwickeln und diese Fixierung im Dienste des Systemerhalts halten“" (Fleischer 1996. 27).

Unter dem Begriff Zweite Wirklichkeit wird in Abgrenzung zum Begriff Kultur Folgendes verstanden: „Die Zweite Wirklichkeit ist [...] die Kultur, jedoch nicht im Sinne des allgemeinen offenen Systems Kultur, sondern im Sinne der gegebenen und funktionierenden Systemausprägungen, d.h. der konkreten Realisation allgemeiner Systemgesetze. Sie liegt also in verschiedenen Ausprägungen vor" (Fleischer 1996, 23). Die Ausprägungen sollen hier in allgemeiner Weise als kulturelle Systeme bezeichnet werden. Kulturelle Systeme basieren auf 
Äußerungen, sie sind ein kognitives Konstrukt (vgl.: Fleischer 1996, 23). Unter dem Begriff Äußerung wird „das sich gegenseitig bedingende Zusammenspiel zwischen der materiell fixierten Form (und dem, was diese zuläßt oder nicht zulaßt) und dem Kommumikat (nach Schmidt 1991], der kollektiven Auffassung über die fixiente Form, wie auch die von ihnen in einer Kultur (und im sozialen System) erfullten Funktionen" (Fleischer 1996, 23) verstanden. Es geht, in der Peirce'schen Terminologie gesprochen, um die Relation zwischen einem Zeichen-Mittel und det kollektiven Auffassung über dieses Zeichen-Mittel, also um einen Zeichen-Interpretanten, der kollektiv generier wird. Da der Begriff Kommunikat nur sprachliche Zeichen umfaßt, kann abgeleitet werden, daß der Begriff Zweite Wirklichkeit ein Phänomen bezeichnet, welches auf Zeichenprozessen beruht, die innerhalb des Zeichensystems der natürlichen Sprache ablaufen. Die Zweite Wirklichkeit wird auf zwei Wegen generiert, einem allgemein-sprachlichen und einem kulturspezifischen. „Der allgemeinsprachliche Weg beruht auf den in der natürlichen Sprache generell vorhandenen und greifenden Semantisierungsmechanismen und -gesetzen (von Wörtern, Sätzen, Texten und allgemein Äußerungen), auf den (nicht wertend verstandenen) manipulativen Verfahren der natürlichen Sprache. [...] Der kulturspezifische Weg betriff jene und nur jene Semantisienungen und sonstige Operationen, die kulturspezifisch sind, die von der jeweiligen Ausprägung einer Kultur abhängen, in ihr generiert werden, kulturspezifische Funktionen aufweisen und zur Erzeugung von Differenzierungen und Zusammenhängen dienen" (Fleischer 1996, 24).

Weiterhin kann zwischen sprachlichen und kulturellen Semantisierungen und sprachlich und kulturell bedingten Aspekten der Zweiten Wirklichkeit unterschieden werden (vgl. Fleischer 1996, 24). Es geht um das Phänomen, daß in unterschiedlichen Subsystemen des Systems Kultur, d.h. in unterschiedlichen kulturellen Systemen, verschiedene (Zusatz-) Bedeutungen zu den allgemein-sprachlichen (Grund-) Bedeutungen von Zeichen generiert werden. Diese Zusatzbedeutungen werden mit dem Begriff kulturelle Bedeutung bezeichnet. Die Unterscheidung von allgemein-sprachlicher (Grund-) und kulturspezifischer (Zusatz-) Bedeutung ist eine, das soll betont werden, theoretische Unterscheidung, denn ein sprachliches Zeichen weist, wenn es generiert wird, gleichzeitig immer beide Bedeutungen auf. Nach Fleischer besitzt die Generierung der Zweiten Wirklichkeit allgemein eine manipulative Funktion: „Die Generierung der Zweiten Wirklichkeit $[(\ldots)]$ dient u.a. auch dazu, Manipulationen an der ersten Wirklichkeit [...] möglich zu machen. ohne sich an die "Tatsachen" halten zu müssen" (Fleischer 1996, 25). Es soll in diesem Zusammenhang die Frage gestellt werden, in welchem Verhältnis der sprachwissenschaftliche Begriff Konnotation (vgl: Bußmann 1990, 410) zu den Begriffen sprachliche und kulturelle Semantisierung steht. Es liegt die Vermutung nahe, daß es sich von einem wissenschaftstheoretischen Standpunkt aus betrachtet, um eine Spezialisienungsrelation handelt 
(vgl: Finke 1982, 161-164) In dieser Hinsicht spezialisieren die Begriffe sprachliche und kulturelle Semantisierung den Begriff Konnotation.

Generell sollten bei der Generienung der Zweiten Wirklichkeit mindestens zwei «Ebenen» der Manipulation unterschieden werden: die erste Ebene ist diejenige, die durch die Metapher des allgemein-sprachlichen Wegs beschrieben wird. Diese Manipulationsart hängt im weitesten Sinne mit dem Modellcharakter der Sprache zusammen. Die zweite Ebene ist die der kulturspezifischen Bedeutungsgenerierung. Dieses Phänomen hängt mit der Bildung sogenannter konsensueller Bereiche zusammen, auf die in Abschnitt 2.6. eingegangen wird. Wird im weiteren Verlauf der Begriff Manipulation verwendet, dann bezieht er sich auf die zweitgenannte Ebene.

Im Hinblick auf die Ausprägungen der Zweiten Wirklichkeit werden als wichtigste Elemente Interdiskurse, Diskurse und Weltbilder genannt (vgl.: Fleischer 1996, 23). Innerhalb dieser sind u.a. Kollektiv- und Diskurssymbole sowie Stereotype und Normative fur die Generierung der $Z$ weiten Wirklichkeit verantwortlich.

\subsection{Kulturstratifikation}

Das System Kultur wird als in vier ruckgekoppelten, vernetzten und systemisch verbundenen Subsystemen funktionierend augefaßt (vgl:: Fleischer 1994, 174). Die vier Subsysteme der Kultur werden als (i) kulturelle Gruppen, (ii) Subkulturen, (iii) Nationalkulturen und (iv) Interkulturen bezeichnet (vgl.: Fleischer 1994, 175) und folgendermaßen charakterisiert

.(i) Kulturelle (iruppen (z.B Freundeskreise, Arbeitsgemeinschaften, Studentengruppen, Cliquen usw ) stellen Bestandteile von Subkulturen dar, in denen Diskurselemente produzien und Diskurse entworfen werden, ohne daß jedoch Sicherheit bezuglich ihrer Durchsetzung besteht [( . .)]. Kulturelle Gruppen sind ein wichtiger „Ort« von Diskursmanipulationen, an dem noch die meisten Freiheitsgrade vorhanden sind, sie stellen [...] ein System maximaler Freiheit dar. Die Diskurspoduktion spielt eine untergeordnete Rolle; für die Speicherung von Diskursen sind komplexere Systeme verantwortlich.

(ii) Subkulturen bestehen aus kulturellen Gruppen unterschiedlicher Anzahl und Größe und werden durch einen Normen-, Verhaltens- oder Regelkatalog als System generiert. Sie bilden einen für sie spezifischen, wenn auch in dieser Subkultur nicht den einzig ausgeprägten Diskurs Sie sind in Abgrenzung zu anderen Subkulturen eines kulturellen Raumes und in Abgrenzung zur nachsthöheren Einheit - der Nationalkultur - gegeben. Die Produktion von Diskursen stellt hier die dominante Komponente dar. Die Speicherung des subkulturellen Diskurs-Fonds (als Spezifikum einer bestimmten Subkultur) ist deutlicher ausgeprägt (als in 
kulturellen Gruppen), die Diskurs-Manipulation dagegen schwächer. Die Manipulation dient der Auseinandersetzung mit den "Anderen«, sie festigt die eigene Subkultur, hält sie aber für externe Einflüsse offen und ermöglicht die Anreicherung und Modifizierung ihrer selbst.

(iii) Die Nationalkultur (oder Einzelkultur) umfaßt alle auf einem geopolitischen (Staats-) Gebiet vorhandenen Subkulturen dieses Bereiches, beinhaltet aber auch subkulturell oder diskurshaft benachbarte nicht-eigene nationale Subkulturen [...]. Hier dominiert eindeutig die Bewahrung von Diskursen, die aus den jeweils weniger komplexen Systemen selektiv in den Gesamtbereich eingehen, ihn jedoch nicht ganzlich ausmachen. D.h.: in der Nationalkultur sind nicht alle Diskurse aller Subkulturen enthalten und sie (die Nationalkultur) beinhaltet Diskurse, die in keiner Subkultur auftreten. Die Produktion ist sehr schwach ausgeprägt; es können und werden nur solche Diskurse bzw. Diskurselemente produzien, die allgemein "konsensfähig" sind bzw. die Eigenart einer Nationalkultur repräsentieren.

Am schwächsten ausgeprägt ist der Bereich der Diskurs-Manipulation. Das Bewahren steht auf dieser Ebene im Mittelpunkt, die Neuproduktion betriff nur bestimmte Bereiche, die von Subkulturen, [kulturellen] Gruppen nicht "geliefert» oder abgedeckt werden können. [...]

(iv) Die Imterkultur macht jenen Bereich aus, der einige wenige Nationalkulturen umfaßt. die sich als ähnlich, benachbart, gleichartig u dgl verstehen (z.B.: EG [(..)], angelsächsische, deutschsprachige, slavische Kulturen usw.).

Was die Korrelation der Speicherung, der Produktion und der Manipulation betrifft, so ist hier ein relativ ausgeglichenes Verhältnis zu beobachten. Die Manipulation bezieht sich auf das "Zurechtschneidern" vorhandener (sowohl eigner als auch fremder) Diskurse für interkulturelle Zwecke, oder aber auf eine interkulturell bedingte Umkodienung jener Diskurse, die von außerhalb der gegebenen Interkultur kommen und die nun "angepaßt" werden müssen. Gleichzeitig ist $z u$ beobachten. $\mathrm{da} \beta$ interkulturelle Diskurse weniger konstant als nationalkulturelle sind, auch ist der Gesamtbereich der Diskurse sehr großen Schwankungen unterworfen und nicht besonders stabil" (Fleischer 1994, 175-177).

Die Subsysteme des Systems Kultur werden unter Rekurs auf das Objekt Diskurs, welches unten ausfuhrlich erläutert wird, beschrieben, indem aufgezählt wird, welche Funktionen sie jeweils in bezug auf dieses Objekt aufweisen. Folgende Funktionen werden genannt: Bildung. Produktion. Entwurf, Manipulation, Speicherung und Bewahrung. Diese Funktionen setzen voraus, daß die Subsysteme aus einem bestimmten «Material» bestehen, da ansonsten diese Funktionen nicht «ausführban) sind. Als materielle Grundlage der Subsysteme kommen nur zeichenbenutzende und zeichengenerierende Aktanten in Frage, die, je nachdem auf welcher "Systemebene» sie sich bewegen, bestimmte Normen, Regeln u dgl.m. bezuglich der Zeichenverwendung, -produktion, -manipulation etc. beachten. In diesem Sinne kann die 
Nennung von Freundeskreisen, Arbeitsgemeinschaften. Studentengruppen, Cliquen usw. interpretiert werden. Diese Aktanten können als «Schnittstelle» zwischen dem sozialen und dem kulturellen System angesehen werden. Die Kulturwissenschaft, wenn sie der Konzeption der Zweiten Wirklichkeit folgt, beschäftigt sich, wie oben erläutert, ausschließlich mit sprachlichen Zeichenphänomenen. Die zeichenbenutzenden und-generierenden Aktanten sind die Grundlage bzw. Voraussetzung dieser Phänomene, sie sind jedoch explizit aus dem Objektbereich der Konzeption ausgeschlossen. Die Kulturstratifikation besitzt daher den Charakter einer Sozialstratifikation, die für die vorliegende Untersuchung nicht notwendig ist. Auf einen Operationalisienungsversuch wird dementsprechend verzichtet.

\subsection{Die Begriffe Diskurs und Interdiskurs}

Der Begriff Diskurs wird folgendermaßen definiert: „Diskurs ist das systemische Zeichenund genauer Interpretanten-Repertoire und die es organisierenden Generierungs- wie auch Benutzungregeln und -normen einer kulturellen Formation, das die gesellschaftliche und kulturelle Spezifik dieser Formation ausmacht, und das sie differenzierende Merkmalsbündel darstellt" (Fleischer 1994, 168). In der Definition wird zum einen festgelegt, was das «Material» eines Diskurses ist, nämlich vereinfachend gesagt, Zeichen und Regeln bzw. Normen, die sich auf diese Zeichen beziehen Zum anderen wird der Geltungsbereich eines Diskurses festgelegt, indem er an das Objekt kulturelle Formation gekoppelt wird. „Unter [dem Begriff] 'kulturelle[r] Formation' sind die konkreten gruppenspezifischen Ausprägungen eines jeden Subsystems des Suprasystems Kultur zu verstehen" (Fleischer 1994, 168). Auf dieser Grundlage kann die folgende Hypothese abgeleitet werden wenn eine konkrete gruppenspezifische Ausprägung eines Subsystems des Suprasystems Kultur, also eine kulturelle Formation, existiert, dann existient ein für diese kulturelle Formation spezifischer Diskurs, in dem spezifische Regeln der Zeichenverwendung gelten. Diese Hypothese wird durch folgende Aussage gestützt: „Diskurs ist die Art und Weise, wie und mit Hilfe welcher Interpretanten sich eine kulturelle Formation außert, in der Zeichenwelt und $d . h$. in der Kultur zu Wort meldet und ihren Zusammenhang gewährleistet. [..] Der Diskurs kreiert die semiotische und also kulturelle Wirklichkeit einer Formation, er wird von dieser aus dem vorhandenen oder neuen Material [(..)] generiert, er verursacht und gewährleistet ihre Diskretheit. Einfach gesagt: er bildet sie und wird von ihr gebildet" (Fleischer 1994, 168). M.a.W. kann gesagt werden, daß eine kulturelle Formation mittels eines ihr zugehörigen Diskurses kommuniziert. Grundlage kultureller Formationen sind zeichenbenutzende und zeichengenerierende Aktanten, denen diskursspezifische Konventionen in Form von Zeichen und "steuernden» Regeln und Normen bekannt sind, wenn bzw. damit sie in einer kulturellen Formation adäquat interagieren können. Bezogen auf textuelles Material kann in diesem Sinne gesagt werden, daß Aktanten einer bestimmten kulturellen Formation die spezifischen Regeln 
ihres Diskurses befolgen und Texte daher in einer bestimmten Art und Weise generieren. Ein Diskurs besitzt für eine kulturelle Formation bestimmte Funktionen, von denen im vorliegenden Zusammenhang lediglich die Differenzierungsfunktion erwähnt werden soll. Diese Differenzierungsfunktion kann von einem forschungsbezogenen Standpunkt aus genutzt werden, um kulturelle Formationen voneinander abzugrenzen. Die Funktionen einzeiner diskurshafter Phänomene werden zum gegebenen Zeitpunkt erläutert.

Der Begriff Interdiskurs wird wie folgt definiert: .Interdiskurs ist der spezifische Diskurs des Suprasystems einer Nationalkultur, der eine vermittelnde, integrative, kommunikationssichernde Funktion in diesem System ausübt und seinen kulturellen Zusammenhang ausmacht. Er ist nicht der einzige in einer Nationalkultur funktionierende Diskurs, er bildet nur den integrativen Bereich des Systems, auf den sich kulturelle Formationen, wenn notwendig, zurückziehen können, um das System zu sichern. Man kann (mit Einschränkungen) von einem Konsensbereich sprechen, auf den sich kulturelle Formationen nicht nur zurückziehen können, sondern auf den sie sich einigen müssen, um kommunizieren zu können" (Fleischer 1994, 391).

Wie deutlich wird, unterscheiden sich die Begriffe Interdiskurs und Diskurs hinsichtlich des ihnen zugesprochenen Geltungsbereichs. Die Kopplung des Begriffs Interdiskurs an den Begriff Nationalkultur empfiehlt sich aus den erläuterten Gründen nicht. Es liegt vielmehr nahe, den Begriff Interdiskurs an den Begriff Kultursystem (nicht kulturelles System; vgl.: 2.3.) zu koppeln, so daß er den spezifischen Diskurs eines konkreten Kultursystems bezeichnet. In diesem Sinne kann ein Interdiskurs als Suprasystem aufgefaßt werden, dem die Funktionen Vermittlung. Kommunikationssichenung und Integration verschiedener Diskurse zukommen. Diese Funktionen sind einer weiteren Funktion, der der Systemsichenung untergeordnet: durch den Interdiskurs wird das den einzelnen Diskursen übergeordnete Kultursystem gesichert. Diskurse besitzen die genannten Funktionen auf einer anderen Ebene, der Ebene der kulturellen Formationen Ob das Kultursystem oder eine kulturelle Formation gesichen werden soll, "entscheiden" die jeweiligen "partizipierenden», zeichenbenutzenden und zeichengenerierenden Aktanten. Wenn sich diese Aktanten gegen eine Systemsicherung auf der Ebene des Diskurses entschieden, dann müßte die kulturelle Formation, die diesen Diskurs generiert und "benutzt", zerfallen, entschieden sie sich gegen eine Systemsicherung auf der Ebene des Interdiskurses, dann zerfiele das Kultursystem. Abschließend sei darauf hingewiesen, daß mit den Begriffen kulturelle Formation und Kultursystem eine Spezialisierungsrelation in bezug auf den Begriff kulturelles System vorliegt.

\subsection{Der Begriff Weltbild}

Der Begriff Weltbild kann als Versuch einer Spezialisierung der Begriffe Diskurs und Interdiskurs aufgefaßt werden. Ziel der Spezialisienung ist es, eine differenziertere Betrachtung 
derjenigen Regeln und Normen zu ermoglichen, die für die Organisation, Generierung und Benutzung von Zeichen bzw. Zeichen-Interpretanten verantwortlich sind. Es stelk sich die Frage, welches Problemlösungspotential diese Spezialisierung für die vorliegende Analyse besitzt. Ermöglicht die Spezialisienung die Erklänung bisher nicht erklärter Phänomene, dann muß bewertet werden, ob der theoretische Aufwand, der mit der Spezialisienung betrieben wird, durch den Erkenntnisgewinn gerechtfertigt wird. Diese Bewertung geht mit der Frage einer möglichen Operationalisienung im vorliegenden Rahmen einher. Eine Bewertung hinsichtlich des Erklärungspotentials ist, bis auf den Fall der Unmöglichkeit der Operationalisierbarkeit des Begriffs, letztendlich subjektiv, da kein Maß existien, das bei der angesprochenen Bewertung behilflich sein könnte. Die Bewertung soll hier auf einer Analyse der Definition des Begriffs Weltbild basieren, die zum einen aus einer als Arbeitsdefinition bezeichneten Bestimmung des Untersuchungsobjekts (vgl: Fleischer 1996, 129), zum anderen aus zusätzlichen definitorischen Ergänzungen, die in Form von Funktionszuweisungen vorliegen, besteht. In beiden Definitionsteilen werden zum Teil implizit Funktionen angefürt, die dem Phänomen Weltbild zugesprochen werden. Es gilt, die aus der Arbeitsdefinition bzw. aus den definitorischen Bestimmungen abzuleitenden Funktionen zu extrapolieren und hinsichtlich ihres Erkläungspotentials für den vorliegenden Zusammenhang zu bewerten. In der erwähnten Asbeitsdefinition, die auf die für die Analyse wesentlichen Aspekte reduziert ist. wird der Begriff Weltbild folgendermaßen bestimmt:

"Weltbilder sind von der ersten Wirklichkeit beeinflußte, von und in der Zweiten Wirklichkeit produzierte Konstrukte, die zur Generierung, Organisation, ausprägungsspezifischen und -gemäßen Aufrechterhaltung eines Kultursystems, das ihre Beschaffenheit selbst mitbestimmt hat, wie auch zur Steuerung der Kommunikation im gegebenen Kultursystem dienen. Weltbilder sind Regulative im Sinne der Systemtheorie Sie liefern die Semantisierungskriterien für die Konstruktion der Zweiten Wirklichkeit innerhalb eines Kultursystems und entscheiden uber die darauf bezogene Organisation von Äußerungen, die diese Wirklichkeit repräsentieren“ (Fleischer 1996, 129). Des weiteren werden „im Weltbild [..] die tatsächlichen kollektiven Überzeugungen der Kulturteilnehmer und Regeln zur Herstellung dieser Überzeugungen wie auch das, was das intersubjektive, kollektive Handeln im Kommunikationssystem Gesellschaft bestimmt, gespeichert. [ ..] im Weltbild ist das sichtbar, was langfristig. konstant, stabil usf. ist und was das gesamte Kollektiv organisiert. Das Weltbild organisien das gesamte System in langen Zeiträumen und für lange Zeiträume, somit ist es ein Regulativ fur Diskurse auf einzelkultureller Ebene [d.h. für Interdiskurse], das aber in den Diskursen abgeschwächt, instrumentalisiert und strategisch bedingt eingesetzt werden kann. Das Weltbild reguliert die Diskurse und wird gleichzeitig von ihnen generiert. Weltbilder weisen daher zwei generelle Funktionskomplexe auf: Es ist einerseits das Bündel systemsichernder und andererseits das Bündel regulativer Funktionen. [..] Die systemsichernden Funktionen orientieren sich nach Maßgabe der Regulative auf die Erhaltung 
des Systems in der vorhandenen Ausprägung, die regulativen liefern aufgrund der Kenntnis des zu sichernden Zustands die Entscheidungsgnundlage und werden somit von den systemsichernden gespeist" (Fleischer 1996, 129).

Das Phänomen Weltbild weist, der Definition folgend, zwei Funktionskomplexe auf, die systemsichernden und die regulativen. Objekt der Regulienung bzw. Sicherung ist nicht, wie die Definition suggeriert, das Weltbild selber, sondern ein gegebener Diskurs bzw. Interdiskurs. Die regulativen Funktionen des Weltbildes zielen, wie letztendlich alle anderen untergeordneten Funktionen auch, auf die Sicherung eines bestimmten Diskurses bzw. Interdiskurses ab. Den generellen Funktionskomplexen sind weitere Funktionen untergeordnet. Wesentlich für die Evolution eines gegebenen Diskurses ist eine Funktion, die als Generierungsfunktion bezeichnet werden kann. Diese Funktion ist dafur verantwortlich, daß Zeichen adäquat, d.h. den bereits im Diskurs enthaltenen Zeichen entsprechend, semantisiert werden. Diese Generierungsfunktion setzt eine andere Funktion voraus, die Speicherungsfunktion. Diese Funktion bewirkt, daß das Weltbild als eine Art «Filten» fungiert, denn Zeichen können nur in einen Diskurs bzw. Interdiskurs gelangen, wenn sie mit dem bereits vorhandenen, d.h. gespeicherten Zeichen-Repertoire «in Einklang stehen». Die adäquate Organisation der Zeichen ist gleichfalls eine Funktion des Weltbilds. Auf der Grundlage der Funktionszuweisungen kann folgendes festgestellt werden: der Begriff Weltbild bezeichnet kein Objekt, wie beispielsweise die Begriffe Diskurs und Interdiskurs, sondern einen Mechanismus, der auf die genannten Objekte «wirkt». Dieser Mechanismus beinhaltet verschiedene Funktionen, die auf die Sichenung eines bestehenden Zeichensystems und also Kultursystems abzielen. In diesem Sinne soll die Äußenung verstanden werden, daß es sich bei einem Weltbild um ein Konstrukt handelt. Es läßt sich emeut die Frage stellen, welches Erklärungspotential der Begriff für die vorliegende Untersuchung aufweist. Es kann angeführt werden, daß der Begriff nur in diachroner Hinsicht ein Erkläunspotential offeriert. Auf diese Tatsache wird implizit hingewiesen, indem betont wird, daß das Weltbild ein bestimmtes System in langen Zeiträumen und für lange Zeiträume organisiert. In der vorliegenden Untersuchung wird jedoch ein als synchroner Bestand aufgefaßtes Korpus analysiert. Der Begriff Weltbild kann dementsprechend im Rahmen dieser Untersuchung nicht operationalisiert werden. Es wird jedoch zur Kenntnis genommen, daß die dargestellten Funktionen des Weltbilds auf den Diskurs eingewirkt haben, in dem die ausgewählten Texte generiert wurden.

\subsection{Der Begriff Kom munikation}

Im folgenden Abschnitt wird der konstruktivistische Kommunikationsbegriff erläutert, der auf Maturana (vgl: Maturana 1985, 1994) zunückgeht. Die Darstellung basiert auf Fleischers Definition des Begriffs Kommunikation, die im Rahmen der Theorie der Zweiten Wirklichkeit entwickelt wurde. Auf die Verknüpfung mit anderen Phanomenen der Theorie der 
Zweiten Wirklichkeit soll nur am Rande eingegangen werden. Fleischer definier den Begriff Kommunikation folgendermaßen: „Kommunikation ist der Prozeß und der ihm zugrundeliegende Mechanismus, der die aus dem Mechanismus der kognitiven Konstruktion resultierende Anpassung, Absteckung, Auslotung, Überprüfung, Konfrontation sozial bedingter und kulturell intersubjektiv hergestellter und funktionierender konstruktiver Weltbilder gewährleistet und sichen, und zwar zum Zweck der Herstellung und Aufrechterhaltung des Kommunikationssystems Gesellschaft mittels des Kultursystems und zum Zweck der Anwendung innerhalb wie auch der Herstellung. Steuerung und Veränderung der jeweiligen Diskurse. [...] Kommunikation ist nicht auf die Übermittlung von Information ausgerichtet $[(\ldots)]$, sondern auf die mittels der Kommunikate geschehende Anpassung und Überprüfung individueller Konstrukte im Hinblick auf ihre Übereinstimmung mit den kollektiven Konstrukten $[(\ldots)]$ und ihren Komponenten. Die konstruktivistisch verstandene Kommunikation ist ein System und ein Regulativ im Sinne der Systemtheorie und ein evoluierender Prozeß im Sinne der systemtheoretischen Evolutionstheorie“" (Fleischer 1997a, 249).

Offensichtlich bezeichnet der Begriff Kommunikation nach Fleischer zwei miteinander verknüpte Phänomene: einen Prozeß und einen Mechanismus. Der Mechanismus Kommunikation, der im ersten Teil der Definition beschrieben wird, steht im Zusammenhang mit dem Mechanismus Weltbild, da er fur dessen Funktionieren respektive dessen Sicherung zuständig ist. Auf den Mechanismus soll nicht näher eingegangen werden, da eine Operationalisierung des Begriffs Kommunikation in diesem Sinne im vorliegenden Rahmen nicht vorgesehen ist. Vielmehr empfiehlt es sich, auf den Prozeß Kommunikation einzugehen. da auch textuelles Material als Basis eines Kommunikationsprozesses angesehen werden kann.

Der Begriff Kommunikation im Sinne des Konstruktivismus steht in Widerspruch zu dem mathematisch-quantitativ geprägten Begriff der Informationstheorie. der den Prozeß der Signalubertragung zwischen zwei informationell-gekoppelten Maschinen bezeichnet, die einen gemeinsamen Zeichenvorrat zur Codierung bzw Decodienung benutzen (vgl.: Fischer 1993, 74). Der informationstheoretische Begriff Kommunikation basiert in dieser Hinsicht auf der Annahme eines manifesten Kommunikationsinhalts. Maturana hingegen geht von lebenden Systemen als autopoietischen Systemen aus, die operational geschlossen aber metabolisch offen sind. „Nun ist diese operationale Geschlossenheit, was die Erkenntnisfähigkeit des Systems betriff, auch kogmitiv (informationstheoretisch) geschlossen, d.h das System nimmt keine Information aus der „Außenwelt“ auf“" (Fischer 1993, 77). Autopoietische Systeme sind dementsprechend zu dem, was in der Informationstheorie als informationelle Kopplung bezeichnet wird, nicht in der Lage $\mathrm{D} \mathrm{h}$. .Information entsteht also erst im jeweiligen lebenden System, sie wird dort erzeugt und außerhalb der Systeme existiert nur Rauschen" (Fischer 1993, 77). Es geht bei dem Prozeß Kommunikation also nicht um Informationsübertragung. sondern um die Schaffung bzw. Aufrechterhaltung eines sogenannten konsensuellen 
Bereichs (vgl: Fischer 1993, 79). Hierbei handelt es sich um einen „Bereich ineinandergreifender Interaktionen zwischen Systemen, die durch strukturelle Kopplung strukturell isomorph geworden sind" (Maturana 1982, 290; zit. nach Fischer 1993, 79). Wird von zwei autopoietischen Systemen, $\mathrm{AS}_{1}$ und $\mathrm{AS}_{2}$, ausgegangen, so läßt sich der Kommunikationsproze $B$ auf der Basis eines konsensuellen Bereichs folgendemaßen darstellen:

„AS 1 orientiert $\mathrm{AS}_{2}$ auf einen Teil seines Interaktionsbereichs (nämlich von $\mathrm{AS}_{2}$ ), wobei der Interaktionsbereich (Kognitionsbereich) des orientierten $\mathbf{A S}_{2}$ mit dem des orientierenden $\mathbf{A S}_{1}$ "vergleichbar" ist. Evolutionäre Voraussetzung dafür ist allerdings, daß beide ASe in ihrem Interaktionsbereich weitgehend übereinstimmen" (Fischer 1993, 80).

In bezug auf sprachliche Kommunikation kann aus dem Gesagten Folgendes geschlossen werden: „Sprachliches Verhalten ist „Orientienungsverhalten“, d.h. wenn wir von zwei miteinander kommunizierenden Systemen ausgehen, dann wird der zu Orientierende (Empfänger, Hörer) auf eine Interaktion innerhalb seines kognitiven Bereiches orientiert. Dies ist unabhängig von der orientierenden Interaktion (des Sprechers, des Senders), der sprachlichen Äußerung selbst" (Fischer 1993, 82). Als Voraussetzung für das Orientienungsverhalten muß die gemeinsame Interaktion lebender Systeme angesehen werden, auf deren Grundlage ein konsensueller, intersubjektiver Bereich entsteht, der als konsensuelle Wirklichkeit bezeichnet weren kann (vgl.: Fischer 1993, 84).

In der vorliegenden Arbeit geht es in diesem Sinne um die konsensuelle Wirklichkeit einer kulturellen Formation, die aus sprachlichen Äußerungen in Form von Texten abgeleitet und (re-) konstruiert werden soll. Es wird davon ausgegangen, daß Kommunikation ein System darstellt, d.h. einzelne Komponenten sich aufeinander beziehen. Das Kommunikationssystem, welches evoluiert, zielt auf die Sicherung eines anderen Systems ab. Bei diesem System muß es sich um einen bestimmten Diskurs bzw Interdiskurs und somit um eine bestimmte kulturelle Formation bzw. ein Kultursystem handeln.

\subsection{Kultursymbole: Diskurssy mbole und Kollektivsymbole}

Diskurs- und Kollektivsymbole sollen unter dem Begriff Kultursymbole zusammengefaßt betrachtet werden. Kultursymbole sind Elemente der Zweiten Wirklichkeit, die, wie bereits angedeutet, wichtige Funktionen für ihren Geltungsbereich übernehmen. Im Sinne der Systemtheorie sollen Kultursymbole als Regulative aufgefaßt werden (vgl: Fleischer 1994, 380). Neben der Regulationsfunktion scheinen Kultursymbole weitere Funktionen zu besitzen. Welche Funktionen dies sind, und ob Diskurs- und Kollektivsymbole immer die selben Funktionen aufweisen, ist bis dato ungeklärt (vgl.: Fleischer 1994, 194). Die Funktionen der Kultursymbole beruhen auf einer besonderen "Absolvierung" des allgemein-sprachlichen und des kulturspezifischen Weges, der sogenannten diskurshaften Semantisierung, aufgrund derer sie von anderen Zeichen unterscheidbar sind. 
Das Gesagte kommt in der Definition des Begriffs Kollektivsymbol zum Ausdruck: "Kollektivsymbole sind Zeichen, die einen derart und dermaßen ausgeprägten Interpretanten besitzen, daß er eine kulturelle Bedeutung aufweist, die für die gesamte Nationalkultur [hier: ein Kultursystem] gilt, und der Interpret auf besondere Kenntnisse bezüglich des Bedeutungsund hauptsächlich Zeichen-Interpretanten angewiesen ist" (Fleischer 1994, 193). Die kulturelle Bedeutung eines Zeichens ist „eine solche, die jeweils nur für eine bestimmte kulturelle Formation gilt und die Bedeutung eines Zeichens [(...)] für diese konkrete Formation ausmacht“ (Fleischer 1994, 193). Der Bedeutungs-Interpretant „umfaßt [...] die lexikalischen Bedeutungen, jene, die sich auf die einschrankende Relation des Zeichen-Mittels zum -Objekt beziehen und ein im Zeichen-Objekt prinzipiell gegebenes Möglichkeitsfeld einengen“ (Fleischer 1994, 194). Hier drängt sich die Frage der Operationalisierbarkeit des angesprochenen Begriffs auf: die kulturelle Bedeutung kann, wie von Fleischer u.a. gezeigt (vgl: Fleischer 1994, 197ff;: Tüschau 16 1996, 1998, 1998b), vermittels der Methode der semiotischen Diskursanalyse für kulturelle Formationen empirisch ermittelt werden. Wie aber soll dies für den Bedeutungs-Interpretanten eines Zeichens möglich sein? Diese Frage kann im Rahmen der vorliegenden Analyse nicht beantwortet werden, sie soll lediglich kurz angeführ werden.

Die Definition des Begriffs Diskurssymbol ist mit der Definition des Begriffs Kollektivsymbol bis auf die Bestimmung des Geltungsbereichs identisch. Kollektivsymbole "gelten» bzw. "funktionieren» in einen Interdiskurs und diesem «untergeordneten» Diskursen, d.h. in einem Kultursystem. Der Geltungs- bzw. Funktionsbereich von Diskurssymbolen ist der Diskurs einer bestimmten kulturellen Formation Von der Struktur her sind Kollektiv- und Diskurssymbole identisch, sie bestehen aus einzelnen Wörtern (types).

\section{Funktionen von Kultursymbolen}

Kultursymbole sind im Sinne der Systemtheorie Regulative. Ihre Funktion ist die Regulation, d.h. die Sicherung eines Systems bzw. eines Geltungsbereichs In Form von Hypothesen fuhn Fleischer weitere Funktionen von Kollektivsymbolen an (vgl. Fleischer 1994, 379-382), die nicht einzeln, sondern in zusammengefaßter Form erläutert werden sollen. Eine Analyse der Hypothesen ergibt zwei Funktionen, die auf der sprachlichen Ebene eine Realisierung der Sicherungsfunktion darstellen und zwar eine, die als Manipulationsfunktion und eine andere, die als Markierungsfunktion bezeichnet werden kann. Die gewählte Einteilung in eine Manipulations- und Markienungsfunktion genügt nicht den Kriterien richtig oder falsch, sie dient lediglich der Komplexitätsreduzienung. Andere Einteilungsvarianten sind denkbar 


\subsubsection{Manipulationsfunktion (Zusammenfassung der Hypothesen 3, 4, 6,} $11,13,14,16)$

Kollektivsymbole üben eine manipulative Funktion in einem Interdiskurs bzw. Diskurs aus, indem sie den Zustand der Normalität in bezug auf einen Sachverhalt postulieren bzw. implizieren. Normalität wird hier im Sinne einer a priori Bewertung eines bestimmten Sachverhalts verstanden. Vermittels dieser Eigenschaft können Argumentationen außer Kraft gesetzt bzw. vermieden werden, denn über einen Sachverhalt, der kollektiv als normal angesehen wird, muß nicht (mehr) diskutiert, d.h. argumentiert werden. Kultursymbole sind Ausdruck einer Polarisierung bzw. Oppositionsgenerierung: eine kulturelle Formation, die einen bestimmten Normalitätszustand als nicht gültig für sich anerkennt, bildet eine Opposition zu einer kulturellen Formation, die eben jenen Zustand als normal auffaßt. Gleichzeitig bieten Kultursymbole die Möglichkeit, auf Differenzierungen zu verzichten (vgl.: Fleischer 1994, 380). Ein Zustand ist normal oder er ist es nicht; er ist es aber nicht weniger und nicht mehr. Darüber hinaus können mit Kultursymbolen Zusammenhänge hergestellt werden, die sich nach den im jeweiligen Diskurs geltenden Semantisierungen richten (vgl: Fleischer 1994, 381). Wenn ein Sachverhalt von einer kulturellen Formation positiv oder negativ bewertet wird, dann kann diese Eigenschaft über die Herstellung eines Zusammenhangs auf einen anderen Sachverhalt «übergehen».

\subsubsection{Markierungsfunktion (Zusammenfassung der Hypothesen 1, 5, 7, 17)}

Kultursymbole markieren Außenungen (vgl: Fleischer 1994, 379). Aufgrund der in einem Diskurs adäquaten Anwendung eines Kultursymboles ist es einem zeichenbenutzenden und -generierenden Aktanten möglich, sich als zu einer kulturellen Formation zugehörig auszuweisen. Werden Kultursymbole hingegen inadäquat angewendet, wird die Außerung und der die Äußerung generierende Aktant als nicht dazugehörend erkannt und möglicherweise ausgeschlossen

\subsubsection{Zur Operationalisierbarkeit}

Fleischer hat mit Hilfe einer Erhebung 34 Wörter ermittelt, welche die erläuterten Funktionen im russischen Kultursystem übernehmen können (vgl.: Fleischer 1997b, 97). Diese Wörter gilt es zunächst in den ausgewählten Texten nachzuweisen. Anschließend muß geprüft werden, ob es sich bei den ermittelten Wörtern auch funktional um Kultursymbole handelt. Hierzu muß der textuelle Kontext herangezogen werden. Ein besonderes Augenmerk soll auf die Manipulationsfunktion gelegt werden, da sich diese im Gegensatz zur Markienungsfunktion aus dem Kontext ableiten läßt. 


\subsection{Der Begriff St ereoty p}

Der Begriff Stereotyp (vgl: Fleischer 1996, 99-102) wird im Rahmen der Theorie der Zweiten Wirklichkeit in einer als Arbeitsdefinition bezeichneten Bestimmung erläutert. Der Begriff Arbeitsdefinition impliziert, daß es sich bei der zu diskutierenden Bestimmung um eine revidierbare Annäherung an das Phänomen Stereotyp handelt. Es wird zudem deutlich, daß der Forschungsproze $B$ in bezug auf dieses Objekt im Rahmen der in Rede stehenden Theorie als nicht abgeschlossen angesehen werden muß. Es gilt zu prüfen, welche Operationalisierung die folgende Definition ermöglicht bzw. welche definitorischen Veränderungen für eine Operationalisierung ratsam erscheinen:

.Stereotype sind [...] aufgrund nicht-wissenschafticher Kriterien kognitiv typisierte bzw. typisierende Aussagen, die zur Erreichung einer funktionellen, strategischen, diskurshaft ausgerichteten und diskurshaft bestimmten Verallgemeinerung in einer gegebenen Kulturausprägung dienen. Sie benötigen zweierlei Legitimation: sie müssen häufig auftreten und also feststellbar sein und sie müssen eine systemsichernde oder -abgrenzende oder -differenzierende Funktion erfüllen“ (Fleischer 1996, 112). Ein Stereotyp in diesem Sinne ist die Aussage „Polen klauen Autos“ (vgl: Fleischer 1996, 110).

Die Arbeitsdefintion beinhaltet, wie auch das angeführte Beispiel zeigt, die Auffassung von Bartmiński und Panasiuk, nachdem „Ein wesentliches Merkmal des [...] Stereotyps [...] die unpräzise subjektive Generalisierung, d.h. eine logisch unberechtigte Zuschreibung eines Merkmals allen Elementen einer Klasse, die mit dem gegebenen Namen bezeichnet werden“ (Bartmiński; Panasiuk 1993, 369; zit. nach Fleischer 1996, 101) ist.

Bevor mit der Diskussion begonnen wird, die sich sowohl auf die Struktur als auch die Funktion des Stereotyps bezieht. soll betont werden, daß die beiden angeführten Zitate als einander erganzend aufgefaßt werden, auch wenn unterschiedliche Urheber für sie verantwortich sind

Zunachst soll die Struktur des Stereotyps betrachtet werden. Ass Strukturmerkmal des Stereotyps wird die Form der Aussage angefuhr. Es wird davon ausgegangen, daß der Begriff Aussage mit dem aus der formalen Logik stammenden Begriff Proposition synonym verwendet wird. Ein Stereotyp muß in diesem Sinne nicht notwendigerweise Satzcharakter aufweisen. Ein für diesen Zusammenhang wesentliches Merkmal von Propositionen ist die Eigenschaft, daß sie aus mehreren Bestandteilen bestehen. Im Falle des Stereotyps aus mindestens zwei aufeinanderbezogenen Teilen: erstens einer mehrere Aktanten umfassenden Gemeinschaft oder Klasse (die Polen) und zweitens einem dieser Gemeinschaft logischungerechtfertigt zugeschriebenen Merkmal, d.h. einer Attribuierung. Nebenbei sei in diesem Zusammenhang bemerkt, daß eine Gemeinschaft gleichfalls durch ein in konstruktivistischer Hinsicht «typisches» Mitglied bezeichnet werden kann (der Pole). In diesem Sinne fallen auch Pars-pro-toto-Relationen unter den diskutierten Begriff 
Es soll versucht werden, den Prozeß der Kategorisienung und anschließenden Stereotypisierung kurz in abstrakter Form darzustellen: eine Gemeinschaft $A$ setze sich aus Trägem eines bestimmten Merkmals x zusammen. Das Merkmal x erfült für die Gemeinschaft A die Funktion des Klassifizierungsmerkmals. Wird der Gemeinschaft $A$, und somit allen Merkmalsträgern von $x$, ein weiteres Merkmal y zugeschrieben, dann liegt von der Struktur her ein Stereotyp vor. Bartmiński und Panasiuk folgend ist die Zuschreibung des Merkmals (y/A) logisch unberechtigt. Da es im vorliegenden Zusammenhang um kognitive Phänomene geht, kann die Wahrscheinlichkeit, mit der y utatsächlich» zutriff, vernachlässigt werden. Gleiches gilt für den sogenannten «wahren Kerns von Stereotypen (vgl.: Fleischer 1996, 110). Ob mit (y/A) auch funktional ein Stereotyp vorliegt, kann ermittelt werden, indem geprüft wird, welche Funktion das Merkmal $y$ in bezug auf $A$ erfullt. Hierzu muB eine weitere Gemeinschaft eingeführt werden, die für die Merkmalszuschreibung (y/A) verantwortlich ist, die diese generiert hat. Diese Gemeinschaft sei $B$ mit dem Klassifizienungsmerkmal z. Kann sich B aufgrund des Merkmals y von A abgrenzen und somit die eigene Gemeinschaft, d.h. letztendlich das eigene System sichern, dann handelt es sich bei der Merkmalszuschreibung (y/A) durch B auch in funktionaler Hinsicht um ein Stereotyp. Es tritt bei der Untersuchung des in Rede stehenden Phänomens an dieser Stelle das Objekt-Subjekt-Problem (vgl: Fleischer 1996, 99) auf. Letztendlich muß ein Subjekt entscheiden, ob Merkmal y die angesprochenen Funktionen übernehmen kann. Das Subjekt kann seine Entscheidung auf einen textuellen und/oder außertextuellen Kontext stützen. Wenn, wie im Beispiel „Polen klauen Autos“, kein textueller Kontext gegeben ist, dann muß die Entscheidung aufgnund des außertextuellen Kontextes in Form des Diskurses, in dem die Proposition generiert worden ist, getroffen werden Der außertextuelle Kontext besagt in diesem Zusammenhang, daß der Vorgang des Klauens negativ zu bewerten ist (vgl: Fleischer 1996, 110).

Das in der Definition angesprochene Kriterium des haufigen Auftretens von Stereotypen soll im Rahmen der vorliegenden Untersuchung aufgrund seiner Relativităt ausgespart werden.

Auf der Grundlage der Diskussion kann abschließend dargestellt werden, wie der Begriff Stereotyp in der vorliegenden Untersuchung operationalisien wird. Durch die Darstellung der Operationalsienung soll das zugrundeliegende Verständnis des Begriffs verdeutlicht werden.

Zunächst soll der Objektbereich auf Gemeinschaften beschränkt werden. Diese Beschränkung wird in den angefuihrten Zitaten nicht expliziert, kann aber als Usus in der Stereotypenforschung angesehen werden (vgl: u.a. Quasthoff, 1989). In einem ersten Untersuchungsschritt muß festgestellt werden, welche Gemeinschaften in einem bestimmten Text genannt werden. Anschließend gilt es für jede Gemeinschaft gesondert zu prüfen, ob neben dem Klassifizienungsmerkmal weitere Merkmale der Gemeinschaft genannt werden. Für jedes Merkmal muß geprüft werden, ob es die Funktion der Systemabgrenzung und somit -sichenung übernehmen kann. Kann ein bestimmtes Merkmal diese Funktion ubernehmen, dann liegt sowohl von der Struktur als auch von der Funktion her ein Stereotyp 
vor. Um von einem Stereotyp sprechen zu können, soll sowohl das Struktur- als auch Funktionskriterium erfült sein.

\subsection{Der Begrifi Normativ}

Es geht in diesem Abschnitt darum, den Begriff Normativ hinsichtlich seiner Operationalisierbarkeit für den vorliegenden Rahmen zu betrachten. In diesem Zusammenhang ist es notwendig, auch auf den Bereich der Normativik einzugehen. Es muß betont werden, daß eine Beschränkung auf die wesentlich erscheinenden Merkmale des Normativs und der Normativik erfolgt, so daß sowohl die basalen Konzeptionen von Riedl (1975) und Willke (1976, 1994) als auch die Vernetzung mit den bisher erörterten Phänomenen der Zweiten Wirklichkeit vernachlässigt werden. Da das Normativ als Grundelement der Normativik aufgefaßt wird, soll zunächst der Begriff Normativik eingeführt werden:

,Normativik bezieht sich auf den kulturbedingten und ausprägungsabhängigen Bereich des Normalen, der Normalität, auf jene Elemente oder Erscheinungen, die in einem Kultursystem als 'normal', 'richtig', 'wahrhaft', 'echtes/wahres x' usf. dargestellt bzw. postuliert werden. [...] Es interessiert, was eine Kultur oder Subkultur [d.h. ein Kultursystem oder eine kulturelle Formation] als den Normalbereich bestimmt und wie sie es bestimmt, aus welchen Elementen dieser Bereich besteht [...]" (Fleischer 1996, 179).

Es wird postuliert, daß jedes Kultursystem bzw. jede kulturelle Formation einen Normalbereich aufweist, der sichtbar wird, wenn bestimmte Elemente oder Erscheinungen, vermittels von Zeichen, die als Merkmalsträger bezeichnet werden können, semantisch um die Merkmale normal, richtig etc. erweitert werden. Die Merkmalsträger sind Zeichen, die mit dem Begriff Normativ belegt werden .Normarive sind Grundelemente der Normativik, in denen oder durch die ein spezieller oder engerer (System-) Bereich als normal festgelegt wird. an dem sich andere Erscheinungen orientieren müssen, um in das gegebene Kultursystem [bzw die gegebene kulturelle Formation] so und nicht anders aufgenommen zu werden [...]" (Fleischer 1996, 179).

Aus dem Gesagten kann abgeleitet werden, daß Normative eine regulative Funktion besitzen. In methodischer Hinsicht geht es darum. Normative zu ermitteln und den Normalbereich eines betrachteten Kultursystems bzw. einer kulturellen Formation zu (re-) konstruieren Es soll hier wiederum die Struktur und die Funktion des definierten Objekts betrachtet werden. Da die Struktur der Normative nicht in den Definitionen thematisiert wird. soll ein ergänzendes Zitat angeführt werden

„Normative [ $\ldots]$ sind $[\ldots]$ Wörter, die die Fähigkeit aufweisen müssen, Maßstäbe manifestieren zu können. Dies kann in expliziter, deskriptiver Form geschehen, dann handelt es sich um Ausdrücke oder Sätze [Propositionen], und es kann in impliziter Form 
(stillschweigend) vor sich gehen, dafür reichen wiederum Einzelworte aus" (Fleischer 1996, 109).

Hinsichtlich der Struktur der Normative kann kein Unterscheidungskriterium zu anderen diskursiven Phänomen festgestellt werden, da sowohl Propositionen als auch einzelnen Wörtern ein normativer Charakter zugeschrieben wird. Dennoch kann gesagt werden, daß ein Normativ immer im Zusammenhang mit anderen Zeichen aufteten muß. Ein Normativ als Merkmalsträger des Normalen wird, da es auf ein anderes Zeichen bezogen ist, ein Merkmal dieses Zeichens. Aufgrund des Normativs wird die Normalität des bezogenen Zeichens deutlich. Abweichend von der in der Definition geäußerten Auffassung, wird davon ausgegangen, daß Normative nicht primär der Konstruktion des Normalbereichs eines kulturellen Systems dienen, sondern nur ein Ausdruck dieses Bereichs sind. Dies geht einher mit der Auffassung, daß Normative Regulative darstellen. Es liegt in diesem Sinne ein Phänomen vor, welches dem des "weißen Schimmels» ähnlich ist: den Mitgliedern eines kulturellen Systems ist die Tatsache bewußt, daß ein bestimmter Sachverhalt normal ist, dennoch wird der Sachverhalt mittels eines Normativs hervorgehoben. Für die Beziehung Normativ - Zeichen bietet sich der Begriff kulturelle Tautologie an. Wie der Begriff Tautologie implizien, zeichnet sich die genannte Beziehung durch eine gewisse Redundanz aus. Gerade durch diese Redundanz wird es möglich, den Normalitätsbereich eines kulturellen Systems zu erkennen und zu rekonstruieren. Es ist leicht nachvollziehbar, daß mit der Ermittlung von Normativen und der Beobachtung ihrer Beziehung zu anderen Elementen nur ein Teil des Normalitätsbereichs eines kulturellen Systems erfaßt werden kann. Dennoch ist es möglich, kulturelle Systeme aufgrund des sichtbaren Normalitătsbereichs voneinander abzugrenzen. Wie vermutlich mit allen diskursiven Objekten kann auch mit dem Normalitätsbereich "gespielt» werden: es kann ironisch mit dem vorhandenen Normalitătsbereich umgegangen werden, es kann ein Normalitätsbereich behauptet werden, den es nicht gibt u.dgl.m. Diese Möglichkeiten des Umgangs mit Normalităt sind nur sehr schwierig wissenschaftlich nachauweisen, da hierzu Insiderinformationen notwendig sind. Es gilt dennoch der Frage der Operationalisierbarkeit des Begriffs Normativ nachzugehen. In einem ersten Schritt müssen Zeichen (Wörter) oder Zeichenkomplexe (Propositionen) ermittelt werden, die andere Zeichen in der Weise attribuieren, daß deren Normalität deutlich wird. Anschließend kann aufgrund der bezogenen Zeichen der Normalitätsbereich des untersuchten kulturellen Systems rekonstruiert werden.

\subsection{Semiotische Diskursanalyse als Grundlage empirischer Systemforschung}

In den vorangehenden Abschnitten wurden die für den vorliegenden Zusammenhang als wesentlich erachteten Aspekte der Theorie der Zweiten Wirklichkeit erläutert. Es folgt eine kurze, allgemeine Darstellung der auf der Theorie beruhenden Analysemethode, die als 
semiotische Diskursanalyse bezeichnet wird. Mittels der semiotischen Diskursanalyse, die sowohl Textanalysen als auch empirische Erhebungen umfaßt, sollen die semiotischen Systeme von kulturellen Formationen bzw. Kultursystemen u.a. rekonstruierbar sein (vgl: Fleischer 1994, 168). Es geht also um die Untersuchung des Zusammenhangs von Kultursystemen und Interdiskursen bzw. kulturellen Formationen und Diskursen:

"Texte [sollen] im Hinblick auf die in ihnen enthaltenen oder sich durch sie manifestierenden Gesetzmäßigkeiten [untersucht werden], mit der Absicht, etwas über die - so und nicht anders - Texte benutzenden Kulturteilnehmer zu erfahren“ (Fleischer 1997, 227).

Genauer kann das Forschungsziel folgendermaßen definiert werden: „Bei der [semiotischen] Diskurs-Analyse geht es nicht bzw. nicht primär um die Aussage, um den wie auch immer verstandenen Inhalt und auch nicht um das Besondere eines Textes, sondern [...] um seine diskurshafte Färbung und als das Typische, Wiederholbare, Kollektive, das die Differenzienungsqualität eines Diskurses ausmacht. [...] Es geht [...] darum, was in dem konkret und einmalig Gesagtem auf allgemeine, für eine Subkultur [hier: kulturelle Formation] oder Nationalkultur [hier: Kultursystem] typische Merkmale hinweist“ (Fleischer 1994, 197).

Aus dem Gesagten folgt die Notwendigkeit, ein Maß zu konstruieren, welches das Merkmal typisch bzw. charakteristisch operationalisierbar macht. Ein Versuch in diese Richtung wird in Abschnitt 5.2. dargestellt. Gleichzeitig müssen Kriterien ermittelt werden, die es ermöglichen, kulturelle Formationen und Kultursysteme zu bestimmen und voneinander abzugrenzen Bei diesen Kriterien muß es sich um solche handeln, die auf der unterschiedlichen kollektiven Verwendung und Generierung von Zeichen und Zeichensystemen basieten. Kriterien dieser Art liegen bisher nicht vor, so daß gesagt werden kann, daß auf der Grundlage des heutigen Kenntnisstands keine Aussagen über die faktische Existenz von kulturellen Formationen und Kultursystemen möglich sind. Alle Äußerungen bezüglich der Existenz kultureller Systeme sind, dies triff auch auf die vorliegende Untersuchung zu, Spekulationen. auf denen konkrete Forschungsvorhaben aufgebaut werden können (vgl.. Tuschau 101996, 1998b). Für die vorliegende Untersuchung wird, wie erläutert, die Existenz einer kulturellen Formation, die mittels eines bestimmten Diskurses kommunizier, postulativ und also axiomatisch festgelegt Die Methoden der Textanalyse und der empirischen Erhebungen brauchen hier nicht erläutert werden. da bereits gezeigt wurde. wie ausgewählte Theorieelemente im vorliegenden Rahmen operationalisiert werden.

\subsection{Zusammenfassung}

Die Existenz von Diskursen bzw. Interdiskursen und kulturellen Formationen bzw. Kultursystemen wird im Rahmen der Theorie der Zweiten Wirklichkeit vorausgesetzt. Bis auf den Begriff Weltbild, der einen Mechanismus bezeichnet und die sogenannte Kulturstratifikation, können die verbleibenden, dargestellten Theorieelemente, also die zusammenfassend als Kul- 
tursymbole bezeichneten Kollektiv- und Diskurssymbole, Stereotype sowie Normative für den vorliegenden Zusammenhang aufgrund ihrer Operationalisierbarkeit genutzt werden. Es wird davon ausgegangen, daß bestimmte diskursive Elemente in Texten konstruiert, andere in diesen deutlich werden. Nicht konstruiert werden diejenigen Elemente, denen eine regulative Funktion zugesprochen wird, d.h. Kultursymbole und Normative. Durch die vorliegende Untersuchung soll die faktische Verwendung von Kultursymbolen in einer kulturellen Formation beobachtet werden. Darüber hinaus wird der Versuch unternommen, den Normalitätsbereich dieser Formation zu rekonstruieren. Konstruiert werden dahingegen alle diskursiven Elemente, die keine regulative Funktion für Diskurse übernehmen. Es handelt sich um das bereits dargestellte Element Stereotyp sowie um weitere, die in Abschnitt 5. ff. eingeführt werden. In diesem Zusammenhang soll ergänzend erwähnt werden, daß Zeichen, die in kultureller Hinsicht funktionalisiert sind, d.h. die in der Funktion von Kultursymbolen, Stereotypen etc. eingesetzt werden, in kultureller Hinsicht für das betroffene kulturelle System relevant sind. Zeichen, die nicht in kultureller Hinsicht funktionalisiert sind, besitzen dementsprechend keine kulturelle Relevanz fur das betroffene kulturelle System.

Wie angedeutet, ist die Theorie der Zweiten Wirklichkeit keine abgeschlossene, sondern eine in der Entwicklung befindliche. Als Grundlage der vorliegenden Arbeit birgt sie ein großes heuristisches Potential, welches ermöglicht, weitere Objekte zu definieren und somit die Theorie zu erweitern. Dennoch fallt bei der Analyse der Theorie auf, daß der qualitative Aspekt der in Rede stehenden Phänomene im Vordergrund steht, da es primär um kultursystembedingte Semantisierungen geht. Es mu $B$ jedoch davon ausgegangen werden, da $B$ auch auf der quantitativen Ebene Hypothesen über die erörterten Phănomene ableitbar sind. Auch dieser Aspekt soll in der vorliegenden Arbeit berücksichtigt werden.

Ein großes Problem ist nicht nur im Rahmen der Theorie der Zweiten Wirklichkeit die Beziehung zwischen Untersuchungsobjekt und untersuchendem Subjekt. Dieses Problem kann durch Offenlegung von Schlüssen bzw. Ableitungen relativiert werden. In diesem Sinne wird in der vorliegenden Untersuchung eine maximale intersubjektive Objektivität angestrebt. 
Martin Bergmann - 9783954790531

Downloaded from PubFactory at 01/10/2019 02:45:57AM

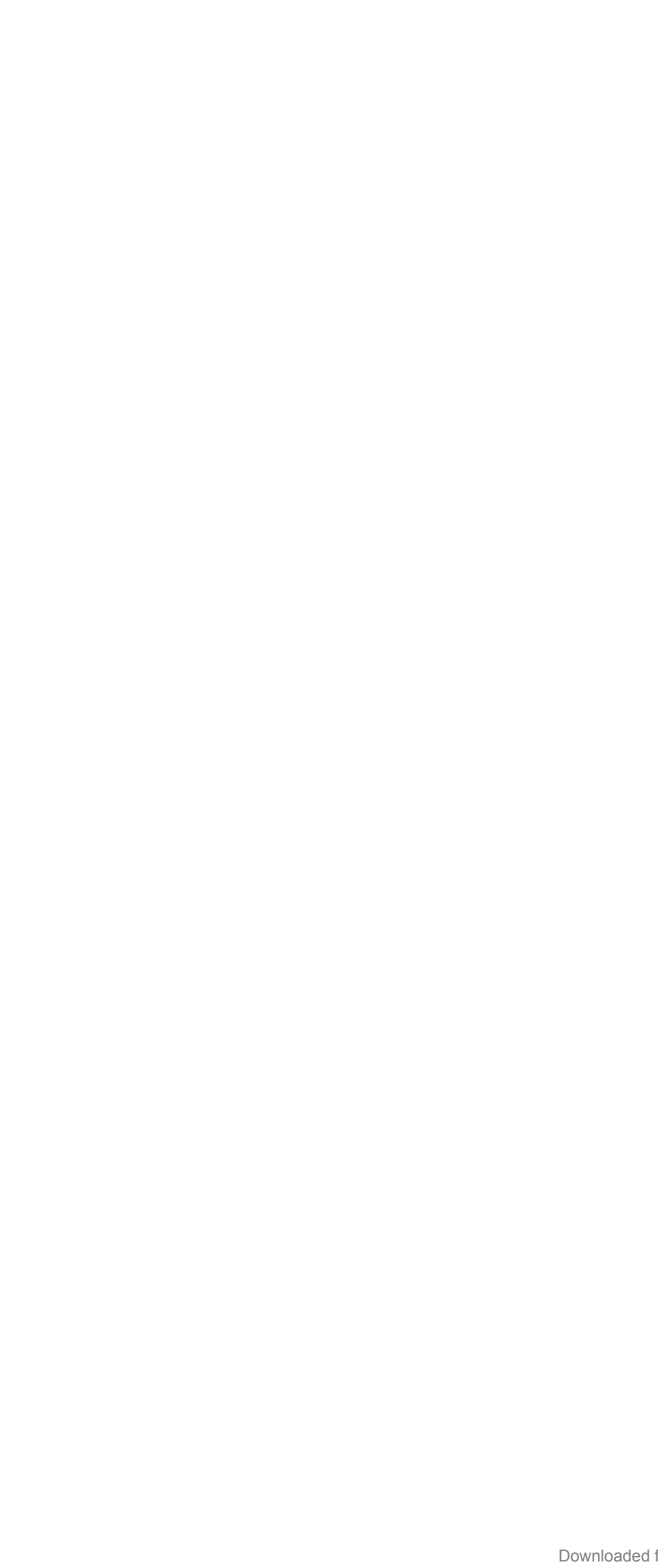




\section{Inhaltsanalyse}

\subsection{Allgemeines}

Die Inhaltsanalyse bzw. einige ausgewählte analytische Verfahren können als Ergänzung zu dem im vorangehenden Abschnitt dargestellten diskursanalytischen Instrumentarium angesehen werden Da es um eine Ergänzung des Untersuchungsinstrumentariums auf der quantitativen Analyseebene geht, stehen die quantitativen inhaltsanalytischen Verfahren im Mittelpunkt der folgenden Darstellung. Zunächst wird dennoch in allgemeiner Hinsicht auf die sozialwissenschaftliche Inhaltsanalyse eingegangen.

\subsection{Theoretischer Ausgangspunkt}

Theoretischer Ausgangspunkt der Methode der Inhaltsanalyse ist das Axiom, daß der Mensch ein bedeutungs- bzw. sinngenerierender Organismus ist. Objektbereich der Inhaltsanalyse sind menschliche Kommunikationsprozesse, wobei sprachliche Prozesse und Produkte im Mittelpunkt des Interesses stehen. Gleichfalls gehören auch Filme, Bilder, Verkehrszeichen und Musikstücke zum Objektbereich der Inhaltsanalyse. Der klassische Schwerpunkt der Inhaltsanalyse liegt im semantischen Bereich sprachlicher Kommunikation, d.h. in der Beschreibung der durch Texte übermittelten Bedeutung (vgl:: Groeben; Rustemeyer 1995, 523/524), wobei der Inhaltsanalyse im Sinne von Groeben und Rustemeyer ein informationstheoretischer Kommunikationsbegriff zugrunde liegt.

Inhaltsanalytische Untersuchungen von Massenmedien basieren auf zwei Massenkommunikationsmodellen, dem Repräsentationsmodell (die Reflektionshypothese) und dem Instrumentalmodell (die Kontrollhypothese), die aus der Frage resultieren, ob Massenmedien die sozialen Verhältnisse widerspiegeln oder Auslöser gesellschaftlicher Veränderungen sind. Beiden Modellen liegen unterschiedliche Auffassungen über die Bedingungen und Möglichkeiten zugrunde, gültig von einem Text auf einen Kontext zu schließen. Der Begriff des Schließens bedeutet in diesem Zusammenhang folgendes: „Unter Schließen ist hier die theoretische Inferenz von Merkmalsausprägung des Textes auf Merkmalsausprägungen der sozialen Wirklichkeit zu verstehen“ (Merten 1995, 110). Das Repräsentationsmodell geht davon aus, .daß manifeste Texte ein verläßliches Abbild oder Korrelat des Kontextes abgeben, so daß der InferenzschluB von Text auf den Kontext legal wird“" (Merten 1995, 112). Das Modell beruht u.a. auf den Überlegungen des Psycholinguisten Osgood, der ..unterstellt, daß die Verwendung bestimmter Worte ein gültiger Ausdruck bestimmter mentaler Zustände des Kommunikators ist" (Merten 1995, 112). Die Kontrollhypothese, die die Basis des Instrumentalmodells darstellt, ..unterstellt, daB die Medien Vorreiter sind und damit gesellschaftliche Strukturen gerade eben 
nicht reflektieren, sondern kontrollieren bzw. verändern" (Merten 1995, 112). Gleichgültig welches Kommunikationsmodell einer Inhaltsanalyse zugrunde liegt, wird davon ausgegangen. daß vermittels von Texten Informationen und somit Bedeutungen übermittelt werden. In dieser Hinsicht unterscheidet sich der Kommunikationsbegriff der Inhaltsanalyse von dem konstruktivistischen und somit von dem der zugrundegelegten Diskurstheorie (vgl: : 2.7.).

Nach Groeben und Rustemeyer stellt die Inhaltsanalyse insgesamt nur eine Methode zur Systematisierung des alltäglichen Verstehens dar: „Ihre Ausarbeitung geht [...] von der methodologischen Implikation aus, daß eine solche Systematisierung notwendig ist, um za einer adaquaten (verstehenden) wissenschaftlichen Beschreibung der jeweils vorliegenden Bedeutungs- bzw. Sinngenerierung zu gelangen" (Groeben; Rustemeyer 1995, 524). Diese Implikation besitzt a) einen quantitativen und b) einen qualitativen Aspekt: .Unter quantitativer Perspektive geht es darum, daß es ohne eine Systematisierung des Verstehens nicht möglich ist, mehrere Texte im Hinblick auf (semiotische) Gemeinsamkeiten bzw. Unterschiede zusammenzufassen. [...] Unter qualitativer Perspektive geht es um die Gefahr subjektiver Verzerrung, die bei einem nicht-systematisierten Verstehen immer gegeben ist" (Groeben; Rustemeyer 1995, 524). Verzerrungen dieser Art resultieren aus der sogenannten kognitiven Konstruktivität des Menschen. Unter kognitiver Konstruktivität wird in diesem Zusammenhang die Fähigkeit des Menschen verstanden, im Prozeß des Verstehens von Texten, diese nicht nur hinsichtlich der in ihnen enthaltenen Informationen zu dekodieren, sondern diese Information mit dem eigenen Sprach- und Weltwissen in Verbindung zu bringen, so daß als Verstehensprodukt immer eine Verschmelzung von text-generierten und rezipienten-generierten Informationen entsteht. Die kognitive Konstruktivität ist somit dafür verantwortlich, daß verschiedene Rezipienten wegen ihres unterschiedlichen Sprach- und Weltwissens den gleichen Text unterschiedlich verstehen konnen. Die Integration des Phänomens der kognitiven Konstruktivitat in den Rahmen der Inhaltsanalyse kann als Annaherung an den Kommunikationsbeyriff des Konstruktivismus gewertet werden, gleichwohl aut den Standpunkt der Informationsübertragung während eines Kommunikationsprozesses behart wird

Zusammenfassend kann festgestellt werden, daß ein „komplexitatsreduzierender Überblick (über Textbedeutungen) sowie intersubjektive Ubereinstimmung (als Elimination von Verzerrungsverfahren) [...] den wissenschaftstheoretischen Ausgangspunkt für die methodologischen Kriterien der Inhaltsanalyse dar[stellen]“" (Groeben; Rustemeyer 1995, 526).

Durch die Systematisierung des alltäglichen Verstehens soll dementsprechend ein (komplexitätsreduzierender) zusammenfassender Überblick uber verschiedene Texte ermöglicht werden und zwar in einer intersubjektiven Übereinstimmung. die individuelle (wissens-, konzept- bzw. einstellungsbedingte) Verzerrungen des einzelnen Rezipienten weitestgehend vermeidet. In dieser Hinsicht ist die hier formulierte Zielsetzung mit den metatheoretischen Werten, die für die vorliegende Arbeit Gültigkeit besitzen, kompatibel. 


\subsection{Die Stellung der Inhaltsanalyse zwischen Hermeneutik und Empirie}

Groeben und Rustemeyer folgend, wird bereits in der klassischen Lasswell-Formel (Wer sagt was in welchem Medium zu wem mit welchem Effekt?) der Versuch der Systematisienung des alltäglichen Verstehens sichtbar. Dieser Versuch steht immer in der Spannung zwischen zwei gegenläufigen Polen: „Dem Pol der intersubjektiven Systematik (als Mittel zur annaherungsweisen Erreichung von 'Objektivität') steht das Bemühen entgegen, [...] eine möglichst große Relevanz der entsprechenden [...] Aussagen zu erreichen“ (Groeben; Rustemeyer 1995, 526). M.a.W. nimmt die Inhaltsanalyse eine Mittelstellung zwischen qualitativem und quantitativem Paradigma ein, wobei sie von der Systematik her der empiriewissenschaftlichen Methode näher als der hermeneutischen steht. Der größte methodologische Unterschied beider Konzeptualisierungen liegt darin, daß bei der hermeneutischen Interpretation eine „subjektiv für richtig gehaltene Interpretationsweise ... formuliert und dann nach möglichst vielen bestätigenden Belegen gesucht" (Früh 58; zit nach Groeben; Rustemeyer 1995, 528) wird. Im empiriewissenschaftichen Paradigma hingegen „werden in der Regel lineare Vorgehensweisen festgelegt, die von allen möglichen (kompetenten) Forscher/innen gleichermaßen durchgeführ werden (sollten)" (Groeben; Rustemeyer 1995, 528). Im empiriewissenschaftichen Paradigma besitzt das Messen, d.h. die Überführung einer empirisch vorliegenden Relation in eine homomorphe Datenstruktur, den Charakter einer Zielfunktion. Dies bedeutet nicht, daß das empiriewissenschaftiche Paradigma ausschließlich quantitative Schlüsse zuläßt. Vielmehr geht es darum, methodische Vorgehensweisen offenzulegen, so daß mit qualitativen Aussagen eine maximale intersubjektive Objektivität erreicht wird

\subsection{Die klassische Inhaltsanalyse: Auswahl-, Analyse und Kontexteinheit}

Die sogenannte klassische Inhaltsanalyse steht im Zusammenhang mit Berelson. der 1952 folgende Definition von Inhaltsanalyse formulierte. "Content analysis is a research technique for the objective, systematic, and quantitative description of the manifest content of communication" (Berelson 18; zit. nach Groeben, Rustemeyer 1995, 529). Kommunikationsinhalt ist für Berelson das "Insgesamt von (verbalen, musikalischen, bildlichen, plastischen und geistigen) symbolischen Bedeutungen, das die Kommunikation als solche hervorbringt" (Berelson 1954 [sic! 1952] 488; in der Übersetzung von LAMNEK 1989, 180; zit. nach Groeben; Rustemeyer 1995, 529/530). Besonderheit der klassischen Inhaltsanalyse ist die Spezifizierung des Kommunikationsinhalts als manifest. Diese Spezifizierung „schließt explizit alle [...] Schlußfolgerungen über den jeweiligen Zeicheninhalt aus [...]; und selbst in der semantischen (bzw. syntaktischen) Dimension des jeweiligen Kommunikationsinhaltes ist damit eine relativ weitgehende Beschränkung auf das unmittelbar Ausgesagte, die 'Oberflächenstruktur' der Kommunikation verbunden" (Groeben; Rustemeyer 1995, 530). Gleichzeitig bedeutet die Konzentration auf den manifesten Kommunikationsinhalt den Ausschluß 
von latenten Bedeutungsgehalten. „Für die klassische Konzeption von BERELSON ist diese Beschränkung auf manifeste Kommunikationsinhalte allerdings weitgehend als eine Konsequenz zu sehen, welche die Realisierung der zentralen methodologischen Kriterien 'Objektivität', 'Systematik' und 'Quantifizienung' ermöglichen soll" (Groeben, Rustemeyer 1995, 530).

Objektivität bedeutet in diesem Zusammenhang intersubjektive Nachprüfbarkeit und Übereinstimmung, die durch die Systematik des inhaltsanalytischen Vorgehens gewährleistet werden soll. Das Kriterium der Systematik besteht darin, daß ein zu analysierender Text in einzelne Teile aufgeteilt wird, hinsichtlich derer dann leichter und sicherer festgestellt werden soll, ob sie bestimmte, theoretisch festgelegte Bedeutungsaspekte enthalten. Die beiden wichtigsten, methodologischen Probleme der klassischen Inhaltsanalyse beziehen sich daher auf die Festlegung der zu kategorisierenden Textteile (das Problem der Einheiten-Festlegung) und die Explikation der thematischen Bedeutungsaspekte in einem sogenannten Kategoriensystem, welches den eigentlichen Kern einer Inhaltsanalyse bzw. genauer gesagt, einer Themenanalyse, darstellt. In einem Kategoriensystem sollen die relevanten Bedeutungsdimensionen bzw. -aspekte explizit und präzise festgehalten werden können. Die Ergebnisse des Kategorisienungsvorgangs, d.h. der Zuordnung von Texteinheiten zu den Kategorien durch sogenannte Kodierer, werden abschließend für eine Gesamtbeschreibung der Textbedeutung(en) zusammengefaßt Dies geschieht beispielsweise mittels Häufigkeitsanalysen (Frequenzanalysen z.B. zur Frage, welche Themen wie häufig in einem Textkorpus angesprochen werden), Kontingenzanalysen (zur Frage, welche Themen miteinander verbunden sind) und endet bei der Bedeutungsfeld-, Symbol- und Bewertungsanalyse. Insgesamt erlaubt die Inhaltsanalyse eine sehr flexible Anwendung des ihr zugehörigen, analytischen Instrumentariums, da, je nach der Fragestellung und des zu analysierenden Textkorpus, ein spezifisches Kategoriensystem entwickelt werden muß. Hinsichtlich der in einer klassischen Inhaltsanalyse betroffenen Texteinheiten wird zwischen Auswahl-, Analyseund Kontexteinheit unterschieden. Als Auswahleinheit (auch. sampling unit, Erhebungseinheit) wird diejenige Einheit bezeichnet. die in die jeweilige Inhaltsanalyse einbezogen werden soll. Die Auswahleinheit ist hierbei eine formal (physikalisch) definierte Einheit (vgl.: Merten 1995, 281), wie z.B. eine Zeitschrift. Die Analyseeinheiten (auch. Kodiereinheiten) werden aus den Auswahleinheiten ausgewahit. Es sind diejenigen Teile der Auswahleinheiten, die zur Analyse herangezogen werden. Die Festlegung der Analyseeinheiten läßt sich unter formaler oder unter inhaltlicher Perspektive vornehmen (formal: Worte, Sätze, Abschnitte etc.; inhaltlich: Symbole, Werte/Wertungen. Themen, Ideen usw.) Als Kontexteinheit wird derjenige Textteil bezeichnet. auf den die Kodierer bei der Einordnung der konkreten Analyseeinheiten unter die jeweiligen Kategorien des inhaltsanalytischen Kategoriensystems zuruckgreifen können und sollen, um eventuelle Verstehensunklarheiten zu beseitigen

Zusammenfassend kann festgehalten werden, daß die klassische Inhaltsanalyse, wie bereits erwähnt, den metatheoretischen Werten verpflichtet ist, die für die vorliegende Arbeit als gültig 
anerkannt werden. Die eingefuhrte Begrifflichkeit in bezug auf Texteinheiten impliziert die Möglichkeit, nicht nur die Analyseeinheiten, sondern auch, wie dies im Rahmen der vorliegenden Diskursanalyse geschehen soll, weitere Einheiten in eine Analyse einzubeziehen.

Von einem theoretischen Standpunkt aus gesehen, muß das Postulat des manifesten Kommunikationsinhalts kritisch beurteilt werden. Wird der Kommunikationsinhalt als manifest postuliert, dann scheiden die im Rahmen der Theorie der Zweiten Wirklichkeit erörterten diskurshaften Phänomene, die auf dem erläuterten Prozeß der diskurshaften Semantisierung beruhen, aus dem Objektbereich der Inhalts- und Diskursanalyse aus. Die Abstraktion geht somit auf der qualitativen Betrachtungsebene fur die vorliegende Untersuchung zu weit. In diesem Sinne ist eine Konzentration auf quantitative inhaltsanalytische Untersuchungsmethoden ratsam, zu denen im weitesten Sinne auch die Themen- und die Kontingenzanalyse gezählt werden können.

\subsection{Ausgewählte inhaltsanalytische Verfahren}

\subsubsection{Syntaktische Analyse}

Syntaktische Analysen sind rein formale Analysen, auf denen u.a. verschiedene Verfahren der Stilanalyse beruhen. Es geht danum, zu klären, „ob aus syntaktisch einfachen oder komplexen Variablen (Zahl der Wörter oder Silben pro Satz, Relation von Haupt- und Nebensätzen etc.) bestimmte Kennwerte ermittelt werden können, die für bestimmte Autoren resp. für bestimmte Eigenschaften von Kommunikation typisch sind" (Merten 1995, 124). In diesem Zusammenhang soll darauf hingewiesen werden, daß syntakische Analysen auch für die Identifikation des journalistischen Stils und zur Charakterisienung unterschiedlicher Textsorten herangezogen werden können (vgl: : Merten 1995, 129).

\subsubsection{Die Wort-Satz-Relation: WSR}

Ein sehr einfaches, quantitatives Textmerkmal ist die Wort-Satz-Relation, d.h. die durchschnittliche oder mittlere Satzlänge (in Wörtern). Rudolf Flesch, der sich in den 40-er und 50-er Jahren im Rahmen der Lesbarkeitsforschung eingehend mit Texten beschäftigte und ein nach ihm benanntes. mathematisches Modell entwickelte, berücksichtigt den angesprochenen Wert, neben anderen, zur Berechnung der Lesbarkeit. Er kommt zu dem Schluß, daß, je kürzer die Sätze sind und je weniger Silben die Wörter umfassen, desto lesbarer und dementsprechend verstehbarer wird ein Text (vgl: Lisch; Kriz 1978, 181). Hinsichtlich der angesprochenen Lesbarkeit in bezug auf die durchschnittliche Satzlänge bildet Flesch Kategorien, die in Tabelle 1 zu sehen sind (vgl.: Lisch; Kriz 1978, 181). Trotz massiver Kritik am Flesch-Modell, weiches insgesamt 8 Untersuchungsschritte umfaßt, soll der WSR-Wert im vorliegenden Zusammenhang berücksichtigt werden, da er als quantitativer Kennwert im vorliegenden Rahmen geeignet erscheint (vgl: : 3.6.). 
Tabelle 1

\begin{tabular}{|c|c|}
\hline Mittlere Satzlinge in Wortern: & Beschreibung des Stils: \\
\hline 29 & schr schwicrig \\
\hline 25 & schwierig \\
\hline 21 & jemlich schuierig \\
\hline 17 & normal \\
\hline 14 & ziemlich einfach \\
\hline 11 & einfach \\
\hline 8 & schr einfach \\
\hline
\end{tabular}

\subsubsection{Das Type-Token Verhältnis (Type-Token-Ratio: TTR)}

Der durch das Type-Token-Verhältnis ausgedrückte formale Indikator, der zu den Indices verbaler Diversifikation gerechnet wird, ist, analog zum WSR-Wert, ein einfaches Mittel zur Beschreibung eines Texts. Das Type-Token-Verhältnis benht auf der unterschiedlichen Bedeutung von Wörtern. d.h. auf der Verschiedenartigkeit semantischer Entitäten. Durch den TTRWert wird das Verhältnis verschiedener Wörter (types) in einem Text zu der Gesamtzahl auftretender Wörter (tokens) ausgedrückt. Es gibt zahlreiche Anwendungen und Interpretationen des TTR-Wertes: so wird er ,als Maß fur die Flexibilität der Wortwahl verwendet [und] soll auch mit Intelligenz korrelieren“ (Lisch; Kriz 1978, 121). Des weiteren „korrespondiert [er] [...] mit einer Reihe personaler Daten, [und] läßt sich daher als psycholinguistisches Verfahren der Inhaltsanalyse benutzen" (Merten 1995, 136). Erwähnt werden muß, daß das Type-TokenVerhältnis mit der Größe der Analyseeinheit variiert. Bei vergleichenden Analysen müssen die Texte bzw. Analyseeinheiten vom Umfang her dementsprechend gleichartig sein

\subsubsection{Semantische Analyse}

Der Begriff der semantischen Analyse ist, analog zum Begriff der syntaktischen Analyse, ein Oberbegriff Sämtliche Verfahren, die unter ihm subsumier werden, bencksichtigen die semantische Qualitat von Texten. Sie können dementsprechend nicht mehr ohne weiteres als rein quantitative Verfahren bezeichnet werden.

\subsubsection{Themenanalyse}

Die Themenanalyse ist zum einen das am häufigsten angewandte. inhaltsanalytische Verfahren (vgl. . Merten 1995, 147), zum anderen sind die Anwendungbereiche und damit die mit ihr realisierbaren Untersuchungsziele fast unerschöpflich (vgl: : Merten 1995, 154). Auf der Themenanalyse basieren weitere Verfahren, wie die sogenannte Symbolanalyse, die sogenannte Bewertungsanalyse etc., auf die hier nicht naher eingegangen wird. Um eine Themenanalyse durchführen zu können, müssen zunächst mehrere wesentliche Vorarbeiten geleistet werden. Zum einen muß ein sogenanntes Sample gebildet werden. Anschließend muß festgelegt werden, welche Einheiten als Auswahl- und welche als Analyseeinheit fungieren sollen Des weiteren muß das bereits erwähnte Kernstück der Themenanalyse, das Kategoriensystem. entwickelt 
werden Der Begriff Kat egorie wird in diesem Zusammenhang folgendermaßen verstanden: „Kategorien sind Klassen eines übergeordneten und damit abstrahierenden Klassifikationsschemas" (Merten 1995, 147).

Bei der Definition des Kategorienschemas gilt es, folgendes zu beachten

„1. Das Kategorienschema soll theoretisch abgeleitet sein, d.h. es soll mit den Zielen der Untersuchung korrespondieren.

2. Das Kategorienschema soll vollständig sein, d.h. es soll die Erfassung aller möglichen Inhalte gestatten

3. Die Kategorien sollen wechselseitig exklusiv angelegt sein.

4. Die Kategorien sollen voneinander unabhängig sein.

5. Die Kategorien sollen einem einheitlichen Klassifikationsprinzip genügen.

6. Die Kategorien sollen eindeutig definiert sein“" (Merten 1995, 147-148).

Nachdem ein Sample gebildet und ein Kategorienschema entwickelt worden ist, kann mit der eigentlichen Themenanalyse begonnen werden. In dieser werden die Analyseeinheiten den Kategorien zugeordnet. Anschließend können die einzelnen Kategorien ausgezählt und vermittels verschiedener statistischer Verfahren analysiert werden. Erwähnt werden sollen in diesem Zusammenhang univariate statistische Maßzahlen (z.B. Mittelwerte, Streuungswerte etc.). Diese eignen sich zur Deskription und ermöglichen bei vergleichenden Betrachtungen die Bildung von Standardwerten und Toleranzbereichen. Kritisiert wird an den Anwendungen der Themenanlayse, daß „die Validiät der Inferenz nur sehr selten geprüft resp. das Reprasentationsmodell als vollgültig angesehen" (Merten 1995, 151) wird.

\subsubsection{Kontingenzanalyse (auch: Assoziationsanalyse)}

Merten versteht unter dem Begriff Kontingenzanalyse „ein inhaltsanalytisches Verfahren, bei dem im Text geprüft wird, welche Symbole überdurchschnittlich oft im Zusammenhang mit vorgegebenen (theoretisch relevanten) Symbolen aufteten" (Merten 1995, 157). Da der Begriff Symbol sehr vieldeutig ist, empfiehlt es sich stattdessen allgemeiner von Worten, types oder it ems zu sprechen, die miteinander assoziiert sind. Hierbei kann in einer Inhaltsanalyse sowohl die Assoziation verschiedener Kategorien (items) als auch einzelner Wörter (types) untersucht werden.

Ausgangspunkt der Kontingenzanalyse ist die aus der Linguistik stammende Erkenntnis, „daß diejenigen Wörter, die im Gehirn der Versuchspersonen gemeinsame Bedeutungselemente besitzen, öfter und rascher assoziiert werden" (Meier 553; zit. nach Merten 1995, 165). D.h. gleichzeitig, daß die Kontingenzanalyse die Gültigkeit des Repräsentationsmodells voraussetzt, da „sie unterstellt, daß die festgestellte Kontingenz zweier Items $X$ und $Y$ Ausdruck einer analogen Kontingenz im Kopf des Kommunikators ist“" (Merten 1995, 157). 
Erwahnenswert ist bei diesem Verfahren die Vorgehensweise bei der Definition der Analyseeinheiten. Diese sollten in Anlehnung an Osgood zwischen 120 und 210 Wörter umfassen (vgl:: Merten 1995, 165). Es muß hierbei jedoch bedacht werden, daß die Größe der Analyseeinheit und, wenn die Assoziation von Kategorien untersucht wird, die Anzahl der Kategorien einen unmittelbaren Einfluß auf die Starke der Assoziation besitzt (vgl: Merten 1995, 161). Der genannte Umfang der Analyseeinheiten muß daher als pragmatische Größe verstanden werden.

\subsection{Zusammenfassung}

Auf den zentralen Unterschied zwischen der gewählten Diskurstheorie und der (klassischen) Inhaltsanalyse, den jeweils zugnundeliegenden Kommunikationsbegriff, wurde bereits hingewiesen (vgl: 3.2.). Da es letztendlich für die ausgewähiten inhaltsanalytischen Verfahren zweitrangig ist, ob der Kommunikationsinhalt manifest ist, stellt der genannte Unterschied kein Hindernis dar, die erlauterten Verfahren als Ergänzung des dargestellten diskursanalytischen Instrumentariums aufzufassen

Im Rahmen der Diskursanalyse wird sowohl das Repräsentationsmodell als auch das Instrumentalmodell als gültig anerkannt. Wie bereits erwähnt wurde (vgl: 2.12.), werden bestimmte diskursive Elemente hinsichtlich ihrer kulturellen Bedeutung in Texten konstruiert (u.a. Stereotype), so daß das Instrumentalmodell gilt. Andere Elemente, die Kultursymbole sowie der Normalitätsbereich, liegen bereits konstruiert vor, sie werden in Texten lediglich "sichtban", so daß das Reprasentationsmodell zutriff. Es wird in diesem Zusammenhang deutlich, daß sich die Modelle im Rahmen der Diskursanalyse ergänzen

Daruber hinaus sollen die dargestellten Verfahren genutzt werden, um die Frage zu beantworten, inwiefern. bezogen auf das Korpus, von einer homogenen bzw heterogenen Textsammlung gesprochen werden kann Dies leitet zu det Frage uber, inwiefern der untersuchte Diskurs und somit die untersuchte Formation als homogen bzw. heterogen aufgefaßt werden konnen Die Beantwortung dieser Fragen basiert selbstredend nicht nur auf den Ergebnissen der syntaktischen und semantischen Analysen, sondern auf allen Analysen, die sich im vorliegenden Rahmen als produktiv erweisen

Konkret kann vermittels der Wort-Satz-Relation (WSR) festgestellt werden, ob eine bestimmte Satzlange bzw ein bestimmter Wertebereich dem Bewertungskriterium charakteristisch (vgl.: 5.2.) genugt. Das Type-Token-Verhaltnis (TTR) gibt Auskunft darüber, ob eine bestimmte Kennzahl oder ein bestimmter Wertebereich gleichfalls als charakteristisch fur das Korpus aufgefaßt werden kann. Die Themenanalyse erlaubt einerseits einen Einblick in das Themenspektrum der untersuchten Formation, andererseits ermöglicht sie die Bewertung eines Themenkomplexes als charakeristisch Die Kontingenzanalyse zielt abschließend auf die Ermittlung charakteristischer Assoziationen zwischen relevanten types ab 


\section{Sčepanskaja: Simvolika molodeżnoj subkul'tury. Opyt etnograficeskogo issledovanija sistemy 1986-1989 $\mathrm{gg}$.}

\subsection{Allgemeines}

In den folgenden Abschnitten sollen ausgewählte Aspekte der Arbeit Simvolika molodeznoj subkul'tury in zusammengefaßter Form dargestellt werden. Die Zusammenfassung orientiert sich an der Frage, wie Bestandteile der Arbeit für die durchzuführende Diskursanalyse genutzt werden können.

Das Untersuchungsobjekt der angesprochenen Arbeit ist eine sogenannte Jugendsubkultur (molodeznaja subkul'tura), die unter der Bezeichnung Sistema bekannt ist und die Mitte der achtziger Jahre in das Blickfeld der Sozialwissenschaften sowie der Medien (vgl.: Scepanskaja 1993, 47) gelangte. Der Analysezeitraum erstreckt sich auf die Jahre 1986 bis 1989 . Sämtliche Beobachtungen wurden in Leningrad und Moskau gemacht.

Zentrale Bedeutung fallt in der vorliegenden Arbeit dem Begriff Symbol zu, der in Anlehnung an Charles W. Morris verwendet wird. Als ein theoretischer Eckpfeiler der vorliegenden Arbeit muß dementsprechend die Pragmatische Semiotik und die darauf beruhende sogenannte Morris'sche Handlungstheorie aufgefaßt werden, die in der Sowjetunion v.a. von der Moskauer und Tartuer semiotischen Schule ${ }^{7}$ rezipiert wurde und so in der Wissenschaftlergemeinde der Sowjetunion einen gewissen Bekanntheitsgrad erlangen konnte. Einen weiteren theoretischen Eckpunkt stellt die Ethnographie und in diesem Zusammenhang der Begriff Ritual dar (vgl.: Scepanskaja 1993, 9)

Scepanskaja bezeichnet itre Arbeit als empirisch, so daß auf dieser Ebene von einer Kompatibilität mit der für die durchzuführende Diskursanalyse gewählten Objekttheorie ausgegangen werden soltte Dies ist jedoch nicht der Fall, da eine unterschiedliche Auffassung des Begriffs empirisch vorliegt. Scepanskaja geht von vorgefertigten, theoretischen Konstrukten (u.a. Symbolen) aus, fur die in der "Realitat", d.h. "empirisch», Beweise gesucht werden. Diese Vorgehensweise wurde im Rahmen dieser Arbeit bereits mit dem Begriff hermeneutisch belegt (vgl: 3.3.). Zur Erfüllung des metatheoretischen Wertes Empirizität muß, im Gegensatz zu der skizzierten Vorgehensweise, eine umgekehrte Vorgehensweise ge-

\footnotetext{
6 Neben dem Begriff molodeznaja subkkul'lura finden sich weitere Begriffe für das Sistema in der Literatur. Scepanskaja weist auf die folgenden hin: subkul'tura (vgl.: Scepanskaja 1993. 8 und Gilinskij 1996. 286). andergraund (vgl.: Scepanskaja 1993. 13). kontrkul'tura (vgl:: Scepanskaja 1993. It und Sikeric 1996. 332) und neformaly (vgl : Secpanskaja 1993. 14).

${ }^{7}$ Der Moskauer und Tanuer Schule sind zahlreiche Arbeiten gewidmet (vgl.: Eimermacher 1974. 1986). Auch entstanden in ihrem Rahmen zahlreiche theoretische (vgl. u.a. Lotman 1973) und anuendungsbezogene semiotische Arbeiten. die sich u.a. mit strukturalistischen und semiotischen Aspekten von künstlerischen und nichtkünstlerischen Texte beschăfigen. An dieser Stelle sei auf die Veroffentlichungsreihe der Schule Truḍ po znakorym sistemam hingewiesen.
} 
wähit werden, indem theoretische Konstrukte auf der Basis von empirisch ermittelten Daten entwickelt werden

Die Arbeit Scepanskajas ist dementsprechend im vorliegenden Rahmen nicht aufgrund der von ihr vertretenen Theorie bzw. der von ihr angewendeten Analysemethode von Interesse, die unter der Bezeichnung einschließende Beobachtung (vkljucennoe nabljudenie) firmiert und deren Elemente zum einen die aktive Teilnahme am Gemeinschaftsleben (tusovaja zizn'), zum anderen freie, nicht-standardisierte Interviews sind, sondern aufgrund des von ihr betrachteten Objekts, welches mit hoher Wahrscheinlichkeit mit der kulturellen Formation des roksamizdat assoziient ist.

Die angedeutete Auswahl der Aspekte, die im vorliegenden Rahmen dargestellt werden, beschränkt sich somit lediglich auf Beschreibungen des Objekts. Qualitative Schlußfolgenungen, die Scepanskaja auf der Grundlage ihrer Beschreibungen zieht, in diesem Sinne auch hinsichtlich des Symbolcharakters einzelner Phänomene, sollen nicht beachtet werden.

\subsection{Sistema: der Versuch einer allgemeinen Bestimmung}

Der Begriff Sistema, der in der in Rede stehenden Jugendsubkultur generiert und zur Selbstbezeichnung herangezogen wird, wird in der soziologischen Literatur nicht einheitlich aufgefaßt (vgl.: Pilkington 1996, 213). Einheitlichkeit herrscht dahingehend, daß er eine sowjetische Hippie- bzw. Post-Hippie-Bewegung (chippovskaja i postchippovskaja tusovka) (vgl: Sicpanskaja 1993, 8) bezeichnet. Scepanskaja erweitert den Bereich der Mitglieder auf Punker und Heavy Metal Fans (vgl. : Scepanskaja 1993, 73, 198) Die Jugendsubkultur Sistema wird von Scepanskaja in diesem Sinne als eine Art Sammelbecken für alternative (neformalye) Jugendliche aufgefaßt, die offensichtlich westlich-orientiert sind Die Verwendung des Begriffes molodeznaja subkul'tura in bezug auf das Objekt ermöglicht eine grobe Altersbestimmung der Sistema-Mitglieder. „Junesko otnosit $k$ molode $\lambda i$ lic $v$ vozraste mezdu 17 i 25 godami, sovetskie sociologi - mezdu 16 i 30" (Vorob'ev 1990, 33). Hinsichtlich des Entstehungszeitpunktes bzw -raumes liegen keine genauen Erkenntnisse vor, Siepanskaja datient die Entstehungsphase auf einen Zeitraum zwischen 1960 und 1970 (vgl.: Scepanskaja 1993, 69) Eine ungefähre Mitgliederzahl, die, so Scepanskaja, starken Schwankungen unterliegt, wird nicht genannt Da die genannte Subkultur einen Bereich umfaßt, in dem sowohl Hippies, Punker und Heavy Metal Fans assimilient sind, liegt die Vermutung nahe, daß es sich um eine großere subkulturelle Bewegung handelt Gleichzeitig liegt die Vermutung nahe. daß ein urbanes Phanomen vorliegt, wie die Bezeichung als gorodskaja tusovka (vgl Siepanskaja 1993, 9) suggeriert.

Die Jugendsubkultur Sistema ist, so Scepanskaja, von anderen Gruppierungen abgrenzbar (vgl: : Scepanskaja 1993, 10), was, so kann die vorliegende Arbeit in zusammengefaßter Form interpretiert werden, mit Eigentümlichkeiten hinsichtlich der Sprache, dem äußeren Erschei- 
nungsbild, zu dem ikonische Zeichen gezählt werden sollen und der (Freizeit-) Beschäftigung der Mitglieder zusammenhängt. Auf diese Aspekte soll im weiteren Verlauf der vorliegenden Zusammenfassung näher eingegangen werden. Zunächst aber gilt es, den Zusammenhang zwischen der genannten Subkultur und dem Objekt der Diskursanalyse, dem Diskurs der kulturellen Formation des rok-samizdat, offenzulegen, um die folgende Zusammenfassung, von einem metatheoretischen Standpunkt aus betrachtet, zu legitimieren.

\subsection{Sistema und rok-samizdat}

Es soll bei der folgenden Darstellung, deren Ziel es ist, Zusammenhänge bzw. Gemeinsamkeiten zwischen Sistema und rok-samizdat aufzuzeigen, nicht von Diskursen ausgegangen werden. Vielmehr sollen beide Phänomene in einem sozialwissenschaftichen Sinne, also als Ansammlung von Aktanten, d.h. als Gemeinschaft, aufgefaßt werden. Von einem sozialwissenschaftichen Standpunkt aus betrachtet, fält es nicht schwer, sowohl rok-samizdat als auch Sistema jeweils als Gemeinschaft zu bezeichnen. In bezug auf Diskurse, d.h. auf der Grundlage der Theorie der Zweiten Wirklichkeit, fält diese Abgrenzung beim jetzigen Kenntnisstand wesentlich schwerer bzw. ist sie ohne empirische Daten erst gar nicht möglich (vgl: 2.11.). Diese Abgrenzung kann umgangen werden, indem die Existenz der Diskurse postuliert wird. Dann würden im folgenden Abschnitt jedoch zwei postulierte Phänomene hinsichtlich ihrer Gemeinsamkeiten verglichen. Es scheint, von einem methodischen Standpunkt aus betrachtet, sinnvoller zu sein, diejenigen Phänomene hinsichtlich etwaiger Assoziationen zu untersuchen, deren Existenz nicht postuliert werden muß. Wenn in einem ersten Schritt Gemeinsamkeiten zwischen den Gemeinschaften festgestellt werden können, dann kann in einem zweiten Schritt der Frage nachgegangen werden, ob es Gemeinsamkeiten auch in kultureller Hinsicht gibt. Es kann in diesem Zusammenhang die Hypothese formuliert werden, daß der rok-samizdat- und der Sistema-Diskurs in gewisser Hinsicht identisch sind. Zumindest kann angeführt werden, daß es in Scepanskajas Arbeit Hinweise gibt, die diese Hypothese stützen. Es gilt, diese Hinweise zu erläutern

Zunächst kann festgestellt werden, daß Scepanskaja den rok-samizdat in ihrer Arbeit nicht thematisiert. Dies kann verschiedene Gründe haben, von denen zwei genannt werden sollen: zum einen besteht die Möglichkeit, daß es keine für die Autorin offensichtliche Beziehung zwischen den Phänomenen gibt, der rok-samizdat somit nicht dem Objektbereich ihrer Arbeit zugerechnet werden kann. Zum anderen existieren enge Beziehungen zwischen rok-samizdat und Sistema, die Autorin geht aber aus bestimmten Gründen nicht auf diese Beziehungen ein.

Wie gezeigt werden kann, existieren einerseits Beziehungen in Form von Gemeinsamkeiten. die mit dem Phänomen Rock-Musik bzw., weiter gefaßt. Rock-Kultur (rok-kul'tura) zusammenhängen, andererseits verlaufen beide Phänomene zeitlich nahezu parallel die erste Veröffentlichung des rok-samizdat kann auf das Jahr 1967 datiert werden; er hat den höchsten quan- 
titativen Output zwischen 1986 und 1989; ab diesem Zeitraum gehen die Veröffentlichungszahlen so weit zurück, daß seine Existenz spätestens nach 1994 fragwürdig ist (vgl: 6.4.). Die angesprochene Jugendsubkultur entsteht, wie bereits erwähnt, in den sechziger bis siebziger Jahren; ab 1987, mit dem massiven Auftreten der ljubera (vgl : 4.7.), setzt ein Konsolidierungsprozeß im Bereich der Jugendlichen ein, der zu einer Erhöhung der Mitgliederzahl der Subkultur führt; ab 1990 bzw. 1991 verringert sich die Mitgliederzahl stark, so daß sogar von einem Verschwinden gesprochen wird (vgl. : Scepanskaja 1993, 8).

Auf den Stellenwert der Rock-Kultur fur den rok-samizdat muß nicht gesondert hingewiesen werden, da dieser als Teil der rok-kul'tura aufgefaßt werden kann. Den Stellenwert der Rock-Kultur für die angesprochene Jugendsubkultur formuliert Scepanskaja folgendermaßen: "[...] rok-kul'tura - karkas Sistemy“ (Scepanskaja 1993, 57) bzw. ,[...] mir Sistemy nerazryvno svazan s fenomen rok-kultury" (Scepanskaja 1993, 71). Wenn der rok-samizdat als Bestandteil der rok-kul'tura aufgefaßt wird, dann muß er, diesen Aussagen folgend, als Teil des "Gerusts» der Jugendsubkultur Sistema aufgefaßt werden. Wird der rok-samizdat hingegen lediglich als sekundäres Phänomen aufgefaßt, welches auf der Grundlage der rok-kul'tura entstanden ist. dann besteht die Gemeinsamkeit zwischen Sistema und rok-samizdat darin, daß ihnen eine gemeinsame Basis eigen ist. Zwischen beiden Gemeinschaften ist, gleichgültig welche der beiden genannten Hypothesen als gültig akzeptiert wird, aufgrund der zentralen Bedeutung des Phänomens rok-kul'tura für beide Gemeinschaften, eine Gemeinsamkeit offensichtlich. In Scepanskajas Arbeit werden des weiteren verschiedene (Musik-) Stilrichtungen (muzykal'nye tecienija), Rock-Gruppen und Aktanten genannt, die als Bestandteil der rok-kul'tura angesehen werden mussen, die in der interessierenden Subkultur eine besondere Stellung einnehmen. Inwiefern sie diese Stellung auch im rok-samizdat einnehmen, soll in der durchzufuhrenden Diskursanalyse ermittelt werden $\mathrm{Zu}$ den bedeutenden Stilrichtungen gehoren ..Chippi [ ] s psichodeliceskim rokom, panki - estestvenno s pank-rokom, metallisty - s muzykoj «tjayelogo metalla"“ (Scepanskaja 1993, 71). Einflußreiche Rock-Gruppen nennt Scepanskaja nur wenige, und zwar Alisa, Bitlz (Beatles) und Akvarium (vgl : Scepanskaja 1993, 71, 111) Bei den Persönlichkeiten, die ein hohes Ansehen genießen, handelt es sich um die sogenannten lider der Gruppen Alisa und Akvarium, Konstantin Kincev und Boris Grebenscikov: „V 1986 - 1987 gg. byl rascvet ob"edinenija alisomanov, poklonnikov rok-gruppy «Alisa", kumirom kotorych byl « lider K. Kincev. [...] Dovol'no dolgoe vremja podobnuju rol' v Sistemnoj srede igral B Grebenscikov, lider gruppy "Akvarium" (Scepanskaja 1993, 111-112).

Zusammenfassend kann festgestellt werden, daß neben der temporaren Überschneidung eine Gemeinsamkeit in Form der Bedeutung der rok-kul'tura für die interessierenden Gruppen vorliegt. Ob weitere, auf dieser Ebene aufbauende Gemeinsamkeiten vorhanden sind, gilt es anhand spezifischer Details, wie sie Musik-Richtungen, Rock-Gruppen und Aktanten darstellen, zu prufen 


\subsection{Sprachliche Aspekte der Jugendsubkultur Sistema}

Nach Scepanskaja verfügt die angesprochene Jugendsubkultur uber einen spezifischen Argot (argo; vgl.: Scepanskaja 1993, 73) bzw. sleng ${ }^{8}$. Die Jugendsubkultur bedient sich des weiteren eines Genres zanr, den sogenannten telegi (vgl.: Scepanskaja 1993, 41). Darüber hinaus gibt es standardisierte Aussagen', die in allgemeiner Weise als Sprüche bezeichnet werden können.

Die sistema-spezifische Sprachvariante in Form des sleng ermöglicht, so die von Scepanskaja vertretene Auffassung, die Abgrenzung von anderen Gruppierungen, denn ,sleng - sredstvo markirovki vyskazyvannij kak «svoich», Sistemnych" (Ścepanskaja 1993, 37). In einem ihrer Arbeit angehängten Wörterbuch sind diejenigen sleng-Elemente aufgeführt, die, so Scepanskaja, nur in der interessierenden Subkultur Verwendung fänden (vgl.: Scepanskaja 1993, 303). Diese Elemente sollten die angesprochene Markierungs- und Abgrenzungsfunktion folglich in eineindeutiger Weise übernehmen. Die dieser Aussage zugrundeliegende Einschätzung hinsichtlich der Exklusivität bestimmter Sprachelemente ist v.a. unter dem synchronen Gesichtspunkt fragwürdig, da sich Sprachsysteme, wie bekannt, verändern und einzelne Elemente ihre Exklusivität verlieren können. Ein Nachweis hinsichtlich der angesprochenen Exklusivităt ist darüber hinaus nur durch eine empirische Erhebung möglich. Wie eine Erhebung diesbezüglich aussehen könnte, ist in Bergmann 1997 dargestellt. Des weiteren kann bezweifelt werden, daß es Scepanskaja mit der Methode der einschließenden Beobachtung gelungen ist, das vollständige, exklusive sleng-Repertoire zu erfassen. Eine weitere Unzulänglichkeit bei der Betrachtung des sprachlichen Aspekts der Subkultur ist die Tatsache, daß nicht eindeutig zwischen mündlichen und schriftlichen Varianten des sleng, der telegi und der Sprüche differenzient wird, so daß unklar ist, in welcher Form sie verbreitet sind bzw. verbreitet werden 10 .

Trotz des fehlenden empirischen Nachweises hinsichtlich der Markierungs- und somit Abgrenaungsfunktion. der Vollständigkeit des sleng-Repertoires und der fehlenden Differenzierung zwischen mündlicher und schriftsprachlicher Realisienung, soll auf Scepanskajas Äusführungen hinsichtlich der angesprochenen Phänomene, die über ihre gesamte Arbeit verstreut aufzufinden sind, eingegangen werden, da sie für die Diskursanalyse mit hoher Wahrscheinlichkeit nutzbar sind.

Zunächst soll auf den etymologischen Aspekt des sleng eingegangen werden, wobei eine Konzentration auf die Herkunft einzelner Elemente erfolgt. Anschließend wird auf einige thematische Bereiche eingegangen, in denen sleng-Elemente besonders häufig auftreten.

${ }^{8} \mathrm{Zu}$ den Begrifien argo und sleng sowic au dem nicht genannten. komplementäen Begriff zargon siche: Mineeva. 1996. 348-349.

9 Zum Teil handelt es sich bei den standardisienen Aussagen um Phraseologismen. Zum Themenkomplex Phraseologismen in der Jugendsprache vgl:: Mineeva 1996. 359.

10 Nach Koester scheint das Auftreten von Anglizismen im Jugendjargon beispiclsweise cin mündliches Phanomen darzustellen (vgl.: Koester 1983). 
Ein wesentlicher Teil des sleng-Repertoires rekrutiert sich nach Scepanskaja aus Anglizismen, die das gesamte Sprachsystem durchdrängten: „buk - kniznyj magazin [...]; gerla - devuska [...]; suzy - obuv' [...]; nakonec, samonazvanie - pip" (Scepanskaja 1993, 57). Darüber hinaus impliziert eine Verwendung von Anglizismen die Sistema-Zugehörigkeit des Anwenders, denn ..Anglicizmy v etoj srede daze bolee "svoich" cern rodnoj jazyk" (Scepanskaja 1993, 57). Als Hinweis in bezug auf den Stellenwert der Anglizismen kann zusätzlich die genannte Selbstbezeichnung ,pipl' der Sistema-Mitglieder gewentet werden. Als Begründung für den Stellenwert bzw. die hohe Frequenz von Anglizismen verweist Scepanskaja auf die Englisch-Sprachigkeit der erwähnten rok-kul'tura (vgl.: Scepanskaja 1993, 71). Im sleng können des weiteren Elemente nachgewiesen werden, die aus dem Kriminellen- und Drogen-Milieu stammen: „Nekotoraja cast' [...] stengovych slov proischodit iz kriminal'noj i narkomanskoj subkul'tur. [...] pricem upominaja chanki (marichuany), Iravki (ee ze), koles (narkoticeskich tabletok), masin (spricev dlja in"ekcij narkotikov), lomki (abstinentnogo sindroma) i proc." (Scepanskaja 1993, 55). Gleichfalls werden in der interessierenden Subkultur Archaismen verwendet: „S odnoj storony, pisut slova $s$ dorevoljucionnym «jat'», Leningrad nazyvali Piterom zadolgo do oficial'nogo pereimenovanija ego v Sankt Peterburg, obrascalis' drug k drugu: "sudar'» ili "gospoda" ('Scepanskaja 1993, 58/59). Die genannte Jugendsubkultur schöpft ihr sleng-Repertoire darüber hinaus aus verschiedenen literarischen und cineastischen Quellen. S'epanskaja verweist in diesem Zusammenhang auf den Film Stalker von Tarkovskij, aus dem einige der als prozvisca bezeichneten Namen stammen, auf die weiter unten eingegangen wird.

In thematischer Hinsicht verweist Scepanskaja auf die Bereiche Selbstmord (samoubijstvo) und Verrucktheit (sumassestvie), in denen verschiedene Ausdrucke, fur den jeweiligen Sachverhalt existieren ..Sleng fiksiruet samoubijstvo [ ] - kımur 'sja - pokoncit' s soboj. pokocat'

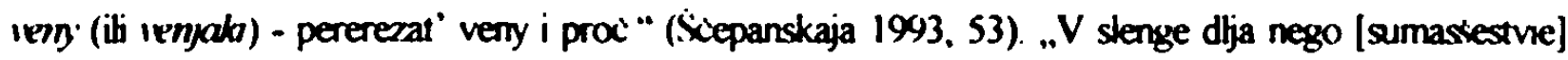
celyj rjad terminov krelzi - sumassedsij. krejza- sumassestvie, sizoval'sja - vesti sebja nenormal'no, siza - bred ili bredoobraznye projavlenija. knysa peexala (edet) - psichiceskie narusenija it d." (Scepanskaja 1993, 54)

Daruber hinaus existieren sistema-spezifische Ausdrucke für Bewertungen (ocenocnye sußdenija), für das "Zusammensein» (vzaimodejstvie) sowie für Personen und Orte (antropo- i toponima). Es können sowohl positive (,kajf, ,otpad', ,ulet', ,krutnjak'), negative (,ne v temu', ,tufta', ,laza', ,oblom', , ne v kajf) als auch indifferente Bewertungen (,do feni', ,ne tascit', , ne cepljaet') ermittelt werden (vgl: S'cepanskaja 1993, 308-339). Die typischen Formen des "Zusammenseins» bzw des gemeinsamen Zeitvertreibs werden als tusovka, sejsn, trassa, ask und vpiska bezeichnet (vgl : Scepanskaja 1993, 74, 186-189). Eine besondere Bedeutung besitzen die als prozvisca bezeichneten (Spitz-) Namen, da jede Person, die sich der Subkultur zugehörig fühlt, vermutlich einen solchen Namen besitzt (vgl . Scepanskaja 1993, 76), und die Personen mitunter nur unter diesen Namen bekannt sind (vgl Scepanskaja 1993, 38) Einige 
auffallige prozvisca sind beispielsweise ,Romaska', ,Dzoker', ,Al'fa', ,Ryba' und .Magister' (vgl.: Scepanskaja 1993, 76). Ähnlich bedeutsam sind Ortsnamen, denn „Sistemnaja toponimija otnositsja k mestam tusovok“ (Siepanskaja 1993, 75). Treffpunkte in diesem Sinne sind „Sajgon (populjarmyj togda v Leningrade kafeterij na peresecenii Nevskogo i Vladimirskogo prospektov [...]), Pikovaja Dama, Torser, Krysa, Kasan', Mikroklimat, Ogryzok, Sambala, Rotonda i proc.“ (Ścepanskaja 1993, 75-76).

Eine weitere Auffäligkeit ist die Verwendung einer als «semejnaja» terminologija bezeichneten Begrifflichkeit (vgl. Scepanskaja 1993, 178). Scepanskaja versteht hierunter den Aufbau fiktiver Familienverhältnisse unter Verwendung der Worte mat', syn und otec.

Bisher erfolgte eine Konzentration auf einzelne Sprachelemente. Im folgenden Abschnitt werden komplexere Formen betrachtet und zwar die telegi sowie Sprüche. Scepanskaja geht nur kurz auf das Wesen der telegi ein: „telega - specificeskij zanr - polupritca, poluanekdot" (Scepanskaja 1993, 41). Im Verlaufe ihrer Arbeit gibt sie einige telegi (vgl. u.a.: Scepanskaja 1993, 57) wieder, so daß auf dieser Grundlage folgende Eigenschaften festgestellt werden können: es handelt sich bei den telegi um Kurzgeschichten, deren Helden Mitglieder der genannten Subkultur sind und die offensichtlich in mündlicher Form innerhalb der Subkultur kursieren. Die Geschichten sind nicht in einer persönlichen Perspektive verfaßt, vielmehr geht es um die Erlebnisse anderer. Der Wahrheitsgehalt der telegi kann als gering eingestuft werden (vgl : Scepanskaja 1993, 58). Der Handlungsfaden ist sehr einfach: den Helden der Geschichten passiert etwas Ungewöhnliches, Bemerkenswertes aber Positives. Nach Scepanskaja besitzen die telegi bestimmte Funktionen, auf die hier nicht naher eingegangen werden muß, da es an dieser Stelle lediglich um eine Beschreibung von sprachlichen Eigenschaften der interessierenden Subkultur geht. Gleiches gilt für die Funktion der Sprüche, die Scepanskaja in ihrer Arbeit zitiert. Es handelt sich hierbei zum größten Teil um Zitate, die einerseits aus unterschiedlichen Glaubensrichtungen übernommen wurden (z B aus der Bibel. ...'Zivite kak deti“ - v mire, spokojstvii, ne gonites' za prizracnymi cennostjami" (Scepanskaja 1993, 180)), andererseits ihren Ursprung in Liedern populärer Musikgruppen haben (.."Panki ljubjat grjaz’, a chippi cvety”“ (Scepanskaja 1993, 158)). Daruber hinaus existieren Sprüche, deren Urheberschaft von Ścepanskaja nicht erläutert wird.

Zusammenfassend kann festgestellt werden, daß mit dem sleng, den telegi und den Sprüchen sprachliche Phanomene vorliegen, die einen Ansatzpunkt für eine Diskursanalyse liefern. Wie die genannten Phänomene im Rahmen der durchzuführenden Diskursanalyse operationalisiert werden, wird in Abschnitt 4.8. erläutert.

\subsection{Aspekte des äuBeren Erscheinungsbilds der Sistema-Mitglieder}

Das außere Erscheinungsbild der Mitglieder der Jugendsubkultur Sistema kann für eine Diskursanalyse, wie sie hier verstanden wird, nur genutzt werden, wenn es Zeichencharakter 
zugewiesen bekommt. Dies bedeutet für den vorliegenden Zusammenhang. daß es in den Texten des rok-samizdat thematisiert werden muß. Da in Scepanskajas Arbeit das außere Erscheinungsbild nicht als Klassifizienungsmerkmal der Sistema-Mitglieder fungiert, handelt es sich bei den folgenden Merkmalen um Stereotype im weiter oben erläuterten Sinne (vgl.: 2.9.). Es gilt in diesem Zusammenhang zu bedenken, daß die genannte Subkultur, wie bereits angedeutet, in gewisser Hinsicht ein Sammelbecken fur neformalye darstellt, so daß ein uniformes Äußeres unwahrscheinlich ist.

Das bedeutendste äußere Merkmal (vgl: Scepanskaja 1993, 51), welches Scepanskaja nennt, ist das der Haare: „Devuski neredko sovsem ili pocti lysy - a molodye ljudi dlinnovolosy" (Ścepanskaja 1993, 59), wobei die männlichen Mitglieder häufig einen Bart tragen (vgl: : Scepanskaja 1993,60). Des weiteren wird von der Autorin auf die Kleidung hingewiesen: „U devic meskovatye svitera i dzinsy - i u molodych ljudej“ (Scepanskaja 1993, 59) und ,dzincy narocno zaplatannye, niz ich machritsja, dorogich ukrasenij prakticeski ne vstretis" "“ (Scepanskaja 1993, 60). Als weitere Besonderheit stell Scepanskaja die sogenannten feneckj oder fen'ki heraus. Diese besitzen in der Funktion von Freundschaftsbändern einen besonderen Stellenwert und werden anscheinend von allen Sistema-Mitgliedern getragen (vgl : Scepanskaja 1993, 125-136). Ebenfalls zu den äußeren Erscheinungsmerkmalen können die ikonischen Zeichen (graficeskie simvoly) gezahlt werden, die in den verschiedensten Varianten, so als Schmuck, an Kleidungsstücken oder als Wandmalereien (graffiti) nachgewiesen werden können (vgl: : Scepanskaja 1993, 37). Die frequentesten ikonischen Zeichen sind: " "pacifik» (izobra/enie gohubinoj lapki vnutri knuga), "anarchija» («A» v knuge), [...] «chippovskjj krest" [...]. Poklonniki tjazelogo metalliceskogo roka prinesli $v$ Sistemu izobrazenie kosoj molnii. Populjarny v nastennoj i nad rinsovoj grafike motivy cvetka, pticy $[(\ldots)]$, dereva [(...)], glaza. sveci. U metallistov pojavljajutsja cerepa, inogda sataninskie motivy“ (Scepanskaja 1993, 37). Des weiteren gehort das „drevnekitajskij simvol: «in'-jan” (vgl: S'Cepanskaja 1993, 99) zum Repertoire der ikonischen Zeichen.

Der Darstellung Scepanskajas folgend, weisen die Sistema-Mitglieder sowohl hinsichtlich ihres Äußeren als auch hinsichtlich der Verwendung ikonischer Zeichen Besonderheiten auf. Es gilt in der Diskursanalyse zu prüfen, wie diese Besonderheiten, die, offensichtlich bezogen auf die Sistema-Mitglieder, als Stereotype funktionieren, in den Texten des rok-samizdat verwendet werden

\subsection{Aspekte der (Freizeit-) Beschäfigung der Sistema-Mitglieder}

Für die im folgenden Abschnitt dargestellten sogenannten charakteristischen Formen des Zeitvertreibs (vgl : Scepanskaja 1993, 186) gilt das bereits in bezug auf das äußere Erscheinungsbild der Sistema-Mitglieder Gesagte: zum einen erhalten die Formen des Zeitvertreibs im vorliegenden Rahmen nur Relevanz, wenn ihnen Zeichencharakter zugesprochen wird, d.h., wenn 
sie in den Texten des rok-samizdat thematisiert werden. Zum anderen handelt es sich bei den Zeitvertreibsformen um solche, die in gewisser Hinsicht allen Sistema-Mitgliedern zugeschrieben werden, so daß es sich in bezug auf diese um ein stereotypisierendes Merkmal handelt

Bezogen auf berufliche Tätigkeiten nennt Scepanskaja keine charakteristischen, die von Sistema-Mitgliedern bekleidet werden. Es scheint jedoch «Berufe» zu geben, die ein hohes, respektive niedriges Ansehen genießen: „Prestizno zdes' byt' chudoznikom [...] ili artistom, poetom, filosofom [...]. I soversenno neprestizno rabotat' na zavode ili, vo vzjakom slucae, govorit' ob etom. Rabota takogo roda priznaetsja tol'ko kak vynuzdennaja“ (Scepanskaja 1993. 94-95). Der berufliche Aspekt stellt in diesem Sinne einen tabuisierten Bereich dar, über dessen «realitătsseitige» Ausprägung offensichtlich nicht gesprochen wird. Dementsprechend liegt die Vermutung nahe, daß der Freizeitbereich d.h. die Beschäftigungsformen tusovka, sejśn, trassa, ask und vpiska fur die angesprochene Subkultur eine großen Stellenwert besitzen. Mit dem Begriff tu sovka liegt in sprachwissenschafticher Hinsicht ein Polysem vor: „Tusovka eto odnovremenno: krug obscenija; stil’ obscenija; mesto obscenija“ (Ścepanskaja 1993, 8). In der vortiegenden Zusammenfassung wurde der Begriff bisher im ersten Sinne verwendet. Hier geht es um die zweite Bedeutung. Auf die dritte Komponente wurde bereits kurz im Zusammenhang mit Ortsbezeichnungen eingegangen. Scepanskaja beschreibt die Vorgänge, die als tusovka bezeichnet werden und die an den genannten Treffpunkten vor sich gehen. folgendermaßen: „Sobirajutsja $\mathrm{i}$ nicego ne delajut: prosto sidjat [...], peregovarivajutsja, perechodjat ot odnoj gruppy k drugoj - i tak casami. [...] Citajut drug drugu stichi, pojut pod gitary i flejtu, obsußdajut filosofskie problemy, knigi, sporjat o Boge“ (Scepanskaja 1993, 186).

Der Begriff sejsn [engl: session] zeichnet sich ebenfalls durch seine Vieldeutigkeit aus, wobei sich für ihn der sprachwissenschafliche Begriff Homonym anbietet, denn .Pod sejsnom mozet ponimat'sja ljuboe interesnoe vremjapreprovozdenie, ot rok-koncerta ili vystavki avangarda do religioznoj ceremonii i da.e universitetskoj lekcii“" (Sccepanskaja 1993, 187).

Unter dem Begriff trassa wird folgendes verstanden. .eto putesestvija po raznym gorodom avtostopom" (Scepanskaja 1993, 159). Diese Reise als Anhaiter wird zum Teil mit, zum Teil jedoch auch gänzlich ohne Ziel durchgeführ (vgl.. Scepanskaja, 1993 187). Scepanskaja schreibt der angesprochenen Freizeitbeschäftigung, dies sei am Rande bemerkt, eine wichtige Funktion bei der Inauguration neuer Personen, die als novicki bzw. pionery bezeichnet werden, zu (vgl. Scepanskaja 1993, 159-170).

Der Begriff ask (engl:: ask) bezeichnet eine Tätigkeit, die Scepanskaja auch Bettelei (poprosajnicestvo) nennt. Es geht bei der Tätigkeit nicht nur darum Geld zu erhalten, sondern auch Essen, Zigaretten u.dgl.m. . Es ist unklar, ob nur Sistema-Mitglieder oder auch auch AuBenstehende angesprochen werden. Aufgrund der Darstellungen der Autorin, liegt jedoch der Schluß nahe, daß ask ein sistema-interner Zeitvertreib ist (vgl. Scepanskaja 1993, 188-189).

Vpiska bezeichnet eine Tätigkeit, bei der es im weitesten Sinne darum geht, eine Einladung zu erhalten bzw. eine Einladung auszusprechen ..Vpisat' na flet - dat' prijut, pustit' perenocevat' 
[...] Vpisyvajutsja ne tol'ko na flet, no i na sejśn - nachodjat sposob popast' (besplatno, razumeetsja) na rok-koncert ili drugoe interesnoe meroprijatie“ (Scepanskaja 1993, 189).

In der durchzuführenden Diskursanalyse gilt es der Frage nachzugehen, ob die angesprochen Tätigkeiten bzw. die am Rande erwähnten «Berufe» in den ausgewählten Texten des roksamizdat thematisiert werden.

\subsection{Sonstiges}

Im folgenden Abschnitt soll kurz auf weitere erwähnenswerte Aspekte der Jugendsubkultur Sistema eingegangen werden, die bisher entweder nur am Rande bzw. noch nicht angesprochen wurden. Es handelt sich zum einen um konkrete Treffpunkte der tusovsciki, um bestimmte (Feier-) Tage, die, Scepanskaja folgend, einen besonderen Stellenwert besitzen und um die ljubera, eine Gruppierung, die nicht mit der interessierenden Subkultur assimiliert ist.

Neben den bereits im Zusammenhang mit sleng-Bezeichnungen erwähnten Orten (vgl: 4.4.) nennt Scepanskaja zwei weitere, an denen sie ihre Beobachtungen gemacht hat: „V Moskve eto Bulgakovskij dom na Sadovoj ulice. $v$ Leningrade - Rotonda [...]. Eto paradnaja doma na nabereznoj r. Fontanki“" (Scepanskaja 1993, 45). An bzw. in diesen Häusern sind bzw. waren, so Scepanskaja, u.a. die erwähnten ikonischen Zeichen und die hier als Sprüche bezeichneten Aussagen zu sehen.

Als Feientage gelten der 1. Juni (Den' zascity detej) und der 1. Mai (den' Otkrytija Treugol'nika).

Die außerst gewalttätige Gruppierung der ljubera. entstand Anfang bis Mitte der achtziger Jahre im Moskauer Vorort Ljubercy, von dem sich die Bezeichnung für die Gruppierung herleitet (vgl S Scepanskaja 1993, 200; Pilkington 1994) Aufgrund der Aktionen dieser Gruppierung. deren Miglieder sich mit Bildern Lenins, roten Sternen und Flaggen sowie Pionterhalstuchern schmuckten (vgl. Scepanskaja 1993, 200), setzte, so \$cepanskaja, ein Konsolidienungsprozeß ein, der dazu fuhrte, daß die genannte Subkultur ein Sammelbecken für westlich orientierte Jugendliche wurde (vgl. : Scepanskaja 1993, 200). Antreibende Idee und zugleich Ziel der ljubera war: "«ocistit'» Moskvy (a zatem i Leningrad i voobsce Rossiju) ot «necisti» $i$ "antisocial'nych elementov" vrode chippi" (Scepanskaja 1993, 200). Die Aktionen gestalteten sich derart, daß die ljubera zunächst von ihrem Heimatort aus nach Moskau fuhren, um dort den ihrer Meinung nach ,asozialen Elementen“ aufzulauern, zu denen die genannten Hippies, aber auch Punker und Heavy Metal Fans gehörten. Wurden diese angetroffen, so „bili za chair, publicno i nasil'no strigli, sryvaly "pacifiki" i drugie znacki s odezdy" (Sicepanskaja 1993, 210).

Es wird deutlich, daß eine Assimilation mit den ljubera, die die Sistema-Mitglieder offensichtlich als ihre Feinde betrachteten, nicht möglich war. Dennoch oder gerade aufgrund dieses negativen Verhältnisses ist eine Thematisierung der ljubera in den Texten des rok-samizdat nicht ausgeschlossen 


\subsection{Zusammenfassung}

Es kann festgestellt werden, daß Sistema und rok-samizdat sowohl zeitlich als auch auf der Ebene der rok-kul'tura miteinander assoziiert sind. In diesem Sinne besitzen Scepanskajas Beschreibungen ein heuristisches Potential in bezug auf die durchzufuhrende Diskursanalyse: auf der Grundlage der Arbeit können «Elemente» definiert werden, deren Vorkommen in den ausgewählten Texten geprüft werden kann. Mit einer solchen Prüfung kann zum einen festgestellt werden, ob die «Elemente» charakteritisch für den rok-samizdat sind, zum anderen kann der Frage nachgegangen werden, wie die Beziehung zwischen den genannten Gruppen konkret aussieht. Die «Elemente», die zur Beantwortung der aufgeworfenen Fragen herangezogen werden, sind

1. sleng: Mit dem sleng des Sistema liegt in diskurstheoretischer Hinsicht eine nicht-interdiskursive Sprachvariante vor. Da Scepanskaja, wie erwähnt, die Veränderbarkeit des sleng nicht berücksichtigt und zudem Zweifel hinsichtlich der Vollständigkeit des von ihr ermittelten sleng-Repertoires bestehen, sollen sämtliche nicht-interdiskursiven Sprachelemente, die in den ausgewählten Texten ermittelt werden können, unter dem Begriff Diskursiva (vgl: 5.4.) subsumiert werden. Es geht in diesem Sinne nicht darum, die von Scepanskaja ermittelten Worte und Ausdrücke in den genannten Texten zu ermitteln. Vielmehr geht es darum, festzustellen, ob die Verwendung nicht-interdiskursiver Sprachelemente ein charakteristisches Merkmal dieser Texte ist.

2. Anglizismen (anglicizmy): Anglizismen stellen einen Unterpunkt der Diskursiva dar Da ihnen sowohl von Scepanskaja als auch von anderen Autoren (vgl.: Koester 1983; Mineeva, 1996, 357) ein besonderer Stellenwert in der Jugendsprache (molodeznyj zargon) zugesprochen wird, sollen sie, wenn es sich als produktiv erweist, separat betrachtet werden.

3. "Namen»: Aufgrund von Siepanskajas Darstellung kann davon ausgegangen werden, daß onomastisches Material, also Ortsnamen. Personennamen. Bezeichnungen für Gemeinschaften, Markennamen. Institutionen u.dgl.m. innerhalb der dargestellten Jugendsubkultur eine besondere Funktion besitzen. Für die angefuhrten Namen bietet sich zusammenfassend der Begriff Index an, der in Abschnitt 5.6. ausführlich erläutert wird.

4. Sprüche: Es kann aus Scepanskaja Ausführungen abgeleitet werden, daß es sich bei den Sprüchen im weitesten Sinne um «Volksweisheiten» handelt. Für Volksweisheiten dieser Art wird der Begriff Sentenz (sententia) gewählt, der in Abschnitt 5.11. dargestellt wird.

5. Es geht des weiteren darum, diejenigen Aspekte des Sistema diskursanalytisch zu erfassen, die nicht primär auf der sprachlichen Ebene angesiedelt sind. Aspekte dieser Art sind u.a. das außere Erscheinungsbild bzw. die (Freizeit-) Beschäftigung der Sistema-Mitglieder. Für die angesprochenen Aspekte wird der Begriff Formationsspezifika eingefürt. der in Abschnitt 5.12. erläutert wird. 


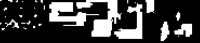

is

I.

* 1

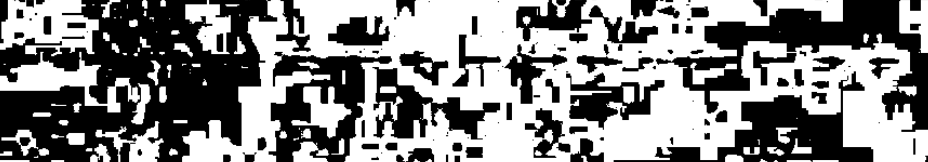
ifo in II

1)

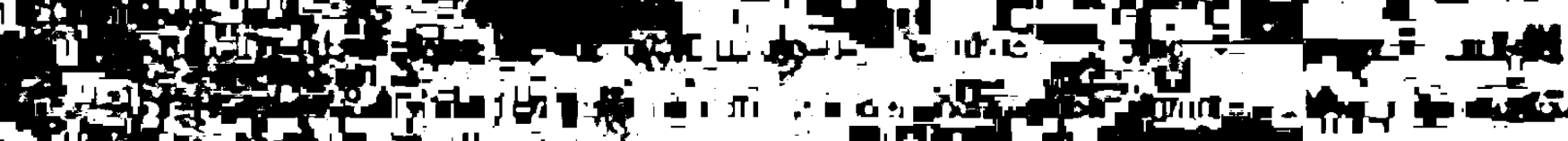
J4

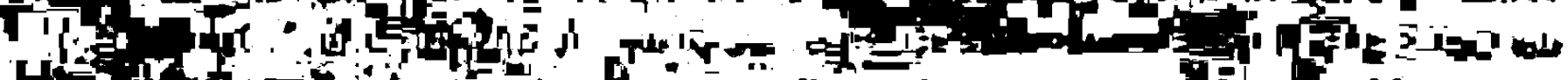

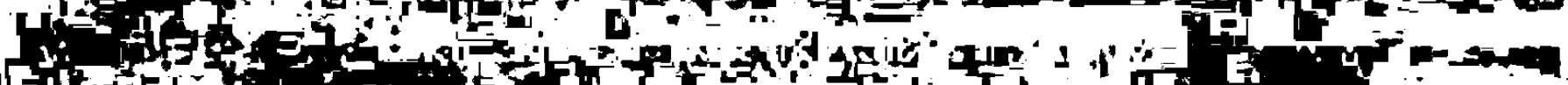

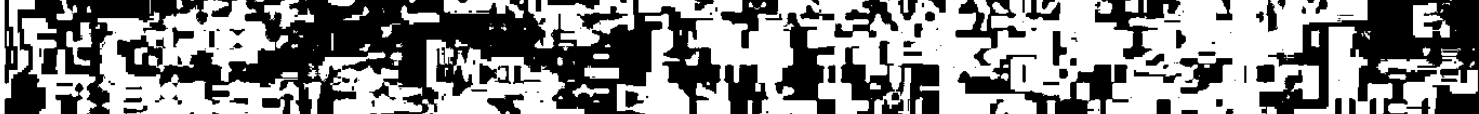

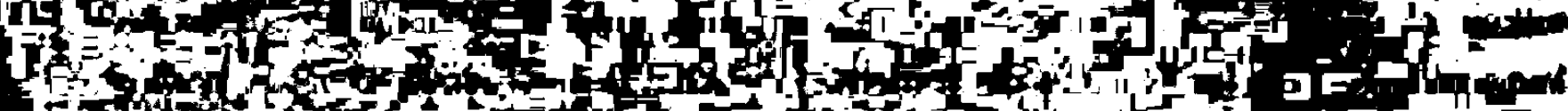

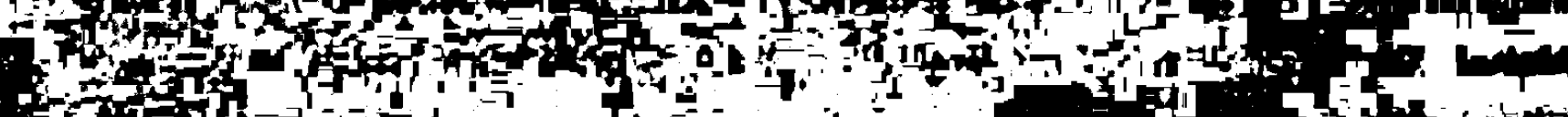

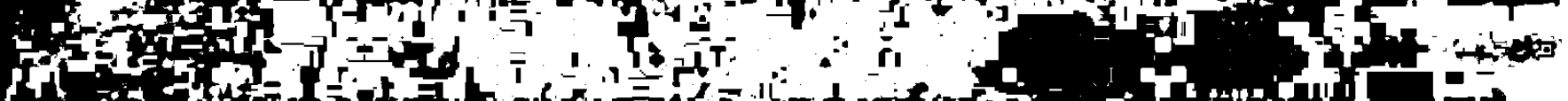
Hot 150

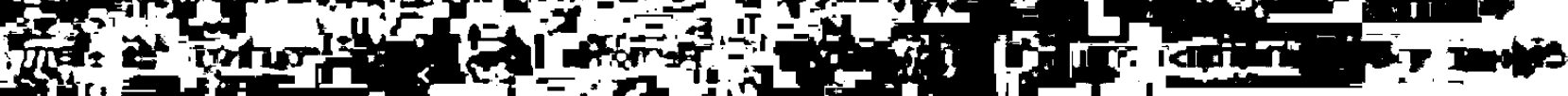
Hit.

Fin

$\therefore=6$ if

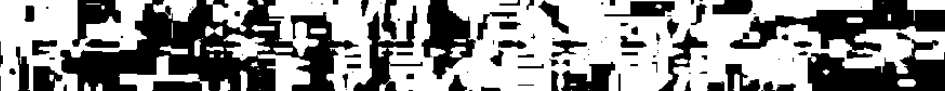

1

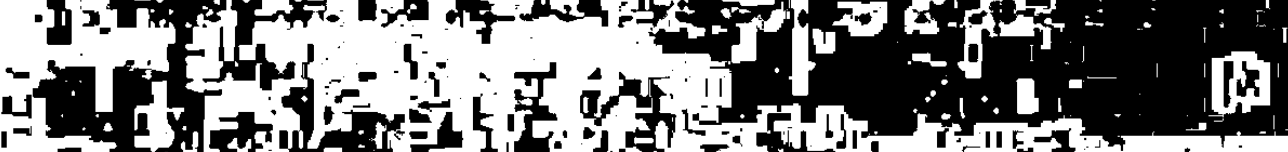

$1 \frac{1}{10}$

年

Ir :

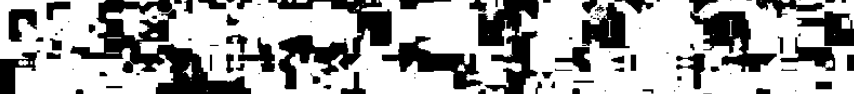

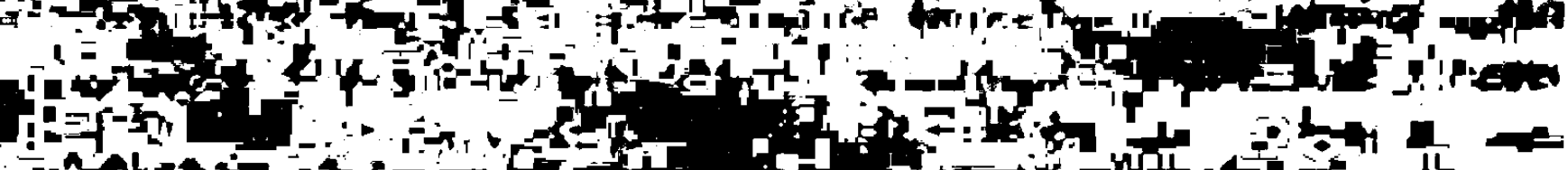

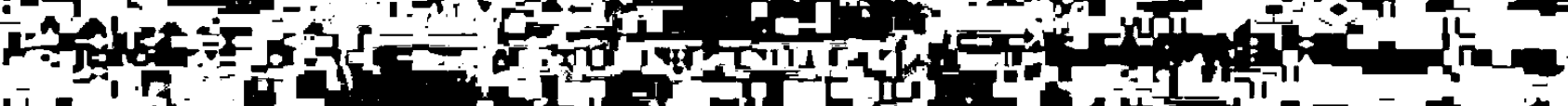

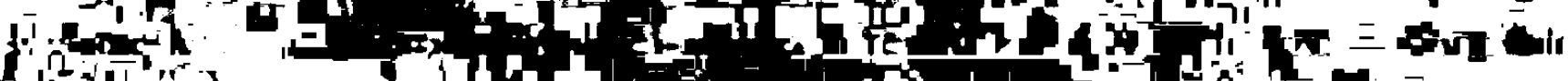

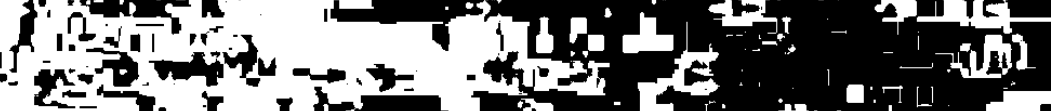

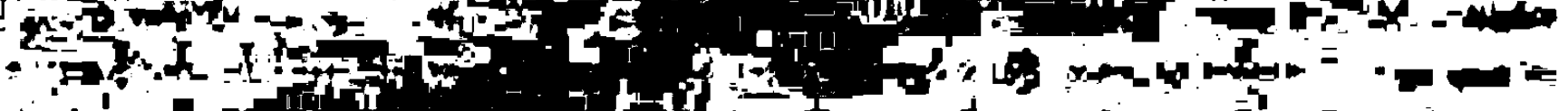

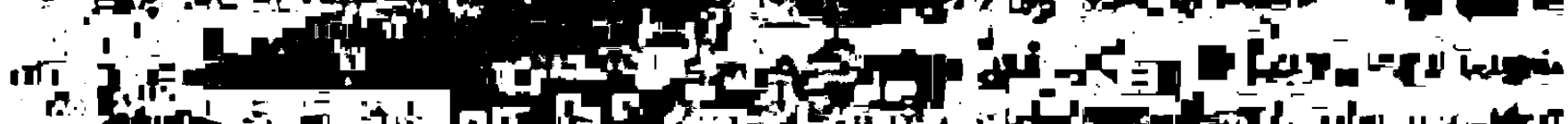

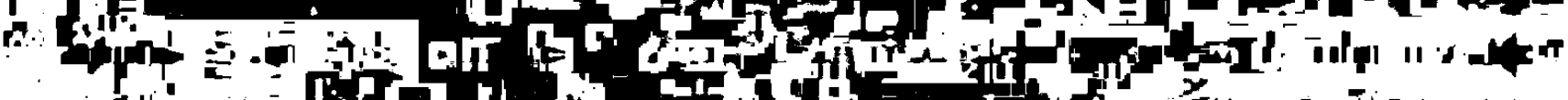

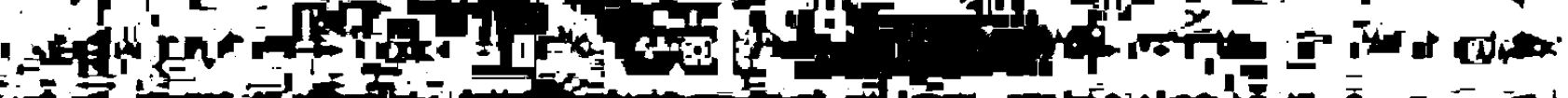

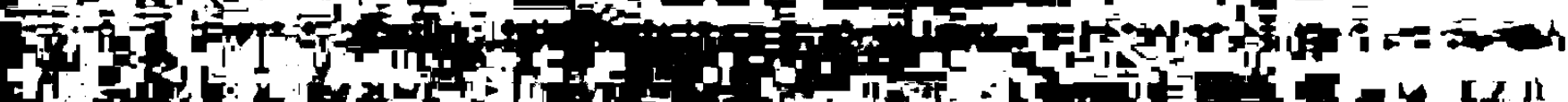
fi.

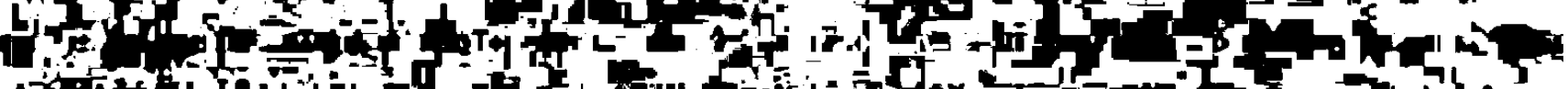

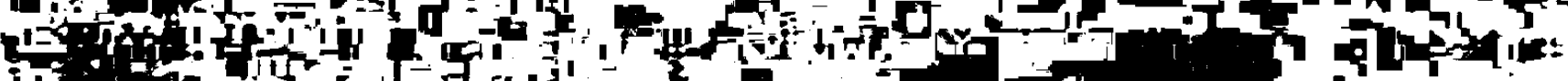

f 1

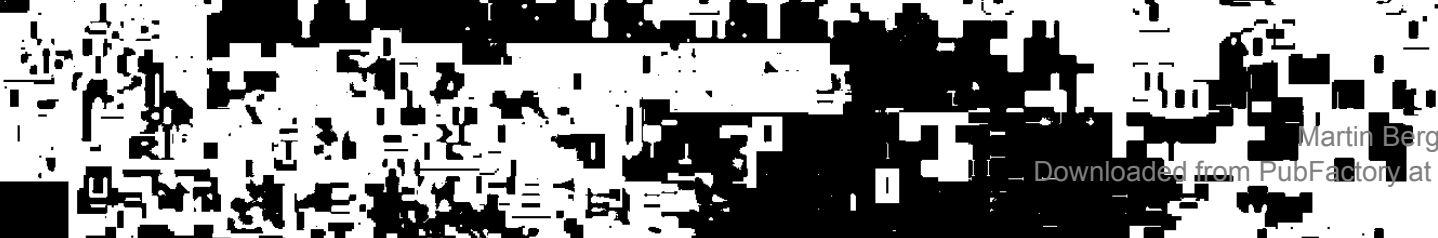




\section{Ergänzungsvorschläge}

\subsection{Allgemeines}

Das bisher erläuterte diskursanalytische Instrumentarium besteht aus Kultursymbolen (Kollektiv- und Diskurssymbolen), Stereotypen. Normativen (kulturellen Tautologien), den syntaktischen Analyseverfahren Wort-Satz-Relation (WSR) und Type-Token-Ratio (TTR) sowie den semantischen Analyseverfahren Themenanalyse und Kontingenzanalyse.

Aufgrund der Kenntnis der im vorangehenden Abschnitt dargestellten Arbeit, des Korpus sowie dieser Arbeit vorangehender Überlegungen (vgl: Bergmann 1997), werden in den folgenden Abschnitten Ergänzungsvorschläge hinsichtlich des zu verwendenden analytischen Instrumentariums gemacht.

Zunächst gilt es, zwei Bewertungskriterien zu konstruieren: einerseits ein Kriterium, welches erlaubt, Objekte, wie beispielsweise Kultursymbole bzw. Analyseergebnisse, wie z.B. WSR-Werte, als charakteristisch für das vorliegende Korpus zu klassifizieren. Andererseits ein Kriterium, welches ermöglicht, die Homo- bzw. Heterogenität des Korpus, des Diskurses und letztendlich der untersuchten Formation zu bestimmen.

Des weiteren werden nicht-interdiskursive Sprachelemente, die als Diskursiva bezeichnet werden, Anglizismen (anglicizmy), Indices, Oppositionen, Antagonismen, die Erzählperspektive, die rhetorische Frage (interrogatio), die Autorität (auctoritas), die Sentenz (sententia) und Formationsspezifika definitorisch bestimmt.

Es soll der Frage nachgegangen werden. welche Funktionen die angeführten Phänomene in einem Diskurs erfullen. Gleichfalls soll im Verlauf der Diskursanalyse festgestellt werden, ob die genannten Phänomene, d.h. Merkmale, für das Korpus charakteristisch sind bzw. inwiefern auf ihrer Grundlage von einer Homogenitat des Korpus, des Diskurses und der betrachteten Formation gesprochen werden kann.

\subsection{Das Bewertungskriterium $\mathrm{ch}$ a rakteristisch}

Das Bewertungskriterium charakteristisch dient in der vorliegenden Analyse der Bestimmung charakteristischer Merkmale des Korpus, um diese als charakteristisch für den untersuchten Diskurs zu klassifizieren. Unter einem charakteristischen Merkmal soll ein Merkmal verstanden werden, welches mit einer Wahrscheinlichkeit von $>0,7$ (70\%) im Korpus nachweisbar ist. Um diese Wahrscheinlichkeit oder Assoziation zu berechnen, empfiehlt es sich, drei Variablen zu berücksichtigen: Variable $A$ umfaßt alle Analyseeinheiten des Korpus. sie ist die Grundgesamtheit (35); Variable B ist ein beliebiges Merkmal (z.B Kultursymbol). 
welches hinsichtlich des Kriteriums charakteristisch geprüft werden soll; die Häufigkeit", mit der Variable B in den Analyseeinheiten des Korpus nachgewiesen werden kann, ist Variable C [z.B.: 32] Es gilt zu berechnen, welche Assoziation zwischen den Ausprägungen von Variable $A$ und Variable $B$ besteht. Zunächst kann angeführt werden, daß die Assoziationen folgendermaßen interpretiert werden 12 :

Tabelle 2

\begin{tabular}{|c|c|}
\hline Formen der Assoziation: & Interpretation: \\
\hline 0.0 bis $<0.2$ & kcine Assoziation \\
\hline 0.2 bis $<0.4$ & niedrige Assoziation \\
\hline 0.4 bis $<0.7$ & mittlere Assoziation \\
\hline 0.7 bis $<1.0$ & hohe Assoziation \\
\hline 1.0 & perfektc Assoziation \\
\hline
\end{tabular}

Um von einem charakteristischen Merkmal zu sprechen, wird, wie angedeutet, für die vorliegende Arbeit eine hohe Assoziation mit einer Wahrscheinlichkeit von mindestens 0,7 postuliert. Die Höhe der Assoziation zwischen den Variablen A und B kann folgendermaßen berechnet werden: man setzt die Variablen $C$ und $A$ in Verhältnis zueinander [32/35] und erhält die Assoziation zwischen den Variablen A und B (hier: 0,91). Dieser Wert besagt, daß das interessierende Merkmal mit einer Wahrscheinlichkeit von 0.91 (91\%) im Korpus nachweisbar ist. Das angesprochene Merkmal ist dementsprechend für das vorliegende Korpus charakteristisch. Darüber hinaus kann gesagt werden, daß die Verwendung dieses Merkmals eine charakteristische Textgenerierungsregel des betrachteten Diskurses darstellt.

\subsection{Das Bewertungskriterium homogen}

Das Bewertungskriterium homogen soll genutzt werden, um die Homogenität und Heterogenität des vorliegenden Korpus, des Diskurses und der untersuchten Formation zu bestimmen. Die Erläuterung des Bewertungskriteriums crfolgt in beaug auf ein Korpus. cin Korpus bestehe aus vier Analyseeinheiten A, B, C und D. Die Homogenität dieses Korpus kann über die Assoziation der in der Diskursanalyse berücksichtigten Phänomene mit dem Korpus bestimmt werden. Ist ein Phanomen $\mathrm{x}$ in den Texten $\mathrm{A}$ und $\mathrm{B}$ nachweisbar, in den Analyseeinheiten $C$ und D nicht, so liegt fur $x$ eine Assoziation von 0,5 (50\%) mit dem Korpus vor. Das Korpus kann unter Berücksichtigung der in Tabelle 2 genannten Interpretationen als in mittlerer Hinsicht homogen und in mittlerer Hinsicht heterogen bezeichnet

"Um die Berochnung der Hăufigkeiten nicht unnötig zu komplizieren. wird im Rahmen dieser Untersuchung mit Dummy-Variablen (0 und 1) gerochnet. Dic absolute Hăufigkeil des Auftretens ciner bestimmten Ausprägung von Variable B wird vernachlässigt. Es wird lediglich nur Kenntnis genommen. dab cin bestimmtes Merkmal im Korpus vorhanden (1) oder nicht vorhanden (0) ist.

12 Je nach Untersuchungsobjekt und wissenschaflicher Dissiplin existieren verschicdenc Interpretationskomventionen. Benninghaus beispielsueise unterscheidet lediglich zwischen den Fällen $k$ eine. schuachc. slarkc und perfeklc Bezichung (vgl: Bcnninghaus 1996. 184-185). Dic in Tabellc 2 aufgefiihrten Interpretationen und Einteilungen sollten in diesem Sinne als Vorschlag verstanden werden. der darauf abriclt. dic anzustellenden Interpretationen nachvollzichbar $\pi u$ gestalten. 
werden. Wenn Merkmal $x$ in drei Analyseeinheiten auftäte, dann ergäbe sich eine hohe Homogenität und eine niedrige Heterogenität. Wird die Betrachtung auf das Merkmal y ausgedehnt, welches in den Analyseeinheiten $A$ und $D$ nachweisbar ist, dann kann von keiner Homogenität und einer perfekten Heterogenität gesprochen werden.

Im Rahmen der Diskursanalyse soll die Homogenität und Heterogenität des Korpus, des Diskurses und der Formation auf der Grundlage derjenigen Phänomene berechnet werden, deren Beruicksichtigung sich als produktiv erweist.

\subsection{Nicht-interdiskursive Sprachelemente: Dis ku rsiv a}

Der Begriff Diskursiva wird fur nicht-interdiskursive lexikalische und syntaktische Sprachelemente vorgeschlagen. Unter dem Begriff Diskursiva werden somit, von einem sprachwissenschaftlichen Standpunkt aus betrachtet, verschiedene Phänomene subsumiert, wie z.B. Vulgarismen, onomatopoetische Ausdrücke, Archaismen, Neologismen. Phraseologismen u.dgl.m. Da die kulturelle Formation des rok-samizdat mit hoher Wahrscheinlichkeit ein Phänomen darstellt, welches mit der sowjetischen bzw. postsowjetischen Jugend assoziiert ist, empfiehlt es sich, auf die Sprache der Jugendlichen einzugehen, die, wie bereits erwähnt, als molodeznyj zargon, argo oder sleng (vgl.: Mineeva 1996, 348-349) bezeichnet wird. Hier wird im weiteren Verlauf der Darstellung für die Sprachvariante der Jugendlichen der Begriff molodeznyj zargon gewähit.

Unter dem Begriff molodeznyj zargon werden verschiedene jugendsprachliche Varianten subsumiert: "Suscestrujet neskol'ko zargonov vnutri sobstvenno molodełnogo zargona" (Mineeva 1996, 351). Mineeva fúhrt in diesem Zusammenhang die spezifischen Varianten der Hippies, der rokery-motociklisty sowie der Anhänger verschiedener Musikrichtungen an (vgl.: Mineeva 1996, 351). Im folgenden Abschnitt soll es nicht um diese spezifischen Varianten, sondern um allgemeine etymologische Charakteristika des molodeznyj zargon sowie um seine Funktionen gehen Auf den Aspekt der produktiven Wortbildungsmodelle, die u.a. auf Affixierung beruhen, soll nicht eingegangen werden.

Eine der wesentlichen Funktionen des molodeznyj zargon, die als Markienungsfunktion bezeichnet werden kann, liegt darin begründet, daß mittels seiner Verwendung die Zugehörigkeit zu einer bestimmten Referenzgruppe, d.h. zu einer kulturellen Formation, angezeigt werden kann. Diese Funktion wird durch die folgenden Prozesse bedingt: zum einen zeigt ein Individuum aufgrund der Verwendung bestimmter Jargonismen an, daß es sich einer bestimmten kulturellen Formation zugehörig fuhlt, zum anderen vermeidet es in dieser Formation tabuisierte Jargonismen. Eine weitere Funktion des molodełnyj zargon ist eine, die als kreativnaja bezeichnet wird, die auf dem sogenannten Spielphänomen (fenomen igrivoj) beruht (vgl.: Mineeva 1996, 351). Es geht in dieser Hinsicht um einen «Wettstreit», der darin besteht, dem Jargon möglichst die eigenen Neuschöpfungen hinzuzufügen. Auf der Grundlage dieses Phanomens entsteht. so Mineeva, eine große Anzahl an Synonymen (Mineeva 1996, 351). Des weiteren besitzt das bekannte Gesetz der Sprachökonomie (zakon ekonomiki jazykovych 
sredstv) im molodeznyj zargon Gültigkeit, so daß Aussagenverkürzungen (sokrascenija vyskazyvanija) beobachtbar sind (vgl.: Mineeva 1996, 352). Darüber hinaus führt Mineeva die Möglichkeit der emotional-expressiven Färbung (emocional'no-ekspressivnaja okraska) von Aussagen an, wobei Mineeva zwischen ironischer (ironiceskaja), spaßhaft-familiärer (sutlivofamil'jarnaja), mißbilligender (neodobritel'naja) und drohender (ugrołajuscaja) Färbung unterscheidet (vgl.: Mineeva 1996, 353).

Die materielle Basis des molodełnyj zargon stellt die sogenannte Literatursprache (literatumyj jazyk) dar, die durch den molodeznyj zargon qualitativ und quantitativ erweitert wird. Von einer qualitativen Erweiterung kann insofern gesprochen werden, da bestimmte Wörter und Ausdrücke durch zusätzliche Semantisierungen ausgezeichnet werden; von einer quantitativen Ergänzung, da dem vorhandenen Repertoire gänzlich neue Elemente hinzugefugt werden. Vorob'ev füht in diesem Zusammenhang an, daß die quantitative Erweiterung 600 Wörter ausmache, wobei es sich bei diesen Wörtern au einem großen Teil um Anglizismen handele (vgl: : Vorob'ev 1990, 40). Aufgrund der Bedeutung der Anglizismen für den molodermyj zargon soll auf diese in Abschnitt 5.5. gesondert eingegangen werden. Neben englischsprachigen Elementen stammen Worte und Ausdrücke des molodezmyj zargon aus der sogenannten StadtfolkJore (gorodskoj folklor) und dem tjuremno-lagernyj targon (vgl: Mineeva 1996, 358).

Neben den genannten Aspekten lassen sich im molodeznyj zargon Phraseologismen bzw. feststehende Ausdrücke (ustojcivye vyrazenija) nachweisen ${ }^{13}$. Hierbei handelt es sich zum einen um neue Ausdrücke, zum anderen um sogenannte kal'kirovannye vyrazenija (vgl.: Mineeva 1996, 357). Nach Mineeva besitzen die feststehenden Ausdrücke für jede Sprache eine besondere, expressive Funktion, denn „Frazeologizmy znacitel'no uvelicivajut vyrazitel'nost' ljubogo jazyka“ (Mineeva 1996, 359). Es liegt auf dieser Grundlage die Vermutung nahe, daß Phraseologismen bzw feststehende Ausdrucke nicht nur im molodènyj zargon haufig realisiert werden. In diesem Zusammenhang weist Mineeva auf eine von ihr durchgefuhrte Untersuchung im studentischen Milieu hin, in der sie feststellen kunnte. daß Phraseologismen und feststehende Ausdrucke die Hälfte der nachweisbaren Jargonismen ausmachen (vgl : Mineeva 1996, 359). Aufgrund der Bedeutung der Phraseologismen und feststehenden Ausdrücke soll auf diese in allgemeiner Hinsicht gesondert in Abschnitt 5.11. eingegangen werden.

Der molodeżnyj zargon stellt, wie jedes andere Sprachsystem auch, ein sich veränderndes System dar, dessen primäre Funktion in bezug auf eine kulturelle Formation und somit auf einen Diskurs mit hoher Wahrscheinlichkeit die der Markierung ist. In der Diskursanalyse sollen Elemente des genannten Systems in den ausgewählten Analyseeinheiten ermittelt werden Es muß an dieser Stelle betont werden, daß z.Zt. unklar ist, inwiefern dieses System schriftsprachlich realisiert wird. Um Elemente als systemisch klassifizieren zu können, kann auf verschie-

\footnotetext{
${ }^{13}$ Es soll darauf hingewiesen werden. daB Mineera die Bcgriffe frazcologizm und ustojcivoe vyrazenie synomm venwendet (vgl.: Mineeva 1996. 359). Dies ist legitım (vgl: BuBmann 1990. 320). Der Begriff frazcologizm kann aber auch aufgrund von rusät7lichen Bodcutungskomponenten (vgl: Bußmann 1990 . 320) als Sperialisierung des Begriffes ust ojeiv oc vy ra r enic aufgefaBt werden
} 
dene Wörterbücher ${ }^{14}$ zurückgegriffen werden. In diesem Sinne kann für einzelne Elemente geprüft werden, ob sie dem molodeznyj zargon zugerechnet werden können. Da sich das System fortwahrend verandert, eine Erfassung des vollständigen Repertoires auch im Nachhinein nahezu ausgeschlossen ist, wird ein anderer Weg eingeschlagen: es werden sämtliche Elemente erfaßt, die nicht literatursprachlich, d.h. die nicht-interdiskursiv sind. Diese Vorgehensweise bietet zwei Vorteile: einerseits muß fur einzelne Elemente nicht geprüft werden, ob sie aus dem molodełnyj zargon oder aus anderen Sprachvarianten stammen, andererseits werden auf einer materiellen Ebene alle Elemente erfaßt, die aufgrund ihrer Nichtzugehörigkeit zur Literatursprache einen Text in diskursiver Hinsicht markieren können.

\subsection{Anglizismen (anglicizmy)}

Anglizismen treten im molodeznyj zargon seit etwa 1960 auf (vgl.: Koester 1983, 82), wobei „eta gruppa v nastojascee vremja imeet tendenciju k bystromu uveliceniju“ (Mineeva 1996, 357). Einige Anglizismen werden originalgetreu dem englisch-sprachigen Vorbild sowohl hinsichtlich der Aussprache als auch der Flexion übernommen, andere werden durch Suffixierung "russifiziert". Es existieren verschiedene Themenkomplexe, in denen Anglizismen besonders haufig auftreten, wobei für den vorliegenden Zusammenhang besonders auf die Bereiche Musik und Freizeit (vgl: Koester 1983, 83) sowie den Bereich der Selbstbezeichnungen (vgl : Stadler 1992, 118) hingewiesen sei. Koester weist darauf hin, daß „anglojazyčnych slov v molodełnom zargone svidetel'stvujut o tom, cto anglicizmy zanimajut central'noe mesto $v$ zargone molode ${ }^{\prime} i$ i ich rasprostranenie dovol'no siroko" (Koester 1983, 83-84). Es ist unklar, inwiefern die Verwendung von Anglizismen ein Phänomen der Umgangssprache (razgovornyj jazyk) und somit ein mündliches Phänomen darstellt: Koester spricht vom molodełnyj خargon implizit als mündlichem Phänomen (vgl: Koester 1983, 82, 84), wobei sie jedoch anhand von Beispielen den Ubergang von Anglizismen in die Schriftsprache demonstriert (vgl : Koester 1983, 83). Da sich die große Anzahl der Anglizismen in der Jugendsprache anscheinend erhoht hat bzw. weiterhin erhöht, ein Phänomen welches sicherlich mit dem Prozeß der «Verwestlichung» der Jugendlichen zusammenhängt («Vesternizacija»; vgl:: Sikevic 1996, 337), die spätestens 1986 's begonnen hat und aufgrund ihrer zentralen Stellung in der Jugendsprache, kann die Vermutung geäußert werden, daß Anglizismen in schriftsprachlicher Realisierung in den ausgewählten Analyseeinheiten nachweisbar sind.

\subsection{Indices}

Der Begriff des Index stammt aus der Peirce'schen Zeichentheorie. Er bezeichnet dort „ein Zeichen. das direkte, kausale, reale Beziehung zu seinem Objekt hat. das direkt auf das Objekt

14 Ein Worncrbuch. welches der Jugendsprache gewidmet ist. stellt Fajn. A.: Lur'e. V. 1991. cin der Umgangssprache gewidmetes Koester-Thoma. S.: Rom. E. 1985. dar

15 Das Jahr 1986 brw. der 27 Paneitag der KPdSU. der im Febnuar des genannten Jahres abgehalten uurde. kann grob als Anfang der Perestrojka. also der Offnung nach "Westen», gewertet werden (vgl.: Lane 1992. 6). 
hinweist, es anzeigt" (Bense. Walther 1973, 40). Diese Definition, die eine Zeit- und Ortsabhängigkeit eines indexikalischen Zeichens suggeriert, wird übernommen.

Indices werden als ein wichtiger materieller, da funktionalisierbarer Bestandteil von Diskursen und Weltbildern aufgefaßt. Sie können unmittelbar als Indikatoren fur spezifische Diskurse und Weltbilder und somit für kulturelle Formationen gelten. Sie funktionieren in diesem Sinne auf der Ebene von Diskursen und Weltbildern, d.h. auf der kulturellen Ebene, wie Jargonismen auf der sprachlichen Ebene: allein durch ihre Verwendung bzw. Vermeidung zeigt ein Individuum seine Zugehörigkeit zu einer kulturellen Formation an. Es soll an dieser Stelle darauf hingewiesen werden, daß nahezu jedem Zeichen eine hinweisende Funktion zugesprochen werden kann. Bei den hier als Indices bezeichneten Zeichen wird jedoch eine näher zu bestimmende hinweisende Qualitat in bezug auf Diskurse, kulturelle Formationen bzw. Kultursysteme vermutet, als bei anderen Zeichen. Indices sind in diesem Sinne ein Phänomen, welches erlaubt, Diskurse. Weltbilder und kulturelle Systeme voneinander abzugrenzen.

Auf der semantischen Ebene handelt es sich bei Indices zum einen um onomastisches Material, also um Ortsnamen, Namen von Aktanten, Bezeichnungen für Gemeinschaften, Markennamen, Namen von Institutionen u.dgl.m. Zum andern umfaßt der Begriff Bezeichnungen für «historische» Ereignisse' ${ }^{16}$. Was als ein solches Ereignis aufgefaßt wird, ist abhängig von einer kulturellen Formation bzw. einem Kultursystem: im deutschen Kultursystem stellt der Index ,der Zweite Weltkrieg' ein solches Ereignis dar, in der kulturellen Formation des rok-samizdat das ,V lenigradskij rok-festival" (vgl.: Kusnir 1994, 10). Ereignisse in diesem Sinne unterscheiden sich von anderen Phanomenen dadurch, $\mathrm{daB}$ uber sie berichtet wird. Sie werden in diesem Sinne kognitiv konstruiert, da auf das Ereignis "an sich» eine Anschlußhandlung erfolgt Ein Ereignis, uber welches nicht berichtet wird, stellt dementsprechend keines der hier interessierenden Ereignisse dar, da es nicht kulturell relevant ist. Das genannte $V$ lenigradskij rok-festival' ist in diesem Sinne für das deutsche Kultursystem kein kulturell relevantes Ereignis. Die Gemeinsamkeit der Elemente, die unter dem Begriff Index subsumiert werden, liegt auf der strukturellen Ebene in dem Vorhandensein einer konkreten, kurzen, d.h. nur wenige Worte umfassenden und dementsprechend pragnanten Bezeichnung. die einen hohen Wiedererkennungswert garantiert. Von einem diskurstaktischen Standpunkt betrachtet, ist bedeutsam, wie bestimmte Indices attribuiert und also konstruiert sind bzw. werden und wie sich die Attribuierung und also Konstruktion in zeitlicher Hinsicht verändert. In diesem Zusammenhang bieten sich verschiedene Differenzierungen an. Zunachst kann zwischen kulturell funktionalisierten und kulturell nicht-funktionalisierten Indices unterschieden werden. Nicht kulturell funktionalisiert sind beispielsweise Namen von Orten, die nur im Sinne einer Ortsbestimmung genannt werden, die also keine kulturelle Bedeutung und somit keine Relevanz aufweisen. Kulturell funktionalisierte Indices hingegen, die auch als kulturell relevante Indices bezeichnet werden können, besitzen eine kulturelle Bedeutung, die entweder

16 Der Begriff Ercignis wird hicr nicht in cinem «breiten" Sinne wic von der Gruppe Tüschau 16 (vgl. Tuschau 16 1998. 71) verstanden. Er stellt hier cinen Unterpunkt der Indices dar. 
expliziert oder nicht expliziert wird. In bezug auf diejenigen, deren Bedeutung nicht expliziert wird, kann von einer abgeschlossenen Bedeutungskonstruktion im Rahmen des sie thematisierenden kulturellen Systems ausgegangen werden. Diejenigen Indices, die attribuiert werden, befinden sich im Gegensatz dazu im Prozeß der Bedeutungskonstruktion. Auf der Grundlage des Gesagten liegt der Schluß nahe, daß mit dem diskursiven Verfahren der IndexKonstruktion, ein äußerst produktives Manipulationsverfahren vorliegt: das Ereignis ,Zweiter Weltkrieg" hat unbestreitbar stattgefunden. Je nachdem welche kulturelle «Position» ein Aktant, eine Gemeinschaft, eine kulturelle Formation bzw. ein Kultursystem in bezug auf das angesprochene Ereignis einnimmt, wird er bzw. sie das genannte Ereignis nicht thematisieren bzw es in bestimmter Hinsicht konstruieren bzw. konstruiert haben. Wenn das genannte Ereignis thematisiert wird, dann ist es, wie angedeutet, entweder bereits konstruiert, oder das Konstrukt wird innerhalb einer Äußerung vermittels von Attributen generiert. D.h. m.a.W., daß nicht nur das Vorkommen bestimmter Indices ein Hinweis auf Diskurse, Weltbilder und kulturelle Formationen ist, sondern auch die Konstruktion der Indices, so daß auch unterschiedliche Konstruktionen Abgrenzungen auf der kulturellen Ebene ermöglichen.

\subsection{Oppositionen und Antagonismen}

Im folgenden Abschnitt geht es um die Struktur und die Funktion sprachlicher und kultureller Gegensätze. Beide Gegensatzarten stellen Konstrukte dar, so daß ihre Ausprägungen von kulturellen Formationen bzw. Kultursystemen abhängen. Sprachliche Gegensätze zeichnen sich in struktureller Hinsicht durch ein scheinbar semantisch diametrales Verhälnis ihrer Elemente aus: .schwarz' vs. ,weiß', gut' vs. ,böse', ,Natur' vs. ,Kultur' etc. Für Gegensätze dieser Art wird der Begriff $O$ p position verwendet.

Ausgangspunkt der Überlegungen in bezug auf kulturelle Gegensatze ist die Hypothese, $\mathrm{da}$ B Mitglieder von kulturellen Systemen explizit eine Abgrenzung von bestimmten Aktanten, Gemeinschaften bzw von anderen kulturellen Systemen anstreben. um das eigene System zu festigen bzw. zu sichern und um interne Differenzierungen vorzunehmen. Diese expliziten Arten der Systemabgrenzung sollen unter dem Begriff Ant agonismus subsumiert werden.

Es scheint verschiedene sprachliche Mittel zu geben, die eine solche Systemabgrenzung ermoglichen: auf Stereotype wurde bereits im Rahmen der Darstellung der Theorie der Zweiten Wirklichkeit (vgl. 2.9.) eingegangen. In funktionaler Hinsicht können auch Oppositionen dem Aufbau von Antagonismen dienen. Ein Beispiel, bei dem von der Opposition ,deutsch'vs. ,polnisch' ausgegangen wird. soll dies verdeutlichen: zunachst soll festgehalten werden, daß es sich bei dem genannten Beispiel um eine Opposition handelt, da die Elemente des Gegensatzpaares in einem scheinbar semantisch diametralen Verhältnis zueinander stehen. $\mathrm{Ob}$ es sich um einen Antagonismus handelt, kann nur anhand des Kontextes gepruft werden, indem der Frage nachgegangen wird. ob die Opposition eine Funktion hinsichtlich der Systemabgrenzung erfullt. Nur wenn die Opposition darauf ausgerichtet ist, eines der Systeme gegen das andere abzugrenzen, dann liegt ein Antagonismus vor. 
Neben Stereotypen und Antagonismen, die auf Oppositionen basieren, existieren vermutlich weitere sprachliche Mittel, die der Systemabgrenzung und also Sicherung dienen. Im vorliegenden Zusammenhang soll nicht primar der Frage nachgegangen werden, mit welchen Mitteln Antagonismen generiert werden. Vielmehr geht es darum, festzustellen, welche Aktanten, Gemeinschaften, kulturellen Systeme u.dgl.m. aus der interessierenden Formation ausgegrenzt bzw. welche internen Differenzierungen vorgenommen werden.

\subsection{Die Erzählperspektive}

Im Rahmen dieser Untersuchung sollen zwei basale Erzählperspektiven unterschieden werden: zum einen eine Perspektive, die aus der Sicht, ich'bzw. ,wir' verfaßt und durch das Vorkommen der Personalpronomen ja“ und ,my' bzw. deklinierter Formen derselben gekennzeichnet ist. Diese Perspektive wird hier als persönliche Perspektive bezeichnet. Zum anderen die unpersönliche Perspektive, welche die verbleibenden Erzählperspektiven umfaßt

Im Rahmen der persölichen Perspektive soll auf eine besondere Ausprägung eingegangen werden, die als Insiderperspektive bezeichnet wird. Die Insiderperspektive ist dadurch gekennzeichnet, daß der Verfasser eines Textes einem Rezipienten suggeriert, daß er ein sogenannter Insider ist und somit über Insiderinformationen verfügt. Insiderinformationen besitzen den Ruf, besser, umfassender und detaillierter zu sein, als andere Informationen, so daß sie geeignet sind, das "Vertrauen» des Rezipienten zu gewinnen. Es gilt zu bedenken, daß es nicht um "wahre" Informationen geht, sondern um den Aufbau eines Konstrukts, das Konstrukt des Insiders. Mit diesem Konstrukt scheint ein außerst produktives manipulatives Verfahren vorzuliegen. Es gilt der Frage nachzugehen, wie das Konstrukt des Insiders generiert wird. Dies ist über Propositionen in der Art von „Ich bin ein Insider und besitze Informationen uber $x$, die sonst niemand besitzt" moglich. Es ist fragwurdig. ob der direkte Hinweis auf den genannten Status effektiv ist. Vielmehr liegt die Vermutung nahe, daß indirekte Hinweise auf den Insiderstatus effektiver und also manipulativer sind. In diesem Sinne geht es darum, Signalworte zu ermitteln, die einen indirekten Hinweis auf den Status des Verfassers geben. In der russischen Sprache handelt es sich vermutlich bei den folgenden Worten bzw. Formulierungen um die angesprochenen Signalworte: .kstati', vprocem”, mimochodom', mèdu tem', ,krome-togo", ,na samom dele', fakticesko' und ,dejstvitel'no'. Es wird davon ausgegangen, daß diese Signalworte in Verbindung mit einer Proposition die geschilderte Wirkung erzielen. Es sei darauf hingeweisen, daß der Verfasser, wenn er den Status eines Insiders im Bewußtsein des Rezipienten erreicht hat, diesen vermutlich über die gesamte Lange eines Textes beibehalt, so daß der Status nicht auf die konstruietende Proposition begrenzt ist.

Ein weiterer Punkt, der vermittels der persönlichen Erzählperspektive betrachtet werden kann, ist der der Generierung von Stereotypen, die sich auf die Wir-Gemeinschaft beziehen (sogenannte Autostereotype) Aufgrund der Definition des Begrifts Stereotyp (vgl. 2.9.) sind für selbiges in struktureller Hinsicht zwei Merkmale notwendig. die sich auf eine Gemeinschaft beziehen ein Klassifizienungsmerkmal und ein Merkmal, welches das Stereotyp letztendlich 
ausmacht Bezogen auf das Phänomen des Autostereotyps muß zunächst ein Klassifizierungsmerkmal vorliegen, da ansonsten unklar ist, wen die Gemeinschaft umfaßt. Das stereotypisierende Merkmal wird dann der durch das Klassifizierungsmerkmal bezeichneten Gemeinschaft zugeordnet. Gelingt aufgrund dieser Zuordnung eine Abgrenzung von anderen Gemeinschaften, dann liegt ein Autostereotyp vor.

In der folgenden Analyse gilt es, festzustellen, welche Erzählperspektive in der interessierenden Formation präferiert wird. Darüber hinaus soll festgestellt werden, ob die Insiderperspektive verwendet wird.

\subsection{Die rhetorische Frage (interrogatio)}

Die Stilfigur der rhetorischen Frage wird allgemein hin zu den Sinnfiguren gerechnet (vgl: Ueding 1992, 67; Göttert 1994, 58). Diese Figuren, zu denen u.a. Vergleiche, Ausrufe, Ironie und Evidenz gezählt werden, stellen "Strategien der Rede" dar bzw. dienen bestimmten .argumentativen Manövern“ (vgl:: Göttert 1994, 58). Da rhetorische Fragen in bezug auf die durchzuführende Diskursanalyse als produktiver und operationalisierbarer eingeschätzt werden, als die übrigen genannten Sinnfiguren, erfolgte eine Konzentration auf diese. Es werden verschieden Anwendungsarten der thetorischen Frage unterschieden: "-die ungeduldigpathetische Frage; -die Frage, die eine Tatsache besonders nachdrücklich aussprechen will [...] -die nur schwer oder gar nicht beantwortbare Frage, die Ungläubigkeit oder Staunen ausdrückt [...] -die Frage, die einen strengen Befehl oder eine Auffordenung enthät [...]-Fragen, die der Redner an sich selbst richtet [...]" (Ueding: Steinbrink 1994, 310-311).

Es soll nicht auf Besonderheiten dieser Anwendungsarten eingegangen werden. Vielmehr geht es um allgemeine Funktionsmerkmale der Figur.

Der Begriff rhetorische Frage bezeichnet allgemein eine „Frage, auf die der Redner keine Antwort erwartet und in die er eine Aufforderung oder Aussage kleidet, wenn er besonders eindringlich und emotional wirken will" (Ueding, Steinbrink 1994, 310). Obwohl im vorangehenden Zitat der Aktant Redner eingefuhrt. was eine Begrenzung des Objektbereichs auf mündliche Kommunikationsprozesse suggeriert, gelten die Überlegungen, wie die von Ueding und Steinbrink genannten Beispiele belegen (Ueding; Steinbrink 1994, 310-311), auch fur schriftliche Kommunikationsprozesse

Zunächst kann festgehalten werden, daß der Figur generell eine manipulative Funktion zugesprochen wird. Betont werden muß, daß es sich bei der rhetorischen Frage in qualitativer Hinsicht nur um eine Stilfigur handelt. Von besonderer Bedeutung für den Verfasser eines Textes und möglicherweise auch für eine kulturelle Formation bzw. ein Kultursystem ist die zugrundeliegende Aussage bzw. Aufforderung. Für den Verfasser eines Textes besitzt die Aussage bzw. Aufforderung zweifellos einen besonderen Stellenwert, da er ansonsten die Figur nicht bemühte. Ob die Aussage bzw. Aufforderung auch fur ein kulturelles Sytsem relevant ist, kann gepruft werden, indem rhetorischen Fragen zugrundeliegende Aussagen bzw. Aufforderungen in qualitativer Hinsicht analysiert werden. 


\subsection{Die A u torität (auctoritas)}

Im folgenden Abschnitt geht es um eine Art der beispielhaften Argumentation: die Autorität. Bestandteile dieses beispielhaften Arguments sind zum einen die Autorität, zum anderen das Beispiel, welches der Autorität zugesprochen wird. Die angesprochene Argumentationsart wird im Rahmen der Rhetorik als wirkungsvolles Überzeugungsmittel angesehen, wobei die Uberzeugungskraft der Autorität mit derem besonderen, gesellschaftlichen Ansehen begründet wird (vgl:: Ueding; Steinbrink 19924, 268). Die dargestellte Auffassung hinsichtlich der Überzeugungskraft bestimmter Phänomene, die auch im Rahmen einer Diskursanalyse als produktiv angesehen wird, läßt sich jedoch nicht ohne weiteres auf eine diskursanalytische Untersuchung übertragen, da der Begriff des kulturellen Ansehens nicht mit dem der kulturellen Bedeutung identisch ist. Ausgangspunkt der Überlegungen im Rahmen der Rhetorik ist die Auffassung, daß nur Personen, die ein kulturelles Ansehen genießen, die dementsprechend kulturell relevant sind, als Autorität eingesetzt werden konnen. Dies setzt voraus, daß die Autoritäten in der entsprechenden Gesellschaft bekannt sind. Dies scheint jedoch in diskursanalytischer Hinsicht nicht notwendig zu sein, da auch Autoritäten Konstrukte darstellen. Es ist in diesem Sinne aus manipulationstaktischen Gründen möglich, die Bekanntheit einer Autorität vorzutäuschen. Die Bedeutung bzw. Bekanntheit einer Autorität sollte deshalb im Rahmen der Diskursanalyse nicht als Eigenschaftsmerkmal angeführt werden. Zunächst gilt es zu klären. von welchen Eigenschaftsmerkmalen der Autorität in einem diskursanalytischen Sinne ausgegangen werden kann Zum einen muß eine Autorität theoretisch wiederholbar als Autorität einsetzbar sein, so daß sie auf der strukturellen Ebene eine Form aufweisen muß, die eine Wiedererkennbarkeit garantiert. Am geeignetsten scheinen in dieser Hinsicht die bereits erläuterten Indices zu sein (vgl : 5 6.). Die Autorität unterscheidet sich von diesen in struktureller Hinsicht dahingehend, daß sie die Eigenschaft der Zitierfahigkeit aufweist, die durch ein beigestelltes Zitat offenkundig wird

In funktionaler Hinsicht wird das Einbringen von Beispielen. $\mathrm{d}$ h dic Berufung auf Autoritaten im Rahmen der Rhetorik zu den kunstgemaßen Beweisen (probationes artificiales) gerechnet Die Autoritäten funktionieren in diesem Sinne als Beweis für eine Proposition, so daß diese den Wahrheitswert wahr erhält. Es geht letztendlich darum, einen Rezipienten dazu zu bewegen, diesen Wahrheitswert als gültig anzuerkennen. In diskursanalytischer Hinsicht weisen die Autoritäten bzw. Zitate neben der Erhöhung des Wahrheitswerts von Propositionen vermutlich weitere Funktionen auf. Es ist beispielsweise moglich, die kulturelle Bedeutung einer Autoritat mittels eines Zitats zu konstruieren, indem beispielsweise der Autoritat eine diskreditierende Aussage zugesprochenen wird. die in dem entsprechenden kulturellen System abgelehnt wird. Es entsteht ein Antagonismus, der auf die Ausgrenzung der Autoritat abzielt. Darüber hinaus kann ein Text mit hoher Wahrscheinlichkeit durch das Einbringen von Autoritaten an Authentizität gewinnen Die Aussage der Autorität besitzt in dieser Hinsicht keine kulturelle Funktion, sondern lediglich eine textuelle. Erklärungsziel der Diskursanalyse ist es zum einen, in quantitativer Hinsicht zu klären. welche 
Autoritäten in der interessierenden Formation funktionieren. Daruber hinaus gilt es, die kulturelle Bedeutung der kulturell relevanten Autoritäten zu rekonstruieren.

\subsection{Die Sentenz (sententia)}

Es geht im folgenden Abschnitt um die bereits erwähnten, feststehenden Ausdrücke (ustojcivye vyrazenija) (vgl.: 5.4.), für die sich der rhetorische Begriff Sentenz anbietet. Unter dem Begriff Sentenz wird ein "kurz, faßlich, präzise und epatierend formulierte[r] Sinnspruch“ (Ueding; Steinbrink 1994, 268) verstanden. Die Sentenz wird zu den Überzeugungsmitteln gezahlt, so daß ihr in allgemeiner Hinsicht eine manipulative Funktion zugesprochen werden kann. Wesentliche Merkmale der Sentenz sind die Allgemeingültigkeit ihrer Aussagen und ihr hoher Wiedererkennungswert (vgl.: Ueding; Steinbrink 1994, 269). Sentenzen sind entweder anonym entstanden oder können einem bestimmten Verfasser, d.h. einer Autorität (vgl: : 5.10.), zugesprochen werden. Sie sind darüber hinaus variabel: "In der konkreten Anwendungssituation brauchen Sentenzen [...] nicht in ihrer eigenständigen Form $[(.)$.$] in das$ Redekontinuum eingefügt werden, sondern können kontextabhängig [...] mehr oder minder stark [...] variiert werden“ (Ueding; Steinbrink 1994, 269).

Es kann in diesem Zusammenhang die Frage gestellt werden, inwiefern sich Sentenzen von den aus der Sprachwissenschaft bekannten Phraseologismen unterscheiden. Mit hoher Wahrscheinlichkeit handelt es sich lediglich um ein Objekt. Hier sollen alle feststehenden Ausdrücke unter dem Begriff Sentenz gefaßt werden, so daß nicht weitere, ähnliche bzw. gleiche Objekte, die auf der Grundlage anderer Forschungsansätze definiert werden, eingeführt werden müssen. In diskursiver Hinsicht wird davon ausgegangen, daß die primäre Funktion der Sentenzen mit hoher Wahrscheinlichkeit nicht in ihrer Aussage begrundet ist. Statt dessen liegt zum einen die Vermutung nahe, daß ein Text vermittels einer Sentenz in diskursiver Hinsicht markiert wird. Zum anderen, hierauf aufbauend und den Wiedererkennungswert der Sentenz berücksichtigend. steht ein Rezipient dem Verfasser bei «richtigen Anwendung positiv gegenuber, da die gemeinsame, kulturelle «Herkunft» offen gelegt ist. Vermutlich ermöglicht die Sentenz dem Verfasser, den Rezipienten in eine "positive Grundstimmung») zu versetzen, so daß derselbe fur den eigentlichen Sachverhalt manipulationsanfalliger wird. Sentenzen sind in diesem Sinne in diskursiver Hinsicht funktionalisiert und daher kulturell relevant. Ob die Urheber in kultureller Hinsicht relevant sind. ist vermutlich vom Verbreitungsgrad der Sentenz abhänig. Es soll in diesem Zusammenhang die Hypothese formuliert werden, daß eine negative Korrelation zwischen dem Verfasser, d.h. dem Urheber einer Sentenz und deren Verbreitungsgrad existiert. Anders ausgedruckt: je verbreiteter eine Sentenz ist, desto weniger bekannt ist ihr Urheber. In diesem Sinne bußt der Urheber mit Erhöhung des Bekanntheitsgrades einer Sentenz an kultureller Bedeutung ein. Je verbreiteter eine Sentenz ist, desto weniger kann sie gleichfalls den bestimmten Diskurs einer bestimmten kulturellen Formation markieren. Relativ weit verbreitete Sentenzen sind daher zwangslaufig ein Bestandteil des Interdiskurses. In der vorliegenden Arbeit wird das im Korpus vorhandene Sentenzen-Repertoire ermittelt. Anschließend wird der 
Frage nachgegangen, inwiefern von einer kulturellen Bedeutung und Relevanz der jeweiligen Urheber gesprochen werden kann.

\subsection{Formationsspezifika}

Es soll davon ausgegangen werden, daß jede kulturelle Formation über spezifische, vermutlich stereotypisierte, nicht primar zeichenhafte Eigenheiten verfugt, die hier als Formationspezifika bezeichnet werden. Unter diesem Begriff werden verschiedene Phänomene, wie z.B. das äußere Erscheinungsbild, die Herkunft, Tätigkeiten u.dgl.m. subsumiert. Diese Eigenheiten können im Rahmen einer Diskursanalyse berücksichtigt werden, wenn ihnen Zeichencharakter zugesprochen wird: ,Zeichen ist alles, was zum Zeichen erklärt wird und nur was zum Zeichen erklärt wird. Jedes beliebige Etwas kann (im Prinzip) zum Zeichen erklärt werden" (Bense, 1967, 9). Im vorliegenden Fall erhalten Formationsspezifika Zeichencharakter, wenn sie in den ausgewählten Analyseeinheiten nachweisbar sind. Formationspezika können in Texten, wenn es sich um Stereotype handelt, diskursive Funktionen übernehmen, sie müssen es aber nicht

In der nachfolgenden Diskursanalyse soll darauf geachtet werden, welche Formationsspezifika in den Analyseeinheiten nachweisbar sind. Es soll auf dieser Grundlage der Frage nachgegangen werden, ob neben den erläuterten Assoziationen zwischen rok-samizdat und Sistema (vgl : 4.3.) weitere vorhanden sind

\subsection{Zusammenfassung}

Das in Abschnitt 5.1 genannte diskursanalytische Untersuchungsinstrumentarium wurde in den vorangehenden Abschnitten um zehn Elemente erweitert. Daruber hinaus konnten die Bewertungskriterien charakteristisch und homogen eingefuhrt werden. Es liegt somit das Instrumentarium vor, mit dem dic Diskursanalyse durchgefuhrt wird

An dieser Stelle soll auf einen Aspekt hingewiesen werden, der die Kombinierfahigkeit bzw die Spezialisierungsrelation bestimmter Phänomene betriff. Die Phänomene Autorität und Index sowie Formationsspezifika und Stereotyp stehen in einem Spezialisierungsverhălnis zueinander, wobei die Autorität den Index und die Formationsspezifika das St er e ot yp spezialisieren bzw. spezialisieren können.

Die erlauterten Phanomene sind darüber hinaus frei kombinierbar. So können beispielsweise Normative in Antagonismen verwendet werden. Kultursymbole können in Sentenzen auftreten usw. Soweit Kombinationen häufig im Korpus ermittelt werden können, wird auf diese hingewiesen 

Martin Bergmann - 9783954790531

Downloaded from PubFactory at 01/10/2019 02:45:57AM

(1)

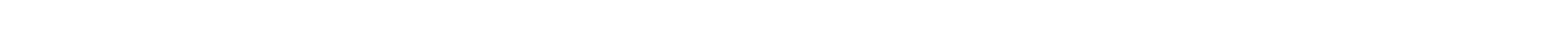




\section{Das Sample}

\subsection{Allgemeines}

Die Analyse des Samples, die auf der Auswertung des Buchs Zolotoe podpol'e. Polnaja illjustrirovannaja enciklopedia rok-samizdata (1967 - 1994) von Aleksandr Kusnir (Kusnir 1994a) beruht, wird durchgeführ, um einige der Rahmenbedingungen festzuhalten, unter denen Texte in der kulturellen Formation des rok-samizdat generiert werden. Bevor mit der Darstellung der Analyseergebnisse begonnen wird, sollen einige allgemeine Worte über dieses 1994 erschienene Buch gesagt werden. Das Buch, welches eine Auflage von 5000 Exemplaren aufweist, umfaßt zum einen zwei Artikel, die sich mit dem Phänomen des rok-samizdat in der ehemaligen Sowjetunion und deren Nachfolgestaaten beschäftigen. Der erste ist von Sergej Gur'ev (Gur'ev 1994), der auf philosophische Aspekte des rok-samizdat eingeht und seine «kulturelle» Bedeutung hervorhebt. Kusnir (Kusnir 1994b) selbst stellt die zeitliche Entwicklung des interessierenden Phänomens im zweiten Artikel dar. Zum anderen enthält das Buch eine Enzyklopädie (enciklopedija), die Informationen über 239 rok-samizdat-Publikationen beinhaltet sowie eine Anthologie (antologija), die 53 Texte aus diesen Publikationen umfaßt. Des weiteren sind verschiedene zeitgenössische Dokumente im Buch enthaiten, die nicht in der angesprochenen Formation entstanden sind, dennoch in gewisser Hinsicht einen Einfluß auf die Entwicklung des betrachteten Phanomens haben. Es soll auf eine Resolution der Moskauer Kulturverwaltung (Postanovlenie Upravlenija Kul'tury g. Moskvy ot 28 sentjabrja 1984) hingewiesen werden, aufgrund derer zahlreiche, namentlich genannte, in- und ausländische Interpreten und Musikgruppen in Moskau verboten wurden sowie auf einen Artikel von B. Zemcov, der am 05.03.87 in der Komsomol'skaja pravda unter der Überschrif Ctivo iz podvorotni erschien und mit hoher Wahrscheinlichkeit den Anfangspunkt eines offenen Konfliktes zwischen der kulturellen Formation des rok-samizdat und dem übergeordneten Kultursystem markiert.

Im Zusammenhang mit den 239 enzyklopädisch erfaßten Publikationen und den 1142 Ausgaben dieser Publikationen, die, den Worten des Verfassers folgend, den vollständigen Bestand des rok-samizdat darstellen, sollen zwei Anmerkungen gemacht werden: Recherchen haben ergeben, daß weitere Publikationen im «Umfeld» der erfaßten existieren (vgl.: Anhang 1). Inwiefern Rock-Musik und damit zusammenhängende Phänomene zum Themenspektrum dieser Publikationen gehören, ist ungeklärt. Darüber hinaus wird Kusnir implizit vorgeworfen «zu viele» Publikationen erfaßt zu haben: „Andrei Burlaka, the former editor of the once-influential "RIO" magazine and one of the most ardent critics of the new book. argues that there existed no more than a dozen "serious" music magazines, which were comparable to the West's "Rolling Stone" and "NME" [New Musical Express], while the rest 200-plus of the 
publications described in "Golden Underground" [Zolotoe podpol'e], were "mere artifacts and marginal rags"“ (Cंernov 1996).

Die Kritik resultiert aus der Tatsache, daß Kusnir sowohl die Auswahlkriterien hinsichtlich der Enzyklopädie als auch der wiedergegebenen Texte nicht offenlegt. Des weiteren fallen in der Enzyklopädie Ungenauigkeiten in bezug auf bestimmte Datenbestände auf. Einheitliche Informationen liegen weitestgehend furr die jeweiligen Erstausgaben vor, so daß auf diese ein besonderes Augenmerk gelegt wird.

\subsection{Umfang, Auflage, Herstellungs- und Vervietraltigungsart der Erstausgaben}

In den folgenden Abschnitten sollen die von Kusnir erfaßten Erstausgaben hinsichtlich der vier Variablen Umfang. Auflage, Herstellungs- und Vervielfaltigungsart betrachtet werden. Die Entwicklung der Variablen im Zeitverlauf wird vernachlässigt.

\subsubsection{Umfang}

In Tabelle 3 sind die den Umfang betreffenden Angaben aufgefürt. Aufgrund der verhälnismäßig großen Variabilität der Werte wird darauf verzichtet, die genauen Umfangszahlen wiederzugegeben, da lediglich eine Tendenz hinsichtlich des Bewertungskriteriums charakteristisch abgeleitet werden soll: eine Einteilung in Zehner-Schritten scheint in dieser Hinsicht angemessen. Da nicht alle Daten auf diese Art gruppiert werden können, wird eine Residualkategorie $(>100)$ gebildet. In bezug auf einige Publikationen liegen darüber hinaus keine bzw. ungenaue Angaben vor, so daß zusatzlich zwei Kategorien, unbekannt und approximativ, eingefuhrt werden Als approximativ werden einerseits Mittelwerte, andererseits Angaben, die nicht in die ubrigen Kategorien eingeordnet werden können (z.B zu großer Range), eingestuft

Tabelle 3

\begin{tabular}{|c|c|c|c|c|c|c|c|c|c|c|c|c|c|c|}
\hline$I$ & 1.10 & 11.20 & 21.30 & $31-0$ & 41.50 & si-e & 61.70 & 71.00 & $81-90$ & 91.100 & $\geq 100$ & conck & c.aver & ; \\
\hline Anen & 20 & 33 & 26 & 24 & 21 & 9 & 5 & 8 & 1 & 7 & 9 & 23 & 53 & 239 \\
\hline$\%$ & 8.4 & 13.8 & 10.9 & 10.0 & 8.8 & 3.8 & 2.1 & 3.4 & 0.4 & 2.9 & 3.8 & 9.6 & 22.2 & 100.1 \\
\hline
\end{tabular}

Die in der Kategorie approximativ erfaßten Angaben sollen vermittels einer weiteren Tabelle differenzierter betrachtet werden. Hierzu wird eine Einteilung in $\leq 50, \leq 100,>100$ und approximativ gewählt.

Tabelle 4

\begin{tabular}{|l|c|c|c|c|c|}
\hline 10 & 550 & 5100 & $>100$ & 202 & 2 \\
\hline 1 & 80 & 8 & 4 & 21 & 53 \\
\hline
\end{tabular}


Tabelle 3 und 4 zusammen betrachtet, lassen erkennen, daß keine der gewählten Kategorien dem genannten Bewertungskriterium genügt. Werden Kategorien subsumiert, dann wird deutlich, daß die Erstausgaben zum größten Teil $(60,3 \%)$ einen Umfang von weniger als 50 bzw. 100 Seiten (76,3\%) aufweisen. Nur ein geringer Prozentsatz (5,5\%) besitzt einen Umfang von mehr als 100 Seiten. Da die Werte zwischen 1 und 100, die in Tabelle 3 zu sehen sind, um das arithmetische Mittel 33,7 mit einer Standardabweichung s von 23,4 stark streuen, (nur 113 Publikationen (47,3\%) liegen bezogen auf das Sample innerhalb eines Toleranzbereichs der mit Hilfe von Standardabweichung und arithmetischem Mittel konstruiert wird) kann dieser Wertebereich nicht als charakteristisch fur das Sample bezeichnet werden.

\subsubsection{Auflage}

In bezug auf die Auflagenhöhe der Erstausgaben soll der Analyse die Anmerkung vorangestelit werden, daß nur die Auflagenzahlen der Originalkopien vorliegen: diese Zahlen sagen über die Verbreitung der Publikationen wenig aus, da die Originalkopien entweder weitergereicht oder in Form von Abschriften und Fotokopien weiterverbreitet werden. Trotz dieser geringen Aussagekraft in bezug auf die Verbreitung, soll das Analyseergebnis diskutiert werden. Es wird, wie bereits bei der Darstellung des Umfangs, eine Gruppierung der Werte in Zehner-Schritte gewählt. Daran anschließend wird eine Gruppe gebildet, die Publikationen umfaßt, die Auflagenstärken zwischen 101 und 499 Exemplaren aufweisen. Die Publikationen, die eine Auflage von mehr als 500 Exemplaren besitzen, werden in einer weiteren Gruppe ( 2500$)$ zusammengefaßt. Diese Einteilung wird im Hinblick auf die Betrachtung der Vervielfältigungsart gewählt: zwischen 101 und 499 Exemplaren bieten sich verschiedene Arten der Vervielfaltigung an, wohingegen ab einer Auflage von ungefahr 500 Exemplaren aus Kostengründen nur die Vervielfaltigung im Druckverfahren sinnvoll erscheint. Weitere Gruppen umfassen Publikationen. die nicht veroffentlicht sind bzw. uber deren Auflagenhohe keine bzw. nur ungenaue Angaben vorliegen.

Tabelle 5

\begin{tabular}{|c|c|c|c|c|c|c|c|c|c|c|}
\hline NAI & $1-10$ & 11.20 & $21 \cdot 30$ & $31-6$ & 41.50 & $51-6$ & $61-70$ & $71=0$ & $n-\infty$ & $91-10$ \\
\hline NM & 83 & 18 & 12 & 1 & 16 & 0 & 0 & 3 & 0 & 11 \\
\hline$\%$ & 34,7 & 7.5 & 5.0 & 0,4 & 6.7 & 0 & 0 & 1,3 & 0 & 4.6 \\
\hline A & $<\operatorname{sen}$ & 2500 & wer. & Datk & $=0.00$ & $\Sigma$ & & & & \\
\hline A & 9 & 7 & 4 & 22 & 33 & 239 & & & & \\
\hline$\%$ & 3.8 & 2.9 & 1.7 & 9.2 & 22.2 & 100 & & & & \\
\hline
\end{tabular}

Wiederum sollen die Werte der Kategorie approximativ differenzierter betrachtet werden: 
Tabelle 6

\begin{tabular}{|c|c|c|c|c|c|c|}
\hline Ances & 550 & 5100 & $<\operatorname{sen}$ & $\geq \operatorname{sen}$ & crove & $\Sigma$ \\
\hline Anew & 27 & 0 & 7 & 0 & 19 & 53 \\
\hline$\%$ & 11.3 & 0 & 2.9 & 0 & 8.0 & 22.2 \\
\hline
\end{tabular}

Keine der Kategorien genügt dem Bewertungskriterium charakteristisch. Bei $65,6 \%$ der erfaßten Publikationen ist die Auflage, wenn Tabelle 5 und 6 addiert werden, kleiner als 50, bei 71,5\% kleiner als 100 Exemplare. Das arithmetische Mittel hinsichtlich der Publikationen, die in Tabelle 5 mit einer Auflagenhöhe zwischen 1 und 100 Exemplaren erfaßt werden, beträgt 20,9 mit einer Standardabweichung s von 26,4. Es kann mit diesen Werten ein Toleranzbereich gebildet werden, der von 0 bis 47,3 reicht. Innerhalb dieses Bereiches liegen, bezogen auf das Sample, die Auflagenhöhen von 130 Publikationen (54,4\%), so daß nicht von einem charakteristischen Wertebereich gesprochen wird.

\subsubsection{Herstellungs- und Vervielnaltigungsart}

Sowohl bei der Betrachtung der Herstellungs- als auch der Vervielfätigungsart erfolgt eine Konzentration auf den textuellen Teil der Publikationen. Hierbei gilt es, folgendes a beachten: es sind Mehrfachnennnungen möglich, so daß eine Summe erzielt wird, die nicht mit dem Sample-Umfang identisch ist; die Prozentangaben werden dennoch auf das Sample bezogen.

Um die Herstellungsart adäquat erfassen zu können, werden vier Kategorien gebildet: Handschrif, Schreibmaschine. Computer und unbekannt. Die Verteilung, die sich für das Sample ergibt, ist in Tabelle 7 abgebildet

Tabelle 7

\begin{tabular}{|c|c|c|c|c|}
\hline 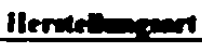 & 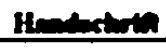 & 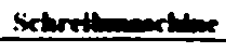 & Comper & كthenct \\
\hline NAm & 28 & 160 & 38 & 36 \\
\hline$\%$ & 11.7 & 67.0 & 15.9 & 15.1 \\
\hline
\end{tabular}

Keine Kategorie in Tabelle 7 genügt dem Bewertungskriterium charakteristisch. Es wird dennoch deutlich, daß der Großteil der Texte (67,0\%) mit dem Medium Schreibmaschine erstellt wird. Darüber hinaus ist der Wert für handschriflich gefertigte Texte, dem der mit Hilfe von Computern erstellten ähnlich. Am Rande bemerkt sei in diesem Zusammenhang, daß handschrifliche Texte über den gesamten Untersuchungszeitraum nachgewiesen werden können, so daß hypothetisch von einer gewissen Tradition dieser Texte in der untersuchten Formation gesprochen werden kann.

Für die Erfassung der Vervielfältigungsart werden 6 Kategorien eingeführt: Handschrift. Schreibmaschine, Computer, Fotokopie. Druck und unbekannt. 14 Publikationen, die mit einer Auflage von jeweils nur einem Exemplar erscheinen, werden in der nachfolgenden Tabelle 8 , die die Verteilung hinsichtlich der Vervielfältigungsart zeigt. nicht berucksichtigt. 
Tabelle 8

\begin{tabular}{|c|c|c|c|c|c|c|}
\hline Verviktaritene & Hemexturn & 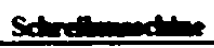 & Cerier & Popolerse & Derets & Denk \\
\hline Anell & 3 & 56 & 9 & 100 & 16 & 45 \\
\hline$\%$ & 1.3 & 23.4 & 3.8 & 41,8 & 6.7 & 18.8 \\
\hline
\end{tabular}

Keine Vervielfätigungsart kann als charakteristisch für das Sample bezeichnet werden: der größte Teil der betrachteten Publikationen $(41,8 \%)$ wird vermittels von Fotokopierem vervielfältigt. In insgesamt $24,7 \%$ der Fälle werden die Publikationen abgeschrieben, wobei dieses in 1,3\% der Fälle handschriftlich, in 23,4\% schreibmaschinell erfolgt. Der Computer als Vervielfältigungsmedium findet mit 3,8\% nur in geringem Maße Verwendung. Selbiges gilt für die Vervielfältigung im Druckverfahren: diese Vervielfältigungsart setzt aufgrund entstehender Grundkosten eine gewisse Auflagenstärke voraus, die, wie bereits verdeutlicht (vgl.: 6.2.2.), in bezug auf die betrachteten Publikationen nur in vergleichsweise geringem Maße gegeben ist.

\subsection{Erscheinungsorte}

Bei der Betrachtung der Erscheinungsorte hinsichtlich der Publikationshäufigkeit wird nicht nur die Anzahl der verschiedenen Publikationen, sondern auch die Anzahl der Ausgaben pro Erscheinungsort berücksichtigt: die Berücksichtigung von zwei Variablen ermöglicht es, gesichertere Hypothesen über die «Bedeutung» einzelner Orte für die untersuchte Formation zu formulieren, als dieses auf der Grundlage von einer Variablen möglich ist. Zunächst sollen die Analyseergebnisse hinsichtlich der Verschiedenartigkeit der Publikationen, anschließend die Anzahl der Ausgaben pro Erscheinungsort dargestellt werden.

\subsubsection{Die Anzahl verschiedener Publikationen pro Erscheinungsort}

Das Sample streut auf 87 Erscheinungsorte, wobei in 47 Orten, die aufgrund ihrer offensichtlich geringen Bedeutung für den rok-samizdat nicht namentlich genannt werden sollen, nur eine Publikation erstellt wird. Am Rande bemerkt sei in diesem Zusammenhang, daß zwei Publikationen, die dem betrachteten Phänomen zugerechnet werden, im Ausland, in der damaligen Tschechoslowakei (Prag: NEW DIAMOND) bzw. in Polen (Warschau: YDRIŻKA (in ukrainischer Sprache)), erscheinen.

Die verbleibenden Publikationen verteilen sich auf die folgenden Erscheinungsorte ${ }^{17}$ : Mit 34 Veröffentlichungen (14,2\%) ist Moskva häufigster Erscheinungsort, gefolgt von Leningrad mit $20(8,4 \%)$. Jeweils $9(3,8 \%)$ Publikationen werden in Novosibirsk, Rostov-na-Donu und Kazan' erstellt; in Archangel'sk, Kiev und Tjumen' jeweils 8 (3,3\%). Riga und Sverdlovsk können jeweils $6(2,5 \%)$. Tallinn $5(2,1 \%)$. Niznij Novgorod. Char'kov und Vladivostok je-

17 Da die Darstellung auf Kusnirs Angaben basiert. sollen nicht die deutschen. sondern die ion Kusnir angegetenen Ortsbezcichnungen in transliterienter Form wiedergegeben werden. 
weils 4 (1,7\%), Omsk. Celjabinsk, Kenigsberg. Murmansk, Voronez und Tula jeweils $3(1,3 \%)$ Publikationen zugerechnet werden. Jeweils 2 Veröffentlichungen $(0,8 \%)$ erscheinen in Rjazan', Isim, Izevsk, Novoisetskoe, Krasnojarsk, Vjatka, Perm, Chabarovsk, Ivanovo, Ufa, Tblisi, Samara, Saratov, Kaunas, Taskent, Baku, Alma-Ata, Krasnodar, Velikie Luki und Magdan.

\subsubsection{Die Anzahl verschiedener Ausgaben pro Erscheinungsort}

Für drei Publikationen liegen keine Angaben bezüglich etwaiger Ausgaben vor: HAS SKLON und OSINOV KOL aus Novosibirsk und KOT-BEGEMOT aus Moskva. In der folgenden Darstellung werden nur diejenigen Erscheinungsorte berücksichtigt, in denen mindestens zwei Publikationen erstellt werden In diesen, die im vorangehenden Abschnitt genannt sind, erscheinen 897 Ausgaben. Mit 158 Ausgaben (13,8\%) liegt Moskva wiederum an der Spitze, Platz zwei mit 102 Ausgaben (8,9\%) nimmt Leningrad ein. Mit 57 Ausgaben (5,0\%) folgt Archangel'sk, mit 42 Ausgaben (3,7\%) Tjumen' und mit 38 (3,3\%) Rostov-na-Donu. 36 Ausgaben $(3,2 \%)$ werden sowohl in Niznij Novgorod als auch in Velikie Luki, 34 in Vladivostok $(3,0 \%), 32$ in Kiev (2,8\%), jeweils 30 in Novosibirsk und Kazan' (2,6\%) publiziert. Sverdlovsk und Tula sind Publikationsort von 22 Ausgaben (1,9\%), Alma-Ata von 20 (1,8\%). Die Orte, in denen weniger als 20 Ausgaben publizient werden, sind Baku $19(1,7 \%)$, Tallinn $15(1,3 \%)$, Char'kov $14(1,2 \%)$, Tblisi $14(1,2 \%)$, Celjabinsk $13(1,1 \%)$, Izevsk $13(1,1 \%)$, Kaunas 13 $(1,1 \%)$, Vorone $10(0,9 \%)$, Isim $10(0,9 \%)$, Novoisetkoe $8(0,7 \%)$, Perm' $8(0,7 \%)$, Omsk 7 $(0,6 \%)$. Murmansk $7(0,6 \%)$, Magdan $7(0,6 \%)$. Krasnojarsk $5(0,4 \%)$. Samara $5(0,4 \%)$. Kenigsberg $4(0,4 \%)$. Chabarovsk $4(0,4 \%)$. Ivanovo $4(0,4 \%)$. Ufa $4(0,4 \%)$, Saratov 4 $(0,4 \%)$, Rjazan' $3(0,3 \%)$. Taskent $3(0,3 \%)$, Vjakta $2(0,2 \%)$ und Krasnodar $2(0,2 \%)$.

\subsection{Die Entwicklung des rok-samizdat: Erstveröfrentlichungsjahre und Publikationsdauer}

Die folgende Darstellung der zeitlichen Entwicklung des rok-samizdat beruht einerseits auf der quantitativen Auswertung der genannten Enzyklopadie, andererseits auf dem bereits erwännten Artikel Kusnirs (Kusnir 1994b), in dem auch qualitative Veranderungen des gewählten Untersuchungsgegenstands angesprochen werden. Die quantitative Entwicklung wird unter Berucksichtigung des Jahres der Erstveröffentlichung (JdE) und der Publikationsdauer (Pd) in Form von Tabelle 9 dargestellt. Die Publikationsdauer 0 besagt in diesem Zusammenhang. daß von den jeweiligen Publikationen, die dieser Kategorie zugeordnet werden, nur jeweils eine Ausgabe existiert. 
Tabelle 9

\begin{tabular}{|c|c|c|c|c|c|c|c|c|c|c|c|c|c|c|c|c|c|c|c|c|c|c|}
\hline $\begin{array}{l}\text { JaE. } \\
\text { PU }\end{array}$ & 67 & 74 & 77 & 79 & 80 & 81 & 82 & 83 & 84 & 85 & 86 & 87 & 88 & 89 & 90 & 91 & $\boldsymbol{n}$ & 93 & 94 & 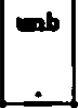 & $\Sigma$ & $\%$ \\
\hline$\theta$ & . & . &. &. &. & - & . & 1 & 1 & 3 & 1 & $s$ & 7 & 12 & 9 & 8 & 7 & 4 & 1 & 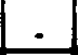 & 99 & 247 \\
\hline$\leq 1$ & 2 & 1 & - & $=$ & 1 & 1 & - & 1 & 2 & 1 & 2 & 2 & 7 & $s$ & 8 & 6 & 3 & 6 &. &. & 48 & 20.1 \\
\hline$\leq 2$ & 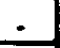 & - & - & 1 & - & - & 1 & $\therefore$ & 1 & 1 & 2 & 9 & 9 & 8 & 9 & 3 & 3 & 4 &. &. & 53 & 222 \\
\hline$\leq 3$ &. &. & - & . &. &. & $=$ & $\therefore$ & $\therefore$ & 1 & 2 & 7 & 5 & 7 & 5 & 2 & 3 &. & $\therefore$ & $=$ & 32 & 13,4 \\
\hline$\leq 4$ &. & - &. & $\therefore$ & 1 & $\therefore$ &. & 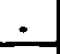 & - & 1 & 5 & 1 & 6 & 3 & 1 & 1 & . & - & - & $=$ & 19 & 79 \\
\hline$\leq 5$ &. & - & - & - & . & $=$ & $=$ &. &. &. & 1 & 3 & 3 & 1 &. &. & . &. &. &. & 8 & 3,3 \\
\hline$\leq 6$ & $\therefore$ &. & . &. & . & $=$ & 1 &. &. & $=$ & 1 &. & 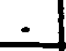 & 1 &. &. &. & $=$ & - &. & 3 & 13 \\
\hline 58 & 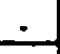 & - & - & 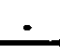 &. & $\dot{-}$ & - & - & - & 1 & 1 & 1 & 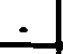 & - & - & - & - & - & . & . & 3 & 1.3 \\
\hline 59 & $\dot{ }$ & - & $\cdot$ & - & - &. & - & $=$ & 1 & $\dot{-}$ & 1 & $=$ & 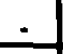 &. &. &. & . &. & - & - & 2 & 0.8 \\
\hline$\leq 14$ & - & $=$ & 1 & 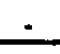 & - & - & - & - & 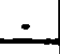 & - & 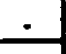 & $=$ & - & - & - & - & - & - & - & - & 1 & 0.4 \\
\hline$\rightarrow$ & . & $=$ & $=$ & - & $=$ & 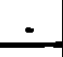 & $=$ & . &. & - & 1 & 1 & 1 & 2 & 1 & 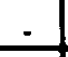 & 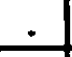 & 1 & $\dot{-}$ & 4 & 11 & 46 \\
\hline$\Sigma$ & 2 & 1 & 1 & 1 & 2 & 1 & 2 & 2 & 5 & 8 & 17 & 29 & 38 & 39 & 33 & 22 & 16 & 15 & 1 & 4 & 239 & \\
\hline$\%$ & 0.8 & 0.4 & 0.4 & 0.4 & 0.8 & 0.4 & 0.8 & 0.8 & 2.1 & 3.4 & 7,1 & 121 & 159 & 16.3 & 138 & 9.2 & 6,7 & 6.3 & 0.4 & 1.7 & & \\
\hline
\end{tabular}

Das Jahr 1967 markiert den Anfang der untersuchten, kulturellen Formation. Es entstehen zwei Publikationen: das Magzin BIT-ECHO in Char'kov und das ,rok-bjulleten'“ NEW DIAMOND von Artem Troickij in Prag. Obwohl beide weniger als ein Jahr lang existieren, liegen sie jeweils in mehreren Ausgaben vor. Abgesehen von einer kurzzeitigen Veröffentlichung $^{18}$ aus dem Jahre 1974 in Rostov-na-Donu mit dem Namen ALLO, die Kuśnir nicht erwähnt, sind erst ab 1977 neue Veröffentlichungen zu verzeichnen: „v Leningrade okoloakvariumistskaja tusovka vo glave s Grebenscikovym nacinaet izdavat' legendarnyj masinopisnyj zurnal «Roksi». Nesmotrja na tjagu redakcii $k$ vsevozmoznym formam literaturnogo analiza, eto bylo pervoe sovetskoe izdanie. celikom orientirovannoe na subkul'turu i otecestvennyj rok" (Kusnir 1994b, 9). Neben den hervorgehobenen, qualitativen Eigenschaften zeichnet sich die Veröffentlichung. wie in Tabelle 9 zu sehen ist. durch die làngste Publikationsdauer aus. 1979 und 1980 erscheinen insgesamt drei Magazine (AU in Kaunas. ORGAN BEKO in Riga, DISKO-START in Tblisi), auf die Kušnir unter dem Hinweis, daß es sich um Veröffentlichungen aus Litauen bzw. Georgien handelt, nicht näher eingeht (vgl : Kusnir 1994b, 9), obwohl das Magazin ORGAN BEKO für einen Zeitraum von mehr als drei Jahren erscheint. 1981 ensteht in Moskau das Magazin ZERKALO, welches bereits Ende desselben Jahres verboten, seit 1982 unter dem neuen Namen UCHO fortgeführt wird. In thematischer Hinsicht beschränkt sich das UCHO „na posledovatel'nom osvescenii sobytij moskovsko-leningradskoj podpol'noj sceny, a idejnyj vektor rumala napravljaetsja $v$

\footnotetext{
${ }^{18}$ Dic Publikationsdauer streut auf insgesamt 10 Wenc. Für die Werte 52 wird die Bezcichnung ku rzzcilig für die verbleibenden die Bezeichnung lăngerfristig vorgeschlagen. Die Grenzjiehung an besagter Siclle Ist subjektiv. sie sollte in einem heuristischen Sinne verstanden werden.
} 
storony esce tolkom ne oformivsegosja v Rossii pank-roka" (Kusnir 1994b, 9). Gleichfalls wird seit 1982 das Magazin KOT-BEGEMOT herausgegeben, welches eine Publikationsdauer von mehr als 5 Jahren aufweist und somit die Ereignisse von 1983 überdauert: durch einsetzende, restriktive Maßnahmen des KGB markiert das genannte Jahr einen Zeitpunkt, an dem massive Veränderungen für die untersuchte kulturelle Formation zu verzeichnen sind: „V tecenie polutora let fakticeski vsja andergraundnaja rok-pressa okazalas' razognannoj, unictozenoj ili dostatocno plotno opekaemoj“ (Kusnir 1994b, 9). Erst Ende 1984 bzw. Anfang 1985 entstehen neue Publikationen: trotz zu befüchtender Repressalien, entstehen in Moskau nahezu zeitgleich die Magazine URLAJT, SMORCOK und ZOMBI. Die Publikationsdauer streut in den Jahren 1984 und 1985 zusammengenommen auf 7 Jahre, wobei sich eine Tendenz zu kurzzeitigen Publikationen (69,2\%) abzeichnet. Dennoch überdauern Publikationen dieses Zeitraumes mehr als 8 (URLAJT) bzw. 9 (ZOMBI) Jahre. Bereits mit Beginn der Perestrojka erhöht sich die Anzahl der erscheinenden Magazine von 8 im Jahre 1985 auf 17 im Jahre 1986 Im Gegensatz zu den Jahren 1984 und 1985 ist in diesem Zusammenhang bemerkenswert, daß $64,7 \%$ der 1986 zum ersten Mal erscheinenden Publikationen eine Publikationsdauer von mehr als zwei Jahren aufweisen. Insgesamt sind die Jahre 1986 bis 1989 durch einen stetigen Anstieg der Erstveröffentlichungszahlen gekennzeichnet: im genannten Zeitraum entstehen, wie Tabelle 9 zeigt. $51,4 \%$ der in der Enzyklopädie erfaßten Veröffentlichungen $39,8 \%$ dieser Publikationen können zu den kurzzeitigen, 60,2\% zu den längerfristigen gezählt werden. Bei den kurzzeitigen Publikationen sticht eine Gruppe hervor, die diejenigen umfaßt, die nur einmal veroffentlicht werden (33,8\% der kurzzeitigen Publikationen). Die längerfristige Orientierung der Mitglieder der untersuchten Formation findet ihren Ausdruck in zwei Treffen, beim funften Leningrader Rock-Festival 1987 (V leningradskij rok-festival') und in Sverdlovsk 1988, die zur Grundung der sogenannten Rok-federacii führen, auf deren Grundlage uberregionale Kommunikationsstrukturen und somit Kooperationsmoglichkeiten entstehen Seit 1990 kann ein stetiger Ruckgang der Neuerscheinungen festgestellt werden, die im Jahre 1993 mit is Publikationen in der niedrigsten Erstausgabenrate seit 1985 mundet. Da Kusnir das Jahr 1994 nur bis zur Jahresmitte erfaßt (vgl: Kuśnir 1994b, 10), soll dieses aus der Betrachtung ausgeklammert werden. Die im Zeitraum von 1990 bis 1993 entstehenden Publikationen zeichnen sich durch ihre extrem kurzzeitige Publikationsdauer aus. $65,1 \%$ der Neuveroffentlichungen erscheinen weniger als ein Jahr lang. Im betrachteten Zeitraum kommt es, neben dem dargestellten Rückgang und der Tendenz zu kurzen Publikationszeiten, zu qualitativen Veränderungen der Publikationen, die mit dem Prozeß der Kommerzialisienung zusammenhängen. Der Prozeß fün zu einer Spaltung der kulturellen Formation, die bewirkt. daß sowohl kommerzielle als auch nicht-kommerzielle Publikationen entstehen bzw «alte» Publikationen sich verandem Einer Kommerzialisienung widerstehen u.a. die Publikationen KONTR KUL'T UR'A. SUMELA" MYS', PALEVO und DVR (vgl. Kusnir 1994b, 10). Es können in diesem Sinne verschiedene Typen von Publikationen unterschieden werden 
„[Zurnali] S otkrovenno primitivstkoj estetikoj «nazad k kornjam» - napisannych i razmnozennych vrucnuju $[(\ldots)]$ i, nakonec, vozniknoveniem ocerednoj volny novych izdannij, specializinjuscichsja na issledovanii cut' li ne vsego okolomuzykal'nogo spektra" (Kuśnir 1994b, 10). Wie erwähnt, endet Kusnirs Erfassung in der Mitte des Jahres 1994. Dies heißt nicht, daß das betrachtete Phänomen nicht mehr existiert. Es liegen z.Zt. jedoch keine Informationen darüber vor. Ein starker Rückgang der Publikationstätigkeit ist dennoch wahrscheinlich.

Werden die Ergebnisse der zeitlichen Betrachtung des rok-samizdat zusammengefaßt, dann kann festgestellt werden, daß die Hauptphase mit dem Jahr 1986 beginnt. Wann diese Phase endet, kann nicht genau abgegrenzt werden. Da ab 1990 einerseits die Anzahl der Neuveröffentlichungen zurückgeht, andererseits, im Gegensatz zu den vorangehenden Jahren, eine Tendenz zu einer kurzzeitigen Publikationsdauer offensichtlich wird, soll hier die Hauptphase bereits mit dem Jahr 1989 als beendet angesehen werden. In die Hauptphase fallen dann, wie bereits erwähnt, 51,4\% der Erstveröffentlichungen.

\subsection{Zusammenfassung}

In bezug auf den Umfang der Publikationen kann kein charakteristischer Wert oder Wertebereich ermittelt werden. Dennoch kann festgestellt werden, daß 47,3\% der Publikationen einen Umfang aufweisen, der sich in einem aus arithmetischem Mittel $(33,7)$ und Standardabweichung $(23,4)$ konstruierten Wertebereich befindet. Gleichfalls existiert keine charakteristische Auflagenhöhe. Innerhalb eines Wertebereichs, der wiedenum mit Hilfe des arithmetischen Mittels $(20,9)$ und der Standardabweichung $(26,4)$ konstruiert wird, liegen die Werte von $54,4 \%$ aller Publikationen. Von charakteristischen Herstellungsverfahren kann nicht gesprochen werden, obwohl die Herstellung per Schreibmaschine $(67,0 \%)$ überwiegt. Die Vervielfaltigungsmethoden lassen gleichfalls kein Verfahren erkennen, welches dem angefuihrten Maß genugt. Der Großteil $(41,8 \%)$ wird, dies sei am Rande bemerkt, mit Hilfe von Fotokopierern vervielfaltigt. Gleichfalls laßt sich kein Erscheinungsort feststellen, der als charakteristisch aufgefaßt werden kann: Moskau und Leningrad weisen jedoch die höchsten Erscheinungszahlen sowohl in bezug auf die Verschiedenartigkeit der Publikationen als auch in bezug auf die Anzahl der Ausgaben pro Erscheinungsort auf. Bezüglich der zeitlichen Entwicklung des roksamizdat soll festgehalten werden, daß der Zeitraum von 1986 bis 1989 als Hauptphase angesehen wird Des weiteren kann von einer Tendenz hin zu einer kurzzeitigen Publikationsdauer gesprochen werden: $67 \%$ der erfaßten Veröffentlichungen existieren weniger als 2 Jahre lang.

Es wird deutlich, daß fur die untersuchte Formation gewisse Rahmenbedingungen vorhanden sind, die mit hoher Wahrscheinlichkeit einen Einfluß auf die Textgenerienung ausüben. Innerhalb eines durch die Rahmenbedingungen definierten Bereichs kann eine gewisse Heterogenität verzeichnet werden, die ihren Ausdruck darin findet, daß keines der betrachteten Merkmale dem Bewertungskriterium charakteristisch genügt 
Martin Bergmann - 9783954790531

Downloaded from PubFactory at 01/10/2019 02:45:57AM

via free access

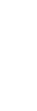

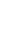




\section{Publikationen und Analyseeinheiten}

\subsection{Allgemeines}

Werden die Ergebnisse der Sample-Analyse (vgl.: 6.ff.) bei der Bestimmung der Auswahl- und Analyseeinheiten berücksichtigt, dann ist offensichtlich, daß eine "repräsentative» 19 Auswahl der genannten Einheiten nahezu ausgeschlossen werden kann bzw. werden muß. Die Auswahl hängt, bedingt durch die relativ geringe Anzahl der Publikationen und Ausgaben sowie der kleinen Auflagenhöhen, von der Zugänglichkeit des Untersuchungsmaterials ab. Da in der Anthologie Kusnirs 53 Texte wiedergegeben sind, kann einerseits auf dieses Material zurückgegriffen werden. Andererseits ist es im Rahmen von Recherchen im Archiv der Forschungsstelle Osteuropa an der Universität Bremen gelungen, folgende Ausgaben der von Kusnir erfaßten Publikationen zu erhalten 20: URLAJT, 9/10; URLAJT, 1 (19); URLAJT, 3 (21); URLAJT, 4 (22); URLAJT, 5 (23); URLAJT, 6 (24); URLAJT, 7; URLAJT, 8, Al'manach pod redakciej Il'i Smimova, Alma-Ata; DVR, 10; DVR, 11; KONTR KUL'T UR'A, 2; KONTR KUL'T UR'A, 3; CHERR (S MOSLOM), 3 und CHERR (BEZ MOSLA), 4.

Bevor Analyseeinheiten ausgewählt werden, sollen einige Vorüberlegungen bezüglich des Auswahlverfahrens angestellt werden bzw. Festlegungen erfolgen:

1 Für die Analyse werden Einheiten ausgewählt, die in nussischer Sprache verfaßt sind;

2. Interviews werden nicht berücksichtigt: Interviews stellen Niederschriften mündlicher Rede dar, so daß in ihnen mit hoher Wahrscheinlichkeit textuelle Merkmale enthalten sind, die aus diesem Kommunikationsprozeß resultieren Mundliche Kommunikationsprozesse sollen in dieser Arbeit nicht als Analyseobjekt fungieren

3. Von jedem Verfasser sollte nur ein Text berücksichtigt werden, so daß die Möglichkeit ausgeschlossen wird. den personlichen Stil eines Verfassers zu untersuchen. Dieses Auswahlkriterium kann aufgrund des Zugänglichkeitsproblems nicht eingehalten werden. Zudem ist haufig unklar, welcher Verfasser fur einen Text verantwortlich ist, da Publikationsbeitrage haufig anonym veroffentlicht bzw. mit Pseudonymen unterschrieben sind (vgl.: 7.4.).

4. Idealerweise sollte aus jeder Publikation nur ein Text ausgewählt werden: auch Publikationen weisen vermutlich einen eigenen Stil auf, der hier nicht als Untersuchungsgegenstand gewählt wird Auch dieses Auswahlkriterium kann aufgrund des angesprochenen $\mathrm{Zu}$ ganglichkeitsproblems nicht eingehalten werden statt dessen wird so verfahren, daß aus jeder Ausgabe nur eine Analyseeinheit ausgewahlt wird. Eine Ausnahme stellen zwei Texte (Analyseeinheit 8 und 26; vgl.: 7.4.) dar, die in einer Doppelnummer des Magazins URLAJT

\footnotetext{
19 Dic Frage. ob eine im sorialwissenschaftıchen Sinne repräsentative Ausuahl fur cine kulturuissenschafliehe Arteit. wie sie eine Diskursanalyse darstellt. moglich und v.a. notwendig ist. soll lediglich angesprochen. nicht aber diskutien werden. Dieses Problem wird ausfuhrlich in Fleischer 1997a. 225-239 crorten.

20 Es wird die in der Enryklopadic veruendete. bereits standardisiente Schreibweise in berug auf die Namen der Publikationen übernommen.
} 
(9/10; April 1986) zu finden sind Es ist erst nach Abschluß des Großteils der Analysen bemerkt worden, daß die genannte Ausgabe eine Doppelnummer darstellt.

5. Da Texte von der Wortanzahl her unterschiedlich sind, müssen in quantitativer Hinsicht Analyseeinheiten definient werden, um eine Vergleichbarkeit der Analyseergebnisse zu gewährleisten. Es wird auf die von Osgood für Analyseeinheiten vorgeschlagene Größe von 120 bis 210 Worten zurückgegriffen (vgl: 3.5.2.2.). In diesem Zusammenhang ist auf zwei Dinge zu achten: erstens muß es sich bei einer Analyseeinheit um einen zusammenhängenden Text handeln; zweitens muß mit dem letzten Satz der Analyseeinheit möglichst die Grenze von 210 Worten erreicht werden.

6. Als Analyseeinheiten werden Textpassagen ausgewählt, die auf einer formalen Ebene, auf der z.B. Anfangs-, Mittel- und Endpassagen unterschieden werden können, gleichartig sind. Es wird eine Entscheidung zugunsten von Anfangspassagen getroffen, wobei Überschriften nicht im Rahmen der syntaktischen Analysen benücksichtigt werden

Auf der Grundlage dieser Beschrankungen werden 35 Analyseeinheiten und 34 Auswahleinheiten bestimmt, denen 15 Publikationen ${ }^{21}$ zugrunde liegen. Zunächst werden im folgenden Abschnitt die Publikationen in chronologischer Reihenfolge beschrieben, bevor die Analyseeinheiten genannt werden. Auf die Auswahleinheiten wird nicht eingegangen.

\subsection{Die berücksichtigten Publikationen}

\subsubsection{Allgemeines}

Die folgende Beschreibung der Publikationen basiert auf der bereits erwahnten Enzyklopädie (Kusnir 1994a) Nicht alle Aspekte, die im Verlauf der dortigen Charakterisierungen der Publikationen angesprochen werden, sind fur den vorliegenden Zusammenhang relevant Als relevant erachtet werden neben formalen Figenschaften die «inneren Entwicklung der Publikationen sowie Veränderungen im Umfeld, die mit hoher Wahrscheinlichkeit einen Einfluß auf die untersuchte Formation haben. In bezug auf die «innere» Entwicklung wird ein besonderes Augenmerk auf die an der Erstellung der Publikationen beteiligten Personen gelegt In diesem Zusammenhang soll der Frage nachgegangen werden, inwiefern es zwischen einzelnen Publikationen Kontakte gibt wenn keine personalen Kontakte nachweisbar sind. die Publikationen sich nicht in irgendeiner Weise gegenseitig beeinflussen. sondern in völliger Isolation entstehen, dann sind Zweifel angebracht. ob das zugrundegelegte Axiom haltbar ist. Auf inhaltliche Aspekte sowie die «kulturelle» Bedeutung der Publikationen wird nicht eingegangen.

\footnotetext{
21 Úber dic erste Analysocınheıl (anon. [Slavorosor. Arkadij: Suto:. Scrgej]. 1982. Kanon.) licgen abweıchende Informationen vor. Kusnir schreibi diesen Text ciner Publikation zu. die den Namen DETI PODZMEL'JA tragt. In sciner Enñklopadic ist diesc Publikation jedoch nicht erfaBt. Fajn und Lur c gethen davon aus. daB DETI PODZMEL'JA ein Pseudonym ist. welches die Autoren nur Kennzcichnung ihres Beitrages. der runáchst als Blattsammlung verbreitet. spaicr u.a. in den Publikationen URLAST. TUSOVKA. UGOLOVNOE DELO No. wiederieroffentlicht uird gewahlt haben ('gl.: Fajn: Lur ${ }^{\circ}$ 1991. 83) Hicr soll die Position von Fajn und Lur'e übernommen werden.
} 


\subsubsection{ROKSI}

Das Leningrader Magazin ROKSI erscheint zwischen 1977 und 1990. Es existieren 15 Ausgaben, wobei die ersten drei mit einem Umfang von 20, die Ausgaben vier bis sieben mit einem Umfang von 60 und die nachfolgenden mit einem Umfang von ungefähr 100 Seiten publiziert werden. Das Magazin wird schreibmaschinell erstellt und mit einer Auflage von jeweils etwa 15 bis 20 Exemplaren verbreitet. Die Gründer des ROKSI, die die ersten drei Ausgaben herausgeben, sind Boris Grebenscikov (vgl.: Analyseeinheit 2), Sänger der Gruppe Akvarium, Michail Naumenko, ein Musiker, der unter dem Namen Majk bekannt ist, Nikolaj Vasin, Natasa Vasil'eva sowie Jurij Il'cenko, ein Musiker, der u.a. bei der Gruppe Masina Vremeni spielt.

Nachdem die Gruppe Akvarium in den Jahren 1978 und 1979 häufig in Leningrad sowie anderen Orten auftritt und die Zeit für die Erstellung des Magazins nicht mehr ausreicht, wird die Verantwortung an Oleg Resetnikov, Michail Bruk und Aleksandr Andreev übergeben, die die vierte Ausgabe im Dezember 1980 verbreiten. Etwa zu diesem Zeitpunkt wird in Leningrad unter der Schirmherrschaft des Dom samodejatel'nogo tvorcestva der erste sowjetische rokklub gegründet. Nach dem Tod von Bruk im November 1983 gesellen sich Boris Malysev (vgl.: Analyseeinheit 3), der bereits an der Erstellung der dritten Ausgabe beteiligt war, und Aleksandr Starcev, der für die weitere Entwicklung der Publikation in entscheidendem Maße verantwortlich ist, zu den verbliebenen Herausgebern. Diese nehmen Abstand von einer weiteren Zusammenarbeit mit Starcev aufgrund von Meinungsverschiedenheiten bezüglich der konzeptuellen Ausrichtung des Magazins nach der sechsten Ausgabe, die im Dezember 1983 unter der Bezeichnung rok-bjulleten' als offizielles Organ des Leningrader rok-klub erscheint. Dieses erscheint nicht in Heftorm, sondem wird als Wandaushang in den Räumen des rok-klub publizien. Nach dieser Ausgabe gelingt es Starcev und Malysev neben Jule Pyskevic Anatolij Gunizkij und Igor' I.eonov als neue Mitarbeiter zu gewinnen. Die beiden letztgenannten werden in den Vorstand des rok-klub gewăhlt. was dazu führ, daß sich das ROKSI in thematischer Hinsicht auschließlich auf Belange des Clubs beschränkt. Starcev sieht sich in dieser Zeit massiver Repressalien seitens des KGB ausgesetzt, der zu verhindern versucht, da $B$ bestimmte Beitrage publiziert werden. Aufgrund dieser und anderer Zensurmaßnahmen entsteht auf Initiative von Andrej Burlak eine unabhängige Publikation mit dem Namen RIO. Mit Beginn der Perestrojka nimmt der Druck auf das ROKSI ab. Seit der zwölften Ausgabe (Frühling 1987), die vorwiegend auf dem funften Leningrader rok-festival' verbreitet wird, erscheint das Magazin wieder unter seinem ursprünglichen Namen. Der Großteil der Artikel, die sich weiterhin lediglich mit dem rok-klub beschäftigen, stammen von Starcev und Gunickij, die diese unter verschiedenen Pseudonymen verfassen. Erst seit Beginn des Jahres 1988 erweitert sich das Themenspektrum. Gleichfalls versuchen Starcev und Gunickij neue Verbreitungswege fur ihre Publikation zu finden: es werden einerseits Veranstaltungen inszenien, die Pressekonferenzen ahneln. andererseits hält Starcev Vorträge, in deren Verlauf Videoeinspielungen verschiedener Konzerte erfolgen, die im rok-klub stattgefunden haben Die vierzehnte Ausgabe 
(Januar/April 1988) ist die letzte, die gemeinsam von verschiedenen Personen angefertigt wird. Im Herbst 1990 publiziert Starcev abschließend die letzte Ausgabe des Magazins allein.

\subsubsection{ZERKALO}

In Moskau erscheint zwischen März und November 1981 das Magazin ZERKALO mit einem Umfang von jeweils etwa 80 bis 120 Seiten. Von den vier publizierten Ausgaben werden die ersten beiden Ausgaben mit einer Auflage von 10, die verbleibenden mit einer Auflage von 20 Exemplaren verbreitet. Hinsichtlich der Gestaltung verandert sich das ZERKALO bestandig, da der schreibmaschinelle Teil zunächst um Fotos, im folgenden auch um Zeichnungen ergänzt wird.

Das ZERKALO ist mit dem klub Rokuella Kenta eng verbunden, der seit etwa 1979 im Umfeld des Moskovskij Inzenernyj Fisiceskij Institut (MIFI) angesiedelt ist und Lesungen, Konzerte. Ausstellungen etc. veranstaltet. In diesem Rahmen publiziert Dmitrij Vrubel' ein Magazin mit dem Namen LICEJ, welches sich vorwiegend mit Literatur beschäftigt. Zum Umfeld des klub und des LICEJ gehören Il'ja Smirnov (vgl: Analyseeinheit 5, 27, 28, 29, 31, 33), Evgenij Matusov (vgl: Analyseeinheit 26) und Il'ja Kricevskij, die sich nach Beendigung eines seminar disk-zokeev, welches im Rahmen des Kurses Iskusstvo i esteticeskoe vospitanie am MIFI abgehalten wird und in dessen Verlauf sie Artem Troickij (vgl: Analyseeinheit 4) kennenlernen, entschließen, ein Magazin herauszugeben, welches sich mit Rock-Musik beschaftigen soll. Die erste Ausgabe ist vollständig der Gruppe Masina Vremeni, die zweite der Gruppe Akvarium gewidmet Die dritte Ausgabe, an der u.a. Sergej Gur'ev (vgl: Analyseeinheit 10) mitarbeitet. beschaftigt sich hauptsachlich mit der Gruppe Voskresen'e. Die vierte Ausgabe hingegen beinhaltet Beitrage uber verschiedene Gruppen und Themenkomplexe. Die genannten Personen veranstalten zwischen März und November 1981 daruber hinaus mehrere akustische Konzente. während derer u.a. Boris Grebenscikov sowie Andrej Makarevic von der Gruppe Masina Vremeni auftreten Ende 1981 wird die weitere Publikation des Magazins seitens der Leitung des MIrI untersagt Da mehre Versuche scheitern, das Magazin offiziell zu publizieren, erscheint es illegal und mit einem stark veranderten Aussehen im April 1982 unter dem Namen UCHO

\subsubsection{UC.HO}

Vom Nachfolgemagazin des ZERKALO entstehen zwischen April 1982 und Dezember 1983 sieben Ausgaben, die jeweils einen Umfang von bis zu 60 Seiten und eine Auflage von bis zu 10 Exemplaren aufweisen. Die ersten beiden Ausgaben konnen sowohl von den beteiligten Personen, vom inhaltlichen Schwerpunkt als auch von der Herstellungsar her, als direkte Fortsetzung des ZERKALO aufgefaßt werden

In der zweiten Ausgabe (September 1982) veröffentlichen Smirnov, Kricevskij und der neue Mitarbeiter Michail Sigalov (vgl.: Analyseeinheit 6) unter dem Pseudonym M. Nikolaeva einen Artikel, der sich mit einem von Troickij verfaßten Beitrag beschäftigt und zur Auflösung des bis dato tatigen Mitarbeiterstabs fuhr Smirnov und Sigalov werden zu den Hauptakteuren 
des UCHO und publizieren die dritte Ausgabe nahezu allein. An den folgenden Ausgaben arbeiten dann u.a. Sergej Zarikov (vgl: Analyseeinheit 7), Hauptakteur der Gruppe DK, der seit 1985, nachdem er für kurze Zeit im Magazin URLAJT publiziert hatte, sein eigenes Magazin SMORCOK herausgibt, I. Ivanov, Jurij Nepacharev und A. Pavljucenko mit. Da eine dieser Personen vermutlich Kontakte zum KGB besitzt, sehen sich die übrigen einem wachsenden Druck ausgesetzt, der Smimov dazu bewegt, die Publikation Anfang 1984 einzustellen.

\subsubsection{ZOMBI}

Zwischen Herbst 1984 und November 1992 erstellt Natalija Komarova in Moskau gemeinsam mit verschiedenen Mitarbeitern 16 Ausgaben des Magazins ZOMBI. Von den Ausgaben 1 bis 8 wird jeweils nur ein einziges Exemplar angefertigt, von den Ausgaben 9 bis 16 jeweils bis zu fünfzehn. Eine Ausnahme stellt die zwölfte Ausgabe dar, die mit einer Auflage von ungefahr 1000 Exemplaren verbreitet wird. Das ZOMBI besitzt ein einfaches Design: Texte werden mit der Schreibmaschine geschrieben und mit Photos sowie Zeichnungen und Collagen verziert.

Die erste Ausgabe beschäftigt sich fast ausschließlich mit den Gruppen Akavrium und Zoopark, die zweite mit Konstantin Kincev und der Gruppe Alisa. In diesem Zeitraum veranstaltet Komarova darüber hinaus Konzerte und organisiert Auftritte für die Gruppe Nocnoj prospekt. Sowohl die durchgeführten Konzerte als auch die genannte Gruppe, bei der Ivan Sokolovskij (vgl. Analyseeinheit 25) spielt, werden ein fester, thematischer Bestandteil der folgenden Ausgaben. In besonderem Maße sprechen sich Komarova und ihre Mitstreiter, zu denen anfangs Vladimir Marockin (vgl.: Analyseeinheit 9) und Valerij Kandakov zählen, gegen die Etablierung der Rock-Clubs aus, die in verschiedenen Städten gegründet werden. In diesem Zusammenhang ist auch der klub samodejatel'noj pesni (KSP) massiver Kritik ausgesetzt. Ende 1985 bzw. Anfang 1986 bahnt sich eine engere Zusammenarbeit mit dem Magazin URLAJT und Il'ja Smimov an. die bereits nach kurzer Zeit wieder beendet wird Nach der siebten Ausgabe (September 1986), die sich verstarkt der Gruppe Nll Kosmetika zugewendet hatte, verlaßt Marockin das Magazin und tritt der Moskauer Variante der Rock-Clubs bei, die unter dem Namen rok-laboratorija firmiert. Dennoch sind in der achten Ausgabe einige seiner Arbeiten enthalten. Mitte 1987 arbeiten Sergej Gur'ev und V. Mironenko, der bei der Gruppe Muchomor spielt, an der Publikation mit. Zu diesen gesellen sich seit der elften Ausgabe (Frühjahr 1988) u.a. Zaforom Chasitov und Vadim Tarmakovskij, die aus Zelenograd berichten. Bereits seit Anfang des genannten Jahres kommt es zu verstärkten Feindseligkeiten zwischen dem offiziellen Rock-Laboratorium und den «Inoffiziellen", die einen Konsolidierunsgprozeß innerhalb der untersuchten Formation bewirken. Aufgrund dieses Prozesses erhoht sich der Bekanntheitsgrad des ZOMBI, was Komarova veranlaßt, die folgende zwolfte Ausgabe in einer weit höheren Auflage als bisher zu produzieren. Im Sommer 1988 sind 50000 Exemplare gedruckt, die, nachdem der Direktor der Druckerei eine Kopie studiert hatte, bis auf 1000 Exemplare vernichtet werden. Das im Dezember 1988 vom ZOMBI offiziell veranstaltete Festival Syrok, an dem mehr als zwanzig der führenden Gruppen des Landes teil- 
nehmen, markiert einen Wendepunkt in der Entwicklung des ZOMBI, da verschiedene Personen aus dem Mitarbeiterstab des Magazins ausscheiden. Die folgenden Ausgaben 13 (Mai 1989) und 14 (Frühjahr 1991) sind die letzten, die als Publikationen der untersuchten Formation angesehen werden, da die Ausgaben 15 (Anfang 1992) und 16 (November 1992) keinen thematischen Bezug mehr zum betrachteten Phänomen aufweisen.

\subsubsection{URLAJT}

Das Magazin URLAJT wird zwischen 1985 und 1992 in Moskau mit 23 Ausgaben publiziert. Da mehrmals die Numerierung der Ausgaben verändert wird, einige Ausgaben zudem nicht veröffentlicht sind, trägt die letzte Ausgabe die Nummer 8. Die ersten 15 Ausgaben $(\mathrm{Nr}$. I bis 18; 6 und 8 existieren nicht; 17 ist unveröffentlicht; die Doppelnummer 9/10 zählt als zwei Ausgaben) werden zwischen 1985 und 1987 mit einer Auflage von 20 bis 30 Exemplaren und einem Umfang von bis zu 40 Seiten erstellt. Die Ausgaben dieses Zeitraumes enthalten schreibmaschinell gefertigte Texte sowie Fotos. Zwischen April 1988 und September 1989 erscheint das URLAJT mit einer leicht veranderten Schreibweise des Namens (UR LAJT) und neuer bzw. doppelter Numerierung. In diesem Zeitraum werden sechs Ausgaben (Nummer 1 (19) bis 6 (24)) mit einer Auflage zwischen 100 und 200 Exemplaren und einem Umfang von 100 bis 150 Seiten produziert. Neben schreibmaschinell gefertigten Texten und Fotos finden sich Zeichnungen und Collagen in den einzelnen Ausgaben, die in Form von Fotokopien verbreitet werden. Eine Ausnahme sowohl hinsichtlich der Vervielfaltigungsart als auch der Auflagenhohe stellt Nummer 5 (23) dar, die mit einer Auflage von 25000 Exemplaren gedruckt wird 1990 erscheint die siebte Ausgabe (Nr. 22) mit einem Unfang von 84 Seiten und einer Auflage von zunachst 50 Exemplaren, die 1991 mit einer Auflage von 10000 Exemplaren nachgedruckt wird 1992 entsteht die 109 Seiten umfassende Nummer 8 (Nr. 23), die mit einer Auflage von 30000 gedruckt wird

Abgesehen von den bereits dargestellten Vorlaufern, ZERKALO und UCHO, beginnt die Geschichte des URLAJT etwa Anfang 1985 als Aleksandr Belonosov, der zeitweilig bei der Gruppe DK spielt. Sergej Zarikov und Il'ja Smirnov mit seinen Kommilitonen Oleg Osetrov und Kamil Musin bekannt macht und die genannten Personen den Entschluß fassen, ein neues Magazin zu grunden. Bereits in den ersten Ausgaben sprechen sich die Herausgeber gegen den Leningrader rok-klub und fur die neueren, unabhangigen Gruppen DDT, DK, Oblacnyj kraj sowie die Gruppen um Jule Roza und Aleksandr Baslacev aus. Nach der ersten Ausgabe verlaßt \%arikov das URLAJT, um das Magazin SMORCOK zu grunden. Smimov aktiviert daraufhin seine ehemaligen Mitstreiter, Evgenij Matusov und Sergej Gur'ev, wieder, die seit der vierten Ausgabe zum festen Mitarbeiterstab des Magazins zählen. Nach der siebten Ausgabe verlassen Belonosov, Osetrov und Musin das URLAJT Etwa Mitte 1985 kommt es zur Grundung des von der städtischen Kulturverwaltung (Gorodskoe Upravlenie Kul'tury) getragenen, bereits weiter oben erwahnten Moskauer Rock-Laboratoriums (Moskovskaja roklaboratorija (MRL)), welches versucht, alle Aktivitaten des ZOMBI und des URLAST zu 
unterbinden. Die Ausgaben 14 und 15, die im September bzw. Dezember 1986 erscheinen, werden als Provokation mit der Bezeichnung Bjulleten' rok-laboratorii No 1 bzw. 2 veröffentlicht. Gleichfalls kommt es in diesem Jahr zu verbalen Auseinandersetzungen mit dem Magazin SMORCOK. Im April 1987 nähert sich das URLAJT dem offiziellen Magazin Junost' an und gewinnt dort neue Mitarbeiter, namentlich Artem Lipanov, Aleksandr lonov (vgl: Analyseeinheit 17) und Kostja Preobrazenskij. Seit Mitte des Jahres veranstalten die Magazine URLAJT und ZOMBI gemeinsam verschiedene Konzerte. Aufgrund dieser Aktivitäten und zahlreicher Publikationen von Smirnov in offiziellen Organen (neben Junost' in Znanie - sila, Moskovskie novosti, Moskovskij komsomolec) sowie eines verstärkten Engagements Gur'evs für das Magazin ZOMBI, hat das URLAJT zu diesem Zeitpunkt (etwa Oktober 1987) sein Erscheinen faktisch eingestellt.

Angeregt durch Andrej Volkov, entschliessen sich Smimov und Gur'ev im April 1988, das Magazin, gemeinsam mit Lipanov, Ionov, Preobrazenskij und Marina Pimaseva, mit dem Ziel wiederzubeleben, eine professionelle Publikation mit einer höheren Auflage und einem breiteren Themenspektrum als bisher zu produzieren. Die ersten vier Ausgaben der neuen Redaktion werden bis Herbst 1988 veröffentlicht, wobei in besagtem Zeitraum gleichfalls verschiedene Konzerte veranstaltet werden. Einen Höhepunkt in dieser Hinsicht stellt ein Gedächtniskonzert zu Ehren von Aleksandr Baślacev dar, dem ein Großteil der Nummer 23 (5) gewidmet ist, die mit einer Auflage von 25000 Exemplaren verbreitet werden soll. Bevor es zur geplanten Veroffentlichung kommt, wird die Auflage in der Dnuckerei beschlagnahmt: eine unbekannte Anzahl von Exemplaren kann gerettet und verbreitet werden. Nach dieser Publikation, von der zahlreiche Teile in anderen Publikationen nachgedruckt werden, ist die Redaktion aufgnund des Versuchs der stärkeren Politisienung und Kommerzialisierung durch Smimov polarisiert. Kurz vor Fertigstellung der funfundzwanzigsten (siebten) Ausgabe kommt es aufgrund des Artikels Stat'ja No 108 von lonov, der später im Magazin KONTR KUL'T UR'A veroffentlicht wird. am 17 September 1989 zum Eklat und zum Austritt von Smirnov aus dem Mitarbeiterstab des URLAJT. Smirnov, der den Namen URLAJT weiterhin fur seine Publikation verwendet, gibt die besagte Ausgabe Anfang 1990 unter der Mitarbeit von Oleg Kovriga (vgl : Analyseeinheit 11, 30), Pimaseva, Jurij Menacharov, Preobrazenskij und V. Ivanov mit einer Auflage von zunachst 50 Exemplaren heraus. Diese Ausgabe, die sich bereits weit vom Themenkomplex Rock-Musik entfernt hat, stellt die letzte Nummer des URLAJT dar, die im samizdat publiziert wird. Fast zwei Jahre später wird die angesprochene Ausgabe mit einer Auflage von 10000 Exemplaren erneut aufgelegt. Der letzten Ausgabe widerfahrt ein ähnliches Schicksal, wie bereits der Nummer 23 (5): die Auflage wird beschlagnahmt und nur ein geringer Teil kann verbreitet werden. 


\subsubsection{TUSOVKA (ROK-VESTNIK)}

Das Magazin TUSOVKA aus Novosibirsk erscheint zwischen 1986 und 1989 mit einem Umfang von jeweils ungefahr 100 bis 200 Seiten. Es existieren 6 Ausgaben, wobei die Auflage der ersten vier Ausgaben, die schreibmaschinell erstellt werden, nicht mehr als 20 Exemplare, die der verbleibenden Ausgaben, die per Computer produzient und vervielfaltigt werden, zwischen 100 und 200 betragt.

Am Beginn der Entwicklung des Magazins steht Ende 1985 bzw. Anfang 1986 der Entschluß, aus einer halboffiziellen Vereinigung, die unter der Bezeichnung rok-sekcija firmiert und deren zentrale Persönlichkeiten Boris Komarov und Aleksandr Kirilov sind, einen offiziellen rok-klub und ein dazugehorendes Organ zu grunden. Nachdem die erste Ausgabe in Form einer Wandzeitung mit dem Namen ROK-VESTNIK publiziert ist, übernimmt Valerij Murzin (vgl:: Analyseeinheit 15) die Verantwortung für das Magazin. Die Gründung des rokklub findet zeitgleich mit einem Festival, dem die zweite Ausgabe des ROK-VESTNIK gewidmet ist. im März 1986 statt. Die dritte Ausgabe erscheint Ende 1986 unter dem Namen TUSOVKA Von dieser Ausgabe werden zwei Varianten erstellt: eine «offizielle», die dem KGB zugespielt wird und eine «inoffizielle», unzensierte, die im Rahmen der betrachteten Formation verbreitet wird. Gleichzeitig werden die drei führenden Gruppen des Clubs, Kalinov Most. Strachovoj polis und Lombard, zu Gastspielen nach Leningrad eingeladen. Da Murzin an dieser von Januar bis September 1987 dauernden Reise teilnimmt, erscheint die folgende Ausgabe als Sammelband. der die Nummern 4, 5 und 6 enthalt Nach dieser Ausgabe gewinnt Murzin A. Belikov und V Lysenko, die an der Erstellung des vom KGB zerschlagenen Magazins ID beteiligt waren, als Mitarbeiter. Die siebte Ausgabe enthält neben Beiträgen der genannten Personen u.a. Artikel von Artem Troickij, Sergej \%arikov und Sergej Gur'ev, die Murzin in der Zwischenzeit kennengelernt hatte. Zu diesem Zeitpunkt zeichnet sich bereits das Ende des Novosibirsker Rock-Clubs ab 1989 erscheint die letzte Ausgabe des Magazins

\subsubsection{DVR (DALNEVOSTOCNYJ RADIO)}

Das Magazin DVR aus Vladivostok erscheint zwischen 1987 und 1991 mit einem durchschnittlichen Umfang von 100 Seiten mit zwolf Ausgaben Die ersten funf werden mit einer Auflagenstärke von maximal 7, die folgenden drei mit maximal 20 und die verbleibenden mit bis zu 150 Exemplaren produzient In bezug auf die Vervielfaltungsart kann eine beständige Entwicklung beobachtet werden: die ersten Ausgaben sind Unikate, die aus schreibmaschinell erstellten Texten und Zeichnungen einzeln zusammengestellt werden Erst seit der sechsten Ausgabe wird dazu ubergegangen, die einzelnen Ausgaben zu kopieren. Seit der elften Ausgabe wird mit dem Computer gearbeitet.

Ausgangspunkt des DVR, welches im Herbst 1986 entsteht, ist das vom seit 1984 existierenden Vladivostoker rok-klub herausgegebene Magazin FUNIKULER, an dem Maksim Nemcov (vgl. Analyseeinheit 18), Ol'ga Nemcova (vgl : Analyseeinheit 22), Igor' Davydov 
und Aleksandr Demin (vgl: Analyseeinheit 23) mitarbeiten. Dieses Magazin wird etwa im Juli 1987 in DVR umbenannt und beschäftigt sich in seinen ersten beiden Ausgaben hauptsächlich mit der untersuchten Formation in Vladivostok. Die dritte Nummer des DVR, die Ende 1987 erscheint, ist u.a. den örtlich neu entstandenen Gruppen (u.a. dem ersten Auftritt der Gruppe Televizor) sowie einem Konzert der Gruppe Alisa in Vladivostok gewidmet. Seit der vierten Ausgabe hat sich ein fester Mitarbeiterstab etabliert, zu dem die Geschwister Nemcov, Aleksej Voronin und Michail Pavin zählen. Im Laufe der Zeit kommt es einerseits zu Kontakten zum Kiever Rok-Artel', die zu Auftritten der Gruppe Rabboty Cho in Vladivostok führen sowie andererseits zu einem informellen Austausch mit der Kiever Publikation GUCNOMOVEC. Nach Veröffentlichung der siebten und achten Ausgabe im Dezember 1989 lernt Ol'ga Nemcova, die bereits vorher versucht hatte, Kontakte zum Moskauer URLAJT aufzubauen, die Herausgeber des neuen Moskauer Magazins KONTR KULT' UR'A, Sergej Gur'ev und Aleksandr Volkov, kennen, die für die Vervielfaltigung und das Design der folgenden Ausgaben 9 und 10 verantwortlich sind. Aufgrund externer Probleme stellt die Nummer 11, die Ende 1990 erscheint, einen qualitativen Rückschritt dar. Mit der Einsicht, daß die Zeit des samizdat beendet ist, wird das Erscheinen des DVR mit der zwölften Ausgabe eingestellt.

\subsubsection{SUB"EKTIKON}

Über das Kiever Magazin SUB"'EKTIKON, welches zwischen 1987 und 1989 füfmal mit einem Umfang von 50 Seiten und einer Auflage von fünf Exemplaren, die schreibmaschinell erstellte Texte sowie Zeichnungen enthalten, erscheint, ist wenig bekannt. Alle Informationen bezuglich der Mitarbeiter sind unsicher, da die einzelnen Beiträge entweder anonym veroffentlicht bzw. mit Pseudonymen unterschrieben sind. Hauptakteur des Magazins ist vermutlich Grigoryj Pavlov Mitgearbeitet bzw in einzelnen Ausgaben publiziert haben u.a. Aleksandr Pipa. Vladimir Satraj, Aleksandra Semenova sowie Vadim Maleskevic. Für die Gestaitung des SUB" KTIKON scheint A. Ovsjannikov verantwortich zu sein.

\subsubsection{NOVYJ CHE'M}

Sechs Ausgaben des Heavy-Metal-Magazins NOVYJ CHE'M werden zwischen 1988 und 1989 in Moskau von Egor Zajcev (vgl: Analyseeinheit 14) publiziert. Die erste Ausgabe erscheint mit einem Umfang von 40 Seiten und einer Auflage von 50 Exemplaren, die letzte mit 60 Seiten und 150 Exemplaren. Das in Form von Fotokopien verbreitete Magazin enthält schreibmaschinell verfaßte Texte sowie Fotos.

Bevor Zajcev das Magazin NOVYJ CHE'M herausgibt, publizient er unter dem Namen CHE' $M$ einzelne Blätter, die neben Texten der Gruppen DDT, Oblacnyj kraj und Krematorij Außerungen enthalten, in denen er sich gegen das Moskauer Rock-Laboratorium (MRL) ausspricht. Anfang 1988 entsteht das Magazin NOVYJ CHE'M, welches im Umfeld des vom KGB kontrollierten Clubs Vitjaz' bzw. des Dom Kul'tury im. Gor'kogo zirkuliert. Nicht nur dort, sondern auch in russischen Emigrantenkreisen, in Frankreich und in den USA, ist das 
Magazin belıebt, was dazu führt. daß die dritte und vierte Ausgabe auschließlich diesem Personenkreis gegen Devisen zuganglich gemacht wird. Weiterhin bemerkenswert ist die enge Verbindung zwischen Zajcev und dem KGB, der ihm erlaubt, die sechste Ausgabe auf seinen Fotokopierern zu vervielfältigen.

\subsubsection{TIF (TEKUSCCAJA INFORMACIJA FILOFONISTA)}

Das Magazin TIF aus Archangel'sk erscheint zwischen April 1988 und Dezember 1992 sechzehnmal. In diesem Zeitraum wird sowohl die Auflage (von 5 Exemplaren auf etwa 50) als auch der Umfang (von 10 Seiten auf 40) gesteigen. Erstellt wird das Magazin mit der Schreibmaschine, verbreitet wird es in Form von Fotokopien.

Das Magazin, welches zunächst von Grigorij Valov allein, später zusammen mit Andrej Turusinov (vgl: Analyseeinheit 16) herausgegeben wird, entsteht im Umfeld des klub filofonistov. Seit etwa Ende 1989 bzw. Anfang 1990 wird die Publikation TIF weit uber die Grenzen des Clubs hinaus bekannt. Einige Artikel werden in der Zeitung Severnoj komsomolec wiederveroffentlicht, andere werden exklusiv fur die Publikationen KONTR KUL'T UR'A und SPID" verfaßt. Die vierzehnte Ausgabe ist die letzte, die die beiden Herausgeber gemeinsam erstellen. Turusinov gründet im Anschluß an die Trennung von Valov das Magazin REZUL'TATY, während dieser zwei weitere Ausgaben der angesprochenen Publikation produziert

\subsubsection{KONTR KUL'T UR'A}

Die aus Moskau stammende Publikation KONTR KUL'T UR'A erscheint zwischen 1990 und 1991 mit drei Ausgaben Die Auflagenhohe der ersten Ausgabe ist unbekannt. die der zweiten betragt 330, die der letzten, die in gedruckter Form erscheint, 10000 . Der Umfang der einzelnen Ausgaben bewegt sich jeweils zwischen 170 und 200 Seiten. Die einzelnen Ausgaben wej. sen sowohl schreibmaschinell als auch mit dem Computer erstellte Texte auf, sie beinhalten Fotos, Zeichnungen und Collagen

Ausgangspunkt der Publikation ist der 17 September 1989, an dem sich die Redaktion des Magazins URLAJT kurz vor Fertigstellung der Nummer 25 (7) in zwei Fraktionen spaitet. Aleksandr Volkov und Sergej Gur'ev entschließen sich, ein neues, radikaleres Magazin herauszugeben, für welches sie die programmatische Bezeichnung KONTR KUL'T UR'A wahlen Neben den Initiatoren arbeiten im Verlaufe der folgenden zwei Jahre Lev Goncarov, Aleksej Koblov, Aleksandr Kusnir, Verfasser der zugnundegelegten Enzyklopadie, Aleksandr Pigarev, Aleksandr Ionov und Artem Lipanov an der Erstellung dieser Publikation mit. Grundlage der ersten Ausgabe, die mit einer Auflage von 500 Exemplaren in Tjumen' gedruckt werden soll, ist die bereits erwähnte Nummer 25 (7) des URLAJT ohne die Beiträge von Il'ja Smimov. Aufgrund externer Einflusse weigen sich die Druckerei, die Auflage herauszugeben. so daß die erste Ausgabe mit Verspatung erst seit dem 31.0190 in Form von Fotokopien vorliegt In diese Zeit fallen weitere Aktivitaten: Volkov und Gur'ev besuchen die Herausgeber des Ma- 
gazins DVR in Vladivostok, was zu einer intensiven Zusammenarbeit führ. Des weiteren werden verschiedene Konzerte und Festivals veranstaltet. Nach der Publikation der ersten Ausgabe des KONTR KUL'T UR'A wird gemeinsam mit dem Kiever Magazin GUĆNOMOVEC' an einer bisher unveröffentlichten Ausgabe dieses Magazins gearbeitet. Am 17. September 1990 erscheint die zweite Ausgabe des KONTR KUL'T UR'A, die zum ersten Mal Beiträge von Kostja Uvarov und Leonid Afonskij (vgl.: Analyseeinheit 24) enthält und in der sich ein gewisser Hang zur Elitisierung abzeichnet. Im Anschluß an die Publikation wird neben der Materialsammlung für die dritte Ausgabe und der Produktion des Moskauer Magazins SUMELA" MYS' das Festival Indjuki organisiert. Es zeichnen sich konzeptuelle Divergenzen hinsichtlich der weiteren Entwicklung des Magazins ab, die au dem Entschluß führen, die Publikation mit der folgenden Ausgabe einzustellen, die dennoch in einer weit höheren Auflage als bisher üblich in gedruckter Form verbreitet werden soll. Im Dezember 1991 wird diese Ausgabe auf einem Festival in Vjatka präsentiert.

\subsubsection{CHERR (S MOSLOM) bZw. CHERR (BEZ MOSLA)}

Das Magazin CHERR (S MOSLOM) bzw. CHERR (BEZ MOSLA) aus Orecho-Zuevo etscheint zwischen Dezember 1990 und Sommer 1993 füfmal. Das Magazin besitzt einen Umfang von bis zu 100 Seiten und eine durchschnittliche Auflage von 100 Exemplaren, wobei die letzte Ausgabe mit einer Auflage von nur 5 Exemplaren erscheint. Das Magazin enthäh Texte, die mit dem Computer erstellt sind, Fotos sowie Collagen und wird in Form von Fotokopien verbreitet.

Herausgeber der Publikation sind Vladimir Tichonov und Jurij Gusev, die zeitgleich in der Gruppe Slucainie svjazj spielen. Aufgrund dieses Umstands steht ein Großteil der Publikationsbeitrage im Zusammenhang mit dieser Gruppe. Darüber hinaus widmen sich die Herausgeber der Beobachtung der allgemeinen Entwicklung der Rock-Presse. Abgesehen von eigenen Artikeln, finden sich solche in der Publikation, die bereits anderweitig, in den Magazinen VOPROSY OLIGOFRENII, URLAST und KONTR KUL'T UR'A veroffentlicht sind.

\subsubsection{SUMELA" MYS'}

Das Moskauer Magazin SUMELA" MYS', das schreibmaschinell erstellte Texte, Zeichnungen und Fotos enthatt und in Form von Fotokopien verbreitet wird. erscheint 1991 mit zwei Ausgaben Die erste, die einen Umfang von 150 Seiten aufweist, wird mit einet Auflage von 23 Exemplaren publiziert, die zweite Ausgabe mit ebenfalls 150 Seiten und einer Auflage von 15 Exemplaren.

Die Grundung des Magazins geht auf das Jahr 1988 zuruck, als Boris Usov (vgl.: Analyseeinheit 20) gemeinsam mit Igor' Puchov sowie später Boris Rudkin (vgl.: Analyseeinheit 19) ein Magazin erstellt, das sich ausschließlich mit dem klub ljubitelej fantastiki (KLF) und esoterischer Literatur beschäftigt. Nachdem Usov und Rudkin Mitte 1990 die erste Ausgabe des KONTR KUL'T UR'A erhalten hatten, verlagern sie den Themenschwerpunkt des Magazins auf Rock-Musik. Die erste Ausgabe wird im Februar 1991 auf dem in Tjumen' durchgeführten Rock-Festival Belaja Pojana sowie kurze Zeit spater im Majakovskij Museum in 
Moskau auf einer samizdat-Ausstellung prasentiert. Die zweite Ausgabe, die im Oktober erscheint, ist vorwiegend dem genannten Festival gewidmet. Trotz der kleinen Auflage erhält diese Ausgabe eine große Resonanz, die dazu fuhrt, daß Teile in den Publikationen KONTR KUL'T UR'A, OKOROK (aus Mogilev) und Erec' (aus Ventspils) sowie in der Zeitung Gumanitarnyj fond nachgedruckt werden. Nach dieser Publikation tritt Usov im Dezember 1990 auf dem bereits erwähnten Festival in Vjatka auf. Seit 1992 wenden sich die beiden Herausgeber des Magazins verstärkt der Organisation von Konzerten sowie eigenen musikalischen Projekten zu. Nachdem sie im Herbst 1992 gemeinsam mit Valerij Murzin und Sergej Gur'ev eine Zeitschrift mit dem Namen ZVERINEC herausgegeben hatten, stellen sie die Publikation ihres Magazins ein. Seit Dezember 1993 gibt Usov gemeinsam mit Sachar Muchin (vgl: : Analyseeinheit 21), der an der Publikation des Magazins PODROBNOSTI VZRYVA beteiligt ist, das Magazin SVJAZ' VREMEN heraus

\subsubsection{PODROBNOSTI VZRYVA}

Das Moskauer Magazin PODROBNOSTI VZRYVA erscheint im Jahr 1993 zweimal mit einer Auflage von jeweils funf Exemplaren. Die erste Ausgabe, die im Februar erscheint, weist einen Umfang von 150, die zweite, die im August erscheint, einen von 36 Seiten auf. Im Magazin finden sich neben handschriftich und schreibmaschinell erstellten Texten Zeichnungen. Fotos und Collagen.

Das Magazin wird v.a. von den Mitgliedern der Punk-Rock-Gruppe Lisickin chleb erstellt, die zu besagtem Zeitpunkt zwischen 14 und 16 Jahre alt sind. Da von den Mitgliedern der Gruppe und den weiteren Mitarbeitern zum größten Teil nur die Spitznamen bekannt sind. wird auf eine namentliche Nennung derselben verzichtet. In der ersten Ausgabe drucken die Verfasser u.a ihre Unzufriedenheit mit der letzten Nummer des KONTR KUL'T UR'A aus. die zweite zeigt eine starke Veranderung nicht nur in bezug auf den Umfang der Themenkomplex Rock-Musik ist nahezu vollstandig verschwunden Im Sommer 1994 sind die Herausgeber mit der Erstellung der dritten Ausgaben beschäftigt.

\subsubsection{SVJAZ' VREMEN}

Das Magazin SVAZ' VREMEN erscheint zwischen Dezember 1993 und Marz 1994 zweimal mit einer durchschnittlichen Seitenzahl von 50 und einer Auflage von 10 Exemplaren.

Hauptakteur des SVAZ' VREMEN ist Boris Usov, der u a. von Sachar Muchin unterstutz wird. Das Magazin, welches zum Umfeld des anarchistischen Vsemoskovskij patrioticeskij pank-klub gerechnet wird, zeichnet sich durch die in ihm formulierten, radikalen Ansichten sowie einer ablehnenden Haltung v.a gegenuber dem Moskauer Rock-Underground aus Ob weitere Ausgaben des Magazins existieren, ist nicht bekannt 


\subsubsection{Zusammenfassung}

Die vorangehende Beschreibung der den Auswahleinheiten zugrundeliegenden Publikationen zeigt zum einen, daß ein großer Teil der Publikationen nicht isolier voneinander entsteht und publiziert wird. Zum anderen wird deutlich, daß es sich bei diesen Publikationen nicht um Projekte einzelner Personen handelt, sondern fast ausschließlich um Gemeinschaftsprojekte. Trotz großer raumlicher Entfernungen kann von Kontakten einzelner beteiligter Personen ausgegangen werden. Gleichzeitig sind einzelne Publikationen bzw. Ausgaben trotz geringer Auflagenzahlen $^{22}$ in räumlicher Hinsicht relativ weit verbreitet.

Es liegt mit der kulturellen Formation des rok-samizdat offensichtlich ein System vor, in dem es im weitesten Sinne u.a. (literarische) Verarbeitungshandlungen (vgl.: Schmidt 1980, 274) gibt. Dies untermauert das Axiom, daß mit dem rok-samizdat eine kulturelle Formation und somit ein Diskurs gegeben ist. $\mathrm{Zu}$ dieser Formation können in personaler Hinsicht zum einen die Mitarbeiter der verschiedenen Publikationen gezählt werden, zum anderen gehören dieser kulturellen Formation auch bestimmte Personen an, über die in den Publikationen berichtet wird. Am Rande bemerkt sei, daß einige Personen, wie z.B. Boris Grebenscikov und Sergej 7arikov, sowohl zu den Herausgebern als auch zu den Objekten der Berichterstattung zählen. Wie viele Personen der kulturellen Formation angehören, ist ungewiß. Es liegt die Vermutung nahe, daß es sich um einen begrenzten Personenkreis handelt. Des weiteren kann festgehalten werden, daß zwischen «offiziellen» und «inoffiziellen» Publikationen differenziert werden sollte. $\mathrm{Zu}$ den "offiziellen» können diejenigen Publikationen gezählt werden, die im Rahmen der mehrfach erwahnten Rock-Clubs entstehen, zu den «inoffiziellen» solche, die unabhängig und illegal verbreitet werden.

\subsection{Die Analyseeinheiten}

Aus den dargestellten Publikationen werden 35 Analyseeinheiten (vgl.: Anhang 2) ausgewählt, die ein Korpus bilden, welches mittels des dargestellten diskursanalytischen Instrumentariums untersucht sowie hinsichtlich seiner Homogenität und Heterogenität betrachtet wird. Auf der Grundlage dieser Analyse sollen Schlußfolgerungen uber die untersuchte kulturelle Formation und deren Diskurs gezogen werden. Daß die Anwendung der dargestellten Analysemethode nicht nur empirisch erfolgen kann, soll anhand einer Analyse eines ungekürzten Textes gezeigt werden, die der empirischen Analyse vorangestellt wird. Ausgehend von diesem Text können Hypothesen uber die diskursive Funktion und die kulturelle Bedeutung verschiedener Elemente in der interessierenden kulturellen Formation und somit über die kulturelle Formation formuliert werden

22 Es soll betont werden. daB nur die Auflagenzahlen der Onginalkopien vorliegen. In berug auf die tatsachliche Verbreitung der Publikationen bzw: einzelner Ausgaben besitzen dicse Zahlen wenig Aussagekraft (vgl.: 6.2.2.) 
Bevor der fur die Beispielanalyse gewahlte Text und die Analyseeinheiten genannt werden, sollen einige Hinweise gegeben werden:

I Da das Korpus als «Ganzes» aufgefaßt wird, ist keine systematische Reihenfolge fur die Nennung notwendig;

2. die folgende Numerierung der Analyseeinheiten wird im Verlauf der Analyse beibehalten;

3. zunächst wird der in der jeweiligen Publikation deklarierte Urheber einer Analyseeinheit genannt. Diesem wird der "reale» Autor, soweit dieser bekannt ist, in eckigen Klammern nachgestellt;

4. die Seitenangaben der Analyseeinheiten sind unvollständig, so daß von einer Nennung abgesehen wird.

Der Text, der für die Beispielanalyse gewählt wird, ist:

anon. [Smimov, II'ja], 1990, Kolonka redaktora. In: URLAJT, 7.

Das Korpus setzt sich aus folgenden Analyseeinheiten zusammen:

1. DETI PODZEMEL'JA [Slavorosov, Arkadij; Sutov, Sergej], 1982, Kanon.

2. B.G. [Grebenscikov, Boris], 1977, O vrube. In: ROKSI, 1.

3. anon. [Malysev, Boris], 1978, O łazde muzyki i vozmoznych metodach ee utolenija. In ROKSI, 3

4. Troickij. Artem, 1981, Rebjata lovjat svoj kaif. In: ZERKALO, 2.

5. Monachov, Igor' [Smimov, Il'ja], 1983, Novyj Gesiod. In UCHO, 4

6. Razuverov, I [Sigalov, Michail], 1983, Cerno-belyj muzon. In: UCHO, 5

7. Baraskov, L. [\%arikov, Sergej], 1985, Folklor, etnografija i rok-muzyka. In: URLAJT, 1.

8 Ataman, S. [Zvezdocetov, Ataman], 1986, Muzon katit. In URLAJT, 9/10

9. Marockin, Vladimir, 1986, Sagi ne tuda (istonja diplomatiki muzyki rok i dvirenija KSP) In ZOMBI, 6

10 anon. [Gur'ev, Sergej], 1985, Welcome to the machine' In URLAJT, 4.

11. Morosov, D [Kovriga, Oleg], 1987, Derzat'sja kornej In: URLAJT, 16

12 Otec Fedor [Saparov, Igor'], 1987, O novoj humalistike ili nam nado pobol'se plochich grupp In ROKSI, 13.

13 Roza D. Kal'vados [unbek ], 1988, Byla kogda-to tetja Miansarova. In: SUB'EKTIKON, 3.

14 Zajcev, Egor, 1989, Umiraem, no ne sdaemsja Boevaja chronika stolicnoj metallurgii In. NOVYJ CHE'M, 6

15. Murzin, Valerij, 1989, Vniz po lestnice $v$ nebo Po motivam Btoroj vsesojusnoj konferencii «Rok-muzyka kak sociokul'tumyj fenomen» Sverdlovsk, 16-18.11 88 In TUSOVKA. 8

16 Turusinov, A., 1989, Hec'estnost' In: TIF, 11.

17 Ser'ga. Aleksandr [Ionov, Aleksandr], 1990, Stat'ja No 108 In: KONTR KUL'T UR'A. I

18. Nemcov, Maksim, 1991, Svoja aziatskaja roła. In: DVR, 12. 
19. Rudkin, Boris, 1991, Do redakcii. In: SUMELA" MYS', 2.

20. Usov, Boris, 1993, C'to takoe rok-poezija i kak s nej borot'sja. In: SVJAZ' VREMEN, 1.

21. Muchin, Zachar, 1993, Uchodjat produkty. In: PODROBNOSTI VSRYVA, 2.

22. anon. [vermutlich: Nemcova, Ol'ga], 1990, Gospod' daroval nam eti dosugi. Popytka klasternogo analiza. In: DVR, 10.

23. Demin, V., 1990, Kul'tura i samorazrusenie. In: DVR, 11.

24. Afonskij, Leonid, 1990, Putem tepla. Kratkij ocerk istorii russkoj kontrkul'tury. Popytka sravnitel'nogo analiza. In: KONTR KUL" UR'A, 2.

25. Sokolovskij, Ivan, 1991, Konec andergraunda. In: KONTR KUL'T UR'A, 3.

26. anon. [Matusov, Evgenij], 1986, Novyj vrag - pidorostija. In: URLAJT, 9/10.

27. anon., [Smirnov, Il'ja], 1988, Novye gorizonty svobody. In: URLAJT, I (19).

28. anon., [Smirnov, Il'ja], 1988, Pered vami, deti, utka ... In: URLAJT, 3 (21).

29. anon., [Smirnov, Il'ja], 1988, Veselye i ne-veselye kartinki uz istorii DK. In: URLAJT, 4 (22).

30. Morozov, D [Kovriga, Oleg], 1989, Cas priliva. In: URLAJT, 5 (23).

31. anon., [Smirnov, Il'ja], 1989, Kolonka redaktora. In: URLAJT, 6 (24).

32. Romanov, Ju., 1989, Dzikija. In: URLAJT, 7.

33. anon. [Smimov, Il'ja], 1991, Vspominaja o klube «Antares». Chippi. In: URLAJT, 8.

34. anon., 1991, Anons. Żivo! Pis'! In: CHERR (S MOSLOM), 3.

35. anon., 1991/1992, Sejs̀n u Sergeica. In: CHERR (BEZ MOSLA), 4. 
8. Eine diskursanalytische Betrachtung von: anon. [Il'ja Smirnov/, 1990, Kolonka redaktora. In: URLAJT, 7.

\section{Kolonka redaktora}

Segodnja, $v$ preddverii zakona o pecati, ton nasich oficial'nych lic po otnoseniju $k$ nezavisimoj presse menjaetsja na glasach. No te sravnitel'no blagozelatel'nye obzori, kotorye prisli na smenu "Citivu"*, toze stradajut izvestnoj bestolkovost'ju. A chotelos' by znat', cto proischodit $s$ nezavisimoj pressoj na samom dele. Pozaluj, problem u nee ne men'se, cem u pressy oficial'noj, a perspektivy mogut okazat'sja takimi te bezotradnymi. kak u otecestvennogo rok-dvizenie, chlynuvsego $v$ proslom godu na stadiony $v$ edinom poryve panurgova stada baranov I cem dol'se my budem tesit' sebja illjuzijami o tom, cto my-de "ne takie, kak oni", "my lucse po samoj svoej prirode", tem soknusitel'nee okazutja posledstvija.

Prezde vsego, $v$ silu osobennostej sistemy rasprostranenija i otsutstvija obratnoj svjazi "redakcija" - "citatel'" (pocty, podpiski), u nezavisimich izdanij neizbezno sformirovalas' orientacija na "svoj krug", na "tusovku" so svemi vytekajuscimi. A vytekaet, prezde vsego, "svetskaja chronika" $v$ toj ili inoj forme - to est' informacija, zavedomo predstavljajuscaja interes dlja nebol'soj gruppy ljudej, - i podbor materialov po principu, izvinite, $\dot{\mathbf{S}} \mathbf{R}$. Rasidova: "Nu. Vasja Pomidorov poet (muzykant, chudoznik), konecno, cherovatyj ... - no muzijk otlicnyj, vcera mne moju stat'ju pochvalil (varianty: televidenie tesce pocinil, stakan nalil). Nado ego napecatat' (variant: otreklamirovat', sravnit' s Polom Makkartni i F. M. Dostoevskim)." Vse eto vmeste nazyvaetsja odnim slovom - "neprofessionalizm" ili po-russki "chaljava". I do tech por, poka oficial'naja pressa rabotala po tem te samym zakonam (tol'ko scet sel ne na stakany, a na clenskie bilety SP SSSR i gos. premii), a nezavisimaja byla okruzena opasnym. no pocetnym oreolom oppozicionnosti - do tech por problema professionalizma ne vstavala pered podpol'nymi redaktorami. Kakoj tut. k certovoj materi. maket i ssylki na istocniki, kogda vcera uze tretyj raz ucastkovyj prichodil s kakim-to murikom v statskom! No vot situacija izmenilas' $S$ oficial'nych organov snjati prakticeskij vse ogranicenija na temy publikacij $i$ imena avtorov Drugie ogranicenija ostalis', i deficit sovesti vovse ne rassosalsja za tri goda - no vse-taki zurnal, pecatajuscij Solzenicyna. eto ne sovsem to te samoe, cto hurnal, zapolnennyj Markovym i Proskurinym Eto uze ne "parallel'noe" (neperesekajusceesja) javlenie Eıo KONKURENT Pricem. konkurent rastusiij, ovladevajuscij na glasach tem. cto sostavljalo do sich por nase monopol'noe preimuscestvo

I tut vyjasnjaetsja cto nezavisimaja pecat' gotova $k$ takomu povorotu sobytij ne bol'se. cem nasi rokery - $k$ gastroljam v Amerike Publikuemaja nize recenzija M Timasevoj na 29 nomer "Glasnosti", naprimer, dostatocno ubeditel'no svidetel'stvujet o tom, cto etot zurnal bez lejbla "ZAPRESCENNOE" - prodavaemyj $v$ kioskach "Sojuspecati" - ne tol'ko ne nasel by pokupatelej po svoej nynesnej cene, no i pri ravnych cenach ne sostavil bi konkurencii ni "Ogon'ku", ni "Novomu miru". A pricina zdes' odna, pervaja, ona te poslednaja - nedostatok masterstva No "Glasnost'" esce iz lucsich primerov Podavljajuscee bol'sinstvo prodavaemoj na "Puske" produkcii po stilju i, cto samoe strasnoe. po urovnju myslenija voschodit k "Pravde" daze ne sovremennoj, a pjatiletnej davnosti, poskol'ku zapolneno ot korki do korki ZDRAVICAMI i PROKLJATIJAMI. Me`du tem, na Zapade pressa slu>it pre de vsego dlja rasprostranenija novoj informacii i novych idej A dlja populjarizacii lozungov bol'se vsego podchodit zabor - tam mołno pisat' "Kolja + Olja = Ljubov'" ili "Petja durak", soverienno bezbojaznenno - nikto ne potrebuet dokazatel'stv togo, cto sam pisusij namnogo prevoschodit Petju intellektom

Nel'zja skazat', cto casa sija minovala nas UR LAJT (chotja problemu kacestva my osoznali vse-taki ran'se bol'sinstva kolleg, $i$ imenno eto pobudilo nas poltora goda nazad tak 
rezko izmenit' vnesnij vid izdanija). I k nam toze stali postupat' daleko ne chvalebnye otzyvy citatelej na takie opisanija koncertov, gde perecisljalos', $v$ kakom rjadu kakoj tusovscik sidel i s kakoj damoj udalilsja. Eto ne fakticeskaj osibka, netocnost', ljap, kotoryj mozet slucit'sja $v$ ljubom izdanii - eto brak soznatel'nyj, kotoromu my ne sobiraemsja iskat' "ob" "ektivnych" opravdanij

Da, ot pravdy nikuda ne denes'sja: my vsegda gotovy trebovat' ot vlastej, ctoby oni upravljali nami po-civilizovannomu, no ne ocen' torpimsja sami brat' primer s togo, kak rabotajut $v$ civilizovannych stranach (anglijskie, amerikanskie, japonskije zurnalisty) - daze togda, kogda nas vrode by nikto ne zastavljaet gnat' parasu.

Vprocem, eto grustnoe umozakljucenie otnositsja ne tol'ko $k$ zurnalistike. Tak cto delat' necego - esli my chotim ne slyt', a byt' dejstvitel'no nezavisimymi ljud'mi, pridetsja prorubat' okno $v$ Evropu, a ne $v$ zopu ( $v$ zopu nam vsem i tak davno okno prorubleno $v$ rezul'tate izvestnych istoriceskich sobytij $i$ bez vsjakogo nasego na to soglasija).

Redkollegija zurnala «Urlajt»

" "Civo iz podvorotni" - "Koms. "pravda", 5.03.87

Bevor mit der diskursanalytischen Betrachtung des obigen Textes begonnen wird, sollen zwei Hinweise erfolgen: erstens ist es nahezu ausgeschlossen, einen Text vollständig diskursanalytisch zu betrachten, da fast jedes Wort bzw. jede Wortverbindung Schlüsse über ein kulturelles System zulassen kann. Es können in diesem Sinne nur Phänomene betrachtet werden, die mittels des verwendeten Untersuchungsinstrumentariums «sichtban» werden. Ein Problem in dieser Hinsicht, dies ist der zweite Hinweis, stellt das "Spiel» mit diskursiven Objekten dar (vgl:: 2.10). Es ist bisher nicht gelungen dieses "Spiel», also Ironie, Sarkasmus u.dgl.m., diskursanalytisch zu erfassen (vgl.: Tüschau 16, 1998 und 1998b).

Unter diesen Voraussetzungen wird der ausgewählte Text mit dem in den vorangehenden Kapiteln dargestellten diskursanalytischen Instrumentarium betrachtet, wobei die syntaktischen Verfahren sowie die Themen- und Kontingenzanalyse nicht angewendet werden, da diese nur im Rahmen einer diskursanalytischen Korpusanalyse sinnvoll sind. Der Text ist der 22. Ausgabe der Publikation URLAJT entnommen. die mit der Numerienung 7 (25) erscheinen sollte Diese Ausgabe ist die erste. die nach der weiter oben erwăhnten Spaltung der Redaktion erscheint (vgl.: 7.2.1.5.). Der Text, der mit Redkollegija zurnala «Urlajt» unterschrieben ist. stammt von Il'ja Smirnov, der der untersuchten Formation seit spätestens 1981 angehört. Neben der langen Zugehörigkeit zu der interessierenden Formation zeichnet sich Smimov u.a. durch seine Mitarbeit an den Publikationen ZERKALO (vgl: 7.2.1.2.), UCHO (vgl: 7.2.1.3.) und URLATT (vgl: : 7.2.1.5.) aus.

In dem Text. dem Vorwort der genannten Ausgabe, geht es um Probleme der inoffiziellen Presse, die aus der Verändenung der allgemeinen politischen Verhältnisse resultieren. Smirnov weist in diesem Zusammenhang darauf hin, daß das Problem der Qualität, v.a. im Vergleich zur offiziellen Presse zentral sei. Nach Erkennen dieses Problems sei, bezogen auf das URLAJT, das äußere Erscheinungsbild der Publikation verändert worden. Inhattlich hingegen weise das 
URLAJT keine Qualitätsmängel auf, da die Veröffentlichung auch «schlechten» Artikel bewußt geschähe.

Hinsichtlich seiner kulturellen Herkunft markiert Smirnov den Text durch die Verwendung von Diskursiva und Indices. Zu den als Diskursiva bezeichneten Elementen werden die folgenden Wörter bzw. Wortverbindungen gezählt: tusovka, cherovatyj, chaljava, idti na stakany, mutik v statskom. lejbl, Puska, parasa, zopa und tusovscik, wobei lediglich das Wort lejbl einen Anglizismus darstellt. Unter den Begriff Index werden die folgenden Elemente gefaßt:

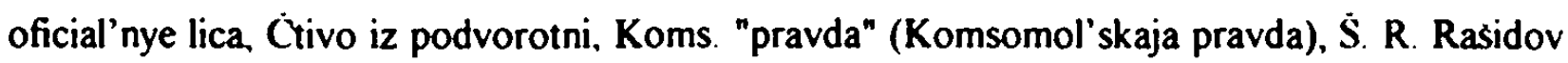
(Saraf R. Rasidov), Vasja Pomidorov, Pol Makkartni (Paul McCartney), F. M. Dostoevskij (Fëdor M. Dostoevskij), SP SSSR (Sojus Pisatelej SSSR), Solzenicyn (Aleksandr Solzenicyn), Markov (Georgij M. Markov), Proskurin (Pëtr L. Proskurin), nasi rokery, Amerika, M. Timaseva (Marina Timaseva), Glasnost', Sojuspecat', pokupateli, Ogonek, Novyj mir, Puska (Puskinskaja Ploscad'), Pravda, Zapad, Kolja, Olja, Petja, URLAJT, kollegi, civilizovannye strany, anglijskie zurnalisty, amerikanskie zurnalisty, japonskie zurnalisty und Evropa.

Insofern diese Elemente neben der Markierungsfunktion weitere kulturelle Funktionen aufweisen, wird auf diese im Verlauf der nachfolgenden Analyse erneut eingegangen.

Verfaßt ist der Text in der persönlichen Erzählperspektive, genauer aus einer Insiderperspektive heraus, die bereits im dritten Satz konstruiert wird: „A chotelos' by znat', cto proischodit's nezavisimoj pressoj na samom dele [Hervorhebung: MB]". Es handelt sich bei diesem Satz um eine rhetorische Frage, die nicht explizit als solche gekennzeichnet ist, die in diskurstaktischer Hinsicht eingesetzt wird, um den Insiderstatus des Verfassers zu unterstreichen Der nachfolgende Text stellt in diesem Sinne die Antwort auf die genannte Frage dar Ein zweites Mal wird die Insiderperspektive im Zusammenhang mit der Konstruktion des Index Zapad deutlich. .Mezdu tem [Hervorhebung: MB]. na Zapade pressa sluzit prède vsego dlja rasprostranenija novoj informacii i novych idej“ Gegen Ende des Textes wird die angesprochene Erzahiperspektive erneut sichtbar .my vsegda gotovy trebovat' ot vlastej, ctoby on upravljali nami po-civilizovannomy, no ne ocen' torpimsja sami brat' primer s togo, kak rabotajut $v$ civilizovannych stranach $[(\ldots)]$ - daze togda, kogda nas vrode by nikto ne zastavljaet gnat' parasu 'procem [Hervorhebung: MB], eto gmstnoe umozakljucenie otnositsja ne tol'ko k hurnalistike [...]"

Auf der Grundlage des Insiderkonstrukts werden verschiedene Ab- bzw. Ausgrenzungen vorgenommen Der basale Antagonismus, der durch die Personalpronomen my und oni gekennzeichnet ist, basiert auf der temporären Opposition segodnja $v$ preddverii zakona o pecati vs do tech/sich por, da Smimov aus dieser einen Handlungsbedarf ableitet Dieser Antagonismus wird uber den gesamten Text konstruiert und erreicht seinen Höhepunkt gegen Ende, wenn nezavisimye ljudi und vlast' gegenübergestellt werden Die aus der untersuchten kulturellen Formation ausgegrenzte Seite oni, die zum Teil mittels des Attributs oficial'nyj gekennzeichnet ist, umfaßt oficial'nye lica, pressa oficial'naja, oficial'nye organy, die 
Publikationen Komsomol'skaja pravda und Pravda sowie vlast'. Einige dieser Elemente werden in negativer Hinsicht konstruiert, indem ihnen die nachfolgenden Merkmale zugeschrieben werden: bestolkovost', neprofessionalizm, chaljava, deficit sovesty und uroven' myslenija pjatiletnej davnosti. In bezug auf oficial'nye lica wird in diesem Zusammenhang ein Stereotyp generiert, welches die momentane Situation verdeutlichen soll: „ton nasich oficial'nych lic po otnoseniju $k$ nezavisimoj presse menjaetsja na glasach". Das Eigenschaftsmerkmal bestolkovost', das sich u.a. auf den bereits erwähnten Artikel Ctivo iz podvorotni bezieht (vgl : 6.1.), bildet zusammen mit dem Normativ izvestnyj eine kulturelle Tautologie, so daß an dieser Stelle ein Aspekt des Normalitätsbreichs der untersuchten Formation deutlich wird. Aufgrund der relativ umfangreichen Konstruktion der genannten Elemente kann die Schlußfolgerung gezogen werden, daß die Konstruktion der kulturellen Bedeutung des genannten Bereichs im Rahmen der untersuchten Formation als nicht abgeschlossen angesehen werden kann. Möglicherweise wird dieser Bereich erst aufgrund zu erwartender Veränderungen für die untersuchte kulturelle Formation bzw. die Publikation URLAJT relevant.

Die Bedeutungskonstruktion der einzelnen Elemente der Seite my hingegen ist nahezu abgeschlossen, wie die geringe Merkmalszuschreibung zeigt. Der im Text offensichtliche Konstruktionsprozeß zielt vielmehr auf die Konsolidienung der Seite ab. Zunächst kann in diesem Zusammenhang festgestellt werden, daß sich hinter dem genannten Personalpronomen zwei Gruppen verbergen Einerseits bezeichnet das Pronomen die Redaktion der Publikation URLAJT (redkollegija zurnala URLAJT), um dessen wirtschaftliche Interessen es geht. Andererseits bezeichnet es den gesamten Bereich des Unabhängigen: nezavisimaja pressa, nezavisimaja pecat' und nezavisimye ljudi $\mathrm{Zu}$ den Unabhängigen werden dariber hinaus die in einem Oppositionsverhalitnis stehenden redakcija und citatel' sowie tusovka (svoj krug, hierzu gehören tusovscik und dama), nasi rokery und kollegi gezählt. Neben dem in Form eines Autostereotyps nachweisbaren Merkmal des Illusionismus bezuglich des moralischen Selbstwerts (..I cem dol'se my budem tesit' sebja illjuzijami o tom, cto my-de "ne takie, kak onj", "my lucse po samoj svoej prirode", tem sokrusitel'nee okazutsja posledstvija“.), findet sich wieder das Merkmal neprofessionalizm, welches somit den Charakter einer Schnittstelle zwischen beiden Seiten erhält. Es liegt die Vermutung nahe. daß diese Schnittstelle der Grund für die Generierung des dargestellten Antagonismus ist. Es gilt im Zusammenhang mit diesem Antagonismus des weiteren der Frage nachzugehen, warum die Seite my zwei Gruppen bezeichnet. wobei eine dieser Gruppen, die Redaktion, in der anderen enthalten ist. Ausgangspunkt der Überlegungen ist der Schluß, daß der Verfasser im Namen der Redaktion versucht, ein bestimmtes Ziel zu erteichen. Er geht dabei nicht den direkten Weg, indem er im Namen der Redaktion spricht. sondern er sammelt "Verbündete» um sich, um auf diese Weise die Bedeutung seiner Aussagen zu erhöhen. Er spricht in diesem Sinne fur eine großere, heterogene Gruppe, die nach und nach im Text differenziert wird (vgl: nasi rokery und kollegi) und erlangt dadurch in konstruktiver Hinsicht einen größeren Einfluß, so daß er seine Interessen besser durchsetzen kann. Daß es im 
vorliegenden Text um die wirtschaftlichen Interessen des Magazin URLAJT geht, wurde bereits weiter oben erwähnt.

Eine weitere Abgrenzung erfolgt von den als Indices nachweisbaren Aktanten Rasidov, der gleichzeitig als Autorität bemüht wird, Markov und Proskurin. Bei den genannten Aktanten handelt es sich um sowjetische Schriftsteller, die zum Teil wichtige Funktionen in der kommunistischen Partei bzw. im sowjetischen Schriftstellerverband (SP SSSR) besaßen (vgl : Prochorov (Hrsg.) 19753). Sie gehören in einem weiteren Sinne, als oficial'nye lica, der ausgegrenzten Seite oni an, sollen hier dennoch separat betrachtet werden, da ihre Ausgrenzung aus dem Zugehörigkeitsbereich der untersuchten Formation mit verschiedenen Mitteln erfolgt. Die beiden letztgenannten werden hinsichtlich ihrer kulturellen Bedeutungen in ein kontradiktorisches Verhältnis zu dem ebenfalls nicht weiter attribuierten, indexikalischen Solzenicyn gesetza. Da die kulturelle Bedeutung von Solżenicyn im Rahmen der untersuchten Formation mit hoher Wahrscheinlichkeit positiv ist, muß die von Markov und Proskurin negativ sein. Da alle an der Kontradiktion beteiligten Aktanten nicht explizit attribuiert sind, kann von einem abgeschlossenen Bedeutungskonstruktionsproze $\beta$ im Rahmen der untersuchten Formation ausgegangen werden

Rasidov, fur dessen Nennung sich Smimov entschuldigt, wird neben dieser Entschuldigung über ein ihm zugeschriebenes Zitat konstruiert. Rasidov lobt in diesem Zitat einen Aktanten mit einem lächerlich anmutenden Namen. Vasja Pomidorov, obwohl weder der «Beruf» des Aktanten (poet. muzykant, chudożnik) noch dessen lobenswerte Tat, für die ebenfalls drei Varianten angeboten werden, bekannt ist $D$ a dieses Zitat vermutlich nicht auf Rasidov zurückgeht, liegt die diskursive Funktion einzig in der Diskreditierung der Autorität. Aus dem Gesagten kann abgeleitet werden, da $B$ der Bedeutungskonstruktionsproze $B$ im Rahmen der untersuchten Formation. der ein negatives Resultat liefern soll, nicht vollständig abgeschlossen ist.

Daruber hinaus erfolgt eine Abgrenzung von der Publikation Glasnost', die offensichtlich ein unmittelbares Konkurrenzprodukt des URLAJT darstellt Die Ausgrenzung erfolgt uber eine relativ ausfuhrliche Bedeutungskonstruktion, die darauf hinweist, daß kein abgeschlossenes Konstrukt vorliegt

Neben der bereits erwähnten Autoritat konnen zwei weitere im Text nachgewiesen werden. my und ein unbekannter Aktant, die mit unterschiedlichen diskursiven Funktionen eingesetzt werden. Die der Autorität my zugeschriebenen Zitate, die von Smimov als Illusionen bezeichnet werden (siehe oben). dienen der Konsolidierung der Seite my des erläuterten. basalen Antagonismus Die Autorität und die erläuterte Seite des Anatgonismus sind trotz der selben Bezeichnung nicht identisch, da die Autorität eine Auffassung vertritt, die es nach Meinung Smimovs abzulehnen gilt Der Leser wird implizit aufgefordert. die Meinung des Verfassers zu übernehmen und so zum Bestandteil der genannten Seite zu werden. Die Zitate, die von unbekannter Urheberschaft sind, werden von Smimov als Losungen (lozungi) bezeichnet Diese involvieren die unspezifischen Personen-Indices Kolja. Olja und Petja sowie das Kollektivsym- 
bol ljubov'. Der Begriff Losung, der im sowjetischen Kontext eine gewisse Seriosität und eine darauf beruhende Bedeutung suggeriert, wird hier ad absurdum geführt ${ }^{23}$, da ihm Banalitäten folgen: „Kolja + Olja = Ljubov““, „Petja durak“. Kurz nach Einführung dieser Losungen wechselt Smirnov das Thema und kommt auf die Qualität seines Magazins zu sprechen, so daß die Funktion in diesem Zusammenhang gesehen werden sollte. In diesem Sinne funktionieren die Losungen als Sentenzen, die den Leser in eine positive Stimmung versetzen sollen, um ihn anschließend mit dem eigentlichen «Problem» zu konfrontieren.

Abgesehen von den bereits erwähnten Indices können die verbleibenden folgendermaßen kategorisiert werden: Personen, Gruppen, Institutionen, Geographica und Marken. Die Kategorie Personen umfaßt McCartney, Dostoevskij und Timaseva, die Kategorie Gruppe nasi rokery, pokupateli, anglijskie zumalisty, amerikanskie zumalisty und japonskije zurnalisty, die Kategorie Institutionen den Index SP SSSR, die Kategorie Geographica Amerika, Puska, Zapad und Evropa und die Kategorie Marken Sojuspecat', Ogonek und Novyj mir. Es gilt der Frage nachzugehen, welche Funktion diese Indices neben der indexikalischen Funktion aufweisen, und welche kulturelle Bedeutung sich jeweils ableiten läßt.

McCartney und Dostoevskij, die in dem der Autorităt Rasidov zugeschriebenen Zitat genannt werden, ermöglichen aufgrund ihrer kulturellen Bedeutung die Aufwertung des Aktanten Vasja Pomidorov. Da sie nicht weiter attribuiert werden, steht ihre kulturelle Bedeutung, die sicherlich positiv ist, für die Mitglieder der untersuchten Formation fest. Timaseva, die in der Ausgabe des URL.AJT, aus der der hier analysierte Text entnommen ist, einen Beitrag verfaßt hat, besitzt im Rahmen der untersuchten Formation vermutlich keine kulturelle Bedeutung, da sie offensichtlich lediglich aus Gründen der Authentizität genannt wird. Aufgrund dieser kulturellen Bedeutungslosigkeit ist eine kulturelle Funktionalisienung mit an Sicherheit grenzender Wahrscheinlichkeit ausgeschlossen. In bezug auf die Gruppe nasi rokery liegt ein Stereotyp vor: .I tut vyjasnjaetsja, cto nezavisimaja pecat' [my] gotova $k$ takomu povoroty ne bol'se, cem nasi rokery - k gastroljam $v$ Amerike“. Dieses Stereotyp wird generiert, um den zentralen Unterschied zwischen der angesprochenen Gruppe und der Gruppe my aufzuzeigen. Das Stereotyp dient in diesem Zusammenhang vermutlich nicht der Ausgrenzung der Gruppe aus der untersuchten kulturellen Formation, sondern deren interner Differenzierung, die u.a. auch in bezug auf kollegi vorgenommen wird. Die Gruppe pokupateli ist mit hoher Wahrscheinlichkeit im vorliegenden Zusammenhang in kultureller Hinsicht nicht funktionalisiert, so daß über ihre kulturelle Bedeutung keine Schlüsse gezogen werden sollen. Die Gruppen anglijskie zurnalisty, amerikanskie turnalisty und japonskije turnalisty werden im Zusammenhang mit den sogenannten zivilisierten Ländern genannt. In diesem Sinne scheinen nicht die Gruppen, sondern die repräsentierten Lander, die offensichtlich eine positive kulturelle Bedeutung aufweisen, von kultureller Relevanz zu sein. In kultureller Hinsicht bemerkenswert ist in diesem Zusammenhang,

23 Diese scheinbare Scriosităt und Bedeutung ideologischer Zeichen uurde Anfang der achuıger Jahre dieses Jahrhunderts auch von den Moskauer Konzeptualisten "entdeckt" und ad absurdum gefünt. 
daß gerade diese Länder als zivilisiert angesehen werden. Des weiteren soll festgehalten werden, daß die zivilisierten Ländern nicht in ein antagonistisches Verhältnis zu dem sogenannten Vaterland gesetzt werden, sondern zu vlast', einem Bereich, der aus der untersuchten Formation ausgegrenzt wird. Das Vaterland «an sich», welches im Text nicht thematisert wird, besitzt, das kann aus dem genannten Antagonismus abgeleitet werden, mit hoher Wahrscheinlichkeit eine positive kulturelle Bedeutung. Die Institution SP SSSR wird eingeführt, um den Unterschied der inoffiziellen und offiziellen Presse, der bis zu dem genannten Zeitpunkt do tech/sich por Bestand hatte, zu verdeutlichen: „scet ne sel na stakany, a na clenskie bilety SP SSSR“. In diesem Sinne gehört die genannte Institution der Seite oni des basalen Antagonismus an. Aufgrund der fehlenden Attribuienung liegt die Vermutung nahe, daß der Bedeutungskonstruktionsprozeß abgeschlossen ist. Der Index Amerika wird herangezogen, um den Unterschied zwischen my und der Gruppe nasie rokery aufzuzeigen. In diesem Zusammenhang wird die genannte Gruppe, wie weiter oben verdeutlicht, stereotypisiert. Da der angesprochene Index geeignet ist, diese Gruppe zu stereotypisieren, kann von einer positiven kulturellen Bedeutung ausgegangen werden, die auf einem abgeschlossenen Bedeutungskonstruktionsproze $\beta$ basiert. Das Geographicum Puska wird zu den als Diskursiva bezeichneten Elementen gezählt. Die Wahl der nicht-interdiskursiven Bezeichnung für Puskinskaja ploscad' und eine fehlende Attribuierung können als Hinweis aufgefaßt werden, daß die kulturelle Bedeutung des Geographicums im Rahmen der untersuchten Formation bekannt ist. Darüber hinaus besitzt dieses im vorliegenden Zusammenhang vermutlich keine, über die indexikalische Funktion hinausgehende, weitere Funktion Der Index Zapad fungiert im vorliegenden Zusammenhang als positives Beispiel in bezug auf die gesellschaftliche Funktion der Presse. Der angesprochene Index muß folglich in kultureller Hinsicht sehr positiv bewertet sein, da er ansonsten nicht als ein solches Beispiel diente In diskursiver Hinsicht bemerkenswert ist hier die Konstruktion des Geographicums, die aus der Sicht des Insiders vorgenommen wird Um den Prozeß dieser Konstruktion ansatzweise verdeutlichen zu konnen, wird vereinfachend davon ausgegangen. daß der Verfasser eine bewußte Manipulation des Lesers intendien Die der folgenden Darstellung zugrunde liegende Abstraktion geht folglich von der Prämisse aus. daß der Verfasser einen Wissenvorsprung gegenuber dem Leser in bezug auf das Geographicum Zapad besitzt Der Verfasser ist sich in dieser Hinsicht zum einen der Tatsache bewußt, daß der Leser um die positive kulturelle Bedeutung des in Rede stehenden Geographicums weiß Er ist sich zum anderen bewußt, daß dem Leser die gesellschaftliche Funktion der Presse dort nicht bekannt ist. da er diese ansonsten nicht zu erläutern brauchte. Auf Grundlage dieses Wissens bzw. Unwissens kann er den Index in beliebiger Weise furr manipulative Zwecke nutzen und konstruieren: „na Zapade pressa sluzit prezde vsego dlja rasprostranenija novoj informacii i novych idej“. Das Geographicum Evropa, das im Text nicht bewertet wird. fungiert als Ziel, welches es zu erreichen gilt. Diese Zielfunktion von Evropa läß sich aus dem letzten Satz des Textes ableiten Evropa muß dementsprechend in der untersuchten Formation eine positive Bedeutung aufwei- 
sen, die vollständig konstruiert ist. Gleichzeitig bedeutet das Ziel Evropa die Überwindung der als negativ bewerteten Vergangenheit (istoriceskie sobitija), die durch den nichtinterdiskursiven Begriff zopa bezeichnet wird. Die Marken Sojuspecat', Ogonek und Novyj mir werden im Rahmen der Bedeutungskonstruktion des Indices Glasnost' eingeführ. Die Marke Sojuspecat' besitzt vermutlich nur eine indexikalische Funktion, da sie ein Verbreitungsmedium von Presseerzeugnissen darstellt und mit dieser Bedeutung im Text genannt wird. Die Publikation Glasnost' hingegen wird mit den Publikationen Ogonëk und Novyj mir verglichen und schneidet bei diesem Vergleich schlecht ab: „etot zurnal [...] ne tol'ko ne na sel by pokupatelej po svoej nynesnej cene, no i pri ravnych cenach ne sostavil by konkurencii ne "Ogon'ku", ni "Novomu miru"“. Da die genannten Publikationen zu den offiziellen gerechnet werden müssen, kann aus dem Vergleich nicht der Schluß gezogen werden, daß sie eine positive kulturelle Bedeutung im Rahmen der untersuchten Formation aufweisen. Vielmehr liegt die Vermutung nahe, daß sie eine negative Bedeutung aufweisen, die von derjenigen der Publikation Glasnost' noch übertroffen werden soll.

Des weiteren können im Text Wörter ermittelt werden, bei denen es sich um Kollektivsymbole handelt: ljubov', pravda und Evropa (vgl.: Fleischer 1997b und 9.5.). Das Wort ljubov', welches, wie die Erhebungen Fleischers belegen (vgl.: Fleischer 1997b), als Kollektivsymbol sehr positiv bewertet ist, wird in den erläuterten lozungi im Zusammenhang mit den Indices Kolja und Olja angewendet. Diese Verwendung legt den Schluß nahe, daß das Wort hier in der Funktion eines Kollektivsymbols gebraucht wird. Somit entsteht in der Losung eine dialektische Spannung zwischen den Polen banal vs. bedeutend, die dazu beiträgt, den bereits erlauterten Effekt zu erzielen. Auch das Wort pravda wird in der Funktion als Kollektivsymbol sehr positiv bewertet. Im vorliegenden Zusammenhang kommt dieser positive Wert durch den Hinweis auf die zu bedauernde Nutzlosigkeit des Wertes zum Ausdruck. Aufgrund der zwar kurzen Reflexion über den Wert pravda, der im Index Komsomol'skaja pravda zudem in Anfuhnungszeichen gesetzt wird, kann im vorliegenden Zusammenhang von der Verwendung des Wortes pravda als Kollektivsymbol ausgegangen werden. In diskursiver Hinsicht kann der Schluß gezogen werden, daß ein allgemeiner Wertewandel stattfindet bzw. stattgefunden hat, der in der untersuchten kulturellen Formation abgelehnt wird. In dieser Hinsicht erfärt sowohl die theoretische Beachtung als auch praktische Umsetzung des Wertes pravda weiterhin positive Anerkennung in sprachlicher Hinsicht fungiert das Kollektivsymbol als Schnittstelle zwischen zwei Themenkomplexen. Der Verfasser nutzt es, um von "seinem» Thema überzuleiten und die Oppositionsgenerienung auf den erwähnten Kulminationspunkt zu treiben. Auf das Wor Evropa wurde bereits im Zusammenhang mit Indices eingegangen. Da Evropa, wie gezeigt werden kann, die Funktion eines Ziels besitzt, muß es sich im vorliegenden Fall bei Evropa um ein Kollektivsymbol handeln.

Der Normalitatsbereich der untersuchten Formation wird durch drei kulturelle Tautologien deutlich izvestnyj bestolkovost', vsegda gotovy trebovat' ot vlastej. ctoby oni upravljali nami 
po-civilizovannomu und izvestnye istoriceskie sobytie. Die kulturelle Tautologie konecno cherovatyj ist eine von Smirnov generierte und der Autorität Rasidov zugesprochene, die keinen Einblick in den Normalitätsbereich der untersuchten Formation gestattet. Es handelt sich an dieser Stelle mit hoher Wahrscheinlichkeit um ein "Spiel» (siehe oben) mit dem gegebenen Normalitätsbereich. Auf die erste kulturelle Tautologie wurde bereits im Zusammenhang mit der Seite oni des basalen Antagonismus eingegangen, so daß eine Konzentration auf die verbleibenden erfolgt. Die zweite Tautlogie, in der das Normativ vsegda verwendet wird, verdeutlicht einerseits, daß sich die Mitglieder der untersuchten Formation von der Macht nicht zivilisiert behandelt fühlen, andererseits, daß die Mißbilligung dieses Sachverhalts als selbstverständlich gilt. In der dritten Tautologie wird deutlich, daß bestimmte, nicht näher bezeichnete, historische Ereignisse samt ihrer kulturellen Bedeutung als bekannt vorausgesetzt werden. Es handelt sich bei diesen Ereignissen mit hoher Wahrscheinlichkeit um die sowjetische Herrschaft, die, als zopa bezeichnet, eine negative Bedeutung aufweist.

Im vorliegenden Text können des weiteren mehrere Elemente nachgewiesen werden, die als Hinweis auf die Jugendsubkultur Sistema aufgefaßt werden können. Es handelt sich einerseits um die der Person Vasja Pomidorov zugeschriebenen «Berufe» poet, muzykant und chudoznik, die in der genannten Jugendsubkultur ein besonderes Ansehen genießen (vgl.: 4.6.), andererseits um die als Diskursiva bezeichneten Begriffe tusovka und tusovscik, wobei tusovka im vorliegenden Zusammenhang im Sinne von krug obscenija (vgl.: 4.6.) verwendet wird. Es gilt der Frage nachzugehen, auf welches Verhältnis zwischen der untersuchten kulturellen Formation und der angesprochenen Jugendsubkultur geschlossen werden kann, wenn die genannten Elemente als Hinweis aufgefaßt werden. Die «Benfe» werden in dem die Autorität Rasidov diskreditierenden Zitat eingeführt, um die Person Vasja Pomidorov naher zu bestimmen. Da diese Person bereits aufgrund ihres Namens lächerlich wirkt, werden die «Berufe» mit hoher Wahrscheinlichkeit nicht eingesetzt, um die Seriosität der Person zu betonen Sie sollten vielmehr als ein erganzendes, diskreditierendes Mittel angesehen werden. In diesem Sinne kann nicht von einer positiven Assoziation zwischen den genannten "Gruppierungen" ausgegangen werden Dennoch zieht Smirnov den Begriff tusovka heran, um sein personales Umfeld zu bezeichen, wobei die Person tusovscik zu diesem Umfeld gezählt werden kann. Die genannten Begriffe weisen folglich auf eine positive Assoziation zwischen den angesprochenen "Gruppierungen» hin. Auf der Grundlage des Gesagten kann die Hypothese formulien werden, daß eine partielle Überschneidung zwischen der untersuchten kulturellen Formation und der Jugendsubkultur Sistema vorliegt

Mit dieser Feststellung soll die diskursanalytische Betrachtung des ausgewählten Textes beendet werden 


\section{Ergebnisse der diskursanalytischen Betrachtung des Korpus}

\subsection{Die Wort-Satz-Relation: WSR}

Das Analyseergebnis hinsichtlich der Wort-Satz-Relation, d.h. der durchschnittlichen Satzlänge der ausgewählten Analyseeinheiten (AE), ist in Tabelle 10 abgebildet.

Tabelle 10

\begin{tabular}{|l|c|c|c|c|c|c|c|c|c|c|c|c|c|c|c|c|c|c|}
\hline AE & 1 & 2 & 3 & 4 & 5 & 6 & 7 & 8 & 9 & 10 & 11 & 12 & 13 & 14 & 15 & 16 & 17 & 18 \\
\hline WSR & 17.4 & 12.8 & 10.4 & 16.1 & 14.3 & 14.9 & 14.9 & 9,2 & 19.1 & 16.4 & 20.7 & 8.3 & 8.1 & 21.6 & 16.5 & 21.4 & 19.0 & 14.4 \\
\hline AE & 19 & 20 & 21 & 22 & 23 & 24 & 25 & 26 & 27 & 28 & 29 & 30 & 31 & 3224 & 33 & 34 & 35 & \\
\hline WSR & 22.1 & 12.4 & 7.4 & 23.3 & 17.0 & 21.4 & 29.0 & 9.9 & 18.4 & 24.9 & 23.6 & 21.6 & 20.9 & 13.9 & 16.0 & 13.9 & 17.4 \\
\hline
\end{tabular}

Der niedrigste Wert der Verteilung ist 7,4, der höchste 29,0, der Range folglich 21,6. Wird für die Verteilung das arithmetische Mittel berechnet, so ergibt sich ein Wert von 16,8, wobei die Standardabweichung s 5,1 beträgt. Unter Berücksichtigung des arithmetischen Mittels und der Standardabweichung wird ein Toleranzbereich festgelegt, der durch die Werte 11,7 und 21,9 begrenzt wird. Innerhalb dieses Toleranzbereiches liegen 24 Analyseeinheiten (in der Tabelle kursiv), was einem Anteil von 68,6\% entspricht. Wird dieser Wert im Sinne des Bewertungskriteriums charakteristisch interpretiert, so kann gesagt werden, daß im Korpus ke in charakteristischer Wert bzw. Wertebereich für die durchschnittliche Satzlänge ermittelbar ist. Es liegt folglich kein auf der durchschnittlichen Satzlänge beruhendes, charakteristisches Textgenerierungsmerkmal im Rahmen des untersuchten Diskurses vor.

\subsection{Das Type-Token-Verhältnis (Type-Token-Ratio: TTR)}

Aufgrund unterschiedlicher grammatikalischer Formen, die u.a. durch Deklination und Konjugation bedingt werden, ist es bei der Betrachtung des Type-Token-Verhältnisses, bei der semantische Entitäten die Analysegrundlage bilden, notwendig, eine Form, d.h. einen «kleinsten gemeinsamen Nennen der types zu finden, auf den die tokens «reduziert» bzw. zuruckgeführt werden Nomen. Adjektive und Numeralien werden in der Form des Nominativ Singular maskulinum in die Analyse einbezogen. Adverbien werden als Adjektive aufgefaßt und gleichfalls im Nominativ Singular maskulinum analysiert. Pronomen werden ebenfalls auf den Nominativ reduziert, hinsichtlich des Geschlechts und des Numerus wird unterschieden. Verben werden auf den Infinitiv reduziert. Eine reflexive Endung von Verben wird

\footnotetext{
24 Analysecinheit 32 weist eıne Besonderheıt auf. dic im vorliegenden Zusammenhang enuahnt werden soll: vermullich aufgrund eines technischen Fehlers bei der Erstellung des Beitrags ist Sat7. 11 nicht vollstándig. so daB unklar ist. wic vicle Worte er umfassen sollte Bei der Bestimmung des WSR-Werts vird con der ermittelbaren Wortan/ahl ausgegangen.
} 
berücksichtigt, wenn sie nicht dem Ausdruck des Passiv dient. Partizipien werden auf das ihnen zugrundeliegende Verb reduziert. Eine Unterscheidung der Verben hinsichtlich des Aspekts bzw. der Aktionsart kann vernachlässigt werden, wenn darauf geachtet wird, daß innerhalb einer Analyseeinheit nur ein Aspekt berücksichtigt wird.

Ob die dargestellte Reduktion, von einem grammatikalischen Standpunkt aus betrachtet, richtig ist, kann vernachlässigt werden: einerseits geht es um unterschiedliche semantische Entitäten, andererseits gilt es, eine praktikable Losung zu finden, die es erlaubt das TypeToken-Verhältnis produktiv in der vorliegenden Arbeit einzusetzen. Da auf der Grundlage des Analyseergebnisses, welches in Tabelle 11 zu sehen ist, keine qualitativen Schlußfolgenungen über Generierungsregeln des untersuchten Diskurses gezogen werden, ist eine Beurteilung der vorgeschlagenen Vorgehensweise mittels der Kriterien richtig oder falsch unangemessen, da furr alle Analyseeinheiten dieselbe Vorgehensweise gewählt wird.

Tabelle 11

\begin{tabular}{|c|c|c|c|c|c|c|c|c|c|c|c|c|c|c|c|c|c|c|}
\hline $\mathbf{A E}$ & 1 & 2 & 3 & 4 & 5 & 6 & 7 & 8 & 9 & 10 & 11 & 12 & 13 & 14 & 15 & 16 & 17 & 18 \\
\hline wes & 147 & 135 & 131 & 157 & 138 & 165 & 144 & 165 & 250 & 159 & 153 & 139 & 140 & 155 & 151 & 136 & 173 & 154 \\
\hline 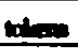 & 191 & 205 & 187 & 209 & 200 & 208 & 209 & 144 & 210 & 197 & 207 & 190 & 202 & 173 & 198 & 193 & 209 & 202 \\
\hline TIR & 0.77 & 0.66 & 0.70 & 0.75 & 0.69 & 0.79 & 0.69 & 0.85 & 0.71 & $(0,81$ & 0.74 & 0.73 & 0,69 & 0,90 & 0.76 & 0,71 & 0,83 & 0,76 \\
\hline $\mathbf{A} \mathbf{E}$ & 19 & 20 & 21 & 22 & 23 & 24 & 25 & 26 & 27 & 28 & 29 & 30 & 31 & 32 & 33 & 34 & 35 & \\
\hline na & 147 & 148 & 148 & 145 & 163 & 158 & 131 & 147 & 150 & 157 & 128 & 130 & 128 & 145 & 148 & 145 & 142 & \\
\hline ald & 199 & 199 & 201 & 186 & 204 & 193 & 203 & 188 & 202 & 199 & 165 & 194 & 167 & 195 & 208 & 195 & 191 & \\
\hline TIR & 0.71 & 0.74 & 0.74 & 0.78 & 0.8 & 0,82 & 0.65 & 0.78 & 0.74 & 0.79 & 0.78 & 0,67 & 0.77 & 0.74 & 0.71 & 0.71 & 0.74 & \\
\hline
\end{tabular}

Der Range der Verteilung beträgt 0,24. Fur das arithmetische Mittel ergibt sich ein Wert von 0,75 , für die Standardabweichung s ein Wert von 0,05 . Innerhalb eines Toleranzbereichs, der unter Berücksichtigung des arithmetischen Mittels und der Standardabweichung konstruient wird (Grenzwerte 0,7 und 0,8), liegen die Werte von 24 Analyseeinheiten (in der Tabelle kurs $)$, was cinem Anteil von $68,6 \%$ des Korpus entspricht. Analog zum WSR-Wer ist dic Streuung der Verteilung zu groß, so daß im Korpus kein Wert bzw. Wertebereich fur das Type-Token-Verhältnis diagnostizien werden kann, welcher dem Bewertungskriterium charakteristisch genügt. Es liegt in diesem Sinne kein auf dem Type-Token-Verhältnis beruhendes, charakteristisches Textgenerierungsmerkmal vor.

\subsection{Themenanalyse}

Vor der Durchführung der Themenanalyse gilt es festzulegen. welche Einheit als Analyseeinheit fungieren und welche Kategorien das Kategoriensystem umfassen soll. Im Rahmen der vorliegenden Untersuchung konnen sowohl die bisher als Analyseeinheiten bezeichneten Einheiten. als auch kleinere Einheiten wie Absätze, Sätze oder Worte als Analyseobjekt genutzt werden Vor Festlegung des Analyseobjekts soll allgemein die Frage gestellt werden, welche 
Schlusse aufgrund einer korpusbezogenen Themenanalyse auf die kulturelle Formation, in der die zu analysierenden Einheiten generiert werden, gezogen werden können. Zunächst gilt es zu bedenken, daß eine Themenanalyse durch zwei Abstraktionsprozeße gekennzeichnet ist, die einen wesentlichen Einfluß auf das Ergebnis der Analyse ausuben. Die Definition der Kategorien, die unter verschiedenen Gesichtspunkten erfolgen kann, stellt einen dieser Prozesse dar, denn je nachdem wie die Kategorien definiert werden, verändert sich das Ergebnis der Analyse. Der zweite Abstraktionsprozeß ist durch die Zuordnung der Analyseeinheiten zu den definierten Kategorien gekennzeichnet. Bei dieser Zuordnung muß von der möglichen thematischen "Breite» und "Tiefe» einer Analyseeinheit abstrahiert und das zentrale Thema der Einheit extrapoliert werden. Diese Extrapolation ist mit hoher Wahrscheinlichkeit durch subjektive Verzerrungen gekennzeichnet, die selbstredend einen Einfluß auf das Analyseergebnis besitzen.

Aufgrund der skizzierten Abstraktionsprozeße scheint das Verfahren der Themenanalyse "an sich» nur unsichere Schlüsse auf den Kontext einer Analyseeinheit, d.h. auf die generierende kulturelle Formation zuzulassen. Im Rahmen dieser Arbeit sollen die Ergebnisse der Themenanalyse dementsprechend nicht "überbewertet" werden. Die Themenanalyse soll genutzt werden, um einen annaherungsweisen Überblick über das Themenspektrum des Korpus zu erhalten. Es wird davon ausgegangen, daß das Ergebnis auf die untersuchte Formation übertragen werden kann, so daß es die in der untersuchten Formation gegebene thematische Homogenität bzw. Heterogenität widerspiegelt

Das Kategoriensystem, welches die Kategorien Musik, Tradition, Gesellschaft, Selbstreflexion. Freundschaft und Sonstiges umfaßt, wird im folgenden Abschnitt kurz erlautert.

Der Kategorie Musik wird der Themenkomplex zugeordnet, der im Zusammenhang mit Rock-Musik und deren Umfeld steht Unter der Kategorie Tradition werden Analyseeinheiten subsumier, deren Haupthema nicht-zeitgenossische Brauche. Gewohnheiten und Ereignisse sind. Analyseeinheiten. die sich mit zeitgenössischen, sozialen Gegebenheiten, Entwicklungen, Ereignissen etc. beschaftigen, werden der Kategorie Gesellschaft zugeordnet. Die Kategorie Selbstreflexion umfaßt Analyseeinheiten, die sich hauptsachlich mit der untersuchten Formation selbst beschaftigen In die Kategorie Freundschaft werden Analyseeinheiten eingeordnet, die sich auf einer personlichen Ebene mit zwischenmenschlichen Beziehungen beschaftigen Als Residualkategorie fungiert die Kategorie Sonstiges.

Aufgrund dieses Kategoriensystems wird das in Tabelle 12 dargestellte Ergebnis erzielt. 
Tabelle 12

\begin{tabular}{|l|l|c|r|}
\hline Kategorie & AE & $\Sigma$ & $\%$ \\
\hline Musik & $1.2 .3 .4 .5,6.8 .9 .11 .13 .15 .16 .17 .19 .20 .29 .35$ & 17 & 48.6 \\
\hline Gesellschaft & 10.21 .23 .27 .28 .30 .31 .32 & 8 & 22.9 \\
\hline Selbstreflexion & 12.14 .18 .22 .25 & 5 & 14.3 \\
\hline Tradition & 7.24 & 2 & 5.7 \\
\hline Sonstiges & 26.34 & 2 & 5.7 \\
\hline Freundschaft & 33 & 1 & 2.9 \\
\hline
\end{tabular}

Die am haufigsten belegte Kategorie ist die Kategorie Musik. Aufgrund des ermittelten Werts ist es dennoch nicht möglich, diesen Themenkomplex als charakteristisch für das Korpus aufzufassen. Das Korpus und somit die untersuchte kulturelle Formation sind vielmehr durch ein in mittlerem Maße homogenes und heterogenes Themenspektrum gekennzeichnet.

\subsection{Kontingenzanalyse}

Wie bereits erläutert (vgl : 3.5.2.2.), wird bei einer Kontingenzanalyse der Frage nachgegangen. welche Symbole überdurchschnittlich oft im Zusammenhang mit vorgegebenen (theoretisch relevanten) Symbolen auftreten. Aufgrund der Vieldeutigkeit des Begriffs Symbol wird im vorliegenden Zusammenhang nicht dieser, sondern der Begriff t ype verwendet. Bevor die Kontingenzanalyse durchgefuhrt werden kann. geht es in einem ersten Schritt zunächst darum. die theoretisch relevanten types zu bestimmen. Als theoretisch relevant werden die types aufgefaßt, die fur das Korpus charakteristisch sind. Da, wie Tabelle 13 zeigt, kein type dem genannten Bewertungskriterium genugt. soll die Analyse auf diejenigen types ausgedehnt werden, die mit dem Korpus in irgendeiner Form assoziiert sind Tabelle 2 (vgl.: 5.2.) verdeutlicht. daß bereits bei einem Wert von $\geq 0.2$ von einer Assoziation gesprochen werden kann, so daß der genannte Wen als Grenzwert fungieren soll. Die types, die mit einer Wahrscheinlichkeit von $\geq 0,2(20 \%)$ im Korpus nachweisbar sind. werden in diesem Sinne als relevant erachtet In einem zweiten Schritt gilt es festzulegen, was unter einem gemeinsamen Auftreten von types verstanden werden soll. Dieser Schritt zielt auf die Festlegung der Analyseeinheiten ab: als Analyseeinheiten können die bisher als Analyseeinheit verwendeten Einheiten fungieren, denkbar sind wiederum auch Absätze, Sätze u.dgl.m. Als Analyseeinheit werden die bisher verwendeten Einheiten gewählt. Ferner soll die Kontingenzanalyse auf Substantive beschränkt werden Diese Beschrankung ist genau wie die Festlegung der Analyseeinheiten in theoretischer Hinsicht weder richtig noch falsch, sie ist in diesem Rahmen notwendig. um Erkenntnisgewinn und Aufwand fur diesen Erkenntnisgewinn in ein angemessenes Verhältnis zueinander zu setzen

In Tabelle 13 sind einerseits die relevanten types aufgefuhr, andererseits ist in der Tabelle mittels Dummy-Variablen festgehalten, in welchen Analyseeinheiten die in Rede stehenden 
types nachweisbar sind. Die absolute Häufigkeit des Auftretens ist den Dummy-Variablen in eckigen Klammern nachgestellt.

Tabelle 13

\begin{tabular}{|c|c|c|c|c|c|c|c|c|c|}
\hline$A E^{\text {type }}$ & rok & god & slovo & vremja & delo & gruppa & den' & 'zisn' & $\Sigma$ \\
\hline 1 & $1[3\rfloor$ & 0 & 1 & 0 & 0 & 0 & 0 & 0 & 2 \\
\hline 2 & 0 & 0 & 0 & 0 & 0 & 1 & 0 & 0 & 1 \\
\hline 3 & $\overline{0}$ & 1 & 1121 & 0 & 0 & 0 & 0 & 0 & 2 \\
\hline 4 & 1 & 0 & 0 & 0 & 1 & 0 & 1 & 0 & 3 \\
\hline 5 & 1 & 0 & 1121 & 0 & 0 & 0 & 0 & 0 & 2 \\
\hline 6 & 1 & 1 & 0 & 0 & 0 & 1 & 1 & 0 & $t$ \\
\hline 7 & 0 & 1 & $1|2|$ & 0 & 1 & 0 & 0 & 0 & + \\
\hline 8 & 0 & $1[2]$ & $1 \mid 21$ & 0 & 0 & 0 & 0 & 0 & 2 \\
\hline 9 & $1[4]$ & $1[4]$ & 0 & $1[3]$ & 0 & 0 & 1 & 0 & 4 \\
\hline 10 & 0 & $1[2]$ & 0 & 0 & 1 & 1 & 0 & 1 & $t$ \\
\hline 11 & 0 & 1 & 0 & 0 & 0 & 0 & 0 & 0 & 1 \\
\hline 12 & 1 & 0 & 0 & 0 & 0 & 0 & 0 & $\overline{0}$ & 1 \\
\hline 13 & $11+1$ & 0 & 0 & 0 & 0 & 0 & 0 & 0 & 1 \\
\hline 14 & 0 & 1 & 0 & 0 & 0 & 0 & 0 & 0 & 1 \\
\hline 15 & 1 & 1 & 0 & 0 & 1 & 0 & 0 & 1 & 4 \\
\hline 16 & 0 & 0 & 0 & 1 & 0 & 1 & 0 & 0 & 2 \\
\hline 17 & $1[3]$ & 0 & $1[3]$ & 0 & 1 & 1 & 0 & 0 & 4 \\
\hline 18 & 0 & 0 & 1 & 0 & 0 & 0 & 0 & 1 & 2 \\
\hline 19 & 0 & 0 & 1 & 0 & 1 & 0 & $1[2]$ & 1 & 4 \\
\hline 20 & 1121 & 0 & 0 & 0 & 0 & $1[2]$ & 0 & 0 & 2 \\
\hline 21 & 0 & 0 & 0 & 0 & 0 & 0 & 1 & 0 & 1 \\
\hline 22 & 0 & 0 & 0 & 1 & 0 & 0 & 0 & 0 & 1 \\
\hline 23 & 0 & 0 & 1121 & 1 & 1 & 1 & 0 & 0 & 4 \\
\hline 24 & 1 & 1 & 0 & 1 & 0 & 1 & 1 & $I[3\rfloor$ & 6 \\
\hline 25 & 0 & 0 & 0 & $1 \mid 2\rfloor$ & 1 & 0 & 0 & 0 & 2 \\
\hline 26 & $1 \mid 21$ & 0 & 0 & 1 & 1 & 0 & 1 & 0 & 4 \\
\hline 27 & 1 & 0 & 1 & 1 & 0 & 0 & 1 & 0 & 4 \\
\hline 28 & 0 & 0 & 0 & 0 & 0 & 1 & 0 & 0 & 1 \\
\hline 29 & 1 & 0 & 0 & 1121 & 0 & 0 & 0 & 1 & 3 \\
\hline 30 & 0 & 0 & $1[3]$ & 0 & 1 & 0 & 0 & 0 & 2 \\
\hline 31 & 0 & 0 & 0 & 0 & 0 & 0 & 0 & 0 & 0 \\
\hline 32 & 0 & 1 & $1[3]$ & 1 & 0 & 0 & 0 & 1 & 4 \\
\hline 33 & 0 & 0 & 0 & 0 & 0 & 0 & 0 & 0 & 0 \\
\hline 34 & 0 & 1 & 0 & 1 & 0 & 0 & 0 & 0 & 2 \\
\hline 35 & 0 & 1 & 0 & 1 & 0 & 0 & 0 & 0 & 2 \\
\hline$\Sigma$ & $1+|26|$ & $13|18|$ & $12[23]$ & $12[16]$ & $10[10]$ & $9(10)$ & 8191 & $7[9]$ & 86 \\
\hline Assoz. & 0.4 & 0.372 & $0.3+3$ & 0.343 & 0.286 & 0.257 & 0.229 & 0.2 & \\
\hline
\end{tabular}

Bevor die Assoziation der relevanten types auf der Grundlage der Dummy-Variablen vermittels der Prozentsatzdifferenz $\mathrm{d} \%$ berechnet wird, soll Tabelle 13 betrachet werden: lediglich 8 types weisen eine Assoziation mit dem Korpus auf; kein type genugt dem eingefuhrten Bewertungskriterium; 25 Analyseeinheiten $(71,4 \%$ ) weisen mehr als ein relevantes type auf.

Gemäß des Assoziationskriteriums $\geq 0,2$ sind die folgenden types miteinander assoziiert: [rok/den']: $45,4 \%$; [den'/rok]: 33,3\%; [god/zizn']: $25 \%$; [slovo/delo] $22 \%$ und [vremja/den']: 
20,4\%. Es liegen somit eine mittlere und vier niedrige Assoziationen einzelner types im Korpus vor. Die mittlere Assoziation besagt im vorliegenden Fall, daß das type rok zu 45,4\% häufiger mit dem type den' auftritt als ohne. Wird dieses Ergebnis kritisch betrachtet, so scheint der Erkenntnisgewinn relativ gering zu sein, da das genannte Paar zudem kein charakteristisches Texgenerierungsmerkmal des untersuchten Diskurses darstellt. Darüber hinaus gilt es zu bedenken, daß das Ergebnis auf einem vergleichsweise hohen Untersuchungsaufwand basiert. Aus dem Gesagten soll der Schluß gezogen werden. daß eine Kontingenzanalyse, deren Ziel es ist, die Assoziation einzelner types zu berechnen, im Rahmen einer Diskursanalyse nicht produktiv ist. Dies bedeutet nicht, daß die Kontingenzanalyse als Analysemethode ungeeignet ist, vielmehr ist die hier vorgeschlagene Anwendung offensichtlich nicht sinnvoll. In diesem Sinne wird das erzielte Ergebnis bei der Berechnung der Homogenität bzw. Heterogenitat des Korpus, des Diskurses und der untersuchten kulturellen Formation nicht berucksichtigt

\subsection{Das Kollektivsymbol-Repertoire}

\subsubsection{Allgemeines}

Die Ermittlung von Kollektivsymbolen des russischen Interdiskurses ist Bestandteil einer umfassenden Vergleichsanalyse der deutschen, polnischen und russischen Zweiten Wirklichkeit. Im folgenden Abschnitt erfolgt eine Konzentration auf die in Rußland durchgefuihre Erhebung. Es werden in diesem Zusammenhang die Vorgehensweise der Erhebung sowie deren zeitliche und raumliche Modalitaten dargestellt, da sich hieraus eine Relativierung des Interpretationsspielraums bezogen auf das betrachtete Korpus ergibt. Die Vorgehensweise soll nicht diskutiert werden, da Fleischer ausfuhrlich auf die kritischen Punkte seiner Untersuchung hinweist (vgl. Fleischer 1997b, 75-78). Gleichfalls soll an dieser Stelle erwahnt werden, daß uber die Frequenz von Kollektivsymbolen in Texten bisher keine Daten vorliegen, so daß keine Vergleiche in dieser Hinsicht angestellt werden können.

\subsubsection{Zur Erhebung des Materials}

Die Erhebung des kollektivsymbolischen Materials, die aus drei sich erganzenden, empirischen Schritten besteht, wurde zwischen November-Dezember 1995 und Dezember 1996 vor allem in den Stadten Moskau. St. Petersburg. Novosibirsk. Tver' und Ivanovo durchgefuhrt. In zeitlicher Hinsicht ergibt sich somit keinerlei Überschneidung mit der hier betrachteten kulturellen Formation und deren Diskurs. In räumlicher Hinsicht hingegen wurde die Erhebung u.a. in den fur die Formation bedeutendsten Stadten, Moskau und St Petersburg, durchgefuhrt (vgl.: 6.3.), so daß von einer Überschneidung gesprochen werden kann. Inwiefern der raumliche und zeitliche Aspekt bezogen auf einen Interdiskurs einen Einfluß auf die Anwendung von 
Kollektivsymbolen besitzen, ist ungewiß. Um den Einfluß des zeitlichen Aspekts zu ermitteln, plant Fleischer die Wiederholung der Erhebung in einem fünjährigen Turnus. Hier wird davon ausgegangen, daß beide Variablen einen Einfluß auf die Entwicklung bzw. Ausprägung des kollektivsymbolischen Repertoires haben, so daß die zu erzielenden Ergebnisse keine Schlüsse uber die quantitative Verbreitung von Kollektivsymbolen in einer kulturellen Formation zulassen. Wie bereits weiter oben erwähnt, besteht die Erhebung aus drei Schritten, auf die im folgenden Abschnitt näher eingegangen wird.

Der erste Schritt der Erhebung dient der Ermittlung des potentiellen kollektivsymbolischen Materials. Um dieses Material zu erheben, werden Versuchspersonen $(V p n)$ aufgefordert, zwei Aufgaben zu bearbeiten. Die erste Aufgabe besteht darin, $V p n$ ihrer Meinung nach positive und negative Worte und Ausdrucke für die Gesamtheit der Menschen in Rußland nennen zu lassen:

"Napisite, pozalujsta, nize te polozitel'no ili otricatel'no okraśennye slova ili vyrazenija, kotorye po Vasemy mneniju, vażny dlja ljudej v Rossii. Po vozmoznosti nazovite kak możno bol'se slov $i$ vyrazenij, daze esli Vy ne sovsem uvereny $v$ ich vałnosti“" (Fleischer 1997b, 74).

Den Vpn werden Antwortfelder zur Verfugung gestellt, so daß zwischen positiven und negativen Nennungen differenziert werden kann

Die Grundlage der zweiten Aufgabe bilden diskursiv markierte und manipulierte Texte, die eine Vielzahl an vermuteten Kollektivsymbolen enthalten. Aufgabe der Vpn ist es, die ihrer Meinung nach für die Menschen in Rußland positiven bzw. negativen Wörter oder Ausdrücke durch Unterstreichung bzw. Durchstreichung zu markieren. Da die Vpn die Texte vermutlich aufgrund der in ihnen enthaltenen Aussagen en gros ablehnten bzw. befurworteten, nicht einzelne Elemente sondern komplette Abschnitte bzw. Texte markierten, nahm Fleischer Abstand von der Erfassung und Berucksichtigung dieses Aufgabenteils in der weiteren Untersuchung.

Der zweite Schritt zielt auf die Ermittlung der Hierarchie der im ersten Schritt ermittelten Wörter und Ausdrucke ab Grundlage des zweiten Schrittes sind drei Häufigkeitslisten, die sich aus der Auswertung des ersten Schrittes ergeben: eine allgemeine Haufigkeitsliste, eine Liste der positiven und eine der negativen Nennungen. Für den zweiten Schritt werden die häufigsten positiven und negativen Worte bzw. Ausdrücke ausgewählt, die um einige Elemente erganzt werden, die sich in der deutschen und polnischen Umfrage als markant erwiesen haben. Vermittels der folgenden Aufgabenstellung werden die $\mathrm{Vpn}$ aufgefordert die 34 ausgewählten Elemente. die alphabetisch geordnet uber drei Kolumnen verteilt prasentiert werden, zu bewerten.

„Ocenite, potalujsta, sledujuscie slova $i$ vyrazenija po skale +100 ( $\propto c^{2}$ ' $^{\prime}$ polozitel'noe slovo) do -100 (ocen' otricatel'noe slovo). Pozalujsta, stav'te ocenku spontanno, dare esli Vy ne sovsem uvereny" (Fleischer 19976, 79).

Im dritten und abschließenden Schritt soll die kulturelle Bedeutung der ausgewahiten Elemente ermittelt werden 
"Ċo, po Vasemu mneniju, oznacajut dlja ljudej $v$ Rossii sledujuscie slova i vyrazenija? Nazovite 5 slov ili vyrazenii, kotorye charakterizujut znacenie dannych slov. Resajte, poialujsta, spontanno, daze esli Vy ne sovsem uvereny" (Fleischer 1997b, 79).

Die Schritte zwei und drei zusammengefaßt, liefern die Kriterien zur Bestimmung derjenigen Wörter und Ausdrücke, die als Kollektivsymbole aufgefaßt werden: es handelt sich um Wörter bzw. Ausdrücke mit einer starken positiven bzw. negativen Färbung und einer kulturellen Bedeutung. Diesen Kriterien gemaß, handelt es sich bei den in der ersten Spaite von Tabelle 14 aufgelisteten 34 Elementen um Kollektivsymbole (vgl:: Fleischer 1997b, 97).

Tabelle 14

\begin{tabular}{|c|c|c|c|c|c|}
\hline -positiv & $\mathbf{A E}$ & $\Sigma$ & Negntion & $\mathbf{A E}$ & $\bar{\Sigma}$ \\
\hline dobrota & - & 0 & - & - & 0 \\
\hline $\operatorname{mir}$ & - & 0 & - & - & 0 \\
\hline stoboda & $27[3]$ & 1 & - & - & 0 \\
\hline dom & - & 0 & - & - & 0 \\
\hline druba & 32 & 1 & - & - & 0 \\
\hline sem ija & - & 0 & - & - & 0 \\
\hline Jubos & 3 & 1 & - & - & 0 \\
\hline dcti & - & 0 & - & - & 0 \\
\hline spravedlivost & 27 & 1 & - & - & 0 \\
\hline kul'tura & $10.17(2) 19.23 .32$ & 5 & kontrkul tura & $17(2) .24|2|$ & 2 \\
\hline cest 1 cestnost & - & 0 & nocestnost ${ }^{\circ}$ & 16 & 1 \\
\hline rodina & 21.34 & 2 & - & - & 0 \\
\hline rabota & - & 0 & - & - & 0 \\
\hline pravda & 23.24 .29 & 3 & - & - & 0 \\
\hline Rossija & 24 & 1 & - & - & 0 \\
\hline nc/avisimost & - & 0 & - & - & 0 \\
\hline Icrpimost" & - & 0 & - & - & 0 \\
\hline vera & - & 0 & - & - & 0 \\
\hline patrioti/m & 17 & 1 & - & - & 0 \\
\hline tradicija & 24 & 1 & - & - & 0 \\
\hline den'pi & 18 & 1 & - & - & 0 \\
\hline Evropa & - & 0 & - & - & 0 \\
\hline demokratija & 29.34 & 2 & - & - & 0 \\
\hline narod & $7[31.8$ & 2 & - & - & 0 \\
\hline gosudarstio & 1.23 & 2 & - & - & 0 \\
\hline kapitalism & - & 0 & - & - & 0 \\
\hline \multicolumn{6}{|l|}{-negetiv } \\
\hline kommunizm & - & 0 & - & - & 0 \\
\hline nacionalizm & - & 0 & - & - & 0 \\
\hline $10 \%$ & - & 0 & - & - & 0 \\
\hline berrabolica & - & 0 & - & - & 0 \\
\hline nenavist & - & 0 & - & - & 0 \\
\hline nasilic & - & 0 & - & - & 0 \\
\hline vojna & - & 0 & - & - & 0 \\
\hline
\end{tabular}

Tabelle 14 verdeutlicht, in welchen Analyseeinheiten die als Kollektivsymbole diagnostizierten Wörter auch in der Funktion von Kollektivsymbolen eingesetzt werden, wobei 
die in eckigen Klammern genannte Zahl die absolute Häufigkeit angibt. Ob es sich in einem konkreten Fall um ein Kollektivsymbol handelt, beruht auf einer Interpretation, die einerseits die Funktion, andererseits die Bedeutung eines Wortes an einer bestimmten Position innerhalb einer Analyseeinheit berücksichtigt.

In 15 Analyseeinheiten (42,9\%) können, Tabelle 14 folgend, 14 verschiedene bzw. insgesamt 29 Kollektivsymbole nachgewiesen werden. Die Häufigkeit der Verwendung schwankt. bezogen auf das Korpus, zwischen 0 und 5, wobei die einmalige Verwendung mit $20 \%$ nach der keinmaligen am wahrscheinlichsten ist. Am häufigsten tritt das Kollektivsymbol kul'tura auf, welches in fün verschiedenen Analyseeinheiten (14,3\%) sechsmal nachgewiesen werden kann. Wird dieses Ergebnis auf der Grundlage des Bewertungskriteriums charakteristisch interpretiert, dann wird deutlich, daß weder die allgemeine Verwendung noch die bestimmter Kollektivsymbole ein Textgenerierungsmerkmal darstellt, welches dem genannten Kriterium genügt.

Hinsichtlich der Verwendung fallt auf, daß in den Analyseeinheiten ausnahmslos Kollektivsymbole mit ihrer interdiskursiven, positiven Färbung eingesetzt werden. In bezug auf den untersuchten Diskurs kann folglich festgestellt werden, daß die ermittelten Kollektivsymbole in itrer interdiskursiven Ausprägung funktionieren. Dies bestätigt die Hypothese, daß Kollektivsymbole sowohl im Interdiskurs als auch in den jeweils untergeordneten Diskursen funktionieren (vgl: 2.8.). Die kulturelle Bedeutung der Kollektivsymbole wird offensichtlich in der untersuchten kulturellen Formation nicht verändert bzw. negiert. Darauf weist der Begriff kontrkul'tura hin, der auf der sprachlichen Ebene eine Negation des Begriffs kul'tura darstellt. Im betrachteten Diskurs weisen beide Begriffe dagegen eine positive kulturelle Bedeutung auf, sie stehen nicht in einem antagonistischen Verhältnis. Auf der kulturellen Ebene scheint der Begriff kontrkul'tura, der vermutlich ein Diskurssymbol darstellt, den Begriff kul'tura um einen Bedeutungsaspekt zu erweitern, er stellt in diesem Sinne eine Bedeutungskomponente des Konstrukts kul'tura dar.

Auch der Begriff necestnost' steht nur auf der sprachlichen Ebene mit den Begriffen cest'/ cestnost' in einem oppositionellen Verhältnis, auf der kulturellen Ebene gilt diese Negation nicht, d.h. der Begriff wird nicht positiv bewertet. Der Begriff necestnost' scheint gleichfalls eine Bedeutungskomponente der genannten Konstrukte zu sein.

Zusammenfassend kann festgestellt werden, daß die von Fleischer ermittelten Kollektivsymbole im Korpus nachweisbar sind. Am häufigsten tritt das Kollektivsymbol kul'tura auf. Die Kollektivsymbole funktionieren als Kollektivsymbole, d.h. sie werden nicht mit einer abweichenden kulturellen Bedeutung angewendet. Wörter, die Kollektivsymbole auf der sprachlichen Ebene negieren, stehen auf der kulturellen Ebene nicht in einem Negationsverhältnis zu diesen. Es liegt die Vermutung nahe, daß die Negationen im betrachteten Diskurs einen Bestandteil des jeweiligen Konstrukts darstellen 


\subsection{Das Diskursiva-Repertoire}

Das Diskursiva-Repertoire umfaßt Wörter und Ausdrücke, die im Korpus nachgewiesen werden können, die nicht bzw. nicht mit der im Korpus verwendeten Bedeutung in den folgenden literatursprachlichen Wörterbüchem aufgeführt sind:

K. Lejn (Hrsg.). 1989, Russko-nemeckij slovar' (osnovnoj). Ok. 53000 slov. 10. verbesserte und erweiterte Aufl. Moskva.

K. Lejn (Hrsg.). 1992, Nemecko-russkij (osnovnoj) slovar'. Ok. 95000 slov. Moskva

Ozegov, S. I., 1972, Slovar' russkogo jazyka. Ok. 57000 slov. 9. verbesserte und erweiterte Aufl. Moskva.

Das Repertoire besteht aus 192 verschiedenen bzw. insgesamt 227 Wörtem und Ausdrucken, die in Tabelle 15 aufgefürt sind. Die Ubersetzung orientiert sich an folgenden Werken

Drummond, D. A.; Perkins, G., 1987, Dictionary of russian obscenities. Third revised edition Oakland.

Fajn, A.; Lur'e, V, 1991, Vse v kajf. Slovar'. Moskva.

Glasnost', Michail, 1988, 100 [Hundert] schmutzige russische Wörter. Kyrillisch, Lautschrif, deutsch. Frankfurt/Main.

Marder, Stephen, 1992, A Supplementary Russian-English Dictionary. Ohio.

Fünf der in Tabelle 15 aufgeführten Wörter und Ausdrücke können unter Berücksichtigung der genannten Werke nicht übersetzt bzw. identifiziert werden; in bezug auf vier weitere können nur Vermutungen angestellt werden

Tabelle 15

\begin{tabular}{|c|c|c|}
\hline$\overline{\mathbf{A E}}$ & Diskursiva & Bedeutung \\
\hline 1 & 7 opa & Arsch \\
\hline 1 & An & cngl: an \\
\hline 1 & chip |2| & Hippie \\
\hline 1 & knt po maten & unter Veruendung des mal schimpien \\
\hline 1 & POP & Pop-Musik \\
\hline 1 & rok [1]. ROK [2] & Rock-Musik \\
\hline 1 & Underground [2] & cngl.: underground \\
\hline 2 & vrub & das Verstehen: das Kapieren \\
\hline 2 & Irubat sja [5] & verstehen: kapieren \\
\hline 3 & fan & cngl.: fan \\
\hline 3 & pogrct'sja & sich bercichern \\
\hline$\frac{3}{3}$ & svistok & Pfcife (Mensch) \\
\hline 3 & V Puskine & v Puskinskom Domc kul 'tun \\
\hline 4 & bardak & Chaos \\
\hline 4 & bills & engl. Beatles: cuphemistische Sammelbe/cıchnung fur Musikgruppen \\
\hline 4 & bljus. & Blues-Musik \\
\hline 4 & idti na /chuj| & auf den Schwan kommen, rum Teufel gehen \\
\hline
\end{tabular}




\begin{tabular}{|c|c|c|}
\hline 4 & Karma & Karma: Schicksal \\
\hline 4 & Klon & Klon: Nachahmer \\
\hline 4 & lovit' kajf [2] & SpaB haben \\
\hline 4 & ne ponjat' ni chrena & überhaupt nichts verstchen \\
\hline 4 & po-A-ski & in der Form von der Gruppe Akvanum \\
\hline 4 & poluakusticeskij & halbakustisch \\
\hline 4 & polupankoryi & zur Haliftc aus Punk-Musik bestchend \\
\hline 4 & rok & Rock-Musik \\
\hline 5 & cuvak & Kerl: Typ \\
\hline 5 & bardak & Chaos \\
\hline 5 & dogon & Hohepunkt \\
\hline 5 & igrat' mesto tol'ko y ZEKe & nur im ZEK spielen dürfen/können \\
\hline 5 & klevyj & geil \\
\hline 5 & oblom & Tiefpunkt \\
\hline 5 & rok & Rock-Musik \\
\hline 5 & roker & Rockcr, Rockmusikcr \\
\hline 6 & bodrjacek & jmd. der alt ist. aber jugendlich crscheinen mochtc \\
\hline 6 & disko & Disko-Musik \\
\hline 6 & muzon & Musik \\
\hline 6 & novaja vol'na & New-Wave-Musik \\
\hline 6 & pank & cngl.: punk \\
\hline 6 & panoptikum-songs & pejorative Sammelbezcichnung für Licder \\
\hline 6 & rok & Rock-Musik \\
\hline 6 & rok-klub & Rock-Club \\
\hline 6 & teleradiokorolevstio & Fcrnsehkonigreich \\
\hline 6 & VIA & Vokal 'nyj Instrumental'nyj Ansambl' \\
\hline 7 & delo y obrazc tcltom i blestjascem & Geld \\
\hline 7 & f fignjal & Nichts: Unsinn \\
\hline 8 & cacaca & Onomatopoesic \\
\hline 8 & Lopa & Arsch \\
\hline 8 & bugi-iugi & Boogic-Woogic-Musik \\
\hline 8 & diky & fett: voll; wild \\
\hline 8 & katit [2] & ctu /jmd ist geil \\
\hline 8 & korborskij & Coutor- \\
\hline 8 & la/a & Lügc. Schroll \\
\hline 8 & manjjak & pencerser Morder \\
\hline 8 & muson 121 & Musik \\
\hline 8 & mstyak & 10ll \\
\hline 8 & odno sestaja vscgo mira & Sorctskij Sojus \\
\hline 8 & otara & Schafherde (menschlich) \\
\hline 8 & perdenic & Fur? \\
\hline 8 & popka & Hintern \\
\hline 8 & posemu & Archaismus poctomu \\
\hline 8 & poten kivanic & Bewegung \\
\hline 8 & rilm-cnd-bljus & Rhithm-and-Blues-Musik \\
\hline 8 & rok-n-roll & Rock'n'Roll (Tan $/$ ) \\
\hline 8 & smerdjascij & stinkend \\
\hline 8 & strcmins & spannend \\
\hline 8 & Lara-para-param & Onomatopoesie \\
\hline 8 & tara-rara & Onomatopocsic \\
\hline 8 & tataro-slavjanskaja orda & dic sowjetische Jugend \\
\hline 8 & tirim-lirim & Onomatopocsic \\
\hline 8 & turum-lurum & Onomatopocsic \\
\hline 8 & uchar" & Morder: Ungeheukr \\
\hline 9 & folk- & Folk- \\
\hline 9 & KSPSnik & Mitglied b/.u. Teilnchmer des klub samodejatel noj pesn \\
\hline
\end{tabular}




\begin{tabular}{|c|c|c|}
\hline 9 & \begin{tabular}{|l|l|} 
KSP $|6|$ \\
\end{tabular} & klub samodcjatcl'noj pesni \\
\hline 9 & rok $[4]$ & Rock-Musik \\
\hline 10 & rok-klub & Rock-Club \\
\hline 11 & ENMC & unbek. \\
\hline 11 & laboratorija & Moskorskaja rok-laboratorija \\
\hline 11 & MEI & Moskorskij Energeticeskij Institut \\
\hline 11 & MELZ & unbek. \\
\hline 11 & rok-laboratorija & Moskovskaja rok-laboratorija \\
\hline 11 & starsie to ariscij $\vee$ p & hohe Parteifunktionäre der KPdSU \\
\hline 11 & V Yukorskom & vermutl.: v Zukovskom Dome kul tun \\
\hline 11 & V Izmajloro & V Izmajlovskom parke \\
\hline 11 & v Kaucukc & unbek. \\
\hline 11 & vint & Überfall: Razyia \\
\hline 12 & chard-rokovaja komanda & Hard-Rock-Gruppe \\
\hline 12 & CHMR [2] & Heavy-Metal-Rock-Musik \\
\hline 12 & HMR & Heavy-Mctal-Rock-Musik \\
\hline 12 & krutoj & toll \\
\hline 12 & metalliceskaja komanda & Healy-Metal-Gruppe \\
\hline 12 & metallosistema & Heavy-Metal-Szene \\
\hline 12 & muzkritik-simfonist & Musikkritiker für ernste Musik \\
\hline 12 & padla & Verrăter. Bastard \\
\hline 12 & ROK & Rock-Musik \\
\hline 12 & rok-turnal & Rock-Musik-Journal \\
\hline 13 & cuvak & Kerl: Typ \\
\hline 13 & ponimat' ne figa & nichts verstehen \\
\hline 13 & rok $|+|$ & Rock-Musik \\
\hline 13 & siganui $v$ kojkx & ins Bett springen/Sex haben \\
\hline 14 & bespredel & $\begin{array}{l}\text {.1) bez:zakonie: 2) clo-l. soversaemoc bey mery: 3) bol'scaja tolpa } \\
\text { molodezi" (Fajn: Lur' c 1991, 94). }\end{array}$ \\
\hline 14 & britogolonyj & Skinhead \\
\hline 14 & chunvcjbing & Schut/gclderpresser \\
\hline 14 & getbesnyj & KGB- \\
\hline 14 & gopmecstio & Pack: Abschaum \\
\hline 14 & lycek & Spit/el \\
\hline 14 & mctallurgija & Heavy-Mctal-S/cnc \\
\hline 14 & molozaryj & jugendlich \\
\hline 14 & musornyj- & Polizci-: Bullen- \\
\hline 14 & paryugend & junge Plonicre \\
\hline 14 & partkassa & Pancikassc \\
\hline 15 & dokument NN & vermutl: Gchcimdokument \\
\hline 15 & klciyj & geil \\
\hline 15 & rok & Rock-Musik \\
\hline 15 & Rok-fedcracii [2] & Rock-Föderation: Vereinigung der Rock-Clubs \\
\hline 15 & rok-knjai & Rock-Fürst: führende Persónlichkeit in der Rock-Musik-S \\
\hline 15 & rokovij 2 | & - \\
\hline 16 & Stat & Soodinennyc Stats Ameriki \\
\hline 16 & cuvak & Kerl: Typ \\
\hline 16 & L.A. & Los Angeles \\
\hline 17 & cems-lo gros cena & etw. hat keine Bodeutung \\
\hline 17 & filarmo-metallist & Anhänger ciner spe/ischen Stilrichtung der Heasy-Metal-Musk \\
\hline 17 & ne machal posle drakı kulakami & $\begin{array}{l}\text { nicht nach dem Kampf mit den Făusten schwingen: was vorbe: ist. } \\
\text { ist vorbei. es kann daran nichts mehr geänden werden }\end{array}$ \\
\hline 17 & Post-rok & Post-Rock-Musik \\
\hline 17 & rok & Rock-Musik \\
\hline 17 & rok- & Rock-Musik- \\
\hline 17 & sorok & Sorctskij Sojus \\
\hline
\end{tabular}




\begin{tabular}{|c|c|c|}
\hline 18 & andergraund & engl:: underground \\
\hline 18 & byt'v kur'se & Bescheid wissen; Ahnung haben \\
\hline 18 & dzentl menskjj nabor & Gundausstattung \\
\hline 18 & ded & engl: dead \\
\hline 18 & draiv [2] & engl : drive \\
\hline 18 & fanorskij & engl.: fan \\
\hline 18 & liud & Neologismus: Singular von ljudi \\
\hline 18 & podnadocst & gelangueilt werden/scin \\
\hline 18 & popsa & Popmusikfan \\
\hline 18 & rablcrianskij & unbek. \\
\hline 18 & rokcnrol'nyj & Rock'n'Roll-Musik- \\
\hline 18 & rokenroll & Rock'n'Roll-Musik \\
\hline 18 & samisdatovskij & Selbstverlag- \\
\hline 18 & ranadvorskaja komada & Hinterhofkapcllc \\
\hline 18 & zatasict'sja y pol'nyj rost & Spaß haben bis zum Umfallen \\
\hline 19 & Ldat' svocyo casa & auf seine Stunde brw. Chance warten \\
\hline 19 & andergraund & engl.: underground \\
\hline 19 & chaljavnyj kser & billiges Gericht \\
\hline 19 & KLF & klub ljubitclcj fantastiki \\
\hline 19 & posemu & Archaismus: poctomu \\
\hline 19 & prikolot'sja po tizni & SpaB/Lust am Leben bekommen \\
\hline 19 & rok-dvizenic & Rock-Musik-Beucgung \\
\hline 19 & rok-samizdat & Publikationen uber Rock-Musik, die im Selbstverlag entstehen \\
\hline 19 & rokenrol inyi & Rock'n'Roll-Musik- \\
\hline 19 & vernut sja is jador. & von der Armee rurückkchren \\
\hline 20 & alisoman & Anhänger der Gruppe Alisa \\
\hline 20 & rastafarianskij & Rastafari- \\
\hline 20 & rastamanskij & Rastafari- \\
\hline 20 & rok $|2|$ & Rock-Musik \\
\hline 20 & rok-poctija |3] & Rock-Poesic \\
\hline 20 & stado & Herde (menschlieh) \\
\hline 21 & Scerbakor & vcrmull Socrbakonskaja ulica \\
\hline 21 & sc10 [2] & Onomatopocsic: cto \\
\hline 21 & sctob & Onomatopoesic: ctoby \\
\hline 21 & Alcksecvskaja & icrmutl Alekseevskaja ulica \\
\hline 21 & chocist & Onomatopoesic: 2. Pers. Sg. Pras. von chotet \\
\hline 21 & kalcjooskopina & Fotoapparai \\
\hline 21 & kirovex & Schüler der Kiron skoc loennoc ucilisce \\
\hline 21 & palatocnik & Kioskbesituer \\
\hline 21 & sluscij & Onomatopoesic: Imp von slusat \\
\hline 21 & svoloc & Bastard: Arschloch \\
\hline 21 & tibe & Onomatopoesic: Dat. Sg. von ts \\
\hline 22 & bloknot & Kritiker \\
\hline 22 & jankki poganyc & Dreckskcrlc \\
\hline 22 & lejka & Pcrson. dic Popmusik macht \\
\hline 22 & ment & Polizist: Bulle \\
\hline 22 & molodeska & Jugendzcitschrift \\
\hline 22 & nakatannaja kolcja & der ausgetretene/einfache Wcg \\
\hline 22 & prosny & verstockt \\
\hline 22 & N-skij & unbck. \\
\hline 22 & rok-scen3 & Rock-Musik-Srene \\
\hline 22 & sovgavanskjj & Sovet-Havanna- \\
\hline 22 & sormcdifa & sor etskaja medija \\
\hline 22 & tusonscik & Person. dic an als tusorka be/cichneten Treffen teilnimmt \\
\hline 23 & kol & Archaismus: kotory \\
\hline 24 & dcrimo & Dreck: Scheißc \\
\hline
\end{tabular}




\begin{tabular}{|c|c|c|}
\hline 24 & cdakij & Archaismus: nekji \\
\hline 24 & rok & Rock-Musik \\
\hline 25 & andergraund & cngl.: underground \\
\hline 25 & drais & engl : drive \\
\hline 25 & kaif & Spaß \\
\hline 25 & littalant & literaturnyj talant \\
\hline 25 & rok-kritika & Rock-Musik-Kritik \\
\hline 25 & rok-n-roll & Rock'n'Roll (Tanz) \\
\hline 25 & rok-pressa [2] & Rock-Musik-Pressc \\
\hline 25 & tusovka [2] & $v g l .: 4.6$. \\
\hline 26 & CRU & $\begin{array}{l}\text { Central'noe Raxiedyatel'noe Upravlenie [Central Intelligence } \\
\text { Agency: CIA] }\end{array}$ \\
\hline 26 & rok [2] & Rock-Musik \\
\hline 26 & rok-muakant & Rock-Musiker \\
\hline 26 & sortira & Klo: ScheiBhaus \\
\hline 27 & fan & cngl: fan \\
\hline 27 & rok & Rock-Musik \\
\hline 27 & rok-zumal & Rock-Musik-Journal \\
\hline 27 & sejsen & cngl.: session \\
\hline 27 & skurvit'sja & verschwinden: krimincll sein \\
\hline 28 & dzenll'menskij nabor & Grundausstattung \\
\hline 28 & goluboj & schwul \\
\hline 28 & gomosck & gomoseksualist \\
\hline 28 & putana & Prostituicre \\
\hline 29 & rok-kul'tura & Rock-Musik-Kultur \\
\hline 29 & rok/Rok [2] & Rock-Musik \\
\hline 30 & sontira & Klo: SchciBhaus \\
\hline 33 & chm 121 & Onomatopoesie \\
\hline 33 & mod & modicinskij institul \\
\hline 34 & siza & \begin{tabular}{|l|} 
Wahnsinn \\
\end{tabular} \\
\hline 34 & be/alabernost & Schlamperei \\
\hline 34 & grafiti & engl: grafiniti \\
\hline 34 & las at $^{\circ}$ & verraten \\
\hline 34 & mctalliccskaja komanda & Hcavy-Mctal-Gruppe \\
\hline 34 & ony & Archaismus: clo \\
\hline 34 & olbuvat pocetnyju pov innost' & einc Haftstrafe absilzen \\
\hline 34 & pisat & pinkeln: pissen \\
\hline 34 & raspizdjajstio & Abgespanntheil \\
\hline 34 & rcka & Gefangener \\
\hline 35 & florofag & fanatisch in berug auf Natur \\
\hline 35 & nakny'sja zdorovennoj vaginoj & in eine riesıge Vagina verschwinden: miBlingen \\
\hline 35 & ony & Archaismus: cto \\
\hline 35 & rok-i/danie & Rock-Musik-Ausgabed-Zeitschrift \\
\hline 35 & rokofil & fanatisch in berug auf Rock-Musik \\
\hline 35 & scjsyn & engl: scssion \\
\hline
\end{tabular}

In der folgenden Besprechung des Analyseergebnisses in bezug auf nicht-interdiskursive lexikalische Entitäten soll der sprachwissenschaftliche Aspekt, hinsichtlich dessen das vorliegende Repertoire betrachtet werden kann, nicht im Vordergrund stehen. Lediglich die Verwendung von Anglizismen, die, wie erläutert (vgl.: 5.4. und 5.5.), einen großen Anteil am Repertoire des molodeznyj zargon ausmachen, wird detaillierter betrachtet. Im Mittelpunkt des Interesses steht der Versuch, Schlüsse über Textgenerierungsregeln im untersuchten Diskurs zu ziehen, die vermutlich auf die Markierungsfunktion der in Tabelle 15 aufgeführten Elemente 
zuruckgeführt werden können. Insofern diese Elemente neben der Markierungsfunktion weitere Funktionen aufweisen, wird auf diese in den entsprechenden Abschnitten eingegangen

In sprachwissenschaftlicher Hinsicht soll dennoch zunächst kurz auf einige Eigenschaften des Repertoires hingewiesen werden: alle in Abschnitt 5.4. erwähnten Phänomene, also Vulgarismen (z.B.: zopa, der'mo), onomatopoetische Ausdrucke (z.B.: cacaca), Archaismen (z.B.: posemu, onyj), Neologismen (z.B.: sovok) und Phraseologismen (z.B.: idti na ... [chuj], igrat' mesto tol'ko v ŻEKe) sind im Korpus nachweisbar. Es wirkt das Gesetz der Sprachokonomie, so daß Aussageverkürzungen (z.B.: littalant statt literaturnyj talant, med statt medicinskij institut) festgestellt werden können. Die Literatursprache wird einerseits qualitativ erweitert, indem neue Wortbedeutungen (z.B.: svistok, musornyj) generiert werden, andererseits quantitativ, indem dem vorhandenen Repertoire gänzlich neue Wörter (z.B.: britogolovyj) hinzugefügt werden. Anglizismen sind entgegen der Erwartung im untersuchten Diskurs nicht sehr frequent: lediglich 9 verschiedene bzw. insgesamt 18 dieser Elemente können ermittelt werden, was einem Anteil von 4,7\% bzw. 7,9\% entspricht. Nachgewiesen werden die angesprochenen Elemente in 10 verschiedenen Analyseeinheiten (28,6\%), so daß die Verwendung von Anglizismen nicht als charakteristisches Textgenerierungsmerkmal des untersuchten Diskurses aufgefaßt wird. Unter Berücksichtigung der Abschnitte 5.4. und 5.5. liegt auf dieser Grundlage die Vermutung nahe, daß die Verwendung von Anglizismen ein mündliches Phänomen darstellt, insofern davon ausgegangen wird, daß die untersuchte Formation mit Hilfe des molodełnyj targon kommuniziert

In bezug auf weitere Textgenerierungsregeln des betrachteten Diskurses kann festgestellt werden. daß die interessierenden Elemente in 33 Analyseeinheiten $(94,3 \%)$ nachweisbar sind, so da $B$ deren Verwendung als eine charakteristische Regel angesehen wird. Die Hauufigkeit der Verwendung schwankt in den einzelnen Analyseeinheiten zwischen 0 und 26, wobei die Verwendung von 4 und 10 Elementen mit jeweils 14,3\% bezogen auf das Korpus am wahrscheinlichsten ist. In diesem Sinne existien keine Haufigkeit, die dem eingeführten Bewertungskriterium genugt.

Mehrmals in verschiedenen Analyseeinheiten finden die folgenden Elemente Verwendung: rok/ROK (14), andergraund/Underground (4) cuvak (3), bardak (2), drajv (2), dzentl'menskij nabor (2), klevyj (2), metalliceskaja komanda (2), muzon (2), onyj (2), posemu (2), sejsen/sejsyn (2), sortira (2), rok-n-roll (2), rokenrol'nyj (2), rok-klub (2), rok-zurnal (2) und topa/liopa (2). Wie bereits in Abschnitt 9.4 festgestellt werden konnte, existieren im Korpus keine charakteristischen types, so daß auch keine charakteristischen Elemente des betrachteten Repertoires ermittelbar sind

Abschließend sollen die Schlüsse, die uber die Textgenerierungsregeln im untersuchten Diskurs gezogen werden können zusammengefaßt werden die Verwendung von Elementen des dargestellten Repertoires genugt dem genannten Bewertungskriterium. Die Verwendung 
von Anglizismen genügt diesem Kriterium nicht, gleiches gilt fur bestimmte Verwendungshäufigkeiten.

\subsection{Das Oppositionen-Repertoire}

Unter dem Begriff Opposition werden, wie erläutert (vgl.: 5.7.), sprachliche Gegensätze verstanden, die sich durch ein scheinbar semantisch diametrales Verhältnis ihrer Elemente, von denen in struktureller Hinsicht mindestens zwei erforderlich sind, auszeichnen. Oppositionen sind von kulturellen Systemen abhängig, da sie in diesen generiert werden, «natürliche» Oppositionen existieren in diesem Sinne nicht. Aufgrund dieser Tatsache unterscheiden sich kulturelle Systeme hinsichtlich ihrer Oppositionen-Repertoires. Diese Repertoires können, von einem theoretischen Standpunkt aus betrachtet, genutzt werden, um kulturelle Systeme voneinander abzugrenzen. So ist beispielsweise die Opposition muzyka vs. tekst, die in Analyseeinheit 20 nachgewiesen wird, eine, die mit hoher Wahrscheinlichkeit nicht in jedem kulturellen System generien wird. Im Gegensatz zu Antagonismen, deren kulturelle Funktion die der Ausbzw. Abgrenzung ist, ist die der Oppositionen unklar. Es wird davon ausgegangen. daß Oppositionen primär ein sprachliches Phänomen darstellen, die in kultureller Hinsicht lediglich im Zusammenhang mit anderen kulturellen Phänomenen auftreten. In sprachlich-kognitiver Hinsicht kann eine Funktion in der Vereinfachung von Zusammenhängen gesehen werden. Grundlage einer Opposition ist daher immer ein AbstraktionsprozeB. Bestimmte Zusammenhange können offensichtlich auf ein Minimum reduziert werden, so daß zum Schluß des Reduktionsprozesses nur zwei Elemente ubrigbleiben, die eine Opposition bilden. Unklar ist in diesem Zusammenhang gleichfalls, inwiefern die Verwendung bestimmter Elemente im Rahmen von Oppositionen mit der kulturellen Relevanz dieser Elemente zusammenhangt, d h. inwiefern die in Oppositionen verwendeten Elemente in kultureller Hinsicht funktionalisiert sind. Da Oppositionen als sprachliches Phanomen aufyefaßt werden, liegt die Vermutung nahe. daß die Verwendung von bestimmten Elementen im Rahmen von Oppositionen nicht zu deren kultureller Funktionalisierung beitragt Elemente, die dennoch eine kulturelle Relevanz aufweisen, erhalten diese nicht aufgrund der angesprochenen Verwendung, sie weisen diese unabhängig davon auf. Soweit einige Voruberlegungen in bezug auf die Auswertung des Analysergebnisses, welches in Tabelle $16 \mathrm{zu}$ sehen ist

Tabelle 16

\begin{tabular}{|c|c|c|c|c|}
\hline $\mathbf{A E}$ & \multicolumn{3}{|r|}{ Opposition } & \multirow{2}{*}{\begin{tabular}{|l|} 
Konstruktion \\
Rok - nc iskusstio. ne nciskusstio.
\end{tabular}} \\
\hline 1 & Iskusstio & is. & nciskusstio & \\
\hline 3 & pecalen & is. & dobryj & $\begin{array}{l}\text { Chot' ischod etogo sobytija i pecalcn. no vse zc ono } \\
\text { moret ostanit' v pampat dobryc vospominanija i ulytku. }\end{array}$ \\
\hline 3 & noiyj & is & stanj & $\begin{array}{l}\text { "Znas, Y Puskine bilety budut novy | I Po stanym } \\
\text { nikogo ne pustjatl }\end{array}$ \\
\hline 3 & Icgal nyj & is. & nclcgal'nyj & $\begin{array}{l}\text { I. I pro scbja prikidwaju: lcgal mych biletor - stuk trista. } \\
\text { nclcgal 'mch - } 3(x)|\ldots| \text {. }\end{array}$ \\
\hline
\end{tabular}




\begin{tabular}{|c|c|c|c|c|c|c|}
\hline 5 & dogon & is. & oblom & & & Kak svjazany te ili inye trorceskic dogom ili oblomy $\mid . .$.$] .$ \\
\hline 6 & cernyj & vs. & belyj & & & Cernyj-belyj muzon. \\
\hline 7 & buduscec & is. & prosloe & & & 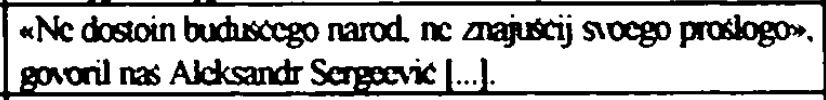 \\
\hline 9 & scjcas & is. & bylo vremja & & & $\begin{array}{l}\text { Scjcas I... I dvitenic KSP kazctsja cem-to melkim i } \\
\text { neznacitelnym. no bylo vremja. kogda ono zapolnjalo } \\
\text { iremja i prostranstio tak te. kak sejcas zapolnjaet } \\
\text { rok. }\end{array}$ \\
\hline 9 & mir & is & antimir & & & $\begin{array}{l}\text { [... I roobsce nado skarat'. cto v mire vse proischodit } \\
\text { prakticeski odnov remenno. i my s vami ne v antimire } \\
\text { zivem } \mid . . . \text {. }\end{array}$ \\
\hline 9 & v 60 e gody & is & scjcas & & & $\begin{array}{l}\text { V } 60-\text { gody KSP bylo takim ze "podzemnym", kak } \\
\text { rok sejtas. }\end{array}$ \\
\hline 12 & stranicy & is. & Labon & & & $\begin{array}{l}\text { Kogda na cogo [ROKSI] stranicach my uvideli sokrascenic } \\
\text { CHMR (HMR). Kuda poertnoc, cem na zaborach. } \\
\end{array}$ \\
\hline 12 & nedavno & is & scgodnja & & & 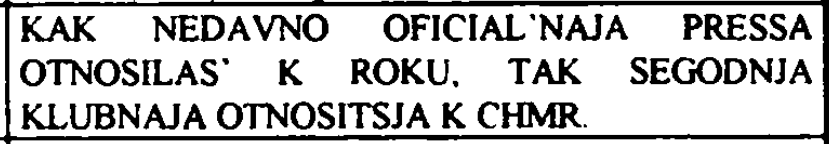 \\
\hline 13 & byto kogatato & is. & teper' & & & $\begin{array}{l}\begin{array}{l}\text { Byla kogda-to tetja Miansarova } \\
\text { pojavilsja rok. }\end{array} \\
\text { pol a teper' vol } \\
\end{array}$ \\
\hline 13 & modnyj & is & nemodnyj & & & $\begin{array}{l}\text { Chotja psichom sejcas byt modno. No ja tore } \\
\text { nemodmj... }\end{array}$ \\
\hline 17 & mertvjaki & is & rive & & & Ne nado ni priparok mertvjakami. ni medalej minym |... L \\
\hline 17 & mertvjaki & is & mertive & & & Mertvjaki i mertvye - protivopolozmye vesci. \\
\hline 20 & mu/yka & $\mathbf{v s}$ & tekst & & & 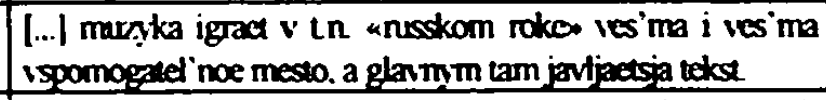 \\
\hline 23 & scgodnja & is. & vse $\operatorname{ran} \propto$ & & & $\begin{array}{l}\text { Scgodnja mnogo govoritsja i delactsja v pol zu razlicij } \\
\text { i suverennosti cego by to ni bylo. [... I Vse rance } \\
\text { podavijascesja vosstact i obnanuzivaet gigantskic } \\
\text { rasstojanija. na kotorye my udalem drug of druga. }\end{array}$ \\
\hline 23 & terminy & is. & meindy & & & Eto spor uze ne o terminach. a o metodach $[. .$.$] .$ \\
\hline 24 & zakon & is. & sorest ${ }^{\circ}$ & & & \begin{tabular}{|l|l|}
$1 \ldots$ tisni "ne po zakonu. a po sovest". \\
\end{tabular} \\
\hline 25 & nastojascox & is & prosloc & is. & buduscec & 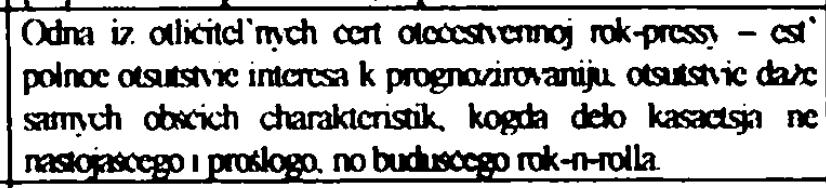 \\
\hline 25 & rostorarenno & is & orloblenno & & & 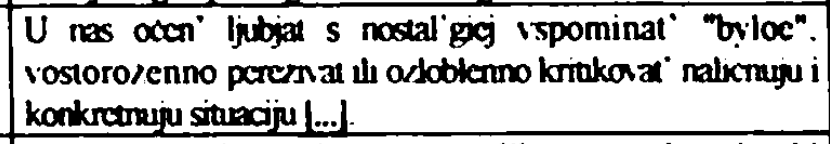 \\
\hline 25 & rosperat & is. & kritikovat" & & & 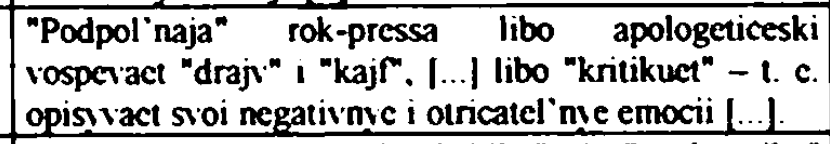 \\
\hline 25 & kritika & is & apologetika & & & $\begin{array}{l}\text { Vo vsjakom slucac i "kritika" i "apologetika" } \\
\text { morfologiceski odnotipny. }\end{array}$ \\
\hline 25 & pocherrdenix & is & otricanic & & & 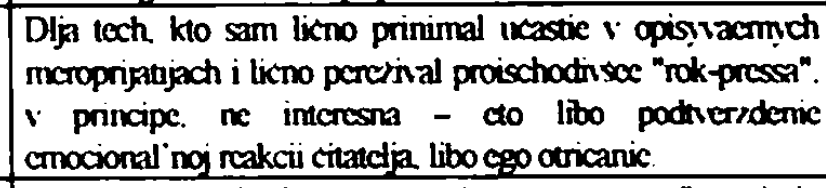 \\
\hline 25 & nravitsja & is & ne nravitsja & & & $\begin{array}{l}\text { [... I ve proischodit na prostcjsem urornc - "nravitsja } \\
\text { - ne nravitsja" }\end{array}$ \\
\hline 27 & modreodnj & vs & inutrenmyj & & & $\begin{array}{l}\text { l... menja v nej IV situaciejl zainteresoval ne } \\
\text { stol ko "merdunarodnyj". skol "ko samyj cto ni na } \\
\text { cst" "vnutrennuj" aspekil.. }\end{array}$ \\
\hline 27 & terminy & is & $\begin{array}{c}\text { politiceskic } \\
\text { jarlyki }\end{array}$ & & & $\begin{array}{l}\text { No s nekotorych por ja starajus izbegat podobmech } \\
\text { terminov |ckstremist| - ibo oni. I suscnosti. i ne } \\
\text { termini vorse. a politiceskic jarlyki. }\end{array}$ \\
\hline
\end{tabular}




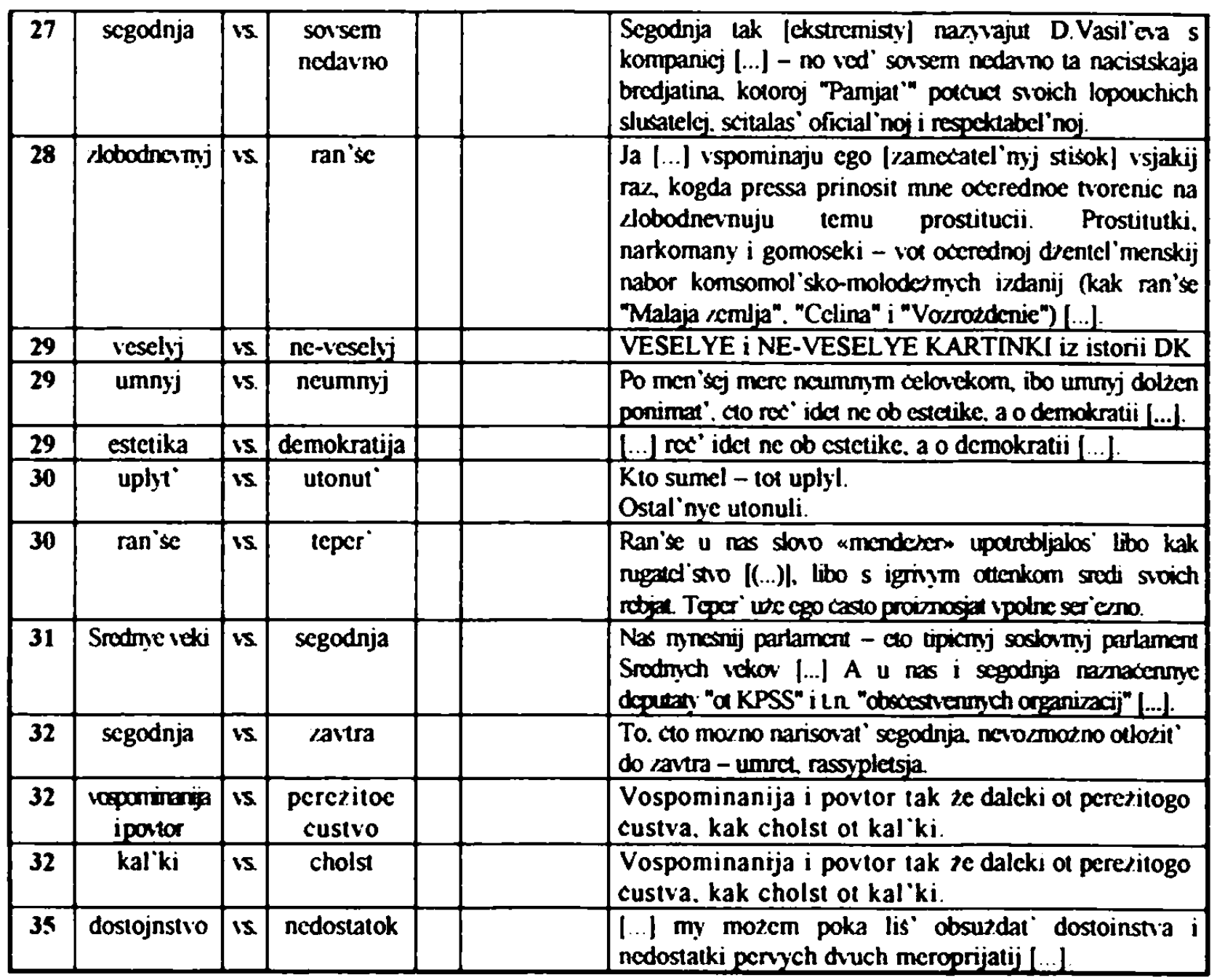

Im Korpus können insgesamt 40 Oppositionen, von denen jede jeweils nur einmal generiert wird, in 20 Analyseeinheiten (57,1\%) nachgewiesen werden. Die Verwendung von Oppositionen kann dementsprechend nicht als charakteristisches Textgenerierungsmerkmal des untersuchten Diskurses angesehen werden. Die Häufigkeit der Verwendung schwankt zwischen 0 und 6, wobei die keinmalige Verwendung mit 42,9\%, gefolgt von der einmaligen mit $25,7 \%$, am wahrscheinlichsten ist. Bei den ermittelten Oppositionen handelt es sich bis auf eine, die drei Elemente umfaßt (nastojascee, prosloe, buduscee), um Binäroppositionen. Insgesamt finden 71 verschiedene Elemente in den ermittelten Oppositionen Berücksichtigung. Von diesen können die folgenden mehrmals in verschiedenen Analyseeinheiten ermittelt werden: segodnja $(12,23,27,31,32)$, buduscee $(7,25)$, prosloe $(7,25)$, ran'se $(28,30)$, teper' $(13,30)$ und terminy $(23,27)$. Es wird deutlich, daß temporäre Oppositionen vergleichsweise häufig auftreten In insgesamt 12 Fällen (30\%) liegen diese Oppositionen vor, so daß auf diese ein besonderes Augenmerk gelegt wird. Es geht in diesem Zusammenhang vor allem um die kulturelle Bedeutung der in die temporären Oppositionen integrierten Elemente, um die Frage zu beantworten, ob bestimmte, abgeschlossene Zeitkonstrukte im Rahmen der untersuchten Formation nachweisbar sind. 
In Analyseeinheit 12 wird die temporäre Opposition nedavno vs. segodnja im Rahmen eines Vergleichs eingefürt. Verglichen wird an dieser Stelle das Verhältnis der offiziellen Presse zur Rock-Musik mit dem Verhältnis der Rock-Club-Presse (klubnaja pressa) zum CHMR. Da die offizielle Presse mit hoher Wahrscheinlichkeit negativ in der untersuchten Formation bewertet wird (vgl: 8.), überträgt sich diese Bewertung vermutlich auf die Rock-Club-Presse. Beide temporären Zustände werden in dieser Hinsicht, abhängig von den Objekten mit denen sie zusammenhängen, vermutlich negativ bewertet. Die Opposition segodnja vs. vse ranee, die in Analyseeinheit 23 generiert wird, steht im Zusammenhang mit gesellschaftlichen Veranderungen. Es liegt die Vermutung nahe, daß segodnja an dieser Stelle einen positiv bewerteten Zustand bestimmt, vse ranee einen negativen. In Analyseeinheit 27 dient die Opposition segodnja vs. sovsem nedavno der Verdeutlichung der Veränderung der interdiskursiven kulturellen Bedeutung von D. Vasil'ev und dessen Partei Pamjat'. Gleichfalls wird der Zustand, der mit dem Temporalwort segondja bezeichnet wird positiv, der mit sovsem nedavno negativ bewertet. Die Opposition Srednye veki vs. segodnja dient der Charakterisierung des sowjetischen Parlaments, welches mit Standesparlamenten gleichgesetzt wird. Beide temporären Zustände besitzen im vorliegenden Zusammenhang, abhängig von ihrem Bezugsobjekt, eine negative kulturelle Bedeutung. In Analyseeinheit 32 wird die Opposition segodnja vs. zavtra ermittelt, die in temporärer Hinsicht nicht rückwärts gewandt ist, wie die bereits dargestellten Oppositionen. Das Temporalwort segodnja wird in diesem Zusammenhang mit hoher Wahrscheinlichkeit positiv, zavtra negativ bewertet. Die Opposition buduscee vs. prosloe ist Bestandteil einer Sentenz, die auf Aleksandr Puskin zurückgeht (vgl.: 9.11.). Beide Temporalwörter werden in diesem Zusammenhang auf narod bezogen. Puskin betont an dieser Stelle die Bedeutung der Vergangenheit für die Zukunft eines Volkes, so daß die Zukunft in diesem Sinne von der Vergangenheit abhängig ist. Beide temporären Zustände werden als bedeutsam angesehen, so daß jeweils von einer positiven kulturellen Bedeutung ausgegangen wird. In Analyseeinheit 25 wird die temporale Bestimmung buduscee im Rahmen einer dreigliedrigen Opposition den Bestimmungen nastojascee und prosloe gegenübergestellt. An der angesprochenen Stelle wird der Mangel an zukunftsorientierter Berichterstattung der vaterlandischen Rock-Presse in bezug auf Rock-Musik kritisiert. Durch diesen Mangel erhalt buduscee eine besondere Bedeutung. Bis auf diese Diagnose kann auf die kulturelle Bedeutung der gegenübergestellten Elemente nicht geschlossen werden. Die Opposition zlobodnevnyj vs. ran'se dient der Verdeutlichung der Veränderungen hinsichtlich der Themenauswahl der offiziellen Jugendpresse (komsomol'sko-molodeznye izdanija). Beide Zustände werden abgelehnt, so daß im vorliegenden Zusammenhang von einer negativen kulturellen Bedeutung der genannten Temporalworter ausgegangen wird. In Analyseeinheit 30 tritt die Opposition ran'se vs. teper' auf. In diesem Fall wird ran'se positiv, teper' negativ bewertet. Die temporale Bestimmung teper' wird in Analyseeinheit 13 der Bestimmung bylo kogda-to gegenubergestellt. An dieser Stelle wird teper' positiv, bylo-kogda-to negativ bewertet. 
Weitere temporäre Oppositionen werden in Analyseeinheit 9 ermittelt: sejcas vs. bylo vremja und v 60-e gody vs. sejcas. Eine vermutlich positive kulturelle Bedeutung in bezug auf den in der genannten Analyseeinheit thematisierten KSP besitzen die temporalen Bestimungen bylo vremja und $v 60$-e gody. In bezug auf den KSP besitzt die Bestimmung sejcas eine negative, in bezug auf Rock-Musik eine positive kulturelle Bedeutung

Es wird deutlich, daß keine einheitlich kulturelle Bedeutung der ermittelten temporalen Bestimmungen nachweisbar ist. Diese Bestimmungen sind hinsichtlich ihrer kulturellen Bedeutung vielmehr von den Objekten abhängig, auf die sie bezogen werden. Es existieren in diesem Sinne in der kulturellen Formation des rok-samizdat keine abgeschlossenen temporalen Konstrukte, die beispielsweise besagen, daß der Zeitpunkt segondja immer eine positive kulturelle Bedeutung aufweist.

Neben den temporären Oppositionen soll auf weitere, ausgewählte Oppositionen eingegangen werden. Die Auswahl beschränkt sich hierbei auf solche Oppositionen, in die mit hoher Wahrscheinlichkeit entweder kulturell funktionalisierte Elemente integriert sind oder die sich auf kulturell funktionalisierte Objekte beziehen

Vermutlich weisen die drei Elemente rok, iskusstvo und neiskusstvo, die in Analyseeinheit 1 aufeinander bezogen werden, eine kulturelle Funktionalisierung und somit Relevanz auf Das Wort rok besitzt für die untersuchte Formation mit Sicherheit eine positive kulturelle Bedeutung. Möglicherweise handelt es sich sogar um ein Diskurssymbol. In bezug auf das Phänomen rok wird an dieser Stelle eine dialektische Auffassung deutlich: für die untersuchte Formation ist das Phänomen vermutlich Kunst, für das übergeordnete Kultursystem das Gegenteil, nämlich Nicht-Kunst. Die Opposition pecalen vs. dobry, die in Analyseeinheit 3 eingefuhrt wird. bezieht sich auf ein «historisches» Ereignis (vgl : 9.9.6.) Die genannte Opposition tragt in dieser Hinsicht zur Konstruktion des Ereignisses bei. In Analyseeinheit 6 bezieht sich die Opposition cernyj vs. belyj auf muzon, also Musik Das Wort myzon, welches ein Element des Diskursiva-Repertoires darstellt, besitzt vermutlich eine positive Bedeutung im Rahmen der untersuchten Formation. In Analyseeinheit 9 wird durch die Opposition mir vs. antimir ein Teil des Autokonstrukts der Mitglieder der untersuchten Formation deutlich. Die Betonung der Tatsache, daß diese nicht in einer Gegenwelt leben, kann als Hinweis darauf gewertet werden, daß diese Meinung im Rahmen der untersuchten Formation existiert bzw vorherrscht Auf die Opposition muzyka vs. tekst wurde bereits weiter oben hingewiesen: zum einen scheint die Opposition eine Abgrenzung zu anderen kulturellen Systemen zu ermöglichen, zum anderen enthält sie das vermutlich kulturell relevante Wort muzyka. Die Opposition terminy vs. metody ermöglicht vermutlich ebenfalls eine Abgrenzung kultureller Systeme. Es liegt die Vermutung nahe, daß die genannte Opposition ihren Ursprung in einem "Wissenschaftsdiskurs» hat. Trifft diese Vermutung zu, dann ist sie vermutlich nicht in allen Diskursen nachweisbar. Die Elemente zizn', zakon und sovest', die in Analyseeinheit 24 in einen Zusammenhang gestellt werden, besitzen vermutlich jeweils eine kulturelle Funktion Möglicherweise handelt es sich 
um Diskurs- bzw. Kollektivsymbole. Ebenfalls aus einem "Wissenschaftsdiskurs» stammt vermutlich die Opposition terminy vs. politiceskie jarlyki, die in diesem Sinne nicht in allen Diskursen nachweisbar sein dürfte. Die Opposition umnyj vs. neumnyj, die in Analyseeinheit 29 auf Personen bezogen wird, dient, wie in Abschnitt 9.12.1 gezeigt wird, dem Aufbau eines Antagonismus. Die in Analyseeinheit 29 gegenübergestellten Begriffe estetika und demokratija besitzen mit hoher Wahrscheinlichkeit ebenfalls eine diskurs- bzw. kollektivsymbolische Funktion.

Wird das Gesagte hinsichtlich der kulturellen Funktion von Oppositionen zusammengefaßt, dann wird deutlich, daß keine einheitliche Funktion zu erkennen ist, da sie nicht zwangsläufig mit kulturellen Phänomenen in Zusammenhang stehen. Oppositionen stellen in diesem Sinne ein sprachliches Phänomen dar, welches in kultureller Hinsicht funktionalisiert werden kann, jedoch nicht funktionalisiert werden muß.

\subsection{Die Erzählperspektive}

Im folgenden Abschnitt geht es um die Frage, ob eine der in Abschnitt 5.8. eingeführten Erzählperspektiven ein Textgenerierungsmerkmal im untersuchten Diskurs darstellt, welches dem eingeführten Bewertungskriterium genügt. Um diese Frage zu beantworten, ist in Tabelle 17 dargestellt, in welchen Analyseeinheiten die persönliche Erzählperspektive (1) und in welchen die unpersönliche $(0)$ verwendet wird.

Tabelle 17

\begin{tabular}{|c|c|c|c|c|c|c|c|c|c|c|c|c|c|c|c|c|c|c|}
\hline AE & 1 & 2 & 3 & 4 & 5 & 6 & 7 & 8 & 9 & 10 & 11 & 12 & 13 & 14 & 15 & 16 & 17 & 18 \\
\hline EP & 1 & 0 & 1 & 1 & 1 & 1 & 1 & 1 & 0 & 1 & 1 & 1 & 1 & 0 & 1 & 1 & 1 & 1 \\
\hline AE & 19 & 20 & 21 & 22 & 23 & 24 & 25 & 26 & 27 & 28 & 29 & 30 & 31 & 32 & 33 & 34 & 35 & \\
\hline EP & 1 & 1 & 1 & 1 & 1 & 1 & 1 & 1 & 1 & 1 & 1 & 1 & 1 & 0 & 1 & 0 & 1 \\
\hline
\end{tabular}

Es wird deutlich, daß die personliche Perspektive im rok-samizdat-Diskurs präferiert wird, da diese in 30 Analyseeinheiten $(85,7 \%$ ) nachweisbar ist. Die Verwendung dieser Perspektive kann dementsprechend als charakteristisches Textgenerierungsmerkmal des rok-samizdatDiskurses aufgefaßt werden. Des weiteren soll der Frage nachgegangen werden, inwiefern die Insiderperspektive, die auf der Grundlage der persönlichen Erzählperspektive generiert wird, als Manipulationsverfahren in den vorliegenden Analyseeinheiten eingesetzt wird. Tabelle 18 zeigt, in welchen Analyseeinheiten das Insiderkonstrukt generiert wird. 
Tabelle 18

\begin{tabular}{|c|c|c|}
\hline $\mathbf{A E}$ & Signal & Proposition \\
\hline 9 & $\begin{array}{l}\text { pravil noc bylo by } \\
\text { scitat }\end{array}$ & $\begin{array}{l}\text { Ljubitcli KSP yvodjat svoju rodoslovnuju iz gorodskogo romansa XIX veka. } \\
\text { odnako pravil nee bỵlo by scitat vremenem ich rosdenija } 60 \text {-e gody nascgo veka } \\
\text { l...l. }\end{array}$ \\
\hline 11 & dejstvitel'no & $\begin{array}{l}\text { I... I ncuzeli nas dejstvitel no ostavili v pokoc? Neuzeli nas perestali } \\
\text { zagonjat' v rjady stroitelej svetlogo buduscego. i my, nakonec. mozem } \\
\text { spokojno. mirno sosuscestvovat'? }\end{array}$ \\
\hline 12 & kstati & $\begin{array}{l}\text { Kstati. klubmyc tumalisty vnacale otkazalis ego } \\
\text { prokommentirovat. }\end{array}$ \\
\hline 24 & fakticeski & Fakticeski nasc Otocestvo vot uze sest' stoletij javljaetsja Stranoj Cudes. \\
\hline 29 & iprocem & $\begin{array}{l}\text { Vprocem. v poslednee vremja situacija s rok-muzykoj v SSSR (ne tol'ko } \\
\text { idcologiceskaja. no prezde vsego ekonomiceskaja i pravovaja) nacinact } \\
\text { menjat'sja. }\end{array}$ \\
\hline 30 & dejstvitel no & $\begin{array}{l}\text { Esli to. o cem ty pytaes'sja napisat'. tebja dejstvitel 'no donimact, to navemjaka } \\
\text { eto donimaet ne tol ko tebja. i kto nibud ure vyrazil cto v suscestienno bolee } \\
\text { korotkoj i suscestienno bolec vysokochudotestvennoj formc. }\end{array}$ \\
\hline 30 & na samom dcle & $\begin{array}{l}\text { Poetomy. poka vse eto abrodit» } u \text { tebja } v \text { golove. tam navernjaka vsplyiet i } \\
\text { lagolovok. kotoryj na samom dele uze davno suscestryet i ne } v \text { edinstiennom } \\
\text { variante. }\end{array}$ \\
\hline 31 & iprocem & 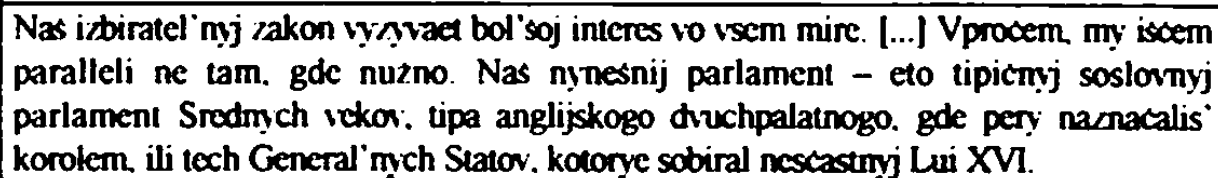 \\
\hline
\end{tabular}

In sieben Analyseeinheiten (20\%) generieren die Verfasser mit Hilfe von 8 Propositionen das Konstrukt des Insiders. Dementsprechend stellt die Insiderperspektive kein charakteristisches Textgenerienungsmerkmal des untersuchten Diskurses dar. Das Insiderkonstrukt ist nicht von bestimmten Verfassern oder Publikationen abhängig, da es von fünf verschiedenen Autoren in vier unterschiedlichen Publikationen generiert wird (vgl.: 7.3.). Auf die jeweilige Proposition in der das Konstrukt generiert wird, soll nicht eingegangen werden, da das Konstrukt mit hoher Wahrscheinlichkeit fur den gesamten Text generiert wird Vielmehr soll betrachtet werden, in welchen Themenbereichen das angesprochene Konstrukt funktionient. Hierzu kann auf das Ergebnis der Themenanalyse zuruckgegriffen werden (vgl. 9.3.). Lediglich in der Residualkategorie und in der Kategorie Freundschaft ist die Insiderperspektive nicht nachweisbar. Das Insiderkonstrukt findet sich dreimal in der Kategorie Musik. zweimal in der Kategorie Gesellschaft und jeweils einmal in den Kategorien Selbstreflexion und Tradition. Bezogen auf das Korpus und die kulturelle Formation funktioniert die Insiderperspektive folglich in nahezu allen thematischen Bereichen.

In bezug auf die aufgeworfenen Fragen soll zusammenfassend festgehalten werden, daß die personliche Erzählperspektive dem Bewertungskriterium charakteristisch genügt Im Rahmen dieser Perspektive wird das Konstrukt des Insiders nahezu themenunabhangig generiert, es stellt dennoch kein charakteristisches Textgenerierungsmerkmal dar. 


\subsection{Indices}

\subsubsection{Allgemeines}

Der Begriff Index ist auf der semantischen Ebene sehr weitläufig, da unter ihm unterschiedliches Wortmaterial, d.h. unterschiedliche Index-Typen subsumiert werden: es wurde bereits darauf hingewiesen, daß es sich bei Indices einerseits um onomastisches Material, andererseits um «historische» Ereignisse handelt (vgl.: 5.6.). Um die Analyseergebnisse bezüglich der Verwendung von Indices im rok-samizdat-Diskurs produktiv interpretieren zu können, ist es angebracht, daß ermittelte Material zu kategorisieren. Eine Kategorisierung erleichtert u.a. die Beantwortung der Frage, inwiefern die Mitglieder der untersuchten Formation «über den Tellerrand schauen» bzw. inwiefern eine Introspektive, d.h., eine Konzentration auf die eigene Formation bzw. das übergeordnete Kultursystem vorliegt. Es werden die folgenden Kategorien gebildet: Personen, Gruppen, Institutionen und Geographica, Marken, Ereignisse sowie eine Residualkategorie Sonstige.

In die Kategorie Personen werden Aktanten eingeordnet, die namentlich, d.h. konkret benannt werden bzw. deren Eigennamen in eindeutiger Weise aus dem Kontext erschlossen werden können. Da Indices Konstrukte darstellen, ist es irrelevant ob ein «reales» Äquivalent existiert. In bezug auf Personen-Indices sollen verschiedene Komplexitätsgrade unterschieden werden, die sich auf den Wiedererkennungswert des Aktanten beziehen. Ein Personenname setzt sich, bezogen auf das russische Kultursystem, aus drei Elementen zusammen: dem Vornamen (imja), dem Vatersnamen (otcestvo) und dem Familiennamen (familija). Der Vatersname soll hier aus den Uberlegungen ausgeklammert und als Bestandteil des Kontexts aufgefaßt werden. Am relevantesten für den Wiedererkennungswert eines Aktanten scheint der Familienname zu sein. Wird der Familienname um den Vornamen ergänzt, so erhöht sich der Wiedererkennungswert, so daß mit der Kombination Vor- und Familiennamme der höchste Komplexitätsgrad vorliegt. Für diese Kombination sowie einen alleinstehenden Nachnamen wird der Begriff spezifische Personen-Indices vorgeschlagen. Alle anderen Formen sollen als weniger komplex aufgefaßt und mit dem Begriff unspezifische PersonenIndices bezeichnet werden. Die Unterscheidung zwischen spezifischen und unspezifischen Personen-Indices scheint in kultureller Hinsicht produktiv zu sein, da diese Indices mit hoher Wahrscheinlichkeit in unterschiedlicher Weise funktionalisiert werden.

In bezug auf die Kategorie Gruppe gilt es zunächst zu klären, was unter dem Begriff Gruppe verstanden wird. Der Begriff bezeichnet hier einerseits Gemeinschaften, die sich aus mindestens zwei Aktanten zusammensetzen, andererseits involviert er Pars-pro-totoRelationen. Es gilt zu klären, welche Gruppenbezeichnungen eine indexikalische Funktion ubernehmen können. Eine Gemeinschaft muß in dieser Hinsicht eine konkrete, kurze Bezeichnung aufweisen, die einen hohen Wiedererkennungswert garantiert. In diesem Sinne 
sollen ad hoc gebildete Gemeinschaften, die nur in einem bestimmten Moment eine Gemeinschaft darstellen (z.B.: auditorija, citateli), nicht als Index aufgefaßt werden. Weiterhin kann als Kriterium angesehen werden, daß eine Gemeinschaft stereotypisierbar sein muß. Es muß in diesem Sinne möglich sein, der Gemeinschaft neben dem Klassifizienungsmerkmal ein weiteres Merkmal zuzuschreiben. Es liegen somit zwei Kriterien vor (1. nicht ad hoc gebildete Gemeinschaft und 2. Stereotypisierbarkeit), die es erlauben Gruppen-Indices zu bestimmen. In diesem Zusammenhang wird deutlich, daß die Bestimmung von Gruppen-Indices auf einer Interpretation basiert, da das zweite Kriterium allein auf dem Wissen bzw. der Intuition des Untersuchenden basiert. Es ist bisher nicht gelungen, ein «objektiveres» Kriterium zur Bestimmung von Gruppen-Indices zu definieren. Im Rahmen der erläuterten Kategorie kann gleichfalls zwischen spezifischen und unspezifischen Indices differenziert werden. Als spezifisch sollen diejenigen aufgefaßt werden, die einen «personalen Charakten aufweisen, deren Mitglieder in diesem Sinne theoretisch namentlich ermittelbar sind. Als unspezifisch können im Gegensatz dazu diejenigen Indices aufgefaßt werden, deren einzelne Mitglieder nicht namentlich ermittelbar sind. Diese Differenzierung scheint, gleichfalls wie die bei den Personen-Indices vorgeschlagene, produktiv zu sein, da spezifische Gruppen-Indices vermutlich andere kulturelle Funktionen übernehmen, als unspezifische.

In die Kategorie Institutionen und Geographica werden einerseits Namen von Orten, andererseits Bezeichnungen von Organisationen eingeordnet. Unter dem Begriff Geographica werden Ortsbezeichnungen, d.h. Namen von Ländern, Städten, Plätzen, Straßen etc. verstanden. Der Begriff Institution umfaßt hingegen das, was allgemein unter dem Begriff öffentliche Einrichtung verstanden wird. Da in der vorliegenden Untersuchung von einem Uberschneidungsbereich der genannten Index-Typen ausgegangen wird, wird eine Kategorie gebildet, die beide Typen umfaßt: die Bezeichnung Puskinskij Dom kul'tury beispielsweise steht einerseits furr einen Ort. genauer fur einen Veranstaltungsort, andererseits verbirgt sich hinter der Bezeichnung eine offentliche Einrichtung, die den Veranstaltungsort leitet bzw betreibt. Zwischen beiden sozialen Funktionen kann ohne weiteres nicht explizit differenzien werden, so daß sich eine beide Funktionen berücksichtigende, abstrahierende Kategorie anbietet. In diese werden daruber hinaus nur diejenigen Indices eingegliedert, die «unmittelban auf eine Institution bzw. ein Geographicum hinweisen. $\mathrm{Zu}$ den unmittelbaren Indices sollen Metaphem und Antonomasien gezählt werden. Aus der Betrachtung werden «mittelbare» Indices ausgeklammert, wie sie einige der den anderen Kategorie zugeordneten Indices darstellen (z.B.: evropejcy, indeecy). Es geht vermittels der hier besprochenen Kategorie u.a. darum, eine kognitive "Landkarte" einer konkreten kulturellen Formation zu rekonstruieren. Die mittelbaren Indices dienen vermutlich nicht primär der Konstruktion dieser Landkarte, sondern erfüllen 
in kultureller Hinsicht andere Funktionen ${ }^{25}$. Im Rahmen der angesprochenen Kategorie soll daruber hinaus nicht zwischen spezifischen und unspezifischen Indices unterschieden werden.

Der Kategorie Marken werden jegliche Formen von Konsumgütern zugeordnet, die eine konkrete Bezeichnung aufweisen. Der von dieser Kategorie erfaßte Objektbereich ist aufgrund der Vielzahl an Konsumgütern und Marken sehr heterogen. Im vorliegenden Rahmen werden in die Kategorie folgende Index-Typen gegliedert: Schallplatten, schrifliche Publikationen, also Zeitungen, Zeitschriften und Bücher, Lieder, Fernsehsendungen u.dgl.m. In bezug auf Musikgnuppen, deren Name gleichfalls als Marke aufgefaßt werden kann, wird die Entscheidung getroffen, diese in die Kategorie Gruppe zu ordnen.

Auf die Kategorie Ereignisse wurde bereits an anderer Stelle eingegangen (vgl.: 5.6.), so $\mathrm{da} B$ auf eine erneute Charakterisienung der Kategorie an dieser Stelle verzichtet wird.

Die Residualkategorie umfaßt diejenigen Indices, die in keine der erläuterten Kategorien geordnet werden konnen.

\subsubsection{Personen}

Die im Korpus nachgewiesenen Personen-Indices und die durch sie bezeichneten Aktanten sind in Tabelle 19 aufgelistet.

Tabelle 19

\begin{tabular}{|c|c|c|}
\hline $\mathbf{A E}$ & Index & Aktant \\
\hline 2 & Drcjms Last & James Last \\
\hline 2 & Ket Stivens & Cat Sterens \\
\hline 4 & A. Makarevic & Andrej Makarevic \\
\hline 4 & B. Grebenscikor: Grebenscikov: BG & Boris Grebenscikor [3] \\
\hline 4 & Borja & vermutl. Alcksandr Ljapin \\
\hline 4 & Djusa & Andrej Romanov \\
\hline 4 & Fagor & Alcksandr Alcksandron \\
\hline 4 & Sera & Sera Gakkel \\
\hline 5 & Gesiod & Hesiod \\
\hline 5 & Majk & Michail Naumenko [2] \\
\hline 6 & Dro Dassen: Dassen & Joc Dassen [2] \\
\hline 6 & 1. Kochanon skij & Igor' Kochanorskij \\
\hline 6 & Iysejuscic staricki-bodnackı & losif Kobzon \\
\hline 7 & Aleksandr Sergeevic & Alcksandr Puskin \\
\hline 7 & Dantes & Georges D'Anthes \\
\hline 8 & Gudzon & Gudzon \\
\hline 8 & Dorian Grej & Dorian Gray \\
\hline 9 & Bob Zimmerman & Bob Dilan \\
\hline 9 & Dulor & Alcksandr Dulor \\
\hline 9 & Okudyara & Bulat Okudzava \\
\hline 11 & GB & Boris Grebenscikor \\
\hline
\end{tabular}

25 In diesem Punkt unterscheidet sich die gewählte Vorgehensweise von der in Abschnitt 8. in berug auf dic Gruppen-Indices anglijskic zurnalısty. amerikanskic zurnalist! und japonskic numahsty gewählien. Im vorlicgenden Zusammenhang ergäbe sich bei einer "doppelicn" Katcgorisicrung cin erhöhtes MaB an Rodundan, so daß davon abgesehen wird. 


\begin{tabular}{|c|c|c|}
\hline 11 & geroima rasckara Zoscenko *Tragikomotija & geroinja raskara Zoscenko * Tragikomodiax \\
\hline 11 & Sven Gundlach & Sven Gundlach \\
\hline 11 & Zoscenko & Michail Zoscenko \\
\hline 12 & Alck Zander & Alcksandr Starcev \\
\hline 12 & O. F. (Otec Fedor) & Igor' Saparov \\
\hline 13 & Klara Ivanoina & Klara Ivanowna \\
\hline 13 & Miansarova & Tat'jana Miansarova \\
\hline 13 & Petr Semenovic & Petr Semenovic \\
\hline 13 & Russos: Demis & Demis Russos [2] \\
\hline 14 & Karl Marks & Karl Marx \\
\hline 15 & Dzordz (Anatolij Gunckij) & Anatolij Gunickij \\
\hline 16 & Ljapin & Alcksandr Ljapin \\
\hline 16 & $\begin{array}{l}\text { BORIS GREBENSHIKOW [1]: } \\
\text { Grebenscikov [1]: BG [3] }\end{array}$ & Boris Grebenscikov [5] \\
\hline 16 & Dejv Sijuan & Dave Stewan \\
\hline 17 & Sercuk & Vasyl' Sevcuk \\
\hline 17 & Bondare & Jurij Bondarev \\
\hline 17 & Egor Letor: & Egor Letor \\
\hline 17 & Iskander & Fazil' Iskander \\
\hline 17 & Kobzon & Iosif Kobzon \\
\hline 17 & kozlorskij & Alcksej Kozlov \\
\hline 17 & Mezclajtis & Eduardas Mcrclajtis \\
\hline 17 & Michalkov & Sergej Michalkov \\
\hline 18 & Belinskij & Vissarion Belinskij \\
\hline 18 & Egor & Egor \\
\hline 18 & Gur'ex & Sergej Gur'ev \\
\hline 18 & Kennedi & John F. Kennedy \\
\hline 18 & Lipa & Lipa \\
\hline 18 & Troickij & Artem Troickij \\
\hline 19 & A. Djuma-otec & Alexandre Dumas \\
\hline 19 & D. Sclivanor: & D. Sclivanon \\
\hline 19 & E. Letor & Egor letov \\
\hline 20 & Del'fin & Del'fin [Musiker der Gruppe Dra Divi/n] \\
\hline 20 & Gera & Gera [Musiker der Gruppe Dha Divizn] \\
\hline 20 & Kincer & Konstantin Kincer \\
\hline 21 & Scerbako & Dimitrij Scerbakor \\
\hline 22 & Umeskij & Umcskij \\
\hline 22 & bolscoj arab & Alcksandr Puskin \\
\hline 22 & Andrcj Burlak & Andrcj Burlak \\
\hline 22 & Rok-Advokal & Rok-Advokal \\
\hline 22 & Sasa Sokolor & Sasa Sokolor \\
\hline 24 & Gol'dman & Gol dman \\
\hline 24 & lvanov & Ivanor \\
\hline 24 & Klykov & Vjaccslav Klyko \\
\hline 24 & Krupin & Vladimir Krupin \\
\hline 24 & Sergej 7 arikor & Scrgej /arikor \\
\hline 24 & Scrgcj Radonc/skij & Sergej Radonerskij \\
\hline 26 & Alcksandr Simonor & Aleksandr Simonov \\
\hline 26 & L. Doros & L. Doros \\
\hline 27 & A. Galic & Alcksandr Galic \\
\hline 27 & Bori Zcmcov & Bori Zcmcor \\
\hline 27 & D. Vasil'cy & D. Vasil'o \\
\hline 27 & M. C. Gorbacer & Michail Gorbacer \\
\hline 27 & Troickij A. & Artem Troickıj \\
\hline 28 & Zort: Djun & Gerard Duru \\
\hline
\end{tabular}




\begin{tabular}{|c|c|c|}
\hline 29 & An. Ivanov & Anatolij Ivanov \\
\hline 29 & Proskurin & Petr Proskurin \\
\hline 29 & Tvardovskij & Aleksandr Tvardovskij \\
\hline 29 & Vladimir Kur.'min & Vladimir Kuz'min \\
\hline 30 & Baslacer: & Alcksandr Baslacer \\
\hline 31 & Christo & \begin{tabular}{|l|} 
Jesus \\
\end{tabular} \\
\hline 31 & Lui XVI & Lui XVI \\
\hline 32 & Charms & Daniil Charms $|2|$ \\
\hline 32 & Dzikija [3]: Sasa & Alcksandr Drikija $[+]$ \\
\hline 32 & Dostoevskij & Fedor Dostoevskij [2] \\
\hline 32 & Mikelandzelo & Michelangelo \\
\hline 32 & Puskin & Aleksandr Puskin \\
\hline 33 & Feliks [2] & Feliks [2] \\
\hline 33 & Inna & Inna \\
\hline 34 & Cernenko & Konstantin Cernenko \\
\hline 34 & Alckscj "Stiv" Karkacy: Stiv & Alekscj Karkaev (2) \\
\hline 35 & Sergej & Sergej \\
\hline
\end{tabular}

Im Korpus können 98 verschiedene Personen-Indices, die 85 Aktanten bezeichnen in 30 Analyseeinheiten $(85,7 \%)$ nachgewiesen werden, so daß die Verwendung der angesprochenen Indices als ein charakteristisches Textgenerierungsmerkmal des rok-samizdat-Diskurses aufge$\mathrm{faßt}$ wird. Die Häufigkeit der Verwendung verschiedener Indices schwankt zwischen 0 und 8, wobei die Verwendung von 2 Indices des angesprochenen Typs mit 25,7\% am wahrscheinlichsten ist. Diese Verwendungshäufigkeit stellt in diesem Sinne kein charakteristisches Textgenerierungsmerkmal dar. 8 verschiedene Personen-Indices (8,2\%: Egor, Feliks, Klara Ivanovna, Inna, Lada, Lipa, Petr Semenovic und Sergej) werden als unspezifisch, die verbleibenden 77 als spezifisch aufgefaßi.

16 verschiedene Aktanten (18,8\%: James Last, Cat Stevens, Joe Dassen, Georges D'Anthes, Gudzon, Dorian Gray, Bob Dylan, Demis Russos, Karl Marx, Dave Stewar, John F. Kennedy, Aleksander Duma, Gerard Duru. Jesus, Lui XVI und Michelangelo) entstammen nicht dem sowjetischen bzw. russischen Kultursystem, so daß in bezug auf Aktanten zunachst von einer Introspektive auf das sowjetische bzw. postsowjetische System gesprochen wird. Der untersuchten Formation können 23 Aktanten (27,1\%: Aleksandr Aleksandrov, Andrej Makarevic, Aleksandr Ljapin, Boris Grebenscikov, Andrej Romanov, Seva Gakkel, Michail Naumenko, Sven Gundlach, Aleksandr Starcev, Igor' Saparov, Anatolij Gunickij, Egor Letov, Artem Troickij, Sergej Gur'ev, Del'fin, Gera, Konstantin Kincev, Andrej Burlak, RokAdvokat, Sergej Zarikov, Aleksandr Baslacev, Feliks und Sergej) zugerechnet werden, so daß keine Introspektive auf die untersuchte kulturelle Formation diagnostizient wird.

Die ermittelten Indices können unter weiteren quantitativen Gesichtspunkten betrachtet werden, wie z.B. der Geschlechterverteilung, der Zugehhörigkeit der Aktanten zu bestimmten "Gruppen» (Politiker, Literaten, fiktive Personen etc.) u.dgl.m. Eine solche Betrachtung soll nicht durchgeführt werden. da sie vermutlich nur wenige Schlüsse uber die betroffene 
kulturelle Formation zuläßt. Vielmehr sollen diejenigen Aktanten, die mehrmals im Korpus genannt werden, hinsichtlich ihrer kulturellen Relevanz und Bedeutung betrachtet werden.

In jeweils drei Analyseeinheiten können die Aktanten Boris Grebenscikov $(4,11,16)$ und Aleksandr Puskin $(7,22,32)$ nachgewiesen werden.

Grebenscikov wurde bereits im Verlauf der Sistema-Darstellung erwähnt (vgl: 4.3.). Er steht im Mittelpunkt der Gruppe Akvarium (vgl.: 9.9.3.), die in Analyseeinheit 4 in positiver Weise konstruiert wird. Grebenscikov selbst wird in diesem Zusammenhang nicht attribuiert. In Analyseeinheit 11 erfolgt seine Nennung im Zusammenhang mit einem von den ljubera (vgl.: 4.7. und 9.9.3.) gestörten Konzert, wobei seine Teilnahme und die Störung von den ljubera dieses Konzert mit hoher Wahrscheinlichkeit zu einem «historischen» Ereignis machen. In Analyseeinheit 16, in der es um eine speziell fur das Ausland hergestellte Soloveröffentlichung geht, wird Grebenscikov äußerst negativ konstruiert, wie folgendes Beispiel verdeutlicht: „Chitryj on cuvak, etot BG. Chitryj i ocen' umnyj, umejuscij delat' krasivye zesty, gramotno i ubeditel'no argumentirovat' svoe povedenie (tak, cto i vozrazit' nikak nel'zja), i $v$ etom vozdvignutom im tumane postupat' tol'ko tak, kak nuzno emu odnomu. Eto prosto ne krasivo". Grebenscikov ist offensichtlich für die untersuchte kulturelle Formation relevant, da er funktionalisiert wird und eine kulturelle Bedeutung aufweist, die in Analyseeinheit 4 nicht ausführlich expliziert wird, über den Zusammenhang mit der Gruppe Akvarium aber hergeleitet werden kann. Daruber hinaus trägt er in Analyseeinheit 11 zur Konstruktion eines Ereignisses bei, was die Hypothese der kulturellen Relevanz und Bedeutung stützt. Die Bedeutungskonstruktion ist offensichtlich zum Zeitpunkt der Erstellung der beiden genannten Analyseeinheiten abgeschlossen, da keine Merkmalszuschreibung erfolgt. Zum Zeitpunkt der Erstellung von Analyseeinheit 16 hingegen befindet sich die Konstruktion in einem Prozeß, wie die ausfuhrliche Attribuienung verdeutlicht.

Puskin, der in Analyseeinheit 7 als Autorität verwendet wird, wird im Korpus nicht konstruier, so dab seine Konstruktion in der untersuchten kulturellen Formation als abgeschlossen angesehen werden kann. Da er funktionalisiert ist, besitzt er kulturelle Relvanz.

In jeweils zwei Analyseeinheiten sind die Aktanten Artem Troickij (18, 27), Egor Letov $(17,19)$ und losif Kobzon $(6,17)$ ermittelbar. Da nicht sicher ist, ob mit dem Spitznamen Borja in Analyseeinheit 4 Aleksandr Ljapin gemeint ist, soll auf ihn nicht eingegangen werden.

Troickij, der bereits im Zusammenhang mit der ersten Publikation, die der untersuchten Formation zugerechnet wird (NEW DIAMOND), enwähnt wurde (vgl: 6.4.), wird in Analyseeinheit 27 in der Funktion einer Autorität eingesetzt. Troickij wird an dieser Stelle nicht attribuiert, so daß von einer abgeschlossenen Konstruktion ausgegangen werden kann. Die ihm zugeschriebene Aussage weist in diesem Zusammenhang auf eine negative Bedeutung hin. In Analyseeinheit 18 wird er ebenfalls in negativer Weise attribuiert, so daß die Bedeutungskonstruktion zumindest zum Zeitpunkt der Erstellung von Analyseeinheit 18 nicht abgeschlossen ist. Die Funktionalisierung von Troickij weist darauf hin, daß er kulturell relevant ist. 
Egor Letov, der 1985 in Omsk die Punk-Gruppe Grazdanskaja Oborona bzw. GrOb gegründet hat (vgl: Mazin 1996), wird in Analyseeinheit 17 in der Funktion einer Autorität und in Analyseeinheit 20 äußerst positiv attribuiert. Letov besitzt in diesem Sinne eine positive kulturelle Relevanz im Rahmen der untersuchten Formation, wobei die Bedeutungskonstruktion offensichtlich nicht abgeschlossen ist.

Iosif Kobzon, ein Schlagersänger, wird in Analyseeinheit 6 nicht namentlich genannt, sondern in diskreditierender Weise eindeutig beschrieben. In Analyseeinheit 17 wird er darüber hinaus der sovkovo-oficiosnaja kul'tura zugesprochen, die in ein antagonistisches Verhältnis zur kontrkul'tura gesetzt wird. Auch Kobzon besizzt in diesem Sinne für die untersuchte kulturelle Formation Relevanz. Die kulturelle Bedeutung, die offensichtlich negativ ist, ist mit hoher Wahrscheinlichkeit vollständig konstnuiert, da Kobzon nicht attribuiert wird.

Die Aktanten, die jeweils nur einmal im Korpus nachgewiesen werden können, sollen an dieser Stelle nicht detaillierter betrachtet werden. Auf einige der funktionalisierten und somit kulturell relevanten Aktanten wird in den Abschnitten 9.10. und 9.12. eingegangen.

\subsubsection{Gruppen}

Das Analyseergebnis hinsichtlich der Ermittlung von Gruppen-Indices und durch sie bezeichnete Gemeinschaften ist in Tabelle 20 abgebildet.

Tabelle 20

\begin{tabular}{|c|c|c|}
\hline $\mathbf{A E}$ & Index & Gemeinschaft \\
\hline 1 & chip: CHIP & chippi \\
\hline 2 & Jethro Tull & Jethro Tull \\
\hline 3 & Masina Vremcni [6]: Masina [3] & Masina Vremeni $[9]$ \\
\hline 4 & $\mathbf{A}$ & Akvarium [t] \\
\hline 4 & Masina Vremeni & Masina Vremenı \\
\hline 5 & Kamaval & Karnaral |2] \\
\hline 5 & livanec-siit & livanoc-siit \\
\hline 5 & livanoc-maronit & livanec-maronit \\
\hline 5 & Moraika & Moraika [2] \\
\hline 5 & nasi roken & nasi roken \\
\hline 5 & Zoopark & Zoopark \\
\hline 6 & Akvarcli & Akvareli \\
\hline 6 & Aricl $^{*}$ & Aricl \\
\hline 6 & Golubyc moloda & Golubic moloda \\
\hline 6 & Kaskad & Kaskad \\
\hline 6 & Krasnyc sscty & Krasnyc sict \\
\hline 6 & Lira & Lira \\
\hline 6 & molodyc slusateli & molodyc slusatcli \\
\hline 6 & Oreol & Oreol \\
\hline 6 & Pojut unitan & Pojut unitan \\
\hline 6 & Ralli & Ralli \\
\hline 6 & Sinjaja s... & Sinjaja S... \\
\hline 6 & Sjabn & Sjabri \\
\hline 6 & slusatcli sredncgo vorrasta & slusateli sredncgo vorrasta \\
\hline
\end{tabular}




\begin{tabular}{|c|c|c|}
\hline 6 & Supersprint & Supersprint \\
\hline 6 & Zemlja i ncbo & Zemlia i nebo \\
\hline 6 & Zemlja. Vecter i Ogon ${ }^{\circ}$ & Earth. Wind and Fire \\
\hline 7 & nasi babuski & nasi babuski \\
\hline 7 & nasi dedy & nasi dedy \\
\hline 7 & nasi vnuki & nasi vnuki \\
\hline 7 & neandertal $a$ & ncandertal'cy \\
\hline 7 & papuasy & papuasy \\
\hline 7 & rjadovoj truacnik & jadovoj trutenik \\
\hline 8 & anglo-saksonskaja raza & anglo-saksonskaja raya \\
\hline 8 & indeocy & indeocy \\
\hline 8 & nacija & nacija \\
\hline 8 & nainme aborigeny odnoj sestoj vsego mira & die Einwohner der UdSSR \\
\hline 8 & negn & negry \\
\hline 8 & nordiceskaja raza & nordiceskaja raza \\
\hline 8 & tataro-slavjanskaja orda & dic soujetische Jugend \\
\hline 9 & KSP'̌niki & $\begin{array}{l}\text { dic Mitglieder bzw. Teilnehmer des klub } \\
\text { samodejatel noj pesni }\end{array}$ \\
\hline 9 & ljubitcli KSP & $\begin{array}{l}\text { dic Mitglieder bzw. Teilnchmer des klub } \\
\text { samodejatel noj pesni }\end{array}$ \\
\hline 9 & milicejskic narjady & milicija \\
\hline 9 & naucnaja molodcz' & naucinaja molodes: \\
\hline 9 & studenceskaja molodet & studenceskaja molodes: \\
\hline 9 & tvorceskaja moloder & tvorceskaja molodct: \\
\hline 10 & Urfin Dzjus & Urfin Dijus \\
\hline 11 & Ccment & Ccment \\
\hline 11 & ljubera & ljubera [2] \\
\hline 11 & milicija & milicija \\
\hline 11 & starsic tor arisci v plykorych sapkach & hohe Partcifunktionare der KPdSU \\
\hline 11 & stroitch svctlogo buduscego & stroitcli svetlogo buduscego \\
\hline 11 & Vecelve kartinki & Vecelyc kartinki \\
\hline 11 & Zuvki Mu & Zıuki Mu \\
\hline 12 & Akvarium & Akvarium \\
\hline 12 & klubnye rurnalisty & Journalisten der Rock-Clubs \\
\hline 14 & sturmoniki pravogo fronta & sturmoviki pravogo fronta \\
\hline 14 & agenty ochranki & agcnty ochranki \\
\hline 14 & gcbainyc glavan & KGB-Führer/Ont/lerc \\
\hline 14 & ka/anc & kazanc \\
\hline 14 & ljubcra & ljubcra [2] \\
\hline 14 & mclkij cho/jajcik & melkij cho/jajkik \\
\hline 14 & musornyc lycki & Polizcispit/cl \\
\hline 14 & olocesticnnic chumvejbiny & olocestrennyc chunvejbiny \\
\hline 14 & partjugend & partjugend \\
\hline 14 & sorestkic britogolonyc & soujetische Skinhcads \\
\hline 14 & sportivnyc fanaty 121 & sportivnse fanaty 121 \\
\hline 14 & srodnyj obr vatcl & srodny jobyvatcl \\
\hline 15 & adminustraty & administran \\
\hline 15 & muxkorcds & muxkoved \\
\hline 15 & prezidents & preridenty \\
\hline 15 & rok-knja/i & $\begin{array}{l}\text { führende Personlichkeiten in der Rock- } \\
\text { Musik-Szene }\end{array}$ \\
\hline 15 & sociologi & sociologi \\
\hline 16 & Akvarium & Akvarium \\
\hline 17 & filarmo-mctallisty & $\begin{array}{l}\text { Anhänger ciner spe/ifischen Stilrichlung } \\
\text { der Heavy-Metal-Musik }\end{array}$ \\
\hline
\end{tabular}




\begin{tabular}{|c|c|c|}
\hline 17 & Laskovyj maj & Laskouyj maj \\
\hline 17 & nasie citatelja & die Leser der Publikation KONTR KUL T UR'A \\
\hline 17 & NJUANS & Njuans \\
\hline 17 & poety & pocty \\
\hline 17 & VEZLLIVYJ OTKAZ & Veslivyj otkaz \\
\hline 18 & popsy & Popmusikfans \\
\hline 18 & PRESTUPNYE ELEMENTY & Prestupmye clementy \\
\hline 18 & STUDZZIS & Stooges \\
\hline 19 & filosofy & filosofy \\
\hline 19 & istoriki & istoriki \\
\hline 19 & roditcli & roditcli \\
\hline 20 & \begin{tabular}{|l|} 
srednestatisticeskij zritel \\
rastamanskich koncertor
\end{tabular} & $\begin{array}{l}\text { srednestatisticeskij zritel' rastamanskich } \\
\text { koncertov }\end{array}$ \\
\hline 20 & alisomany & Anhănger der Gruppe Alisa \\
\hline 20 & Dia Divizn & Dra Divitn \\
\hline 20 & rok-poety & rok-poety \\
\hline 21 & demokraty & demokraty \\
\hline 21 & kirova & Schüler der Kiroviskoe voennoe ucilisce \\
\hline 22 & bloknoty & Kritiker \\
\hline 22 & Icjki & Personen, dic Popmusik machen \\
\hline 22 & sluzascij sormodii & sluzascij sovmedii \\
\hline 23 & protivniki obnovienija & protivniki obnovlenija \\
\hline 24 & DK & DK \\
\hline 24 & nasi skol'nye ucitcli istorii & nasi skol myc ucitcli istorii \\
\hline 26 & rok-muzykanty & rok-muzikanty \\
\hline 27 & starye drü ja & starye druz'ja \\
\hline 27 & rjadoryc sturmovikı & rjadorye sturmoviki \\
\hline 28 & nasi 7orzi Djurua & rurnalisty \\
\hline 28 & golubye & Homosexuellc \\
\hline 28 & gomoseki & Homoscxuelle \\
\hline 28 & narkoman & narkomany 121 \\
\hline 28 & prostitutki |2]: prostitutka & prostitutki \\
\hline 28 & putam & prostitutki \\
\hline 29 & DK & DK \\
\hline 29 & mysljascaja cast auditorii & mysljascaja cast auditorii \\
\hline 29 & munkant & muzykanty \\
\hline 29 & OBLACNYJ KRAJ & Oblacnu kraj \\
\hline 29 & TELEVIZOR & Telcizor \\
\hline 29 & ucenye muri & ucenye mui \\
\hline 30 & menedren & menedken \\
\hline 31 & deputaty "ol KPSS" & deputaty "ol KPSS" \\
\hline 31 & deputan "ot obscestiennych organizacii & deputaty "ol obscestiennych organizacu \\
\hline 31 & evropejcy & cropeja \\
\hline 33 & bandity & bandity \\
\hline 33 & chippi & chippi [2] \\
\hline 33 & dzentl'meny & dzentl meny \\
\hline 33 & MUCHOMOR & Muchomor \\
\hline 34 & bolgarka & bolgarka \\
\hline 34 & chudorniki & chudoz niki \\
\hline 34 & momentalisty [2] & momentalist [2] \\
\hline 34 & rabocij klass & rabocij klass \\
\hline 34 & vord ja mirovogo proletariata & vozd ja mirovogo prolctariata \\
\hline 35 & Natasa Rostova & Natasa Rostova \\
\hline 35 & Usastyc Usi & Usastye Usi \\
\hline 35 & Vtorticnie lane & Vtorzemie lane \\
\hline
\end{tabular}


Innerhalb des Korpus können 120 verschiedene Gemeinschaften, die durch 128 verschiedene Indices bezeichnet werden, ermittelt werden. Diese Indices verteilen sich auf 32 Analyseeinheiten $(91,4 \%)$, so daß mit der Verwendung dieser Indices ein charakteristisches Textgenerierungsmerkmal des untersuchten Diskurses vorliegt. Die Häufigkeit der Verwendung schwankt zwischen 0 und 16, wobei die Verwendung von einem Index dieses Typs mit 22,9\% am wahrscheinlichsten ist. Es läßt sich in diesem Sinne keine charakteristische Verwendungshäufigkeit bestimmen.

Um die Frage hinsichtlich einer möglichen Introspektive zu beantworten, erfolgt eine Konzentration auf Musikgruppen, da diese die einzigen spezifischen Indices des betrachteten Typs ausmachen. Im Korpus werden 37 Musikgruppen durch 39 Indices bezeichnet. 3 dieser Gruppen (Jethro Tull, Stooges und Earth, Wind and Fire: 8,1\%) stammen nicht aus der Sowjetunion bzw. deren Nachfolgestaaten, so daß zunächst eine Introspektive auf den genannten Bereich diagnostiziert wird. Der kulturellen Formation können 18 Musikgruppen (Masina Vremeni, Akvarium, Zoopark, Urfin Dzjus, Cement, Veselye kartinki, Zvuki Mu, Njuans, Vezlivyj otkaz, Prestupnye elementy, Dła Divizn, DK, Oblacnyj kraj, Televizor, Muchomor, Natasa Rostova, Usastye Usi und Vtorołenie izvne: $48,6 \%$ ) zugerechnet werden, so daß keine Introspektive auf die untersuchte Formation vorliegt.

Wie bereits im Verlauf der Betrachtung der Personen-Indices und der durch sie bezeichneten Aktanten erwähnt ( $\mathrm{vgl}$ : 9.9.2.), können die ermittelten Indices bzw die durch sie bezeichneten Gemeinschaften unter weiteren quantitativen Gesichtspunkten betrachtet werden. Von einer solchen Betrachtung wird Abstand genommen, da sie nur wenige Erkenntnisse über die untersuchte Formation und deren Diskurs verspricht. Vielmehr sollen diejenigen Gemeinschaften hinsichtlich ihrer kulturellen Relevanz und Bedeutung betrachtet werden, die mehrmals in verschiedenen Analyseeinheiten nachweisbar sind Hierbei handelt es sich um: Akvarium (4, 12. 16). chıpp I $(1,33)$, DK $(24,29)$, ljubera $(11,14)$, Masina Vremeni $(3,4)$, und milicija $(9,11)$

Im Mittelpunkt der Gruppe Akvarium, die durch zwei Indices bezeichnet wird (Akvarium und A), steht der bereits mehrfach erwähnte Boris Grebenscikov (vgl.: 4.3. und 9.9.2.). Die Gruppe wird in der vierten Analyseeinheit ausfuhrlich positiv konstruiert. In Analyseeinheit 12 wird sie aus der Sicht eines opytnyj muzkritik-simfonist «bewertet», was zu der angesprochenen, positiven Konstruktion der Gruppe beiträgt. In Analyseeinheit 16 wird die Gruppe im Zusammenhang mit der Konstruktion von Grebenscikov erwähnt, wobei sie selbst nicht konstruiert wird. Es liegt der Schluß nahe, daß die Gruppe Akvarium in positiver Hinsicht relevant ist. In den Analyseeinheiten 4 und 12 befindet sich die Bedeutungskonstruktion in einem Prozeß, wahrend in Analyseeinheit 16 vermutlich ein abgeschlossenes Konstrukt vorliegt.

Die Gemeinschaft chippi, die durch drei Indices bezeichnet wird (chip. CHIP und chippi). wird weder in Analyseeinheit 1 noch in Analyseeinheit 33 ausführlich konstruiert. Lediglich in Analyseeinheit I wird sie in stereotypisierter Form eingeführt (vgl. 9.12.2.), was auf eine 
kulturelle Relevanz hinweist. Darüber hinaus wird die kulturelle Bedeutung der Gruppe in diesem Zusammenhang vermittels einer Anspielung auf die sowjetische Nationalhymne (Velikij i mogucij!) hervorgehoben. Da die Gruppe ansonsten nicht konstruiert wird, kann die Vermutung geäußert werden, daß die Konstruktion im Rahmen der betrachteten Formation abgeschlossen ist. Es kann weiterhin vermutet werden, daß die Gemeinschaft fü die untersuchte Formation in positiver Hinsicht relevant ist.

Der Index DK bezeichnet eine Musikgruppe, in der Sergej \%arikov spielt, der bereits im Zusammenhang mit der Publikation URLAJT erwähnt wurde (vgl.: 7.2.1.5.). Die Gruppe wird in Analyseeinheit 24 unter Verwendung des Normativs populjarnyj konstruiert, was eine kulturelle Relevanz impliziert. In Analyseeinheit 29 wird die Gruppe, abgesehen von der Überschrif, nicht genannt, so daß keine Konstruktion diagnostizierbar ist. In diesem Sinne lassen sich keine Hypothesen über die kulturelle Bedeutung der Gruppe formulieren, außer, daß sie mit hoher Wahrscheinlichkeit positiv im Rahmen der untersuchten kulturellen Formation bewertet wird.

Die Gemeinschaft der ljubera, die bereits im Verlaufe der Sistema-Darstellung ansatzweise charakterisiert wurde (vgl.: 4.7.), trägt vermutlich, wie bereits im Zusammenhang mit dem Aktanten Grebenscikov erläutert (vgl: 9.9.2.), zur Konstruktion eines Ereignisses bei, so daß sie zweifellos kulturell relevant ist. Sie wird in dieser Analyseeinheit, gleichfalls wie in Analyseeinheit 14, äußerst negativ konstruiert, indem sie in einen Zusammenhang mit der "Obrigkeit» (KPSS, KGB) gebracht und als deren Handlanger dargestellt wird. Die kulturelle Bedeutung der ljubera ist nicht in allen Einzelheiten konstruiert, wie die relativ ausfürliche Darstellung in den genannten Analyseeinheiten verdeutlicht. Möglicherweise ist der Bedeutungsaspekt des Handlangers neu und noch nicht vollends in das Konstrukt der ljubera eingepaßt.

Die Gruppe Masina Vremeni, die durch zwei Indices bezeichnet wird (Masina Vremeni und Masina), wird in der dritten Analyseeinheit ausfuhrlich positiv konstruiert. „osnovnaja tema etogo povestvovanija [...] est' vpolne ponjatnaja ljubov' nasej publiki k muzyke «Masiny», a mołet byt', i k nim licno". In Analyseeinheit 4 kann erneut von einer positiven Konstruktion ausgegangen werden, da die Gruppe in der Funktion einer «Übergruppe», an der sich andere Gruppen orientieren, dargestellt wird. Es ist offensichtlich, daß die angesprochene Gruppe eine positive Relevanz und Bedeutung in der untersuchten kulturellen Formation aufweist. In beiden Analyseeinheiten liegt ein nahezu abgeschlossenes Konstrukt vor.

Die Gemeinschaft milicja, die gleichfalls durch zwei Indices bezeichnet wird (milicejskie narjady und milicija), wird weder in Analyseeinheit 9 noch in Analyseeinheit 11 attribuiert und also konstruiert. Die Konstruktion ist in diesem Sinne innerhalb des untersuchten Diskurses abgeschlossen. Da die Gruppe zum Bereich der "Obrigkeit» gezählt werden kann, liegt die Vermutung nahe, daß sie zum einen in kultureller Hinsicht relevant ist, zum anderen im Rahmen der untersuchten kulturellen formation negativ bewertet wird. 
Auf die kulturelle Relevanz und Bedeutung derjenigen Gemeinschaften, die nur einmal im Korpus nachgewiesen werden können, soll an dieser Stelle nicht eingegangen werden. Da ein großer Teil dieser Gemeinschaften in Form von Stereotypen (vgl.: 9.12.2.) und als Bestandteil von Antagonismen (vgl.: 9.12.1.) auf der kulturellen Ebene funktionalisiert wird, wird in den entsprechenden Abschnitten auf die kulturell relevanten Gemeinschaften eingegangen.

\subsubsection{Geographica und Institutionen}

Im Verlauf der Analyse stellte sich heraus, daß sehr häufig Antanomasien wie otecestvo, gosudarstvo, imperija und obscestvo für das Geographicum Sovetskij Sojus im Korpus anzutreffen sind. Soweit die indexikalischen Antanomasien eindeutig fur bestimmte Geographica und Institutionen stehen, werden sie erfaßt. Das Analyseergebnis hinsichtlich der angesprochenen Indices und der durch sie bezeichneten Geographica und Institutionen ist in Tabelle 21 dargestelit.

Tabelle 21

\begin{tabular}{|c|c|c|}
\hline$\overline{\mathbf{A E}}$ & Index & Geographicum und Institution \\
\hline 1 & gosudarstio & Soretskij Sojus \\
\hline 3 & Puskinskij Dom kul'tun: Puskin & Puskinskij Dom kul tury |2] \\
\hline 4 & Ccrnogolovka & Comogolovka \\
\hline+ & Gosfilarmonija & Gosudarstiennaja filamonija \\
\hline 4 & Moskia & Moskva \\
\hline 4 & Tblisi & Tolisi \\
\hline 5 & ZEK & Ziliscno-Ekspluatacionnaja Kontora \\
\hline 5 & moskorksij kafe * Lira» & moskorksij kafe «Lira» \\
\hline 5 & Moskva & Moskva \\
\hline 5 & Zapad & Lapad \\
\hline 6 & Leningrad & Leningrad \\
\hline 6 & gorodskoj rok-klub & I cmingradskij rok-klub \\
\hline 7 & Afrika & Afrnka \\
\hline 8 & odna sestaja v sego mira & Sorctskij Sojus \\
\hline 9 & KSP & klub samodejatel'noj pesni \\
\hline 9 & Zapad & Zapad \\
\hline 10 & Dom samodcjatcl nogo tworcestva & Dom samodcjatcl nogo thorcestia \\
\hline 10 & leringradskij opit & Lcmingradskij rok-klub \\
\hline 10 & Moskor skij rok-klub & Moskovskaja rok-laboratorija \\
\hline 10 & Moskva, zlatoglavaja stolica & Moskva [2] \\
\hline 10 & nase obscestvo & Sovetskij Sojus \\
\hline 10 & Upravicnic kul'tun Mossovcta & Upravlenic kul'tury Mossoveta \\
\hline 11 & 7ukorskij & vermutl.: "/ukovskij dom kul 'tun \\
\hline 11 & ENMC & unbek. \\
\hline 11 & Izmajlovo & 1zmajlorskij park \\
\hline 11 & Kaucuk & unbek. \\
\hline 11 & Kreml & Kreml' \\
\hline 11 & MEI & Mockovskij Encrgeticeskij Institut \\
\hline 11 & MELZ & unbek. \\
\hline 11 & rok-laboratorija: laboratorija & Moskor-shaja rok-laboratorija [2] \\
\hline 14 & $\mathrm{Krcml}^{\circ}$ & Krcm! \\
\hline
\end{tabular}




\begin{tabular}{|c|c|c|}
\hline 14 & Moskva & Moskva \\
\hline 14 & sgnivsaja imperija & Sorctskij Sojus \\
\hline 14 & Sverdlovsk & Sverdlorsk \\
\hline 15 & \begin{tabular}{|l} 
Verchovinye Sovety \\
\end{tabular} & Verchormyj Sovet \\
\hline 15 & Vsesojusraja Rok-federacija: Rok-foderacija & Vsesojusnaja Rok-foderacija [2] \\
\hline 16 & L.A. & Los Angeles \\
\hline 16 & N'ju-Jork & New York \\
\hline 16 & Staty & Socdinennyc Staty Ameriki \\
\hline 16 & Zapad & Zapad \\
\hline 17 & \begin{tabular}{|l|} 
u nas $v$ strane \\
\end{tabular} & Sovetskij Sojus \\
\hline 18 & \begin{tabular}{|l|} 
DK ^Chimik» \\
\end{tabular} & Dom kul'tur $\propto$ Chimik \\
\hline 18 & DK «Fizik* & Dom kul tur «Fizik» \\
\hline 19 & KLF & klub ljubitclcj fantastiki \\
\hline 21 & Scerbakor & vermutl.: Scertakov skaja ulica \\
\hline 21 & Aleksecrskaja & vermut : Alckscer skaja ulica \\
\hline 21 & Moska & Moskva \\
\hline 21 & Podmoskor'e & Podmoskov'e \\
\hline 21 & Rodina & Sorctskij Sojus \\
\hline 21 & Universam & Universam \\
\hline 22 & dvorec Molodezi & dvorec Moloderi \\
\hline 22 & $\mathrm{~N}$-skij & unbek. \\
\hline 22 & Sibir' & Sibir \\
\hline 23 & nase obscestio & Sovetskij Sojus \\
\hline 24 & Moskva & Moskva \\
\hline 24 & Rossija [2]: gosudarstio: nase Otecestvo & Rossija [4] \\
\hline 24 & Zapad & Zapad \\
\hline 26 & CRU & Central 'noc Rañoduatel 'noe Uprantenie \\
\hline 26 & obscestio & Sor ctskij Sojus \\
\hline 27 & Bundes-Respublika Germanija & Bundesrepublik Deutschland \\
\hline 29 & $\mathrm{CCCP}$ & Sorctskij Sojus \\
\hline 31 & curopcjskaja strana & erropcjskaja strana \\
\hline 31 & Gencral nye Stats & Gencral'nye Stats \\
\hline 31 & KPSS & KPSS \\
\hline 31 & nasc sovremennoc dvorjanstio & Sorctskij Sojus \\
\hline 31 & Verchornyj Sovet & Verchooinyj Sorct \\
\hline 33 & Antares & Antares [3] \\
\hline 34 & Liaz. & Liaz. \\
\hline 34 & nasa rodina & Sorctskij Sojus \\
\hline 35 & aciatskic gosudarstia & aciatskic gosudarstia \\
\hline 35 & DK na ploscadi Puskına & Dom kul'tur na ploscadi Puskin \\
\hline 35 & Orochovo-Zuerskij rajon & Orochovo-Zuciskij rajon \\
\hline
\end{tabular}

Im Korpus werden 50 verschiedene Geographica und Institutionen genannt, die durch 72 Indices bezeichnet werden. Die angesprochenen Indices werden in 27 Analyseeinheiten $(77,1 \%)$ registriert, so daß deren Verwendung als charakteristisches Textgenerienungsmerkmal des untersuchten Diskurses angesehen wird. Die Haufigkeit der Verwendung der angesprochenen Indices schwankt zwischen 0 und 8 , wobei eine einmalige Nennung mit $28,6 \%$ am wahrscheinlichsten ist. Die Nennungshaufigkeit genügt dem eingeführten Bewertungskriterium in diesem Sinne nicht. 
Bei den 50 Geographica und Institutionen betreffen 10 (Afrika, aziatskie gosudarstva, Bundesrepublik Deutschland, Central'noe Razvedyvatel'noe Upravlenie, evropejskaja strana, General'nye Staty, Los Angeles, New York, Soedinennye Staty Ameriki und Zapad: 20\%) nicht den Bereich der Sowjetunion bzw. deren Nachfolgestaaten, so daß in bezug auf Institutionen und Geographica von einer Introspektive auf den genannten Bereich ausgegangen wird. Zum Bereich der untersuchten kulturellen Formation gehören mit hoher Wahrscheinlichkeit die folgenden vier Geographica und Institutionen (8\%): moskovskij kafe «Lira», Leningradskij rok-klub, Moskovskaja rok-laboratorija und Vsesojusnaja Rok-Federacija, so daß keine Introspektive auf die untersuchte Formation diagnostizierbar ist.

Aus weiter oben genannten Gründen (vgl: 9.9.2. und 9.9.3.) wird die quantitative Betrachtung des Analyseergebnisses an dieser Stelle beendet. Wiederum soll auf diejenigen Geographica und Institutionen hinsichtlich ihrer kulturellen Relevanz und Bedeutung eingegangen werden, die mehrmals in verschiedenen Analyseeinheiten nachweisbar sind. Es handelt sich um: Sovetskij Sojus $(1,8,10,14,17,21,23,26,29,31,34)$, Moskva (4, 5, 10, 14, 21 , 24), Zapad (5, 9, 16, 24), Kreml' $(11,14)$, Leningradskij rok-klub (6, 10), Moskovskaja roklaboratorija $(10,11)$ und Verchovnyj Sovet $(15,31)$.

Das Geographicum Sovetskij Sojus, das in 10 Analyseeinheiten nachweisbar ist, wird in Form von 10 verschiedenen Indices eingeführt. Allein die Wahl des Indices läßt vermutlich Schlüsse uber die Bewertung und somit Bedeutung des Geographicums im Rahmen der untersuchten kulturellen Formation zu. In Analyseeinheit 1 wird der Begriff gosudarstvo gewählt und in Verbindung mit dem Begriff rok gebracht. Das Geographicum wird in negativer Weise konstruiert, da die Verstandnislosigkeit, die innerhalb des angesprochenen Bereichs in bezug auf das Phanomen rok herrsche, bedauert wird In Analyseeinheit 8 kann die indexikalische Antonomasie odna sestaja vsego mira ermittelt werden, die, bezogen auf die Gemeinschaft aborigeny, dem Aufbau eines Stereotyps dient (vgl 9.12.2.). Der Index selbst wird an dieser Stelle nicht attribuiert. Synivsaja imperija, der Index, der in Analyseeinheit 14 gewahlt wird, verdeutlicht ohne weitere Attribuierung eine stark negative Bewertung des betrachteten Geographicums. In Analyseeinheit 17 hingegen erfolgt keine explizit negative Konstruktion Das Geographicum wird mittels des neutral anmutenden Indices u nas v strane ausgedruckt. die den räumlichen Rahmen eines Antagonismus abgibt, der aus drei Elementen besteht: sovkovo-oficioznaja kul'tura, Kul'tura und kontrkul'tura (vgl.: 9.12.1.). Der Verfasser tritt für dasjenige Element, die kontrkul'tura, ein, die es seiner Meinung nach im angesprochenen Bereich nicht gibt. Aufgrund dieser Ansicht sollte von einer negativen Bewertung des Geographicums ausgegangen werden. Der Index Rodina, der vermutlich auf eine positive Bedeutung hinweist, kann in Analyseeinheit 21 ermittelt werden. Negativ hingegen wird das diskutierte Geographicum in Analyseeinheit 23 konstruiert, wie folgendes Beispiel verdeutlicht: .Poka obscestvo nase nachoditsja v sostojanii vozbuzdennych potrebnostej vygody". In Analyseeinheit 26 dient das Geographicum, das in diesem Fall durch gosudarstvo ausgedrückt 
wird, der Attribuierung des Aktanten Aleksandr Simonov, wobei es selbst nicht attribuiert wird. In Analyseeinheit 29 dient der Index CCCP, der ebenfalls nicht attribuiert wird, lediglich als Ortsbestimmung, so daß er in diesem Sinne einen nicht funktionalisierten Index darstellt. Im Zusammenhang mit dem negativ bewerteten Wahlgesetz wird das diskutierte Geographicum mittels des Indices nase sovremmenoe dvorjanstvo bezeichnet. Dieser Index weist auf eine negative Bewertung hin. Nicht attribuiert und daher konstruiert wird das Geographicum in Analyseeinheit 34, da es lediglich im Zusammenhang mit dem momentanen Aufenthaltsort des in dieser Analyseeinheit konstruierten Aktanten eingeführt wird. Zusammenfassend kann festgestellt werden, daß das Geographicum Sovetskij Sojus im Großteil der dargestellten Fälle funktionalisiert wird und daher kulturell relevant ist. Die kulturelle Bedeutung ist mit hoher Wahrscheinlichkeit negativ, beschränkt sich vermutlich jedoch nur auf Teilaspekte des Konstrukts. Geht es um die generelle kulturelle Bedeutung, wie offensichtlich in Analyseeinheit 21, dann ist diese mit hoher Wahrscheinlichkeit nicht negativ.

Das Geographicum Moskva kann in 6 Analyseeinheiten in Form von zwei Indices (Moskva und zlatoglavaja stolica) nachgewiesen werden. Das Geographicum dient in den Analyseeinheiten 4, 5, 14 und 24 lediglich der Ortsbestimmung und erfahrt keinerlei weitere Attribuierung, so daß es in den genannten Analyseeinheiten nicht funktionalisiert und daher kulturell relevant ist. In Analyseeinheit 10, in der das angesprochene Geographicum zweimal verwendet wird, dient es einerseits als Ortsbestimmung, andererseits wird es in der Variante zlatoglavaja stolica funktionalisiert. Der Index kann an dieser Stelle im Sinne einer ironischen Anspielung auf die Konservativität der Institutionen Dom samodejatelogo tvorcestva und Upravlenie kul'tury Mossoveta, die in den folgenden zwei Sätzen eingeführt werden, aufgefaßt werden Das Geographicum selbst wird an dieser Stelle nicht negativ bewertet. In Analyseeinheit 21 wird Moskva aufgrund des Vorhandenseins von Feinden (vragi) negativ konstruiert. Es liegt die Vermutung nahe, daß sich die negative Beurteilung an dieser Stelle lediglich auf diesen Teilaspekt des Konstrukts bezieht. Zusammenfassend kann festgestellt werden, daß das Geographicum Moskva fast auschließlich der Ortsbestimmung dient, so daß auf dieser Grundlage nicht von einer negativen Bewertung innerhalb des rok-samizdat-Diskurses ausgegangen wird. Vielmehr scheint sich die negative Beurteilung des Geographicums nur auf Teilbereiche des Konstrukts zu beziehen

Das Geographicum Zapad wird in vier verschiedenen Analyseeinheiten eingefuhrt. In den Analyseeinheiten 5, 9 und 24 stellt es einen Bestandteil eines Antagonismus dar, wobei es lediglich in Analyseeinheit 24 negativ attribuiert wird (vgl.: 9.12.1.). In Analyseeinheit 16 dient das diskutierte Geographicum lediglich als Ortsbestimmung. Es liegt die Vermutung nahe, daß die primäre Funktion des Geographicums Zapad die der Abgrenzung ist, die aber offensichtlich nicht der Unterstützung durch negative Attribute bedarf.

Der Index Kreml' kann zweimal innerhalb des Korpus ermittelt werden Im ersten Fall, in Analyseeinheit II, fungiert er als Geographicum, da bemerkt wird, daß in einem Kilometer 
Entfernung ein Konzert stattgefunden hat. Das Geographicum trägt in diesem Zusammenhang zur Generierung eines Ereignisses bei, da gerade die Entfernung zum Kreml' das angesprochene Konzert kulturell relevant werden läßt (vgl:: 9.9.6.). Darüber hinaus wird das Geographicum nicht attribuiert. In Analyseeinheit 14 hingegen wird der Index im Sinne einer Institution eingesetzt und äußerst negativ konstruiert, da er als Kommandozentrale der ljubera (vgl.: 4.7. und 9.9.3.) und kazancy dargestellt wird. In diesem Sinne besitzt der Index Kreml', wenn er ein Geographicum bezeichnet, in der kulturellen Formation des rok-samizdat eine positive kulturelle Bedeutung, wenn er eine Institution bezeichnet, eine negative.

Die Institution Leningradskij rok-klub, auf die bereits im Zusammenhang mit der Publikation ROKSI eingegangen wurde (vgl.: 7.2.1.1.), wird in zwei Analyseeinheiten genannt. In Analyseeinheit 6 erfolgt ihre Nennung im Zusammenhang mit dem als negativ beurteilten Ereignis Pesnja-83 (vgl: 9.9.6.). Da Gruppen des Leningradskij rok-klub anscheinend an diesem Ereignis teilnahmen, liegt die Vermutung nahe, daß er negativ bewertet wird. Eine ironische Position gegenuber dem Leningradskij rok-klub wird in Analyseeinheit 10 eingenommen, wie vermittels des gewählten Indices slavnyj leningradskij opyt belegt werden kann. Die genannte Antonomasie kann als Hinweis auf eine ablehnende Haltung aufgefaßt werden. In diesem Sinne besitzt die angesprochene Institution im Rahmen der untersuchten Formation mit hoher Wahrscheinlichkeit eine negative kulturelle Bedeutung.

Die Institution Moskovskaja rok-laboratorija wird im Korpus ausführlich konstruiert. In Analyseeinheit 10 fallt diese negativ aus, da die angesprochene Institution als Produkt der negativ bewerteten Institutionen Dom samodejatel'nogo tvorcestva und Upravlenie kul'tuy Mossoveta aufgefaßt wird In Analyseeinheit 11 wird ergänzend ausfuhrlich geschildert, in welchen Zusammenhangen die Institution Zensur ausgeubt hat Die ausfuhrliche Attribuierung weist daraufhin, daß die negativ ausgerichtete Konstruktion der Institution nicht abgeschlossen ist.

Die Institution Verchovnyj Sovet wird, wie die vorangehenden Indices, in zwei Analyseeinheiten genannt. In Analyseeinheit 25 erfolgt keine Attribuierung, so daß von einer abgeschlossenen Konstruktion ausgegangen wird. In Analyseeinheit 31 erfolgt im Zusammenhang mit der Darstellung des Wahlgesetzes eine negative Attribuierung. In diesem Sinne besitzt die angesprochene Institution im Rahmen der untersuchten Formation mit hoher Wahrscheinlichkeit eine negative kulturelle Bedeutung.

Auf die verbleibenden Geographica und Institutionen soll nicht naher eingegangen werden. Angemerkt werden soll lediglich, daß die folgenden 20 Geographica neben den bereits genannten die nachweisbare, kognitive Landkarte der untersuchten kulturellen Formation ausmachen Cernogolovka. Tblisi, Leningrad, Afrika, Izmajlovskij park, Sverdlovsk, Los Angeles, New York, Soedinennye Staty Ameriki, Scerbakovskaja ulica, Alekseevskaja ulica, Podmoskov'e, N-skjj, Sibir', Rossija, Bundesrepublik Deutschland, evropejskaja strana. General'nye Staty, aziatskie gosudarstvo sowie Orechovo-Zuevskij rajon. 
Zusammenfassend kann festgestellt werden, daß die betrachteten Institutionen im Rahmen der betrachteten kulturellen Formation auschließlich eine negative kulturelle Bedeutung und somit Relevanz aufweisen. In bezug auf Geographica beschränkt sich die negative Bedeutung mit hoher Wahrscheinlichkeit lediglich auf bestimmte Teilaspekte des jeweiligen Konstrukts.

\subsubsection{Marken}

Die in Tabelle 22 aufgeführten Indices bezeichnen die der gleichnamigen Kategorie zugeordneten Marken.

Tabelle 22

\begin{tabular}{|c|c|c|}
\hline $\mathbf{A E}$ & Index & Marke \\
\hline 2 & Songs from the wood & Songs from the wood \\
\hline 4 & Bljue svin i v usach & Bljuz svin "i v usach \\
\hline$t$ & Geroi & Geroi \\
\hline 4 & Luc sveta v carstic & Luc sveta v temnom carstice \\
\hline 4 & Marina & Marina \\
\hline+ & Minus 30 & Minus 30 \\
\hline 4 & Molodaja giardija & Molodaja gvardija \\
\hline 4 & Tarclka & Tarelka \\
\hline 4 & Tclckaster & Tclckaster \\
\hline 5 & Mifologiceskaja cnciklopedija & Mifologiceskaja cnciklopedija \\
\hline 6 & Korolev stio krivech zerkal & unbck. \\
\hline 6 & VIA & Vokal'myj Instrumental'my Ansambl' \\
\hline 9 & bul dozer & Bulldozer \\
\hline 10 & \$.51 po Farengcjitu & 451 po Farengejtu \\
\hline 10 & Welcome to the machine! & Welcome to the machine! \\
\hline 11 & Tragikomodija & Tragikomcdija \\
\hline 12 & Roksi & ROKSI $[4]$ \\
\hline 13 & Sub" cktikon & SUB ${ }^{\circ}$ EKTIKON \\
\hline 16 & RADIO SILENCE & Radio Silence \\
\hline 18 & Nasic sov remenniki & Nasic sorremenniki \\
\hline 18 & Neulorymyc mstitcli & Neulonymyc mstutelı \\
\hline 18 & Orecstucnnye rapiski & Otocstiennye /apiski \\
\hline 19 & $\mathrm{~S}^{*} \mathrm{M}$ & SUMELA“" MYS" \\
\hline 19 & Istorija KLF Prisclec & Istorija KLF Prisclec \\
\hline 19 & K.K.URa & KONTR KUL'T UR'A \\
\hline 19 & O tckuscem momentc & O tekuscem momentc \\
\hline 24 & Rock-n-roll na Rusi & Rock-n-roll na Rusi \\
\hline 24 & Strana cudes & Alice in Wonderland \\
\hline 26 & Inestija & Inestija \\
\hline 26 & Komu podygnval intellektual "nvi rok? & Komu podygnval intellektual 'mj rok? \\
\hline 26 & Vocernyj Kisener & Vocernyj Kisener \\
\hline 27 & made in Germam & made in German \\
\hline 27 & Munzkal'nyj tizn' & Muzykal nyj zizn" \\
\hline 27 & Pamjat' & Pamjat' [2] \\
\hline 28 & Celina & Cclina \\
\hline 28 & Gruppy riska & Gruppri riska \\
\hline 28 & Malaja zemlja & Malaja zcmlja \\
\hline 28 & Pcred vami. deti. utka & Pcred vami. deti. utka [2] \\
\hline 28 & Vozrozdenic & Volrozdenic \\
\hline
\end{tabular}




\begin{tabular}{|c|c|c|}
\hline 29 & Assa & Assa \\
\hline 29 & Moskovskij komsomolec & Moskovskij komsomolec \\
\hline 29 & Sojuspecat" & Sojuspocat ${ }^{\circ}$ \\
\hline 33 & URLATT & URLAST \\
\hline 34 & Djadjuska zck 1 久cllom sarfikc & Djadjuska zck v zcltom sarfikc \\
\hline 35 & Bambuk & Bambuk [3] \\
\hline 35 & Cherr s moslom. CHSM [2] & CHERR S MOSLOM $|3|$ \\
\hline 35 & TAZ - "Telefonnoj Agentury Zueva" & $\begin{array}{l}\text { TASS - "Telegrafnoc agentstio Sovetskogo } \\
\text { Sojwa" }\end{array}$ \\
\hline
\end{tabular}

Im Korpus können 48 Indices ermittelt werden, die 47 Marken bezeichnen. Die angesprochenen Indices werden in 20 Analyseeinheiten $(57,1 \%)$ eingeführt, so daß ihre Verwendung kein charakteristisches Textgenerierungsmerkmal des untersuchten Diskurses darstellt. Die Indices werden mit einer Häufigkeit von 0 bis 8 eingesetzt, wobei, abgesehen von der Häufigkeit 0 (42,9\%), die Häufigkeit 1 mit 25,7\% am wahrscheinlichsten ist. Dennoch genügt keine Nennungshäufigkeit dem eingefuhrten Bewertungskriterium.

7 Marken (Songs fom the wood, Telekaster, Bulldozer, Welcome to the machine. Alice in Wonderland, made in Germany und Gruppy riska: 14,9\%) haben ihren Ursprung außerhalb des Bereichs der Sowjetunion bzw. deren Nachfolgestaaten, so daß auch im vorliegenden Fall von einer Introspektive auf den genannten Bereich gesprochen werden kann. Ihren Ursprung in der untersuchten Formation haben mit hoher Wahrscheinlichkeit 15 Marken (Bljuz svin'i $v$ usach, Geroi, Marina. Minus 30, Tarelka, 451 po Farengejtu26, ROKSI, SUB' EKTIKON, Radio silence, SUMELA' MYS, KONTR KUL'T UR'A, Rock-n-roll na Rusi, URLAJT, Bambuk und CHERR S MOSLOM: $31,9 \%$ ), so daß keine Introspektive auf die untersuchte Formation diagnostizien wird

Auf die im Korpus in kultureller Hinsicht funktionalisierten Indices und Marken, die nicht an anderer Stelle im Rahmen dieser Arbeit besprochen werden, soll im folgenden Abschnitt eingegangen werden. Auf kulturell nicht-funktionalisierte Indices und Marken wird nicht eingegangen Es geht in diesem Sinne um die folgenden Marken Vokal'nyj Instrumental'nyj Ansambl', Otec'estvennye zapiski, Nasi sovremenniki, Malaja zemlja, Celina, Vozrozdenie. Assa und Moskovskij komsomolec

Ende der sechziger Jahre erfolgte in der Sowjetunion von staatlicher Seite aus eine Unterteilung in offizielle und inoffizielle Musikgruppen. Hinter der Bezeichnung Vokal'nyj Instrumental'nyj Ansambl' (kurz: VIA) verbergen sich die offiziellen Gruppen, die, zensiert durch diverse Kulturbehörden, genotigt waren "positive», sozialistisch-realistische Lieder zu verfassen. Die bekannteste Gruppe der VIAs trägt. um diesem Ziel gerecht zu werden, dementsprechend den programmatischen Namen Veselye rebjata. Die angesprochene Marke wird in Analyseeinheit 6 im Zusammenhang mit dem Ereignis Pesnja-83 eingefuhrt. Es wird im dorti-

\footnotetext{
${ }^{26}$ Der Index 451 po Farengejtu bercichnet im Korpus ein Licd der Gruppe Urfin Drjus. niche den Roman von Ray Bradbun. Da nicht der Roman sondern das Lied indexikalischen Charakter besitat. wird der Index nicht dem Bereich des Auslands sondern der untersuchten Formation zugeordnet.
} 
gen Zusammenhang nicht bedauert, daß die Gruppen der genannten «Marke» verschwunden sind. Darüber hinaus erfolgt keine weitere Attribuierung, so daß von einem abgeschlossenen Bedeutungskonstruktionsproze $B$ im Rahmen der untersuchten Formation ausgegangen werden kann. Mit hoher Wahrscheinlichkeit wird die kulturelle Bedeutung der Gruppen der angesprochenen «Marke» negativ sein.

Bei den Marken Oteciestvennye zapiski und Nasi sovremenniki handelt es sich um offizielle Zeitschriftenreihen, die in Analyseeinheit 18 als Beispiel dienen und somit indirekt in negativer Weise konstruiert werden. Über die Publikationsreihen selbst werden in diesem Zusammenhang keine Angaben gemacht, so daß gleichfalls von einem abgeschlossenen Bedeutungskonstruktionsprozeß, der ein negatives Ergebnis liefert, ausgegangen wird.

Malaja zemlja, Celina und Vozrozdenie, die in Analyseeinheit 28 ebenfalls als Beispiel genannt und indirekt negativ konstruiert werden, sind die Titel von literarischen, stark autobiographischen Werken Leonid Breznevs. In bezug auf diese Werke scheint die Bedeutungskonstruktion abgeschlossen zu sein, da keine weitere Attribuierung erfolgt. Wiederum kann von einer negativen kulturellen Bedeutung ausgegangen werden.

Assa ist der Titel eines in der Sowjetunion erfolgreichen Films von Sergej Solovjev, der 1988 in die Kinos kam. Ein personaler Zusammenhang mit der untersuchten kulturellen Formation liegt in zweierlei Hinsicht vor: zum einen stammt die Filmmusik von Grebenscikov, zum anderen spielt der Musiker Viktor Coj von der Gruppe Kino eine Hauptrolle und zwar sich selbst. Der Film wird in Analyseeinheit 29 als Kitsch (kitc) bezeichnet, was auf eine negative kulturelle Bedeutung hinweist. Die Bedeutungskonstruktion in bezug auf den Film ist offensichtlich zum Zeitpunkt der Erstellung der Analyseeinheit nicht abgeschlossen.

Der Marken-Index Moskovskij komsomolec, der die neben der Pravda wahrscheinlich bedeutendste Tageszeitung der ehemaligen Sowjetunion bezeichnet, wird in Analyseeinheit 29 ebenfalls als negatives Beispiel eingefuhrt. Die negative Bedeutungskonstruktion im Rahmen der untersuchten kulturellen Formation kann in bezug auf die Zeitung als abgeschlossen angesehen werden, da keine weitere Attribuierung an der genannten Stelle erfolgt.

Zusammenfassend kann festgehalten werden, daß die besprochenen Marken häufig in der Funktion von negativen Beispielen eingesetzt werden. Die kulturelle Bedeutung muß, um diese Funktion erfüllen zu können, dementsprechend im Rahmen der untersuchten Formation feststehen 


\subsubsection{Ereignisse}

Die Indices, die «historische» Ereignisse bezeichnen und die durch sie bezeichneten Ereignisse sind, soweit sie rekonstruiert werden können, in Tabelle 23 aufgefuhrt. Da es sich um Ereignisse handelt, die sich durch die Anschlußhandlung Bericht und somit durch eine kulturelle Konstruktion auszeichnen, kann jeweils von einer kulturellen Relevanz des Ereignisses ausgegangen werden, auch wenn es, wie in vier Fällen, nicht stattgefunden hat. Gerade diese Tatsache bewirkt vermutlich, daß diese Ereignisse zu «historischen» Ereignissen werden.

Tabelle 23

\begin{tabular}{|c|c|c|}
\hline $\mathbf{A E}$ & Index & Ereignis \\
\hline 3 & $\begin{array}{l}\text { okjabr` } 1977 \text { goda: odin iz poslednych priczdor } \\
\text { «Masiny Vremeniw }\end{array}$ & $\begin{array}{l}\text { Leningrad. 15.10.1977, «Masina Vremeni», koncert v } \\
\text { Puskinskom Dome kul'tun }\end{array}$ \\
\hline+ & festival v Cernogolovku & hat nicht stattgefunden \\
\hline 6 & Pesnja-83 & Leningrad. 1983 , vsenarodnaja peredaca $\propto$ Pesnja-83w \\
\hline 7 & $\begin{array}{l}1985 \text { god [za oknom resennimi nuc jami nalicactsja } \\
1985 \text { god. i. kak skazal by "ncrass. "nnastal cas } \\
\text { rozonjix]. }\end{array}$ & vermull.: Sovetskij Sojus. 1985 \\
\hline 10 & otknuija Moskorskogo rok-kluba & Moskva. 1985. otkntija Moskovskoj rok-laboratorii \\
\hline 11 & Sven Gundlach vystupact v kilometre of Kremlja & $\begin{array}{l}\text { Moskva. vermutl.: 1987, Sven Gundlach vystupaet v } \\
\text { kilometre of Kremlja }\end{array}$ \\
\hline 11 & Riyskij $\approx$ Ccmenl $*$ v $\propto$ Kau & hat nicht stattgefunden \\
\hline 11 & festival' v MELZe & hat nicht statlgefunden \\
\hline 11 & festival v fevrale $v$ MEI & \begin{tabular}{|llll}
$\begin{array}{l}\text { Moskva. vermutl.: 02.1987. festival } \\
\text { (Moskovskij Encrgcticeskij Institut) }\end{array}$ & MEI \\
\end{tabular} \\
\hline 11 & «Vesclye kartinkı" v Zuko skom & $\begin{array}{l}\text { vermul.: Moskva. vermutl.: 1987. «Vesctyc kartinki». } \\
\text { vermul.: v 'Yukonskom Donx kul' tury }\end{array}$ \\
\hline 11 & koncert v Izmajlovo s ucasticm GB & $\begin{array}{l}\text { Moskva. vermull: 1987. koncen s wcasticm GB } \\
\text { (Boris Grebenscikov) v l/zmajlovskom parke }\end{array}$ \\
\hline 11 & vint 1983 goda & vermutl : Moskva. 1983. vint aljuben \\
\hline 15 & $\begin{array}{l}\text { Vioraja vsesojusnaja konferencija Rok-foderacii } \\
\text { «Rok-munka kak sociokul turnyj fenomen» } \\
\text { Sierdlorsk. 16-18.11.88. }\end{array}$ & $\begin{array}{lll}\begin{array}{l}\text { Sverdlorsk. } \\
\text { konferencija }\end{array} & \text { Rok-foderacii } \\
\text { sociokul 'tumy fenomen } & \text { «Rok-munka kak } \\
\end{array}$ \\
\hline 35 & festival Bambuk & hat nicht staltgefunden \\
\hline 35 & Vudstok & Woodstock. 1969 \\
\hline
\end{tabular}

Im Korpus konnen 15 verschiedene Indices und Ereignisse, die sich auf 8 Analyseeinheiten $(22,9 \%)$ verteilen, ermittelt werden. Die Thematisienung von Ereignissen stellt in diesem Sinne kein charakteristisches Textgenerierungsmerkmal des betrachteten Diskurses dar. Die Haufig keit der Nennung schwankt zwischen 0 und 7, wobei die Nennung von einem Ereignis mit $17,1 \%$ bezogen auf das Korpus neben dem ganzlichen Fehlen der in Rede stehenden Indices $(77,1 \%)$ am wahrscheinlichsten ist. In diesem Sinne genügt die keinmalige Verwendung der angesprochenen Indices dem Bewertungskriterium charakteristisch

Lediglich das Ereignis Woodstock hat sich nicht auf dem Territorium der Sowjetunion bzw. deren Nachfolgestaaten abgespielt, so daß auch in bezug auf Ereignisse zunachst von einer Introspektive auf den genannten Bereich ausgegangen werden kann Der kulturellen Formation 
des rok-samizdat konnen 11 Ereignisse (73,3\%) zugerechnet werden, die in den Analyseeinheiten 3, 4, 10, 11 (bis auf: vint 1983 goda), 15 und 35 genannt werden. In bezug auf Ereignisse kann folglich nicht nur von einer Introspektive auf den Bereich der Sowjetunion bzw. deren Nachfolgestaaten, sondern vor allem auf die untersuchte kulturelle Formation gesprochen werden.

Auf die kulturelle Bedeutung der ermittelten Ereignisse soll in den folgenden Absätzen eingegangen werden.

Das Ereignis, in dessen Mittelpunkt die positiv bewertete Gruppe Masina Vremeni steht (vgl.: 9.9.3.), wird in Analyseeinheit 3 ausfuhrlich konstruiert. Es kann davon ausgegangen werden, daß das Ereignis im Rahmen der untersuchten kulturellen Formation mit hoher Wahrscheinlichkeit eine positive Bedeutung aufweist.

Das Festival, welches in Analyseeinheit 4 erwähnt wird, zeichnet sich durch ein Verbot aus, welches drei Tage vor Beginn der Veranstaltung ausgesprochen wurde. Im Verlaufe des Festivals sollte neben lokalen Gruppen (provincial'nye bitly) die Gruppe Akvarium (vgl.: 9.9.3.) auftreten. Vermutlich erlangt das angesprochene Festival den Status eines «historischen» Ereignisses aufgrund des Verbots, wobei die genannte Gruppe mit hoher Wahrscheinlichkeit einen Einfluß auf das Konstrukt ausübt.

Das landesweit im Fernsehen übertragene Konzert Pesnja-83 wird in der gesamten sechsten Analyseeinheit in expliziter, negativer Form konstruiert. Der Konstruktionsprozeß ist offensichtlich zum Zeitpunkt der Erstellung der Analyseeinheit nicht abgeschlossen.

Das Jahr 1985, welches in Analyseeinheit 7 genannt wird, steht zum Zeitpunkt der Erstellung der Analyseeinheit kurz bevor. Bemerkenswert ist in diesem Zusammenhang, daß der Verfasser ein negatives Konstrukt generiert. Dieses Konstrukt kann genutzt werden, um Schlüsse über das Konstrukt der vorangehenden Jahre, bezogen auf die Sowjetunion, zu ziehen, da diese in temporärer Hinsicht die Grundlage des angesprochenen Jahres bilden. Es liegt die Vermutung nahe, daß diese im Rahmen der untersuchten kulturellen Formation eine negative Bedeutung aufweisen. In dieser Hinsicht wird das genannte Jahr als Fortsetzung aufgefaßt.

In der gesamten Analyseeinheit 10 geht es um die Eroffnung der Institution Moskovskaja rok-laboratorija, deren kulturelle Bedeutung bereits an anderer Stelle erläutert wurde (vgl: 9.9.4.). In diesem Sinne kann davon ausgegangen werden, daß das Ereignis im Rahmen der untersuchten Formation eine negative Bedeutung aufweist.

Gleichfalls wurde bereits auf das Ereignis, welches durch den Index Sven Gundlach vystupaet $v$ kilometr ot Kremlja bezeichnet wird, eingegangen (vgl.: 9.9.4.). Dieses Konzert erhalt seine Bedeutung und Relevanz vermutlich dadurch, daß es in Zusammenhang mit dem positiv konstruierten Geographicum Kreml' gebracht wird. Ein «normales» Konzert von Sven Gundlach besitzt vermutlich nicht den Charakter eines «historischen» Ereignisses. 
Die Ereignise, die durch die Indices Ritskij «Cement» v «Kaucuke», festival v MELZe, festival v fevrale v MEI, "Veselye kartinki" v Zukovskom und koncert $v$ Izmajlovo s ucastiem GB bezeichnet werden, stehen einerseits in einem Zusammenhang mit der Institution Moskovskaja rok-laboratorija, andererseits mit der Gruppe ljubera. Die Verbote bzw. Störungen machen diese Konzerte mit hoher Wahrscheinlichkeit zu Ereignissen. Aufgrund der negativen kulturellen Bedeutung sowohl der Institution als auch der Gruppe, erhalten die Konzerte vermutlich eine positive kulturelle Bedeutung. Es liegt die Vermutung nahe, daß die genannten Konzerte, ähnlich wie das Konzert von Sven Gundlach, erst unter den genannten Umständen zu Ereignissen werden konnten.

Im Jahre 1983 hat offensichtlich ein Überfall (vint) unter Beteiligung der ljubera stattgefunden, der den Charakter eines Ereignisses aufweist. Dieses Ereignis besitzt eine abgeschlossene. negative kulturelle Bedeutung, da es vermutlich einen «Sieg» der genannten Gruppe markiert.

Das Ereignis, welches in Analyseeinheit 15 ausführlich dargestellt wird, erfahrt vom Autor der genannten Analyseeinheit keine Bewertung. Da der Bedeutungskonstruktionsprozeß nicht abgeschlossen ist und keine negative Bewertung erfolgt, wird von einer positiven kulturellen Bedeutung ausgegangen.

Das festival' Bambuk, welches nicht durchgeführ wurde, steht in Zusammenhang mit der nicht veröffentlichten Publikation BAMBUK, die in einem antagonistischen Verhältnis zu der Publikation CHERR S MOSLOM steht (vgl.: 9.12.1.). Aus der Sicht der zuletzt genannten Publikation wird sowohl das Festival als auch die Publikation negativ bewertet. Da es in der vorliegenden Analyseeinheit vorwiegend um persönliche Differenzen geht, sollen keine Schlüsse hinsichtlich der kulturellen Bedeutung des genannten Ereignisses gezogen werden.

Abschließend soll das Ereignis Woodstock, welches eine abgeschlossene Bedeutungskonstruktion aufweist, betrachtet werden Das Ereignis weist eine positive kulturelle Bedeutung auf, da es genutzt werden kann, um mit Hilfe eines Vergleichs ein anderes Ereignis, das festival' Bambuk, zu diskreditieren.

Hiermit soll die Betrachtung der Konstruktion kulturell relevanter Ereignisse im Rahmen der untersuchten Formation beendet werden

\subsubsection{Sonstige}

Die im Korpus ermittelten Indices, die der Kategorie Sonstige zugeordnet werden, sind in Tabelle 24 zu sehen. Die durch die Indices bezeichneten Objekte und Phanomene werden nicht separat erfaßt 
Tabelle 24

\begin{tabular}{|c|l|}
\hline AE & Index \\
\hline 1 & Armageddon \\
\hline 1 & Karma \\
\hline 5 & srednevekoryj scparatizm \\
\hline 7 & nas industrial nyj vek \\
\hline 7 & vek informacii i nauki \\
\hline 9 & gorodskoj romans XIX vcka \\
\hline 9 & dvizenie k stetlomu buduscemu \\
\hline 10 & sorTemennyj pljuralizm \\
\hline 11 & strojteli svetlogo buduscego \\
\hline 11 & perestrojika \\
\hline 14 & liberalizm \\
\hline 16 & kollektivizm \\
\hline 17 & Bog [2] \\
\hline 22 & Gospod 12 ] \\
\hline 24 & Bolonskaja skola prava \\
\hline 27 & GLASNOST \\
\hline 28 & SPID \\
\hline 28 & celovecestio \\
\hline 29 & demokratija \\
\hline 35 & demokratija \\
\hline
\end{tabular}

Im Korpus können insgesamt 19 verschiedene Indices ermittelt und der Kategorie Sonstige zugeordnet werden. Die Indices verteilen sich auf 15 Analyseeinheiten (42,9\%), so daß die Verwendung der ermittelten Indices kein charakteristisches Textgenerierungsmerkmal des untersuchten Diskurses darstellt. Die Häufigkeit der Verwendung schwankt zwischen 0 und 2 , wobei die einmalige Verwendung der angesprochenen Indices mit 28,6\% nach der keinmaligen mit $57,1 \%$ am wahrscheinlichsten ist. In diesem Sinne genügt keine Verwendungshäufigkeit dem eingefuhrten Bewertungskriterium. In bezug auf die mehrfach in den vorangehenden Abschnitten diagnostizierte Introspektive kann angemerkt werden, daß die folgenden 4 Indices $(21,1 \%)$ einen sowjetischen bzw. postsowjetischen Ursprung besitzen: dvizenie $k$ svetlomu buduscemu, stroiteli svetlogo buduscego, perestrojka und glasnost', so daß im vorliegenden Fall nicht von einer Introspektive ausgegangen wird. Mit hoher Wahrscheinlichkeit weist kein Index unmittelbar auf den untersuchten Diskurs hin. Zwei Indices bzw. durch sie bezeichnete Phänomene, demokratija $(29,35)$ und Bog/Gospod' $(17,22)$, können jeweils zweimal im Korpus ermittelt werden, so daß auf diese zunächst hinsichtlich ihrer kulturellen Relevanz und Bedeutung eingegangen wird.

Der Index demokratija wird in Analyseeinheit 29 folgendermaßen definiert und somit konstruiert: ,pravo govorit' i pravo zatykat' rot'“. In Analyseeinheit 35 wird das bezeichnete System darüber hinaus überschwenglich für die Existenz von zwei rok-samizdat-Publikationen im Bezirk Orecho-Zuevo gelobt. Sowohl die überschwengliche Attribuierung als auch die Definition weisen darauf hin, daß die Bedeutung des funktionalisierten und daher kulturell relevanten Systems nicht vollstandig konstruiert ist. Trotz des merkwurdigen Demokratiever- 
standnisses in Analyseeinheit 29 kann davon ausgegangen werden, da $\beta$ das angesprochene System positiv im Rahmen der untersuchten kulturellen Formation bewertet wird.

Die Indices Bog und Gospod' werden zusammen betrachtet, da sie ein Objekt bezeichnen. Dieses wird in Analyseeinheit 17 zweimal, jeweils im Rahmen eines Phraseologismus genannt (Tragedija, odnim slovom, ej-Bogu, tragedija, [...] pomogi im Bog v poiskach puti), so daß von keiner Funktionalisierung in kultureller Hinsicht gesprochen wird. In Analyseeinheit 22 wird der Index Gospod' einmal in der Überschrift genannt und in Zusammenhang mit dem Hobby (dosug) des Verfassers gesetzt. Das bezeichnete Objekt scheint in diesem Zusammenhang eine kulturelle Bedeutung aufzuweisen; ob diese positiv oder negativ ist, kann nicht diagnostiziert werden. Im zweiten Fall wird der Index ebenfalls im Rahmen eines Phraseologismus (ne privedi Gospod' [...]) eingeführt. Lediglich im Fall der Überschrift sollte dementsprechend von einer Funktionalisierung auf der kulturellen Ebene und von einer kulturellen Relevanz ausgegangen werden.

Des weiteren soll an dieser Stelle auf die funktionalisierten Indices, d.h. diejenigen, die im vorliegenden Zusammenhang mit hoher Wahrscheinlichkeit funktionalisierte Phänomene, die sich durch eine funktionalisierte kulturelle Bedeutung auszeichnen, bezeichnen, eingegangen werden. Es handelt sich um die Indices nas industrial'nyj vek, vek informacii $i$ nauki, dvizenie $k$ svetlomu buduscemu, sovremennyj pljuralizm, stroiteli svetlogo buduscego, perestroijka, liberalizm und SPID

Die Indices nas industrial'nyj vek und vek informacii i nauki markieren den zeitlichen Rahmen eines Antagonismus, in dem die "Gemeinschaften" my und Afrika/papausy/judi gegenübergestellt werden (vgl. 9.12.1.). Der erste Index, der vermittels des Possesivpronomens nas mit der Gemeinschaft my verbunden ist, besitzt aufgrund dieser Verbindung mit hoher Wahrscheinlichkeit eine positive kulturelle Bedeutung, die auch für den zweiten Index angenommen werden kann. Da die Indices jeweils attribuiett sind, kann nicht von einer abgeschlossenen Bedeutungskonstruktion ausyegangen werden.

Der Index dvizenie $k$ svetlomu buduscemu, der in Analyseeinheit 9 eingefuhrt wird, steht in der genannten Analyseeinheit in Zusammenhang mit dem KSP (klub samodejatel'noj pesni), der an dortiger Stelle positiv konstruiert wird. Der in Rede stehende Index wird im zeitlichen Kontext des KSP (60-er Jahre) gesehen, wie durch folgendes Zitat belegt werden kann: „Otxicim znakom KSP byl zdorovyj, mazomyj plus, i ona otrazala umonastroenija togo perioda dvizenija $k$ svetlomu buduscemu“ In der angesprochenen Periode besaß der Index bzw. das bezeichnete Phänomen offensichtlich keine negative Bedeutung. Die Beschrankung auf die Periode legt den Schluß nahe, daß der Index und das bezeichnete Phänomen in zeitgenossischer Hinsicht eine andere Bedeutung aufweisen. Es wird in diesem Zusammenhang deutlich, daß sich die angesprochene, kulturelle Bedeutung mit hoher Wahrscheinlichkeit von den sechziger Jahren bis zum Zeitpunkt der Erstellung der Analyseeinheit stark verändert hat 
Das durch den Index sovremennyj pljuralizm bezeichnete Phänomen wird in der untersuchten Formation positiv bewertet: „Sovremennyj pljuralizm nas ucit [...] mnozestvennosti tocek zrenija na suscestvujuscie problemy". Die Bedeutungskonstruktion des Phänomens ist nicht abgeschlossen, wie das Zitat verdeutlicht. Das Adjektiv sovremennyj weist darauf hin, daß die kulturelle Bedeutung des Phänomens pljuralizm Veränderungen unterworfen war.

In zeitgenössischer Hinsicht besitzt der Index stroiteli svetlogo buduscego, der dem Index dviżenie $\mathbf{k}$ svetlomu buduscemu in semantischer und etymologischer Hinsicht sehr ähnlich ist, eine äußerst negative, explizierte Bedeutung: „Neuželi nas perestali zagonjat' $v$ rjady stroitelej svetlogo buduscgo, i my, nakonec, mozem spokojno, mimo sosuscestvovat'?". Die Bedeutungskonstruktion befindet sich jedoch offensichtlich in einem Prozeß.

Das Phänomen perestrojka wird im vorliegenden Zusammenhang als vyigryšnyj bilet bezeichnet, was auf eine positive kulturelle Bedeutungskonstruktion hinweist, die sich im Aufbau befindet.

Das Phänomen liberalizm hingegen, das in Analyseeinheit 14 eingeführt wird, besitzt eine vermeintlich negative Bedeutung. Die negative Bedeutung bezieht sich vermutlich nur auf die von offizieller Seite aus praktizierte Variante des Phänomens. D.h. der Index, wie er von offizieller Seite aus konstruiert wird, wird im Rahmen der untersuchten Formation abgelehnt. Mit hoher Wahrscheinlichkeit besitzt das angesprochene Phänomen innerhalb der untersuchten Formation eine andere Bedeutung, die in einem oppositionellen Verhältnis zu der offiziellen Bedeutung steht.

Das Phanomen Aids (SPID) wird in Analyseeinheit 28 als tema bezradostnaja bezeichnet. Darüber hinaus wird eine kurze Zusammenfassung von zwei Fernsehbeiträgen, die dem Phanomen gewidmet sind, gegeben. Die Bedeutungskonstruktion, die abgeschlossen zu sein scheint, fallt negativ aus.

Hiermit soll die Betrachtung der angesprochenen Indices beendet werden.

\subsubsection{Zusammenfassung}

In der folgenden Zusammenfassung der Analyseergebnisse in bezug auf die Verwendung unterschiedlicher Index-Typen im untersuchten Diskurs soll festgehalten werden, welche charakteristischen Textgenerienungsregeln feststellbar sind, und inwiefern von einer Introspektive auf den Bereich der Sowjetunion und deren Nachfolgestaaten bzw. die untersuchte Formation gesprochen werden kann.

Aufgrund der relativ geringen Anzahl an unspezifischen Indices wurde der Frage, ob diese anders als spezifische funktionalisient werden, nicht nachgegangen. Es soll auf der Grundlage der Analyseergebnisse dennoch die Vermutung geäußert werden, daß eine unterschiedliche Funktionalisierbarkeit sehr wahrscheinlich ist, da unspezifische Indices offenbar keine kulturelle Bedeutung aufweisen können. 
Die Verwendung von Indices der Kategorien Personen, Gruppen sowie Geographica und Institutionen genügt dem eingefuihrten Bewertungskriterium, so daß jeweils von einer charakteristischen Textgenerierungsregel ausgegangen wird. Die Indices der Kategorien Marken, Ereignisse und Sonstige genügt dem genannten Bewertungskriterium nicht.

Keine Introspektive kann im Fall der Residualkategorie diagnostiziert werden. Ansonsten liegt eine Introspektive auf die kulturelle Formation nur im Fall der Kategorie Ereignisse vor. Die verbleibenden Kategorien zeigen eine Introspektive auf die Sowjetunion bzw. deren Nachfolgestaaten.

\subsection{Autoritäten}

Im folgenden Abschnitt geht es um die im Korpus ermittelten Autoritäten, d.h. um indexikalische Zeichen, die die Eigenschaft der Zitierbarkeit aufweisen. In Tabelle 25 sind die im Korpus nachgewiesenen Autoritäten und die ihnen beigestellten Beispiele (Zitate) aufgeführt.

Tabelle 25

\begin{tabular}{|c|c|c|}
\hline $\mathbf{A E}$ & Autoritat & Beispiel (Zitat) \\
\hline 3 & fany & 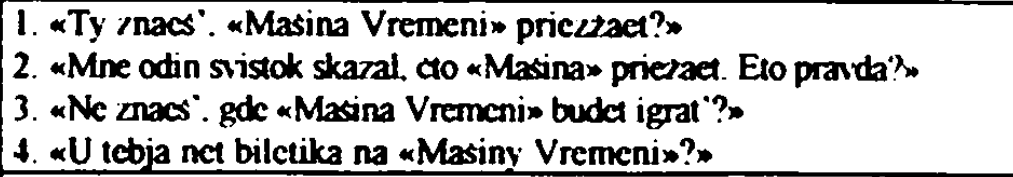 \\
\hline 4 & Boris Grebenscikor & $\begin{array}{l}\text { 1. «Rcbjata lowjat svoj kajf». } \\
\text { 2. «Voera ja scl domoj } \\
\text { Krugom byla vesna } \\
\text { Ego ja vstretil na uglu. } \\
\text { I v nem ne ponjal ni chrena. } \\
\text { Sprosıl on Byi ili ne byt } \\
\text { A ja skayal: Idi ty na } \\
\end{array}$ \\
\hline 5 & Milologeceskaja cnciklopoodija & $\begin{array}{l}\text { Pokolenija deljatsja na pokoleme bogov. pokolenic ljudej I } \\
\text { promesutocnyc pokolenija - cudovisc. velikanov i karlikov }\end{array}$ \\
\hline 6 & Igor Kochanor ski] & 1. „Pustoc slovo vscgda minucl cunstro stydax. \\
\hline 7 & Alcksandr Puskin & 1. «Ne dostoin buduscego narod. ne /najuscij stocgo proslogow. \\
\hline 10 & Urfin D/jus & $\begin{array}{l}\text { 1. Po/arnyc so/dany. } \\
\text { Croby sledit za porjadkom. } \\
\text { Esli narusimsja ras nor csic } \\
\text { Mcydu gorjascim toboj } \\
\text { I okru/ajuscej } \\
\text { Cholodnoj srodoj. }\end{array}$ \\
\hline 11 & $\begin{array}{l}\text { Michail Zoicenko: geroinja rasskara } \\
\text { Zoscenko \&Tragikomedijaw }\end{array}$ & 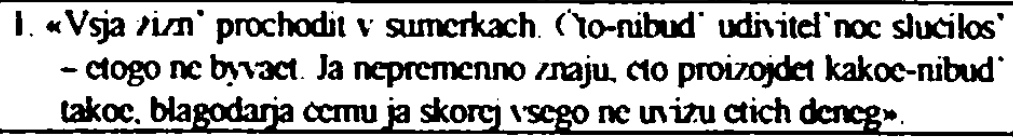 \\
\hline 13 & Klara lvanorna/Petr Scmenovic & 1. «Kakaja naglost ${ }^{\circ}$. \\
\hline 14 & Karl Mari & 1. «Proletarii vsech stran soedinjajtes! ! \\
\hline 15 & Rok-Foderacija & 1. «cto delat ?" \\
\hline 17 & Egor Letor & 1. «Odinocki opasnee dlja sociuma cem celoc dvirenic». \\
\hline 19 & Alcxandrc Dumas & $\begin{array}{l}\text { 1. «Strannaja. nclcgkaja dlja istorikov i filosofov zadaca - nonjat } \\
\text { kak my dosli do risni takoj? }\end{array}$ \\
\hline 19 & D. Sclivanor & 1. akniznaja kul tura mern ax \\
\hline
\end{tabular}




\begin{tabular}{|c|c|c|}
\hline 20 & Dza Divizn & 1. «U menja tvoja golova. v nebe dvizetsja troja golova». \\
\hline 20 & $\begin{array}{l}\text { srednestatisticeskij zritel } \\
\text { rastamanskich koncertov }\end{array}$ & 1. *Otdajte mne moju golovu!». \\
\hline 20 & Konstantin Kincer & 1. «Nastalo vremja menjat'!* \\
\hline 20 & alisomany & 1. «Uraaa! Vremja menjat' imena!» \\
\hline 22 & Sasa Sokolor & $\begin{array}{l}\text { 1. ... Neposredstvenno za dvorom berut nacalo zachorki. Za nimi } \\
\text { ugadtrajutsja: kabak stagbaum. versty razmytogo trakta. ostrog. } \\
\text { Sibir' i pogost. }\end{array}$ \\
\hline 26 & L. Doros & 1. "Komu podygryval intellektual'nyj rok?" \\
\hline 27 & Aleksandr Galic & $\begin{array}{l}\text { 1. Ja vybiraju svobody, } \\
\text { No ne iz boja. a v boj. } \\
\text { Ja vybiraju svobody. } \\
\text { Byt prosto samim soboj. }\end{array}$ \\
\hline 27 & starye druz.ja & 1. "cto eto, mol. ty na sebja nacenil?" \\
\hline 30 & Aleksandr Baslacer & $\begin{array}{l}\text { 1. Cas priliva } \\
\text { Cas priliva probil. } \\
\text { Razbezalis' } \mathrm{i} \text { nymuli. } \\
\text { Kto sumel - tot uplyl. } \\
\text { Ostal 'nye utonuli. } \\
\text { A my s toboj otpolyli } \\
\text { I legli na meli. } \\
\text { My se pocetnom karaule. }\end{array}$ \\
\hline 35 & TAZ: Telefonaja Agentura Zueva & $\begin{array}{l}\text { 1. "27/11-91 v DK na ploscadi Puskina sostojalsja konoent s ucastiem } \\
\text { gnupp: "Natasa Rostova". Usastye Usi" Vtorzenie Iane". }\end{array}$ \\
\hline
\end{tabular}

Zunächst wird das Ergebnis in quantitativer Hinsicht analysiert, bevor auf die kulturelle Bedeutung der einzelnen Autoritäten eingegangen wird. Es können insgesamt 23 verschiedene Autoritäten in 18 Analyseeinheiten $(51,4 \%)$ ermittelt werden, so daß die Verwendung von Autoritäten kein charakteristisches Textgenerierungsmerkmal des untersuchten Diskurses darstellt. Die durch die unspezifischen Indices Klara Ivanovna und Petr Semenovic bezeichneten Aktanten, die in Analyseeinheit 13 genannt werden, werden in diesem Zusammenhang als eine Autorität aufgefaßt, da ihnen nur ein Beispiel zugeschrieben wird. Die Häufigkeit der Verwendung verschiedener Autoritäten in den einzelnen Analyseeinheiten schwankt zwischen 0 und 4, wobei die ein- bzw. keinmalige Verwendung mit $40 \%$ bzw. $45,7 \%$ am wahrscheinlichsten ist. In diesem Sinne liegt keine charakteristische Verwendungshäufigkeit vor.

Lediglich zwei Autoritaten (8,7\%), Karl Marx und Alexandr Dumas, entstammen nicht dem sowjetischen bzw. postsowjetischen Kultursystem, so daß in einem ersten Schritt von einer Introspektive bezogen auf das genannte System gesprochen wird. Mit hoher Wahrscheinlichkeit können 10 verschiedene Autoritäten (43,5\%: fany, Boris Grebenścikov, Urfin Dijus, Egor Letov, Dła Divizn, srednestatisticeskij zritel' rastamanskich koncertov, Konstantin Kincev, alisomany, starye druz'ja und Aleksandr Baslacev) der kulturellen Formation des rok-samizdat zugerechnet werden, so daß in einem zweiten Schritt gleichfalls nicht von einer Introspektive auf die untersuchte Formation gesprochen wird.

Der Reihenfolge der Verwendung nach sollen die ermittelten Autoritäten hinsichtlich ihrer kulturellen Relevanz und Bedeutung analysiert werden. Auf Grebenscikov, Puskin und Letov wird an dieser Stelle nicht eingegangen, da die kulturelle Bedeutung dieser Autoritäten bereits 
im Rahmen der Personen-Index-Analyse erläutert wurde (vgl:: 9.9.2.). Es wird lediglich betrachtet, welche Funktion die jeweiligen Zitate in bezug auf die Analyseeinheit besitzen, in denen die genannten Aktanten verwendet werden.

Der Gruppe fany werden in Analyseeinheit 3 vier verschiedene Zitate zugesprochen, in denen es um ein Gerucht hinsichtlich des Auftritts der Gruppe Masina Vremeni in Leningrad im Oktober 1977 geht. Die Autorität, zu der sich der Autor der Analyseeinheit selbst zähit, wird nicht explizit attribuiert, so daß nicht auf deren kulturelle Bedeutung geschlossen werden kann. Da die Autorität offensichtlich nicht der Steigerung des Wahrheitsgehalts der Analyseeinheit dient, besitzt sie vermutlich primar eine textuelle Funktion: durch sie wird die Analyseeinheit authentischer. In diesem Sinne sind die Autorität sowie die ihr zugeschriebenen Aussagen an dieser Stelle nicht kulturell relevant.

Grebenscikov tritt in Analyseeinheit 4 zweimal als Autorität auf. Die erste Aussage, eine Sentenz (vgl. 9.11.), ist dem Text vorangestellt und dient vermutlich der Erhöhung des Wahrheitswerts des Textes. Grebenscikov und die ihm zugeschriebene Aussage sind an dieser Stelle in kultureller Hinsicht funktionalisiert, sie sind daher kulturell relevant. Im zweiten Fall wird ein Liedtext zitiert. In diesem Fall liegt die Vermutung nahe, daß nicht die Aussage des Textes, sondern das Lied relevant ist: ,ja poezilsja ot sladkogo oscuscenija, cto vpervye slysu natural'nyj rok na russkom jazyke", wie der Verfasser der genannten Analyseeinheit anmerkt. Das Beispiel erfült an dieser Stelle vermutlich primär eine textuelle Funktion, die der Authentizitatssteigerung dient, so daß an dieser Stelle nicht von einer kulturellen Relevanz des Beispiels und der Autorität ausgegangen wird.

Der durch den Marken-Index bezeichneten Autorität Mifologiceskaja enzyklopedija wird in Analyseeinheit 5 ein dem Text vorangestelltes Zitat zugesprochen, in dem es in einem weiteren Sinne um die Dreiteilung der Gesellschaf bzw. der untersuchten Formation geht. Es kann davon ausgegangen werden, daß durch die Angabe einer Autoritat eine Steigerung des Wahrheitsgehalts des Textes erzielt werden soll. Dementsprechend mussen Autoritat und Zitat eine kulturelle Relevanz und eine positive kulturelle Bedeutung besitzen.

Igor' Kochanovskij, ein zeitgenössischer, russischer Schriftsteller, besitzt vermutlich dieselbe Funktion wie die soeben erlauterte Autorität: die ihm zugeschriebene Aussage, die wiederum dem Text vorangestellt ist, bewirkt mit hoher Wahrscheinlichkeit eine Erhöhung des Wahrheitsgehalts des nachfolgenden Textes. Auch Kochanovskij besitzt dementsprechend vermutlich sowohl eine kulturelle Relevanz sowie eine positive kulturelle Bedeutung, die vollständig konstruiert zu sein scheint, da sie nicht expliziert wird.

Die Puskin in Analyseeinheit 7 zugeschriebene Aussage, eine Sentenz (vgl: 9.11.), dient mit hoher Wahrscheinlichkeit der Erhöhung des Wahrheitswertes der Analyseeinheit. Puskin und das Zitat sind dementsprechend an dieser Stelle kulturell relevant.

Die Musikgruppe Urfin Dzjus, die in Analyseeinheit 10 als Urheber eines Beispiels fungiert, wird vermittels des Normativs znamenityj attribuiert, was auf eine kulturelle Relevanz hinweist. 
Es ist sehr wahrscheinlich, daß die genannte Gruppe in der untersuchten Formation eine positive Bedeutung aufweist. Die Aussage, die auf den Roman 451 Grad Fahrenheit von Ray Bradbury zurückgeht, dient ebenfalls der Steigerung des Wahrheitsgehaltes des Textes. Bemerkenswert ist in diesem Zusammenhang, daß nicht Bradbury, sondern die Gruppe als Autorität eingesetzt wird. Die Gruppe ist in diesem Sinne fur die untersuchte Formation in kultureller Hinsicht relevanter als der Autor des Romans.

Die Aussage, die durch den spezifischen Personen-Index geroinja rasskaza Zoścenko «Tragikomedija» vermittelt wird, soll hier dem Urheber des genannten Aktanten, Michail Zoscenko, einem russischen Schriftsteller, zugesprochen werden. Die Aussage dient vermutlich ebenfalls der Steigerung des Wahrheitsgehalts des Textes. Zoscenko selbst, der als kulturell relevant angesehen wird, wird nicht attributiert, was auf einen abgeschlossenen Bedeutungskonstruktionsproze $B$ hinweist, dessen Resultat mit hoher Wahrscheinlichkeit positiv ist.

Die Doppelbelegung der Autorität in Analyseeinheit 13 mit den Aktanten Klara Ivanovna und Petr Semenovic weist darauf hin, daß die dortige Aussage offensichtlich nicht der Steigerung des Wahrheitsgehalts des Textes dient. Es liegt die Vermutung nahe, daß die Autorität und das Beispiel primär eine textuelle Funktion besitzen. Der Absatz, in dem die Autorität genannt wird, stellt einen Einschub dar, der mit dem Rest des Textes inhaltlich nur bedingt in einem Zusammenhang steht. Vermutlich dient der Einschub, der in gewisser Hinsicht eine Anekdote darstellt, der Auflockerung des Textes.

Karl Marx, der nicht attribuiert wird, fungiert in Analyseeinheit 14 als Autorität, indem ihm die Urheberschaft in bezug auf die Sentenz „Proletarii vsech stran, soedinjaites'!“ zugesprochen wird. Die fehlende Attribuierung legt den Schluß nahe, daß die Bedeutungskonstruktion bezogen auf Marx als abgeschlossen angesehen werden kann. Da es in der Analyseeinheit inhaltlich um die Obrigkeit (KPSS, KGB) und deren Handlanger (ljubera, kazancy) geht, Marx in gewisser Hinsicht als Ausloser der in der Analyseeinheit geschilderten Prozesse aufgefaßt wird. sollte von einer negativen kulturellen Bedeutung im Rahmen der untersuchten Formation ausgegangen werden. Das kulturell relevante Beispiel dient in diesem Sinne, bezogen auf den ebenfalls kulturell relevanten Marx, der Diskreditienung.

In Analyseeinheit 15 geht es um die zweite Allunionskonferenz der Institution RokFederacija. Die Institution selbst wird nicht erläutert, es geht in der genannten Analyseeinheit vieimehr um Einzelheiten der Konferenz. Die Bedeutungskonstruktion in bezug auf die diskutierte Institution soll dementsprechend als abgeschlossen angesehen werden. $\mathrm{Ob}$ die kulturelle Bedeutung positiv oder negativ ist, kann aus der Analyseeinheit nicht abgeleitet werden. Das Beispiel, eine Frage, ist in dieser Hinsicht nicht aufschlußreich, da es vermutlich primär die textuelle Funktion der Authentizitätssteigerung erfült. 
Letov fungiert in Analyseinheit 17 als Urheber einer Sentenz (vgl.: 9.11), die vermutlich eingesetzt wird, um den Wahrheitswert der Analyseeinheit zu steigern. Sowohl Letov als auch die Sentenz sind an dieser Stelle für die untersuchte kulturelle Formation relevant.

In Analyseeinheit 19 werden zwei Autoritäten, Alexandre Dumas und D. Selivanov, bemüht. Beide werden nicht attribuiert, so daß von einer abgeschlossenen Bedeutungskonstruktion ausgegangen werden kann. Die den Autoritäten zugeschriebenen Aussagen dienen vermutlich der Steigerung des Wahrheitsgehaltes des Textes, so daß beide eine positive kulturelle Relevanz und Bedeutung aufweisen.

In Analyseeinheit 20 werden vier verschiedene, kulturell relevante Autoritäten bemüht, von denen zwei, die Gruppe Dza Divizn und Konstantin Kincev, seit 1985 Sänger der Leningrader Heavy-Metal Gruppe Alisa, vom Verfasser dem Bereich der rok-poezija zugerechnet werden, den es seiner Ansicht nach zu bekämpfen gilt. Die Guppe Dza Divizn wird, bis auf einige Einzelheiten der von ihr verwendeten Bühnendekoration, nicht attribuiert. Trotz des offensichtlichen Erfolgs, den die Gruppe besitzt, wird sie samt ihrer Fans, bezeichnet als srednestatisticeskij zritel' rastamannskich koncertov, negativ konstruiert. Die banalen Aussagen, die der Guppe und den Fans zugesprochen werden, dienen in diesem Sinne der Diskreditienung. Kincev wird im Gegensatz zu seinen Fans, den alisomany, die als Herde (stado) bezeichnet werden, nicht explizit konstruiert. Emeut wirken die banalen Aussagen, die Kincev bzw. seinen Fans zugesprochen werden, diskreditierend.

In Analyseeinheit 22 ist die Aussage, die der Autorität Sasa Sokolov zugesprochen wird, dem Text vorangestellt. Sokolov, Autor des Romans Skola durakov, wird selbst nicht attribuiert, so daß von einer abgeschlossenen Bedeutungskonstruktion ausgegangen wird. Das Zitat dient, wie bereits mehrfach diagnostiziert, der Steigenung des Wahrheitsgehalts der Analyseeinheit Dementsprechend kann von einer positiven kulturellen Bedeutung der genannten Autoritat ausgegangen werden.

L. Doros wird als Urheber eines Zeitungsartikels genannt, der in Analyseeinheit 26 besprochen wird. Doros ist mit hoher Wahrscheinlichkeit im Rahmen der kulturellen Formation des rok-samizdat unbekannt: „[...] avtor L. Doros. Ljubopytnoe nazvanie“. Er stellt in diesem Sinne einen in kultureller Hinsicht nicht funktionalisierten Aktanten dar, der keine kulturelle Bedeutung aufweist. Die Einfuhrung des Aktanten erfolgt im Sinne der Authentizitätssteigerung der betroffenen Analyseeinheit.

Der Urheber des Zitats, welches Analyseeinheit 27 vorangestellt ist, ist Aleksandr Galic, der zum Umfeld des KSP gehört. Er erhielt für seine Arbeiten verschiedene Preise, wurde jedoch 1974 aus der Sowjetunion ausgebürgert. 1988, nach seinem Tod, wurde er in der Sowjetunion rehabilitiert. In der genannten Analyseeinheit wird Galic nicht attribuiert, die auf ihn bezogene Bedeutungskonstruktion scheint abgeschlossen zu sein. Die zitierte Aussage dient offensichtlich der Wahrheitswertsteigerung des nachfolgenden Textes, so daß Galic mit hoher Wahrscheinlichkeit eine positive kulturelle Bedeutung in der untersuchten Formation 
aufweist. Die zweite Autorität, die in Analyseeinheit 27 bemüht wird, wird durch den unspezifischen Gruppen-Index starye druz'ja bezeichnet. Aufgrund der Aussage, die der Gruppe zugesprochen wird, entsteht ein Antagonismus, in den neben der Gruppe der Verfasser der Analyseeinheit involviert ist. Die Gruppe starye druz'ja wird nicht attribuiert, so daß von einer abgeschlossenen Bedeutungskonstruktion ausgegangen werden kann. Das Zitat selbst kann in textueller Hinsicht als Ausgangspunkt für die nachfolgenden Überlegungen des Verfassers aufgefaßt werden. Es dient in diesem Sinne nicht der Erhöhung des Wahrheitsgehalts des Textes, sondern der Diskreditienung der genannten Gemeinschaft.

Die Überschrift von Analyseeinheit 30 (Cas priliva) geht auf Aleksandr Baslacev zurück, die in der betroffenen Analyseeinheit in erweiterter Form nachweisbar ist und das eigentliche Beispiel darstellt. Basłacev, ein Musiker und Sänger, der 1988 trotz größerer Erfolge Selbstmord beging, wird nicht attribuiert. Seine kulturelle Bedeutung fur die analysierte kulturelle Formation scheint folglich festzustehen. Das Zitat dient wiederum der Wahrheitswerterhöhung des Textes, was für eine positive Bedeutung und Relevanz der diskutierten Autorität spricht.

Das letzte im Korpus nachweisbare Zitat ist eine offensichtliche Falschmeldung, die einer fiktiven Institution namens TAZ - Telefonnaja Agentura Zueva zugesprochen wird (vgl: 9.9.5.). Diese Institution wird nicht attribuiert. Die Meldung ist im Stil der Meldungen der offiziellen Institution Telegrafnoe Agenstvo Sovetskogo Sojusa - TASS verfaßt. Mit hoher Wahrscheinlichkeit stellt die Falschmeldung eine Anspielung auf diese offizielle Institution dar. Sollte diese Vermutung zutreffen, so kann von einer negativen kulturellen Bedeutung der offiziellen Institution im Rahmen der untersuchten kulturellen Formation ausgegangen werden. In diesem Fall dient das Zitat dementsprechend der Diskreditienung der Institution TASS.

Abschließend soll betrachtet werden, ob Regelmäßigkeiten in bezug auf die Funktionalisierung bestimmter Index-Typen als Autoritäten innerhalb des Korpus nachweisbar sind, um die Hypothese der unterschiedlichen Funktionalisierbarkeit von spezifischen und unspezifischen Indices erneut aufzugreifen.

Im Korpus können sieben verschiedene Index-Typen ermittelt werden: spezifische und unspezifische Gruppen-Indices, spezifische und unspezifische Personen-Indices, von denen ein spezifischer nicht funktionalisiert ist, ein Marken-Index, sowie Institutionen-Indices. Die Beispiele, die den Autoritäten zugesprochen werden, erfullen verschiedene Funktionen, die als Wahrheitswertsteigerung des Textes, Diskreditienung der Autorität, Authentizitätssteigerung des Textes und Auflockenung des Textes bezeichnet werden können. In Tabelle 26 ist dargestellt, wie diese Funktionen auf die genannten Index-Typen verteilt sind. 
Tabelle 26

\begin{tabular}{|c|c|c|c|c|c|c|c|c|c|}
\hline & \multicolumn{3}{|c|}{ Personen-Index } & \multicolumn{2}{|c|}{ Gruppen-Index } & \multirow[t]{2}{*}{ Institut. } & \multirow[t]{2}{*}{ Marken } & \multirow[t]{2}{*}{$\Sigma$} & \multirow[t]{2}{*}{$\%$} \\
\hline & spez+fiuld & $\begin{array}{c}\text { spez-t } \\
\text { nicht-fink }\end{array}$ & unspez. & spez. & unspez. & & & & \\
\hline Wt toicuet & 11 & - & - & - & - & - & 1 & 12 & 50 \\
\hline Dewolioung & 2 & - & - & 1 & 3 & 1 & - & 7 & 29.1 \\
\hline Al thentiven & 1 & 1 & - & - & 1 & 1 & - & 4 & 16,7 \\
\hline Al hodering? & - & - & 1 & - & - & - & - & 1 & 4.2 \\
\hline$\Sigma$ & 14 & 1 & 1 & 1 & 4 & 2 & 1 & 24 & \\
\hline$\%$ & 58.3 & 4.2 & 4.2 & 4.2 & 16.7 & 8.3 & 4.2 & & \\
\hline
\end{tabular}

Zunächst wird anhand von Tabelle 26 deutlich, daß keine Funktion auf einen bestimmten Index-Typ beschränkt ist. Dennoch gibt es zwei Regelmäßigkeiten: überdurchschnittlich häufig treten funktionalisierte, spezifische Personen-Indices auf, die somit offensichtlich als Autorität am geeignetsten sind. Diese Autoritäten werden vorwiegend eingesetzt, um den Wahrheitswert eines Textes zu steigern. Lediglich in bezug auf den Wahrheitswert kann die Hypothese, daß unspezifische Indices andere Funktionen übernehmen als spezifische, nicht falsifiziert werden. Die verbleibenden Daten lassen keine Regelmäßigkeiten erkennen, so daß die Analyse in bezug auf die im Korpus ermittelten Autoritäten hiermit beendet wird.

\subsection{Sentenzen}

Bevor mit der Diskussion des Analyseergebnisses in bezug auf Sentenzen begonnen wird, soll darauf hingewiesen werden, daß im Korpus feststehende Ausdrücke erfaßt werden, die in theoretischer, nicht empirischer Hinsicht Sentenzen darstellen. Ob neben der im Korpus festgestellten Anwendung weitere Realisienungen existieren, kann nicht eindeutig gesagt werden, so daß der empirische Beleg bei der Bestimmung der Sentenzen fehlt. Trotz dieser Einschränkung konnen die Ergebnisse in produktiver Weise analysiert werden. In Tabelle 27 ist, soweit dies moglich ist, erfaßt, welche Sentenzen im Korpus nachgewiesen werden konnen, welche Indices für die Urheber eingeführt werden und welche Urheber «tatsächlich» für die Sentenzen verantwortich sind

Tabelle 27

\begin{tabular}{|c|c|c|c|}
\hline $\mathbf{A E}$ & Sentem & Index & Urbeber \\
\hline 4 & Rcbjata lovjat svoj kajf. $|2|$ & B. Grebenscikov: & Boris Grebenscikov \\
\hline 4 & But' ili ne byt'. & - & William Shakespeare \\
\hline 4 & Karma sdelal svoe delo. & - & - \\
\hline 6 & Pustoe slovo vsegda minuet curstro styda. & I. Kochanowskij & Igor" Kochanow skij \\
\hline 7 & $\begin{array}{l}\text { Fol klorno-ctnograficeskoc norcestio - cto istorija } \\
\text { naroda. kul turnyj cgo plast. }\end{array}$ & - & - \\
\hline 7 & Nc dostoin budrecogo narod nc majucij suocso prostogo. & nas Alcksandr Scrgecric & Alcksandr Proskin \\
\hline 10 & Welcome to the machine! & - & Pink Floyd \\
\hline 14 & Umiracm no nc scacmsja. & - & - \\
\hline 14 & Prolctarii vsech stran soedinjajtes'! & Karl Marx & Karl Marx \\
\hline 17 & Odinocki opasnec dlja sociuma. cem celoc dvirenic. & Egor Letov & Egor Lctor \\
\hline 27 & Ja ne verbljud. & - & Vladimir Vysockij \\
\hline
\end{tabular}


Bevor auf die kulturelle Relevanz und Bedeutung der Urheber der ermittelten Sentenzen eingegangen wird, soll das Ergebnis zunächst in quantitativer Hinsicht analysiert werden: es liegen 11 verschiedene Sentenzen vor, von denen eine zweimal in einer Analyseeinheit verwendet wird. Die Sentenzen verteilen sich auf 7 Analyseeinheiten (20\%), so daß deren Verwendung kein charakteristisches Textgenerierungsmerkmal des untersuchten Diskurses darstellt. Die Verwendungshäufigkeit schwankt zwischen 0 und 3, wobei die Häufigkeit 0 mit $80 \%$ am wahrscheinlichsten ist. In diesem Sinne ist das Fehlen von Sentenzen in Texten. die in der untersuchten Formation generiert werden, ein charakteristisches Textmerkmal. Drei Sentenzen (27,3\%: Byt' ili ne byt', Welcome to the machine, Proletarii vsech stran soedinjajtes'!) entstammen nicht dem sowjetischen bzw. postsowjetischen Kultursystem, so daß eine Introspektive auf das genannte System diagnostizient wird. Der untersuchten Formation werden die Sentenzen zugeordnet, die auf Boris Grebenscikov und Egor Letov zurückgeführ werden können. Da es sich hierbei nur um zwei Sentenzen $(18,2 \%)$ handelt, kann keine Introspektive auf die untersuchte Formation festgestellt werden.

In qualitativer Hinsicht wurde bereits angemerkt, daß die primäre, diskursive Funktion der Sentenz nicht in ihrer Aussage, sondern vielmehr in der Textmarkierung begründet ist ( $\mathrm{vgl}$ : 5.11.). Ob die ermittelten Sentenzen diese Funktion hypothetisch erfullen, gilt es zu prüfen. Eine inhaltliche Analyse der ermittelten Sentenzen ist nicht notwendig. Des weiteren soll versucht werden, Schlüsse hinsichtlich der kulturellen Bedeutung der jeweiligen Urheber zu ziehen. Auf Grebenscikov, Kochanovskij. Puskin, Marx und Letov wurde bereits im Zusammenhang mit Personen-Indices (vgl.: 9.9.2.) und Autoritäten (vgl.: 9.10.) eingegangen, so daß an dieser Stelle auf eine erneute Charakterisierung verzichtet wird.

Die Sentenz „Rebjata lovjat svoj kajf“ beinhaltet einerseits ein Element (lovit' kajf), welches als Element des Diskursiva-Repertoires erkannt wurde (vgl: 9.6.), andererseits geht sie auf ein kulturell relevantes Mitglied der untersuchten Formation zurück. In diesem Sinne liegt die Vermutung nahe, daß sie einen Text in diskursiver Hinsicht markient, sie ist in diesem Sinne für die untersuchte Formation relevant

Die Sentenz „Byt' ili ne byt”“ geht auf Shakespeare zurück, die dieser Hamlet, dem Helden der gleichnamigen Tragödie, in den Mund legt. In empirischer Hinsicht kann von einer weiten Verbreitung ausgegangen werden. Es liegt die Vermutung nahe, daß die Sentenz nicht geeignet ist, den rok-samizdat-Diskurs zu markieren, sondern daß sie vielmehr dem Interdiskurs zugerechnet werden muß. Wenn diese Vermutung zutriff, dann besitzt Shakespeare im Rahmen der untersuchten Formation keine kulturelle Bedeutung und Relevanz. Gleiches gilt fur die genannte Sentenz.

Über den Unheber der Sentenz „Karma sdelal svoe delo“ liegen keine Erkenntnisse vor. Möglicherweise stammt sie von Artem Troickij, dem Verfasser der betroffenen Analyseeinheit. Es liegen ebenfalls keine Erkenntnisse uber die empirische Verbreitung der Sentenz vor. In theoretischer Hinsicht ist sie geeignet, den untersuchten Diskurs zu markieren, da sie mit dem 
Element Karma ein Diskursivum (vgl.: 9.6.) und einen Index (vgl.: 9.9.7.) enthät. Dariber hinaus kann an der vorliegenden Stelle von einer kulturellen Funktion ausgegangen werden, da keine textuelle feststellbar ist: die Sentenz ist in diesem Sinne furr die Textkohärenz nicht notwendig. Da der Urheber der Sentenz nicht ermittelbar ist, können keine Schlüsse über dessen kulturelle Bedeutung und Relevanz gezogen werden. In bezug auf die Sentenz hingegen kann von einer kulturellen Relevanz ausgegangen werden.

Auch beäglich der empirischen Verbreitung der Aussage „Pustoe slovo vsegda minuet cuvstvo styda“ liegen keine Erkenntnisse vor. In diskursiver Hinsicht weist sie keine auffalligen Besonderheiten auf, so das sie vermutlich nicht geeignet ist, einen Text in bezug auf den untersuchten Diskurs zu markieren. Wenn die genannte Sentenz den untersuchten Diskurs markiert, dann wird diese Funktion mit hoher Wahrscheinlichkeit von der Autorität Kochanovskij gewährleistet. In diesem Sinne besitzt diese, wie im vorangehenden Kapitel erlautert, eine positive kulturelle Bedeutung und Relevanz. Die Sentenz erfullt ihre Funktion, die als Wahrheitswertsteigerung des Textes bezeichnet wurde (vgl.: 9.10.), aufgrund der Autorität. Im Zusammenhang mit dieser ist sie kulturell relevant, ohne diese ist sie es vermutlich nicht.

Genauso verhält es sich mit der Aussage „Folkjorno-etnograficeskoe tvorcestvo - eto istorija naroda kul'turnyj ego plast", die vermutlich von Sergej Zarikov stammt, dem Autor der betroffenen Analyseeinheit. Zarikov (vgl.: 9.9.3.) ist mit hoher Wahrscheinlichkeit ein kulturell relevantes und bedeutendes Mitglied der untersuchten Formation, so daß er pradestiniert ist, Sentenzen einzubringen. Die Sentenz ist vermutlich nicht geeignet, den untersuchten Diskurs zu markieren, da sie keine diskursiven Besonderheiten aufweist. Sie ist vielmehr geeignet, den russischen Interdiskurs zu markieren, so daß sie für diesen relevant ist.

Die Aussage Puskins, der lediglich durch den Index nas Aleksandr Sergeevic bezeichnet wird, ist ebenfalls nicht geeignet, den untersuchten Diskurs zu markieren. Da zudem von einer hohen Verbreitung ausgegangen werden kann, ist sie, analog zu der Sentenz \%arikovs, geeignet, den Interdiskurs, fur den sie dementsprechend relevant ist, zu markieren.

"Welcome to the machine!" ist der Titel einer Schallplatte der englischen Rock-Gruppe Pink Floyd. Aufgrund der Bekanntheit der Gruppe und der Schallplatte kann von einer weiten Verbreitung der Sentenz in bestimmten kulturellen Formationen ausgegangen werden. Im vorliegenden Fall bezieht sich die genannte Sentenz auf die Eroffnung der Institution Moskovskaja rok-laboratorija (vgl.: 9.9.6.), so daß sie in kultureller Hinsicht funktionalisiert ist Die Sentenz ist in englischer Sprache formuliert und stellt in diesem Sinne kein Element des russischen Interdiskurses dar Da der Zusammenhang mit dem Phanomen Rock-Musik offensichtlich ist, markient die angesprochene Sentenz mit hoher Wahrscheinlichkeit den Diskurs der untersuchten kulturellen Formation. In bezug auf die genannte Rock-Gruppe kann daruber hinaus von einer positiven kulturellen Relevanz und Bedeutung im Rahmen der untersuchten Formation ausgegangen werden. 
Mit der Aussage „Umiraem, no ne sdaemsja“ verhält es sich wie mit der Sentenz, die vermutlich auf "̈arikov zurückgeht: sie zeichnet sich weder durch offensichtliche Auffalligkeiten in diskursiver Hinsicht aus, noch ist etwas über ihre Verbreitung bekannt. Ob Zajcev, Verfasser der angesprochenen Analyseeinheit, auf den die Sentenz vermutlich zurückgeht, bekannt ist und Sentenzen einzubringen vermag, kann nicht gesagt werden. Hypothetisch wird davon ausgegangen, daß die genannte Sentenz den untersuchten Diskurs markiert und fur die untersuchte Formation relevant ist. Die Markienungsfunktion hängt in diesem Sinne von den einzelnen Elementen der Sentenz ab.

Auf die die Autorität Marx diskreditierende Aussage „Proletarii vsech stran soedinjajtes!“ wurde bereits eingegangen (vgl: 9.10.). Die Aussage stellt eine Sentenz dar, die in der ehemaligen Sowjetunion sehr verbreitet war (u.a. zierte sie das Titelblatt der Tageszeitung Pravda). Sie war dementsprechend ein stark funktionalisiertes und relevantes Element des Interdiskurses. Wie gezeigt werden konnte, kann sie im Rahmen der untersuchten Formation, auf der genannten Funktionalisierung aufbauend, eingesetzt werden, um zu diskreditieren, d.h. auszugrenzen. Es ist in diesem Sinne möglich, vermittels ihrer Verwendung, Antagonismen zu generieren. Sie ist in diesem Sinne auch für die untersuchte Formation relevant.

Für die Sentenz, die auf Letov zurückgeht, triff das zu, was bereits im Zusammenhang mit der Aussage von Kochanovskij gesagt wurde, so daß auf die genannte Stelle verwiesen wird.

Abschließend soll die Sentenz, die auf Vysockij zurückgeht, betrachtetet werden. Vysockij ist neben Bulat Okudzava sicherlich der bekannteste russische, regimekritische Sänger der sechziger und siebziger Jahre. Die Sentenz „Ja ne verbljud“, die nicht hinsichtlich ihres Urhebers gekennzeichnet ist, wird im vorliegenden Zusammenhang als Beispiel funktionalisiert. Die Markierungsfunktion beruht mit hoher Wahrscheinlichkeit auf der kulturellen Bedeutung und Relevanz von Vysockij. Es ist fragwürdig, inwiefern die genannte Sentenz geeignet ist, den untersuchten Diskurs za markieren, da von einer weiten Verbreitung ausgegangen wird. Im vorliegenden Fall ist sie funktionalisiert und daher fur die kulturelle Formation relevant.

Wie in den vorangehenden Abschnitten gezeigt werden konnte, sind nicht alle im Korpus nachgewiesenen Sentenzen geeignet, den untersuchten Diskurs zu markieren. Dennoch sind alle Sentenzen im vorliegenden Zusammenhang fur die untersuchte Formation relevant. Bis auf Shakespeare weisen vermutlich alle ermittelbaren Urheber eine kulturelle Relevanz und Bedeutung auf. Hiermit soll die Betrachtung der ermittelten Sentenzen und ihrer Urheber beendet werden. 


\subsection{Abgrenzungen und interne Differenzierungen}

In den folgenden beiden Abschnitten geht es um diejenigen Objekte der Zweiten Wirklichkeit, die primär der Abgrenzung bzw. Differenzierung dienen: Antagonismen und Stereotype, wobei Stereotype eine Untergruppe der Antagonismen darstellen. Mit ihrer Hilfe gelingt es den Mitgliedern kultureller Systeme, sich von Aktanten, Gemeinschaften, Institutionen u.dgl.m. abzugrenzen, $d . h$. diese aus dem eigenen System auszugrenzen und Differenzierungen innerhalb der eigenen Formation vorzunehmen. Da die Elemente, die im Rahmen von Antagonismen aufteten, in kultureller Hinsicht funktionalisiert sind, besitzen sie für das ausgrenzende bzw. differenzierende System kulturelle Relevanz. Es existieren verschiedene sprachliche Moglichkeiten, Abgrenzungen und Differenzierungen vorzunehmen: eine sprachliche ist die Generierung von Stereotypen. Die anderen, auf die in sprachlicher Hinsicht nicht explizit eingegangen wird, werden unter dem Begriff Antagonismus subsumiert

\subsubsection{Antagonismen}

Antagonismen entstehen. wenn ein explizites oder implizites kontradiktorisches Verhältnis zwischen mindestens zwei Elementen konstruiert wird. Ein explizites Verhälnis liegt vor, wenn die entgegengesetzten Elemente benannt werden, ein implizites, wenn ein Element, genauer das ausgrenzende, nicht genannt wird. Die im Korpus nachweisbaren Antagonismen sind in der nachfolgenden Tabelte 28 abgebildet. Die Elemente, aus deren Sicht ausgegrenzt bzw. differenziert wird, sind fett gedruckt, die ausgegrenzten bzw. «an den Rand gedrangten» kursıv. Daruber hinaus existieren Elemente, die genutzt werden konnen, um andere Elemente auszugrenzen. Diese sind in der gewohnten Schriftart

Tabelle 28

\begin{tabular}{|c|c|c|}
\hline $\mathbf{A E}$ & Antaponismes & Konetruktion \\
\hline 2 & $\begin{array}{l}\text { my: } \\
\text { odni: } \\
\text { novyj znakomy } \\
\text { vs } \\
\text { we: } \\
\text { trigie: } \\
\text { stany znationmy }\end{array}$ & 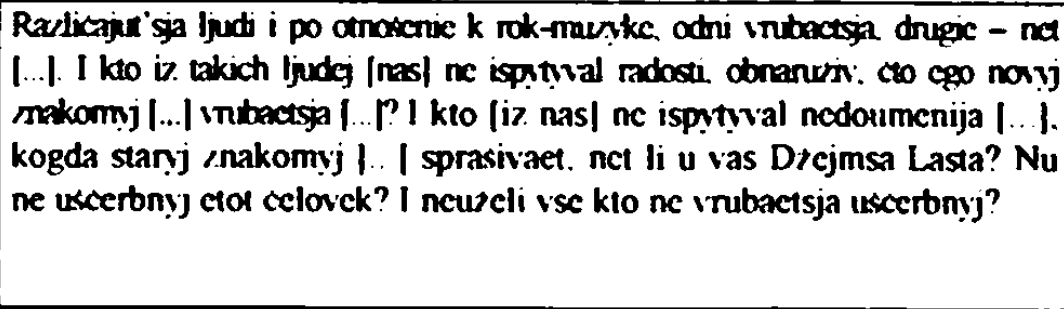 \\
\hline 3 & $\begin{array}{l}\text { my } \\
\text { is. } \\
\text { drugic }\end{array}$ & 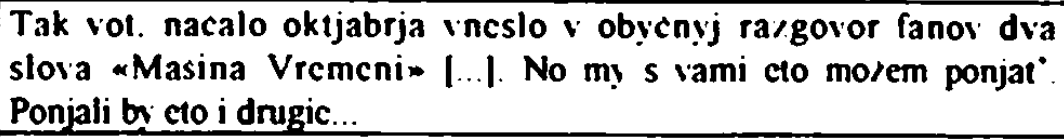 \\
\hline 4 & $\begin{array}{l}\text { ja } \\
\text { is. } \\
\text { prusuncial inte bulls }\end{array}$ & $\begin{array}{l}\text { Sutki ja ne slc/al s apparata. vgonjaja razochotissichsja } \\
\text { provincial mych bitlor obratno vich nory } \mid \text {... I. }\end{array}$ \\
\hline
\end{tabular}




\begin{tabular}{|c|c|c|}
\hline $\mathbf{5}$ & $\begin{array}{l}\text { bol'sinstvo; } \\
\text { Michail Naumenko } \\
\text { vs. } \\
\text { Mozaika: } \\
\text { Karnaval } \\
\end{array}$ & $\begin{array}{l}\text { A na vzgljad bol'sinstva, imenno u Majka [Michail Naumenko] - eto } \\
\text { teksty, eto stichi. a u «Mozaiki» i kKarnavala» zastampovannyj nabor } \\
\text { slov. }\end{array}$ \\
\hline $\mathbf{5}$ & \begin{tabular}{|l|} 
my \\
vs. \\
Zapad
\end{tabular} & $\begin{array}{l}\text { Vod' kuming - cto u nas. cto na Zapad - vornikajut ne sami po sebc } \\
\text { [...]. }\end{array}$ \\
\hline 6 & $\begin{array}{l}\text { my } \\
\text { vs. } \\
\text { Golubye molndcy: } \\
\text { Rrannie crety: } \\
\text { Pojut unitosy } \\
\end{array}$ & 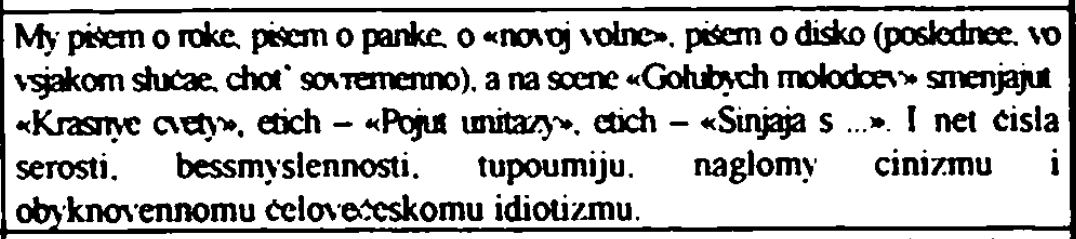 \\
\hline 6 & $\begin{array}{l}\text { ja } \\
\text { vs. } \\
\text { laureaty: } \\
\text { massy: } \\
\text { slusateli iz Leningrada } \\
\end{array}$ & 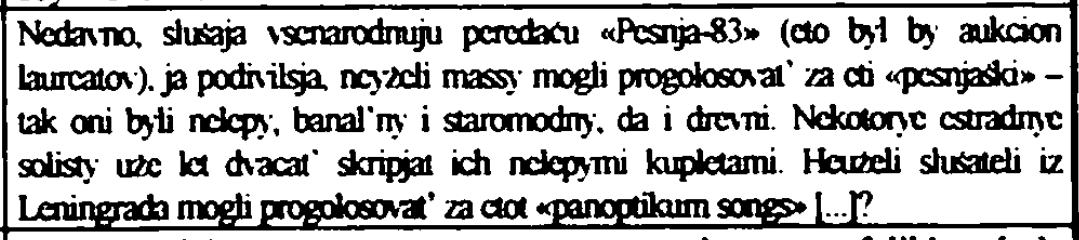 \\
\hline 7 & $\begin{array}{l}\text { my } \\
\text { vs. } \\
\text { oni }\end{array}$ & *Oni napadajut na samoc cennoc. cto u nas est ${ }^{\circ}$, - na nas fol'klor $*[\ldots]$ \\
\hline 7 & $\begin{array}{l}\text { my } \\
\text { vs. } \\
\text { Afrika: } \\
\text { papausy: } \\
\text { ljudi } \\
\end{array}$ & $\begin{array}{l}\text { Etnograficeskaja differenciacija v nas incustrial mj vek imoet mesto lis' } v \\
\text { Afrike i gde-to u papuason. da i v vek informacii i nauki ljudi } \\
\text { ogosudarstvilis' kak nikogda. }\end{array}$ \\
\hline 8 & $\begin{array}{l}\text { ja } \\
\text { is. } \\
\text { narod: } \\
\text { devorki: } \\
\text { otecesniennje snobs: }\end{array}$ & $\begin{array}{l}\text { Narodu mnogo. Devocki. popki. to. se. Stremno slegka i modno diko! } \\
\text { Modno i skucno. Posemy scitaju neobchodimym obrusit'sja na vjju ety } \\
\text { lazu. na tupost mody i stit' s puti poslusnuju otaru olecestiennych } \\
\text { snobor. }\end{array}$ \\
\hline 8 & $\begin{array}{l}\text { my } \\
\text { vs. } \\
\text { amerikanizm }\end{array}$ & 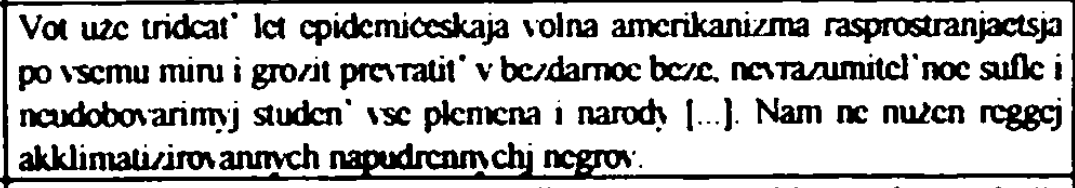 \\
\hline 8 & $\begin{array}{l}\text { my |taturo-sla janskaja ordal } \\
\text { is. } \\
\text { (judzon: } \\
\text {-nordiceskaja raza: } \\
\text { dorianv gret: } \\
\text {-nordiceskaja raza: } \\
\end{array}$ & 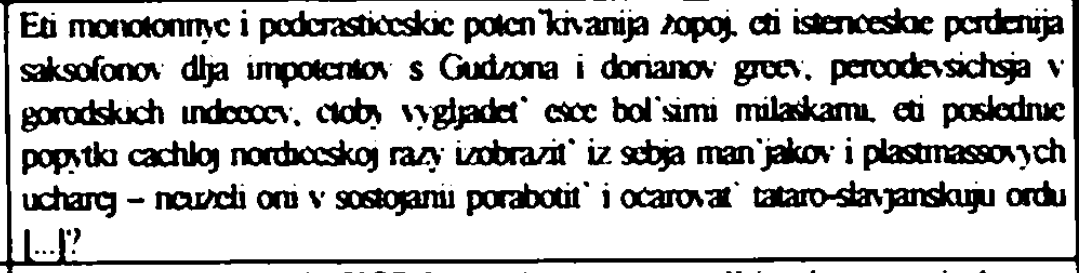 \\
\hline 9 & $\begin{array}{l}\text { rok } \\
\text { vs. } \\
\text { KisP }\end{array}$ & $\begin{array}{l}\text { Sejcas }|\ldots| \text { dvizenie KSP kazetsja cem-to melkim i neznacitelnym. } \\
\text { no bylo vremja. kogda ono zapolnjalo vremja i prostranstvo tak te. } \\
\text { kak sejcas zapolnjact rok }|\ldots| \text {. V } 60 \text {-e gody KSP bylo takim ze } \\
\text { «podzemnym. kak rok sejcas. }\end{array}$ \\
\hline 9 & \begin{tabular}{|l|} 
my \\
vs. \\
Zapad \\
\end{tabular} & 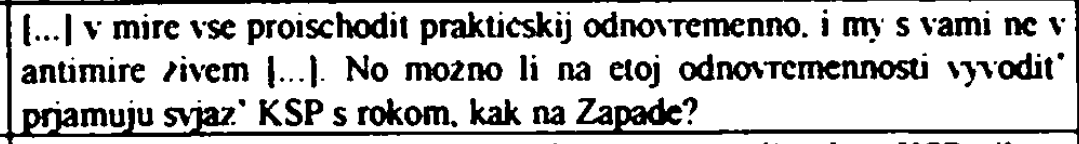 \\
\hline 9 & $\begin{array}{l}\text { KSPSniki } \\
\text { vs. } \\
\text { milicejskie narjady }\end{array}$ & $\begin{array}{l}\text { Ego [KSP] gonjali. zaprescali. primcajali k KSPsnikam } \\
\text { admimstrativ nye men vozdejstriju I... I. Rasskanavajut. kak neredko } \\
\text { festival nyj lager oknuzalsja milicejskim najadom. a zatem bul 'dozery } \\
\text { sgrebali palatki v odnu bol 'suju kucu. }\end{array}$ \\
\hline
\end{tabular}




\begin{tabular}{|c|c|c|}
\hline 10 & 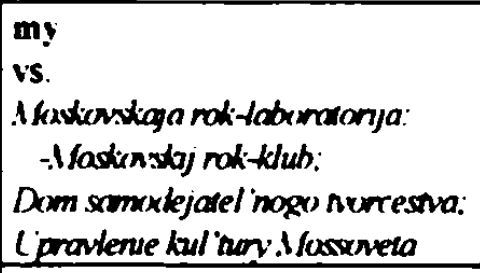 & 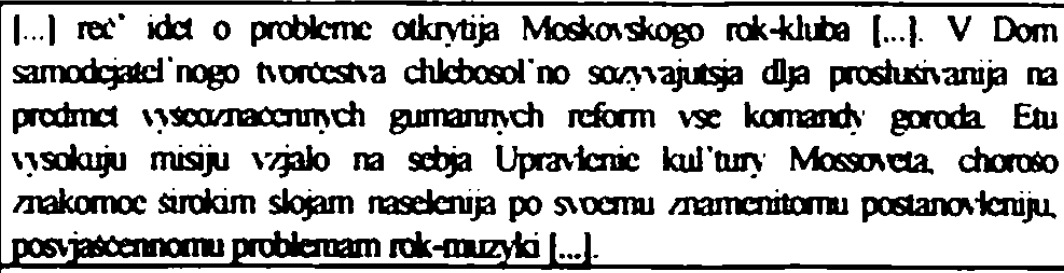 \\
\hline 11 & $\begin{array}{l}\text { my } \\
\text { is. } \\
\text { stroiteli svellogo huduscego }\end{array}$ & $\begin{array}{l}\text { I... I ncuzcli nas dejstvitcl'no ostavili v pokoe? Newzeli nas perestali } \\
\text { zagonjat' v rady stroitclej svetlogo buduscego. i my. nakonoc. motem } \\
\text { spokojno. mimo sosuscestvovat'? }\end{array}$ \\
\hline 11 & $\begin{array}{l}\text { my } \\
\text { vs. } \\
\text { E:Vilc: } \\
\text { Mfoskovisknja rok-laboratorija: } \\
\text {-rok-lahoratorija }\end{array}$ & 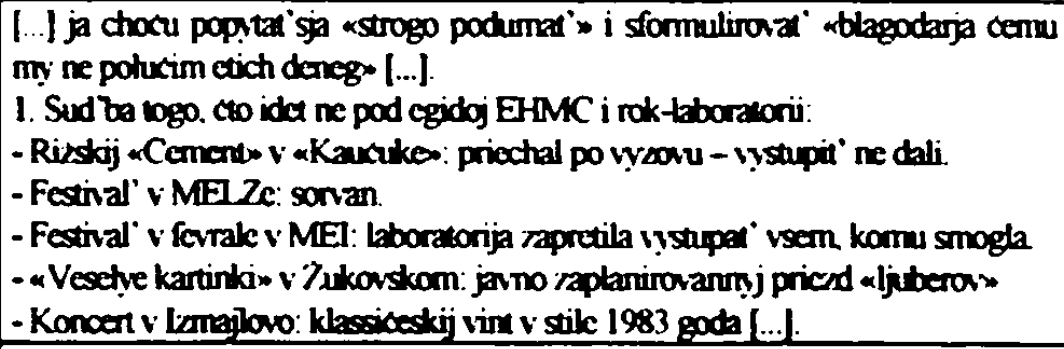 \\
\hline 11 & $\begin{array}{l}\text { muzykanty: } \\
\text { publika } \\
\text { vs. } \\
\text { ljubera: } \\
\text { starsie tovarisci v plizikoiych } \\
\text { sapkach }\end{array}$ & 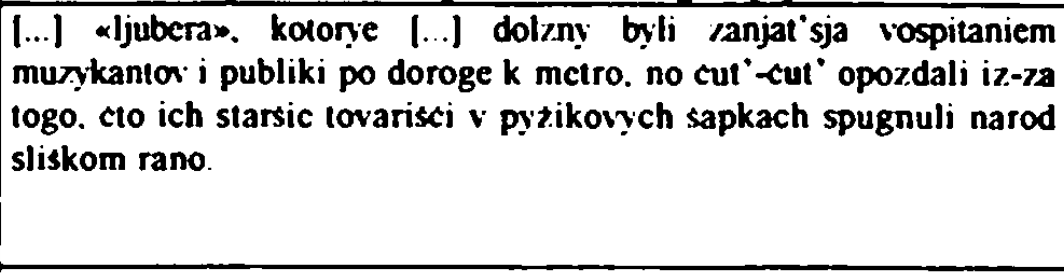 \\
\hline 12 & $\begin{array}{l}\text { ja } \\
\text { is. } \\
\text { Roksi: } \\
\text { CIIIIR (III/R): } \\
\text { oficial noja pressa: } \\
\text { klubnoja prexisa }\end{array}$ & 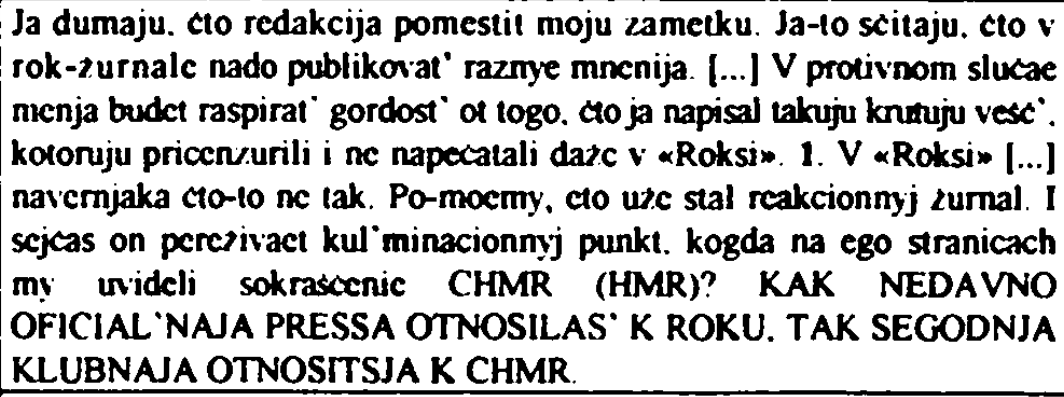 \\
\hline 12 & $\begin{array}{l}\text { Modonki Loodrostkil } \\
\text { is } \\
\text { 1e. komu za iridcal }\end{array}$ & 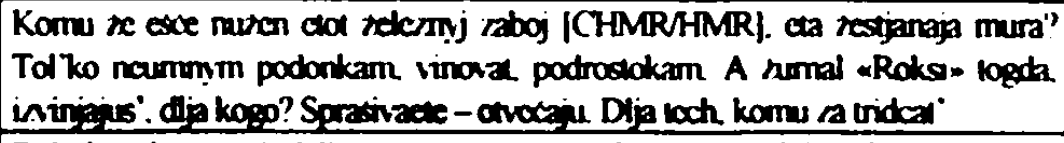 \\
\hline 13 & $\begin{array}{l}\text { rok } \\
\text { vs. } \\
\text { Tat janna Itiansarnina }\end{array}$ & By la kogda-to tetja Miansarova ... a teper' vol pojavilsja rok. \\
\hline 13 & $\begin{array}{l}\text { ja } \\
\text { vs. } \\
\text { sobrat jo }\end{array}$ & $\begin{array}{l}\text { Ja s detstia ucus molcat. inaju po opytu sobrat ja no vidy mogut } \\
\text { sozrat. esli cto ne po nim. }\end{array}$ \\
\hline 13 & $\begin{array}{l}\text { ja } \\
\text { is. } \\
\text { drugic }\end{array}$ & $\begin{array}{l}\text { Cholja ja } v \text { nem Iv rokcl nc ponimaju ni figa } \mid \text { I No drugic } \\
\text { ved ponimajut } v \text { nem esce men'sc. }\end{array}$ \\
\hline 13 & $\begin{array}{l}\text { ostal'nye } \\
\text { vs. } \\
\text { mustkanț: } \\
\text { prichlebatels }\end{array}$ & 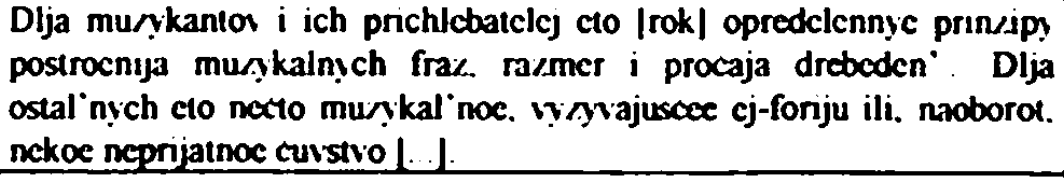 \\
\hline 14 & $\begin{array}{l}\text { agenty ochrunki: } \\
\text { luwhera. } \\
\text {-sturmoviki provogo fronta: } \\
\text { kazancy: } \\
\text {-sturmoviki provogo fronta. } \\
\text { Kreml. }\end{array}$ & 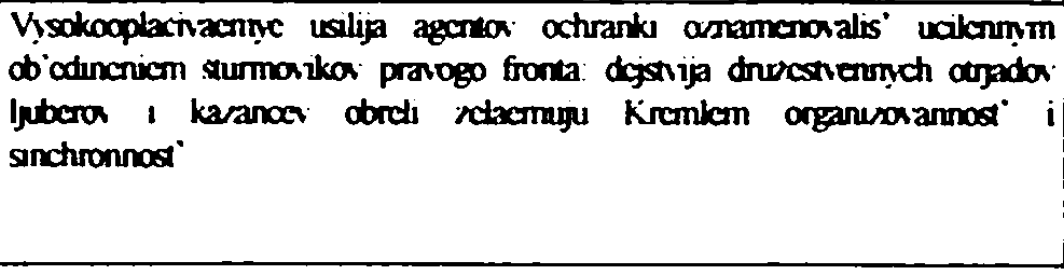 \\
\hline
\end{tabular}




\begin{tabular}{|c|c|c|}
\hline 14 & $\begin{array}{l}\text { sporfiunye fanaty: } \\
\text { ljubera: } \\
\text { musornye lycki }\end{array}$ & 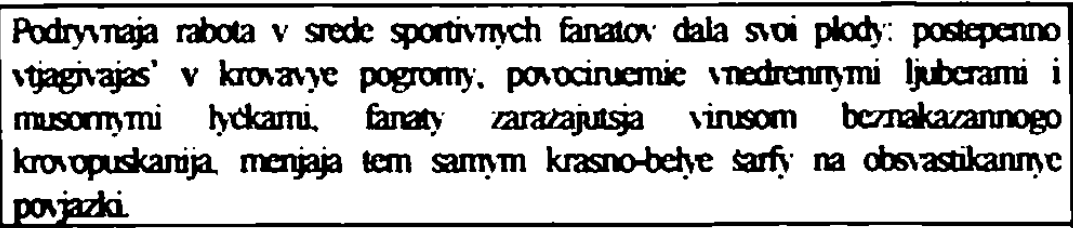 \\
\hline 14 & $\begin{array}{l}\text { ljubera: } \\
\text {-molozavie ubljudki: } \\
\text { kazancy: } \\
\text {-molozave ubljudki: } \\
\text { sportivnue fanaty: } \\
\text {-molozavve ubljudki }\end{array}$ & $\begin{array}{l}\text { Novye zvezdocki padajut s partijnych ncbes na krasnye pogony } \\
\text { molozavych ubljudkov. }\end{array}$ \\
\hline 14 & $\begin{array}{l}\text { olecestuennue chumvejbiny } \\
\text { [boeviki]: } \\
\text { gebesnye glavari }\end{array}$ & $\begin{array}{l}\text { Razdelenie Moskyy na sfery vlijamja pozvolilo otecestvennym } \\
\text { chunvejbinam kvalificirovannee provodit' operacii grabeza i sanitarii, a } \\
\text { popolnenie partkassy razivaetsja operetajuscimi tempami v sravnenii } \\
\text { s raschodom sredsti na stimulirovanie osobo otlicivsichsja boevikov i } \\
\text { ich gebesnych glavarej. }\end{array}$ \\
\hline 14 & boevie stranniki & $\begin{array}{l}\text { Pod pompezmyc znamena ideologii liberalizma sgnivsej imperii vstajut } \\
\text { isc novye boevye stranniki }[. . .1 \text {. }\end{array}$ \\
\hline 15 & $\begin{array}{l}\text { ja } \\
\text { is. } \\
\text { vse }\end{array}$ & $\begin{array}{l}\text { Ja tol'ko dobavil by } k \text { nemu esce odnu poziciju: illjuziju togo. cto } \\
\text { po proctenii etoj stat }{ }^{\circ} \text { i vse srazu vdrug vse pojmut. }\end{array}$ \\
\hline 16 & $\begin{array}{l}\text { my } \\
\text { vs. } \\
\text { Boris Grebenscikov } \\
\end{array}$ & $\begin{array}{l}\text { Pojavlenie etoj recenziji |Radio Silencel v nasem izdanii - sorsem ne } \\
\text { preklonenie pered Grebenscikorym. Naprotiv |...]. }\end{array}$ \\
\hline 17 & $\begin{array}{l}\text { my } \\
\text { vs. } \\
\text { niscie duchi }\end{array}$ & $\begin{array}{l}\text { My prisutsvuem pri istoricestom momente - slovi «rok» terjaet smysl. } \\
\text { Process ego deval'vazii podchodit k tragiceskoj tocke - dal'se idet } \\
\text { prozjabanic razmennoj monety dlja niscich duchom. (...) kotorym gros } \\
\text { cena. }\end{array}$ \\
\hline 17 & $\begin{array}{l}\text { filarmo-metallisty:: } \\
\text { laskoivy maj }\end{array}$ & 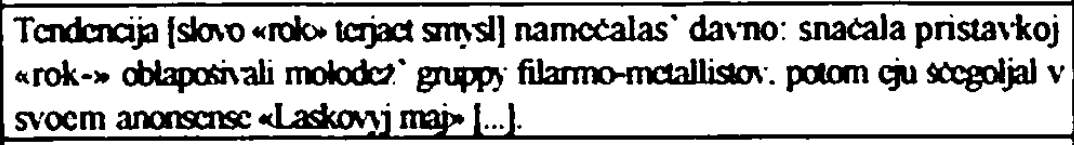 \\
\hline 17 & $\begin{array}{l}\text { my } \\
\text { us. } \\
\text { gerui }\end{array}$ & 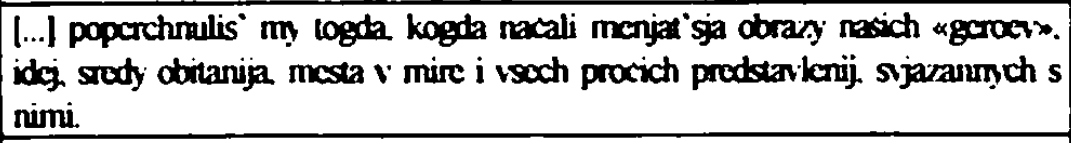 \\
\hline 17 & 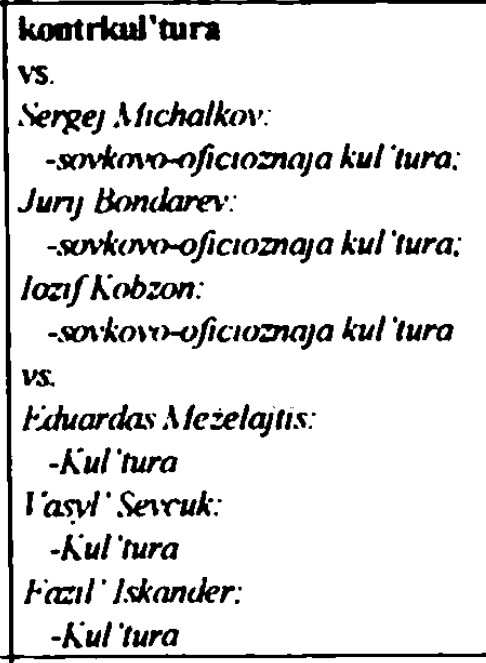 & 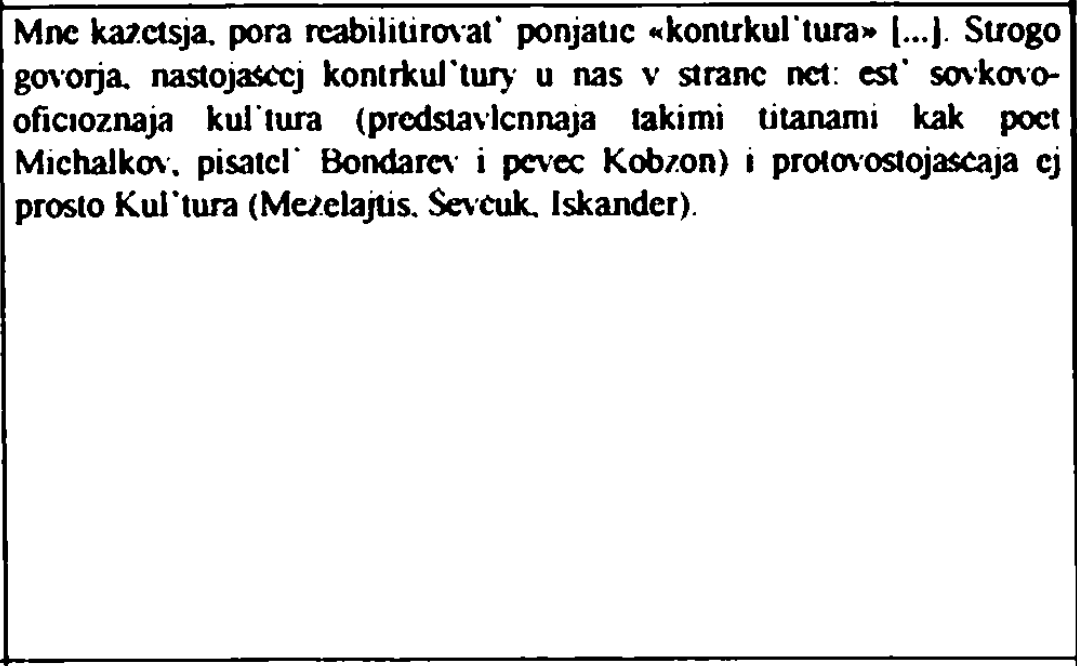 \\
\hline 18 & $\begin{array}{l}\text { Itoraja polovina redakcii } \\
\text { vs. } \\
\text { dostojnaja polovina redakcii }\end{array}$ & 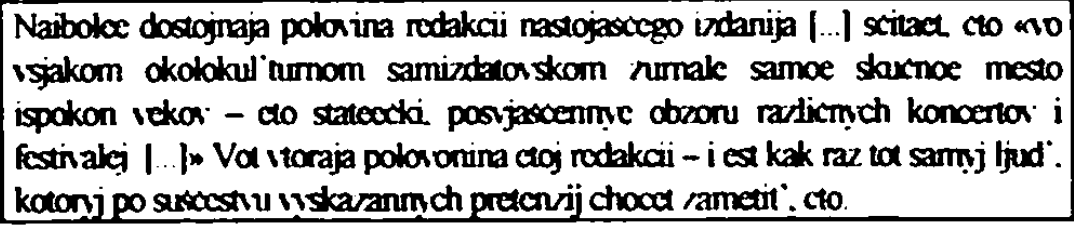 \\
\hline
\end{tabular}




\begin{tabular}{|c|c|c|}
\hline 18 & $\begin{array}{l}\text { chorodie mal'ciki» (nasie) } \\
\text { vs. } \\
\text { mal riki dija bit jon (ne nasie) }\end{array}$ & 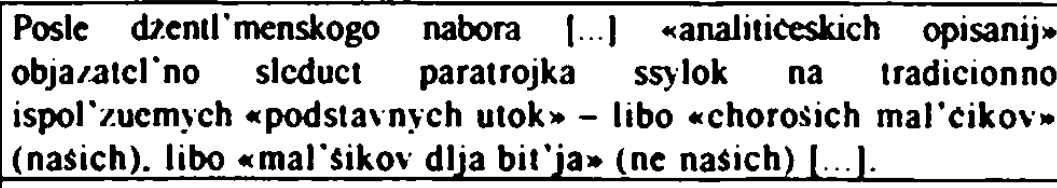 \\
\hline 18 & $\begin{array}{l}\text { my } \\
\text { vs. } \\
\text { popso }\end{array}$ & $\begin{array}{l}\text { Ich Kennedi - dod. a nas - csce net. No popsy na nasej zemle esce } \\
\text { mnogo. }\end{array}$ \\
\hline 19 & $\begin{array}{l}\text { ja [zisnennyj opyt] } \\
\text { vs. } \\
\text { roditcli }\end{array}$ & $\begin{array}{l}\text { Buduci po vospitaniju (vospitaniju ne v smyslc politiki moich. } \\
\text { dopustim. roditclcj. a skoree } v \text { smyslc hisnennogo opyta) } \times \text { kniznym } \\
\text { mal cikom } \mid \text {.... }\end{array}$ \\
\hline 20 & $\begin{array}{l}\text { rok-poezija: } \\
\text { rok-poct: } \\
\text { D:a Divisn }\end{array}$ & 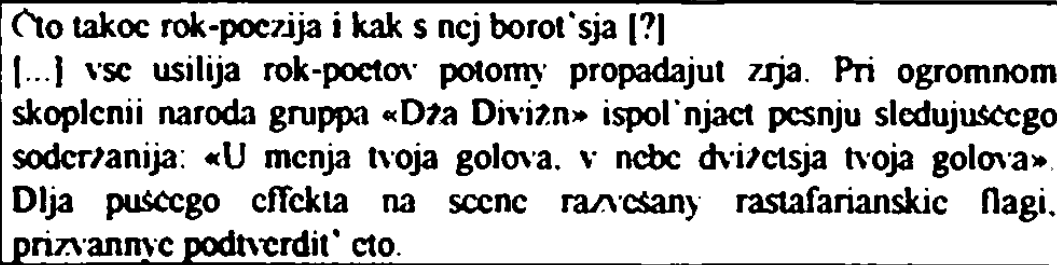 \\
\hline 21 & $\begin{array}{l}\text { ja } \\
\text { is. } \\
\text { oni [sroloci] }\end{array}$ & 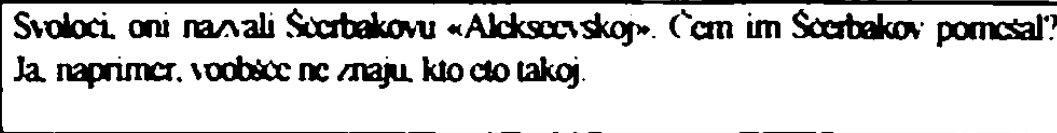 \\
\hline 21 & $\begin{array}{l}\text { ja } \\
\text { vs. } \\
\text { vrag! |balalocnikil| }\end{array}$ & 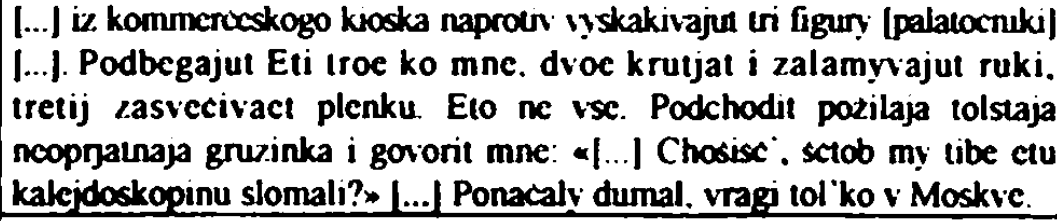 \\
\hline 22 & $\begin{array}{l}\text { my: } \\
\text { Andrej Burlak } \\
\text { vs. } \\
\text { Rok-Adtuokat: } \\
\text {-oficial 'naja pressa: } \\
\text { tenscina iz. I'-skoj molode ski: } \\
\text { oficial 'naja pre.ssa }\end{array}$ & 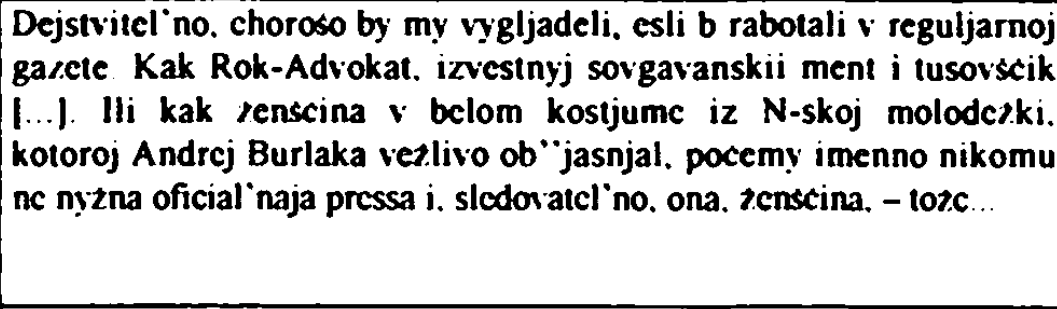 \\
\hline 22 & $\begin{array}{l}\text { my } \\
\text { is. } \\
\text { legkit. } \\
\text {-rok-scena. } \\
\text { blohnoty. } \\
\text {-rok-icena }\end{array}$ & 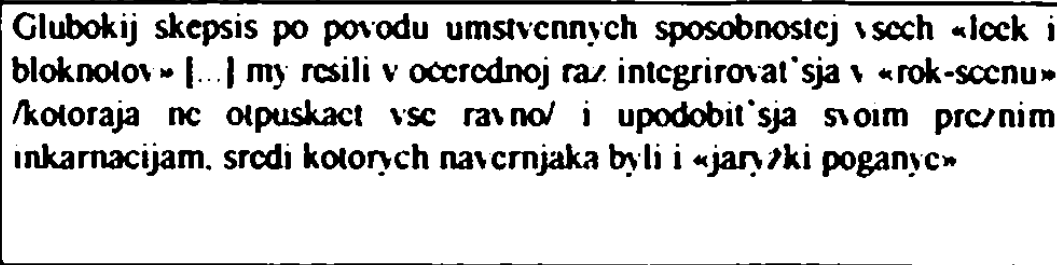 \\
\hline 23 & $\begin{array}{l}\text { my } \\
\text { is. } \\
\text { protivniki obnovlenija. } \\
\text { diletant?. }\end{array}$ & 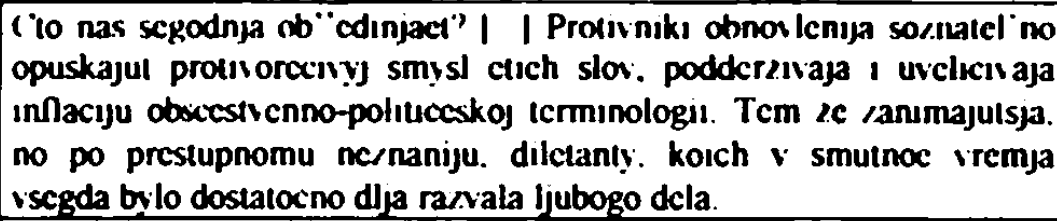 \\
\hline 23 & ljudt & 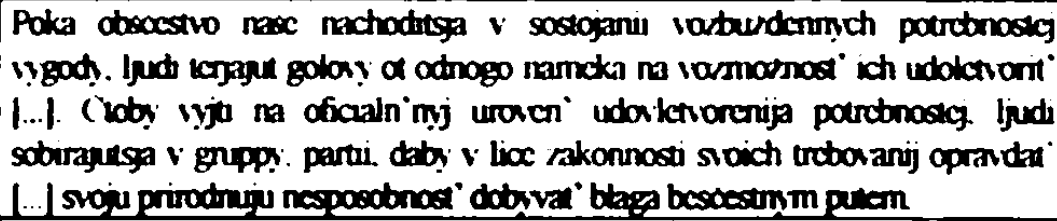 \\
\hline 24 & $\begin{array}{l}\text { my } \\
\text { vs. } \\
\text { \%apad }\end{array}$ & 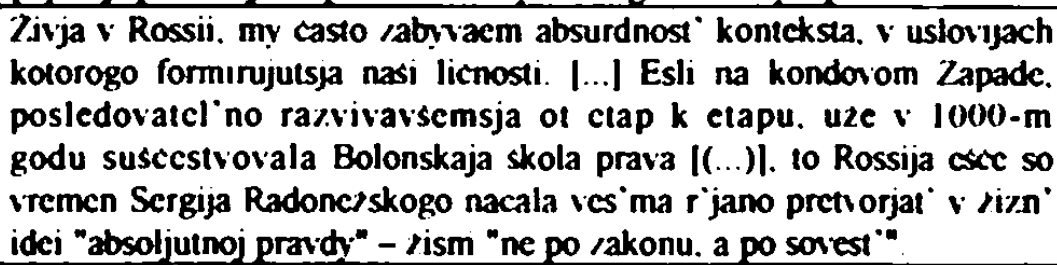 \\
\hline
\end{tabular}




\begin{tabular}{|c|c|c|}
\hline 25 & "podpol 'naja" rok-pressa & 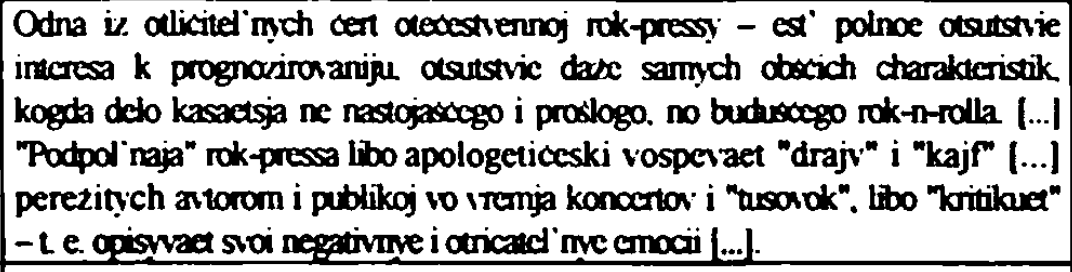 \\
\hline 26 & $\begin{array}{l}\text { ja } \\
\text { vs. } \\
\text { gazety: } \\
\text {-pressa: } \\
\text { radio: } \\
\text {-pressa: } \\
\text { televizor: } \\
\text {-pressa: } \\
\text { L. Doms: } \\
\text {-pressa } \\
\end{array}$ & $\begin{array}{l}\text { No na dnjach moj status kvo byl nanusen. Druz" ja prinesli mne vyrezki } \\
\text { iz dvuch nomerov gazety "Vecernij Kisener" [...]. Stat'ja pod } \\
\text { zagolovkom "Komu podygryval intellektual'nyj rok?" (v krugu loznych } \\
\text { cennostej) avtor L.Doros. }\end{array}$ \\
\hline 26 & $\begin{array}{l}\text { ja } \\
\text { vs. } \\
\text { Aleksandr Simonov }\end{array}$ & 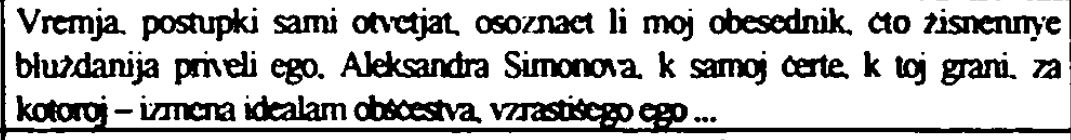 \\
\hline 27 & $\begin{array}{l}\text { ja } \\
\text { vs. } \\
\text { starye druz ja }\end{array}$ & $\begin{array}{l}\text { [...] kogda ja s etim znackom [s portretom M.C. Gorbaceva] na kurtke } \\
\text { pojavilsja za kulisami. nekotorye iz starych druzej vyrazili nedoumenie } \\
\text { i daze negodovanic: "cto cto. mol. ty na sebja nacenil?" [... Obidersis" } \\
\text { na tech. komu ne ponravilsja moj firmennyj znacok. ja mog by v } \\
\text { otmestku obozat" ich. naprimer "ckstremistami". }\end{array}$ \\
\hline 27 & $\begin{array}{l}\text { ja } \\
\text { vs. } \\
\text { Bori Lemcov } \\
\end{array}$ & $\begin{array}{l}\text { Ja ne stal tratit' vremja na perecislenie svoich proslych revoljucionnych } \\
\text { /aslug /ostavljaju etu temy dlja Bori Zemcova/ }[. . .] \text {. }\end{array}$ \\
\hline 27 & $\begin{array}{l}\text { ja } \\
\text { vs. } \\
\text { Irtem Troickij }\end{array}$ & 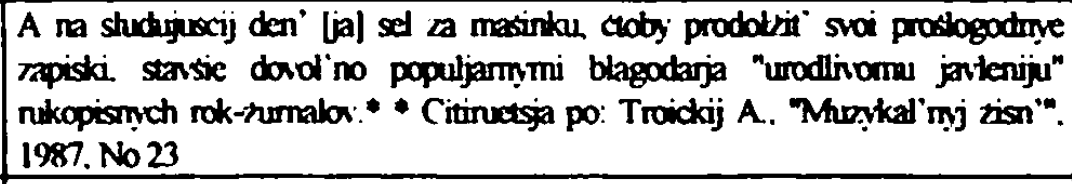 \\
\hline 27 & $\begin{array}{l}\text { D. I'asil'ev: } \\
\text { l'amjat' }\end{array}$ & 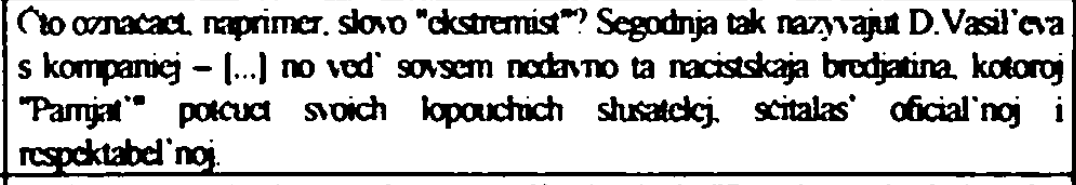 \\
\hline 28 & $\begin{array}{l}\text { pressa: } \\
\text { komsomol skit-molisde =nue } \\
\text { Izdanya }\end{array}$ & 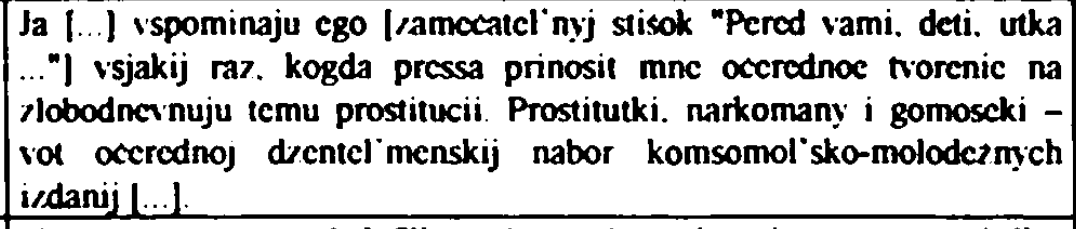 \\
\hline 28 & \begin{tabular}{|l|} 
prostitutka \\
vs. \\
¿umalist \\
\end{tabular} & 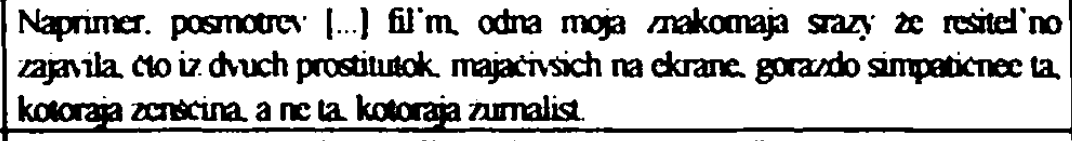 \\
\hline 29 & $\begin{array}{l}\text { te. kto prosto probivaetsja k } \\
\text { "prestiznoj kormuske": } \\
\text { celovek. pisuscij o roke dlja } \\
\text { oficial "nogo izdanija }\end{array}$ & 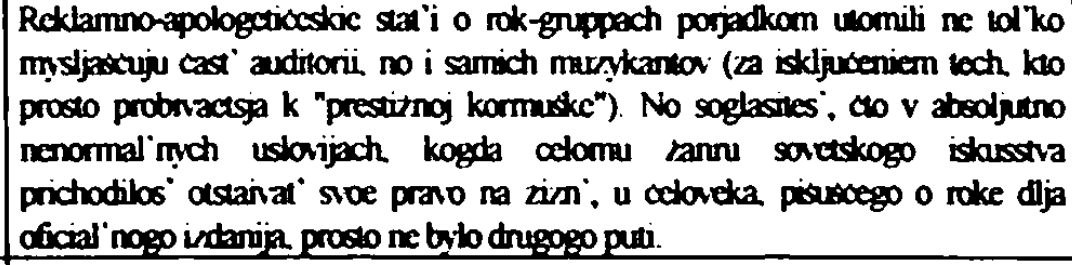 \\
\hline 29 & $\begin{array}{l}\text { umnyj celovek } \\
\text { vs } \\
\text { neumnyj celovek }\end{array}$ & 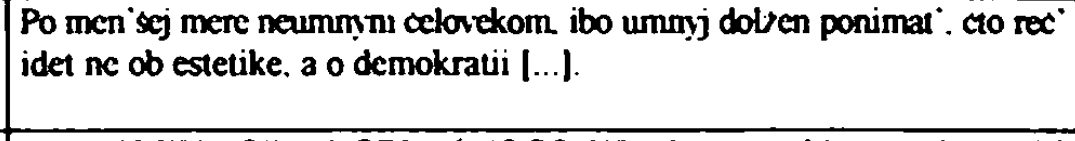 \\
\hline 29 & $\begin{array}{l}\text { TELEVIZOR: } \\
\text { OBLAC NYJ KRAJ } \\
\text { vS. } \\
\text { I Tadimir Kuz min } \\
\end{array}$ & $\begin{array}{l}\text { I... I TELEVIZORA i OBLAC NOGO KRAJA vse-taki ne stoit stavit } \\
\text { na odnu dosku s nayojlivym voschvalcnicm B.Kur. mina [...]. }\end{array}$ \\
\hline
\end{tabular}




\begin{tabular}{|c|c|c|}
\hline 29 & $\begin{array}{l}\text { progressivnye redakcit; } \\
\text { TI: } \\
\text { Sojuzpecal }\end{array}$ & 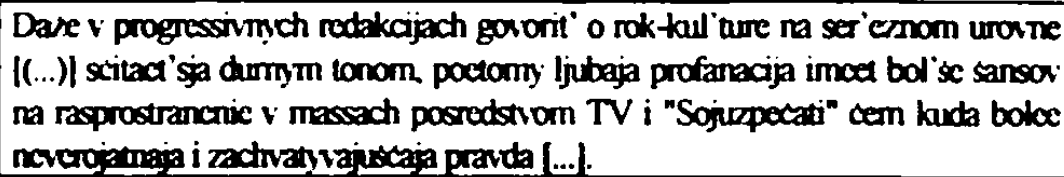 \\
\hline 30 & menediery & 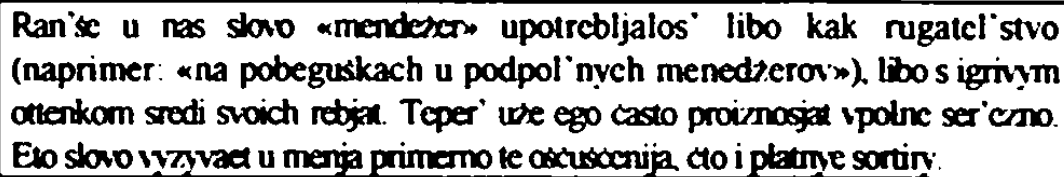 \\
\hline 31 & $\begin{array}{l}\text { my } \\
\text { vs. } \\
\text { ciropejal }\end{array}$ & $\begin{array}{l}\text { Tak cto ciropejcam ne stoit osobenno zadirat' pered nami nos: oni to/c } \\
\text { cere/ vse eto prosli. }\end{array}$ \\
\hline 31 & $\begin{array}{l}\text { ja } \\
\text { vs. } \\
\text { nas nunesnij parlament; } \\
\text { raninji socializm }\end{array}$ & $\begin{array}{l}\text { I ise tc mne ne chotclos' by bezogovorocno osuzdat' etu } \\
\text { srednerckoruju sistemu [nas nynesnij parlament]. Vo-pernych, ona } \\
\text { znamenuct soboj kolossal'myj progress po sravneniju s ranitym } \\
\text { socializmom } \mid . . . \text {. }\end{array}$ \\
\hline 32 & $\begin{array}{l}\text { Fcdor Dostocvskij: } \\
\text { Daniil Charms: } \\
\text { Mikclandrclo } \\
\text { vs. } \\
\text { Ileksandr Dtikja }\end{array}$ & 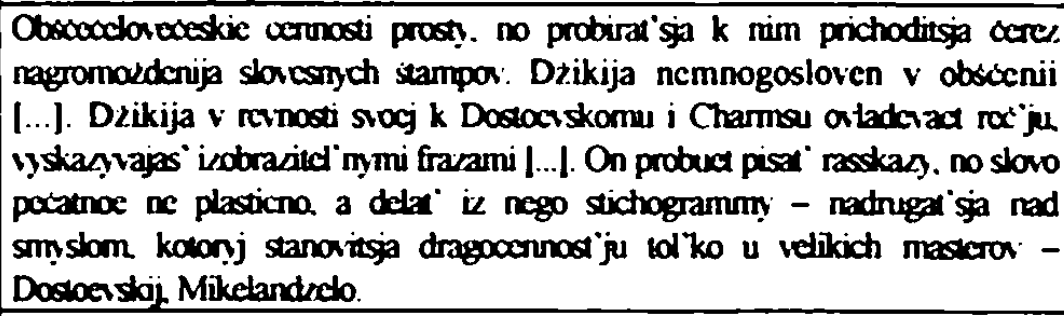 \\
\hline 33 & $\begin{array}{l}\text { URLAJT } \\
\text { vs. } \\
\text { molodeznaja pressa }\end{array}$ & 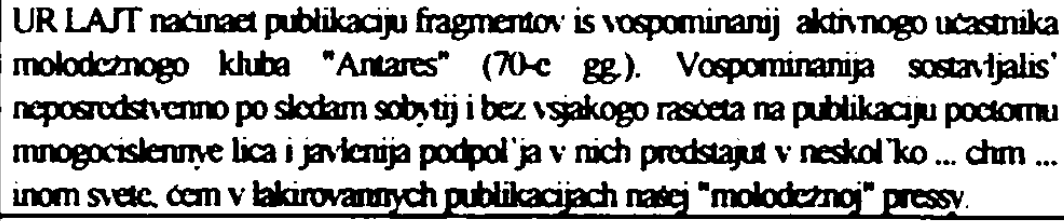 \\
\hline 33 & $\begin{array}{l}\text { my [dtentl'meny] } \\
\text { is. } \\
\text { bandity. }\end{array}$ & $\begin{array}{l}\text { I kakic ze my stali dzemt'meny: da cto vy, da kak sy poedetc } v \text { takuju } \\
\text { noc } v \text { takuju dal', da vokrug tc bandity }|\ldots| .\end{array}$ \\
\hline 34 & vosdja mirovogo proletariata & 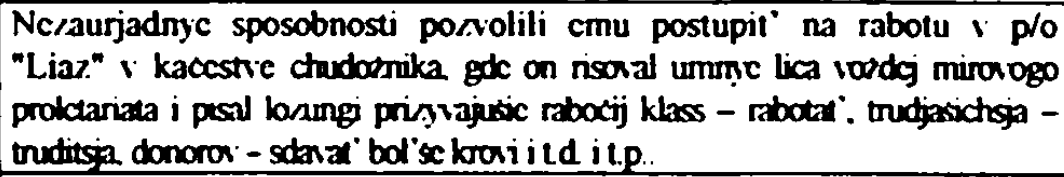 \\
\hline 35 & $\begin{array}{l}\text { my [CHERR S MOSLOM] } \\
\text { vS. } \\
\text { Bambuk }\end{array}$ & 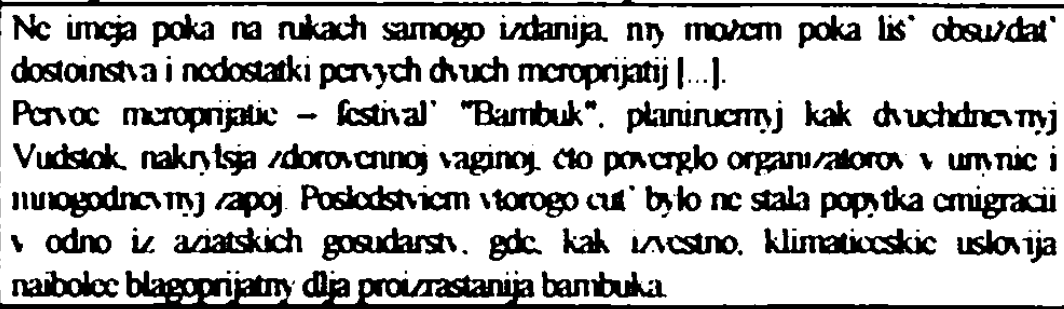 \\
\hline
\end{tabular}

Die Erfassung von Antagonismen bzw. ihr Erkennen basiert auf einer Interpretation und ist daher subjektabhängig. Es wurde in Tabelle 28 versucht, die Interpretation nachvollziehbar zu gestalten und die Antagonismen mit maximaler Deutlichkeit aus den jeweiligen Analyseeinheiten zu extrapolieren. In diesem Sinne lassen sich gewisse Redundanzen nicht vermeiden. In einigen Fälen können Differenzierungen innerhalb der aufgefuhrten Antagonismen vorgenommen werden: beispielsweise lassen sich aus dem einen Antagonismus, der in Analyseeinheit 2 festgestellt wird, problemlos drei Antagonismen "erstellen», namlich my vs. vse, odni vs. drugie und novyj znakomyj vs. staryj znakomyj. Diese Differenzierung ist nicht notwendig. da mit der gewählten Methode alle betroffenen Elemente erfaßt werden. Es geht letztendlich im vorliegenden Rahmen nicht um die Anzahl der Antagonismen im untersuchten Korpus, sondern 
um die betroffenen Elemente, d.h. um diejenigen Elemente, aus deren Sicht eine Abgrenzung bzw. Differenzierung vorgenommen wird, die ausgegrenzt bzw. «an den Rand» der untersuchten Formation "gedrängt» werden. Darüber hinaus gilt es festzustellen, inwiefern die Verwendung von Antagonismen charakteristisch für das untersuchte Korpus ist. Auch für die Beantwortung dieser Frage ist die absolute Anzahl der Antagonismen nicht erforderlich.

Mit der Beantwortung der zuletzt gestellten Frage soll die Zusammenfassung des Analyseergebnisses beginnen. In bezug auf das Korpus kann festgestellt werden, da $B$ in 34 Analyseeinheiten (97,1\%) Antagonismen generiert werden, so daß die kulturelle Ab- bzw. Ausgrenzung dem Bewertungskriterium charakteristisch genügt. Die Generierung von Antagonismen ist in diesem Sinne ein charakteristisches Textgenerierungsmerkmal des untersuchten Diskurses.

Zur untersuchten kulturellen Formation gehören auf der ausgrenzenden Seite 22 verschiedene Elemente, die insgesamt $56 \mathrm{mal}$ verwendet werden. Auf diejenigen, die mehrmals im Korpus nachgewiesen werden können, soll hinsichtlich ihrer kulturellen Bedeutung eingegangen werden. Es handelt sich um die Elemente my (zwanzigmal: $35,7 \%$ ), ja (fünfzehnmal: 26,8\%) und rok (zweimal: 3,6\%). Die kulturelle Bedeutung des Elements my wird nicht explizit thematisiert, so daß von einem nahezu abgeschlossenen Bedeutungskonstruktionsprozeß ausgegangen werden kann. Das Konstrukt wird ledglich um die Elemente tataroslavjanskaja orda, dzentl'meny und CHERR S MOSLOM erweitert. Mit hoher Wahrscheinlichkeit besitzt das angesprochene Konstrukt im Rahmen der untersuchten Formation die Funktion eines Autokonstrukts, so daß sich die Mitglieder der Formation mit diesem identifizieren. In bezug auf das Element ja sollte nicht von einem gruppenbezogenen, sondern von einem personenbezogenen Konstrukt ausgegangen werden. Es liegt die Vermutung nahe, daß die Ausgrenzungen, die aus der Sicht eines Aktanten ja generiert werden, dennoch die Auffassung der untersuchten Formation widerspiegeln (vgl: 3.2.; Repräsentationsmodell). Das Element rok, welches in den Analyseeinheiten 9 und 13 eingesetzt wird, besitzt mit hoher Wahrscheinlichkeit eine positive kulturelle Bedeutung, die im Rahmen der vorliegenden Antagonismen nicht expliziert wird. Es kann in diesem Sinne von einem abgeschlossenen Bedeutungskonstruktionsproze $B$ ausgegangen werden.

Aufgrund ihrer kulturellen Bedeutung werden fünf Elemente (KSPsniki, prostitutka, Daniil Charms, Fedor Dostoevskij und Mikelandzelo) genutzt, um andere Elemente auszugrenzen. Die Gruppe, die durch den Index KSPsniki bezeichnet wird, die auch in stereotypisierter Form (ljubiteli KSP; vgl.: 9.12.2.) im Korpus nachweisbar ist, besitzt eine positive bzw. indifferente kulturelle Bedeutung. Diese kann aus der Konstruktion des KSP in Analyseeinheit 9 abgeleitet werden. Der Aktant prostitutka, der im Sinne einer Pars-pro-toto-Relation eingeführt wird (vgl: : 9.9.3.), besitzt vermutlich ebenfalls eine positive bzw. indifferente kulturelle Bedeutung, da die Mitglieder der repräsentierten Gruppe zu den aus dem übergeordneten Kulturssystem Ausgegrenzten gezählt werden können, zu denen in einem weiteren Sinne auch die Mitglieder 
der untersuchten Formation zählen. Die Bedeutungskonstruktion in bezug auf die repräsentierte Gruppe ist mit hoher Wahrscheinlichkeit abgeschlossen. Diese Vermutung soll auch in bezug auf die verbleibenden Aktanten geäußert werden, die mit hoher Wahrscheinlichkeit eine positive kulturelle Bedeutung aufweisen.

Aus der untersuchten Formation werden 106 verschiedene Elemente, die insgesamt 118 mal im Korpus nachgewiesen werden können, ausgegrenzt. Eine Ausgrenzung von verschiedenen Elementen, die nicht zum sowjetischen bzw. postsowjetischen Kultursystem gezählt werden können, erfolgt in sechs Fälen (5,7\%: Afrika, amerikanizm, doriany grei: nordiceskaja raza, evropejcy, Gudzon: nordičeskaja raza und Zapad). Gleichfalls können Differenzierungen innerhalb der untersuchten Formation festgestellt werden. In diesem Sinne werden die Aktanten Grebenscikov, Troickij und Rok-Advokat, die Gruppen provincial'nye bitly, Dia Divizn und rok-poety, die Institution Moskovskaja rok-laboratorija sowie die Marken ROKSI und BAMBUK an den «Rand» der untersuchten Formation "gedrängt».

Die verbleibenden Antagonismen beziehen sich auf das übergeordnete Kultursystem. In diesem Sinne werden Antagonismen vorwiegend generien, um eine Abgrenzung von diesem System sicherzustellen. Es liegt somit eine Introspektive auf das sowjetische bzw. postsowjetische Kultursystem vor.

In mehreren Analyseeinheiten erfolgt eine Aus- bzw. Abgrenzung von drugie $(2,3,13)$, ljubera $(11,14)$, ljudi $(7,23)$, Moskovskaja rok-laboratorija $(10,11)$, oni/oni [svoloci] $(7,21)$, vse $(2,15)$, televizor: pressa/TV $(26,29)$ und Zapad $(5,9,24)$. Insofern auf diese bisher nicht hinsichtlich ihrer kulturellen Bedeutung eingegangen wurde, soll dieses hier erfolgen. In bezug auf die Gruppe drugie kann in allen drei Fällen von ad hoc Antagonismen ausgegangen werden, so daß sie in diesem Sinne keine kulturelle Bedeutung aufweist. Die Abgrenzung von der Gruppe ljudi, der eine negative kulturelle Bedeutung zugesprochen wird, kann bezogen auf die untersuchte Formation mit dem Begriff Elitismus bezeichnet werden Es ist unklar, wer sich hinter der Gruppe verbirgt, so daß ebenfalls keine kulturelle Bedeutung diagnostizien wird. Hinter dem Personalpronomen oni verbirgt sich eine konkrete Gruppe. die "Obrigkeit", die zum einen über die Folklore im allgemeinen und speziell über die der untersuchten Formation «herfallt» (napast') und die zum anderen für die Umbenennung von Straßen u.dgl.m verantwortlich gemacht wird. Im zweiten Fall wird für die Gruppe gleichfalls die negative Bezeichnung svoloci verwendet In beiden Fallen besitzt die "Obrigkeit» eine negative kulturelle Bedeutung. Bei der Gruppe vse handelt es sich erneut um eine ad hoc generierte, die keine kulturelle Bedeutung aufweist In beiden Fallen kann die Abgrenzung mit dem Begriff Elitismus bezeichnet werden.

Die Ausgrenzungen der Elemente televizor. pressa/TV sollte im Zusammenhang mit der Ausgrenzung des ausdifferenzierten Bereichs der offiziellen Presse gesehen werden, dem insgesamt 13 verschiedene Elemente $(12,1 \%)$ zugerechnet werden können. celovek pisusciij o roke dlja oficial'nogo izdanija, gazety: pressa, komcomol'sko-molodeinye izdanija, L. Doros: 
pressa, molodènogo pressa, oficial'naja pressa, pressa, progressivnye redakcij, radio: pressa, Rok-Advokat: oficial'naja pressa, Sojuspecat' und zenscina iz N-skoj molodezki. Dieser gesamte Bereich besitzt im Rahmen der untersuchten Formation eine negative kulturelle Bedeutung.

Auf weitere Einzelheiten bezüglich der ermittelten Antagonismen soll nicht eingegangen werden. Es ist im Veriauf der Darstellung deutlich geworden, aus welcher Sicht Ausgrenzungen im Rahmen der untersuchten Formation vorgenommen werden, wer ausgegrenzt wird bzw. wer «an den Rand gedrängt» wird. Abschließend soll darauf hingewiesen werden, daß Abbzw. Ausgrenzungen vorgenommen werden, die auf ein elitäres Autokonstrukt der Mitglieder der untersuchten Formation schließen lassen.

\subsubsection{Stereotype}

Im folgenden Abschnitt werden die im Korpus nachgewiesenen Stereotype diskutiert. Es geht um eine Art der Ausgrenzung und Differenzienung, die derart funktioniert, daß einer betroffenen Gruppe neben dem Klassifizierungsmerkmal mindestens ein weiteres Merkmal zugesprochen wird (vgl.: 2.9.). Dieses weitere Merkmal tritt in der Funktion eines stereotypisierenden Elements auf. In struktureller Hinsicht besteht ein Stereotyp aus einem Guppen-Index und einem auf die durch den Index bezeichnete Guppen bezogenen, stereotypisierenden Merkmal. In der nachfolgenden Analyse soll neben der Darstellung des Analyseergebnisses die kulturelle Bedeutung der stereotypisierten Gruppe rekonstruiert werden.

In Tabelle 29 sind die ermittelten Stereotype aufgeführt.

Tabelle 29

\begin{tabular}{|c|c|c|}
\hline $\mathbf{A E}$ & Index & Sterectyp \\
\hline 1 & chip & CHIP rivet na gram vosprijatija (..). \\
\hline 5 & nasi rokery & $\begin{array}{l}\text { Sredi nich [nasich rokerov] gospodstiujut partizanscina i srednevekoryj } \\
\text { separatizm | . . }\end{array}$ \\
\hline 5 & $\begin{array}{l}\text { livanco-maronit: } \\
\text { livanec-siit }\end{array}$ & $\begin{array}{l}\text { No nam tak te slozno pereubedit drug druga. kak livancu-maronitu } \\
\text { livanca-siita. Jazyk u nas vrode by obscij. no vot ponimaem my pod } \\
\text { odnimi i temi te terminami sovsem raznye vesci. }\end{array}$ \\
\hline 6 & $\begin{array}{l}\text { molodye slusateli: } \\
\text { sluxatcli srodncgo vorrasta }\end{array}$ & $\begin{array}{l}\text { Heyzeli slusateli iz Leningrada mogli progolosovat" za etot «panoptukum- } \\
\text { songs», imcja pod rukoj } 50 \text { grupp gorodskogo rok-kluba (dlja molodych) i } \\
\text { pjat" raz Dto Dassena po mestnomu radio (dlja srednego vozrasta) v den`? }\end{array}$ \\
\hline 7 & rjadoroj Inuscnik & $\begin{array}{l}\text { Babki. prjalki. papachi. sapogi. pljaski. tancy. tmancy - tak vosprinimaet } \\
\text { javicnija narodnogo trorcestvo jadovoj truzenik. poputno vspominaja o } \\
\text { pitekantropach, neanderal 'cach i naskal nych rospisjach. }\end{array}$ \\
\hline 8 & $\begin{array}{l}\text { naivnyje aborigeny odnoj } \\
\text { sestoj vsego mira }\end{array}$ & $\begin{array}{l}\text { Naivnye aborigeny odnoj sestoj vsego mira [Sovetskogo Sojusa] toze } \\
\text { stali zertvami kortojskogo uljuljukan'ja. Oni do sich por platjat dorogoj } \\
\text { cennoj za svoju dovercivost. Uze podroslo pokolenie. Iyrazennoe } \\
\text { desjatiletiem bez slov }(60-70 \mathrm{gg}) \text {. }\end{array}$ \\
\hline 8 & nacija & $\begin{array}{l}\text { Desjat let celaja nacija slusala pesni. slor kotonch ne ponimala. Nakonec. } \\
\text { pojavilis' pocti svoi teksty ili udacmc vol me perevody. no nuki ostalis } \\
\text { prezmimi. }\end{array}$ \\
\hline
\end{tabular}




\begin{tabular}{|c|c|c|}
\hline 8 & anglo-saksonskaja rasa & $\begin{array}{l}\text { I... orgazmiceskie stony anemicnoj anglo-saksonskoj rasy, kotoraja } \\
\text { pytactsja gal vanizirovat svoju smerdjascuju mumiju pri pomosci } \\
\text { afrikanskoj spermy. }\end{array}$ \\
\hline 9 & ljubitcli KSP & $\begin{array}{l}\text { Ljubiteli KSP wyodjat svoju rodoslovnuju iz. gorodskogo romansa XIX } \\
\text { veka. odnako pravilnec bylo by scitat vremenem ich rozdenija } 60-e \text { gody } \\
\text { nasego veka } 1 . . . \text {. }\end{array}$ \\
\hline 10 & $\begin{array}{l}\text { potarnye lvse komandy } \\
\text { gorodal }\end{array}$ & $\begin{array}{l}\text { Pozarnue sozdany } \\
\text { Ctobv sledit' za porjadkom }[. . .]\end{array}$ \\
\hline 12 & klubnye turnalisty & $\begin{array}{l}\text { I...] klubnye numalisty inacale otkasalis ego [CHMR/HMR] } \\
\text { prokommentirovat. }\end{array}$ \\
\hline 14 & partjugend & $\begin{array}{l}\text { Ranermytyj bespredel partjugenda ob" jasnjactsja procnym sprascivaniem } \\
\text { transsojuznogo gopnicestia sovetskich britogolowych. }\end{array}$ \\
\hline 14 & $\begin{array}{l}\text { srednij obyvatel: } \\
\text { melkij chorjajcik }\end{array}$ & $\begin{array}{l}\text { Pod pompeznye znamena ideologii liberalizma sgnivsej imperii vstajut vse } \\
\text { novyc boevye stranniki. ocarovannyc scedrost ju darov, uprugim } \\
\text { posobnicestvom vnutrennych organov, snischoditel nym obscestvenym } \\
\text { mneniem srednego obyvatelja i melkogo cho/jajcika. prebyvajuscich v } \\
\text { sladkom sostojanii kommunisticeskogo pochmel'ja i sogrevaemych velikim } \\
\text { kJicem zabludivsegosja mnogo let nazad v debrjach stoego volosjanogo } \\
\text { pokrowa Karla Marksa } \ldots . . . \text {. }\end{array}$ \\
\hline 17 & nasic citatclja & $\begin{array}{l}\text { I... I moja stat ja prodstavljact popytku obratit vmmanic nasich citatclcj na } \\
\text { nekotorye pozabytye storony etogo javlenija /kontrkul tury. }\end{array}$ \\
\hline 20 & $\begin{array}{l}\text { srednestatisticeskij zritel } \\
\text { rastamanskich koncertor }\end{array}$ & $\begin{array}{l}\text { I... posle okoncanija koncerta srednestatisticeskij zritel' rastamanskich } \\
\text { koncertor brosilsja za kulisy, vornalsja v grimerku i potrebonal u liderov } \\
\text { gruppy Gery i Del fina: «Oidajtc mne moju golovu!» }[\ldots] \text { Zritel’ ne verit } \\
\text { pesne i ne trebuet svoju golovu obratno. }\end{array}$ \\
\hline 20 & alisomany & 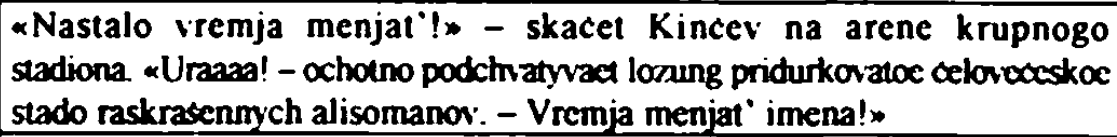 \\
\hline 21 & demokraty & 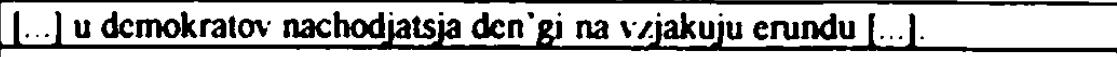 \\
\hline 22 & sluzascij sovmodii & $\begin{array}{l}\text { V obscem. ne privedi Gospod' nastol'ko ne oscuscat' okrurajuscej } \\
\text { dejstvitel 'nosti. naskol ko eto svojstvenno sluascim sovmedii. }\end{array}$ \\
\hline 24 & $\begin{array}{l}\text { nasic skol nye ucitclja } \\
\text { istoni }\end{array}$ & $\begin{array}{l}\text { l... I 1000-m godu suscestiovala Bolonskaja skola prava /kakovoj fakı po } \\
\text { sej den iyzyvaet ne/aslwennuju zevotu nasich skol mych ucitelej Istony } \\
\text { l... }\end{array}$ \\
\hline 27 & jadoryc sturmonikı & 1. . rjadorye sturmovikı ne dostojny svocgo Rema! \\
\hline 28 & $\begin{array}{l}\text { rumalisty: } \\
\text {-nasi 7.orri Djuña }\end{array}$ & 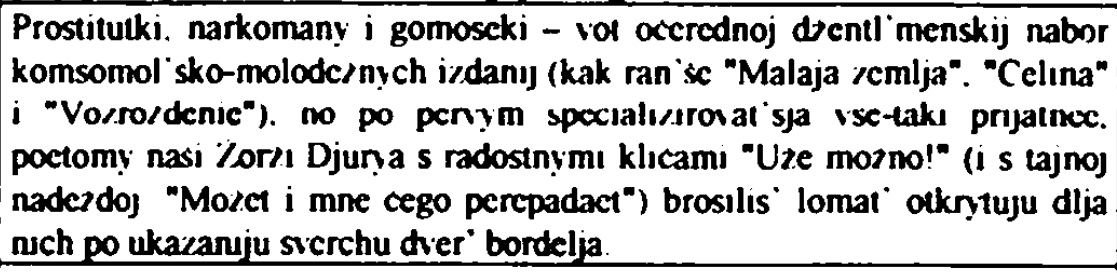 \\
\hline 29 & $\begin{array}{l}\text { musljascaja cast auditorii. } \\
\text { muakant }\end{array}$ & $\begin{array}{l}\text { Reklamno-apologeticeskic stat i o rok-gruppach porjadkom utomili ne } \\
\text { tol'ko mysljascuju cast' auditorii. no i samich munkantor }[(\ldots)] \text {. }\end{array}$ \\
\hline 31 & $\begin{array}{l}\text { depuraty a obcstienmich } \\
\text { organivacj }\end{array}$ & $\begin{array}{l}\text { "Deputaty a obscestvennych organizacij" - nu v kakoj sovremennoj } \\
\text { ertopejskoj strane motno popast' } v \text { parlament ia uspechi v sobiranii } \\
\text { marok ili za ignu v trezrost"? }\end{array}$ \\
\hline 33 & bolgarka & L... strojnaja podpostkoraja figurka. cermuc volos; - pochora na bolgarku. \\
\hline
\end{tabular}

Im Korpus konnen 28 stereotypisierte Gruppen ermittelt werden. Die Stereotype treten in 19 Analyseeinheiten auf $(54,3 \%)$, so $\mathrm{da} B$ die Generierung von Stereotypen kein charakteristisches Textgenerierungsmerkmal des untersuchten Diskurses darstellt. Die Häufigkeit der Verwendung schwankt zwischen 0 und 3, wobei die kein- $(45,7 \%)$ bzw einmalige $(42,9 \%)$ am wahrscheinlichsten ist. Lediglich vier stereotypisierte Gruppen (14,3\%: livanec- 
maronit, livanec-siit, anglo-saksonskaja-raza und bolgarka) zählen zu außersowjetischen bzw. außerpostsowjetischen Kultursystemen, so daß in bezug auf Stereotype eine Introspektive auf das sowjetische bzw. postsowjetische Kultursystem diagnostiziert wird. Der internen Differenzierung der untersuchten Formation dienen mit hoher Wahrscheinlichkeit die folgenden neun Stereotype (32,1\%): chip, nasi rokery, molodye slusateli, klubnye humalisty, nasie citateli, srednestatisticeskij zritel' rastamanskich koncertov, alisomany, mysljascaja cast' auditorii und muzykanty. Es kann in diesem Sinne nicht von einer Introspektive auf die untersuchte Formation gesprochen werden. Offensichtlich werden Stereotype gleichfalls wie Antagonismen generiert, um eine Abgrenzung vom übergeordneten Kultursystem zu gewährleisten.

Im folgenden soll auf die kulturelle Bedeutung der Guppen eingegangen werden, wie sie durch die stereotypisierenden Merkmale zum Ausdruck kommt.

Die durch den Index chip bezeichnete Gruppe chippi wurde bereits im Zusammenhang mit Gruppen-Indices hinsichtlich ihrer kulturellen Bedeutung charakterisiert (vgl.: 9.9.3.). Wegen des stereotypisierenden Merkmals kann nicht unmittelbar abgeleitet werden, ob die Gruppe eine positive bzw. negative kulturelle Bedeutung aufweist. Da keine negative Bedeutung explizien wird, liegt mit hoher Wahrscheinlichkeit an der angesprochenen Stelle eine positive bzw. indifferente Bedeutung vor.

Die Gruppe nasi rokery, die explizit zu der untersuchten kulturellen Formation gerechnet wird, erhält durch das stereotypisierende Merkmal eine negative kulturelle Bedeutung. Die diagnostizierte Bedeutung beschränkt sich vermutlich nur auf Teilaspekte des angesprochenen Konstrukts, da ein umfassenderes Konstrukt der Gruppe mit hoher Wahrscheinlichkeit eine positive Bedeutung aufweist.

Die beiden Gruppen livanec-maronit und livanec-siit dienen im vorliegenden Fall als negatives Beispiel für ein gestortes Kommunikationsverhälnis, welches auf die Gruppe my ubertragen wird. Die genannten Gruppen besitzen in dieser Hinsicht eine negative kulturelle Bedeutung. Implizit liegt ansatzweise ein Autostereotyp vor. Da kein Klassifizienungsmerkmal fur die Gruppe my in der Analyseeinheit genannt wird, fehlt das entscheidende Element fur die genannte Feststellung.

Die im Zusammenhang mit dem Ereignis Pesnja-83 konstruierten Gruppen molodye slusateli und slusateli srednogo vozrasta werden in negativer Weise stereotypisiert, so daß von einer negativen kulturellen Bedeutung ausgegangen wird

Die Gruppe, die sich hinter der Bezeichnung rjadovoj truzenik verbirgt, wird hinsichtlich ihrer Wahmehmung der Volkskunst (narodnoe tvorcestvo) konstruiert. Die unter der Gruppenbezeichnung subsumierten Aktanten werden als einfaltig dargestellt, so daß das Konstrukt jadovoj trutenik in dieser Hinsicht im Rahmen der untersuchten Formation eine negative kulturelle Bedeutung besitzt. Allein die Wahl des Adjektivs jadovoj als Klassifizierungsmerkmal stutzt die geäußerte Vermutung. 
Das stereotypisierende Merkmal, welches auf die Gruppe naivnyje aborigeny odnoj sestoj vsego mira bezogen wird, führt zu einem Autostereotyp, da sich hinter der genannten Gruppenbezeichnung alle Bewohner der Sowjetunion verbergen, also auch der Verfasser der Analyseeinheit bzw. die Mitglieder der untersuchten kulturellen Formation. Die kulturelle Bedeutungskonstruktion fallt offensichtlich negativ aus. Die untersuchte Formation grenzt sich in dieser Hinsicht von dem ihr übergeordneten Kultursystem ab. Diese Position kann mit dem Begriff Elitismus bezeichnet werden. Genauso verhält es sich mit der Gruppe, die sich hinter dem Begriff nacija verbirgt, so daß auf diese nicht gesondert eingegangen wird.

Die Gruppe anglo-saksonskaja raza wird durch das sie stereotypisierende Merkmal außerst negativ konstruiert, so daß von einer negativen kulturellen Bedeutung ausgegangen werden muß

Die ljubiteli KSP werden hinsichtlich der Einschätzung ihrer "Abstammung» (rodoslovnaja) stereotypisient. Der Verfasser der Analyseeinheit bezeichnet diese Einschätzung nicht als falsch, er weist dennoch darauf hin, daß eine andere Einschätzung vermutlich richtiger wäre. Trotz dieser unterschiedlichen Auffassungen wird die genannte Gruppe nicht in negativer Weise konstruiert. Die kulturelle Bedeutung der Gruppe im Rahmen der untersuchten Formation ist mit hoher Wahrscheinlichkeit positiv bzw. indifferent.

In Analyseeinheit 10 geht es um die Eröffnung des Moskovskaja rok-laboratorija durch die Institutionen Dom samodejatel'nogo tvorcestva und Upravlenie kul'tury Mossoveta, die zu diesem Anlaß die Gruppe vse komandy goroda eingeladen haben. Die Stereotypisienung der genannten Gruppe geschieht im vorliegenden Fall durch ein Symbol innerhalb eines Zitats, welches der Gruppe Urfin Dzjus zugeschrieben wird Die Gruppe pozarnye, die als Symbol verwendet wird, wird in dem Zitat nicht in negativer sondern in indifferenter Weise konstruiert. Wird diese Stereotypisierung auf die Gruppe vse komandy goroda ubertragen, so ergibt sich fur diese eine negative kulturelle Bedeutung.

Die Gruppe klubnye zurnalisty wird in indifferenter Weise sterectypisiert. Die kulturelle Bedeutung kann aufgnund des gegebenen Merkmals nicht rekonstruiert werden.

Die Aktanten, die unter dem Begriff partjugend, der seinen etymologischen Ursprung mit hoher Wahrscheinlichkeit im Begriff Hitlerjugend hat, subsumiert werden, sind in außerst negativer Weise konstruiert

Hinter den Gruppenbezeichnungen srednyj obyvatel' und melkij chozjascik verbergen sich zwei Gruppen, die ahnlich wie die bereits erwahnte Gruppe rjadovoj truzenik konstruiert werden. Die gewahlten, gleichfalls klassifizierenden Adjektive weisen auf eine negative kulturelle Bedeutung hin. Wie im genannten Fall, werden die Mitglieder der jeweiligen Gruppe als einfältig dargestellt.

In Analyseeinheit 17 wird das ermittelte Stereotyp nicht direkt mit Bezug auf die Gruppe nasi citatelja generier. Vielmehr wird ein allgemeines Phanomen angesprochen, welches auch auf die genannte Gruppe bezogen werden kann bzw. werden muß. Die Gruppe wird in diesem 
Zusammenhang nicht negativ konstruiert, so daß nicht von einer negativen, sondern von einer positiven kulturellen Bedeutung ausgegangen wird.

Bei der Stereotypisienung und also Bedeutungskonstruktion der Gruppe srednestatisticeskij zitel' rastamanskich koncertov verhält es sich wie mit den bereits erläuterten Gruppen srednyj obyvatel', melkij chozjascik und rjadovoj truzenik, so daß lediglich auf die genannten Abschnitte verwiesen wird.

Die kulturelle Bedeutung der Gruppe alisomany konnte bereits im Zusammenhang mit dem Phänomen Autorität ermittelt werden (vgl:: 9.10.). Der Gruppe wird als stereotypisierendes Merkmal eine banale, diskreditierende Äußerung zugeschrieben.

Die Gruppe demokraty wird im vorliegenden Zusammenhang durch ein negatives, stereotypisierendes Merkmal konstruiert, so daß von einer negativen kulturellen Bedeutung der genannten Gruppe ausgegangen wird. Erwähnenswert ist in diesem Zusammenhang eine Bedeutungsdifferenz zwischen den Begriffen demokratija (vgl.: 9.9.7.) und der hier stereotypisierten Gruppe.

Die Aktanten, die als sluzascij sovmedii bezeichnet werden, weisen in der kulturellen Formation des rok-samizdat eine negative kulturelle Bedeutung auf, wie das stereotypisierende Merkmal verdeutlicht.

Die Gruppe nasie skol'nye ucitelja istorii wird in bezug auf die Rechtsschule von Bologna konstruiert, die die genannte Gruppe angeblich zum Gähnen verleitet. Es ist fraguürdig, inwiefern aufgrund dieses stereotypisierenden Merkmals von einer negativen kulturellen Bedeutung ausgegangen werden kann, so daß diese Frage nicht beantwortet wird.

Die Gruppe jadovye sturmoviki wird negativ stereotypisiert. Wiederum wird ein Adjektiv als begleitendes Klassifizienungsmerkmal verwendet.

Die Gruppe zumalisty wird gleichfalls negativ stereotypisier, so daß von einer negativen Bedeutung im Rahmen der untersuchten kulturellen Formation ausgegangen wird. Erganzend soll in diesem Zusammenhang erwahnt werden, daß es mit hoher Wahrscheinlichkeit nur um Journalisten geht, die fur offizielle Publikationen arbeiten.

In Analyseeinheit 29 werden zwei Gruppen, mysljascaja cast' auditorii und muzykanty, eingefuhrt und anhand eines Merkmals stereotypisiert. Die sich ergebende kulturelle Bedeutung der genannten Gruppen ist positiv. Des weiteren liegt im Fall der erstgenannten Gruppe vermutlich ein Autostereotyp vor.

Die Gruppe deputaty ot obscestvennych organizacij wird negativ stereotypisier, so daß von einer negativen kulturellen Bedeutung ausgegangen wird.

Abschließend soll auf die stereotypisierte Gruppe eingegangen werden, die sich hinter der Bezeichnung bolgarka verbirgt. Die Gruppe wird nur in bezug auf Äßßerlichkeiten stereotypisiert wobei das sich ergebende Konstrukt nicht negativ bewertet wird.

Die Ergebnisse zusammenfassend, soll die Frage gestellt und beantwortet werden, vom wem sich die untersuchte kulturelle Formation explizit abgrenzt. Die neun genannten Gruppen, 
die der untersuchten Formation zugerechnet werden, dienen mit hoher Wahrscheinlichkeit, wie bereits gesagt, der inneren Differenzierung der untersuchten Formation. Die auf sie bezogenen Stereotype dienen nicht der externen Abgrenzung.

Im Rahmen des sowjetischen bzw. postsowjetischen Kultursystems erfolgt eine Abgrenzung von den Gruppen slusateli srednego vozrasta, rjadovoj truzenik, naivnye aborigeny odnoj sestoj vsego mira, nacija, vse komandy goroda, partjugend, srednyj obyvatel', melkij chozjascik, demokraty, sluzascij sovmedij, jadovye sturmoviki, zurnalisty und deputaty ot obsciestvennych organizacij. Werden die genannten Gruppen zu umfassenderen Gruppen zusammengefaßt, so kann festgestellt werden, daß a) eine Abgrenzung von «den Durchschnittsbürgern» (slusateli srednego vozrasta, jadovoj truzenik, naivnye aborigeny odnoj sestoj vsego mira, nacija, srednyj obyvatel' und melkij chozjascik) b) den «Offiziellen» (vse komandy goroda, sluzascij sovmedij, zumalisty und deputaty ot obscestvennych organizacij) und c) von «den Handlangern» (partjugend, rjadovye sturmoviki) erfolgt.

Über die Grenzen des genannten Systems hinweg erfolgt eine Abgrenzung von livanecmaronit, livanec-siit und anglo-saksonskaja raza.

Darüber hinaus kann in mehreren Fällen auf ein elitäres Autokonstrukt geschlossen werden. Hiermit soll die Betrachtung der im Korpus nachgewiesenen Stereotype beendet werden.

\subsection{Normalität}

Es geht im folgenden Abschnitt um das Normalitätskonstrukt der untersuchten Formation, d.h. um den Bereich, den die Mitglieder der untersuchten Formation als «normal» ansehen Normalitat bezieht sich entweder auf das Autokonstrukt der Formation oder auf das des ubergeordneten kulturellen Systems. Normalitat wird in theoretischer Hinsicht sichtbar, wenn ein Element, welches als Normativ bezeichnet wird, auf ein anderes Element bezogen wird Hierdurch wird das Vorhandensein des Bedeutungsaspekts normal innerhalb des Bedeutungskonstrukts des bezogenen Elements hervorgehoben. Der angesprochene Bedeutungsaspekt muß sich nicht auf das vollstandige Konstrukt beziehen, auch Teilbereiche können als normal gelten. Ein Beispiel soll das Gesagte verdeutlichen. Es wird in diesem Zusammenhang dem Untersuchungsergebnis vorweggegriffen. In Analyseeinheit 3 geht es im zweiten Normalitatskonstrukt um die Gruppe Masina Vremeni. Die Normative ne stoit' und eto vy i sami znaete werden in dieser Einheit nicht auf die Gruppe, sondern auf die Popularität der Gruppe in bezug auf die untersuchte Formation bezogen. Es wird auf diese Weise das Vorhandensein des Bedeutungsaspekts normal in bezug auf die Popularitat der Gruppe innerhalb der untersuchten Formation hervorgehoben, nicht generiert. Bedeutsam ist in diesem Zusammenhang. daß der angesprochene Aspekt bereits vorhanden ist, er dennoch genannt wird, so daß sich eine kulturelle Tautologie ergibt. Da die normalisierten Elemente in kultureller Hinsicht funktionalisien sind. besitzen sie für das System, in dem sie generiert wurden, kulturelle Relevanz. 
In Tabelle 30 sind die Untersuchungsergebnisse in bezug auf den angesprochenen Bereich dargestellt.

Tabelle 30

\begin{tabular}{|c|c|c|}
\hline $\mathbf{A E}$ & Normativ & Normalithiskonstrukt \\
\hline 1 & kto iz nas? & CHIP $|\ldots|$ - k1o iz nas ne uznact ego v lico? \\
\hline 2 & kto iz. takich ljudej [nas]? & 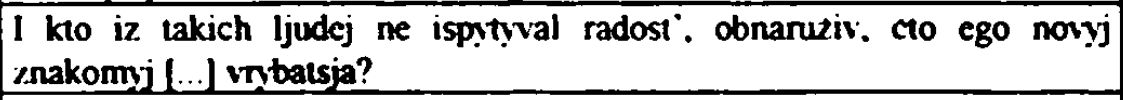 \\
\hline 2 & kto [iz. nas|? & $\begin{array}{l}\text { I kto ne ispytyval nedoumenija. [...] kogda staryj znakomyj [ ... ] sprasivaet. } \\
\text { net u vas Drejmsa Lasta? }\end{array}$ \\
\hline 2 & vo vsjakom slucac & $\begin{array}{l}\text { Vo vsjakom slucac edva li ne samoe otcetlivoe oscuscenie obscnosti }[\ldots] \\
\text { vyzyvaet imenno sormestnoc ucastic } v \text { slusanii rok-muzyki }[\ldots] \text { no ne u } \\
\text { tech, u kogo cego net. }\end{array}$ \\
\hline 3 & \begin{tabular}{|l|} 
my s vami cto motem \\
ponjat
\end{tabular} & $\begin{array}{l}\text { [...] nacalo oktjabrja vneslo v obyem j razgovor fanov dia slova - «Masina } \\
\text { Vremeni }[\text {.... No my s vami eto mozem ponjat'. }\end{array}$ \\
\hline 3 & $\begin{array}{l}\text { ne ctoit; } \\
\text { cto w } \mathrm{w} \text { i sami znacte }\end{array}$ & $\begin{array}{l}\text { O bol'soj populjamosti «Masiny» v nasem gorode tam govorit' ne stoit. } \\
\text { Eto vy. nadejus'. i sami znaete [...]. }\end{array}$ \\
\hline $\mathbf{5}$ & \begin{tabular}{|l|} 
u nas: \\
normal no \\
\end{tabular} & $\begin{array}{l}\text { Jazyk u nas vTode by obscij. no vol ponimaem my pod odnimi i temi ze } \\
\text { terminami sonsem razmye vesci. Voobsce-to, vse eto normal no. }\end{array}$ \\
\hline $\mathbf{5}$ & $u$ nas & $\begin{array}{l}\text { Ved' kumiry - cto u nas. cto na Zapad - voznikajut ne sami po sebe. ich } \\
\text { vynosit social'naja volna. }\end{array}$ \\
\hline 6 & ro vsjakom slucac & $\begin{array}{l}\text { My pisem o roke. pisem o panke. o «noroj volnew. pisem o disko } \\
\text { (poslednee, vo vsjakom slucae, chol' sovremenno) } \\
\end{array}$ \\
\hline 6 & navernoe & 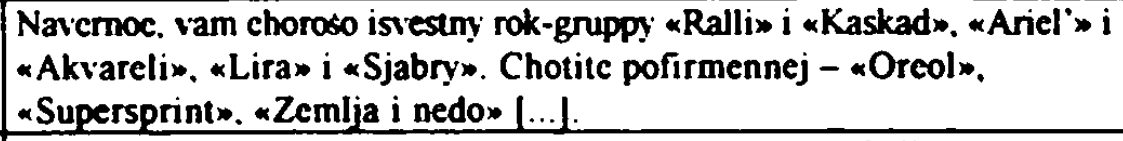 \\
\hline 7 & u nas & «Oni napadajut na samoe cennoe. cto u nas est". - na nas fol 'klor $[\ldots]$. \\
\hline 8 & maloponjatnyj & $\begin{array}{l}\text { Rec' idet o vsjaceskich rok-n-rollach. ritm-end-bljuzach. bugi-vugi } \\
\text { i procich maloponjatnych vescach. }\end{array}$ \\
\hline 10 & /namenityj & 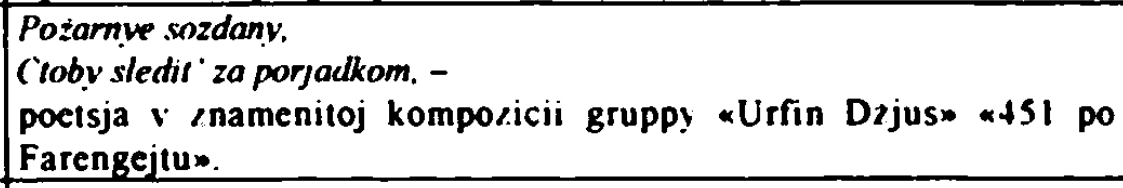 \\
\hline 10 & nas ucit & $\begin{array}{l}\text { Sovremennyj pljuralizm nas ucit. odnako. mnotesticnnosti tocek zrenija } \\
\text { na suscestvujuscic problem }\end{array}$ \\
\hline 10 & po-ridimomu & $\begin{array}{l}\text { Vse. po-ridimomu. ure dogadalis'. cto roc' idet o probleme oktritija } \\
\text { Moskorskogo rok-kluba. }\end{array}$ \\
\hline 10 & znakomyj & $\begin{array}{l}\text { Etu rysokuju misiju vz.jalo na sebja Upravlenie kul'tury Mossoveta. } \\
\text { choroso znakomoe sirokim slojam naselenija }\end{array}$ \\
\hline 10 & znamenițj & $\begin{array}{l}\text { Upravlenie kul'tuñ Mossoveta. choroso inakomoe }[\ldots] \text { po sioemu } \\
\text { znamenitomu postanovleniju. posvjascennomu problemam rok-muạki } \\
\text { l...l. }\end{array}$ \\
\hline 11 & nakonec & $\begin{array}{l}\text { I... I newreli nas dejstvitel'no ostavili v pokoc? Neuzeli nas perestali } \\
\text { zagonjat v radh stroitelej stetlogo buduscego. i my. nakonec. mozem } \\
\text { spokojno. mimo sosuscestiovat? }\end{array}$ \\
\hline 12 & pravil'no & 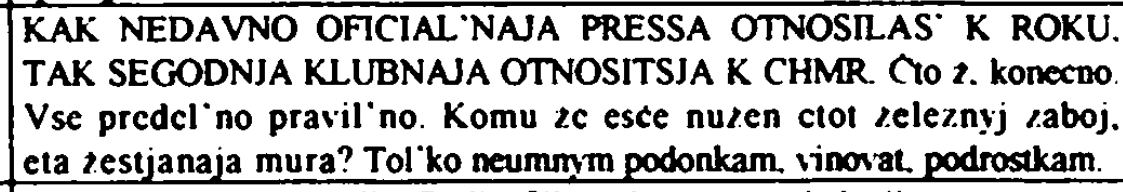 \\
\hline 16 & $\begin{array}{l}\text { samo sobog ranumcetsja: } \\
\text { cto i tak jasno }\end{array}$ & 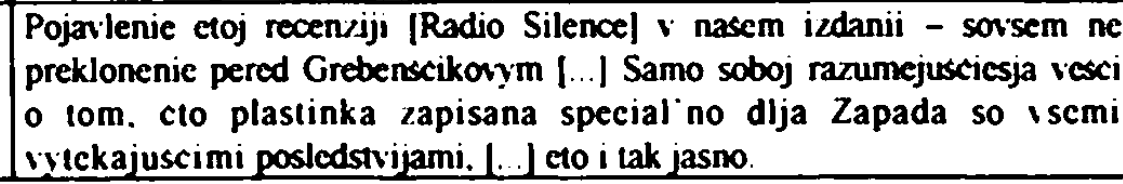 \\
\hline
\end{tabular}




\begin{tabular}{|c|c|c|}
\hline 17 & navernoe & 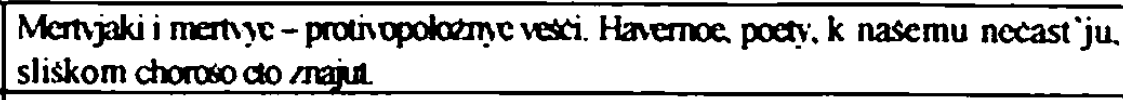 \\
\hline 17 & u nas & $\begin{array}{l}\text { Strogo govorja. nastojascej kontrkul'tury u nas v strane net: est' sovkovo- } \\
\text { oficioznaja kul'tura (predstavlennaja takimi titanami kak poct Michalkov, } \\
\text { pisatel' Bondarev i pevec Kobzon) i protovostojascaja ej prosto Kul'tura } \\
\text { (Mezelajtis. Sevcuk. Iskander). }\end{array}$ \\
\hline 18 & $\begin{array}{l}\text { objazatel'no: } \\
\text { tradicionno }\end{array}$ & 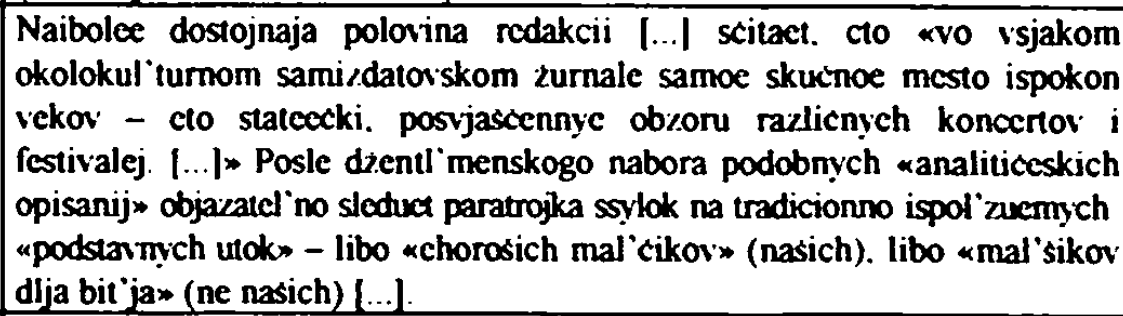 \\
\hline 18 & $\begin{array}{l}\text { toch iz nas: } \\
\text { kak pravilo: } \\
\text { tradicionno }\end{array}$ & 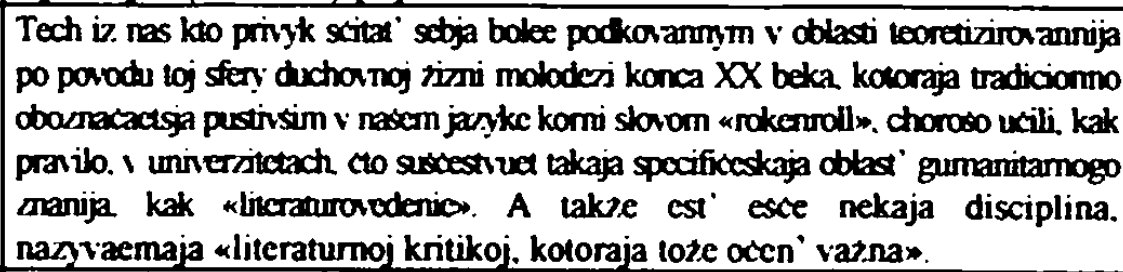 \\
\hline 18 & $\begin{array}{l}\text { samoocteridno: } \\
\text { kto govorit. cto my dolmy }\end{array}$ & $\begin{array}{l}\text { Samoocevidno. cto «Belinskim ot andergraunda» esce tol'ko predstoit } \\
\text { pojavit sja no kto govorit. cto my dolzny iskljucat podobnye popytki iz. } \\
\text { nynesnej rokenrol noj praktiki? }\end{array}$ \\
\hline 20 & obsceizvestno & $\begin{array}{l}\text { Obsceizestno i ne trebuet nikakich dokazatel'stv to. cto rok-muryka } \\
\text { javljaetsja antiobscestvennym zlom. }\end{array}$ \\
\hline 20 & obsceirvestno & $\begin{array}{l}\text { Obsceizvestno [...], cto sobstvenno muayka igraet } v \text { t.n. «russkom roke» } \\
\text { ves'ma i ves'ma vspomogatel'noe mesto. a glavnym tam javljaetsja tekst. }\end{array}$ \\
\hline 21 & navernjaka & $\begin{array}{l}\text { Uchodjat «PRODUKTY } * \text { I ne tol'ko oni. Iscezla kolonna mogucich } \\
\text { «kiroveev». Samyj pronicatel nyj citatel' ponjal navernjaka. cto } \\
\text { rec' ne o ede. REC" O OSCUSCENII. }\end{array}$ \\
\hline 22 & izvestnyj & $\begin{array}{l}\text { Dejstritel'no. choroso by my rygljadeli. csli b rabotali v reguljarnoj gazcte. } \\
\text { Kak Rok-Advokat. izestnyj sorgavanskii ment i tusorscik } \quad \text {..... }\end{array}$ \\
\hline 22 & navcrnjaka & 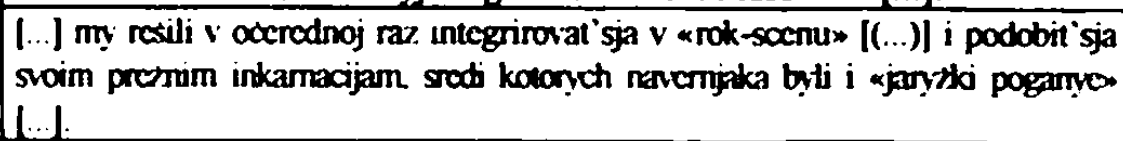 \\
\hline 23 & nas emoc & $\begin{array}{l}\text { Clo nas scgodnja ob"cdinjact? Navernoc. pravda kak obscestvennyj } \\
\text { razdrazitel". }\end{array}$ \\
\hline 23 & irvesimyj & 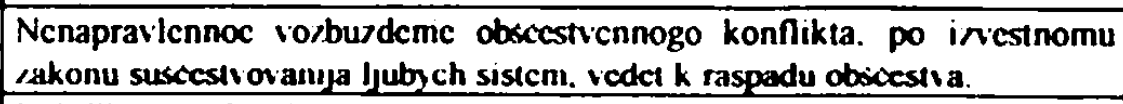 \\
\hline 23 & vsegda & $\begin{array}{l}\text { 1... I dilctanty, koich v smutnoc vremja vsegda bylo dostatocno dlja raniala } \\
\text { ljubogo dela. }\end{array}$ \\
\hline 24 & casto & $\begin{array}{l}\text { Zivja v Rossii. my casto zabyvacm absurdnost' konteksta. v uslovijach } \\
\text { kotorogo forminjutsja nasi licnosti. }\end{array}$ \\
\hline 24 & populjarmyj & $\begin{array}{l}\text { Smeluju popytku takogo roda sdelal nedavno lider populjarnoj gruppy } \\
\text { "DK" Sergej Zarikor } 1 . . .1\end{array}$ \\
\hline 24 & nasumevijij & $\begin{array}{l}\text { Smeluju popylku [... ] sdelal nedarno | ... I Scrgej Zarikov v sicej nasumensej } \\
\text { rabote "Rok-n-Roll na Rusi". }\end{array}$ \\
\hline 25 & u nas & $\begin{array}{l}\text { U nas ocen' ljubjat s nostal' gicj vspominat' "byloc". vostorosenno peretivat ili } \\
\text { ozlobtenno kritikowat nalicmuju i konkretnuju situaciju no ne dajut sebe truda } \\
\text { vzgljanut' na opisyvaemyj fenomen s tocki zrenija ego razvitija. } \\
\text { ego dinamiceskich vozmoznostej. s istoriceskoj tocki zrenija. }\end{array}$ \\
\hline 26 & tradicionno & "Komu podygryval intcllcktual' myj rok?" $1 . .$. A vot vopros tradicionen. \\
\hline 27 & soglasit'sja & $\begin{array}{l}\text { Fany soretskogo roka iz. Bundes-respubliki Germanii. kotorych ja provodil } \\
\text { na sejsen. podarili mne za eto macok s portretom M.C. Gorbacera i } \\
\text { nadpis"ju po-russki "GLASNOST" lodnako "made in Germany"/ } \\
\text { Situacija. soglastes". dovol'no neobycnaja. }\end{array}$ \\
\hline
\end{tabular}




\begin{tabular}{|c|c|c|}
\hline 27 & populjarnyj & $\begin{array}{l}\text { A na sledujuscij den' sel za masinku. ctoby prodolzit' stoi proslogodnye } \\
\text { rapiski. stavsic dovol 'no populjarnymi blagodarja "urodlivomu javieniju" } \\
\text { nukopismych rok-rumalo: }\end{array}$ \\
\hline 27 & segodnja & $\begin{array}{l}\text { Co oznacaet. naprimer. slovo "ckstremist"? Segodnja tak nazyvajut } \\
\text { D. Vasil eva s kompaniej } \mid . . .1 \text {. }\end{array}$ \\
\hline 28 & kto iz. nas [?] & $\begin{array}{l}\text { Pered vami, deti. utka - } \\
\text { Ona bol'saja prosti... } \\
\text { Prostitc. deti. ona malen kaja. } \\
\text { Nu kto iz nas nc pomnit s detstia ctot zamocatel nyj stisok. }\end{array}$ \\
\hline 29 & dolzno & 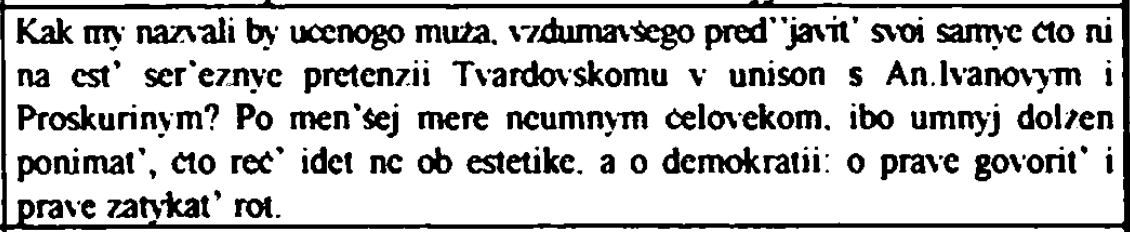 \\
\hline 29 & ne stoit' & $\begin{array}{l}\text { Tak cto reklamu TELEVIZORA i OBLACNOGO KRAJA vse-taki ne stoit } \\
\text { stavit' na odnu dosku s nazojlivym voschvaleniem B.Kuz'mina }|\ldots| . \mid\end{array}$ \\
\hline 29 & soglasit sja & $\begin{array}{l}\text { No soglasitcs', cto } v \text { absoljutno nenormal 'mych uslorijach. kogda celomu } \\
\text { zanru sorctskogo iskusstia prichodilos' otstaivat' swoe pravo na tiz.n'. u } \\
\text { celoveka. pisuscego o roke dija oficial'nogo izdanija. prosto ne bylo } \\
\text { drugogo puti. }\end{array}$ \\
\hline 30 & $\begin{array}{l}\text { u nas: } \\
\text { casto }\end{array}$ & 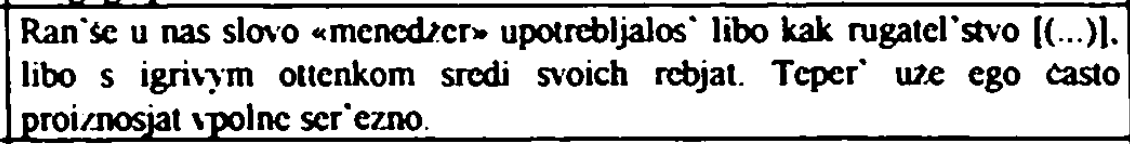 \\
\hline 31 & ne stoit ${ }^{\circ}$ & $\begin{array}{l}\text { Tak cto curopejcam ne stoit osobenno zadirat pered nami nos: oni toze } \\
\text { cerez vse eto prosli. }\end{array}$ \\
\hline 31 & u nas & $\begin{array}{l}\text { A u nas i segodnja naznacennye deputaty "ot KPSS" i t.n. "obscestvennych } \\
\text { organizacij" }\end{array}$ \\
\hline 32 & vsegda & Slovo v russkoj kul 'ture vscegda cenilos' neobycajno vysoko. \\
\hline 35 & iacsino & $\begin{array}{l}\text { Poslodstiicm torogo cut' bylo ne stala popytka emigracii v odno iz. } \\
\text { aziatskich gosudarst: gdc. kak isvestno. klimaticeskic uslovija naiboloc } \\
\text { blagoprijatny dlja proizrastanija bambuka. }\end{array}$ \\
\hline
\end{tabular}

Es wird im Korpus von 50 verschiedenen kulturellen Tautologien ausgegangen, die das Resultat der Generienung des Normalitätsbereichs der untersuchten Formation sind. Diese Tautologien können in 26 Analyseeinheiten (74,3\%) ermittelt werden, so daß ihre Verwendung ein charakteristisches Textgenerierungsmerkmal des untersuchten Diskurses darstellt. Kulturelle Tautologien werden mit einer Haufigkeit, die sich zwischen 0 und 5 bewegt, in den Analyseeinheiten eingesetzt, wobei die Häufigkeit 1 mit $37,1 \%$ am wahrscheinlichsten ist Es können 34 verschiedene Normative ermittelt werden, von denen die folgenden mehrmals im Korpus in verschiedenen Analyseeinheiten Verwendung finden: $u$ nas $(5,7,17,25,30,31)$, kto iz nas/kto [iz nas]/kto iz takich ljudej [nas] $(1,2,28)$, navernoe $(6,17,23)$, ne stoit' $(3,29$, $31)$, casto $(24,30)$ navernjaka $(21,22)$, populjarnyj $(24,27)$, soglasit'sja $(27,29)$, tradicionno $(18,26)$, vo vsjakom slucae $(2,6)$ und vsegda $(23,32)$.

Neben diesen quantitativen Ergebnissen der Korpusanalyse gilt es der Frage nachzugehen, welche Elemente normalisien und somit kulturell relevant sind. Um diese Frage zu beantworten, wird auf die einzelnen kulturellen Tautologien eingegangen.

Vordergrundig geht es in Analyseeinheit 1 um das außere Erscheinungsbild der Mitglieder der Gruppe chippi, die durch den Index CHIP bezeichnet werden und aufgrund besonderer 
Merkmale (vgl.: 4.5.) von den Mitgliedern der untersuchten Formation erkannt werden. Vermutlich geht es in einem weiteren Sinne um das Verstehen der Gruppe. In dieser Hinsicht ist es selbstverständlich, daß das, was sich hinter der äußeren Fassade der Mitglieder der genannten Gruppe verbirgt, von den Mitgliedern der untersuchten Formation verstanden wird. Mit hoher Wahrscheinlichkeit besitzt die angesprochene Gruppe, die vermutlich mit der untersuchten Formation assoziiert ist (vgl.: 4.3. und 9.15.), in diesem Sinne, wie bereits diagnostiziert (vgl: 9.9.3.), eine positive kulturelle Bedeutung im Rahmen der untersuchten Formation.

In Analyseeinheit 2 geht es ebenfalls um das Verstehen, wie bereits die Überschrift der genannten Analyseeinheit ( $O$ vrube) unmißverständlich klarstellt. Es geht um das Verstehen, das «Eindringen» in die Rock-Musik. In den aufgeführten Tautologien werden Einzelheiten bzw. Kriterien dieses Verstehens genannt. In diesem Sinne werden nur Aktanten zur untersuchten Formation gezählt, die den in der Analyseeinheit genannten Kriterien genügen. Die kulturellen Tautologien verdeutlichen folglich die Konsolidierung der Formation

Das Normativ my s vami eto motem ponjat' in Analyseeinheit 3 bezieht sich auf die Fans der Gruppe Masina Vremeni, deren Verhalten im vorliegenden Zusammenhang als verstanden, d.h. als normal beurteilt wird. Wiederum wird in einem weiteren Sinne die Konsolidierung der Formation deutlich. Auf die kulturelle Tautologie, die die Popularität der genannten Gruppe behandelt, wurde bereits weiter oben eingegangen.

Sowohl im Westen als auch in der Sowjetunion bzw. deren Nachfolgestaaten entstünden Gotzenbilder, also das, was mit dem Begriff Star bezeichnet wird, nicht von selbst, sondern aufgrund einer sozialen Welle. Die in dieser Gesetzeshypothese geäßerte Auffassung, die gleichfalls eine Gemeinsamkeit mit «dem Westen» betont, spiegelt Normalität wider. Gleichfalls wird an dieser Stelle ein "normalen Antagonismus generiert, der nicht auf der negativen kulturellen Bedeutung des Geographicums Zapad basiert (vgl : 9. 12.1.).

In Analyseeinheit 5 findet sich eine Gesetzeshypothese, die sich auf das gegenseitige Verstehen bezieht. Es ist, dieser Hypothese folgend, richtig, daß, bezogen auf das russische Kultursystem, eine Sprache gesprochen, dennoch von jedem Kulturteilnehmer etwas anderes verstanden wird. Mit hoher Wahrscheinlichkeit geht es im vorliegenden Zusammenhang um das Verhältnis zum ubergeordneten Kultursystem, welches in diesem Sinne als gestöt aufgefaßt wird

In Analyseeinheit 6 geht es einerseits um diejenigen Musikrichtungen, die zum Gegenstand der untersuchten Formation zählen (rok, pank, novaja volna, sovremennoe disko), andererseits um Musikgruppen, die nicht dazugehoren. Wiederum wird die Konsolidierung der betrachteten kulturellen Formation deutlich

Die Normalität von zwei Elementen wird in Analyseeinheit 7 im Rahmen einer kulturellen Tautologie vermittels des Normativs u nas hervorgehoben. Nebenbei bemerkt sei, daß es sich im vorliegenden Fall um ein Zitat handelt, welches einem Aktanten, der als odin surovyj dejatel' bezeichnet wird, zugesprochen wird. In dieser Aussage wird die Folklore einerseits als 
das Wertvollste dargestellt, was die Mitglieder der untersuchten Formation besitzen, andererseits ist das Herfallen über die Folklore, also das Verbot durch die Gruppe oni, hinter der sich vermutlich die "Obrigkeit" verbirgt ( $v g l$ : 9.12.1.), eine Tatsache, die bedauert wird, aber als normal gilt. Es geht letztendlich erneut um das Verhälnis zum übergeordneten Kultursystem, welches offensichtlich gestört ist.

In Analyseeinheit 8 werden die Musikstile rok-n-roll, ritm-end-bljuz und bugi-vugi zu den wenig verstandenen Dingen gezäht. Diese Zuordnung bezieht sich vermutlich nicht auf die untersuchte Formation, sondern auf das übergeordnete Kultursystem. Die kulturelle Tautologie verdeutlicht einerseits die Konsolidierung der Formation, andererseits wird erneut ein gestörtes Verhältnis zu dem übergeordneten Kultursystem deutlich.

Insgesamt fünf Normative und also kulturelle Tautologien können in Analyseeinheit 10 ermittelt werden. Dreimal wird der Bekanntheitsgrad bestimmter Elemente betont: 451 po Farengejtu, Upravlenie kul'tury Mossoveta und postanovlenija posvjascenomu problemam rokmuzyki. Einmal geht es in einer Gesetzeshypothese, in der das Normativ nas ucit bemüht wird, um die Funktion des zeitgenössischen Pluralismus, der in positiver Weise konstruiert wird. Das Normativ po-vidimomu bezieht sich im vorliegenden Fall auf den Rezipienten. Der Verfasser der Analyseeinheit geht davon aus, genug Hinweise im Text gegeben zu haben, so daß der normale Rezipient problemlos nachvollziehen kann, worum es in der Analyseeinheit eigentlich geht, nämlich um die Eröffnung der Institution Moskovskaja rok-laboratorija, nicht um die gesellschafliche Funktion von Feuerwehrleuten.

In Analyseeinheit 11 wird der Lebensstil definiert, der von den Mitgliedern der untersuchten Formation gewünscht wird: spokojno i mirno. Aufgrund von Repressalien seitens der "Obrigkeit» läßt sich dieser Wunsch zum Zeitpunkt der Erstellung der Analyseeinheit nicht realisieren. Wiederum wird ein gestörtes Verhältnis zum übergeordneten Kultursystem deutlich, welches als normal angesehen wird

Das Verhaltnis der Rock-Club-Presse (klubnaja pressa) zum CHMR wird in Analyseeinheit 12 durch das Normativ pravil'no bezeichnet. Deutlich wird an dieser Stelle die ablehnende Haltung, die dem CHMR entgegengebracht wird. Es wird ein «normalen Antagonismus generiert. der eine interne Differenzienung der untersuchten Formation verdeutlicht (vgl: 9.12.1.).

In Analyseeinheit 16 geht es um eine Soloveröffentlichung von Grebenscikov. Es wird in diesem Zusammenhang offenkundig, daß Unterschiede zu dem als Westen bezeichneten Ausland bestehen, die als normal angesehen werden. Die Tatsache dieser Unterschiedlichkeit wird vermittels von zwei Normativen, samo saboj razumeetsja und eto i tak jasno, hervorgehoben.

Offensichtlich gilt es in der untersuchten Formation als selbstverständlich, daß die Gruppe poety den Unterschied zwischen den Begriffen mertvjaki und mertvye kennt Vermittels des Normativs $u$ nas wird in Analyseeinheit 17 gleichfalls die Tatsache betont, daß es keine kontrkul'tura, sondern lediglich eine sovkovo-oficioznaja-kul'tura und eine Kul'tura gebe. wobei einige Vertreter der beiden letztgenannten Bereiche namentlich genannt werden. Aufgrund 
des antagonistischen Verhältnisses kann von einer positiven kulturellen Bedeutung der kontrkul'tura und einer negativen der verbleibenden Elemente ausgegangen werden (vgl: 9.12.1.). In diesem Sinne liegt wiederum ein Hinweis auf ein gestörtes Verhältnis zum übergeordneten Kultursystem vor.

In Analyseeinheit 18 geht es um bestimmte, negativ bewertete Beiträge, die nach Auffassung des Verfassers der genannten Analyseeinheit in Publikationen des rok-samizdat nachweisbar sind. Die angesprochenen Beitrage enthaten Elemente, deren Verwendung als normal gilt. In der genannten Analyseeinheit spricht sich der Verfasser gegen diese Normalitä aus. Des weiteren geht es in der Analyseeinheit um den Normalitätsbereich von Aktanten, die sich aufgrund ihrer universitären Bildung elităr geben. Dieser Normalitätsbereich wird ebenfalls abgelehnt. In bezug auf den formationsinternen Normalitätsbereich wird betont, daß Aktanten, die einen gewissen Intellekt, namentlich wie Vissarion Belinskij, besitzen, bedauemswerterweise nicht zu der untersuchten Formation zählen. Das Gesagte läuft darauf hinaus, daß der Verfasser der genannten Analyseeinheit intendiert, den momentanen Normalitätsbereich der untersuchten Formation zu verändern und Vorschläge in diese Richtung unterbreitet. Darüber hinaus wird eine interne Differenzierung der untersuchten Formation deutlich.

Das Normativ obsceizvestno wird in Analyseeinheit 20 zweimal angewendet. Zum einen dient es als Nachweis, daß ein gestörtes Verhältnis zum übergeordneten Kultursystem vorliegt. da offensichtlich unterschiedliche Normalitätsauffassungen in bezug auf das Element rokmuzyka existieren. Im zweiten Fall wird eine Gesetzeshypothese in bezug auf das Element russkij rok formuliert, die den Normalitatsbereich der untersuchten Formation widerspiegelt.

In Analyseeinheit 21 funktioniert das Normativ navernjaka wie das Normativ po-vidimomu in Analyseeinheit 10. Der Verfasser geht davon aus, daß der normale Rezipient aufgrund der textinternen Hinweise nachvollziehen kann, worum es in der Analyseeinheit geht

Det Bekanntheitsgrad des Aktanten Rok-Advokat, der eine negative kulturelle Bedeutung aufweist, wird in der nachfolgenden Analyseeinheit als normal dargestell Des weiteren ist das Vorhandensein von jaryzki poganye im Rahmen der Rock-Szene (rok-scena), gemaß der angesprochenen Analyseeinheit, normal. Wiederum wird eine interne Differenzierung der untersuchten Formation sichtbar.

In Analyseeinheit 23 geht es zunächst um die Wahrheit (pravda), deren Funktion (obscestvennyj razdraritel') in bezug auf das übergeordnete Kultursystem dargestellt wird. Dariber hinaus wird in der genannten Analyseeinheit vermittels des Normativs izvestnyj die Bekanntheit eines bestimmten Systemgesetzes betont. Des weiteren wird durch das Normativ vsegda deutlich, daß das Vorhandensein von der Guppe diletanty in bestimmten Situationen als normal angesehen wird.

Zunachst wird in Analyseeinheit 24 vermitteis des Normativs casto ein Aspekt des Normalitatsbereichs des russischen Kultursystems deutlich: die Tatsache der Absurdität, in der sich die dortigen Charaktere entwickeln. Dariber hinaus wird offensichtlich, daß sowohl eine Arbeit von Sergej \%arikov (Rok-n-Rol na Rusi) als auch die Gruppe DK, in der dieser spielt, eine 
positive kulturelle Bedeutung aufweisen. Die Normalität in bezug auf die diagnostizierte Bedeutung kann mit Hilfe der Normative nasumevsij und populjarnyj belegt werden.

In Analyseeinheit 25 wird der Normalitätsbereich der untersuchten Formation abgelehnt, der vermittels des Normativs u nas sichtbar wird. Der Verfasser der genannten Analyseeinheit zielt auf eine Veränderung des angesprochenen Normalitätsbereichs ab.

Die Frage „Komu podygryval intellektual'nyj rok“, die aus dem Interdiskurs stammt, ist gemäß der Auffassung des Verfassers von Analyseeinheit 26 traditionell, d.h. für das übergeordnete Kultursystem normal. Wiederum tritt ein gestörtes Verhältnis zu diesem System offen zu Tage.

In Analyseeinheit 27 wird vermittels des Normativs populjarnyj der Bekanntheitsgrad und die kulturelle Bedeutung der proslogodnoe zapiski, die aus der Feder des Verfassers der genannten Analyseeinheit stammen, deutlich. Es ist fragwürdig inwiefern ein Verfasser den Bekannkheitsgrad und die Bedeutung seines eigenen Werks beurteilen kann. Möglicherweise liegt der Versuch vor, einen falschen Normalitätsbereich zu konstruieren. Das Normativ soglasit'sja erfullt in der genannten Analyseeinheit die Funktion einer thetorischen Frage. Der Rezipient wird im vorliegenden Fall aufgefordert, eine bestimmte Situation zu beurteilen, wobei die Beurteihung eines normalen Rezipienten vorweggenommen wird: dovol'no neobycnaja. Daruber hinaus wird in der genannten Analyseeinheit die Bezeichnung des Vorsitzenden der Partei Pamjat, Vasil'ev, als Extremist im temporaren Kontext (segodnja) als normal hervorgehoben.

Analyseeinheit 28 folgend ist es selbstverständlich, daß das Gedicht Pered vami, deti, utka sowohl den Mitgliedern det untersuchten Formation als auch denen des übergeordneten Systems von Kindheit an bekannt ist. Die Bekanntheit des Gedichts stellt mit hoher Wahrscheinlichkeit einen Aspekt des Normalitätsbereichs des ubergeordneten Kultursystems dar, da zweifellos niemand von Kindheit an zur untersuchten Formation gezählt werden kann

In Analyseeinheit 29 funktioniert das Normativ dolzno wie die Normative po-vidimomu in Analyseeinheit 10 bzw. navernjaka in Analyseeihneit 21 Der Verfasser geht davon aus, daß ein normaler Rezipient unmißverstandlich nachvollziehen kann, worauf er in thematischer Hinsicht abzielt: auf den Themenkomplex demokratija. Des weiteren wird ein Vergleich genannt, der sich im Rahmen der untersuchten Formation nicht gehört, der nicht in den Normalitätsbereich integriert ist. Das Normativ soglasit'sja funktioniert in der angesprochenen Analyseeinheit wie in Analyseeinheit 27: zum Zeitpunkt der Erstellung der genannten Analyseeinheit gibt es keinen Zweifel daran, daß a) sich die gesamte sowjetische Kunst in einer schweren Krise befindet und $\mathrm{da} B$ b) ein Mensch, der für die offizielle Presse tätig ist, in einer bestimmten Weise schreiben $\mathrm{muB}$

In Analyseeinheit 30 wird eine Veränderung des Normalitätsbereichs der untersuchten Formation deutlich. Das Wort menedzer galt früher (ran'se) als Schimpfivort. Es kann heute (teper') gemäß der Aussage des Verfasser der Analyseeinheit völlig seriös verwendet werden. 
In einem weiteren Sinne geht es um gesellschaftiche Veränderungen, die als normal angesehen werden

Das Normativ ne stoit' in Analyseeinheit 31 zielt auf den Normalitätsbereich ab, der der Gruppe evropejcy zugesprochen wird. Dieser Normalitätsbereich wird in der untersuchten Formation offensichtlich abgelehnt. Des weiteren gilt es als normal, daß Parlamentsmitglieder von der KPSS bzw. öffentlichen Organisationen emannt werden. Diese Tatsache wird als falsch bewertet, so daß emeut ein Hinweis auf ein gestörtes Verhältnis zum übergeordneten Kultursystem vorliegt.

Mit Hilfe des Normativs vsegda wird in Analyseeinheit 32 eine allgemeine Gesetzeshypothese formuliert, die sich auf den Normalitätsbereich des übergeordneten Kultursystems bezieht.

In der in Analyseeinheit 35 ermittelten kulturellen Tautologie geht es um die klimatologischen Kenntnisse der Mitglieder der untersuchten Formation: das Wissen, daß in asiatischen Ländern das Klima fur den Anbau von Bambus günstiger ist als in Orecho-Zuevo, gilt als selbstverständlich. Offensichtlich wird im vorliegenden Fall mit dem Normalitätskonstrukt der untersuchten Formation «gespielt».

Die Analyseergebnisse sollen abschließend zusammengefaßt werden. Es soll hierbei darum gehen, inwiefern im Korpus der eigene, formationsinterne Normalitätsbereich bzw. andere Normalitätsbereiche deutlich werden. Bei den anderen Normalitätsbereichen handelt es sich bis auf den des übergeordneten Kultursystems um Konstrukte, die innerhalb der Formation generiert werden. Diese müssen nicht mit den Normalitätsbereichen der entsprechenden Formationen und kulturellen Systeme identisch sein. Weiterhin soll der Frage nachgegangen werden, welche Normalitatsbereiche explizit negiert, d.h. abgelehnt werden. Grundlage der $\mathrm{Zu}$ sammenfassung sind die ermittelten 50 kulturellen Tautologien, die in Tabelle $\mathbf{3 0}$ aufgefuihrt sind. Da es in zwei dieser Tautologien (8 und 11) um jeweils zwei Normalitatsbereiche geht, wird von 52 Hinweisen auf Normalitatsbereiche ausgegangen.

Insgesamt werden funf Normalitätsbereiche erwahnt: der formationsinterne, der des ubergeordneten Kultursystems, der des Westens, der elitärer Aktanten und der von Aktanten, die unter dem Index evropejcy subsumiert werden In 33 kulturellen Tautologien (63,5\%) wird der formationsinterne Normalitätsbereich thematisien, so daß in bezug auf kulturelle Tautologien von einer Introspektive auf die untersuchte Formation gesprochen wird. In 15 Fällen $(28,8 \%)$ geht es um den Normalitätsbereich des übergeordneten Kultursystems, in zwei Fällen $(3,8 \%)$ um den des Westens und in jeweils einem Fall $(1,9 \%)$ um den von elitaren Aktanten bzw. Europäern Durch sechs kulturelle Tautologien (11,5\%) wird eine Negation des ubergeordneten Kultursystems deutlich. D.h. in $40 \%$ der Fälle, in denen Hinweise auf den angesprochenen Normalitätsbereich gegeben werden, erfolgt eine Ablehnung von diesem. In $60 \%$ der Fälle wird dieser Normalitätsbereich dennoch auch als gultig fur die untersuchte Formation akzeptiert. In zwei Fallen (3,8\%) wird der formationsinterne Normalitätsbereich negiert In bezug auf 
bestimmte Aspekte dieses Bereichs herrscht dementsprechend keine Übereinstimmung innerhalb der untersuchten Formation. Darüber hinaus erfolgt eine jeweils einmalige Negation des Normalitätsbereichs der elitären Aktanten und der Europäer. Wenn der Normalitätsbereich dieser Gruppen thematisiert wird, dann wird er, im Gegensatz zum Normalitätsbereich des Westens, abgelehnt.

Hiermit soll die Analyse des Normalitätsbereichs der kulturellen Formation des roksamizdat beendet werden.

\subsection{Rhetorische Fragen}

In funktionaler Hinsicht werden rhetorische Fragen eingesetzt, um relevante Aussagen hervorzuheben, d.h. zu bekräftigen (vgl: 5.9.). In struktureller Hinsicht kann die vom Verfasser eines Textes als relevant erachtete Aussage zum einen als Frage formuliert werden, zum anderen kann sie einer Frage vor- oder nachgestellt sein. In diesem Sinne beziehen sich kurze, wenige Worte umfassende Fragen mit hoher Wahrscheinlichkeit auf ihnen vor- bzw. nachgestellte Aussagen. Im vorliegenden Zusammenhang soll der angesprochene Begriff nicht auf einzelne Fragen beschränkt, sondern auch auf Fragenkomplexe, die sich auf eine Aussage beziehen, erweitert werden, da diese Komplexe vermutlich in ihrer Gesamtheit die genannte Funktion erfüllen.

Das Ergebnis der Korpusanalyse ist in Tabelle 31 abgebildet.

Tabelle 31

\begin{tabular}{|c|c|}
\hline $\mathbf{A E}$ & Rhetoriscbe Frage \\
\hline 1 & CHIP $|\ldots|-k$ to iz nas ne uznaet ego v lico? \\
\hline 2 & 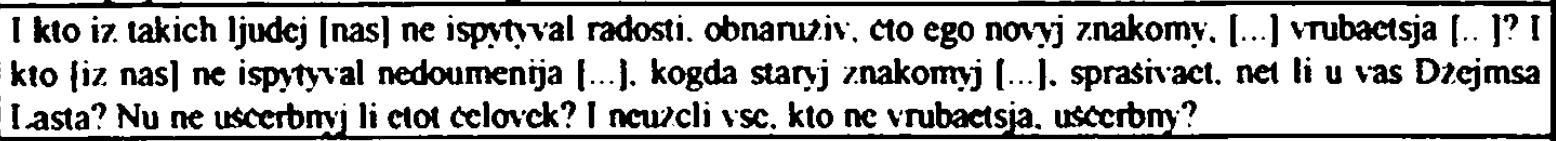 \\
\hline 2 & 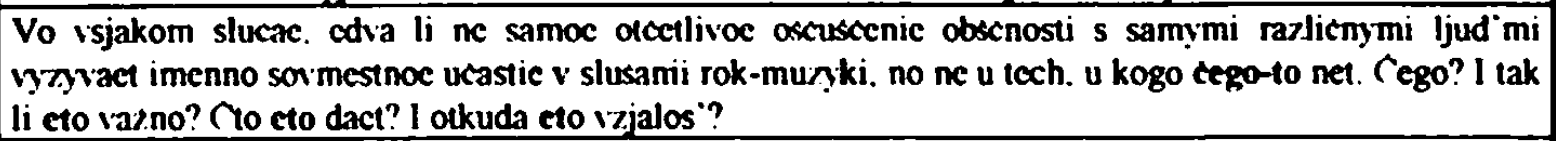 \\
\hline 5 & 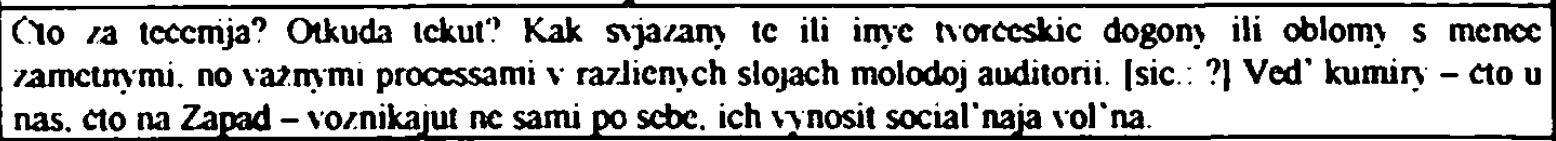 \\
\hline 6 & $\begin{array}{l}\text { "Pustoc slovo vsegda minuet curstio styda» I. Kochanovskij } \\
\text { A dejstvitel'no. minuct li? }\end{array}$ \\
\hline 6 & Heyzeli slusatcli iz Leningrada mogli progolosovat za clol «panoptikum-songs» $[. .$.$] ?$ \\
\hline 7 & $\begin{array}{l}\text { No za oknom vesennimi ruc 'jami nalivactsja } 1985 \text { god. i. kak skazal by «veras». "nastal cas rozoryj». } \\
\text { Ho fol 'klor li cto? (. yo budut govorit' o nas nasi vnuki i cto my smozem im otvetit? } \\
\end{array}$ \\
\hline 8 & 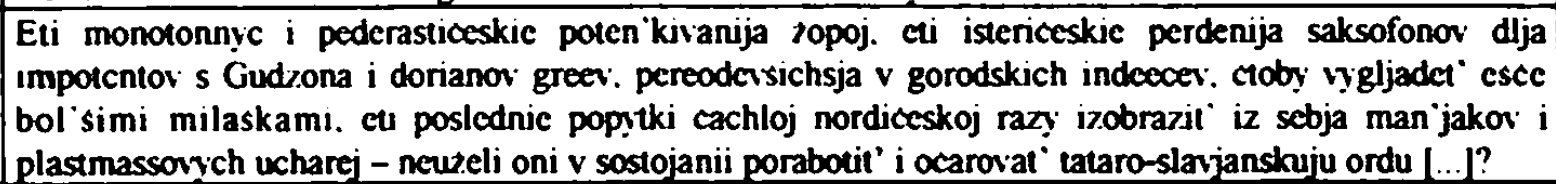 \\
\hline 9 & $\begin{array}{l}\text { I... I v mire vse proischodit prakticeski odnowremenno. i my s vami ne } v \text { antimire tivem [...]. No mozno } \\
\text { li na ctoj odnovtemennosti wrodit' prjamuju svjaz' KSP s rokom. kak na Zapade? Edia li. }\end{array}$ \\
\hline 11 & $\begin{array}{l}\text { [... I neuzcli loni] nas dejstritcl' no ostavili v pokoe? Ncuzeli [oni] nas perestali zagonjat' v rjady } \\
\text { stroitelcj stctlogo buduscego. i my. nakonec. mozem spokojno. mimo sosuscestiovat"? }\end{array}$ \\
\hline 12 & $\begin{array}{l}\text { Po-mocmu. cto [Roksi] ure stal reakcionmj zumal I scjcas on pererivaet kul'minacionnyj punkt. Kogda } \\
\text { na cgo stranicach my uvideli sokrascenic (HMR (HMR)? Kuda pozdnec. cem na raborach. }\end{array}$ \\
\hline
\end{tabular}




\begin{tabular}{|c|c|}
\hline 12 & $\begin{array}{l}\text { Komu te esce nuzen etor zelc/myj zaboj. eta testjanaja mura? Tol 'ko neumnym podonkam. vinovat. } \\
\text { podrostkam. A turnal «Roksi» togda, izvinjajus'. dlja kogo? Sprasivacte - otvecaju. Dlja toch. komu za } \\
\text { tridcat'. }\end{array}$ \\
\hline 13 & O roke pisat"? Pozalujsta $[. . .1$. Vo-penych. cto cst' rok? \\
\hline 16 & 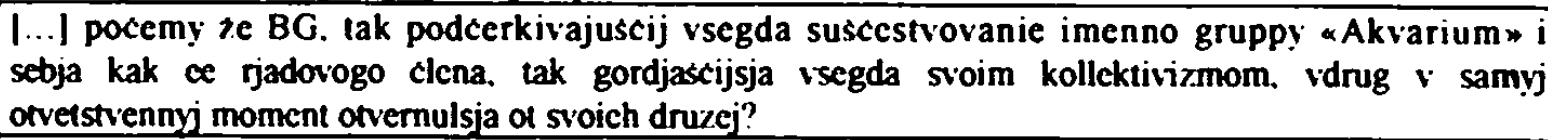 \\
\hline 18 & $\begin{array}{l}\text { Samoocevidno. cto aBelinskim ot andergraundaw esce tol 'ko predstoil pojavit'sja. no kto govorit. cto my } \\
\text { doltny iskljucat podobnye popytki iz mynesnej rokenrol noj praktiki? }\end{array}$ \\
\hline 19 & $\begin{array}{l}\text { «Strannaja. nelcgkaja dlja istorikov i filosofov zadaca - nonjat' }{ }^{*} \text { - pisal A. Djuma-otec. - kak my } \\
\text { dosli do tisni takoj?» Stranno date ne stol'ko potomy, cto do «takoj». skol'ko potomy. cto - «kakm? }\end{array}$ \\
\hline 21 & $\begin{array}{l}\text { Svoloci. oni nazvali Scerbakovu aAlekseevskoj». Cem im Scerbakov pomesal? Ja. naprimer, voobsce } \\
\text { ne znaju, kto eto takoj. }\end{array}$ \\
\hline 23 & Clo nas segodnja ob" odinjact? Navernoc. pravda kak obscestvenmj razdrazitcl". \\
\hline 26 & $\begin{array}{l}\text { "Komu podygryval intellektual nyj rok?" } \\
\text { intellektual nyj rok? Neuzeli opjat A } \text { CRU? }\end{array}$ \\
\hline 27 & O cem eto prodolzenie? Da vse o tom ze - SPRAVEDLIVOSTI \\
\hline 27 & $\begin{array}{l}\text { Co oznacaet. naprimer. slovo "ekstremist"? Segodnja tak naz̧rajut D. Vasil"eva s kompanicj - } \\
\text { pojavilsja daze stamp: "ekstremistskie lidery "Pamjati" |... }\end{array}$ \\
\hline 29 & 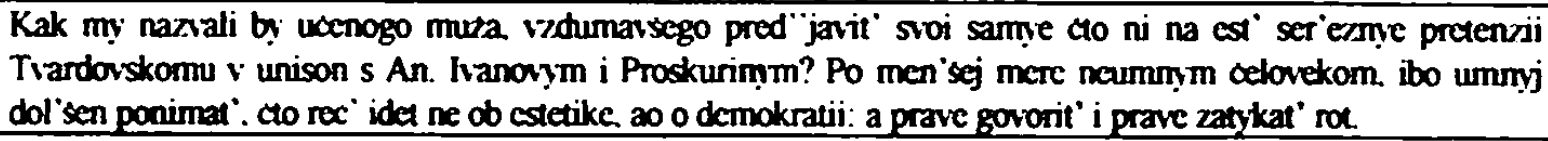 \\
\hline 31 & $\begin{array}{l}\text { Nas izbiratel'nyj zakon vyzyvaet bol soj interes vo vsem mire. "Deputaty ot obscestvennych } \\
\text { organizacij" - nu v kakoj sovremennoj erropejskoj strane mozno popast' v parlament za uspechi v } \\
\text { sobiranii marok ili za ign v trexost"? }\end{array}$ \\
\hline
\end{tabular}

Die nachfolgende Besprechung des Analyseergebnisses geht von 23 rhetorischen Fragen aus, die in 19 Analyseeinheiten (54,3\%) nachgewiesen werden. Die Verwendung der angesprochenen Fragen stellt folglich kein charakteristisches Textgenerienungsmerkmal des untersuchten Diskurses dar. Die Haufigkeit der Verwendung schwankt zwischen 0 und 3, wobei die einbzw keinmalige Verwendung mit jeweils 45,7\% am wahrscheinlichsten ist.

Auf die Aussagen, die durch das angesprochene Stilmittel bekraftigt werden, soll in den folgenden Absätzen eingegangen werden Insofern die Aussagen im Rahmen der vorliegenden Arbeit bereits erlautert werden konnten, erfolgt ein Hinweis auf die entsprechenden Abschnitte

In Analyseeinheit I wird eine kulturelle Tautologie (vgl: 9.13.), in der es vermutlich um das Verstehen der Gruppe chippi geht, in Form einer rhetorischen Frage formuliert. Dementsprechend wird diese Tautologie durch das angesprochene Stilmittel hervorgehoben.

In Analyseeinheit 2 sind zwei Fragenkomplexe nachweisbar, die jeweils zu einer rhetorischen Frage zusammengefaßt werden. Im ersten Fall sind im Komplex zwei kulturelle Tautologien (vgl.: 9.13.) und ein Antagonismus (vgl.: 9.12.1.) enthalten, im zweiten bezieht sich die rhetorische Frage auf eine kulturelle Tautologie (vgl: : 9. 13.).

Die durch die rhetorische Frage bekräftigte Aussage ist in Analyseeinheit 5 der Frage nachgestellt. Es handelt sich um eine kulturelle Tautologie (vgl.: 9.13.), die in Form einer Gesetzeshypothese formuliert ist.

In Analyseeinheit 6 wird die Frage auf ein Zitat bezogen, welches auf die Autoritat Igor' Kochanovskij (vgl: 9. 10.) zurückgeht Da Kochanovskij im Rahmen der untersuchten Formation vermutlich eine positive kulturelle Bedeutung besitzt, dient das Zitat der Steigerung des 
Wahrheitswertes der Analyseeinheit. An der genannten Stelle scheint der Text hinsichtlich seiner Manipulationskraft sehr effektiv aufgebaut zu sein: zunächst wird der Wahrheitswert des Textes durch eine positiv bewertete Autorität gesteigert, anschließend erfolgt eine Bestatigung der autoritären Aussage durch eine thetorische Frage. Wenn ein Rezipient der vom Verfasser vertretenen Auffassung widersprechen will, dann muß er sich gegen beide Manipulationsverfahren «behaupten». In dieser Hinsicht muß er sowohl die Autoritat als auch die Frage negieren. Diese Negation wird einem «normalen» Rezipienten vermutlich nicht gelingen, da er sich in diesem Fall selbst aus der untersuchten Formation ausgrenzt. Die zweite rhetorische Frage in Analyseeinheit 6 stellt einen Antagonismus (vgl.: 9.12.1.) dar. Die Abgrenzung von der Gruppe, die ad hoc generiert wird, wird an dieser Stelle durch die Frage unterstrichen.

In Analyseeinheit 7 steht die rhetorische Frage im Zusammenhang mit dem Ereignis 1985 (vgl.: 9.9.6.) und dem in der Anayseeinheit thematisierten Komplex der Folklore. Vermittels der Frage wird zunächst die Existenz der Folklore bezweifelt. Hieraus werden anschließend pessimistische Zukunftsaussichten abgeleitet.

Im Rahmen der thetorische Frage in Analyseeinheit 8 wird ein Antagonismus, der die Seiten my [tataro-slavjanskaja orda] und Gudzon: nordiceskaja raza; doriany grei: nordiceskaja raza umfaßt, generiert (vgl: : 9.12.1.).

Ebenfalls im Zusammenhang mit einem Antagonismus steht die rhetorische Frage in Analyseeinheit 9 (vgl:: 9.12.1.). Primär dient die Frage an dieser Stelle nicht dem Aufbau des Antagonismus my vs. Zapad, sondern der Hervorhebung der Meinung des Verfassers, die dieser in bezug auf den KSP vertritt. Ein Rezipient soll derart manipuliert werden, daß er diese Meinung übernimmt. da er mit hoher Wahrscheinlichkeit die Meinung des Verfassers in bezug auf den Antagonismus teilt.

In Analyseeinheeit 11 kann im Rahmen der rhetorischen Frage, die verneinend vom Verfasser im Rahmen der Analyseeinheit beantwortet wird, ein Antagonismus (vgl: : 9.12.1.) und eine kulturelle Tautologie (vgl.: 9.13.) ermittelt werden. Trotz der Verneinung der Frage wird sowohl der Antagonismus als auch die kulturelle Tautologie hervorgehoben, die die bevorzugte Lebensweise der Mitglieder der untersuchten Formation betriff.

Die erste rhetorische Frage in Analyseeinheit 12 wird gestellt, um den Zeitpunkt hervorzuheben, an dem die Publikation ROKSI den Höhepunkt des vom Verfasser unterstellten Reaktionismus uberschritten hat. Dieser Punkt wird als derjenige bestimmt, an dem zum ersten Mal uber den CHMR (HMR) berichtet wurde. Einerseits wird an der angesprochenen Stelle eine Abgrenzung zu der genannten Publikation generiert (vgl.: 9.12.1.), andererseits wird die negative kulturelle Bedeutung des CHMR bzw. HMR im Rahmen der untersuchten Formation deutlich. Die zweite thetorische Frage wird genutzt, um die nachfolgende Antwort auf dieselbe hervorzuheben. In dieser wird ein Antagonismus generiert, der die Seiten podonki [podrostki] und te, komu za tridcat' umfaßt (vgl.: 9.12.1.) 
Obwohl im Umfeld der rhetorischen Frage in Analyseeinheit 13 der Antagonismus ja vs. drugie aufgebaut wird, besitzt die Frage vermutlich nur eine textuelle Funktion, da sie vom Verfasser im weitesten Sinne als Grund fur die Textgenerierung eingefuhrt wird.

In Analyseeinheit 16 wird die thetorische Frage verwendet, um dem Konstrukt Grebenscikov eine weitere, negative Bedeutungskomponente hinzuzufügen. Sie dient somit der internen Differenzierung der untersuchten Formation.

Im Rahmen der rhetorischen Frage in Analyseeinheit 18 können zwei Normative und also kulturelle Tautologien (vgl.: 9.13.) nachgewiesen werden, deren Aussage bekräftigt wird. In Analyseinheit 19 liegt ein Textaufbau vor, der dem in Analyseeinheit 5 gleicht: auf ein Zitat, welches einer vermutlich positiv bewerteten Autorität zugesprochen wird (vgl: 9.10.), folgt eine Frage, um die zitierte Aussage zu stützen. Abweichend ist im vorliegenden Fall nur, daß der Verfasser keine Frage bezuglich des gesamten Zitats stellt, sondern lediglich einen Teilaspekt herausgreift

Die rhetorische Frage, die in Analyseeinheit 21 gestellt wird, dient dem Aufbau des Antagonismus ja vs. oni [svoloci] (vgl.: 9.12.1.). In thematischer Hinsicht ist die Frage vermutlich zweitrangig, da sie lediglich in der Funktion eines Beispiels eingesetzt wird, vermittels dessen eine negativ bewertete Handlung der genannten Gruppe aufgezeigt werden soll.

In Analyseeinheit 23 ist die rhetorische Frage einer kulturellen Tautologie vorangestellt (vgl.: 9.13.), die die Antwort auf eben die Frage darstellt. Hervorgehoben wird an dieser Stelle die Tautologie, nicht die Frage.

Die rhetorische Frage in Analyseeinheit 26 zielt auf den Normalitätsbereich des übergeordneten Kultursystems ab, der an der genannten Stelle negiert wird (vgl.: 9. 13.).

Vermittels der ersten rhetorischen Frage in Analyseeinheit 7 wird die kulturelle Bedeutung des Wortes spravedlivost' hervorgehoben. Dieses Wort besitzt an dieser Stelle die Funktion eines Kollektivsymbols (vgl:: 9.5.2.). Gerade durch den Zusammenhang mit einer rhetorischen Frage wird die kollektivsymbolische Funktion des angesprochenen Wortes deutlich. Die Antwort auf die zweite Frage stellt einen Antagonismus dar, der auf die Ausgrenzung von D. Vasil'ev und dessen Partei Pamjat' abzielt (vgl.: 9.12.1.).

Die rhetorische Frage in Analyseeinheit 29 stellt mit hoher Wahrscheinlichkeit nur ein Beispiel dar, durch das die Aussage hervorgehoben wird, die auf die Frage folgt. Es handelt sich hierbei um einen Antagonismus, dem eine kulturelle Tautologie folgt.

Die thetorische Frage in Analyseeinheit 31 dient der Abgrenzang der untersuchten Formation: in ihrem Rahmen wird ein Stereotyp generiert, das auf eine Ausgrenzung der deputaty ot obscestvennych organizacii aus dem Zugehorigkeitsbereich der untersuchten Formation abzieht (vgl: : 9.12.2.).

Werden die genannten Ergebnisse zusammengefaßt, so wird deutlich, daß rhetorische Fragen im Korpus sehr haufig $(91,3 \%)$ im Zusammenhang mit anderen kulturellen Phanomenen nachgewiesen werden können: am haufigsten mit kulturellen Tautologien und Antagonismen, 
aber auch mit einem Kollektivsymbol, einem Stereotyp und zwei Zitaten. Lediglich eine Frage (AE 13) besitzt mit hoher Wahrscheinlichkeit nur eine textuelle Funktion. Eine Frage, die in Analyseeinheit 7 nachgewiesen wird, ist derart konzipiert, daß die durch sie unterstrichene Aussage mit dem vorhandenen Instrumentarium nicht erschlossen werden kann.

Die primäre kulturelle Funktion der rhetorischen Frage liegt, so kann geschlußfolgert werden, mit hoher Wahrscheinlichkeit in der Hervorhebung von kulturell bereits funktionalisierten Phanomenen. In diesem Sinne können rhetorische Fragen als Indikatoren fur kulturell funktionalisierte und daher relevante Phänomene angesehen werden. Nur selten wird ein Element durch eine rhetorische Frage in kultureller Hinsicht erst funktionalisiert. Eine Tatsache, die möglicherweise mit dem erarbeiten Instrumentarium zusammenhängt. Möglicherweise birgt das Beispiel aus Analyseeinheit 7 ein kulturelles Phänomen, welches nicht erkannt wurde. Gleichfalls besitzen rhetorische Fragen nur selten eine textuelle Funktion. Hiermit soll die Besprechung der Analyseergebnisse in bezug auf rhetorische Fragen beendet werden.

\subsection{Sistema und rok-samizdat}

In Abschnitt 4.3. wurde festgestellt, daß basale Gemeinsamkeiten zwischen der sogenannten Jugendsubkultur Sistema und der kulturellen Formation des rok-samizdat nachweisbar sind. Zum einen verlaufen beide Phänomene nahezu zeitgleich, zum anderen sind beide Phänomene in hohem Maße mit der sogenannten rok-kul'tura assoziiert. Es soll im folgenden Abschnitt der Frage nachgegangen werden, ob vermittels der Korpusanalyse weitere Gemeinsamkeiten ermittelt werden können. Es geht in diesem Zusammenhang nicht darum, die Assoziation zwischen den genannten Phänomenen auf der quantitativen Ebene zu bestimmen. Vielmehr geht es um die Frage, ob eine qualitative Assoziation in den Analyseeinheiten deutlich wird, d.h. ob direkte oder indirekte Hinweise auf die genannte Jugendsubkultur in den Analyseeinheiten nachweisbar sind und ob die kulturelle Bedeutung dieser Elemente deckungsgleich ist. Unter einem direkten H linweis soll in diesem Zusammenhang die Nennung der genannten Jugendsubkultur verstanden werden, unter einem indirekten das Vorkommen bestimmter Elemente, die in Tabelle 32 aufgefuhrt sind. Bezogen auf die untersuchte kulturelle Formation und deren Diskurs, geht es im vorliegenden Zusammenhang um die Frage, ob eine Assoziation zwischen den genannten Phänomenen Einfluß auf die Textgenerierung besitzt, d.h. letztendlich, ob aufgnund der Assoziation ein charakteristisches Textgenerierungsmerkmal vorliegt.

Im Verlauf der vorliegenden Arbeit konnten verschiedene kulturelle Phänomene eingefuhrt werden, deren Verwendung im Korpus geprüft wurde. Um die Assoziation mit der Jugendsubkultur Sistema zu verdeutlichen, sollen nur einige, zudem spezifische Elemente, die, wie angedeutet, als indirekter Hinweis auf die genannte Jugendsubkultur bewertet werden, Berücksichtigung finden. Nicht berucksichtigt werden folglich die primar textbezogenen Analyseverfahren Wort-Satz-Relation und Type-Token-Ratio sowie Themen- und Kontingenzanalyse. Auch die Verwendung primar sprachlicher Besonderheiten, wie sie Diskursiva und Oppositionen darstellen, soll nicht geprüft werden Dies hängt damit 
zusammen, daß in bezug auf die Sprachvariante der Mitglieder des Sistema nur ungenaue Erkenntnisse vorliegen (vgl: 4.4.). Die Übereinstimmungen, die zwischen sleng. d.h. der Sistema-spezifischen Sprachvariante und dem ermittelten Diskursiva-Repertoire festgestellt werden können, sollen dementsprechend vernachlassigt werden. Da in bezug auf Sentenzen nur wenig Vergleichsmaterial vorhanden ist, werden gleichfalls keine Überschneidungen der jeweiligen Repertoires ermittelt. Darüber hinaus liegen keine Informationen über die Verwendung von Stereotypen. Marken-Indices, Autoritäten und Kollektivsymbolen in der genannten Jugendsubkultur vor. Ob identische Antagonismen vorliegen, ergibt sich aus einem Vergleich der kulturellen Bedeutungen, so daß Antagonismen nicht separat erfaßt werden. Gleichfalls ist es anhand des vorhandenen Materials nicht möglich, den Normalitätsbereich der genannten Jugendsubkultur zu rekonstruieren. Es effolgt dementsprechend eine Konzentration auf Personen-, Gruppen-, Geographica-, Institutionen- und Ereignis-Indices sowie auf Formationsspezifika. In der Jugendsubkultur sind darüber hinaus kulturell relevante Elemente ermittelbar, die unter der Bezeichnung Sonstiges zusammengefaßt werden. Es handelt sich hierbei um «Berufe», die eine besondere kulturelle Bedeutung aufweisen, Musikstile, die von den Sistema-Mitgliedern präferiert werden. Spitznamen (prozvisca) sowie das Genre der telegi. In Tabelle 32 sind die im Korpus zu ermittelnden, indirekt hinweisenden Elemente mit ihrer Sistema-spezifischen Ausprägung aufgeführt. In bezug auf die beiden zuletzt genannten Elemente liegt kein abgeschlossenes Repertoire vor. Die Nennung einer Auswahl möglicher Elemente scheint nicht notwendig zu sein, da beide Elemente erläutert wurden (vgl : 4.4 ) und auf dieser Grundlage ermittelbar sind Da die in Tabelle 32 aufgeführten Elemente im Rahmen der genannten Jugendsubkultur in kultureller Hinsicht funktionalisien sind, besitzen sie für diese kulturelle Relevanz

Tabelle 32

\begin{tabular}{|c|c|}
\hline "Ekement" & Sistema-sperifixche Auspripang. \\
\hline Pernomen-Indices & Boris Grebenscikov: Konstantın Kincer (vgl +.3.) \\
\hline Gruppen-Indices & 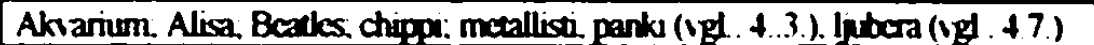 \\
\hline $\begin{array}{l}\text { Geopraphicar und Institutionen- } \\
\text { lodices }\end{array}$ & 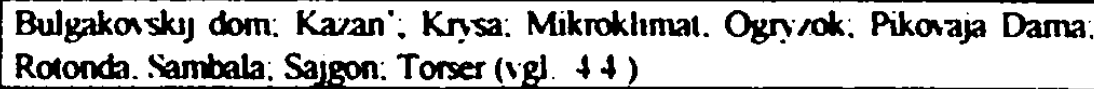 \\
\hline Ereipaise & -Den Okntija Treugol nika (1. Mai): -Den rascin detej (1. Juni) $(v g l .+7$. \\
\hline Formationssperifika & 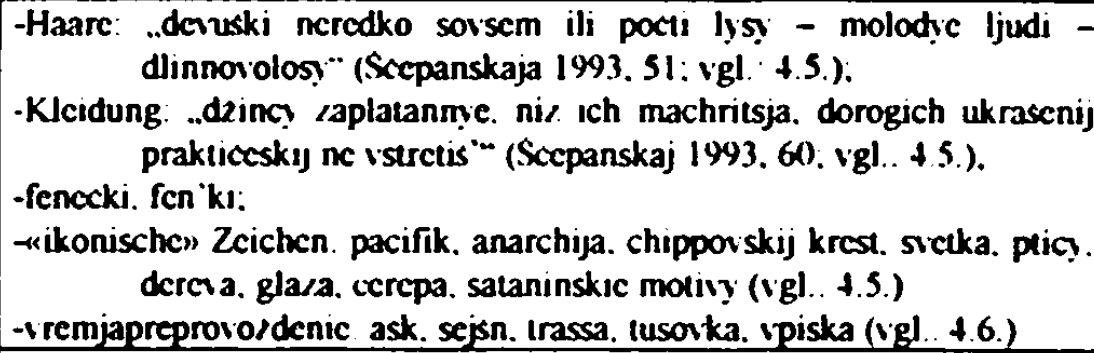 \\
\hline Sonstiges & 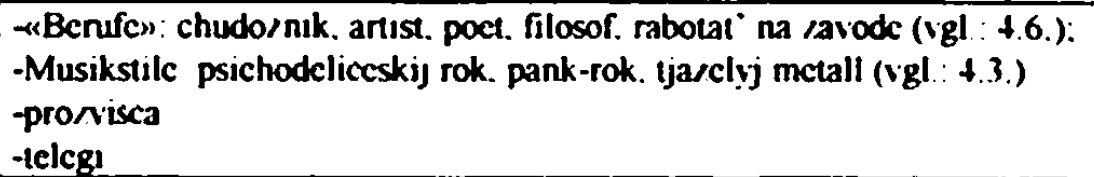 \\
\hline
\end{tabular}


Es gilt zu erlautern, in welcher Form die in Tabelle 32 aufgefuhrten Elemente als Hinweis auf die Jugendsubkultur Sistema aufgefaßt werden sollen. In diesem Zusammenhang muß bedacht werden, daß die aufgefuhrten Elemente mit hoher Wahrscheinlichkeit nicht exklusiv in der genannten Jugendsubkultur und der untersuchten kulturellen Formation verwendet werden. Sie besitzen jeweils eine indexikalische Funktion in bezug auf die genannten Phanomene, dennoch ist es möglich, daß sie diese Funktion beispielsweise auch in bezug auf das übergeordnete Kultursystem besitzen. Bedeutsam ist dementsprechend nicht nur das Vorhandensein des Elements, sondern, wie bereits angedeutet, auch eine deckungsgleiche kulturelle Bedeutung, um hypothetisch von einer Assoziation sprechen zu konnen. In bezug auf die Jugendsubkultur Sistema besitzen alle aufgefuhrten Elemente eine positive kulturelle Bedeutung. Eine Ausnahme stellt die Gruppe der ljubera und der «Beruf» rabotat' na zavode dar. Des weiteren sollte in bezug auf prozvisca und telegi von keiner kulturellen Bedeutung ausgegangen werden. Diese ergibt sich erst, wenn die genannten Elemente auf ein konkretes Objekt bezogen werden. Bei der Ermittlung der aufgeführten Elemente im Korpus wird so vorgegangen, daß ein relativ breiter Toleranzbereich hinsichtlich der gewählten Variante akzeptiert wird. Sobald ein Element zweifellos auf die Sistema-spezifische Ausprägung zurückgeführt werden kann, wird es als Hinweis auf eine Assoziation gewertet. In bezug auf «ikonische» Zeichen muß betont werden. daß keine ikonischen Zeichen ermittelt werden. Es geht darum festzustellen, ob die genannten ikonischen Zeichen auf der textuellen Ebene vorhanden sind.

In Tabelle 33 ist dargestellt, in welchen Analyseeinheiten die in Tabelle 32 aufgefuihrten Elemente nachweisbar sind.

Tabelle 33

\begin{tabular}{|c|c|c|}
\hline$\overline{\mathbf{A E}}$ & Sistema-specaifixche Ausprisgung & tolerierte rok-anmizdat Variante \\
\hline 1 & chippi & chip: CHIP (vgl: 9.9.3.) \\
\hline 4 & Boris Grebenscikor & B. Grebenscikor: Grebensciko: BG (vgl.: 9.9.2.) \\
\hline 4 & Akvarium & $A[4](v g]: 9.9 .3)$. \\
\hline 4 & pank-rok & polupankovij \\
\hline 4 & pronisca & Fagor: Borja: Djusa: Seva \\
\hline 5 & pronisca & Majk [2] \\
\hline 6 & pank-rok & pank \\
\hline 11 & Boris Grcbenscikor & GB (vgl: : 9.9.2.) \\
\hline 11 & ljubcra $|2|$ & \\
\hline 12 & Akvarium & \\
\hline 12 & metallist & ncuninyc podonki [podrostki] \\
\hline 12 & ljaclyj nkitall & mctalliceskie komand \\
\hline 12 & tjaxely nretall & metallosistema \\
\hline 12 & tjaretyj metall & CHMR (HMR) \\
\hline 12 & prozisca & Alek Zander: O. F. [Orec Fedor] \\
\hline 14 & ljubera & \\
\hline 14 & tjazclyj metall & metallurgija \\
\hline 15 & prozisca & Drord? \\
\hline 16 & Boris Grebenscikor & $\begin{array}{l}\text { BORIS GREBENSHIKOV: Grebensciko: BG }|3| \\
\text { (vgl: 9.9.2.) }\end{array}$ \\
\hline 16 & Akvarium & \\
\hline 17 & poet & poet Michalko:. poen \\
\hline 17 & tjarclyj mctall & filarmo-metallist \\
\hline
\end{tabular}




\begin{tabular}{|c|c|c|}
\hline 19 & filosof & filosofy \\
\hline 20 & Konstantin Kincer & Kincen (vgl.: 9.9.2.) \\
\hline 20 & Alisa & alisomany \\
\hline 20 & prozisca & Del'fin: Gera \\
\hline 20 & poct & rok-poety \\
\hline 22 & tusovka & tusoviscik \\
\hline 22 & pronisca & Rok-Adrokat \\
\hline 25 & tusovka & tusovki [2] \\
\hline 27 & scjsn & scisen \\
\hline 32 & chudoznik & Dzikija [...] Ego rec' - ego kartinki. \\
\hline $\mathbf{3 3}$ & chippi & \\
\hline 33 & ask & poprosit' sigaretku \\
\hline 34 & chudoznik & chudoznik: chudozniki-momentalisty \\
\hline 34 & tazclyj metall & metalliceskic komandy \\
\hline 34 & prosisca & Stiv \\
\hline 35 & sejsn & $\operatorname{sejsin}$ \\
\hline
\end{tabular}

In 19 Analyseeinheiten $(54,3 \%)$ sind die für die Jugendsubkultur Sistema relevanten Elemente nachweisbar. Die Verwendung dieser Elemente kann dementsprechend nicht als charakteristisches Textgenerierungsmerkmal des untersuchten Diskurses angesehen werden. Die Häufigkeit der Verwendung schwankt zwischen 0 und 6, wobei hinter der keinmaligen Verwendung, die einmalige mit $25,7 \%$ am wahrscheinlichsten ist. Verwendet werden 16 verschiedene Elemente, auf deren kulturelle Bedeutung im Rahmen der untersuchten Formation hingewiesen werden soll, um die Frage hinsichtlich der Deckungsgleichheit dieser Bedeutungen zu beanworten. Die Verwendung von Spitznamen in 7 verschiedenen Analyseeinheiten wird zur Kenntnis genommen, auf die kulturelle Bedeutung der bezogenen Aktanten wird nicht eingegangen, da diese fur die Jugendsubkultur Sistema nicht als relevant erkannt wurden.

Wie die Analyse in Abschnitt 9.9.2. zeigt, kann in bezug auf Grebenscikov nicht von einer einheitlichen Bedeutungskonstruktion im Rahmen der untersuchten Formation gesprochen werden Bis zu einem bestimmten Zeitpunkt, der Veröffentlichung seines Soloalbums, sind die kulturellen Bedeutungen deckungsleich, danach verandert sich die Bedeutung im Rahmen des rok-samizdat. Kincev, Sanger der Heavy-Metal Gruppe Alisa, erfullt im Rahmen der untersuchten Formation die Funktion einer Autorität (vgl: 9.10.), die mit hoher Wahrscheinlichkeit aufgrund der Zuordnung zum Bereich der Heavy-Metal-Musik keine positive kulturelle Bedeutung aufweist. In dieser Hinsicht sind die Bedeutungen zwischen den verglichenen Phänomenen nicht deckungsgleich. Die Gruppe Akvarium ist in positiver Hinsicht relevant für die Formation des rok-samizdat (vgl.: 9.9.3.). Die kulturellen Bedeutungen sind dementsprechend identisch. Die Musikgruppe Alisa wird im Korpus nicht direkt genannt. Vielmehr werden Kincev sowie die Fans der Gruppe in Analyseeinheit 20 eingeführ. Über die Bedeutung derselben kann eine negative kulturelle Bedeutung der Gruppe im Rahmen der untersuchten Formation hergeleitet werden. Wiederum sind die Bedeutungen nicht deckungsgleich. Zweimal ist die Gruppe chippi im Korpus mit einer vermutlich positiven kulturellen Bedeutung nachweisbar, so daß von einer Identitat der Bedeutungen ausgegangen wird. Eine explizit negative kulturelle Bedeutung besitzt die Gruppe metallisty, deren Mitglieder als neumnye podonki bzw. podrostkj bezeichnet 
werden. Es liegt in diesem Sinne keine Deckungsgleichheit der kulturellen Bedeutungen vor. In bezug auf die Gruppe ljubera kann eine Identität der kulturellen Bedeutungen in der Jugendsubkultur Sistema und der untersuchten Formation festgestellt werden. Es erfolgt jeweils eine massive Abgrenzung von der Gruppe. Des weiteren sind drei Formen des Sistema-spezifischen Zeitvertreibs im Korpus nachweisbar: ask, sejsn und tusovka. In Analyseeinheit 33 wird eine Handlung geschildert (poprosit' sigaretku), die als ask bezeichnet werden kann. Die Handlung wird nicht ausfuhrlich dargestellt, da sie offensichtlich keine Besonderheit darstellt. Sie gilt in diesem Sinne vermutlich als normal. Triff diese Vermutung $\mathbf{z u}$, dann kann von einer positiven kulturellen Bedeutung ausgegangen werden, so daß eine Deckungsgleichheit mit der SistemaBedeutung diagnostiziert werden kann. In den Analyseeinheiten 27 und 35 wird die Beschäftigung sejśn eingeführt. In beiden Fällen wird die Beschäftigung nicht erläutert. Da keine negative Bedeutung offensichtlich wird, soll von einer positiven Bedeutung ausgegangen werden, so daß eine Deckungsgleichheit der Bedeutungen konstatiert wird. Der Zeitvertreib tusovka wird im Gegensatz dazu mit einer negativen kulturellen Bedeutung eingeführt: einmal im Zusammenhang mit dem Aktanten Rok-Advokat, der, da er u.a. für offizielle Presseorgane tătig ist, abgelehnt wird und einmal im Zusammenhang mit einem Antagonismus, der auf die Ausgrenzung des Bereichs "podpol'naja" rok-pressa abzielt. Es liegt in diesem Sinne keine Identität der Bedeutungen vor. Im Zusammenhang mit den Aktanten Aleksandr Dtikija und Aleksej Karkaev wird der «Beruf» chudoznik eingeführt. Beide Aktanten, die den genannten «Benf) ausüben, werden nicht in positiver Weise konstruiert. Da der berufliche Aspekt ein Teil des Bedeutungskonstukts ist, liegt die Vermutung nahe, daß auch dieser Aspekt keine positive kulturelle Bedeutung aufweist. Emeut sollte nicht von einer Deckungsgleichheit der Bedeutungen gesprochen werden. Der «Beruf» poet kann in zwei Analyseeinheiten ermittelt werden. In Analyseeinheit 17 wird er einerseits auf den ausgegrenzten Aktanten Sergej Michalkov bezogen, andererseits wird allgemein die Kenntnis der Gruppe poety hinsichtlich eines bestimmten Sachverhalts hervorgehoben. An dieser Stelle besitzt der «Beruf» mit hoher Wahrscheinlichkeit eine positive kulturelle Bedeutung. Eine negative Bedeutung besitzt er bezogen auf die Gruppe rok-poety. In allgemeiner Hinsicht, d.h. wenn der «Benuf» nicht auf andere Objekte bezogen wird, besitzt er vermutlich eine positive kulturelle Bedeutung. so daß von einer identischen kulturellen Bedeutung in der Jugensubkultur Sistema und in der kulturellen Formation des rok-samizdat gesprochen wird. Der «Beruf» filosof wird in einem Zitat, welches der Autorität Alexandre Dumas zugesprochen wird, eingefürt. An dieser Stelle kann von einer positiven kulturellen Bedeutung des «Berufs" ausgegangen werden, so daß eine Deckungsgleichheit der Bedeutungen diagnostiziert wird. Der Musikstil pank-rok wird einerseits im Zusammenhang mit der Gruppe Akvarium, andererseits im Zusammenhang mit der Konsolidierung der untersuchten Formation eingefuhrt. In beiden Fällen soll von einer positiven kulturellen Bedeutung ausgegangen werden, so daß eine Identitat der Bedeutungen diagnostizient wird. Der Musikstil tjazelyj metall besitzt in der untersuchten Formation eine 
negative kulturelle Bedeutung, so daß in diesem Sinne keine Deckungsgleichheit mit der Sistema-spezifischen Bedeutung vorliegt.

Zusammenfassend kann festgestellt werden, daß neun der 16 verwendeten Elemente $(56,3 \%)$ vermutlich hinsichtlich ihrer kulturellen Bedeutung deckungsgleich sind. In diesem Sinne sind die Jugendsubkultur Sistema und die kulturelle Formation des rok-samizdat nicht nur quantitativ sondern auch qualitativ mit nahezu identischen Werten (54,3\% und 56,3\%) assoziiert. Es kann auf dieser Grundlage von einer partiellen Übereinstimmung der genannten Phänomene gesprochen werden. Wird die Größe der Phänomene, die durch die jeweiligen Mitgliederzahlen ausgedrückt werden kann (vgl: 4.2. und 7.2.2.), berücksichtigt, dann kann abschließend die Vermutung geäußert werden, daß die untersuchte kulturelle Formation im weitesten Sinne einen Teilbereich der genannten Jugendsubkultur darstellt.

\subsection{Die Homogenität und Heterogenität des Korpus, des Diskurses und der kulturellen} Formation des rok-samizdat

Unter Berücksichtigung der Analyseergebnisse der vorangehenden Abschnitte soll die Frage beantwortet werden, ob mit dem Korpus eine homogene oder heterogene Textsammlung vorliegt. Wird darüber hinaus davon ausgegangen, daß die Ergebnisse der Korpusanalyse auf die untersuchte Formation übertragbar sind, dann kann dieses Ergebnis gleichfalls genutzt werden, um Aussagen bezüglich der Homogenität bzw. Heterogenität dieser Formation und ihres Diskurses zu formulieren.

In einem ersten Schritt geht es zunächst um die Frage, ob in der untersuchten Formation Texte generiert werden, die in gewisser Hinsicht ahnlich sind, oder ob hinsichtlich der ausgewahlten Textgenerienungsmerkmale nur wenige Gemeinsamkeiten zwischen den Analyseeinheiten existieren. Die Beantwortung dieser Frage leitet zu der Frage über, ob von charakteristischen Analyseeinheiten gesprochen werden kann Unter einer charakteristischen Analyseeinheit soll ein Text verstanden werden. der eine bestimmte Anzahl der dargestellten Merkmale aufweist. Daruber hinaus soll die Frage beantwortet werden, ob eine Assoziation (Kontingenz) bestimmter Merkmale vorhanden ist, $\mathrm{d} . \mathrm{h}$. ob bestimmte Merkmale in signifikanter Weise häufig gemeinsam auftreten oder ob bestimmte Merkmale sich ausschließen.

In einem zweiten Schritt soll auf der Gundlage des ersten Schritts anschließend eine Hypothese uber die Einheitlichkeit der Formation, die auf der Einheitlichkeit des Diskurses beruht, formuliert werden

Bevor die genannten Fragen beantwortet werden, sollen zwei Hinweise gegeben werden, die die Methode betreffen, die zur Beantwortung der Fragen gewählt wird: 1. Die durchzuführende Analyse beruht nicht nur auf den Merkmalen, die als charakteristisch fur das Korpus erkannt wurden, sondern auf denjenigen, die sich in der vorliegenden Arbeit als produktiv erwiesen haben. In diesem Sinne wird die Kontingenzanalyse, die in bezug auf bestimmte types durchgefuhrt wurde, nicht zur Beantwortung der aufgeworfenen Fragen herangezogen. 2. Die Merkmale werden nicht explizit "gewichtet», d.h. allen Merkmalen wird für das Vorhandensein 
bzw. das Fehlen in einer Analyseeinheit jeweils ein Wert zugeordnet, nämlich 1 bzw. 0 . Diese Vorgehensweise ist zweifellos problematisch, da u.a. quantitative und qualitative Merkmale «in einen Topf geworfen werden». Da keine andere "Gewichtung» produktiver erscheint, wird die dargestellte Vorgehensweise gewählt. Da mit Dummy-Variablen gerechnet wird, wird das «faktische» Vorhandensein eines Merkmals gleichfalls vernachlässigt.

Die Grundlage der folgenden Ausführungen stellt Tabelle 34 dar.

Tabelle $34^{27}$

\begin{tabular}{|c|c|c|c|c|c|c|c|c|c|c|c|c|c|c|c|c|c|c|c|c|c|c|}
\hline $\mathbf{A E}$ & $\mathrm{MSR}$ & TIR & TA & $\mathbf{K S}$ & D & As & 0 & EP & P-I & G-I & $C_{\infty}$ & $M$ & $\bar{E}$ & $\mathbf{S}$ & $\mathrm{Au}$ & Sen & $\mathbf{A}$ & $\mathbf{S t}$ & $\mathbf{N}$ & $\mathbf{R F}$ & $S / r$ & $\Sigma$ \\
\hline 1 & 1 & 1 & 1 & 1 & 1 & 1 & 1 & 1 & 0 & 1 & 1 & 10 & 0 & 1 & 0 & 0 & 0 & 1 & 1 & 1 & 1 & 15 \\
\hline 2 & 1 & 0 & 1 & 0 & 1 & 0 & 0 & 0 & 1 & 1 & 0 & 1 & 0 & 0 & $\mathbf{0}$ & 0 & 1 & 0 & 1 & 1 & 0 & 9 \\
\hline 3 & 0 & 1 & 1 & 1 & 1 & 1 & 1 & 1 & 0 & 1 & 1 & 0 & 1 & 0 & 1 & 0 & 1 & 0 & 1 & 0 & 0 & 13 \\
\hline 4 & 1 & 1 & 1 & 0 & 1 & 1 & 0 & 1 & 1 & 1 & 1 & 1 & 1 & 0 & 1 & 1 & 1 & 0 & 0 & 0 & 1 & 15 \\
\hline 5 & 1 & 0 & 1 & 0 & 1 & 0 & 1 & 1 & 1 & 1 & 1 & 1 & 0 & 1 & 1 & 0 & 1 & 1 & 1 & 1 & 1 & 16 \\
\hline 6 & 1 & 1 & 1 & 0 & 1 & 1 & 1 & 1 & 1 & 1 & 1 & 1 & 1 & 0 & 1 & 1 & 1 & 1 & 1 & 1 & 1 & 19 \\
\hline 7 & 1 & 0 & 0 & 1 & 1 & 0 & 1 & 1 & 1 & 1 & 1 & 0 & 1 & 1 & 1 & 1 & 1 & 1 & 1 & 1 & 0 & 16 \\
\hline 8 & 0 & 0 & 1 & 1 & 1 & 0 & 0 & 1 & 1 & 1 & 1 & $\mathbf{0}$ & 0 & 0 & 0 & 0 & 1 & 1 & 1 & 1 & 0 & 11 \\
\hline 9 & 1 & 1 & 1 & 0 & 1 & 0 & 1 & 0 & 1 & 1 & 1 & 1 & 0 & 1 & 0 & 0 & 1 & 1 & 0 & 1 & 0 & 13 \\
\hline 10 & 1 & 0 & 0 & 1 & 1 & 0 & 0 & 1 & 0 & 1 & 1 & 1 & 1 & 1 & 1 & 1 & 1 & 1 & 1 & 0 & 0 & 14 \\
\hline 11 & 1 & $\overline{1}$ & $\overline{1}$ & 0 & 1 & 0 & 0 & 1 & 1 & 1 & 1 & 1 & 1 & 1 & 1 & 0 & 1 & 0 & 1 & 1 & 1 & 16 \\
\hline 12 & 0 & 1 & 0 & 0 & 1 & 0 & 1 & 1 & 1 & 1 & 0 & 1 & 0 & 0 & 0 & 0 & 1 & 1 & 1 & 1 & 1 & 12 \\
\hline 13 & 0 & 0 & 1 & 0 & 1 & 0 & 1 & 1 & 1 & 0 & 0 & 1 & 0 & 0 & 1 & 0 & 1 & 0 & 0 & 1 & 0 & 9 \\
\hline 14 & 1 & 0 & 0 & 0 & 1 & 0 & 0 & 0 & 1 & 1 & 1 & 0 & 0 & 1 & 1 & 1 & 1 & 1 & 0 & 0 & 1 & 11 \\
\hline 15 & 1 & 1 & 1 & 0 & 1 & 0 & 0 & 1 & 1 & 1 & 1 & 0 & 1 & 0 & 1 & 0 & 1 & 0 & 0 & 0 & 1 & 12 \\
\hline 16 & 1 & 1 & 1 & 0 & 1 & 0 & 0 & 1 & 1 & 1 & 1 & 1 & 0 & 1 & 0 & 0 & 1 & 0 & 1 & 1 & 1 & 14 \\
\hline 17 & 1 & 0 & 1 & 1 & 1 & 0 & 1 & 1 & 1 & 1 & 1 & 0 & 0 & 1 & 1 & 1 & 1 & 1 & 1 & 0 & 1 & 16 \\
\hline 18 & 1 & 1 & 0 & 1 & 1 & 1 & 0 & 1 & 1 & 1 & 1 & 1 & 0 & 0 & 0 & 0 & 1 & 0 & 1 & 1 & 0 & 13 \\
\hline 19 & 0 & 1 & 1 & 1 & 1 & 1 & 0 & 1 & 1 & 1 & 1 & 1 & 0 & 0 & 1 & 0 & 1 & 0 & 0 & 1 & 1 & 14 \\
\hline 20 & 1 & 1 & 1 & 0 & 1 & 0 & 1 & 1 & 1 & 1 & 0 & 0 & 0 & 0 & 1 & 0 & 1 & 1 & 1 & 0 & 1 & 13 \\
\hline 21 & 0 & 1 & 0 & 1 & 1 & 0 & 0 & 1 & 1 & 1 & 1 & 0 & 0 & 0 & 0 & 0 & 1 & 1 & 1 & 1 & 0 & 11 \\
\hline 22 & 0 & 1 & 0 & 0 & 1 & 0 & 0 & 1 & 1 & 1 & 1 & 0 & 0 & 1 & 1 & 0 & 1 & 1 & 1 & 0 & 1 & 12 \\
\hline 23 & 1 & 1 & 0 & 1 & 1 & 0 & 1 & 1 & 0 & 1 & 1 & 0 & 0 & 0 & 0 & 0 & 1 & 0 & 1 & 1 & 0 & 11 \\
\hline 24 & 1 & 0 & $\mathbf{0}$ & 1 & 1 & 0 & 1 & 1 & 1 & 1 & 1 & 1 & 0 & 1 & 0 & 0 & 1 & 1 & 1 & 0 & 0 & 13 \\
\hline 25 & 0 & 0 & 0 & 0 & 1 & 1 & 1 & 1 & 0 & 0 & 0 & 0 & 0 & 0 & 0 & 0 & 1 & 0 & 1 & 0 & 1 & 7 \\
\hline 26 & 0 & 1 & 0 & 0 & 1 & 0 & 0 & 1 & 1 & 1 & 1 & 1 & 0 & 0 & 1 & 0 & 1 & 0 & 1 & 1 & 0 & 11 \\
\hline 27 & 1 & 1 & 0 & 1 & 1 & 1 & 1 & 1 & 1 & 1 & 1 & 1 & 0 & 1 & 1 & 1 & 1 & 1 & 1 & 1 & 1 & 19 \\
\hline 28 & 0 & 1 & 0 & 0 & 1 & 0 & 1 & 1 & 1 & 1 & 0 & 1 & 0 & 1 & 0 & 0 & 1 & 1 & 1 & 0 & 0 & 11 \\
\hline 29 & 0 & 1 & 1 & 1 & 1 & 0 & 1 & 1 & 1 & 1 & 1 & 1 & 0 & 1 & 0 & 0 & 1 & 1 & 1 & 1 & 0 & 15 \\
\hline 30 & 1 & 0 & 0 & 0 & 1 & 0 & 1 & 1 & 1 & 1 & 0 & 0 & 0 & 0 & 1 & 0 & 1 & 0 & 1 & 0 & 0 & 9 \\
\hline 31 & 1 & 1 & 0 & 0 & 0 & 0 & 1 & 1 & 1 & 1 & 1 & 0 & 0 & 1 & 0 & 0 & 1 & 1 & 1 & 1 & 0 & 12 \\
\hline 32 & 1 & 1 & 0 & 1 & ( & 0 & 1 & 0 & 1 & 0 & 0 & 0 & 0 & 0 & 0 & 0 & 1 & 0 & 1 & 0 & 1 & 8 \\
\hline 33 & 1 & 1 & 0 & $\mathbf{0}$ & 1 & 0 & 0 & 1 & 1 & 1 & 1 & 1 & 0 & 0 & 0 & 0 & 1 & 1 & 0 & 0 & 1 & 11 \\
\hline 34 & 1 & 1 & 0 & 1 & 1 & 1 & 0 & 0 & 1 & 1 & 1 & 1 & 0 & 0 & 0 & 0 & 1 & 0 & 0 & 0 & 1 & 11 \\
\hline 35 & 1 & 1 & 1 & 0 & 1 & 1 & 1 & 1 & 1 & 1 & 1 & 1 & 1 & 1 & 1 & 0 & 1 & 0 & 1 & 0 & 1 & 17 \\
\hline$\Sigma$ & 24 & 24 & 17 & 15 & 33 & 10 & 20 & 30 & 30 & 32 & 27 & 20 & 8 & 16 & 18 & 7 & 34 & 19 & 27 & 19 & 19 & 49 \\
\hline
\end{tabular}

27 Der Wen 1 bercichnet jeweils das Vorhandensein des Merkmals. welches am haufigsten in den jeweiligen Anabseabschnitten crmittelt werden konnte. Die Abkürnungen bedeuten. WSR: Wort-Satz-Relation: TTR: Type-Token-Ratio: TA: Themenanalyse: KS: Kollektivsymbole: D: Diskursiva: Ag: Anglizismen: 0 : Oppositionen: EP: Erzahlperspektive: P-I: Personen-Indices: G-I: Gruppen-Indices: Geo: Geographica- und Institutionen-Indices. M: Marken-Indices E: Ercignis-Indices: S: Sonstige-Indices: Au: Autoritaten: Sen: Sentenzen: A: Antagonismen: St Stereotype: N: Normative: RF: Rhetorische Fragen: S/r: Sistema/roksamirdat-Zusammenhang. 
Die Nachweishaufigkeit der erlauterten Merkmale schwankt zwischen 7 und 19, so daß sich ein Range von 12 ergibt. Das arithmetische Mittel der Verteilung beträt 12,8, die Standardabweichung s 2,8. Vermittels des arithmetischen Mittels und der Standardabweichung wird ein Toleranzbereich gebildet, der von 10 bis 15,6 reicht. In diesem Bereich befinden sich die Werte von 23 Analyseeinheiten (65,7\%). Dieser Bereich kann dementsprechend nicht als charakteristischer Wertebereich angesehen werden. Wird dieses Ergebnis zur Beantwortung der Frage bezüglich der Homogenitat bzw. Heterogenitat des Korpus und des Diskurses der betrachteten Formation herangezogen, so kann festgestellt werden, daß das Korpus bzw. der Diskurs nicht in hohem, sondern in mittlerem Maße homogen ist, die Heterogenitat $(34,3 \%)$ ist dementsprechend niedrig. In diesem Sinne kann hinsichtlich der kulturellen Formation des roksamizdat von einer in mittlerem Maße homogenen kulturellen Formation ausgegangen werden.

Um von einer charakteristischen Analyseeinheit sprechen zu konnen, muß diese eine Assoziation von mindestens $0,7(70 \%)$ mit dem Merkmalrepertoire aufweisen, welches 21 Merkmale umfaßt. Mit anderen Worten ausgedrückt, muß eine Analyseeinheit mindestens 15 Merkmale aufweisen, um dem angesprochenen Bewertungskriterium zu genügen. Wie Tabelle 34 zeigt, erfüllen dieses Kriterium 10 Analysecinheiten $(28,6 \%)$ : 1, 4, 5, 6, 7, 11, 17, 27, 29 und 35.

Die letzte Frage, die in diesem Abschnitt beantwortet werden soll, zielt auf die Assoziation oder Kontingenz einzelner Merkmale ab. Diese Assoziation wird vermittels der Prozentsatzdifferenz. d\% berechnet. Zwischen den in Tabelle 34 aufgeführten Merkmalen liegt in 283 Fallen (67,4\%) keine Assoziation vor, in 108 Fällen $(25,7 \%)$ eine niedrige, in 27 Fallen $(6,4 \%)$ eine mittlere und in zwei Fällen (0,5\%) eine hohe. Perfekte Assoziationen konnen nicht ermittelt werden Im Korpus sind dementsprechend nur zwei Merkmalspaare vorhanden, die in der Weise assoziiert sind, daß von einer charakteristischen Assoziation für das Korpus gesprochen werden kann [G-I/Geo] mit $84,4 \%$ und [A Ag] mit $-73,5 \%$. Es gilt zu erklären, was diese Werte besagen Wie Tabelle 34 zeigt. konnen Gruppen-Indices in 32 Analysecinheiten ermittelt werden Gemeinsam mit diesen treten 27 Geographica- und Institutionen-Indices $(84,4 \%)$ auf In den verbleibenden 3 Analyseeinheiten, in denen keine Gruppen-Indices nachgewiesen werden konnen, treten keine Geographica- und Institutionen-Indices auf (0\%). Es ergibt sich eine Prozentsatzdifferenz d\% von $84,4 \%$. Dieser Wert besagt, daß Geographicaund Institutionen-Indices zu 84,4\% häufiger mit Gruppen-Indices auftreten als ohne. Der negative Wert besagt in diesem Zusammenhang, daß ein gemeinsames Auftreten der betroffenen Merkmale unwahrscheinlich ist, daß diese sich m.a.W. ausschließen. Wie Tabelle 34 zeigt, treten Antagonismen in 34 Analyseeinheiten auf In diesen können gleichzeitig 9 Anglizismen ermittelt werden (26,5\%). In der Analyseeinheit, in der kein Antagonismus nachweisbar ist, tritt ein Anglizismus auf (100\%), so daß sich eine Prozentsatzdifferenz von -73,5\% ergibt. Dieser Wert wiederum besagt, daß Anglizismen im Korpus zu 73,5\% häufiger ohne Antagonismen auftreten, als mit diesen Erneut kann die Frage nach dem Erkenntnisgewinn dieses Ergebnisses ge- 
stellt werden. Dieser scheint, subjektiv beurteilt, recht niedrig zu sein. Auch in diesem Fall hat sich die Kontingenzanalyse im Rahmen der vorliegenden Diskursanalyse als nicht produktiv erwiesen.

Mit diesem Ergebnis wird die Korpusanalyse beendet. 
ond

-1, al

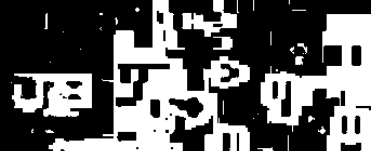

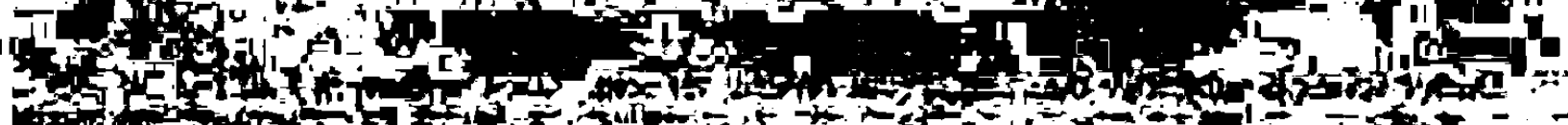

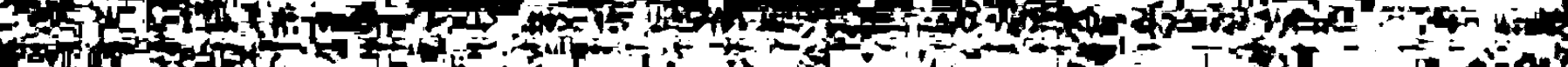
167

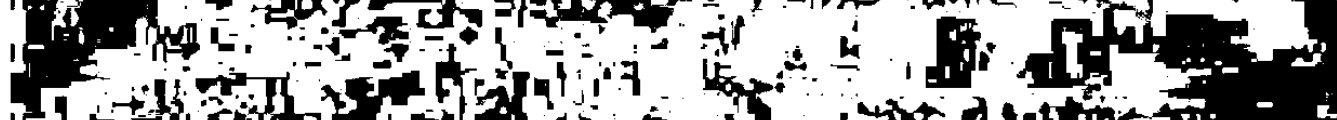

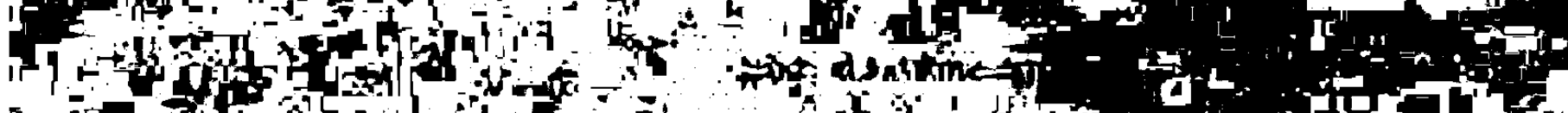

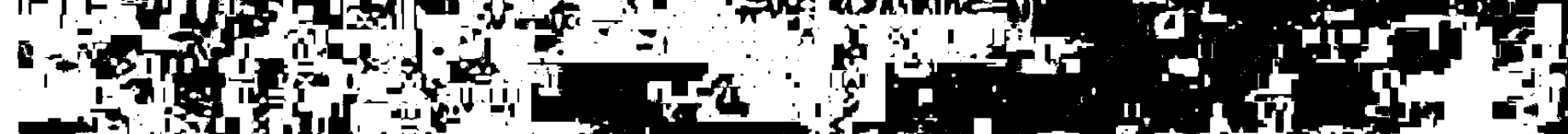
s

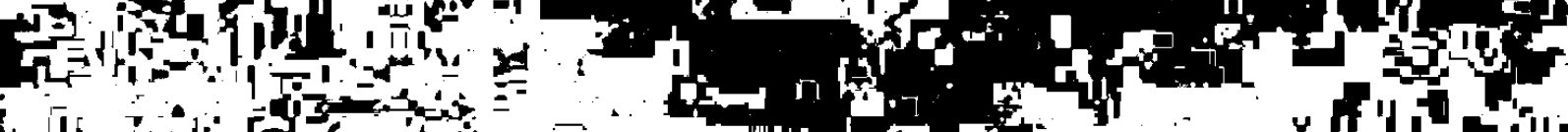

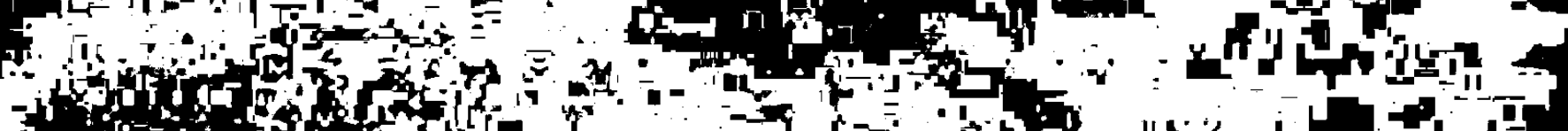

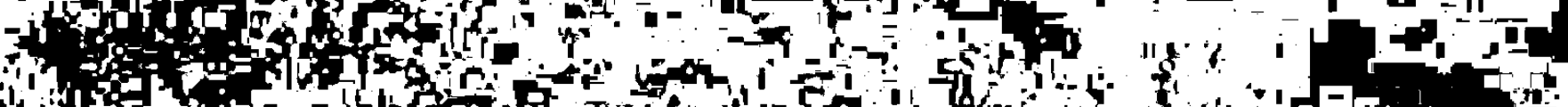
foldse

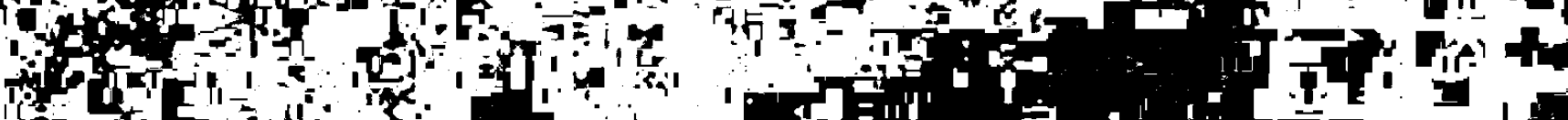
(2)

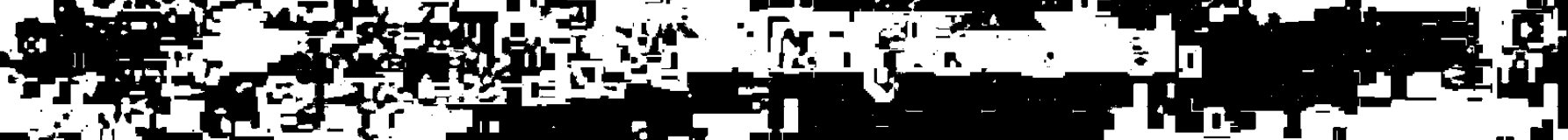
1)

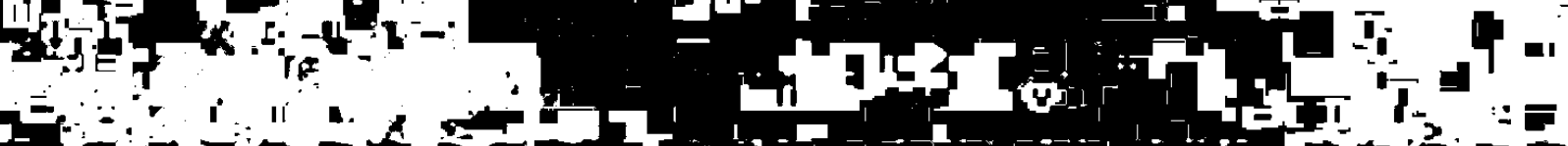

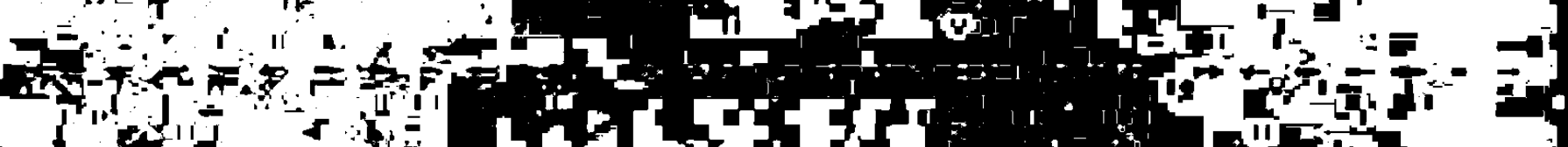
$t_{1}$

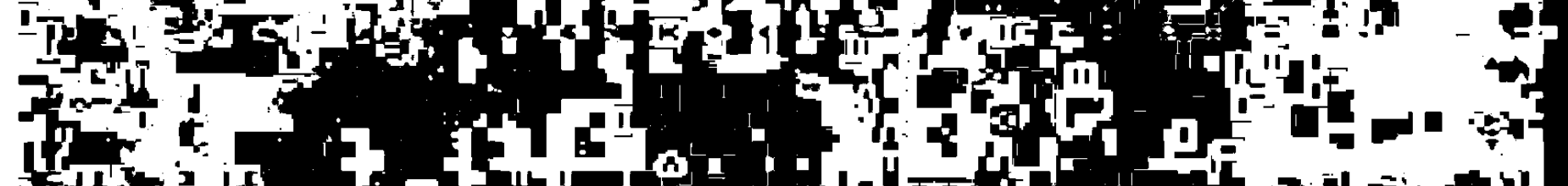

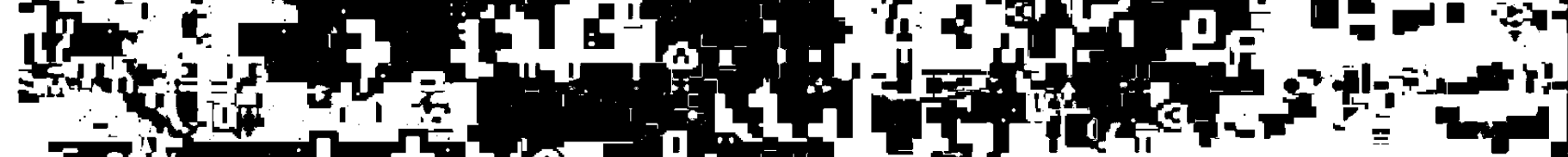
$=5, y_{1}=0$

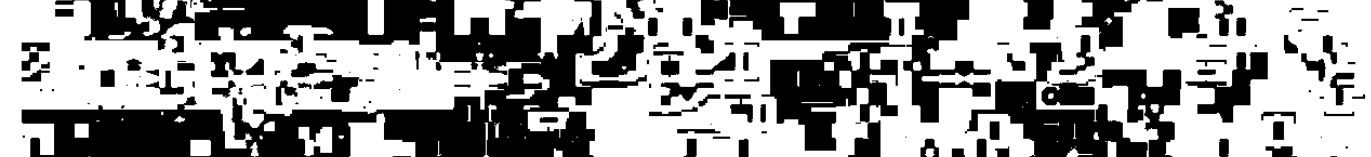
w

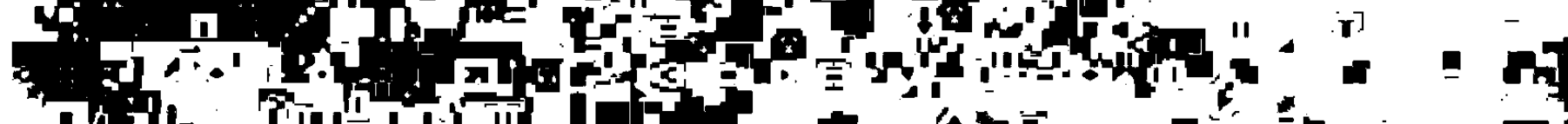

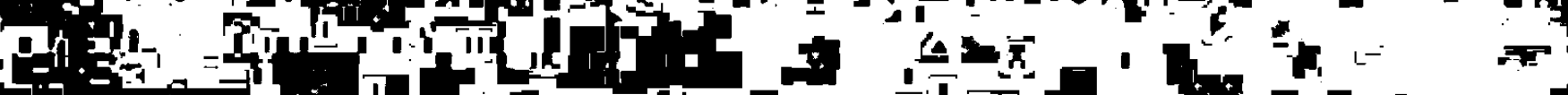

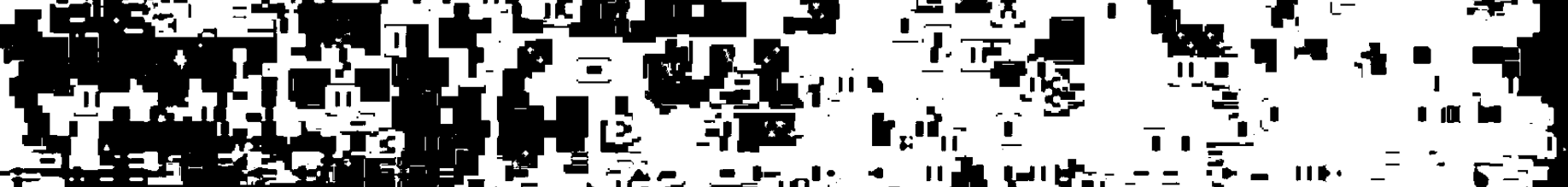

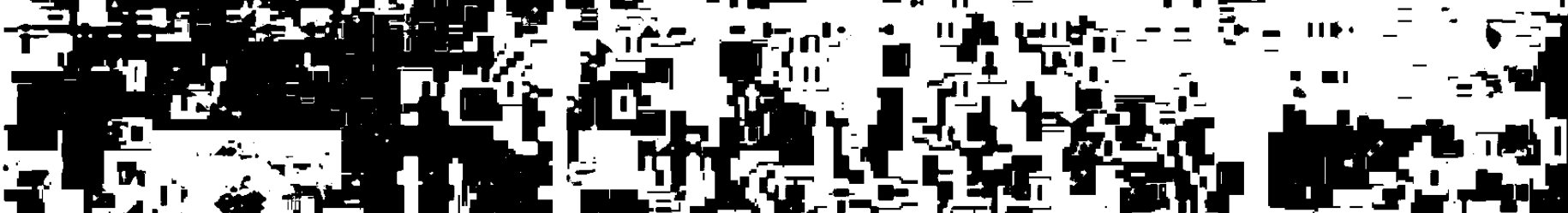

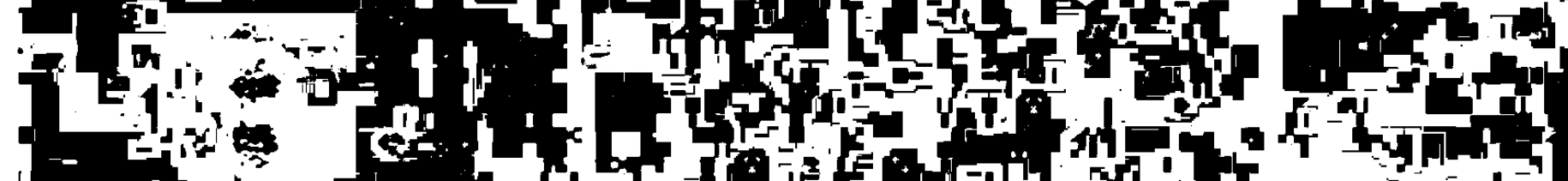

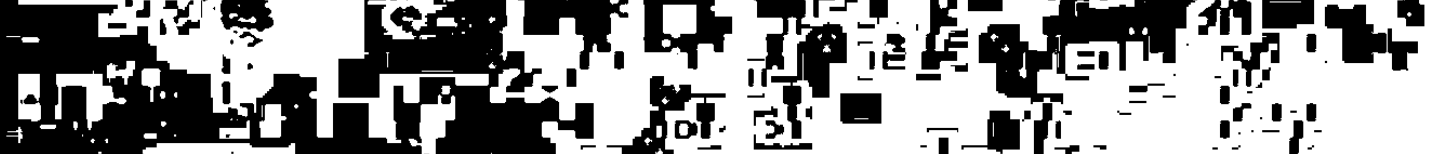

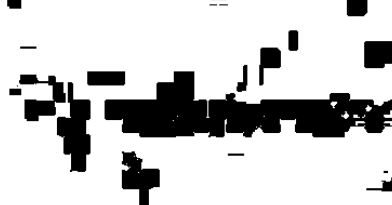

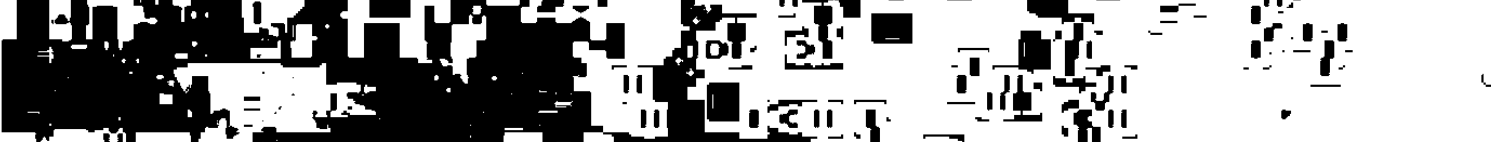

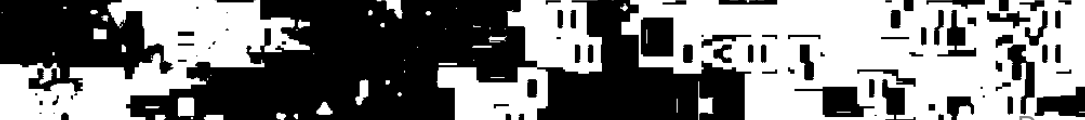

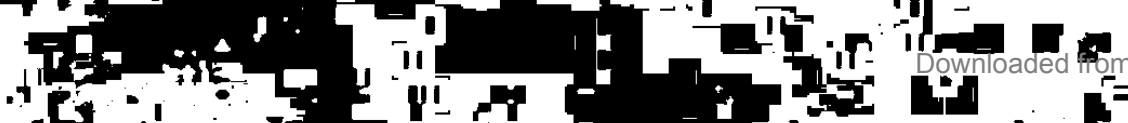




\section{Zusammenfassung}

In der vorliegenden Arbeit ging es in Teil I darum, auf der Grundlage der Theorie der Zweiten Wirklichkeit ein Instrumentarium zu entwickeln, mit dem ein Textkorpus diskursanalytisch betrachtbar ist, so daß Schlüsse über dessen kulturellen und sozialen Kontext gezogen werden können. In Teil II ging es darum, dieses Instrumentarium hinsichtlich seiner Produktivitat an einem konkreten, bisher nicht untersuchten Diskurs zu prüfen und Schlüsse über die kulturelle Formation zu ziehen, die vermittels dieses Diskurses kommuniziert.

Als wissenschaftstheoretische Basis für die Objekttheorie wurde die Konzeption des Konstruktiven Funktionalismus von Finke gewählt, so daß die metatheoretischen Werte Einfachheit, Widerspruchsfreiheit, Anwendbarkeit, Empirizität, Theoretizität und Intersubjektivität Gültigkeit für die vorliegende Arbeit besitzen.

Auf der Basis der genannten Metatheorie entwickelt Fleischer die Theorie der Zweiten Wirklichkeit, eine konstruktivistisch fundierte, empirische und systemtheoretische Kultur- und Diskurstheorie. Da das primäre Erkenntnisinteresse dieser Theorie nicht auf textuelles Material ausgerichtet ist, qualitative Aspekte kultureller Systeme zudem im Vordergrund stehen, wurde in der vorliegenden Arbeit der Versuch unternommen, die genannte Theorie weiterzuentwickeln, $d . h$. neue Theorieelemente einzuführen. Neben den grundlegenden Elementen der angesprochenen Theorie. Diskurs und Interdiskurs sowie kulturelle Formation und Kultursystem, ist es gelungen, die diskursiven Objekte Kollektivsymbol, Diskurssymbol, Stereotyp sowie Normativ (kulturelle Tautologie) fur eine Textanalyse zu operationalisieren.

Da die Inhaltsanalyse, die zwar einen von der genannten Objekttheorie abweichenden Kommunikationsbegriff zugrundelegt, den postulierten metatheoretischen Werten genügt, kann sie als geeigneter Fundus angesehen werden, der es ermöglicht, die Theorie der Zweiten Wirklichkeit auf der quantitativen Betrachtungsebene zu ergänzen. Aus diesem Fundus wurden die syntaktischen Analyseverfahren Wort-Satz-Relation (WSR) und Type-Token-Ratio (TTR) sowie die semantischen Analyseverfahren Themenanalyse und Kontingenzanalyse ausgewählt.

Im Zusammenhang mit der Arbeit Simvolika molodeznoj subkul'tury von Scepanskaja, in der eine Jugendsubkultur untersucht wird, die unter der Bezeichnung Sistema bekannt ist und die offenbar mit der untersuchten kulturellen Formation auf einer basalen Ebene assoziiert ist, ist es gelungen, die Existenz verschiedener diskursiver Objekte abzuleiten und zu definieren. Es handelt sich hierbei um Diskursiva, Anglizismen. Indices, Sentenzen und Formationsspezifika.

Erganzend wurden einerseits zwei Bewertungskriterien, charakteristisch und homogen, eingeführt, die auf die Bestimmung charakteristischer Textgenerierungsregeln des betrachteten Diskurses und auf die Beantwortung der Frage der Homogenitat und Heterogenität des ausgewählten Korpus, des Diskurses und der untersuchten Formation abzielen. Andererseits wurde das Instrumentarium durch Oppositionen. Antagonismen, die Erzählperspektive (die Insiderperspektive), die rhetorische Frage und die Autoritat vervollständigt. 
In Teil II, dem Analyseteil der Arbeit, ging es zunächst um textexterne Rahmenbedingungen der Textproduktion innerhalb der ausgewählten kulturellen Formation: es konnte festgestellt werden, daß die Publikationen, die der genannten Formation zugerechnet werden, einen geringen Umfang und eine kleine Auflagenhöhe aufweisen. Zum größten Teil werden die in den Publikationen enthaltenen Texte schreibmaschinell erstellt und mit Hilfe von Fotokopierem vervielfältigt. Vorwiegend sind die Publikationen in Moskau und Leningrad erschienen. Der Zeitraum von 1986 bis 1989 stellt die Hauptphase der Publikationstätigkeit dar.

Die Charakterisierung der den ausgewählten Texten zugrundeliegenden Publikationen legt den Schluß nahe, daß die Publikationen nicht isoliert voneinander entstehen, da trotz großer räumlicher Entfernungen Kontakte zwischen den jeweils beteiligten Personen nachweisbar sind. Zudem läßt sich auf dieser Grundlage die Hypothese formulieren, daß die untersuchte Formation aus einem begrenzten Personenkreis besteht.

In einer Beispielanalyse konnte anschließend gezeigt werden, wie das eingeführte diskursanalytische Instrumentarium auf einen ungekürzten Text angewendet werden kann.

Im Rahmen der diskursanalytischen Betrachtung des Korpus konnte auf der quantitativen Ebene nachgewiesen werden, daß die Verwendung von Diskursiva. Personen-Indices, Gruppen-Indices, Geographica- und Institutionen-Indices sowie Normativen, die Präferierung der persönlichen Erzählperspektive und der Aufbau von Antagonismen charakteristische Textgenerienungsmerkmale des untersuchten Diskurses darstellen, wobei keine charakteristischen Häufigkeiten bestimmter Elemente nachweisbar sind Unter Berücksichtigung derjenigen Merkmale, die sich im Laufe der Analyse als produktiv erwiesen haben, konnte berechnet werden, daß das Korpus, der Diskurs und die untersuchte Formation in mittlerem Maße homogen und in niedrigem Maße heterogen sind.

Die nachfolgende Zusammenfassung der Ergebnisse, die qualitative Aspekte des untersuchten Diskurses und der untersuchten Formation betreffen, orientien sich an der Reihenfolge der Abschnitte, in denen die Analyseergebnisse dargestellt wurden. Insofern bestimmte Ergebnisse (z.B.. Wort-Satz-Relation, Type-Token-Ration) nicht in qualitativer Hinsicht interpretiert wurden, wird auf diese nicht eingegangen

Mit Hilfe der Themenanalyse (9.3.) konnte festgestellt werden, daß sich die untersuchte Formation durch ein in mittlerem Maße homogenes Themenspektrum auszeichnet. Die thematische Kategorie Musik ist dominant, dennoch können gleichfalls hohe Werte für die Belegung der Kategorien Gesellschaft und Selbstreflexion ermittelt werden.

Kollektivsymbole (9.5.ff.) können im Korpus nachgewiesen werden, wobei das Kollektivsymbol kul'tura am frequentesten ist. Kollektivsymbole werden in der untersuchten Formation mit ihrer interdiskusiven Bedeutung eingesetzt, so daß die Hypothese, daß Kollektivsymbole im Interdiskurs und den jeweils untergeordneten Diskursen funktionieren, nicht falsifiziert werden kann In bezug auf die Kollektivsymbole kul'tura und cest'/cestnost' liegt vermutlich 
eine Erweiterung des jeweiligen kollektivsymbolischen Konstrukts um die Bedeutungskomponente kontrkul'tura bzw. necestnost' vor

Oppositionen (9.7.) sind dem Analyseergebnis folgend ein sprachliches Phänomen, welches in kultureller Hinsicht funktionalisiert werden kann, nicht aber muß, so daß nicht generell von einer kulturellen Funktion ausgegangen wird. Im Korpus treten temporäre Oppositionen häufig auf, so daß der Frage nachgegangen wurde, ob abgeschlossene Zeitkonstrukte im Rahmen der untersuchten Formation vorliegen. Dies ist nicht der Fall, vielmehr ist die kulturelle Bedeutung der Temporalwörter von den Elementen abhängig, auf die sie bezogen werden.

Das Konstrukt des Insiders, welches im Rahmen der persönlichen Erzählperspektive (9.8.) generiert wird, stellt kein charakteristisches Textgenerierungsmerkmal des untersuchten Diskurses dar, obwohl es zu diskurstaktischen Manipulationen nahezu themenunabhängig in der untersuchten Formation eingesetzt wird.

Im Rahmen der Betrachtung der Personen-Indices und der durch sie bezeichneten Aktanten (9.9.2.) kann zunächst eine Introspektive auf das sowjetische bzw. postsowjetische Kultursystem. nicht aber auf die untersuchte Formation diagnostiziert werden. Aufgrund der Häufigkeit ihrer Verwendung fallen die Aktanten Boris Grebenscikov, Aleksandr Puskin, Artem Troickij, Egor Letov und Iosif Kobzon auf, die offensichtlich im Rahmen der untersuchten Formation kulturell relevant sind. Grebenscikov weist keine einheitliche Bedeutungskonstruktion auf, die von Puskin und Letov ist positiv, die von Troickij und Kobzon negativ

Im Zusammenhang mit Gruppen-Indices und durch sie bezeichnete Gemeinschaften (9.9.3.) kann unter Berücksichtigung derjenigen Indices, die Musikgruppen bezeichnen, wiederum von einer Introspektive auf das sowjetische bzw. postsowjetische Kultursystem, nicht aber auf die betrachtete kulturelle Formation gesprochen werden Die jeweils kulturell relevanten Gemeinschaften Akvarium, chippi, DK, ljubera, Masina Vremeni und milicija, die aufgrund ihrer mehrmaligen Verwendung in unterschiedlichen Analyseeinheiten auffallen, wurden hinsichtlich ihrer kulturellen Bedeutung betrachtet. In bezug auf die Gemeinschaften Akvarium, chippi und Masina Vremeni wird eine positive kulturelle Bedeutung, in bezug auf die Gemeinschaften ljubera und milicija eine negative diagnostiziert. Hinsichtlich der kulturellen Bedeutung der Musikgruppe DK können keine Schlüsse gezogen werden.

Geographica- und Institutionen-Indices (9.9.4.) verdeutlichen erneut eine Introspektive auf das sowjetische bzw. postsowjetische Kultursystem, nicht aber auf die untersuchte Formation. Am häufigsten kann das Geographicum Sovetskij Sojus ermittelt werden, dessen Bedeutungskomponenten zum Teil eine negative kulturelle Bedeutung aufweisen. Das Geographicum als "Ganzes" weist im Gegensatz zu den einzelnen Komponenten vermutlich eine positive Bedeutung auf. Das Geographicum Moskva dient im Korpus fast auschließlich der Ortsbestimmung. Eine negative kulturelle Bedeutung beschränkt sich mit hoher Wahrscheinlichkeit lediglich auf Teilaspekte des Bedeutungskonstrukts. Das Geographicum Zapad dient vermut- 
lich der Abgrenzung, wobei die Abgrenzung nicht auf eine negative kulturelle Bedeutung des Geographicums zurückführbar ist Der Index Kreml' weist. wenn er eine Institution bezeichnet, eine negative kulturelle Bedeutung auf, wenn er ein Geographicum bezeichnet, eine positive. Die Institution Leningradskij rok-klub besitzt dem Analyseergebnis folgend eine negative kulturelle Bedeutung, die Institution Moskovskaja rok-laboratorija, deren Bedeutung sich in einem Konstruktionsprozeß befindet, vermutlich ebenfalls. Die Institution Verchovnyj Sovet weist gleichfalls mit hoher Wahrscheinlichkeit eine negative kulturelle Bedeutung auf. In allgemeiner Hinsicht kann festgestellt werden, daß Institutionen ausschließlich eine negative kulturelle Bedeutung und somit Relevanz aufweisen, in bezug auf Geographica beschränkt sich eine negative Bedeutung und Relevanz vermutlich lediglich auf ausgewählte Teilaspekte des jeweiligen Konstrukts

Auf der Grundlage des Analyseergebnisses in bezug auf Marken-Indices (9.9.5.) kann erneut eine Introspektive auf das sowjetische bzw. postsowjetische Kultursystem diagnostiziert werden. Die näher betrachteten, kulturell funktionalisierten Marken Vokal'nyj Instrumental'nyj Ansambl' (VIA), Otec'estvennye zapiski. Nasi sovremenniki, Malaja zemlja. Celina. Vozrozdenie, Assa und Moskovskij komsomolec besitzen jeweils eine negative kulturelle Bedeutung. Indices, die "historische» Ereignisse bezeichnen (9.9.6.), zeigen eine Introspektive auf die untersuchte Formation, nicht aber auf den Bereich der Sowjetunion bzw. deren Nachfolgestaaten. Bis auf die durch die folgenden Indices bezeichneten Ereignisse Pesnja-83, 1985 god, otkrytija Moskovskogo rok-kluba und vint 1983 goda besitzen die Ereignisse, auch wenn sie nicht stattgefunden haben, eine positive kulturelle Bedeutung.

Bei den Indices, die unter der Bezeichnung Sonstige subsumiert werden (9.97.), kann keine Introspektive diagnostiziert werden Die in kultureller Hinsicht funktionalisierten Indices sind demokratija. Bog/Gospod', nas industrial'nyj vek, vek informacii i nauki, dvizenie k svetlomu buduscenı. sovremennyj pljuralizm. stroiteli svetlogo buduscego, perestrojka, liberalizm und SPID Eine positive kulturelle Bedeutung weisen mit hoher Wahrscheinlichkeit die Indices demokratija, nas industrial'nyj vek, vek informacii i nauki, perestrojka und liberalizm auf, eine negative dvizenie $k$ svetlomu buduscemu, stroiteli svetlogo buduscego und SPID In bezug auf Bog/Gospod' kann weder von einer positiven, noch einer negativen Bedeutung gesprochen werden

Im Zusammenhang mit Autoritaten (9 10.) liegt erneut eine Introspektive auf den Bereich der Sowjetunion und deren Nachfolgestaaten vor. Es kann daruber hinaus festgestellt werden, daß die Beispiele (Zitate), die den Autoritäten zugesprochen werden, verschiedene Funktionen erfüllen. Wahrheitswertsteigerung des Textes, Diskreditierung der Autorität. Authentizitätssteigerung des Textes und Auflockerung des Textes Eine Wahrheitswertsteigerung bewirken Zitate, die Autoritäten zugesprochen werden, die eine positive kulturelle Bedeutung aufweisen. Boris Grebenscikov, Mifologiceskaja enciklopedija. Igor' Kochanovskij, Aleksandr Puskin, Urfin D’jus, Michail Zoscenko, Egor Letov, Aleksandr 
Duma. D. Selivanov, Sasa Sokolov, Aleksandr Galic und Aleksandr Baslacev. Durch Zitate diskreditient werden die Autoritäten Karl Marx, Dza Divizn, Konstantin Kincev, srednestatisticeskij zritel' rastamanskich koncertov, alisomany, starye druz'ja und TASS, die dementsprechend eine negative kulturelle Bedeutung im Rahmen der untersuchten Formation aufweisen. Lediglich textuelle Funktionen (Authentizitätssteigerung, Auflockerung) besitzen die Autoritäten fany, Klara Ivanovna/Petr Semenovic, L. Doros und Rok-Federacija. so daß auf dieser Grundlage nicht auf eine kulturelle Bedeutung geschlossen werden kann. In bezug auf Autoritäten kann des weiteren festgestellt werden, daß spezifische Personen-Indices als Autoritäten, die den Wahrheitswert eines Textes sieigern, am häufigsten auftreten.

Bei der Betrachtung des Sentenzen-Repertoires (9.11.) ist erneut eine Introspektive auf das sowjetische bzw postsowjetische Kultursystem, nicht aber die untersuchte Formation nachweisbar. Da keine Informationen über die empirische Verbreitung der ermittelten Sentenzen vorliegen, die Urheber teilweise nicht ermittelbar sind, sind alle Aussagen über die kulturelle Relevanz und Bedeutung der Urheber und der Sentenzen sehr unsicher. In diesem Sinne sollen nur diejenigen Sentenzen genannt werden, die mit hoher Wahrscheinlichkeit den untersuchten Diskurs markieren und somit im Rahmen der untersuchten Formation kulturelle Relevanz besitzen. Es handelt sich um die Sentenzen, die auf die kulturell relevanten Urheber Boris Grebenscikov und Pink Floyd zun̈ckgehen: „Rebjata lovjat svoj kaje“ und „Welcome to the machine!" .

Ab- bzw. Ausgrenzungen, die auf dem Aufbau von Antagonismen (9.12.1.) beruhen, erfolgen zum größten Teil vom übergeordneten Kultursystem. Des weiteren sind Abgrenzungen, die uber die Grenzen dieses Systems hinausgehen und solche. die der internen Differenzienung der untersuchten Formation dienen, nachweisbar. Eine Abgrenzung erfolgt hauptsächlich aus der Perspektive my, ja und rok, wobei mehrmalig eine Abgrenzung von drugie, ljubera, ljudi, Moskovskaja rok-laboratorija, der "Obrigkeit", die als oni und oni [svoloci] bezeichnet wird, vse, televizor pressa/TV, einem Teilbereich des ausdifferenzierten Bereichs der offiziellen Presse, und Zapad nachweisbar ist. Einige Abgrenzungen (z.B. von ljudi und vse) weisen daruber hinaus auf ein elitares Autokonstrukt hin.

Ab- bzw. Ausgrenzungen vermittels von Stereotypen (9.12.2.) erfolgen erneut hauptsächlich vom übergeordneten Kultursystem, aber auch nach «außen» und «innen». Innerhalb des sowjetischen bzw. postsowjetischen Kultursystems wird eine Abgrenzung von «den Durchschnittsburgern», "den Offiziellen» und "den Handlangern» der Obrigkeit deutlich. Emeut kann v.a. im Fall der Abgrenzung von "den Durchschnittsbürgern" auf ein elitares Autokonstrukt geschlossen werden.

Die im Korpus nachweisbaren kulturellen Tautologien (9.13.) weisen auf funf verschiedene Normalitätsbereiche hin: den formationsinternen, den des übergeordneten Kultursystems, den des Westens, den elitärer Aktanten und den von Aktanten, die unter dem Index evropejcy subsumiert werden. Der formationsinterne Normalitätsbereich steht im Vordergrund, so daß eine 
Introspektive auf die kulturelle Formation diagnostiziert wird. Der Normalitätsbereich des übergeordneten Kultursystems wird im Rahmen der untersuchten Formation nicht gänzlich negiert, sondern in $60 \%$ der Fälle als gültig anerkannt Die anderen Normalitätsbereiche, bei denen es sich um Konstrukte handelt, die innerhalb der Formation für andere generiert werden, werden bis auf den des Westens negiert.

Die primäre Funktion rhetorischer Fragen (vgl.: 9.14.) liegt dem Analyseergebnis folgend in der Hervorhebung von kulturell bereits funktionalisierten Phänomenen, so daß durch ihre Betrachtung in qualitativer Hinsicht keine neuen Erkenntnisse über die untersuchte kulturelle Formation gewonnen wurden. Rhetorische Fragen können jedoch offenbar als Indikatoren für kulturell relvante Phänomene aufgefaßt werden.

Eine nähere Betrachtung der Assoziation zwischen der Jugendsubkultur Sistema und der untersuchten Formation des rok-samizdat (9.15.) führt zu der Vermutung. daß die untersuchte Formation im weitesten Sinne einen Teil der genannten Jugendsubkultur darstellt.

Werden die dargestellten Einzelergebnisse zusammengefaßt, dann können auf der Grundlage der Korpusanalyse folgende allgemeine Schlüsse über die untersuchte kulturelle Formation gezogen werden. Die kulturelle Formation des rok-samizdat ist mit hoher Wahrscheinlichkeit ein in mittlerem Maße homogener Bestandteil der heterogenen Jugendsubkultur Sistema, die wiederum ein Bestandteil des sowjetischen bzw. postsowjetischen Kultursystems ist. Die betrachtete Formation ist in kultureller Hinsicht nicht autark, wie vermittels der häufigen Introspektive auf das übergeordente Kultursystem belegt werden kann. Dennoch wird eine Abgrenzung von Teilen dieses Systems angestrebt, wobei diese Abgrenzung teilweise auf ein elitäres Autokonstrukt zurückgeführt werden kann

Abschließend soll der Frage nachgegangen werden, inwiefern sich das gewählte diskursanalytische Instrumentarium in allgemeiner Hinsicht in seiner Gesamtheit im vorliegenden Rahmen bewahrt hat

Als nicht produktiv hat sich das inhaltsanalytische Vertahren der Kontingenzanalyse erwiesen, was vermutlich mit den gewählten Anwendungen zusammenhangt. Das verbleibende Instrumentarium hat sich auf verschiedenen Analyseebenen als produktiv erwiesen und konnte in seiner Gesamtheit genutzt werden, um Schlusse uber die Homogenität und Heterogenitat der untersuchten kulturellen Formation und deren Diskurs zu ziehen. In diesem Sinne ist es mit dem vorhandenen Instrumentarium, welches sowohl auf der quantitativen als auch qualitativen Betrachtungsebene erganzbar ist, möglich zu zeigen, ob bestimmte «Gruppierungen» (z.B.: Studenten, vgl Tuschau 16, 1998b) einen eigenen Diskurs besitzen und somit eine kulturelle Formation darstellen Es liegt dementsprechend erstmalig ein diskursanalytisches Instrumentarium vor, welches auf Textgenerienungsmerkmalen bzw. -regeln aufbauend ermoglicht. Diskurse und somit kulturelle Formationen zu bestimmen. 


\section{Anhang 1}

Evolucionnaja schema moskovskogo molodènogo samizdata. Aus: Kontr Kul't Ur'a, 2, B

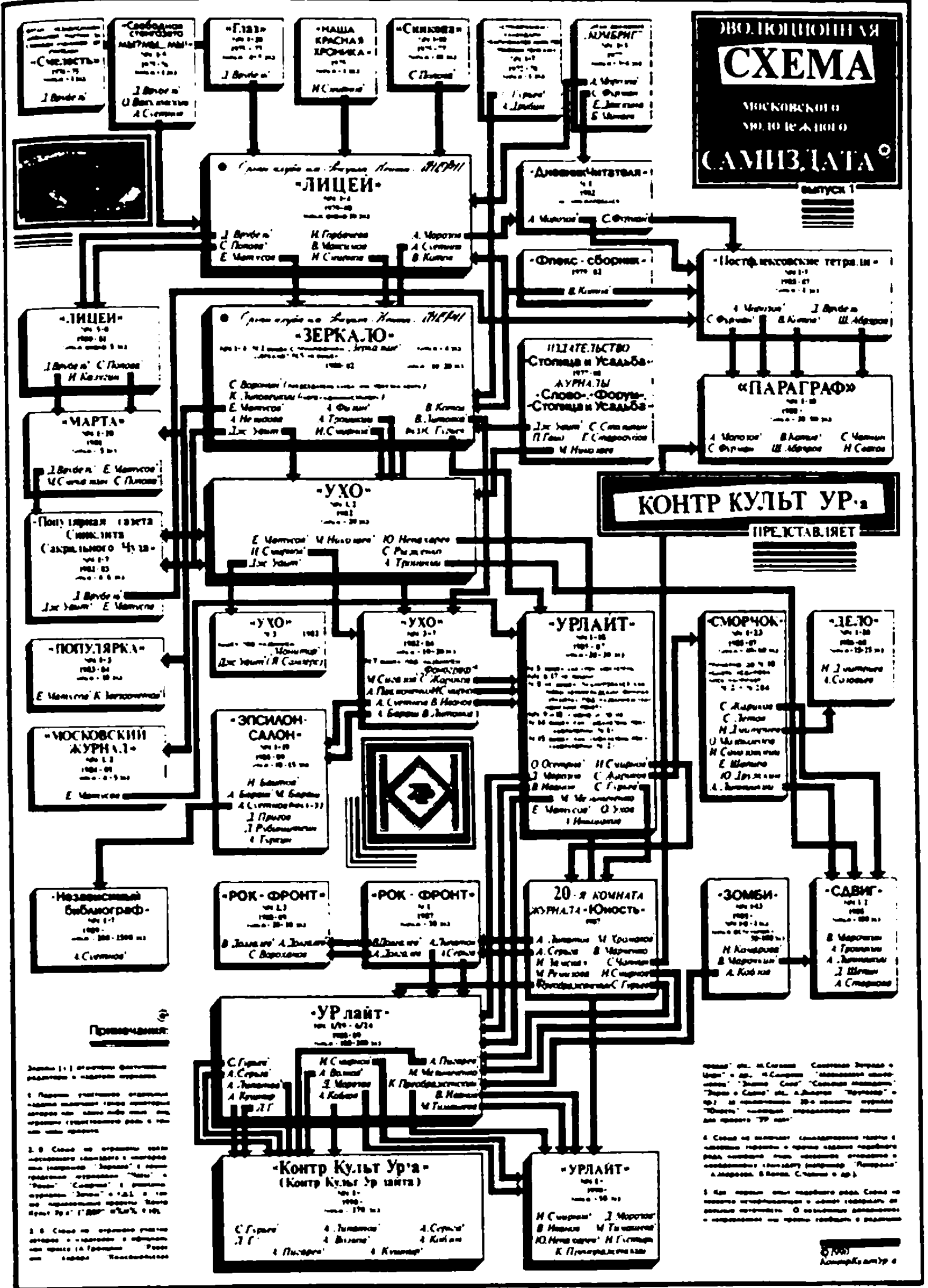




\section{Anhang 2}

\section{Analyseeinheit 1}

Deti podzemel'ja [Slavorosov, Arkadij; Sutov, Sergej], 1982, Kanon

Vmesto predislovija - otstuplenie dlja slaboumnych.

Rok - ne iskusstvo, ne neiskusstvo. Slovo ROK zdec' ispol'zuetsja v svoem naibolee obscem znacenii - ot muzyki do manery splevyvat' skvoz' zuby - kak nekotoraja velicina opredeljajuscaja edinstvo stilja i videnija. forma suśccestvovanija castota vibracii.

Slovecko ROK, $v$ gosudarstve ne prizivseesja tolkom, polupodpol'noe, idiotskoe i polurugatel'noe, okazalos' naibolee emkim, ctoby vmestit' $v$ sebja glubokuju bessmyslicu, istosnyj sepot ulicy, sochraniv nabivsij oskominu skandal'nyj privkus konfrontacii. Ne my vybrali ego, a ono vybralo nas.

POP ili Underground - terminy iz kriticeskich statej i obzorov, zamorskie cucela, mladency v formaline. $\mathrm{K}$ tomu he sliskom mnogo zvannych vpisyvajutsja peresidet' $v$ etom podpol'e termo-jadernuju bombardirovku Armageddona. so vsemi svoimi skarbami i rodstvennikami. Da i fonetika ochromeet na obe nogi ot takogo cudovišca - Underground, - kak perelomannyj $v$ cetyrech mestach skelet iskopaemogo $s$ malen'kim cerepom chippi - a my dolzny scitat'sja so vkusami trecov iskusstva. raz uz. s chodu nacali kryt' po materi. Vot i POP - malo togo, cto l'net $k$ Artu (a eto pachnet sodomiej), no i zvucit voobsce po-russki somnitel'no: to li cerkovnyj mrakobes merescitsja, to li voobsce - \%opa

\section{CHIP خivet na grani vosprijatija - kto iz nas ne uznaet ego $v$ lico? Velikij i mogucij!}

\section{Analyseeinheit 2}

B. G. [Grebenscikov, Boris], 1977. O vrube. In ROKSI, 1

Ljudi razlicajutsja po rassovym priznakam i po nacional'noj prinadle'nosti, suscestrujut bol'sie gruppy ljudej, razlicajusciesja po otnoseniju k sobstvennosti na sredstva proizvodstva Razlicajutsja ljudi i po otnoseniju $k$ rok-muzyke, odni vrubajutsja. drugie - net, poslednych - bol'se Do sich por ostaetsja zagadkoj, ob"edinjaet li tech, kto vrubaetsja. cto-nibud', krome togo cto oni vrubajutsja

Prijatno vstretit' celoveka, sposobnogo ocenit' to neotidannoe obstojatel'stvo, cto Jethro Tull na poslednem al 'bome "Songs From The Wood" okazalis' v neposredstvennom sosedstve s Ketom Stivensom. I kto iz takich ljudej ne ispytyval radosti, obnaruziv, cto ego novyj znakomyj, kotoryj po kakim-to pricinam nikogda nicego ne slysal, vrubaetsja i nacinaet zadavat' voprosy i prosit dat' poslusat' cto-nibud' esce? I kto ne ispytyval nedoumenija, granicascego s neprijazn'ju, kogda staryj znakomyj, byvavsij u vas million raz, sprasivaet. net li u vas Dzejmsa Lasta? Nu ne uscerbnyj li etot celovek? I neuteli vse, kto ne vrubaetsja, usc'erbny? Est' nad cem porazmyslit'.

Maloverojatno, cto otnosenie $k$ rok-muzyke javljaetsja kakim-to iznacal'nym svojstvom celoveceskoj natury. Odnako, opredeljaetsja ono cem-to letascim ves'ma gluboko. Vo vsjakom slucae, edva li ne samoe otcetlivoe oscuscenie obscnosti $s$ samymi razlicnymi ljud'mi vyzyvaet imenno sovmestnoe ucastie $v$ slusanii rok-muzyki, no ne u tech, u kogo cego-to net Cego? I tak li eto vazno? Cto eto daet? I otkuda eto vzjalos'? 


\section{Analyseeinheit 3}

anon. [Malysev, Boris], 1978, O zazde muzyki i vozmoznych metodach ee utolenija. In: ROKSI, 3.

Chot' ischod etogo sobytija i pecalen, no vse ze ono mozet ostavit' $v$ pamjati dobrye vospominanjja i ulybku. «A cem ja?» - sprosite vy.

Ja chocu napravit' nasi vospominanija na odin iz poslednich priezdov (oktjabr' 1977 goda) «Masiny Vremeni».

Atiotaz. vokrug ich priezda nacalsja zadolgo do samogo priezda. $O$ bol'soj populjarnosti «Masiny" $v$ nasem gorode vam govorit' ne stoit. Eto vy, nadejus', i sami znaete, chotja osnovnaja tema etogo povestvovanija $i$ est' vpolne ponjatnaja ljubov' nasej publikj k muzyke «Masiny», a mozet byt', i k nim licno.

Tak vot, nacalo oktjabrja vneslo v obycnyj razgovor fanov dva slova - «Masina Vremeni». Poroj kazalos', cto ves' gorod zuzzit etimi slovami. Tol'ko i slysis': «Ty znaes', «Masina Vremeni» priezzaet?» «Mne odin svistok skazal, cto «Masina» priezzaet. Eto pravda?» «Ne znaes', gde «Masina Vremeni» budet igrat'?» «U tebja net biletika na «Masiny Vremeni»?» Ljubopytno, ne pravda li? No my s vami eto mozem ponjat'. Ponjali by eto $i$ drugie...

I vot tocknaja data izvestna. 15-go pervyj koncert, kotoryj byl namecen v Puskinskom Dome kul'tury $v$ voskresen'e na 12 casov. Bilety ja dostal. « 0 , mnogo sil ja $v$ eto delo polozil». I vdrug pribegaet znakomyj i soobscaet novost': «Znaes", v Puskine bilety budut novye, $t$. $k$. kto-to poddelal sotni biletov, i eto doslo do organisatorov. Po starym nikogo ne pustjat!». Da, dumaju, vot esce kto-to resil pogret'sja na populjamosti «Masiny Vremeni». Polucil novye bilety, no pro sebja prikidyvaju: legal'nych biletov - stuk trista, nelegal'nych - 300, da esce zelajuscich, $u$ kotorych net biletov, - toze celovek sto.

\section{Analyseeinheit 4}

Troickij, Artem, 1981, Rebjata lovjat svoj kajf. In: ZERKALO, 2.

"Rebjata lovjal swoj kajt". B. (irebensicikov"

Ja poznakomilsja s A pri posrednicestve A Makarevica i priglasil ich na festival' v Cernogolovku. Za tri dnja do namecavsegosja otkrytija festival' byl prikryt. Sutki ja ne slezal s apparata. vgonjaja razochotivsichsja provincial'nych bitlov obratno $v$ ich nory, i ne smog dozvonit'sja do A. Karma solal svoe delo Oni priechali v Moskvu i vystupili na $20-m$ etase kombinata redakcii «Molodaja gvardija» s polyakusticeskim-polupankovym repertuarom. Kogda Grebenscikov zapel:

\section{«l'cera ja sel domoj}

Knigom byla vesna

Ego ja vstretil na uglu.

I v nem ne ponjal ni chrena.

Sprosil on: Byt 'ili me byt'?

A ja skazal: Idi ty ma..."

ja poezilsja ot sladkogo oscuscenija. cto vpervye słysu natural'nyj rok na nusskom jazyke. Ne vse bylo tak choroso u A, no na rol' luca sveta v carstve kJonov "Masiny vremeni" oni ocen' daze godilis'. Ja povez ich v Tblisi. Ne znaju, byl li eto zvezdnyj cas $\mathbf{A}$. vse slucilos' stranno, sumburno. glupo (po-A-ski), no... nekaja staruska vo mne so slezoj sepeljavila cto «takogo uze ne budet». Za neskol'ko minut do koncerta ja. prebyvaja v netverdom sostojanii, slomal gitaru BG, i emy prislos' terzat' cu»oj «Telekaster», prodirajas' s nim skvoz' "Marinu», "Tarelku», "Minus 30», "Geroev» i procie netlennosti. 'Turi rastvorilos' pod nadpis'ju "Vychod" esce $v$ seredine programmy. A v konce byl ustroen formennyj bardag («Bljuz svin’i v usach»), kogda Seva perepilival smyckom 
leخascego na polu Borju (s gitaroj), Fagot lupil fagotom kogo popalo, Djusa, kak slepoj kutenok, tykalsja $v$ udamuju ustanovku i t.p. Gosfilarmonija takogo ne videla

\section{Analyseeinheit 5}

Monachov, Igor' [Smimov, Il'ja], 1983, Novyj Gesiod. In: UCHO, 4

Pokolenija deljatsja na pokolenie bogov, pokolenie ljudej i promezutocinye pokolenija - cudovisc. velikanov i karlikov.

"Mifologiceskaja enciklopedija", t. 2. str. 317 .

Nasi rokery malo pochozi na armiju $v$ forme, marsirujuscuju s tamburmałorom. Sredi nich gospodsivujut partizanscina i srednevekovyj separatizm, a porjadka $v$ roke ne bol'se, cem $v$ drevnegreceskich mifach ili $v$ moskovskom kafe "Lira». Takova ob"ektivnaja real'nost', dannaja nam $v$ ob" ektivnom povsednevnom osccusccenii. Kogda klevyj vo vsech otnosenijach cuvak prosto ne mozet slysat' pesen Majka, to on govorit, cto eto prosto nabor slov, to eto marazm kakoj-to, i ego ot takoj muzyki prosto blevat' tjanet Dalee on utverzdaet, cto $v$ Moskve est' profi «Karnaval» $i$ ljubitel'skaja "Mozaika», i eto vescc, a vot «Zooparku» mesto tol'ko v 'Z.EKe igrat' A na vzgljad bol'sinstva. imenno u Majka - eto teksty, eto stichi, a u «Mozaiki» i «Karnavala» zastampovannyj nabor slov. No nam tak te slozno pereubedit' drug druga kak livancu-maronitu livanca-siita Jazyk u nas vrode by obscij, no vot ponimaem my pod odnimi i temi ze terminami sovsem raznye vesci.

Voobsce-to, vse eto normal'no

No ctoby zit' $v$ takom bardake, nado ego poznavat'. Cto za tecenija? Otkuda tekut? Kak svjazany te ili inye tvorceskie dogony ili oblomy s menee zametnymi, no vałnymi processami $v$ razlicnych slojach molodoj auditorii. [sic: ?] Ved' kumiry - cto u nas, cto na Zapad voznikajut ne sami po sebe, ich vynosit social'naja volna.

\section{Analyseeinheit 6}

Razuverov, I [Sigalov, Michail]. 1983, ('erno-belyj muzon In: UCHO. 5

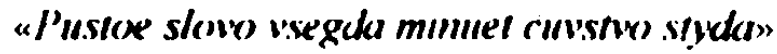
I. Kexhamowsky

A dejstvitel'no, minuet li? My pisem o roke, pisem o panke, o «novoj volne", pisem o disko (poslednee, vo vsjakom slucae, chot' sovremenno), a na scene "Golubych molodcev" smenjajut "Krasnye cvety", etich - «Pojut unitazy", etich - «Sinjaja S ...» I net cisla serosti, bessmyslennosti. tupoumiju. naglomy cinizmu $i$ obyknovennomu celoveceskomu idiotizmu. Nedavno, slysaja vsenarodnuju peredacu «Pesnja-83» (eto byl vrode by aukcion laureatov), ja podivilsja, neyzeli massy mogli progolosovat' za eti «pesnjaski» - tak oni byli nelepy, banal'ny i staromodny, da i drevni. Nekotorye estradnye solisty u'e let dvacat' skripjat ich nelepymi kupletami. Heyteli slusateli iz Leningrada mogli progolosovat' za etot "panoptikum-songs", imeja pod rukoj 50 grupp gorodskogo rok-kluba (dlja molodych) i pjat' raz D ro Dassena po mestnomu radio (dlja srednego vozrasta) $v$ den'? Ved' daze Dassen smotritsja sarmovee. sovremennee, elegantnee, vkusnee etogo sbroda skukmych dev, lysejuscich starickov-bodrjacikov, tichon'ko tolstejuscich' i umil'no tjanuscich "do" otdelenij, rot i polkov odinakovych murickov. A, da cto govorit', teleradiokorolevstvo krivych zerkal $v$ nasem brennom mire. Posmotrim na estradu.

VIA teper' pocti net Neotidanno iz vsech ugolov polezli rok-gnuppy. Fakt vrode by radostnyj, no prijatnogo, $k$ sołaleniju, malo Navernoe, vam choroso isvestny rok- 
gruppy «Ralli» i «Kaskad», «Ariel"» i «Akvareli», «Lira» i «Sjabry». Chotite pofirmennej - «Oreol», «Supersprint», «Zemlja i nedo» (poslednee - nu, pocti cto «Zemlja, Veter i Ogon'»).

\section{Analyseeinheit 7}

Baraskov, L. [Żarikov, Sergej], 1985, Folklor, etnografija i rok-muzyka. In: URLAJT, 1.

"Oni napadajut na samoe cennoe, cto u nas est', - na nas fol'klor", - vyskazyvaet svoju mys' odin surovyj dejatel', no my pozvolim sebe usomnit'sja $v$ ego slovach, ibo dannyj tovarisc, kuluarno, konecno, dlja sebja licno predpocitaet bolee «cennye» dela $v$ obraze bolee zeltom $i$ blestjaścem.

Babki, prjalki, papachi, sapogi, pljaski, tancy, zmancy - tak vosprinimaet javlenija narodnogo tvorcestva rjadovoj trużenik, poputno vspominaja o pitekantropach, neandertal'cach i naskal'nych rospisjach.

A ved' fol'klor - eto bezymjannoe narodnoe tvorcestvo i... vse. Imenno tvorcestvo, improvizacija, i tvorcestvo segodnjašnee. Etnograficeskaja differenciacija $v$ nas industrial'nyj vek imeet mesto lis' $v$ Afrike $i$ gde-to $u$ papuasov, da $i v$ vek informacii $i$ nauki ljudi ogosudarstvilis' kak nikogda.

Lapti, prjatki, utki-gusi i tomu podobnaja utvar' - vse eto ostalos' v istorii i v skazkach nasich babusek. Fol'klorno-etnograficeskoe tvorcestvo - eto istorija naroda, kul'tumyj ego plast.

"Ne dostoin buduscego narod, ne znajuscij svoego proslogo", govoril nas Aleksandr Sergeevic, obnimaja ocerednuju svoju ljubovnicu, gotovjas' $k$ dueli s Dantesom. I my ne mozem ne cenit' i ne uvazat' tvorcestva nasich dedov, vsego nasego naroda, to est' sebja samich, $v$ konce koncov. No za oknom vesennimi nuc'jami nalivaetsja 1985 god, i, kak skazal by «veras", "nastal cas rozovyj». Ho fol'klor li eto? Ćto budut govorit' o nas nasi vnuki i cto my smozem im otvetit? «Chmurit'sja ne nado, Lada»?

Nasa rok-muzyka ili, drugimi slovami, molodeznaja muzykal'naja subkul'tura, nazyvajuscaja sebja rokom, i est' to samoe na bukvy «f».

\section{Analyseeinheit 8}

\section{Ataman, S. [Zvezdocetov, Ataman], 1986, Muzon katit. In: URLAJT, Nr 9/10.}

\section{Tara-para-pararam! Tara-rara! Cacaca!}

Turum-turum, tirim-tirim!

Voobscie nistjak tak. Muzon katit. Narodu mnogo. Devocki, popki, to, se. Stremno slegka i modno diko! Modno i skucno. Posemy scitaju neobchodimym obrusit'sja na vsju ety lazu, na tupost' mody i sbit' s puti poslusnuju otaru otecestvennych snobov.

Rec' idet o vsjaceskich rok-n-rollach, ritm-end-bljuzach, bugi-vugi i procich maloponjatnych vescach. Vot uze tridcat' let epidemiceskaja volna amerikanizma rasprostranjaetsja po vsemu miru i grozit prevratit' $v$ bezdarnoe beze, nevrazumitel'noe sufle $i$ neudobovarimyj studen' vse plemena i narody.

Naivnye aborigeny odnoj sestoj vsego mira toze stali zertvami kovbojskogo uljuljukan'ja. Oni do sich por platjat dorogoj cenoj za svoju dovercivost'. Uze podroslo pokolenie, vyrascennoe desjatiletiem bez slov (60-70 gg.). Desjat' let celaja nacija slusala pesni, slov kotorych ne ponimala. Nakonec, pojavilis' pocti svoi teksty ili udacnye vol'nye perevody, no zvuki ostalis' preznimi. Vernee, ne zvuki, a orgazmiceskie stony anemicnoj anglo-saksonskoj rasy, kotoraja pytaetsja gal'vanizirovat' svoju smerdjascuju mumiju pri pomosci afrikanskoj 
spermy. Eti monotonnye i pederasticeskie poten'kivanija zopoj. eti istericeskie perdenija saksofonov dlja impotentov s Gudzona i dorianov greev, pereodevsichsja v gorodskich indeecev, ctoby vygljadet' esce bol'simi milaskami, eti poslednie popytki cachloj nordiceskoj razy izobrazit' iz sebja man'jakov i plastmassovych ucharej - neuzeli oni v sostojanii porabotit' i ocarovat' tataro-slavjanskuju ordu, vorvavsujusja diskoteki?

Nam ne nut.en reggej akklimatizirovannych napudrennych negrov

\section{Analyseeinheit 9}

Marockin, Vladimir, 1986, Sagi ne tuda (istorija diplomatiki muzyki rok i dvizenija KSP). In: ZOMBI, 6.

Sejcas, s iuravlinugo poleta roka 80-x, thizenie KSP kazietsja cem-to melkim i neznacitelinym. no bylo vremja, kogda ono zapolnjalo vremja i prostransnn tak ze, kak sejcas zapolnjaet rok.

\section{Cast' I. KOORDINATY}

Ljubiteli KSP vyvodjat svoju rodoslovnuju iz gorodskogo romansa XIX veka, odnako pravil'nee bylo by scitat' vremenem ich rołdenija 60-e gody nasego veka, vse-taki stoletie nazad i sreda byla drugaja. i uslovija. A $\vee 50$-e gody po vsemu miru budto folk-povetrie proneslos', voobsce nado skazat', cto $v$ mire vse proischodit prakticeski odnovremenno, i my $s$ vami ne $v$ antimire jivem, chotja uveren - ni Okudłava ni Dulov, pervye bardy, do segodnjasnego dnja ne podozrevajut o suscstvovanii Boba Cimmermana. No mozno li na etoj odnovremennosti vyvodit' prjamuju svjaz' KSP s rokom, kak na Zapade? Edva li.

V 60-e gody KSP bylo takim że "podzemnym", kak rok sejcas. Ego gonjali, zapresćali, primenjali k KSPsnikam administrativnye mery vozdejstvija vplot' do otcislenija iz institutov za ucastie $\vee$ festivaljach. Rasskazyvajut, kak neredko festival'nyj lager' okrułalsja milicejskim narjadom, a zatem bul'dozery sgrebali palatki v odnu bol'suju kucu.

No $v$ 60-e gody - $i$ eto glavnoe - slozilas' estetika KSP. Samodejatel'naja pesnja ob" "edinjala studenceskuju, a także naucnuju i tvorceskuju molodez', ona byla polna romantiki turistskich pochodov $\mathrm{i}$ romantiki budnej. Obxcim znakom KSP byl zdorovyj. may.ornyj pljus, i ona otra/ala umonastroenija togo perioda dvirenija $k$ svetlomu buduscemu

\section{Analyseeinheit 10}

anon. [Gur'ev, Sergej], 1985, Welcome to the machine! In URLAJT, 4

Pozarnye sozdany:

('toby sledit' za porjadkom. -

poetsja v znamenitoj kompozicii gruppy "Urfin Drjus» «45I po Farengejtu». Ocen' glubokaja ideja, raskryvajuscaja glaza na mnogoe. V samom dele. dolyna te suscestvovat' kakaja-to social'naja sluzba na tot slucaj.

Fsli narusimsja rannovesie

Me:chu gorjascim toboj

I okrusajusciej

('holoctno) sredoj -

kak ze inace. Ljuboj procinoj social'noj sisteme ure po prirode ee nadleyit bditel'no bljusti svoju ustojcivost' i operativno reagirovat' na voznikajuscie opasnye o'agi zagoranija $S$ etoj to'ki zrenija, połamye komandy predstavljajut soboj javlenie nesomnenno vałnoe, nułnoe, plodotvornoe i obsciestvenno poleznoe. 
Sovremennyj pljuralizm nas ucit, odnako, mnozestvennosti tocek zrenija na suscestvujuscie problemy. To, cto na etoj steze neslozno pereuserdstvovat', so vsej opredelennost'ju sleduet iz toj situacii, kotoraja slozilas' $v$ Moskve v 1985 godu: koe-kto vnezapno uvidel $v$ pozarnych pokrovitelej iskusstv. Bol'sego smesenija ponjatij, pozalyj, ne proizchodilo za vsju istoriju mirovoj kul'tury.

Vse, po-vidimomu, uze dogadalis', cto rec' idet o probleme otkrytija Moskovskogo rok-kJuba. Sluchi o kazavsejsja sovsem esce nedavno fantasmagoriceskoj vozmoznosti rasprostranenija slavnogo leningradskogo opyta na nasu zlatoglavuju stolicu stanovjatsja vse bolee intensivnymi. V Dom samodejatel'nogo tvorcestva chlebosol'no sozyvajutsja dja proslusivanija na predmet vyseoznacennych gumannych reform vse komandy goroda. Etu vysokuju misiju vzjalo na sebja Upravlenie kul'tury Mossoveta, choroso znakomoe sirokim slojam naselenija po svoemu znamenitomu postanovleniju, posvjascennomu problemam rok-muzyki, vynesennomu god nazad (tekst etogo postanovlenija, prodolłajuscego igrat' fundamental'nuju rol' v zizni nasego obscestva, po dosadnomu nedorazumeniju nigde ranee ne obnarodovannyj, my special'no puplikuem $v$ etom nomere tumala).

\section{Analyseeinheit 11}

Morosov, D. [Kovriga, Oleg], 1987, Derzat'sja kornej. In: URLAJT, 16.

\section{Cast' 1}

Mesjaca dva nazad ja smotrel na proischodjascee vokng menja i ne mog poverit' svoim glazam: neuzeli nas dejstvitel'no ostavili $v$ pokoe? Neuzeli nas perestali zagonjat' $v$ rjady stroitelej svetlogo buduscego, i my, nakonec, mozem spokojno, mimo sosuscestvovat'? «Zvyki Mu" reguljarno igrajut v bol'sich zalach na chorosem apparate, Sven Gundlach vystupaet v kilometre ot Kremlja, a milicija po stenam tmetsja, publika sebja custvuet soversenno rasslablenno, dare smotret' na nee prijatno i t.d. Ocen' chocetsja poverit', no, kak, govorila geroinja rasskaza Zoscenko «Tragikomedija»: "Vsja zizn' prochodit v sumerkach. Ćto-nibud" udivitel'noe slucilos' - etogo ne byvaet. Ja nepremenno znaju, cto proizojdet kakoe-nibud' takoe, blagodarja cemu ja skorej vsego ne uvizu etich deneg". Dolgo zdat', v obscem-to, ne prislos', i teper' ja chocu popytat'sja «strogo podumat'" i sformulirovat' "blagodarja cemu my ne polucim etich deneg", nesmotrja na takoj vyigrysnyj bilet. kak perestrojka. a mozet byt', i blagodarja emu, kto znaet.

I Sud'ba togo, cto idet ne pod egidoj ENMC i rok-laboratorii:

- Rizskij «Cement» v «Kaucuke»: priechal po vyzovu - vystupit’ ne dali.

- Festival' v MELZe. sorvan.

- Festival' v fevrale v MEI: laboratorija zapretila vystupat' vsem, komu smogla.

- "Veselye kartinki» v Tukovskom: javno zaplanirovannyj priezd «ljuberov».

- Koncert v lzmajlovo: klassiceskij vint v stile 1983 goda s ucastiem GB i opjat' ie «ljubera», kotorye, ocevidno, dolzny byli zanjat'sja vospitaniem muzykantov i publiki po doroge $k$ metro, no cut'-cut' opozdali iz-za togo, cto ich starsie tovarisci v pyzikovych sapkach spugnuli narod sliskom rano.

\section{Analyseeinheit 12}

Otec Fedor [Saparov, Igor'], 1987, O novoj turnalistike ili nam nado pobol'se plochich grupp In: ROKSI, 13.

U menja byli nezavisimye zametki na raznye povody, i ja ich ob"edinil v odnu. 
Ja dumaju, cto redakcija pomestit moju zametku. Ja-to scitaju, cto $v$ rok-zurnale nado publikovat' raznye mnenija. Daz.e oppozicionnye, daye rugatel'nye, daze te, s kotorymi ne

soglasen. V protivnom slucae menja budet raspirat' gordost' ot togo, cto ja napisal takuju krutuju vesc', kotoruju pricenzurili i ne napecatali daze $v$ «Roksi».

1. V «Roksi», kak $v$ igre, navernjaka cto-to ne tak. Po-moemu, eto uze stal reakcionnyj zurnal. I sejcas on perezivaet kul'minacionnyj punkt. Kogda na ego stranicach my uvideli sokrašcenie CHMR (HMR)? Kuda pozdnee, cem na zaborach. Kstati, klubnye zurnalisty vnacale otkazalis' ego prokommentirovat'. Choroso, chot' priznali svoju nekompetentnost'. (Alek Zander, «Roksi» No 11). Razetakie nechorosie, daze ne delajut raznicy mezdu chard-rokovymi i metalliceskimi komandami. Repliki na metallosistemu pochozi v zurnale na to, kak mne otozvalsja raz odin opytnyj muzkritik-simfonist:

- Och, uz mne vse eti ANSAMBLI... Chotja, postojte, net... Kazetsja, nedavno ja videl $v$ peredace po televideniju odin ANSAMBL' («Akvarium» - O.F.). I vy znaete - nicego... Krivljajutsja, konecno, padly... No vse ze menja eta muzyka ne razdrazaet Ja mogu eto slusat', ne zakryvaja glaza. Ne tak uz gromko i ne tak uz plocho.

KAK NEDAVNO OFICIAL'NAJA PRESSA OTNOSILAS' K ROKU, TAK SEGODNJA KLUBNAJA OTNOSITSJA K CHMR.

C'to Z, konecno. Vse predel'no pravil'no. Komu ze esce nuzen etot zeleznyj zaboj, eta zestjanaja mura? Tol'ko neumnym podonkam, vinovat, podrostkam. A zumal «Roksi» togda, izvinjajus', dlja kogo? Sprasivaete - otvecaju. Dlja tech, komu za tridcat'.

\section{Analyseeinheit 13}

Roza D. Kal'vados [unbek.], 1988, Byla kogda-to tetja Miansarova. In: SUB'EKTIKON, 3.

a teper' vot pojavilsja rok. I odin cuvak poprosil menja napisat' o nem. Dija etogo usivogo "Sub" 'ektikona" A cego $z$ ne napisat', pisat' my ljubim. Ja s detstva ucus' mokcat', znaju po opytu: sobrat 'ja po vidu mogut sozrat', esli cto ne po nim. No mysli, kotorymi ne obryzgivajut svoich soplemennikov, nakaplivajutsja v podsoznanii, i ot sego mozet prikljucit'sja psichoz. I, ctoby ne stat' psichom, ja stal pisat' Chotja psichom sejcas byt' modno. No ja toze nemodnyj

$O$ roke pisat'? Pozalujsta Chotja ja $v$ nem ne ponimaju ni figa. Sliskom blizko stoju No drugie ved' ponimajut $v$ nem esce men'se. Naskol'ko ja uspel zametit' Ottogo vy citajte, a ja budu pisat'

Vo-pervych, cto est' rok? Dlja muzykantov i ich prichlebatelej eto opredelennye prinzipy postroenija muzykalnych fraz, razmer i procaja drebeden'. Dlja ostal'nych eto necto muzykal'noe, vyzyvajuscee ej-foriju* ili, naoborot, nekoe neprijatnoe cuvstvo, kotoromu $v$ bednom russkom jazyke net nazvanija

Haprimer. $v$ junosti ocerednaja moja passija priznalas' mne, cto siganula ko mne $v$ kojku tol'ko potomu, cto naslusalas' kastrata Russosa. «Postav' mne Demisa, ob' 'jasnjala eta cutkaja natura, - i ja sama razdenus'!» "Kakaja naglost'», - skazet Klara Ivanovna ili Petr Semenovic. Ich vozmuscenie vpolne ponjatno, onj tak davno uze ne mogut. No ne o nich rec'.

Zdec' naprasivaetsja rezjume - rok pomogaet razmnozeniju, a eto patrioticno.

*j-forija - modnoe slovo 


\section{Analyseeinheit 14}

Zajcev, Egor, 1989, Umiraem, no ne sdaemsja. Boevaja chronika stolicnoj metallurgii. In: NOVYJ CHE'M, 6.

Voennoe protivostojanie podotcetnogo perioda charakterizuetsja zametnoj aktivizaciej boevych prikljucenij protivoborstvujuscich storon. Razvernytyj bespredel parijugenda ob"jasnjaetsja procnym sprascivaniem transsojuznogo gopnicestva sovetskich britogolovych.

Vysokooplacivaemye usilija agentov ochranki oznamenovalis' ucilennym ob" 'edineniem sturmovikov pravogo fronta: dejstvija drużestvennych otrjadov ljuberov i kazancev obreli zelaemuju Kremlem organizovannost' i sinchronnost'. Podryvnaja rabota $v$ srede sportivnych fanatov dala svoi plody: postepenno vtjagivajas' $v$ krovavye pogromy, povocinemie vnedrennymi ljuberami i musornymi lyckami, fanaty zarazajutsja virusom beznakazannogo krovopuskanija, menjaja tem samym krasno-belye sarfy na obsvastikannye povjazki. Verbovka prodolżaetsja. Novye zvezdocki padajut s partijnych nebes na krasnye pogony molozavych ubljudkov.

Razdelenie Moskvy na sfery vlijanija pozvolilo otecestvennym chunvejbinam kvalificirovannee provodit' operacii grabeta i sanitarii, a popolnenie partkassy razvivaetsja operezajuscimi tempami $v$ sravnenii s raschodom sredstv na stimulirovanie osobo otlicivsichsja boevikov $\mathrm{i}$ ich gebesnych glavarej. Pod pompeznye znamena ideologii liberalizma sgnivsej imperii vstajut vse novye boevye stranniki, ocarovannye scodrost"ju darov, uprugim posobricestvom vnutrennych organov, snischoditel'nym obscestvenym mneniem srednego obyvatelja i melkogo chozjajcika, prebyvajuscich $v$ sladkom sostojanii kommunisticeskogo pochmel'ja $i$ sogrevaemych velikim klicem zabludivsegosja mnogo let nazad v debrjach svoego volocjanogo pokrova Karla Marksa, dal'novidno vozopivsego: «Proletarii vsech stran soedinjajtes'!»

\section{Analyseeinheit 15}

Murzin, Valerij. 1989, Vniz po lestnice v nebo. Po motivam Btoroj vsesojusnoj konferencii «Rokmuzyka kak sociokul'tumyj fenomen". Sverdlovsk, 16-18 1188 In: TUSOVKA, 8

Svoe vystuplenie na vtorom po scetu sborisce Vsesojuznoj Rok-Federacii Dzordz. (Anatolij Gunickij) nacal's perénja illjuzij, koimi probavljalis' poslednye gody ljubiteli sovetskogo roka. Perecen' kJevyj. Ja tol 'ko dobavil by $k$ nemu esce odnu poziciju: illjuziju togo, cto po proctenii etoj stat'i vse srazu vdrug vse pojmut. I vse ze... pisat' ja nacinaju, v baske bedlam i sum; o cem pisat' - ja znaju, i vse ze... napisu.

Nynesnjaja schodka stala krupnoj vetv'ju na ternistom rokovom puti. S odnoj storony, vrode by, $v$ otlicie ot pervoj konferencii, ne bylo prinjato nikakich resenij, dokumentov NN, bumag $v$ Verchovnye Sovety. Opjat' ze ne bylo elementarnogo kvoruma: prezidentov, administratov, i procych udel'nych rok-knjaz'ev na etot raz s'echalos' vdvoe men'se. Zato okrepli jady sociologov i muzykovedov, i Rok-Federacija zanjalas' voprocami bolee nasusinymi, nezeli pisanie ustavov. A imenno eto:

- sravnitel'nyj analiz polozenie rokovych del $v$ razlicnych regionach,

- mucitel'noe osoznanie fenomena rok-muzyki (sociologiceskie i kul'turologiceskie aspekty);

- kak my dosli do tizni takoj (istoriceskij aspekt);

- i, nakonec, sakramental'noe «cto delat'?".

Dlja menja konferencija byla vażna tem, ćto uporjadocila moi mysli, dosele razroznennye, $v$ dovol'no strojnuju cepocky, kotoruju ja i predlagaju vam kak echo konferencii.

Vnacale o vesciach ocevidnych. 
Stalo soversenno jasno, cto svoeobrazie regional'nych kartinok - svoeobrazie, $v$ obscem-to, mnimoe.

\section{Analyseeinheit 16}

Turusinov, A., 1989, Hecestnost'. In: TIF, 11.

\section{BORIS GREBENSHIKOW "RADIO SILENCE" p 1989 CB MKA 1001}

Pojavlenie etoj recenziji v nasem izdanii - sovsem ne preklonenie pered Grebenscikovym. Naprotiv, neobchodimo tscatel'no proanalizirovat' to, cto vse eto znacit, ibo ne vse tak prosto, kak kazetsja na pervyj vzgljad. Samo soboj razumejusciesja vesci o tom, cto plastinka zapisana special'no dlja Zapada so vsemi vytekajuscimi posledstvijami - upominatsja bol'se ne budut, eto i tak jasno.

Interesno drugoe: pocemy ze BG, tak podcerkivajuscij vsegda suscestvovanie imenno gruppy «Akvarium» $\mathrm{i}$ sebja kak ee rjadovogo clena, tak gordjascijsja vsegda svoim kollektivizmom, vdrug $v$ samyj otvetstvennyj moment otvernulsja ot svoich druzej? Sam on $v$ masse razlicnych interv'ju otvecaet na etot voproz po-raznomy: to pricinoj javilis' material'nye zatrudnenija ( $v$ to ze vremja on $v$ odinocku raz"ezal po Statam of N'ju Jorka do L.A.), to Dejva Stjuarta ne ustroili professional'nye dannye muzykantov (eto Ljanina, $k$ primenu...), to voobsce nicego ob etom ne govorit, nacinaja trepat'sja o privycnoj metafiziceskoj erunde zaputyvaja mozgi interv'jueru do neobchodimoj stepeni.

Chitryj on cuvak, etot BG. Chitryj i o'en' umnyj, umejuscij delat' krasivye testy, gramotno i ubeditel'no argumentirovat' svoe povedenie (tak, cto $i$ vozrazit' nikak nel'zja), i $v$ etom vozdvignutom im tumane postupat' tol'ko tak, kak nuzno emu odnomu. Eto prosto ne krasivo. Tak kazetsja mne

\section{Analyseeinheit 17}

Ser'ga, Aleksandr [lonov, Aleksandr], 1990, Stat'ja No 108 In: KONTR KUL'T UR'A, I.

\section{Vnimanie!}

My prisutsvuem pri istoriceskom momente - slovo "rok" terjaet smysl Process ego deval'vazii podchodit $k$ tragiceskoj tocke - dal'se idet prozjabanie razmennoj monety dlja niscich duchom, popadanie $v$ razjad ponjatij (tipa «soznatel'nost'», «prekrasnoex ili «patriotizm»), kotorym gros cena.

Tendencija namecalas' davno snacala pristavkoj "rok-» oblaposivali molode?' gruppy filarmo-metallistov, potom eju sciegoljal v svoem anonsense «Laskovyj maj», no eto ne perekryvalo kislorod pri proiznesenii rodnogo (anglijskogo «kacajsja») slova, - poperchnulis" my togda, kogda nacali menjat'sja obrazy nasich «geroev», idej, sredy obitanija, mesta v mire i vsech procich predstavlenij, svjazannych s nimi. Tragedija, odnim slovom, ej-Bogu, tragedija.

Ne nado ni priparok mertvjakami*, ni medalej tivym - posle draki kylakami ne masut i «Postanovlenija o merach po ozdorovleniju roka» ne prinimajut. Te, kto pointellektual'nee. manevrinujut v poiskach obchodnych putej. Egor Letov vydvigaet tezic «Odinocki opasnee dlja sociuma, cem celoe dvitenie", VE\%LIVYJ OTKAZ i NJUAS vstupajut $v$ tumannuju kozlovskuju associaciju «Post-rok», kto-to spesno perekljucaetsja s politiki na liriku - pomagi im Bog v poiskach puti. Takie vot dela... Na etom skorbno-pateticeskoe vystuplenie zakancivaetsja. 


\section{Nedobitaja "kontra-"}

Mne kayetsja. pora reabilitirovat' ponjatie «kontrkul'tura», i moja stat'ja predstavljaet popytku obratit' vnimanie nasich citatelej na nekotorye pozabytye storony etogo javlenija Strogo govorja, nastojascej kontrkul'tury u nas v strane net: est' sovkovo-oficioznaja kul'tura (predstavlennaja takimi titanami kak poet Michalkov, pisatel' Bondarev i pevec Kobzon) i protovostojascaja ej prosto Kul'tura (Mezelajtis, Sevcuk, Iskander).

* Mertvjaki i mertvye - protivopolożnye vesci. Havernoe, poety, k nasemu necast'ju, sliskom choroso eto znajut.

\section{Analyseeinheit 18}

Nemcov, Maksim, 1991, Svoja aziatskaja roła. In. DVR, 12.

Naibolee dostojnaja polovina redakcii nastojascego izdanija, naprimer, scitaet, cto "vo vsjakom okolokul'tumom samizdatovskom hurnale samoe skucnoe mesto ispokon vekov - eto stateecki, posvjascennye obzoru razlicnych koncertov $i$ festivalej.» Obycno oni perepolneny fanovskimi zameianijami, tipa: «Na koncerte v DK «Fizik» Egor vygljadel bolee ustalym, cem na koncerte v DK "Chimik». Ili vot tak: "Draiv, ischodivsij ot zanadvorovskoj komandy PRESTUPNYE ELEMENTY, byl by srodni drajvu koncertov STUDŻIS, esli by ne tipicnye gitamye vstavki iz K'JUE, mesavsie mne zatascit'sja v polnyj rost».

Posle dzentl'menskogo nabora podobnych "analiticeskich opisanij» objazatel'no sleduet paratrojka ssylok na tradicionno ispol'zuemych «podstavnych utok» - libo "chorosich mal'cikov " (nasich), libo «mal'sikov dlja bit'ja» (ne nasich): «Rjadom sideli Troickij s Lipoj - «im nado byt' $v$ kurse, oni delajut den'gi»». Ili: "Gur'ev potom skazal, cto podobnaja rablezianskaja spontannost' vpervye osvetila etu scenu, a ruki ego $v$ tot moment otpustili koleno ocerednoj rensciny $i$ stali soversat' $v$ vozduche kontrkul'turnye passy". Tak. Posle etogo polozen nostal'giceski-prozrenceskij vyvod: «Ich Kennedi - ded, a nas - esce net. No popsy na nasej zemle esce mnogo",

Cestno govorja, mne eto porjadkom podnadoelo, da i ne citaju ja kak-to davno uze podobnych festival'nychj obzorov-otcetov. Chotja znaju, est' ljudi, kotorye citajut i na raznych koncach strany ticho radujutsja novym prikljucenijam neulovimych mstitelej. (konec citaty).

Vot vtoraja polovina etoj redakcii - i est' kak raz tot samyj ljud', kotoryj po suscestvu vyskazannych pretenzij chocet zametit', cto.

Tech iz nas kto privyk scitat' sebja bolee podkovannym $v$ oblasti teoretizirovannija po povodu toj sfery duchovnoj zizni molodezi konca XX beka, kotoraja tradicionno oboznacaetsja pustiusim v nasem jazyke korni slovom "rokenroll», choroso ucili, kak pravilo, v univerzitetach. cto suscestvuet takaja specificeskaja oblast' gumanitarnogo znanija kak «literaturovedenie». A tak re est' esce nekaja disciplina, nazyvaemaja "literatumoj kritikoj, kotoraja toze ocen' va:na». Samoocevidno. cto «Belinskim ot andergraunda" esce tol'ko predstoit pojavit'sja, no kto govorit, cto my dol’ny iskljucat podobnye popytki iz nynesnej rokenrol'noj praktiki? Podobnych ambicij, po pravde govorja. uze sejcas chvataet na celye biblioteki "Otecstvennych zapisok» $i$ «Nasich sovremennikov». 


\section{Analyseeinheit 19}

Rudkin, Boris, 1991, Do redakcii. In: SUMELA" MYS', 2.

«Strannaja, nelegkaja dlja istorikov i filosofov zadaca - nonjat'», - pisal A. Djuma-otec, kak my dosli do tisni takoj?

Stranno daze ne stol'ko potomy, cto do «takoj», skol'ko potomy, cto - «kak»?

Rassmotrenie sego global'nogo ( $v$ masstabe S'M, po krajnej mere) voprosa ja predpologal v stol' ze global'noj (gibel'noj) monografii «Istorija KLF Priselec» - sobiralsja ja cerescur dolgo, a teper' u nas rezko posel chaljavnyj kser, ugrozaja so dnja na den' zamolcat' sovsem. Posemu monografija ostaetsja idat' svoego casa, a ja poka ogranicus' kratkoj. po vozmoznosti, zametkoj "o tekuscem momente» (ne bez istoriceskich osmyslennij).

Vernuvsis' v nacale leta odna tysjaca $990 \mathrm{~g}$. «iz rjadov», ja znal o t.n. «rok-dvizenii» stol'ko, cto naibolee tocno eto mozno vyrazit slovom «nicego». Ob andergraunde $i$, tem bolee, roksamizdate, i togo men'se. Poslusav E.Letova i pocitav «K.K.URa No l" ja prikololsja po tisni' i scel «VSE ETO» naibolee dlja sebja interesnym delom.

Buduci po vospitaniju (vospitaniju ne $v$ smysle politiki moich, dopustim, roditelej, a skoree $\checkmark$ smysle zisnennogo opyta) «kniznym mal'cikom» a po tipu psichologii - «literaturnym celovekom", ja, vmeste s tem, i emocional'no, i na praktike (dvizenie KLF) ubedilsja $v$ nezisnesposobnosti literatury v cistom vide, t.e. cto «kniłnaja kul'tura mertva» (D. Selivanov).

Iz vsego etogo (dva predyduscich abzaca) ja sdelal vyvod, cto dlja ozivlenija literatury edinstvennoe sredstvo - peresadka ee na rokenrol'nuju pocvu.

\section{Analyseeinheit 20}

Usov, Boris, 1993, Cto takoe rok-poezija i kak s nej borot'sja. In: SVJAZ' VREMEN, I.

Obsceizvestno i ne trebuet nikakich dokazatel'stv to, cto rok-muzyka javljaetsja antiobscestvennym zlom Obsceizvestno takze, cto sobstvenno muzyka igraet $v$ t.n. "russkom roke» ves'ma i ves'ma vspomogatel'noe mesto, a glavnym tam javljaetsja tekst Takim obrazom. unictoziv samu vozmoznost' sozdavat' poeticeskij material, my likvidiruem «russkij rok" kak klass i załivem nakonec veselo i scastlivo No dlja etogo nado sperva znat' charakterneisie osobennosti rok-poezii i ee ujazvimye mesta

Russkaja rok-poezija ne $v$ meru obrazna. No obrazy, kotoraja ona sozdaet, pri blizajsem rassmotrenii okazyvajutsja ni na cto ne prigodny, vse usilija rok-poetov potomy propadajut zja. Pri ogromnom skoplenii naroda gruppa «Dła Divizn» ispol'njaet pesnju sledujuscego soder $a$ anija: "U menja tvoja golova $v$ nebe dvizetsja tvoja golova». Dlja puscego effekta na scene razvesany rastafarianskie flagi, prizvannye podtverdit' eto. No predpolotim, cto kompozicija avtomaticeski vozymela svoe vozdejstvie, i posle okoncanija koncerta srednestatisticeskij zritel' rastamanskich koncertov brosilsja za kulisy, vorvalsja $v$ grimerku i potreboval u liderov gnuppy Gery i Del'fina: «Otdajte mne moju golovu!». Uvy. Takogo ne proischodit Zritel' ne verit pesne i ne trebuet svoju golovu obratno

Pri etom na koncertach poroj voznikaet illuzija kontakta ispolnitelja $i$ auditorii, a inogda daze fanaticeskogo preklonenija pered ljubimym kumirom. No illuzija eta obmanciva, esli ne skazat: Iziva

«Nastalo vremja menjat'!» - skacet Kincev na arene krupnogo stadiona «Uraaaa! ochotno podchvatyvaet lozung pridurkovatoe celoveceskoe stado raskrasennych alisomanov. Vremja menjat' imena!» Na stadione carit edinenie i edinoobrazie, obycno stol' redkoe dlja etich mest. 


\section{Analyseeinheit 21}

Muchin, Zachar, 1993, Uchodjat produkty. In: PODROBNOSTI VSRYVA, 2.

Uchodjat «PRODUKTY». I ne tol'ko oni. Iscezla kolonna mogucich «kirovcev». Samyj pronicatel'nyj citatel' ponjal navernjaka, cto rec' ne o ede. REC" O OSC'UŚCENII.

Nedavno y menja ukrali Rodinu. Svoloci, oni nazvali Scerbakovu «Alekseevskoj». Cem im Scerbakov pomesal? Ja, naprimer, voobsce ne znaju, kto eto takoj.

Esce nedavno ja naivno polagal, cto vsja kontrrevoljucija po nocam spit, soglasno mescanskim zakonam. Odnazdy, dozdavsis' noci, Ja posel guljat' s fotoapparatom. Vot, vizu svoj staryj Universam. Imenno svoj, my s nim pocti povesniki, mne, scitaj, $v$ nem detskoe pitanie pokupali. V Duse srasy cuvstva vsjakje prinjatnye pojavilis'. Vzvel ja zatvor fotoapparata $i$ dumaju: "Nazovu kadr "Vitriny svetjat dałe noc'ju» ili cto-nibud' v etom rode». Vdrug tam, za steklom pojavljaetsja celovek $s$ raciej i cto-to $v$ nee govorit. Mne ne slyśno. Tol'ko on skazal, iz kommerceskogo kioska naprotiv vyskakivajut tri figury. Vot, dumaju, sistema! Podbegajut Eti troe ko mne, dvoe krutjat i zalamyvajut ruki, tretij zasvecivaet plenku. Eto ne vse. Podchodit pozilaja tolstaja neoprjatnaja gnuzinka i govorit mne: «Sluscij, ti scto, na Krolika rabotaes"? Chosisc', sctob my tibe etu kalejdoskopinu slomali?» A potom parnjam: «Otpustite ego poka no esli scto iz magazina propadet, vo vsem ego obvinim. Lico zapomnite...».

I kak raz togda u menja rodilas' ideja. Esli u demokratov nachodjatsja den'gi na vzjakuju erundu, to pust" nasjut pogonov s nadpisjami "OKP» ("Okkupant») i vsem palatocnikam v objazatel'nom porjadke pris'jut' na odezdu.

Ponacaly dumal, vragi tol'ko v Moskve. Resil poprobovat' uechat' na den' v Podmoskov'e.

\section{Analyseeinheit 22}

anon. [vermutlich: Nemcova, Ol'ga], 1990, Gospod' daroval nam eti dosugi. Popytka klasternogo analiza. In: DVR, 10.

Neposredstvenno za dvorom berut nacalo zadvorki. Za nimi ugadyvajutsja: kabak, slagbaum. versty razmytogo trakta, ostrog. Sibir' i pogost. (Sasa Sokolov)

Dejstvitel'no, choroso by my vygljadeli, esli b rabotali $v$ reguljarnoj gazete. Kak RokAdvokat. izvestnyj sovgavanskii ment $i$ tusovicik, - gde-nibud' v pytosnoj, no pri ziletke. Ili kak zenscina $\mathrm{v}$ belom kostjume iz $\mathrm{N}$-skoj molodezki, kotoroj Andrej Burlaka vezlivo ob" 'jasnjal, pocemy imenno nikomu ne nyłna oficial'naja pressa i, sledovatel'no, ona, zenscina, toze... V obscem, ne privedi Gospod' nastol'ko ne oscuscat' okruzajuscej dejstvitel'nosti, naskol'ko eto svojstvenno sluzascim sovmedii. Glubokij skepsis po povodu umstvennych sposobnostej vsech «leek i bloknotov» chot' $i$ imeet pod soboj ne menee glubokie osnovanija, no k predmetu nastojascego obzora imeet otnosenie lis' postol'ko, poskol'ky my resili v ocerednoj raz integrirovat'sja v «rok-scenu» (kotoraja ne otpuskaet vse ravno) i upodobit'sja svoim preznim inkamacijam, sredi kotorych navernjaka byli $i$ «jaryzki poganye» - i, ubiv takogo bol'sogo araba $v$ samom nacale, nam nicego ne ostaet'sja, kak pustit'sja po nakatannoj kolee sub" ektivnoj opisatel'sciny, kak eto ljubimo s davnich vremen.

Cego ot nas i zdut, sobstvenno govorja.

- A vot zena Umeckogo, skazali nam v tolkucke dvorca Molodezi, uvesannogo resetkami iz fol'gi i raznostilevymi nadpisjami, pocemy-to tjagotevsimi $k$ severokorejskoj ieroglifike. Na chrupkogo, boleznenno vygljadesego cernogo celoveka jadom $s$ nej nikto ne obratil vnimanija 


\section{Analyseeinheit 23}

Demin. V., 1990, Kul'tura i samorazusenie. In DVR, 11.

Segodnja mnogo govoritsja i delaetsja v pol'zu razlicij $i$ suverennosti cego by to ni bylo: nacij, narodnostej, regional'nych ekonomik, jazykovych kul'tur. Vse ranee podavljaseesja vosstaet $\mathrm{i}$ obnarułivaet gigantskie rasstojanija, na kotorye my udaleny drug ot druga.

C'to nas segodnja ob"edinjaet? Navemoe, pravda kak obscestvennyj razdrazitel'. No, okazyvaetsja, to, cto obladaet vseobscim dejstviem, razdeljaet poljarnost'ju reakcij na razdrazitel'. Nenapravlennoe vozbuzdenie obscestvennogo konflikta, po izvestnomu zakonu suscestvovanija ljubych sistem, vedet $k$ raspadu obscest va.

Nyne oblast' primenenija energii razbuzennogo gosudarstva opredeljaetsja $v$ dvuch slovach: vzaimoponimanie i vzaimovygoda, pricem pervoe, bolee sirokoe, ponjatie svoditsja $k$ bolee uzkomu vtoromu i takim obrazom obescenivaetsja. Protivniki obnovlenija soznatel'no opuskajut protivorecivyj smysl etich slov, podderzivaja i uvelicivaja inflaciju obscestvennopoliticeskoj terminologii. Tem ze zanimajutsja, no po prestupnomu neznaniju, diletanty, koich $v$ smutnoe vremja vsegda bylo dostatocno dlja razvala ljubogo dela. Eto spor uze ne o terminach, a o metodach i napravlennijach vychoda iz nestabil'nosti.

Poka obscestvo nase nachoditsja $v$ sostojanii vozbuzdeninych potrebnostej vygody, ljudi terjajut golovy ot odnogo nameka na vozmoznost' ich udovletvorit', i eto malo cem otlicaetsja ot op'janenija zastoem. Ctoby vyjti na oficialn'nyj uroven' udovletvorenija potrebnostej, ljudi sobirajutsja $\vee$ gruppy, partii, daby $v$ lice zakonnosti svoich trebovanij opravdat' (prosu ne putat' opravdanie i smysl) svoju prirodnuju nesposobnost' dobyvat' blaga bescestnym putem.

\section{Analyseeinheit 24}

Afonskij, Leonid, 1990, Putem tepla. Kratkij ocerk istorii russkoj kontrkul'tury. Popytka sravnitel'nogo analiza. In KONTR KUL'T UR'A, 2.

\section{VVEDENIE}

'ijvja v Rossii, my casto zabyvaem absurdnost' konteksta, v uslovijach kotorogo forminujutsja nasi licnosti. Fakticeski nase Otecestvo vot ure sest' stoletij javljaetsja Stranoj Cudes. Esh na kondovom Zapade, posledovatel'no razvivausemsja ot etap $k$ etapu, uze $v$ 1000-m godu suscestvovala Bolonskaja skola prava /kakovoj fakt po sej den' vyzyvaet nezasluzennuju zevotu nasich skol'nych ucitelej istorii, to Rossija esce so vremen Sergija Radonezskogo nacala ves'ma r'jano pretvorjat' v zisn' idei "absoljutnoj pravdy" - risni "ne po zakonu, a po sovest'". Uze pri pervych sagach etoj, s pozvolenija skazat', "dejatel'nosti" absurd nacal sguscat'sja.

Napisali my etot absac, i srazy predstavili sebe, kak skul'ptor Klykov vkupe s pisatelem Krupinym zabegajut po Moskve $v$ poiskach koscunstvennogo evreja, pokusivsegosja na nacional'nuju svjatynju - edakogo Gol'dmana, sosuscego krov' iz trupika mladenca Ivanova. Chotim ich razocarovat': avtor chot' i privyk zit' $v$ der'me, no otnjud' ne stal ot etogo rusofobom - i daje nachodit opredelennoe udovol'stvie v svoem polozenii. Odnako, zitelju paradoksal'nogo gosudarstva chocetsja poroj ponjat' korni svoej udivitel'noj tisni.

Smeluju popytku takogo roda sdelal nedavmo lider populjamoj gruppy "DK" Sergej " arikov v svoej nasumevsej rabote "Rok-n-roll na Rusi". On popytalsja postavit' rok - duchovno-esteticeskoe jadro mirovoj kontrkul'tury - v svjaz' s russkoj kul'turnoj tradiciej i odnovremenno proanalizirovat' $v$ etom plane sovremennuju sociokul'turnuju situaciju 


\section{Analyseeinheit 25}

Sokolovskij, Ivan, 1991, Konec andergraunda. In: KONTR KUL'T UR'A, 3.

Odna iz otlicitel'nych cert otecestvennoj rok-pressy - est' polnoe otsutstvie interesa k prognozirovaniju. otsutstvie daze samych obscich charakteristik, kogda delo kasaetsja ne nastojascego i proslogo, no budusccego rok-n-rolla. U nas ocen' ljubjat s nostal'giej vspominat' "byloe", vostorozenno perezivat ili ozloblenno kritikovat' nalicnuju i konkretnuju situaciju, no ne dajut sebe truda vzgljanut' na opisyvaemyj fenomen s tocki zrenija ego razvitija, ego dinamiceskich vozmoznostej, s istoriceskoj tocki zrenija. "Podpol'naja" rok-pressa libo apologeticeski vospevaet "drajv" i "kajf", ogranicivajas' tsciatel'nym opisaniem bumogo potoka emocij, perezitych avtorom i publikoj vo vremja koncertov $\mathrm{i}$ "tusovok", libo "kritikuet" - t. e. opisyvaet svoi negativnye $i$ otricatel'nye emocii, peretivaemye vo vremja tech te koncertov $i$ "tusovok". Vo vsjakom slucae i "kritika" i "apologetika" morfologiceski odnotipny. I to i drugoe est' opisanie emocij, kotoroe $k$ tomu ze prizvano prodemonstrirovat' nezaurjadnye literatumye talanty "rok-kritika". Dlja tech, kto sam licno prinimal ucastie $v$ opisyvaemych meroprijatijach i licno perežival proischodivsee "rok-pressa", v principe, ne interesna - eto libo podtverzdenie emocional'noj reakcii citatelja. libo ego otricanie. I v tom, i v drugom slucae. vse proischodit na prostejšem urovne - "nravitsja - ne nravitsja", a demonstracija littalanta obycno lis' razdrazaet citatelja, kotoryj, $v$ bol'sinstve slucaev i sam scitaet sebja dostatocno esteticeski $i$ literaturno odarennym dlja togo, ctoby skepticeski otnosit'sja $k$ cużim talantam.

\section{Analyseeinheit 26}

anon [Matusov, Evgenij], 1986, Novyj vrag - pidorostija. In: URLAJT, 9/10.

Samyj lucsij sposob sochranit' zdorov'e i dusevnyj pokoj - ne imet' obsicich del s pressoj: ne citat' gazet, ne slusat' radio, ne smotret' televizor. Ja tak i delaju. Pravda nebol'sie dozy. no $v$ cisto naucnich celjach, ja vse ze prinimaju $-v$ sortire, pri ctenii melkonarezannoj gazety "Izvestija", odnako eto ne $v$ scet.

No na dnjach moj status kvo byl narusen Druz'ja prinesli mne vyrezki iz dvuch nomerov gazety "Vecernij Kisenev" za sentjabr' 1985 g (No 216 i No 217). Stat'ja pod zagolovkom "Komu podygryval intellektual'nyj rok?" (v krugu loznych cennostej) avtor L Doros. Ljubonytnoe nazvanie. "Intellektual'nyj rok" - svez.o. A vot vopros tradicionen. Komu podygryval intellektual'nyj rok? Neuteli opjat' CRU? Podozdi, citatel', uznaes'.

Ne legka rabota u rok-muzykantov, ternist i poroj dramaticen ich zisnennyj put'

My razgovarivaem uze bolee trech casov. Utrennee solnce uspelo podnjat'sja $v$ zenit i sprjatat'sja za stenoj sosednego doma. Prosumel i vysoch na asfal'te dozd'. A neobchodimoj jasnosti v razgovore poka net. Vremja, postupki sami otvetjat, osoznaet li moj sobesednik, cto tisnennye blurdanija priveli ego. Aleksandra Simonova, $k$ samoj certe, $k$ toj grani, za kotoroj - izmena idealam obscestva. vzrastisego ego

Ne legka rabota i u cenzopa 


\section{Analyseeinheit 27}

anon., [Smimov, II’ja], 1988, Novye gorizonty svobody. In: URLAUT, I (19).

Ja vybiraju svobody,

No ne iz boja, a v boj.

Ja vybiraju svobody

Byt' prosto samim soboj.

\section{A. Galic}

Fany sovetskogo roka iz Bundes-respubliki Germanii, kotorych ja provodil na sejsen, podarili mne za eto znacok s portretom M.C.Gorbaceva i nadpis'ju po-russki "GLASNOST'" lodnako "made in Germany"/.

Situacija, soglasites', dovol'no neobycnaja. No menja $v$ nej zainteresoval ne stol'ko "mezdunarodnyj", skol'ko samyj cto ni na est' "vnutrennyj" aspekt - kogda ja s etim znackom na kurtke pojavilsja za kulicami, nekotorye iz starych druzej vyrazili nedoumenie $i$ daze negodovanie: "cto eto, mol, ty na sebja nacenil?"

Ja ne stal tratit' vremja na perecislenie svoich proslych revoljucionnych zashug /ostavljaju etu temy dlja Bori Zemcova/, perechodjasee $v$ samoopravdanie po tipu "ja ne verbljud" /ne kon" "junkturscik, ne kar'erist, ne "skurvilsja" etc./ - chotja i znacka ne snjal. A na sledujuscij den' sel za masinku, ctoby prodolzit' svoi proslogodnye zapiski, stavsie dovol'no populjamymi blagodarja "urodlivomu javleniju" rukopisnych rok-zurnalov. O cem eto prodolzenie? Da vse o tom 2e -

\section{O SPRAVEDLIVOSTI}

Obidersis' na tech, komu ne ponravilsja moj firmennyj znacok, ja mog by $v$ otmestku obozvat' ich, naprimer "ekstremistami" No s nekotorych por ja starajus' izbegat' podobnych terminov - ibo oni, $v$ suscnosti, $i$ ne termini vovse, a politiceskie jarlyki. Cito oznacaet, naprimer, slovo "ekstremist"? Segodnja tak nazyvajut D. Vasil'eva s kompaniej - pojavilsja dase stamp: "ekstremistskie lidery "Pamjati" /kak budto by jadovye sturmoviki ne dostojny svoego Rema!/ - no ved' sovsem nedavno ta nacistskaja bredjatina. kotoroj "Pamjat" potcuet svoich lopouchich slusatelej, scitalas' oficial'noj i respektabel'noj

* Citiruetsja po: Troickij A., "Muzykal'nyj zisn'", 1987, No 23

\section{Analyseeinheit 28}

anon., [Smirnov, Il'ja], 1988, Pered vami, deti, utka ... In: URLAJT, 3 (21).

Pered vami, deti, utka -

Ona bol'saja prosti...

Prostite, deti, ona malen'kaja.

Nu kto iz nas ne pomnit s detstva etot zamecatel'nyj stisok. Ja toze vspominaju ego vsjakij raz. kogda pressa prinosit mne ocerednoe tvorenie na zlobodnevnuju temu prostitucii. Prostitutki, narkomany i gomoseki - vot ocerednoj dzentl'menskij nabor komsomol'skomolodeznych izdanij (kak ran'se "Malaja zemlja", "Celina" i "Vozrozdenie"), no po pervym specializirovat'sja vse-taki prijatnee, poetomy nasi 7orti Djurya s radostnymi klicami "Ułe mołno!" 
(i s tajnoj nadezdoj: "Mozet i mne cego perepadaet") brosilis' lomat' otkrytuju dlja nich po ukazaniju sverchu dver' bordelja. I do togo pri etom vozbudilis', cto zabyli ob elementamoj ostoroznosti. Ja imeju $v$ vidu ne venzabolevanija, a to, cto nelestnye associacii sami soboj rozdajutsja $v$ golove citatelja ili zritelja ich produkcii. Naprimer, posmotrev podobnogo roda fil'm, odna moja znakomaja srazy ze resitel'no zajavila, cto iz dvuch prostitutok, majacivsich na ekrane, gorazdo simpaticnee ta, kotoraja tenscina, a ne ta, kotoraja tumalist

A sovsem nedavno ja popal na obsuzdenie bezradostnoj temy SPIDa. Predvaritel'no auditorii prodemonstrirovali dve videozapisi: nas dovol'no bestolkovyj fil'm "Gruppy riska" (klassiceskaja obojma: sjuzet o narkomanach, sjuzet o putanach, a sjuzet o "golubych") $i$ programmu anglijskogo televidenija: strasnye kadry, snjatye $v$ bol'nicach, gde umirajut molodye ljudi, i prjamoj, cestnyj, bez malejsego naleta chanzestva $i$ licemerija razgovor ob epidemii i o tech problemach, kotorye ona postavila pered celovecestvom.

\section{Analyseeinheit 29}

anon., [Smirnov, Il'ja], 1988, Veselye i ne-veselye kartinki uz istorii DK. In: URLAJT, 4 (22).

Reklamno-apologeticeskie stat'i o rok-gruppach porjadkom utomili ne tol'ko mysljaścuju cast' auditorii, no i samich muzykantov (za iskljuceniem tech, kto prosto probivaetsja k "prestiznoj kormuske"). No soglasites', cto $v$ absoljutno nenormal'nych uslovijach, kogda celomu zanru sovetskogo iskusstva prichodilos' otstaivat' svoe pravo na lisn', u celoveka, pisuscego o roke dlja oficial'nogo izdanija, prosto ne bylo drugogo puti. Kak my nazvali by ucenogo muza, vzdumavsego pred'javit' svoi samye cto ni na est' ser'eznye pretenzii Tvardovskomu v unison s An. Ivanovym i Proskurinym? Po men'sej mere neumnym celovekom, ibo umnyj dolyen ponimat', cto rec' idet ne ob estetike, a o demokratii: o prave govorit' $i$ prave zatykat' rot. Tak cto reklamu TELEVIZORA i OBLACNOGO KRAJA vse-taki ne stoit stavit' na odnu dosku s nazojlivym voschvaleniem B.Kuz'mina, skazem, v "Moskovskom komsomol'ce". Vprocem, v poslednee vremja situacija s rok-muzykoj v SSSR (ne tol'ko ideologiceskaja. no prezde vsego ekonomiceskaja i pravovaja) nacinaet menjat'sja Sledovatel'no, dolzen izmenit'sja i ton publikacij na etu temu - cto toze potrebuet nekotorogo vremeni na preodolenie inercii myslenija. Daze $v$ progressivnych redakcijach govorit' 0 rokkul'ture na ser'eznom urovne (prinjatom $v$ otnosenii teatra ili literatury) scitaet'sja durnym tonom, poetomy ljubaja profanacija imeet bol'se sansov na rasprostranenie $v$ massach posredstvom TV i "Sojuzpecati" cem kuda bolee neverojatnaja i zachvatyvajuscaja pravda. otsjuda i sumnaja kampanija vokrug kinematograficeskich podelok - kitca po imeni "Assa" i udrucajuscego primitiva po imeni "Rok".

\section{Analyseeinheit 30}

Morozov, D. [Kovriga, Oleg], 1989, Cas priliva. In: URLAJT, 5 (23).

Nedavno ja ponjal, cto pojavlenie zagolovka y menja vsegda proischodit po odnoj i toj ze scheme. Esli to, o cem ty pytaes'sja napisat', tebja dejstvitel'no donimaet, to navernjaka eto donimaet ne tol'ko tebja, i kto nibud' ure vyrazil eto $v$ suscestvenno bolee korotkoj i suscestvenno bolee vysokochudozestvennoj forme. Poetomy, poka vse eto «brodit» u tebja v golove. tam navernjaka vsplyvet i zagolovok, kotoryj na samom dele uze davno suscestvyet $i$ ne $v$ edinstvennom variante. Pri etom, pravda vsegda projavljaetsja otstojnyj effekt ozvucivanija plochich fil'mov chorosim pesnjami, tak cto, kogda pises', inogda kayetsja: nu vot, nakonec-to ja smog; a kogda potom citaes, stanovitsja ułasno stydno. No «brozenie»-to, vse ravno idet, a kogda vsja eta produksija vylivaetsja. vrode kak by stanovitsja i legce. 
V dannom slucae ja sam ne sovsem ponimaju, cto mne chocetsja skazat', poetomy i nazvanie ne ocen' podchodit, no tem ne menee, mne kazetsja, cto Baslaceskie slova vse ze imejut sjuda kakoe-to otnosenie:

Cas priliva probil.

Razbezalis' i nyrnuli.

Kto sumel - tot uplyl.

Ostal'nye utonuli.

A my s toboj otpolzli

I legli na meli.

My se pocetnom karaule.

Ran'se u nas slovo "mendezer» upotrebljalos' libo kak rugatel'stvo (naprimer: "na pobeguskach u podpol'nych menedzerov"), libo s igrivym ottenkom sredi svoich rebjat. Teper' uze ego casto proiznosjat vpolne ser'ezno.

Eto slovo vyzyvaet u menja primemo te oscuscenija, cto i platnye sortiry.

\section{Analyseeinheit 31}

anon., [Smirnov, Il’ja], 1989, Kolonka redaktora. In: URLAJT, 6 (24).

Nas izbiratel'nyj zakon vyzyvaet bol'soj interes vo vsem mire. "Deputaty ot obscestvennych organizacij" - nu $v$ kakoj sovremennoj evropejskoj strane mozno popast' $v$ parlament za uspechi v sobiranii marok ili za igru $v$ trezvost'?

Vprocem, my iscem paralleli ne tam, gde nużno. Nas nynesnij pariament - eto tipicnyj soslovnyj parlament Srednych vekov, tipa anglijskogo dvuchpalatnogo, gde pery naznacalis' korolem*, ili tech General'nych Statov, kotorye sobiral nescastnyj Lui XVI. Tak cto evropejcam ne stoit osobenno zadirat' pered nami nos: oni toze cerez vse eto prosli. A u nas i segodnja naznacennye deputaty "ot KPSS" i t.n. "obscestvennych organizacij" /v bol'sinstve svoem fiktivnych - eto ne cto inoe, kak garantirovannoe predstavitel'stvo vyssego soslovija, to est' bjurokratii, nasego sovremennogo dvorjanstva

I vse he mne ne chotelos' by bezogovorocno osuzdat' etu srednevekovuju sistemu. Vopervych, ona znamenuet soboj kolossal'nyj progress po sravneniju s razvitym socializmom, kogda my voobsce ne imeli nikakogo parlamenta i nase gosudarstevennoe ustrojstvo nachodilos' na urovne ne soslovnoj monarchii XV veka n.e., a aziatskoj despotii XV veka do Rozdestva Christova. A Verchovnyj Sovet, kotoryj naznacalsja na vse $100 \%$, bolee vsego napominal sjuzety drevneegipetskich rel'efov: kak vse nomy, soslovija i professii prichodjat vozdat' pocesti faraonu/verojatno, na etich ceremonijach toze sobljudalis' kakie-to normy predstavitel'stva procent ot Nubii, procent ot livijskich kocevnikov, ot ochotnikov na begemotov i t.d.l.

* Palata perov suscestvuet do sich por, no nikakoj real'noj vlasti ne imeet. 


\section{Analyseeinheit 32}

Romanov, Ju., 1989, Drikija. In: URLAJT, 7

Vo vremena pereocenki obscestvennych idealov crezvycajno vażny ljudi, ne utrativsie cistogo, pocti detskogo vzgljada na mir. Obsceceloveceskie cennosti prosty, no probirat'sja k nim prichoditsja cerez nagromozdenija slovesnych stampov.

Dzikija nemnogosloven $v$ obscenii. Ego rec' - ego kartinki. My govorim - on eto vidit i risuet. Slovo v russkoj kul'ture vsegda cenilos' neobycajno vysoko. Ditikija v revnosti svoej $k$ Dostoevskomu i Charmsu ovladevaet rec'ju, vyskazyvajas' izobrazitel'nymi frazami. Charms povlijal na nego sil'nej, cem lubok, otkrityj im mnogo pozdnee tech let, kogda Sasa, kak improvizator Puskina, delal kartinki na ljubuju temu, frazu, slovo, zakazannye prijateljami. Ego risunki na poljach nenapisannych povestej razrastalis' $v$ zakoncennye figurativnye kompozicii, tak cto tekst otodvigalsja v pravyj ugol, stanovjas' nazvaniem.

On probuet pisat' rasskazy, no slovo pecatnoe ne plasticno, a delat' iz nego stichogrammy nadrugat'sja nad smyslom, kotoryj stanovitsja dragocennost'ju tol'ko u velikich masterov Dostoevskij, Mikelandzelo. Ich velicie $v$ tom, cto im poddaetsja ljuboj material, ljuboe prostranstvo, no im malo technologiceskogo soversenstva, oni uvereny - esli oni cego-to ne sdelajut $-v$

U zisni net drugoj poloviny - vse proischodit zdec' i sejcas. To, cto mozno narisovat' segodnja, nevozmozno otlozit' do zavtra - umret, rassypletsja. Vospominanija i povtor tak ze daleki ot perezitogo custva, kak cholst ot kal'ki.

\section{Analyseeinheit 33}

anon. [Smirnov, Il'ja], 1991, Vspominaja o klube «Antares». Chippi. In: URLAJT, 8.

UR LAJT nacinaet publikaciju fragmentov is vospominanij aktivnogo ucastnika molodeznogo kluba "Antares" (70-e gg.). Vospominanija sostavljalis' neposredstvenno po sledam sobytij $i$ bez vsjakogo rasceta na publikaciju poetomu mnogocistennye lica i javlenija podpol'ja $v$ nich predstajut $v$ neskol'ko ... chm ... inom svete, cem $v$ lakirovannych publikacijach nasej "molodeznoj" pressy Imena inogda zameneny klickami ili psevdonimami - v sjuzetach kriminal'nych $\mathrm{i}$ intimnych.

My vybrali tri otryvka. o moskovskich chippi, o kommunarach, o debjute gruppy MUCHOMOR

(istorija nacinaetsja s togo, cto dvoe bojcov Antaresa, osnovatel'no potrepannye v drake. vybirajutsja iz lesa...)

Odnako plasc mne razorvali snizy doverchu, ruka byla razbita i sootvetstvenno vsja v krovi, a volosy dase ot legkogo poglasivanija vypadali puckami, kak pri oblucenii.

My vybralis' na odnu iz allej na territorii bol'nicy i pod fonarem izacali povrezdenija drug druga - Feliks smotrelsja ne lucse, kak vdrug zametili priblitajuscujussja zenskuju figurku. Massiruja razbituju gubu. Feliks napravilsja $k$ nej, ctoby poprosit' sigaretku. Pokurit' to my tak i ne uspeli. I chotja znakomstvo $v$ II casov na allee vozle lesa moglo by nastorozit' daze mastera po boksu, devuska ne ispugalas'. I sigartu u nee nastis', i ko mne oni podosli uze druz'jami.

Pol'skaja korotkaja modnaja kurtka - golubogo cveta s vorotnikom. firmennye dzinsy, strojnaja podpostkovaja figurka, cernye volosy - pochoła na bolgarku.

- Da .. - v svoju ocered' ocenila ona menja - Chipyem. 
Ili net, vru, tak po-svojski ona togda esce ne razgovarivala, a proizvodila vpecatlenie ocen' priličnoj devocki. Tak uz polucilos', cto ona zaderzalas' $v$ bol'nice. A voobsce to ona studentka 2-go meda, 3 kurs, zovut Inna.

I kakie ze my stali dzentl'meny: da cto vy, da kak vy poedete $v$ takuju noc' $v$ takuju dal', da voknug ze bandity, skrommuju devusku mogut obidet'. A u nas zdes' nepodaleky roskosnyj apportament s finskoj mebel'ju (chm...) v tisine i pokoe (da...) vy mozete tam perenocevat'.

\section{Analyseeinheit 34}

anon., 1991, Anons. Żivo! Pis'! In: CHERR (S MOSLOM), 3.

Tol'ko raspizdjajstvom i bezalabernost'ju możno ob'jasnit' fakt razmesenija etoj zametocki na etoj stranicke. A ne $v$ rubrike "siza". Vpred' kljanemsja ne lasłat'.

Predlagaem vasemu vnimaniju 3 raboty Alekseja "Stiva" Karkaeva. Nyne otbyvajusego pocetnuju povinnost' na odnoj iz zastav nasej rodiny.

Tjaga $k$ karandasu pojavilas'sja, kak vodit'sja, v detstve. No za neimeniem onogo (karandasa) rebenok obchodilsja melom ili kuskom kirpica, risuja na stenach domov. $\mathrm{Za}$ cto i byl neodnokratno bit. Nedobityj talant osvaival rez'bu po partam v skole vse 10 let i parallel'no $s$ etim uvlekalsja pod'ezdnym graffiti, specializinujas' na nazvanijach metalliceskich komand.

Nezaurjadnye sposobnosti pozvolili emu postupit' na rabotu v p/o "Liaz" v kacestve chudoznika, gde on risoval umnye lica vozdej mirovogo proletariata i pisal lozungi prizyvajusie rabocij klass - rabotat', trudjasichsja - truditsja, donorov - sdavat' bol'se krovi i t.d. i t.p.. Za cto i polucal zarplatu.

A dlja dusi ... dlja dusi on rabotal doma i v klube ljubitelej muzyki "Rok-Front", gde on sostojal clenom kruzka chudoznikov-momentalistov.

Koncepcija momentalistov - uroduj! Vot oni i urodovali, i nado skazat', neplocho polucalos'! Voz'mut, byvalo, portret Cernenko i urodujut, urodujut, urodujut... takie monstry u nich iz pod britvy i rucki vychodili - krov' styla v zilach!

No vse rete posesajusee vo vremja urodovanija cuvstvo sladostrastija i ekstaza, podviglo Stiva na tvorceskij podvig.

K etomu periodu otnositsja kartina "Djadjuska zek v zeltom sarfike".

\section{Analyseeinheit 35}

anon., 1991/1992, Sejşyn u Sergeica. In: CHERR (BEZ MOSLA), 4.

"27/11-91 v DK na plošcadi Puškina sostojalsja koncert s ucastiem grupp: "Natasa Rostova", Usastye Usi" "Vtorzenie Izvne"

(Po soobseniju TAZ - "Telefonnoj Agentury Zueva")

Ura! Razgul demokratii v Orechovo-Zuevskom rajone pozvolil nam poimet' uze az celych dva nezavizimych, partizanskich rok-izdanija, samovol'no prisvoivsich sebe gordoe nazvanie "zurnal". Pervoe - "Cherr s moslom" - v dekabre 1991 goda otmetilo svoju godovscinu. 
vtoroe - "Bambuk" - v nojabre zajavilo o sebe, kak o perspektivnom proekte, no, pravda, exce ne pojavilos'.

Naskol'ko my, redakcija "CHSM", ponimaem slorivsujus' situaciju, sej proekt, otpockovasijsja v svoe vremja ot "CHSM", vzjal na sebja trud ne tol'ko osvescat' vsevozmoznye meroprijatija, tak ili inace svjazannye $s$ rok-muzykoj, no i periodiceski organizovat' ili uz, na chudoj konec, pomogat' organizovat' onye.

(Vnimanie, uvazaemaja publika! Na scene pojavljajutsja te samye, preslovutye dva zajca! Sejcas vyskocet usatyj, zloj ochotnik i kogo-to spoknet! Esli tol'ko zajcy ne sgovorjatsja i ne spoknut ego samogo...)

Ne imeja poka na rukach samogo izdanija, my mozem poka lis' obsuzdat' dostoinstva i nedostatki pervych dvuch meroprijatij, neposredstvennoe ucastie $v$ organizacii kotorych prinjala demichovskaja gruppirovka rokofilov-florofagov "Bambyk".

Pervoe meroprijatie - festival' "Bambuk", planiruemyj kak dvuchdnevnyj Vudstok, nakrylsja zdorovennoj vaginoj, cto poverglo organizatorov $v$ unynie i mnogodnevnyj zapoj. Posledstviem vtorogo cut' bylo ne stala popytka emigracii $v$ odno iz aziatskich gosudarstv, gde, kak izvestno, klimaticeskie uslovija naibolee blagoprijatny dlja proizrastanija bambuka. 
Martin Bergmann - 9783954790531

Downloaded from PubFactory at 01/10/2019 02:45:57AM

(n)




\section{Literatur}

Bartmiński, Jerzy; Panasiuk, Jolanta, 1993, Stereotypy językowe. In: Barmiński, Jerzy (Hrsg.), Wspókczesny język polski. Wrocław, 363-387.

Benninghaus, Hans, 1996, Einfuhrung in die sozialwissenschaftliche Datenanalyse. 4. Aufl. München/Wien.

Bense, Max: Walther, Elisabeth, 1973, Wörterbuch der Semiotik. Köln.

Bense, Max, 1967, Semiotik. Allgemeine Theorie der Zeichen. Baden-Baden.

Bense, Max, 1971, Zeichen und Design. Semiotische Ästhetik. Baden-Baden.

Berelson, B., 1952, Content Analysis in Communication Research. Glencoe.

Bergmann, Martin, 1997, Die Funktion der Kommunikationssituation und textueller Merkmale bei der diskursadäquaten Bedeutungskonstruktion. Projektskizze einer empirischen Untersuchung. In: Zet. Zeitschrift für Empirische Textforschung, 3 (im Druck).

Bußmann, Hadumod, 1990, Lexikon der Sprachwissenschaft. 2., völlig neu bearbeitete Aufl. Stuttgart.

Cernov, Sergej, 1996, http://alice.ibpm.serpukhov.su/oldfriends/spbweb/lifestyl/88/pages.html

Drews, Axel; Gerhard, Ute; Link, Jürgen, 1985, Moderne Kollektivsymbolik. Eine diskurstheoretisch orientierte Einfuhrung mit Auswahlbibliographie. In: Frühwald, Wolgang, Jäger, Georg; Martino, Alberto (Hrsg.), Internationales Archiv für Sozialgeschichte der deutschen Literatur. 1. Sonderheft Forschungsreferate, 256-295.

Drummond, D. A.; Perkins, G., 1987, Dictionary of russian obscenities. Third, revised edition. Oakland

Eimermacher, Karl (Hrsg.), 1986, Semiotica sovietica 1. Sowjetische Arbeiten der Moskauer und Tartuer Schule zu sekundären modellbildenden Zeichensystemen (1962-1973). In: Aachener Studien zur Semiotik und Kommunikationsforschung Band 5.1.

Eimermacher, Karl, 1974, Arbeiten sowjetischer Semiotiker der Moskauer und Tartuer Schule. Kronberg/Ts.

Fajn, A; Lur'e, V. 1991, Vse v kajf! Slovar'. Moskva.

Finke, Peter, 1982, Konstruktiver Funktionalismus. Die wissenschaftstheoretische Basis einer empirischen Theorie der Literatur. Braunschweig/Wiesbaden.

Fischer, Hans Rudi (Hrsg.), 1993, Information, Kommunikation und Sprache. Fragen eines Beobachters. In: Autopoiesis. Eine Theorie im Brennpunkt der Kritik. 2., korrigierte Aufl. Heidelberg, 67-97.

Fleischer, Michael, 1989, Die sowjetische Semiotik. Theoretische Grundlagen der Moskauer und Tartuer Schule. Tübingen.

Fleischer, Michael, 1994, Die Wirklichkeit der Zeichen. Empirische Kultur- und Literaturwissenschaft (systemtheoretische Grundlagen und Hypothesen). Bochum.

Fleischer, Michael, 1995, Stereotype und Normative aus der Perspektive der Systemtheorie. In: Zet. Zeitschrift furr Empirische Textforschung, 2, 7-17. 
Fleischer, Michael, 1996, Weltbildgesteuerte Wirklichkeitskonstruktion. Beiträge zum Phänomen Weitbild. München.

Fleischer, Michael, 1997a, Weltbildgesteuerte Wirklichkeitskonstruktion. Beiträge zum Phänomen Weltbild. Band 2: Aspekte russischer und polnischer Kultur. München.

Fleischer, Michael, 1997b, Das System der russischen Kollektivsymbolik. Eine empirische Untersuchung München.

Foucault, Michel, 1971, Die Ordnung der Dinge. Frankfurt/M.

Foucault, Michel, 1973, Archäologie des Wissens. Frankfurt/M.

Foucault, Michel, 1974, Die Ordnung des Diskurses. München.

Früh, W., 1989, Inhaltsanalyse. Theorie und Praxis. 2. Aufl. München.

Gilinskij, Ja. I., 1996, Deviantnoe povedenie molodezi, prestupnost' i social'naja praktika. In: V. T. Lisovskij (Hrsg.), Sociologija molodezi: Ucebnik. Sankt-Peterburg, 279-315.

Glasersfeld. Emst von, 1987, Wissen, Sprache und Wirklichkeit. Abeiten zum radikalen Konstruktivismus. Braunschweig/Wiesbaden.

Glasnost', Michail, 1988, 100 [Hundert] schmutzige russische Wörter. Kyrillisch, Lautschrift, deutsch. Frankfurt/M.

Göttert, Kart-Heinz, 1994, Einfuihnung in die Rhetorik. Grundbegriffe - Geschichte - Rezeption. 2., verbesserte Aufl. München.

Groeben, Norbert; Rustemeyer, Ruth, 1995, Inhaltsanalyse. In: König, E; Zedler, P. (Hrsg.), Bilanz qualitativer Forschung. Bd. 2: Methoden. Weinheim, 523-554.

Gur'ev, Sergej, 1994, Pogranicnye stolby rok-samizdata. Opyt opisanija opisanija. In: Kusnir, Aleksandr (Hrsg.), Zolotoe podpol'e. Polnaja illjustrirovannaja encikJopedija rok-samizdata 1967 - 1994. Istorija, Antologija. Bibliografija. Niznyj Novgorod, 5-7.

K. Lejn (Hrsg.), 1989, Russko-nemeckij slovar' (osnovnoj). Ok. 53000 slov. 10. verbesserte und erweiterte Aufl. Moskva.

K. Lejn (Hrsg.), 1992, Nemecko-russkij (osnovnoj) slovar' Ok. 95000 slov. Moskva

Koester, Soia, 1983, Anglicizmy $\vee$ molode/nom zargone. In. Zielsprache Russisch Zeitschrif fur den Russischunterricht, 3, 82-84.

Koester-Thomas, S.; Rom, E., 1985, Wörterbuch der modernen russischen Umgangssprache Munchen.

Kon, I S., 1996, Junost' kak social'naja problema. § I. Vozrastnaja stratifikacija. In: V. T. Lisovskij (Hrsg.), Sociologija molodetii: Ucebnik. Sankt-Peterburg, 81-87.

Kusnir, Aleksandr (Hrsg.), 1994a, Zolotoe podpol'e. Polnaja illjustrirovannaja enciklopedija rok-samizdata 1967 - 1994. Istorija, Antologija, Bibliografija. Niznyj Novgorod.

Kusnir, Aleksandr (Hrsg.), 1994b, Iz pod pressa vremeni. In: 1994, Zolotoe podpol'e. Polnaja illjustrirovannaja enciklopedija rok-samizdata 1967 - 1994. Istorija, Antologija, Bibliografija Niznyj Novgorod. 8-10.

Kuhn, Thomas S., 1973, Die Struktur wissenschaftlicher Revolutionen. FrankfurtM.

Lamnek, S., 1989, Qualitative Sozialforschung. Band 2: Methoden und Techniken. München. 
Lane, David, 1992, Soviet society under perestoika. Completely revised edition. London/New York.

Link, Jürgen; Parr Rolf, 1989, Militarisierung der $>$ Sprache $<$ ? Zum Verhältnis von elementarer Literatur, Mediendiskursen und subjektiver Aufrüstung. In: Förster, Jürgen; Neuland, Eva; Dupp, Gerhard (Hrsg.), Wozu noch Germanistik? Stuttgart.

Link, Jürgen; Link-Herr, Ursula, 1989, Kollektivsymbolik und Orientienungswissen. Das Beispiel des ,Technisch-Medizinischen Vehikel-Körpers'. In: Der Deutschunterricht, 4, 44-55.

Link, Jürgen, 1975, Die Struktur des literarischen Symbols. Theoretische Beiträge am Beispiel der späten Lyrik Brechts.

Link, Jürgen, 1979, Tzvetan Todorov: Théories du symbole. Paris: Seuil, 1977. $8^{\circ} .378$ S. In: Poetica, 3/4, 473-481.

Link, Jürgen, 1982, Kollektivsymbolik und Mediendiskurse. Zur aktuellen Frage, wie subjektive Aufrüstung funktioniert. In: kultuRRevolution, 1, 6-21.

Link, Jürgen, 1983, „Der irre Saddam setzt seinen Krummdolch an meine Gurgel!“ Fanatiker, Fundamentalisten, Irre und Traffikanten - das neue Feinbild Süd. In: Jäger, Siegfried (Hrsg.), Kritische Diskursanalyse. Eine Einfuhrung. Duisburg.

Link, Jürgen, 1983, Elementare Literatur und generative Diskursanalyse. München.

Link, Jürgen, 1986, Noch einmal: Diskurs. Interdiskurs. Macht. In: kultuRRevolution, 11, 47.

Link, Jürgen. 1991, Konturen medialer Kollektivsymbolik in der BRD und in den USA. In: Grzybek, Peter (Hrsg.), Cultural Semiotics: Facts and Facets. Fakten und Facetten der Kultursemiotik. Bochumer Beiträge zur Semiotik, 26, 95-135.

Link, Jürgen, 1992, Die Analyse der symbolischen Komponenten realer Ereignisse. Ein Beitrag der Diskurstheorie zur Analyse neorassistischer Äußerungen. In: Osnabrücker Beiträge zur Sprachtheorie (OBST), 46, 37-52.

Lisch, Ralf; Kriz, Jürgen, 1978, Grundlagen und Modelle der Inhaltsanalyse. Bestandsaufnahme und Kritik. Reinbek.

Lotman, Ju. M., 1973, Die Struktur des künstlerischen Textes. Frankfurt/M.

Marder, Stephen, 1992, A Supplementary Russian-English Dictionary. Ohio.

Maturana, Humberto R. 19852, 1982, Erkennen: Die Organisation und Verkörpenung von Wirklichkeit. Ausgewählte Arbeiten zur biologischen Epistemologie. Braunschweig.

Maturana, Humberto R., 19942, 1987, Kognition. In: Schmidt, Siegfried J. (Hrsg.), Der Diskurs des Radikalen Konstruktivismus. Frankfurt, 89-118

Mayntz, R.; Holm. K.; Hübner, P., 1974, Einführung in die Methoden der empirischen Soziologie. 3. Aufl. Opladen.

Mazin, Kirill, 1996, http://trailerpark.com/phasel/kmazin/civdef/civdef.html

Meerson-Aksenov, Michael (Hrsg.), 1977, The political and religious thought of Russian "Samizdat": an anthology. Belmont.

Meier, Georg F., 1976, Die historischen Wurzeln der Bedeutungsfeldproblematik. In: Phonetik, Sprachwissenschaft und Kommunikationsforschung, 29, 551-555.

Merten, Klaus, 19952, 1983, Inhaltsanalyse. Einführung in Theorie, Methode und Praxis. Opladen. 
Mineeva, Z. I. 1996, Sociologija kul'tury i meznacional'nych otnosenij. § 3. Molodełnyj zargon: igra ili vyzov?. In: V. T. Lisovskij (Hrsg.). Sociologija molodezi: Ucebnik. SanktPeterburg. 348-360.

Nöth, Winfried, 1985, Handbuch der Semiotik. Stuttgart.

Ozegov, S. I., 1972, Slovar' russkogo jazyka. Ok. 57000 slov. 9. verbesserte und erweiterte Aufl. Moskva.

Peirce, Charles S., 19932, 1983, Phänomen und Logik der Zeichen. Hrsg. und übers. von H. Pape. FrankfurtM.

Pilkington, Hilary, 1996, Gender, generation and identity in contemporary Russia. London.

Pilkington, Hilary, 1994, Russia's youth and it's culture: A nation's Constructors and Constructed. London.

Popper, Karl, 19732a, 1972, Objektive Erkenntnis. Ein evolutionärer Entwurf. Hamburg.

Popper, Kart, 1973b, Logik der Forschung. Tübingen.

Prochorov, L. M. (Hrsg.), 1975³, Bol'saja sovetskaja enciklopedija. Moskva.

Quasthoff, U., 1989, Ethnozentrische Verarbeitung von Information: Zur Ambivalenz der Funktionen von Stereotypen in der interkulturellen Kommunikation. In: Matusche, Petra (Hrsg.), Wie verstehen wir Fremdes? Aspekte zur Klärung von Verstehensprozessen. München. 37-62.

Riedl, Rupert, 1975, Die Ordnung des Lebendigen. Systembedingungen der Evolution. Hamburg.

Röttgers, Kur, 1988, Diskursive Sinnstabilisation durch Macht. In J. Fohrmann; H. Müller (Hrsg.), Diskurstheorien und Literaturwissenschaft. Frankfurt/M., 114 - 133.

S'epanskaja. Tat'jana Borisovna, 1993, Simvolika molodernoj subkul'tury. Opyt etnograficeskogo issledovanija sistemy 1986-1989 gg. Sankt-Peterburg

Schmidt, Siegfried J., 1980, Gundriss der Empirischen Literaturwissenschaft. Teilband I Der gesellschaftiche Handlungsbereich Literatur. Braunschweig/Wiesbaden.

Sikevic. Z V. 1996. Sociologija kul'tury i mexnacional'nych otnosenij $§ 1$ Molodesnaja subkul'tura v sovremennoj Rossii. In Sociologija molodezi: Ucebnik. Hrsg. V. T. Lisovskij. Sankt-Peterburg, 332-341

Skilling. Harold Gordon, 1989, Samizdat and an independent society in Central and Eastem Europe. Columbus

Stadler, Michael, Kruse, Peter, 1990, Über Wirklichkeitskriterien. In: Riegas, Volker; Vetter, Christian (Hrsg.), Zur Biologie der Kognition. Ein Gespräch mit Humberto R. Maturana und Beiträge zur Diskussion seines Werkes. Frankfurt/M., 133-158.

Stadler, Wolfgang, 1992, «Rumnata, kaif, tusovka»: Integrierter Landeskundeunterricht am Beispiel «Molodeznaja kul'tura $v$ Rossii». In: Zielsprache Russisch. Zeitschrift für den Russischunterricht, 4, 115-125.

Tüschau 16, 1996, Die Diskurssymbolik in alternativen Subkulturen in Deutschland. Eine Erhebung. In: Zet. Zeitschrift für Empirische Textforschung, 2, 97-102.

Tüschau 16, 1998, Die Darstellung anderer Kulturen. Ermittlung von Stereotypen in deutschen Polen-Reisefuhrem (der Jahre 1990 - 1996). Oberhausen. 
Tüschau 16, 1998b, Die subkulturellen Symbole der Punks. Eine empirische Erhebung. Oberhausen.

Ueding, Gert; Steinbrink, Bernd, 1994, Grundriß der Rhetorik. Geschichte - Technik - Methode. 3., überarbeitete und erweiterte Aufl. Stuttgart/Weimar.

Ueding, Gert, 19962, 1995, Klassische Rhetorik. München.

Vorob'ev, Gennadij Grigorevic., 1990, Molodez v informacionnom obscestve. Moskva.

Weingart, P. (Hrsg.), 1972, Wissenschaftssoziologie I. Wissenschaftliche Entwicklung als sozialer Proze $B$. Ein Reader mit einer kritischen Einleitung des Herausgebers. Frankfurt/M.

Willke, Helmut, 1976, Funktionen und Konstitutionsbedingungen des normativen Systems der Gruppe. In: Kölner Zeitschrift für Soziologie und Sozialpsychologie, 1, 426-450.

Willke, Helmut, 1994, Systemtheorie. Eine Einfuihrung in die Grundprobleme der Theorie sozialer Systeme. 4. überarb. Aufl. Stuttgart/Jena. 


\section{DIE WELT DER SLAVEN SAMMELBÄNDE - СБОРНИКИ}

Herausgegeben von Peter Rehder und Igor Smimov

Band I: Anton P. Čechov

Philosophische und religiöse Dimensionen im Leben und im Werk

Vorträge des Zweiten Internationalen Cechov-Symposiums Badenweiler, 20.-24. Oktober 1994

Herausgegeben von Vladimir B. Kataev, Rolf-Dieter Kluge, Regine Nonejl 1997. Hardcover. XXII. 641 S. 140.- DM. (ISBN 3-87690-675-X)

Band 2 Beiträge der Europäischen Slavistischen Linguistik (POLYSLAV) Band 1

Herausgegeben von Markus Giger und Bjöm Wiemer 1998. Hardcover. X. 212 S. 86.- DM. (ISBN 3-87690-705-5)

Band 3:

Lebenskunst - Kunstleben Жизнетворчество

в русской культуре XVIII - XX вв.

Herausgegeben von Schamma Schahadat 1998. Hardcover. 229 S. 86.- DM. (ISBN 3-87690-706-3)

Band 4:

Beiträge

der Europäischen Slavistischen Linguistik (POLYSLAV) Band 2

Herausgegeben von Katharina Böttger, Markus Giger und Björn Wiemer 1999. Hardcover. VIII. 320 S. I12.- DM. (ISBN 3-87690-738-1)

Band 5:

Festschrift

für Klaus Trost zum 65. Geburtstag

Herausgegeben von E. Hansack. W. Koschmal, N. Nübler, R. Vecerks 1999. Hardcover. 355 S. 120.- DM. (ISBN 3-87690-739.X)

\section{VERLAG OTTO SAGNER}

\section{D-80328 MUNCHEN}

Telefon: (089) 54 218-0 - e-mail: postmaster@kubon-sagner.de 